\title{
THE EFFECTS OF MITOGENIC AND METABOLIC CUES ON CLATHRIN-MEDIATED ENDOCYTOSIS
}

\author{
by \\ Ralph Christian Delos Santos \\ B.Sc.(Hons.), Ryerson University, 2012
}

\author{
A Dissertation \\ presented to Ryerson University \\ in partial fulfillment of the \\ requirements for the degree of \\ Doctor of Philosophy \\ in the Program of \\ Molecular Science
}

Toronto, Ontario, Canada, 2019

(C) Ralph Christian Delos Santos, 2019 


\title{
(C) Copyright by Ralph Christian Delos Santos 2019 All Rights Reserved
}

\author{
AUTHOR'S DECLARATION FOR ELECTRONIC SUBMISSION OF A DISSERTATION \\ I hereby declare that I am the sole author of this dissertation. This is a true copy of the dissertation, \\ including any required final revisions, as accepted by my examiners. \\ I authorize Ryerson University to lend this dissertation to other institutions or individuals for the \\ purpose of scholarly research. \\ I further authorize Ryerson University to reproduce this dissertation by photocopying or by other means, \\ in total or in part, at the request of other institutions or individuals for the purpose of scholarly research.
}

I understand that my dissertation may be made electronically available to the public. 


\title{
The effects of Mitogenic and Metabolic cues on clathrin-mediated endocytosis
}

Ralph Christian Delos Santos

Doctor of Philosophy, 2019

Molecular Science, Ryerson University

\begin{abstract}
The cell 'surfaceome' collectively describes proteins found on the plasma membrane (PM), which functions in fundamental cellular processes including growth and proliferation. The surfaceome undergoes dynamic remodeling via the addition/removal of surface proteins in response to changing environmental conditions. In mammalian cells, surfaceome remodeling is predominantly facilitated by clathrin-mediated endocytosis (CME) which involves the invagination and internalization of PM regions via clathrin-coated structures-removing proteins from the cell surface. As a major regulator of the surfaceome, it is important to understand the underlying mechanisms governing CME, given that its dysregulation has been implicated in human pathologies including neurologic and oncogenic disorders.
\end{abstract}

Cellular cues including mitogenic (e.g. growth factors) and metabolic signals (e.g. cellular energy levels) induce diverse cellular processes (e.g. growth and proliferation) requiring surfaceome/PM remodeling. Precisely how mitogenic and metabolic signals may induce surfaceome remodeling however, is under-examined. As a major regulator of the surfaceome, CME is a likely mechanism through which cellular cues may remodel the PM. Poorly understood, I thus sought to investigate how mitogenic and metabolic signals may regulate CME.

Mitogenic signaling by the epidermal growth factor receptor (EGFR) triggers PLC 11-calcium signals, which I found a requirement for CME of EGFR-likely via calcium control of the Sjn1 protein. Consistently, using TIRF-M imaging coupled to automated software analysis, I demonstrate that inhibition of PLCY1-calcium signals impairs the formation/assembly of EGFR-containing clathrin structures. In addition, I hypothesize that PLC 1 1-calcium signals also regulates the CME of other surface proteins given its robust control of Sjn1-which localizes broadly amongst clathrin-coated structures. 
AMPK is a cellular energy sensor activated by metabolic stress (e.g. starvation). Using TIRF-M imaging coupled to automated software analysis, I found that AMPK activation broadly reduces the formation/assembly of bona fide clathrin-coated pits, without impairing the internalization rates of CME cargoes (e.g. EGFR, TfR and $\beta 1$-integrin). Furthermore, I found that AMPK may regulate CME through control of the Arf6 protein.

Collectively, my findings uncover and provide novel mechanisms by which mitogenic (via EGFRinduced calcium signals) and metabolic signals (via AMPK control of Arf6) may induce regulation of $\mathrm{CME}$-in eliciting global reorganization of the plasma membrane. 


\section{Acknowledgements}

Finally! The last couple of years I have envisioned this moment - the day I finally get to sit down and pen words of gratitude for those who ran alongside my pursuit of the PhD. Six years filled with wonderful memories and unforgettable experiences, I have grown not only as a scientist but also watched myself transform into a whole new person (a better one of course). Graduate work is often regarded as a very lonely road ${ }^{14}$, and so I am extremely grateful for having found myself in a challenging, yet supportive environment; this dissertation is a reflection of that. I am both thankful and proud of my scientific achievements and academic accolades, for they provide tangible metrics justifying my 'Stupid, Stupid Decision to Go to Grad School' (Ruben, 2010). Still, I am most grateful and deeply indebted to the people who made this all possible, many of which duly deserve explicit recognition.

First, I would like to express my deepest appreciation to my supervisor, occasional arch-nemesis and fellow Hakka-cuisine enthusiast, Dr. Costin Antonescu. I don't think I can thank him enough for giving me the opportunity of fulfilling my dreams of becoming a bona fide ECP scientist. He had been a guiding force during difficult times and I am forever indebted to him for his unwavering guidance and support. I couldn't have asked for a better mentor ${ }^{15}$. Special thanks goes out to Dr. Roberto Botelho for constantly providing invaluable insight and constructive feedback to improve my work. His untiring energy and humour provided much-needed boosts in times when academic formalities became dull ${ }^{16}$. I would also like to gratefully acknowledge Dr. Jeffrey Fillingham, Dr. Rene Harrison, Dr. Raffi Karshafian and Dr. Eric Kam. Their helpful advice, insightful comments, and patience cannot be underestimated in the completion of my dissertation. To the program administrators Maria Landau and Sarah Kovacs, a sincere appreciation goes out to them for continually exercising the flexibilities of internal deadlines. I will not be poised to graduate without their help.

To Camilo, Choy, Michael, Krishna, GM, Alejandro and Gurjeet, I am beyond thankful for the support, encouragement, and the genuine camaraderie we shared in our early graduate years. I would not have been as keen to pursue a doctorate were it not for our early days in KHE-319 ${ }^{17}$. You've all taught me that 'Time you enjoy wasting is not wasted time'. To John and Louis, thank you for keeping me sane in the face of intense stress. I am deeply grateful for your tireless encouragements and comic reliefs which motivated me to push through the difficult stages of my dissertation. I look forward to doing the same for you both in the near future ${ }^{18}$. To Chris and Mark, thank you for your unconditional 
friendship and optimism, for believing in me in times of self-doubt, and for inspiring me to always be better. I hope to repay all the bar tabs uponsubmission of this dissertion on ${ }^{19}$. To Stephen and Jeffrey, we've come a long way since Gr.12 biology. I am deeply thankful for your continuous support ${ }^{20}$.

To Denise. Thank you for being there, for listening to my daily rants, for reminding me there is life and normalcy beyond this dissertation, and for always believing and never doubting my capabilities. Thank you for being kind and patient, and for taking on a role you never asked for ${ }^{21}$. You are my partner in crime, best friend, and my hero! To Marie, Bill and Hunter. Thank you for your unwavering excitement for my successes, no matter how small. You have supported me in more ways than you can imagine. Thank you for constantly believing and reassuring me that there is always light at the end of every tunnel $\left.\right|^{22}$.

To my parents Gladys and Rafael, there are no words. You have devotedly supported me during the lowest of my lows. I am deeply grateful for your unconditional love and support. Thank you for teaching me how to be kind, and inspiring in me the courage to shoot for the stars. You are my motivation and the reason for this moment. Your sacrifices are unquantifiable and for all you have done for me, I am forever indebted. To my brother Ralph, as proud as you are of me, I am equally if not more proud of you for doing things I did not. You are my protector who inspires me to be courageous and to be the best of myself every day. You always believed I would live up to my potential even in times when I could not share your certainty. Thank you for letting me be stupid with you and standing by me when the going was tough ${ }^{23}$. To my sister Katrina, thank you for repeatedly reigniting my drive to persevere during the difficult stages of my dissertation. Your fearlessness, boldness and confidence is contagious ${ }^{24}$. You continue to inspire me to work passionately and to face adversity head-on. To my sister Rachel, we share the most inside jokes out of anyone else I know ${ }^{25}$. Thank you for always putting a smile on my face and for always reminding me that 'laughter is poison to fear'. You continue to motivate me in many ways you do not realize. I wish to have as much grit and resilience as you someday. To my best-pal Poncho $^{26}$. You were small and did not make much noise for nine years. Yet, you had a loud impact. You are my blessing and I am better for having known you.

Lastly, to my family ${ }^{27}$, friends ${ }^{28}$ and colleagues ${ }^{29}$ who have-in some way, shape or formpositively influenced me throughout what I can only describe as an arduous test of my mental fortitude, your impact has not gone unnoticed-and for that I am sincerely grateful. 
Dedicated to my Mom and Dad,

who instilled in me the love of learning.

Thank you for your unconditional love and support.

And to my future self,

we did it! 


\section{Table of Contents}

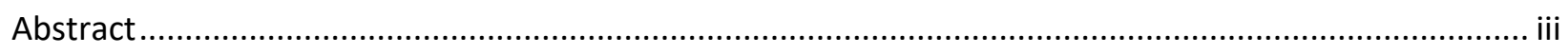

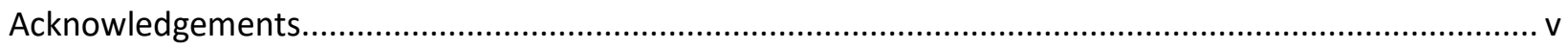

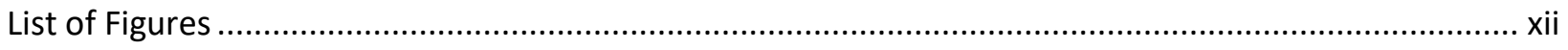

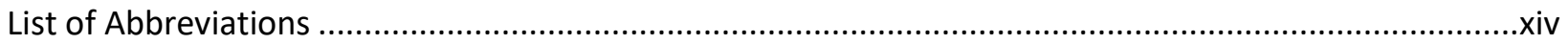

Chapter 1. Introduction ..........................................................................................

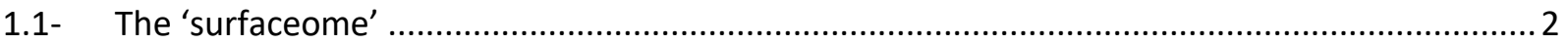

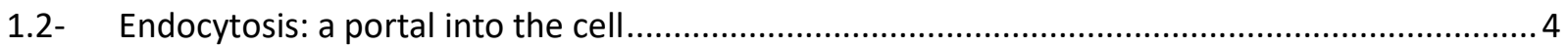

1.2.1 - A conceptual map for the reader: topics to be discussed ........................................... 6

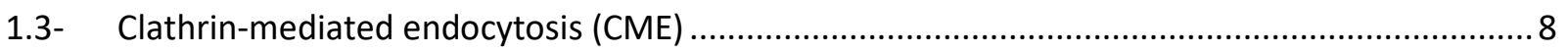

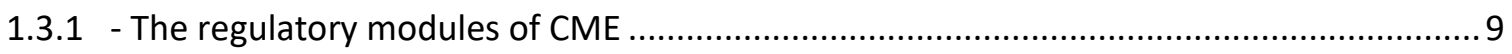

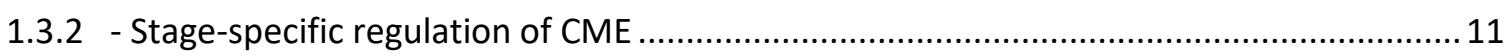

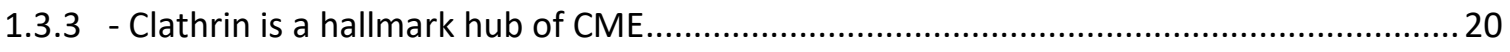

1.3.4 - Adaptor protein-2 (AP2) complex is a major adaptor hub of CME.................................25

1.3.5 - Phosphatidylinositol-(4,5)-bisphosphate $\left(\mathrm{PIP}_{2}\right)$ is a CME lipid hub ................................ 33

1.4- Phospholipase C (PLC) and its potential role in CME regulation ......................................... 40

1.4.1 - The conserved core structure of PLCs facilitates PIP 2 hydrolysis .................................. 41

1.4.2 - PLCs are characterized by distinct domain inserts and extensions .................................. 41

1.4.3 - PLCs induce cytosolic calcium increases via PIP 2 hydrolysis .......................................... 45

1.4.4 - PLCs may regulate CME via direct $\mathrm{PIP}_{2}$ hydrolysis and/or calcium signals .......................46

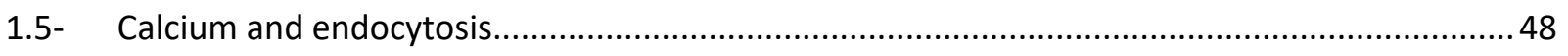

1.5.1 - Calcium gradients are maintained by channels and pumps ............................................ 48

1.5.2 - Calcium signals are decoded by calcium binding proteins (CBPs) .................................50

1.5.3 - Calcium is required for endocytosis in excitable cell systems ....................................... 51

1.5.4 - The potential role of calcium is non-excitable cell systems ..........................................53

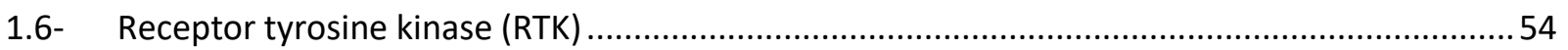

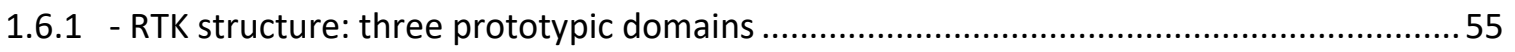

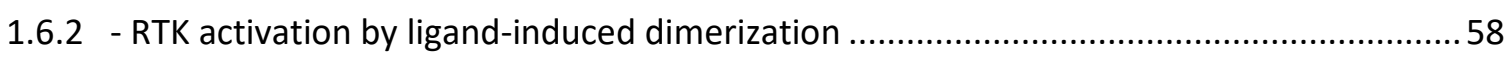

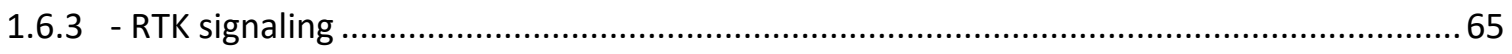

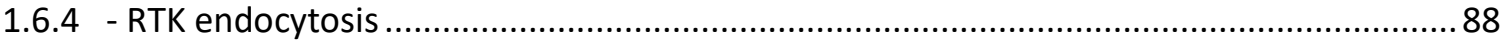


1.7- Metabolic signals may regulate the plasma membrane via CME ........................................95

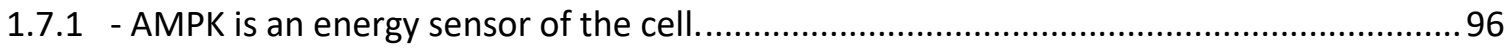

1.7.2 - The Arf family of small GTPases are major regulators of membrane traffic .................100

1.7.3 - AMPK may induce global plasma membrane remodeling via Arf6 ..............................103

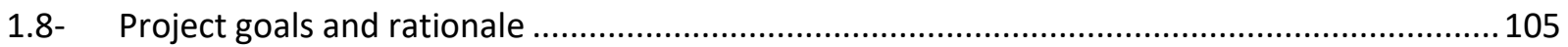

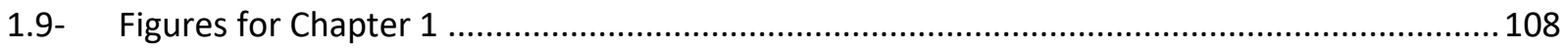

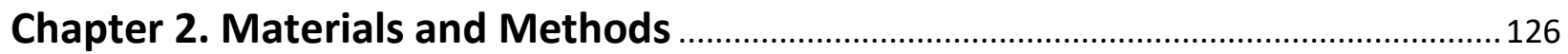

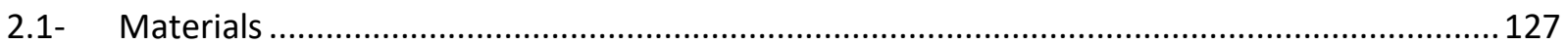

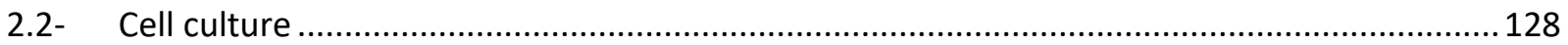

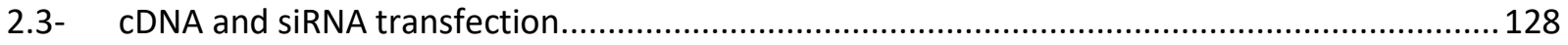

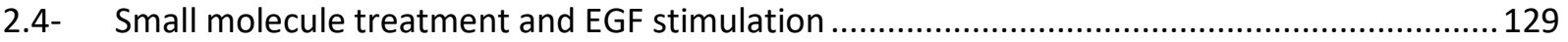

2.5- TIRF-M measurement of cell-surface $\mathrm{PIP}_{2}$ and $\beta 1$-integrin abundance .................................130

2.6- Biochemical measurement of phosphoinositide levels ...................................................... 131

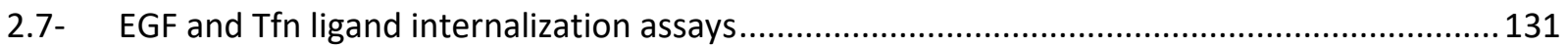

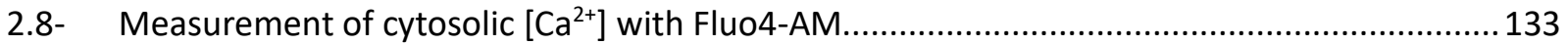

2.9- TIRF-M and automated software image analysis of CLSs in fixed cells ................................. 134

2.9.1 - Fixed sample preparation for CLS detection and analysis by TIRF-M...........................134

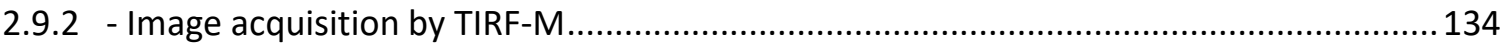

2.9.3 - CLS detection and quantification of protein fluorescence intensity within CLSs ...........135

2.9.4 - Measurement of mean fluorescence of ligand or cytosolic proteins within CLSs..........135

2.9.5 - Generation of two-dimensional histogram of EGF and Tfn fluorescence within CLSs .. 135

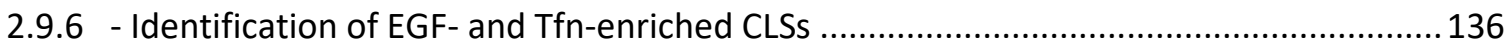

2.10- TIRF-M and automated image analysis of CCP properties (time-lapse imaging).................136

2.11- Whole-cell lysates and Western blotting ........................................................................... 138

\section{Chapter 3. Determining the role of PLC- $ү 1$ in EGFR endocytosis ............................139}

3.1- PLC- $ү 1$ selectively controls clathrin-mediated endocytosis of EGFR ......................................141

3.2- PLCY1-derived intracellular calcium is selectively required for EGFR endocytosis...................142

3.2.1 - PLC- $\gamma 1$ does not broadly regulate plasma membrane PIP $_{2}$ concentrations ...................143

3.2.2 - PLC- $\gamma 1$ regulates EGFR endocytosis via stimulation of intracellular calcium .................144

3.2.3 - PLCY1-calcium control of EGFR endocytosis is mechanistically distinct from that in neuronal systems and may involve PKC. ..... 
3.3- EGFR and TfR localize largely to distinct CLSs ............................................................... 147

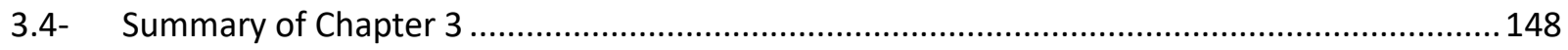

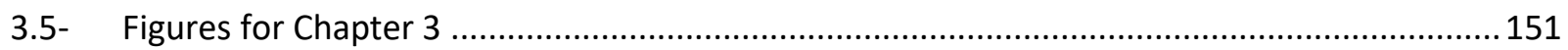

\section{Chapter 4. Understanding how calcium signals impact CME dynamics ..................164}

4.1- $\quad$ EGFR-derived calcium signals regulate CCP initiation and assembly ....................................166

4.1.1 - PLC- $ү 1$ does not regulate the recruitment of EGFR within CLSs ...................................166

4.1.2 - PLC- $\nu 1$ regulates the assembly and maturation of EGFR-containing CCPs....................167

4.2- PLC- $\gamma 1$ regulates EGFR-containing CCPs by removal of Sjn1 .............................................170

4.3- PLC- $\gamma 1$ is required for clathrin-dependent EGFR signaling .................................................173

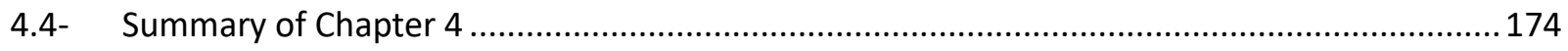

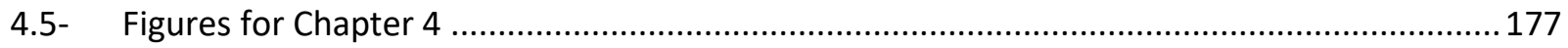

Chapter 5. Understanding the role of metabolic signals in control of CME .........195

5.1- Activation of AMPK elicits global reduction in CCP size....................................................198

5.2- AMPK may regulate CME via control of Arf6 activity ..........................................................199

5.2.1 - AMPK may regulate Arf6 by promoting GTP hydrolysis .............................................200

5.2.2 - Arf6 interacts and localizes within CCPs in a GTP-dependent manner ..........................201

5.2.3 - Regulation of Arf6 controls clathrin assembly and function .......................................203

5.3- $\quad$ AMPK selectively regulates CME of surface cargoes .........................................................204

5.3.1 - AMPK selectively enhances the endocytosis of $\beta 1$-integrin containing CCPs ................204

5.3.2 - AMPK enhances $\beta 1$-integrin recruitment to CCPs and may selectively regulate integrin-

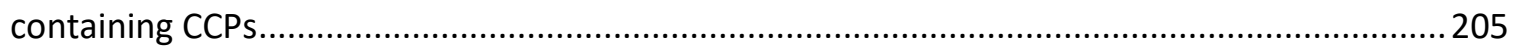

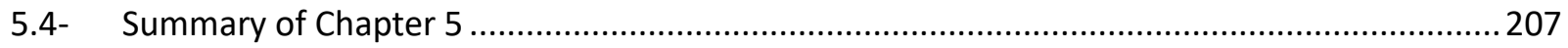

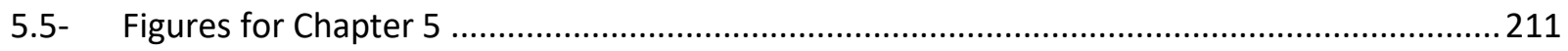

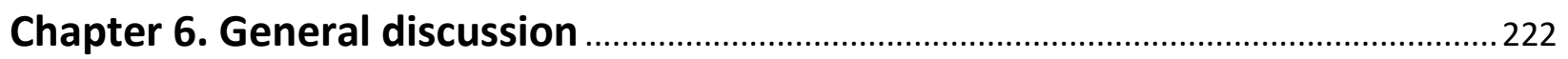

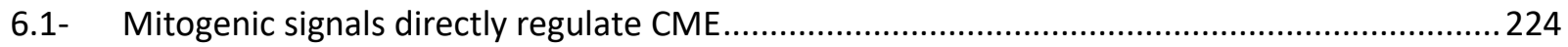

6.1.1 - Regulation of CCPs and clathrin-mediated endocytosis by intracellular calcium ..........224

6.1.2 - Distinct regulation of clathrin-mediated endocytosis of EGFR versus TfR ....................228

6.1.3 - Regulation of EGF-stimulated Akt phosphorylation by PLC $\gamma 1$-derived signals...............230

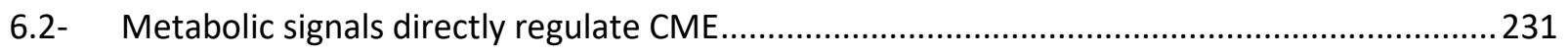

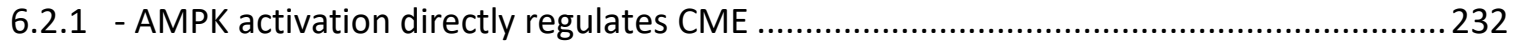


6.2.2 - AMPK may regulate CME via control of Arf6 activity

6.2.3 - AMPK activation may differentially regulate CME of surface cargoes ..........................237

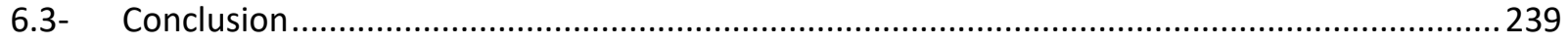

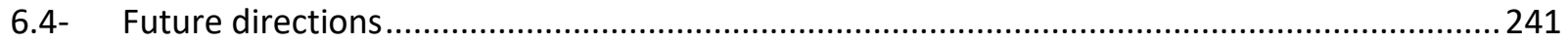

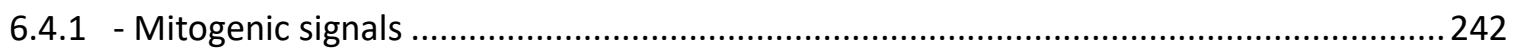

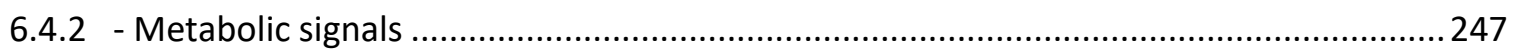

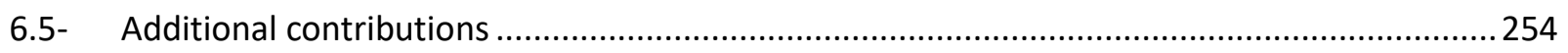

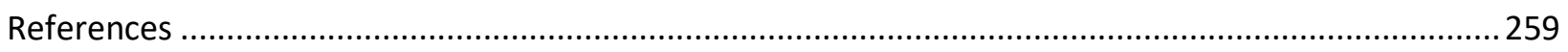




\section{List of Figures}

\section{Chapter 1}

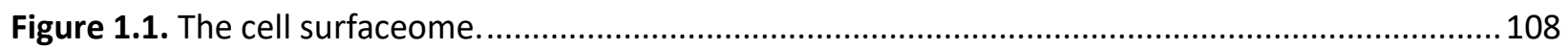

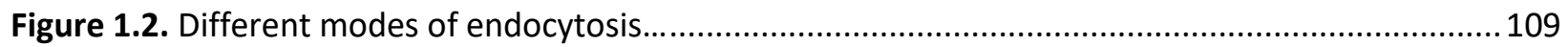

Figure 1.3. Clathrin-mediated endocytosis is defined by morphologically distinct stages. ...................110

Figure 1.4. Clathrin-mediated endocytosis is regulated by three core hubs. ......................................111

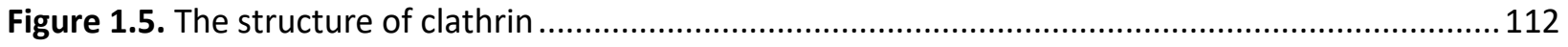

Figure 1.6. The structure of the adaptor protein complex-2 ...........................................................113

Figure 1.7. A large conformational change is required for the activation and stabilization of AP2. .......114

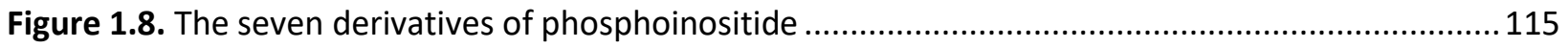

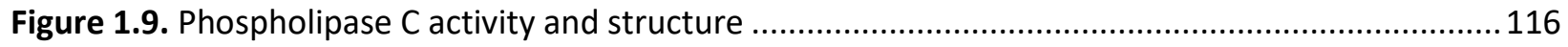

Figure 1.10. Maintenance of the cellular calcium gradient..............................................................118

Figure 1.11. The different families of receptor tyrosine kinases (RTK) ...............................................119

Figure 1.12. The major signaling pathways of EGFR.......................................................................... 120

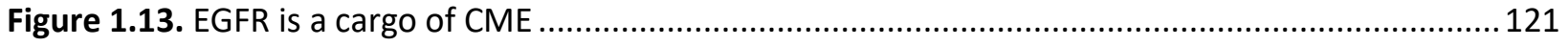

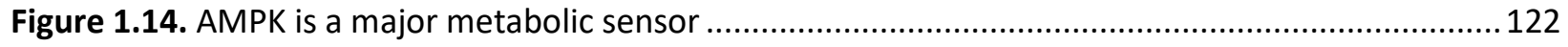

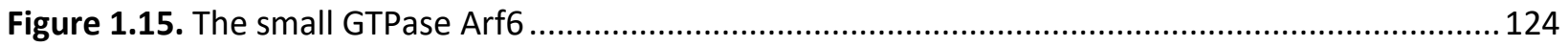

Figure 1.16. Arf6 has been implicated in the regulation of CME .....................................................125

\section{Chapter 3}

Figure 3.1. EGF-stimulated PLC- $\gamma 1$ activation and PLC- $\gamma 1$ silencing in RPE cells ..................................151

Figure 3.2. The pan inhibition of PLC by U73122 decreases EGF endocytosis .....................................152

Figure 3.3. PLC- $\gamma 1$ is required for EGF but not Tfn internalization .................................................. 153

Figure 3.4. Silencing of clathrin broadly reduces EGF internalization .................................................154

Figure 3.5. Silencing of Dyn1 has no effect on EGF internalization ....................................................155

Figure 3.6. EGF stimulation elicits a PLC 1 -dependent increase in cytosolic $\mathrm{Ca}^{2+}$...............................156

Figure 3.7. IP3 receptor and cytosolic Ca2+ are required for EGF but not Tfn internalization...............158

Figure 3.8. BAPTA-AM treatment does not affect EGF ligand binding to EGFR ...................................159

Figure 3.9. PKC, but not calcineurin, is selectively required for EGF internalization. ............................160

Figure 3.10. Cyclosporin A effectively perturbs calcineurin in RPE cells .............................................161 
Figure 3.11. EGF and Tfn are recruited to largely distinct CLSs

Figure 3.12. PLCY1-derived calcium signaling is selectively required for the internalization of EGFR but not TfR. 163

\section{Chapter 4}

Figure 4.1. Cytosolic Ca2+ and PKC regulate CLSs containing EGFR but not those harboring TfR .177

Figure 4.2. Full-size representative TIRF-M images of RPE GFP-CLC cells treated with A555-EGF or A555-

Tfn

Figure 4.3. Cytosolic Ca2+ selectively controls initiation and assembly of CCPs harboring EGFR 180

Figure 4.4. Dynamics of EGF+ sCLSs and CCPs 182

Figure 4.5. Dynamics of Tfn+ sCLSs and CCPs.....

Figure 4.6. mCherry-Sjn1 is depleted from CLSs upon EGF stimulation in a $\mathrm{Ca}^{2+}$ - and PKC-dependent manner......

Figure 4.7. Full-size representative TIRF images of RPE eGFP-CLCa cells transfected with mCherry-

Synaptojanin 1

Figure 4.8. Endogenous synaptojanin 1 is depleted from CCPs upon EGF stimulation in a cytosolic $\mathrm{Ca}^{2+}{ }_{-}$ dependent manner.

Figure 4.9. PLC- $\gamma 1$ is required for clathrin-dependent EGFR signaling

Figure 4.10. Perturbation of intracellular Ca2+ impairs EGF-stimulated Akt phosphorylation..... .193

Figure 4.11. PLCY1-derived calcium signals selectively regulate the efficient formation, assembly and subsequent internalization of EGFR-containing CCPs.

\section{Chapter 5}

Figure 5.1. A7 treatment regulates the rate of initiation and assembly of CCPs .................................212

Figure 5.2. AMPK activation regulates Arf6 GTP/GDP binding ...........................................................213

Figure 5.3. Arf6 associates with clathrin-coated pits in a GTP-dependent manner ..............................214

Figure 5.4. Arf6 regulates CME dynamics in a GTP-dependent manner ............................................215

Figure 5.5. AMPK activation selectively regulates the endocytosis of CME cargoes ............................217

Figure 5.6. AMPK activation differentially regulates integrin+ and integrin- clathrin structures. ...........218

Figure 5.7. AMPK activation induces the modulation of CME dynamics.. ..........................................220 


\section{List of Abbreviations}

\begin{tabular}{|c|c|}
\hline$\mu \mathrm{HD}$ & C-terminal AP2- $\mu$ homology domain \\
\hline AAK1 & $\alpha$-adaptin associated kinase 1 \\
\hline ACC & Acetyl-coA carboxylase \\
\hline ADP & Adenosine diphosphate \\
\hline AID & Autoinhibitory domain \\
\hline A-loop & Activation loop \\
\hline AMP & Adenosine monophosphate \\
\hline AMPK & AMP-activated protein kinase \\
\hline ANTH & AP180 N-terminal homology domain \\
\hline AP2 & Adaptor protein complex 2 \\
\hline Arf & ADP-ribosylation factor \\
\hline $\mathrm{ARH}$ & Autosomal recessive hypercholesterolemia \\
\hline Arp2/3 & Actin-related protein $2 / 3$ complex \\
\hline ATP & Adenosine triphosphate \\
\hline BAR & Bin, amphiphysin and Rvs domain \\
\hline$C-\mu 2$ & C-terminal domain of the AP2 $\mu 2$ subunit \\
\hline CALM & Clathrin assembly lymphoid myeloid leukemia protein family \\
\hline $\mathrm{CaM}$ & Calmodulin \\
\hline CaMKII & Calmodulin dependent kinase II \\
\hline $\mathrm{CaN}$ & Calcineurin \\
\hline $\mathrm{Cbl}$ & Casitas B-lineage lymphoma \\
\hline CBP & Calcium binding proteins \\
\hline CC & Coiled-coil domain \\
\hline CCP & Clathrin-coated pit \\
\hline $\mathrm{CCV}$ & Clathrin-coated vesicle \\
\hline$C D$ & Carboxy-terminal domain \\
\hline CDK5 & Cyclin dependent kinase 5 \\
\hline CDS & Christian Delos Santos \\
\hline $\mathrm{CHC}$ & Clathrin heavy chain \\
\hline CLC & Clathrin light chain \\
\hline
\end{tabular}




\begin{tabular}{|c|c|}
\hline CLS & Clathrin labeled structure \\
\hline CME & Clathrin-mediated endocytosis \\
\hline CsA & Cyclosporin A \\
\hline Dab2 & Disabled homolog 2 \\
\hline DAG & Diacylglycerol \\
\hline DEG & Delayed-early gene \\
\hline Dyn & Dynamin \\
\hline ECD & Ectodomain \\
\hline EGF & Epidermal growth factor \\
\hline eGFP & Enhanced green fluorescent protein \\
\hline EGFR & Epidermal growth factor receptor \\
\hline ENTH & Epsin N-terminal homology domain \\
\hline Eph & Ephrin \\
\hline Eps15 & Epidermal growth factor receptor substrate 15 \\
\hline ER & Endoplasmic reticulum \\
\hline ERK & Extracellular signal-regulated protein kinase \\
\hline ESCRT & Endosomal sorting complexes required for transport \\
\hline F-actin & Filamentous actin \\
\hline $\mathrm{FCHo} 1 / 2$ & Fer/Cip4 homology domain only proteins 1 and 2 \\
\hline FGF & Fibroblast growth factor \\
\hline FGFR & Fibroblast growth factor receptor \\
\hline FKBP12 & FK506 binding protein 12 \\
\hline FRB & Fragment of mTOR that binds FKBP12 \\
\hline FRET & Förster resonance energy transfer \\
\hline FRET-FLIM & Förster resonance energy transfer by fluorescence lifetime imaging \\
\hline Gab1 & Grb2-associated binding protein 1 \\
\hline G-actin & Globular actin \\
\hline GAK & G-associated kinase \\
\hline GAP & GTPase-activating protein \\
\hline GED & GTPase effector domain \\
\hline GEF & Guanine nucleotide exchange factor \\
\hline GFP & Green fluorescent protein \\
\hline
\end{tabular}




\begin{tabular}{|c|c|}
\hline GGA3 & Golgi-localized gamma-ear containing Arf-binding protein 3 \\
\hline GLUT & Glucose transporter \\
\hline GPCR & G-protein coupled receptor \\
\hline Grb2 & Growth receptor-bound protein 2 \\
\hline GTP & Guanosine triphosphate \\
\hline HIP1R & Huntingtin-interacting protein 1-related protein \\
\hline HSC70 & Heat shock cognate 70 chaperone protein \\
\hline IEG & Immediate-early gene \\
\hline Ig-like & Immunoglobulin-like repeats \\
\hline IP3 & Inositol 1,4,5-triphosphate \\
\hline IP3R & Inositol 1,4,5-triphosphate receptor \\
\hline IR & Insulin receptor \\
\hline IRS & Insulin receptor substrate \\
\hline JM & Juxtamembrane region \\
\hline KID & Kinase insert domain \\
\hline LDL & Low density lipoprotein \\
\hline LDLR & Low density lipoprotein receptor \\
\hline LKB1 & Liver-kinase-B1 \\
\hline MAPK & Mitogen-activated protein kinase \\
\hline MEK & MAP/ERK kinase \\
\hline mTOR & Mammalian target of rapamycin \\
\hline$N-\mu 2$ & $\mathrm{~N}$-terminal domain of the AP2 $\mu 2$ subunit \\
\hline NFAT & Nuclear factor of activated T-cells \\
\hline NGF & Nerve growth factor \\
\hline NPF & Asparagine-proline-phenylalanine domain \\
\hline PDGF & Platelet-derived growth factor \\
\hline PDGFR & Platelet-derived growth factor receptor \\
\hline PDK1 & 3-Phosphoinositide-dependent kinase 1 \\
\hline $\mathrm{PH}$ & Pleckstrin homology \\
\hline $\mathrm{PI}$ & Phosphoinositide \\
\hline $\mathrm{PI} 3,4 \mathrm{P} 2$ & Phosphatidylinositol-3,4-bisphosphate \\
\hline PI3K & Phosphatidylinositol 3-kinase \\
\hline
\end{tabular}




\begin{tabular}{|c|c|}
\hline PI3P & Phosphatidylinositol-3-phosphate \\
\hline PI4K & Phosphatidylinositol-4 kinase \\
\hline PI4P & Phosphatidylinositol-4-phosphate \\
\hline PI5P & Phosphatidylinositol-5-phosphate \\
\hline PIP2 & Phosphatidylinositol-4,5-bisphosphate \\
\hline PIP3 & Phosphatidylinositol-3,4,5-trisphosphate \\
\hline PIP3K & Phosphatidylinositol-4,5-bisphosphate 3-kinase \\
\hline PIP5K & Phosphatidylinositol-4-phosphate-5-kinases \\
\hline PIPKI & Type I phosphatidylinositol phosphate kinases \\
\hline PKC & Protein kinase $\mathrm{C}$ \\
\hline PLC & Phospholipase C \\
\hline PMCA & Plasma membrane Ca2+ ATPase pump \\
\hline PRD & Proline-rich domain \\
\hline PTB & Protein tyrosine binding domain \\
\hline Ptdlns & Phosphatidylinositol \\
\hline PTEN & Phosphatase and tensin homolog domain \\
\hline PTP & Protein tyrosine phosphatases \\
\hline pTyr & Phosphotyrosine \\
\hline Raf & Rapidly accelerated fibrosarcoma kinase \\
\hline RHEB & Ras homolog enriched in brain \\
\hline RIM & Regulatory-subunit-interacting motifs \\
\hline ROS & Reactive oxygen species \\
\hline RSK & Ribosomal S6 kinase \\
\hline RTK & Receptor tyrosine kinase \\
\hline sCLS & Sub-threshold clathrin structure \\
\hline $\mathrm{SH} 2$ & Src homology 2 domain \\
\hline SH3 & Src homology 3 domain \\
\hline Shc & Src homology domain-containing adaptor protein $\mathrm{C}$ \\
\hline SiRNA & Small interfering RNA \\
\hline Sjn & Synaptojanin \\
\hline SNX9 & Sorting nexin 9 \\
\hline Sos & Son of Sevenless \\
\hline
\end{tabular}




$\begin{array}{ll}\text { SPH } & \text { Split PH domain } \\ \text { SRG } & \text { Secondary response gene } \\ \text { STAT } & \text { Signal transducers and activators of transcription } \\ \text { TF } & \text { Transcription factor } \\ \text { Tfn } & \text { Transferrin } \\ \text { TfR } & \text { Transferrin receptor } \\ \text { TGF- } \alpha & \text { Transforming growth factor- } \alpha \\ \text { TGN } & \text { Trans-Golgi network } \\ \text { TIRF-M } & \text { Total internal reflection fluorescence microscopy } \\ \text { TKD } & \text { Tyrosine kinase domain } \\ \text { TMD } & \text { Transmembrane domain } \\ \text { TOM1L1 } & \text { Target of yb1 like 1 membrane trafficking protein } \\ \text { TRAIL/DR } & \text { Tumor necrosis factor-related apoptosis-inducing ligand-death receptor } \\ \text { Trk } & \text { Tropomyosin-related kinase receptor } \\ \text { TSC2 } & \text { Tuberous sclerosis complex 2 } \\ \text { UBD } & \text { Ubiquitin binding domain } \\ \text { VEGF } & \text { Vascular endothelial growth factor } \\ \text { VEGFR } & \text { Vascular endothelial growth factor receptor } \\ \text { VOCs } & \text { Voltage-operated Ca2+-selective channels } \\ \text { WASP } & \text { Wiskott-Aldrich syndrome protein } \\ \end{array}$


Chapter 1. Introduction 


\section{1- The 'surfaceome'}

The plasma membrane is a specialized structure surrounding the cell which separates the cellular interior from the external environment (extracellular space). Primarily, the plasma membrane acts as a barrier which protects the cell from harmful physical, biological, and chemical agents present within its surroundings. Amphipathic in nature, the plasma membrane also forms a selective filter around the cell which is impermeable to ions and hydrophilic molecules, thereby acting as an insulator through which transit of material is tightly regulated ${ }^{30}$. Furthermore, it is involved in a variety of cellular processes including cell-cell and cell-environment communication, adhesion and migration, metabolism, and signal transduction ${ }^{30}$. In performing these roles, the plasma membrane recruits a variety of proteins onto the bilayer, creating a dense lipid-protein landscape collectively described as the cell-surface proteome or 'surfaceome' (Figure 1.1) ${ }^{31}$.

Proteins of diverse structure and function make up the cell-membrane surfaceome. Depending on the nature of interaction with the lipid bilayer, membrane proteins can be classified into two broad categories: integral (intrinsic) and peripheral (extrinsic) ${ }^{32}$. Integral proteins are defined as membrane proteins which contain one or more segments directly embedded within the plasma membrane bilayer. Regarded as transmembrane proteins, most integral proteins span the entire bilayer through a hydrophobic domain commonly made up of $\alpha$-helices or multiple $\beta$-strands-which interact with the hydrophobic core of the plasma membrane ${ }^{32}$. Spanning the bilayer, integral proteins form three major domains: an extracellular ectodomain and an intracellular cytosolic domain, separated by the hydrophobic transmembrane domain. In contrast, peripheral proteins do not interact with the hydrophobic core of the phospholipid bilayer. Instead, they reversibly associate with the plasma membrane indirectly through interactions with integral proteins, or directly through interactions with polar lipid head groups ${ }^{33}$. Peripheral proteins include intracellular and extracellular components involved in diverse processes including signal transduction and cytoskeletal rearrangements ${ }^{34}$.

A major function of the plasma membrane involves sensing of the environment, allowing cells to modify their behaviours in adapting to changing extracellular conditions ${ }^{10}$. Cell growth and programmed cell death for instance, are influenced by extracellular nutrient availability and as well as growth factors ${ }^{35,36}$. Both peripheral and integral proteins are involved in cellular adaptation, but the latter are mostly responsible for environmental sensing due to their characteristic transmembrane nature ${ }^{37}$. Integral proteins including signaling receptors for instance-through their extracellular and intracellular 
domains-respectively conduct signal sensing and signal transmitting activities. Receptor tyrosine kinases (detailed in section 1.6) are signaling receptors exemplifying this role, which converts extracellular signals into intracellular responses ${ }^{11}$. Briefly, receptor tyrosine kinases promote cell growth and proliferation in response to extracellular growth signals. Growth signals in the form of polypeptide growth-factors bind the receptor tyrosine kinase ectodomain, which induces a conformational shift within its intracellular structure. This in turn enables the receptor tyrosine kinase in activating proliferative and growth-stimulating intracellular signaling pathways, involved in pro-mitogenic cellular responses ${ }^{11}$. Other integral proteins such as the G-protein coupled receptor (GPCR), also detect extracellular stimuli in the form of hormones, neurotransmitters, ions, photons, and odorants, which they similarly convert into intracellular responses directing cell behavior ${ }^{38}$. Thus, integral proteins including receptor tyrosine kinases and GPCRs contribute to the ability of cells to sense and respond to changes within the environment.

Other integral proteins on the plasma membrane include nutrient transporters which serve as a critical link between the cell and its environment ${ }^{39}$. Several nutrient transporters exists on the plasma membrane which facilitates entry of nutrients and charged molecules into the cell, impermeable to the membrane ${ }^{40}$. The integral solute carrier (SLC) transport proteins for instance, is a large group consisting of over 300 members organized into 51 families $^{41}$. SLCs facilitate the intracellular transport of organic compounds required for anabolic processes including glucose through several glucose transporter (GLUT) families (GLUT-1, -2, -3, -4/SLC2A-1, -2, -3, -4); amino acids through significantly overlapping SLCS (e.g. SLC7A2 for arginine and lysine, and SLC16A10 for tryptophan, tyrosine, and phenylalanine); and lipids through several transporters which recognize various fatty acid lengths (e.g. SLC27A3 for long chain fatty acids $)^{42,43}$. As well, essential macro-minerals including $\mathrm{Ca}, \mathrm{P}, \mathrm{Mg}, \mathrm{Na}, \mathrm{K}, \mathrm{Cl}$, and $\mathrm{S}$ required for numerous signaling activities and proper protein function, are similarly imported into the cell through various SLC transporters ${ }^{43}$. Lastly, micro-minerals such as iron important for biochemical activities including oxygen transport, energy production and cellular proliferation, are brought into the cell by the integral glycoprotein, transferrin receptor ${ }^{44}$. SLCs and glycoproteins are amongst many other membrane components which regulate uptake of material required for cellular growth and survival.

Diverse adhesion molecules such as cadherins, selectins, and integrins also comprise the cell surfaceome where they direct functions in cell-cell and cell-matrix adhesion, cell motility and migration, and cell proliferation ${ }^{45}$. Adhesion molecules anchor cells to their surroundings by connecting 
intracellular cytoskeletal components to extracellular scaffolds, acting as critical sensors towards their environment ${ }^{46}$. As well, these integral proteins provide the necessary traction for dynamic forces required during cell migration, of which some are upregulated and serve as common markers in metastatic cancer types (e.g. CD44 upregulation in endometrial cancer) ${ }^{45,46}$. Due to the dynamic properties of the extracellular environment, adhesion molecule profiles on the plasma membrane are in constant flux, continually adapting to diverse stimuli. This pertains to other integral proteins and the surfaceome as a whole, which exists as a dynamic landscape capable of remodeling itself in response to varying external cues and cellular demands. As such, the surfaceome confers cellular sensing which allows the cells to respond, adapt, survive, and grow within their environments.

The inherent capacity of cells to acutely modify the plasma membrane landscape determines their survival, growth, and proliferation within a given environment. Remodeling the surfaceome requires co-ordination of several processes which participate in protein synthesis/expression and turnover. Increased gene expression and protein synthesis triggered by growth factors for instance, may act in the upregulation of membrane proteins which sustains environmental sensing ${ }^{47}$. Delivery of proteins to the plasma membrane may also be augmented to increase cell sensitivity to low levels of stimuli, or in enhancing nutrient uptake ${ }^{48}$. In contrast, endocytosis is a cellular process which acutely removes proteins from the plasma membrane into the cell. Through endocytosis, cells capture various materials including nutrients and ligands from the extracellular milieu into the cytosol, where they are used in many biological processes. Crucially, endocytosis serve as the main mechanism which attenuates signaling functions of integral proteins, critical in preventing aberrant activities ${ }^{49}$. Indeed, cells tightly regulate endocytic processes in maintaining cellular homeostasis, of which dysregulation manifests in several human pathologies including cancer ${ }^{50}$.

\section{2- Endocytosis: a portal into the cell}

Endocytosis is one of the most important mechanisms for entry of material into the cell. It is a cellular process which engulfs extracellular molecules from the plasma membrane into vesicular carriers that are delivered into the cytosol. These small $(60-120 \mathrm{~nm})$ membrane vesicles transport various cargo molecules including extracellular nutrients, ligands, and as well as surface proteins collectively termed as the endocytic 'cargo' ${ }^{51}$. Aside from its major role in regulating surface protein expression, endocytosis 
facilitates a range of different functions including nutrient uptake, cell signaling, differentiation, cell adhesion, and have also been implicated in entry of various microorganisms ${ }^{10,52}$. Several modes of endocytosis have been described in eukaryotic cells, each defined by distinct molecular mechanisms and specificities for various cargoes (Figure 1.2) ${ }^{10}$. Specifically, distinct endocytic processes are distinguished by unique protein coats which aid cargo recruitment, vesicle budding, and subsequent internalization ${ }^{50}$. Clathrin- and caveolae-mediated endocytosis for instance, respectively require clathrin and caveolin proteins, in providing scaffold support to the structure ${ }^{51,53}$. In addition, endocytic routes with poorly defined coats have been described including macropinocytosis, phagocytosis, and entosis which involve uptake of larger membrane areas relative to coat-dependent mechanisms ${ }^{10,54,55}$. The relative proportions between these endocytic modes differ in different cell types owing to their adaptations for specific functions ${ }^{10}$.

The advent of modern electron microscopy allowed the first visualization of material uptake inside the cell, within vesicles surrounded by proteinaceous coats-later identified as clathrin ${ }^{50}$. Since then, extensive knowledge had been gained about endocytic events in context of mechanisms and distinct biological functions. Particularly, clathrin-mediated endocytosis (CME) had been wellcharacterized in the literature, serving as a prototypic model from which mechanistic details of other endocytic modes may be derived ${ }^{54,56}$. CME is versatile process which mediates internalization of a diverse array of cargoes, implicated in many facets of cell physiology and homeostasis ${ }^{57}$. It is considered as the major endocytic route in mammalian cells, accounting for a large proportion of endocytic events $(>95 \% \text { of total protein endocytic flux })^{10,58}$. Indeed, clathrin perturbations and its central components are often embryonic lethal in multi-cellular organisms such as Drosophila melanogaster, Caenorhabditis elegans, and Mus musculus ${ }^{50}$. The following discussion will therefore focus on $\mathrm{CME}$, interchangeably referred to as 'endocytosis' throughout for simplicity, unless stated otherwise. For discussions of clathrin-independent endocytic pathways, the reader is referred to the following excellent reviews which details both mechanical and biological functions for the distinct processes [52-56].

Conceptually, endocytosis is a simple process involving sequential, partially overlapping steps which may be defined in three stages: 1) coat assembly and pit initiation, 2) pit maturation and fission, and 3 ) intracellular vesicle traffic and delivery ${ }^{51}$. Further detailed in section $1.3, \mathrm{CME}$ is initiated upon clustering of clathrin within distinct areas on the inner leaflet of the plasma membrane ${ }^{51}$. Clathrin is a soluble protein triskelion which polymerizes into geometric shapes defined by hexagonal, and 
pentagonal formations ${ }^{50}$. Cargo proteins are recruited within these coated areas through specific protein adaptors. Together with adaptors and membrane bending components, clathrin assemblies promote membrane curvature which transforms the flat plasma membrane into a 'clathrin-coated pit' (50$200 \mathrm{~nm})^{51}$. Clathrin-coated pits (CCPs) progressively invaginate, recruiting further structure-stabilizing components. Mature CCPs are then released from the plasma membrane into the cytosol forming clathrin-coated vesicles (CCVs), through scission by a dynamin-based molecular machinery ${ }^{50}$. Within the cytosol, CCVs are uncoated of clathrin, with the 'naked' vesicle delivered into distinct cellular compartments for cargo processing, and intracellular traffic and sorting ${ }^{10}$.

This canonical pathway generally describes the mechanistic progression of $\mathrm{CME}$, shared amongst eukaryotes ${ }^{50}$. It is a highly dynamic process which in addition to the hallmark clathrin-coat, involves the co-ordination of functionally diverse set of proteins and lipid components-fine-tuning internalization mechanisms of distinct cargo types ${ }^{12}$. The molecular identities and functional roles of many of these proteins, have been characterized in detail through bio-chemical, -physical, and -structural approaches ${ }^{51}$. Still, the major question of how these proteins are precisely organized in time and space, in regulating endocytic responses based on diverse physiological stimuli, remain unclear and require further study.

\subsection{1 - A conceptual map for the reader: topics to be discussed}

Endocytosis is the primary mechanism responsible for remodeling the plasma membrane landscape which acutely removes surface proteins involved in diverse cellular processes ${ }^{49}$. While several modes of endocytosis exist, $\mathrm{CME}$ is the major endocytic route in mammalian cells, of which regulation and progression is a major focus in this thesis. Specifically, this work aims to investigate and address the potential role of diverse cellular cues including mitogenic (e.g. growth factors), and metabolic signals (e.g. cellular energy levels) in regulating the process of CME. This research question is fundamentally motivated by the diverse range of physiological processes mediated by mitogenic and metabolic signals in mammalian cells, including cellular growth, proliferation, migration, and differentiation. These processes, to some degree, require both local and global plasma membrane remodeling events of which precise mechanisms however, remain relatively unexplored ${ }^{59-61}$. As a major regulator of the mammalian surfaceome, CME may therefore be implicated in facilitating plasma membrane remodeling required for cellular processes. Whether and how mitogenic and metabolic signals may regulate CME however, is not 
well-understood, of which elucidation will provide insight as to how these cellular cues may induce remodeling of the plasma membrane.

In understanding how mitogenic and metabolic cues may regulate $\mathrm{CME}$, a thorough examination of CME and its molecular mechanism is required, initially presented in section 1.3. In this section, I will describe key endocytic proteins and their contributions to the distinct molecular stages of CME. In addition, the three major 'hubs' (the clathrin-coat; the AP2 adaptor complex; and the phospholipid PIP 2 ) governing CME will be discussed in detail, to give the reader some insight in the potential junctures through which CME may be regulated. Special focus will be placed on the plasma membrane-enriched $\mathrm{PIP}_{2}$ hub, due to its indispensable role in CME initiation and progression. As $\mathrm{PIP}_{2}$ is indispensable to CME, cellular processes which regulate $\mathrm{PIP}_{2}$ plasma membrane levels should regulate $\mathrm{CME}$ to some degree. The phospholipase $\mathrm{C}$ enzyme for example, upon activation will result in direct $\mathrm{PIP}_{2}$ reduction, due to its $\mathrm{PIP}_{2}$-hydrolyzing activity. In section 1.4, I will therefore focus on phospholipase $\mathrm{C}$ enzymes in understanding their potential role in CME regulation. In this section, I will emphasize the diversity in phospholipase $\mathrm{C}$ isoforms, and the mechanisms through which they may be activated-in providing insight as to how they may regulate CME via $\mathrm{PIP}_{2}$ hydrolysis. As will be discussed, the hydrolysis of $\mathrm{PIP}_{2}$ by phospholipase $\mathrm{C}$ not only depletes the anionic lipid, but also generates secondary molecules which increases cytosolic calcium concentrations in the cell. Calcium is a ubiquitous signaling molecule of which cytosolic increases is known to upregulate $\mathrm{CME}$-albeit in neurons during neurotransmission events. Whether or not calcium increases generated by phospholipase $\mathrm{C}$ contributes to the regulation of CME in non-neuronal cells however, is not well-understood. Thus, in section 1.5, I will describe the potential role of calcium in non-neuronal cells, drawing parallels from its established role in excitable cell systems such as in neurons.

At this stage, the reader should conceptualize the bimodal role of phospholipase $C$ in the potential regulation of CME either by i) direct $\mathrm{PIP}_{2}$ depletion, or by ii) triggering cytosolic calcium increases. Emphasized in section 1.4, phospholipase C enzymes are commonly activated downstream of many signaling processes, thus, providing these cellular processes with the capacity in potentially regulating CME. Mitogenic signals such as growth factors for instance, bind and activate receptor tyrosine kinases, which in turn activates phospholipase C. Receptor tyrosine kinase activation therefore induces $\mathrm{PIP}_{2}$ hydrolysis and cytosolic calcium increases-providing a possible mechanism through which mitogenic signals may regulate $\mathrm{CME}$; and hence induce plasma membrane remodeling. Whether 
$\underline{\text { mitogenic signaling by receptor tyrosine kinases result in CME modulation via phospholipase } \mathrm{C} \text { activity }}$ however, is not well-understood, and is one of the specific questions I will address in this thesis. Thus, in section 1.6, I will comprehensively describe receptor tyrosine kinase biology, with emphasis on their activation mechanisms and signaling properties, in highlighting their potential regulatory roles in CME.

Having described the probable role of mitogenic signals in influencing CME, I will shift my focus towards understanding how metabolic signals within the cell may similarly affect the process. This thematic shift is largely motivated by previous findings from our laboratory, which indicates the robust cellular remodeling of the surfaceome under perceived metabolic stress; such that of low cellular ATP. Specifically, the AMP-activated protein kinase (AMPK) is the primary energy sensor which is activated by a decrease in ATP levels, and as such, a likely metabolic signal regulating the reorganization of the plasma membrane. It remains unclear however, how AMPK may precisely facilitate plasma membrane remodeling and whether CME is involved in the process; serving as the second specific question I will address in this work. Thus, in section 1.7 I will discuss AMPK biology and its potential role in regulating $\mathrm{CME}$, in response to metabolic signals presented by fluctuations in cellular energy levels. Lastly, I will conclude the chapter by providing rationale and objectives for this thesis in section 1.8.

\section{3- Clathrin-mediated endocytosis (CME)}

$\mathrm{CME}$, as it implies, is an endocytic pathway marked by the clathrin protein which surrounds and coats endocytic vesicles. CME is a major endocytic route for internalization of many surface proteins including transporters and receptors with associated macromolecules, mechanistically conserved amongst eukaryotes ${ }^{50,58}$. CME mediates the uptake of materials through CCPs, which progressively invaginates into the cytosol, forming CCVs upon scission from the plasma membrane. Within the cytosol, CCVs are uncoated of clathrin, revealing a 'naked' vesicle which-contingent on cargo processing requirements - traffic within the different routes of the endomembrane system ${ }^{10}$. Receptor tyrosine kinases for example (see section 1.6.4), are resident cargoes of CME which enters the cell through clathrin-coated structures. Upon uncoating, these vesicles are either routed back to the plasma membrane for receptor recycling, or towards the lysosomes for degradation, depending on distinct receptor identities and activities. As will be examined here, $\mathrm{CME}$ is a versatile process critical for nutrient uptake, signaling, and membrane regulation, and thus regarded as the 'master regulator' of the cell 
surface proteome and processes ${ }^{62}$. In accomplishing this role, CME coordinates assembly of a large number of proteins at the plasma membrane, distinctly regulated in response to diverse physiological stimuli62.

\subsection{1 - The regulatory modules of CME}

An interesting feature of CME in mammalian cells is the recruitment of specific endocytic proteins which assemble on the plasma membrane-in which different regulatory proteins arrive and leave to facilitate the endocytic process ${ }^{51}$. The arrival of specific endocytic proteins corresponds to distinct functional endocytic stages, allowing them to be grouped into functional modules. Three functional modules can be described on the basis of their appearance during defined endocytic events ${ }^{51}$. The first module, regarded as the 'coat module', is responsible for initiating endocytic processes. It includes proteins which make up the clathrin-coat assembly including clathrin, adaptor proteins, and other scaffolding components. Adaptor proteins include the well-characterized hetero-tetrameric adaptor protein 2 complex (AP2; see section 1.3.4), the monomeric adaptor clathrin assembly lymphoid myeloid leukemia protein family (CALM/PICALM), and epsins - which bind to lipids on the plasma membrane and cargo molecules ${ }^{51}$. Scaffold proteins apart from clathrin include the epidermal growth factor receptor substrate 15 (eps15), epidermal growth factor receptor substrate 15 related (eps15R), and intersectins. Some of these scaffold proteins are capable of self-polymerization in addition to individual interactions with clathrin, providing necessary structural support for the maturation of the coat complex ${ }^{51}$. Later discussed, these components are involved in both early and late stages of CME, overlapping with proteins within other modules ${ }^{51}$.

After coat assembly, a network of actin filaments is assembled at endocytic sites, where they provide necessary mechanical forces in the progressive invagination of coated-pits ${ }^{51,63}$. Although the majority of the following mechanistic details were derived from studies of endocytosis in budding yeast, other studies have similarly indicated the role of actin in mammalian endocytosis, which may reflect some of the details described-and thus, warrant inclusion in this section ${ }^{64}$. The 'actin/membrane curvature module' includes both the actin filament network and its regulatory components. Briefly, actin is the most abundant protein in many cell types, of which globular monomers (G-actin) polymerize into long actin filaments (F-actin) ${ }^{65}$. F-actin is spatially organized into a variety of structures including stress fibers, surface protrusions, and cortical actin filament networks involved in endocytosis ${ }^{66}$. 
Polymerization of actin within specific endocytic sites is determined by the recruitment of actin-related protein 2/3 complex (Arp2/3), and a number of other actin-binding proteins (ABPs) ${ }^{51}$. The Arp2/3 complex contains Arp2 and Arp3, which are closely related to related to G-actin and thus, capable of rapidly inducing de novo actin polymerization ${ }^{67}$. ARP2/3 however, exists in an inactive conformation which is relieved by binding to the Wiskott-Aldrich syndrome protein (WASP) family ${ }^{67}$. WASP is a regulatory component of the actin module activated on the plasma membrane upon binding the phospholipid $\mathrm{PIP}_{2}$ (phosphatidylinositol-4,5-bisphosphate; see section 1.3.5), or through interactions with SH3 domain-containing proteins; including BAR domain proteins involved in the third module $\mathrm{e}^{50,68,69}$. WASP also activates actin binding proteins including myosin motors which act as dynamin anchors linking actin filaments to the membrane at the base of invagination. The localization of WASP and Arp2/3 within endocytic sites thus, largely contribute to the production of forces necessary for membrane shaping 51 .

The progressive invagination of CCPs is followed by their subsequent detachment from the plasma membrane, forming CCVs. This process is largely mediated by the 'scission-related protein module' which facilitates constriction and scission of the neck of a mature coated pit. The GTPase dynamin family is the primary component within this module comprising of three isoforms in mammals: dynamin 1 (Dyn1) and Dyn3, which are highly expressed in neurons, and Dyn2 which is ubiquitously expressed ${ }^{70}$. All three share a conserved structure: an N-terminal G domain; a 'middle' or 'stalk' region; a pleckstrin homology domain (PH domain); a GTPase effector domain (GED); and a proline-rich domain (PRD) at the carboxy-terminal region ${ }^{71}$. The stalk domains of two dynamin molecules can dimerize in a cross-like fashion, in where two $\mathrm{G}$ domains orient in opposite directions ${ }^{71}$. Dynamin dimers form the basic unit of dynamin, which further assembles into higher-order helical structures around the neck of budding membranes ${ }^{70}$. The exact mechanism of dynamin-mediated scission is unclear. Recent crystallographic and cryo-electron microscopy (cryo-EM) studies however, have described the process as a 'power stroke', in where GTP hydrolysis by the G domain induces a twisting motion throughout the structure, progressively constricting the membrane towards fission ${ }^{71}$.

Cell-free studies have shown that purified dynamin alone can cut synthetic lipid tubules in presence of GTP ${ }^{50}$. Other factors however, are likely to assist the action of dynamin in vivo due to identification of its several interaction partners. In particular, dynamin is recruited to budding CCVs by BAR (Bin, amphiphysin and Rvs) domain-containing proteins, which through their $\mathrm{SH} 3$ domains, 
recognize the dynamin PRD region ${ }^{72}$. Proteins including amphiphysin and endophilin contain both SH3 and BAR domains, of which the latter is characterized by a crescent conformation, capable of sensing and binding curved membranes; with preference for the highly curved neck of budding vesicles ${ }^{50}$. In addition, the stalk region of dynamin can directly bind F-actin, which further supports its recruitment and assembly onto $\mathrm{CCPs}^{71}$. Lastly, the PH domain of dynamin binds the membrane lipid $\mathrm{PIP}_{2}$, of which enzymatic depletion result in the catastrophic dissociation of dynamin ${ }^{71}$. Thus, dynamin mutants with defective PH domains exert dominant-negative effects on $\mathrm{CME}^{73}$.

These three functional modules drive fundamental events in the progression of CME. This modular organization is conserved amongst eukaryotes, facilitating homologous molecular mechanisms in coat initiation, pit formation, and vesicle scission; exemplified by the endocytic protein homologues in yeast $^{51}$. Specifically, the different protein modules induce five distinct morphological stages in vesicle formation, defined by ultrastructural and cell biological observations-detailed in the next section ${ }^{50}$.

\subsection{2 - Stage-specific regulation of CME}

CME can be defined by five distinct stages: nucleation, cargo selection, clathrin-coat assembly, vesicle scission, uncoating and clathrin component recycling (Figure 1.3 $)^{50}$.

(i) Nucleation represents the first stage which defines plasma membrane sites, from which CME is initiated. Although there is no clear consensus on the exact mechanism directing endocytic site initiation, many publications indicate that the adaptor protein 2 complex (AP2) plays a major role during the process ${ }^{50,51}$. Further discussed in section 1.3.4, AP2 is a highly conserved protein which is recruited to the plasma membrane by binding the anionic phospholipid PIP 2. AP2 is a hetero-tetramer composed of four subunits which in addition to lipid binding, interacts with a diverse set of endocytic accessory proteins, cargo and clathrin. Distinct plasma membrane sites concentrated with $\mathrm{PIP}_{2}$ and/or endocytic cargo, effectively recruit, accumulate and stabilize AP2 leading to the assembly of the initial clathrincoat complex ${ }^{51}$. Cells expressing AP2 mutants deficient in cargo and clathrin binding, thus, fail to form CCPs which are unable to invaginate and rapidly abort ${ }^{5}$.

Although classically regarded as the master initiator of CME, the role of AP2 at the very early stages of endocytosis remains controversial. Several studies demonstrate that RNAi depletion of AP2 do 
not fully ablate clathrin sites, which although smaller and less abundant, still formed cargo-containing $\mathrm{CCPs}^{74}$. Conner and Schmid produced similar results by inhibiting AP2 via mutation of its activating regulatory kinase, AAK1 (described in section 1.3.4) ${ }^{75}$. In another study, depletion of AP2 largely decreased the number of productive endocytic sites-consistent with its embryonic lethal phenotype in mice ${ }^{5,62}$. Small, transient clathrin sites however, were still detected with similar frequency and density compared to control cells, indicating that other factors in addition to AP2 are likely to contribute to the initiation of endocytosis ${ }^{5,62}$. Henne et al., proposed an alternative mechanism which describes the role of the $\mathrm{FCHo} 1 / 2$ protein family (Fer/Cip4 homology domain only proteins 1 and 2 ) in initiating $\mathrm{CME}^{76}$.

$\mathrm{FCHo} 1 / 2$ belongs to the superfamily of BAR domain proteins, which includes other endocytic components such as amphiphysins, endophilins and SNX972. Briefly, proteins with BAR domains influence membrane traffic by generating membrane curvature to sites of recruitment ${ }^{69}$. FCHo1/2 contains a membrane-binding F-BAR domain which is unique to other BAR proteins, in recognizing less extreme membrane curvatures ${ }^{76}$. Through a C-terminal AP2- $\mu$ homology domain $(\mu \mathrm{HD}), \mathrm{FCHo} 1 / 2 \mathrm{co}-$ associate with two endocytic scaffold proteins eps15 and intersectin, of which functions are crucial to clathrin-coat progression. Indeed, the absence of the FCHo/eps15/intersectin complex result in coatdisassembly ${ }^{72,76}$. Recently, $\mathrm{FCHo1} / 2$ has been proposed to be the master initiator of endocytosis, shown to arrive at endocytic sites preceding recruitment of AP2 and clathrin ${ }^{76}$. Henne and colleagues demonstrate that $\mathrm{FCHO} 1 / 2$ sites on the plasma membrane are unaffected by depletion of AP2. In contrast, depletion of FCHo1/2 by RNAi resulted in a loss of endocytic sites marked by AP2 and clathrin-concomitant with inhibition of three CME cargoes: transferrin (Tfn), low-density lipoprotein, and epidermal growth factor ${ }^{76}$. Overexpression of $\mathrm{FCHo1} / 2$ led to a dramatic increase in CCP density which remain dynamic and functional, as demonstrated by enhanced Tfn internalization ${ }^{76}$. Thus, $\mathrm{FCHo} 1 / 2$ is a major component of the initial coat module which is proposed to subsequently recruit AP2 in directing clathrin assembly ${ }^{50}$. The precise mechanism by which $\mathrm{FCHo} 1 / 2$ is activated and recruited to plasma membranes in defining endocytic sites, however, remains to be elucidated ${ }^{51}$.

(ii) Following nucleation and initiation of coat-assembly, is the recruitment of cargo within endocytic sites via adaptor proteins of the putative pioneer module ${ }^{51}$. Adaptors such as AP2, FCHo1/2, eps15, and the CALM family for instance, recognize and bind distinct recognition sequences on the cytosolic domains of transmembrane cargoes, bringing them within endocytic sites ${ }^{51}$. Recognition sequences include post-translational modifications such as ubiquitination and phosphorylation, and 
linear motifs which are distinctly recognized by adaptor proteins ${ }^{77}$. Linear motifs are short sequences of invariant and variant amino acids, that are found in the cytosolic tails of CME cargoes ${ }^{77}$. This includes widely used 'tyrosine-based' motifs such as $\mathrm{YXX} \varnothing$, where $\varnothing$ is any hydrophobic residue and $X$ is any amino acid $^{78}$. The transferrin receptor (TfR) for instance, is a classical cargo of CME which contains the canonical YXXØ motif recognized by the AP2 adaptor ${ }^{78}$. AP2 is the major adaptor which binds and recruits transmembrane proteins containing tyrosine-based motifs, within CCPs; and similarly, proteins bearing "dileucine-based" signals such as the [DE]XXXL[LI] motif ${ }^{79}$. Further detailed in section 1.3.4.4, YXXØ binding activates AP2, leading to the recruitment of clathrin and subsequent coat complex stabilization ${ }^{79}$. Cargo-specific adaptors such as Dab2 (disabled homolog 2) and ARH (autosomal recessive hypercholesterolemia) recognize other cytosolic signal sequences such as the [FY]XNPX[YF] motif present in the low density lipoprotein (LDL) receptor $(L D L R)^{80}$. Recruitment of cargoes represent an 'endocytic checkpoint' in the formation of bona fide CCPs, of which accumulation below a certain threshold lead to coat disassembly, or delay in vesicle budding ${ }^{51}$. As such, cargo recruitment is tightly coupled to the progressive invagination of endocytic pits, serving as a regulatory step in $\mathrm{CME}^{50,51}$.

How exactly adaptors within early endocytic sites bind and recruit cargo proteins remain unclear, although current research indicates two possible mechanisms ${ }^{62}$. In mammalian cells, the fluorescent tracking of TfR and LDLR revealed a passive diffusion mechanism, bringing cargo to endocytic sites in a stochastic manner ${ }^{81}$. Specifically, work by Ehrlich and colleagues showed the passive diffusion of fluorescently tagged TfR and LDLR across the plasma membrane, which became stationary upon collision with pre-formed clathrin sites ${ }^{82}$. The authors showed that this co-localization subsequently promoted further clathrin recruitment and coat assembly-indicated by a gain in fluorescent-clathrin signal. Accordingly, both TfR and clathrin signals simultaneously disappeared from the plasma membrane, indicative of productive receptor endocytosis ${ }^{82}$. Through passive diffusion, physiological cargoes encounter pre-formed cargo-capturing pits, and thus, play a passive role in their own uptake ${ }^{72}$.

TfR and LDLR are CME cargoes internalized in a constitutive manner which do not depend on specific internalization signals ${ }^{62}$. Other cargo including signaling receptors however, require ligandbinding for effective recruitment into clathrin-coated structures. The epidermal growth factor (EGF) receptor (EGFR) for instance, is a cargo of CME which is incorporated within CCPs upon ligand stimulation ${ }^{11}$. Detailed in section 1.6.4, ligand binding induces conformational modifications within the 
cytosolic domains of EGFR, leading to its ubiquitination ${ }^{11}$. In parallel to the EGFR YXXØ motif recognized by $\mathrm{AP} 2$, the post-translational ubiquitin tag is recognized by epsin and ep15 (via ubiquitin interaction motifs), which further augments EGFR recruitment within clathrin-coated structures (see section 1.6.4) ${ }^{10,83}$. Similarly, G-protein coupled receptors (GPCRs) are brought into CCPs by the $\beta$-arrestin protein, which binds phosphorylation sites on intracellular domains of activated GPCR; and as well as to AP2 and clathrin assemblies ${ }^{10}$. Thus, in addition to recruitment by AP2, internalization of functionally distinct surface proteins within clathrin-coated assemblies, are likely to involve unique sets of regulatory adaptors and accessory proteins-producing heterogeneous subtypes of CCP assemblies ${ }^{10}$.

(iii) AP2 and other adaptors promote the clustering of cargoes within endocytic sites. Cargo clustering exerts a positive effect on AP2 stabilization leading to recruitment of clathrin ${ }^{84}$. As cargo accumulates, membrane curvature progressively increases largely due to the action of clathrin assembly and the recruitment of other membrane bending proteins ${ }^{51}$. Emphasized in section 1.3.3, clathrin exists as a triskelion within the cytosol, which self-assembles into the protein coat upon recruitment to the membrane by AP2 and other adaptors ${ }^{85}$. It was postulated that clathrin could induce curvature by imposing the shape of its assembled lattices onto membrane recruitment sites ${ }^{51}$. Exactly how clathrin scaffolds the membrane into curved structures, however, remain controversial as two opposing mechanisms have gained substantial support from various experimental studies (see section 1.3.3.2) ${ }^{51}$.

Briefly, the first model describes clathrin initially forming as flat hexagonal lattices on the inner side of the plasma membrane, which transitions into highly curved pentagonal buds during $\mathrm{CME}^{51}$. Although flat-lattices are directly visualized by electron microscopy, this model presents a questionable scenario due to the extremely energetically costly process - of remodeling clathrin assemblies from hexagonal to pentagonal structures ${ }^{86}$. Thus, it was instead proposed that clathrin polymerizes dynamically onto plasma membranes-growing and accumulating in response to increasing membrane invagination ${ }^{51}$. This second model is supported by studies which demonstrate the absence of clathrin assemblies on plasma membranes subjected to high membrane tension ${ }^{87}$. Nevertheless, electron microscopy studies have demonstrated the direct transition of flat clathrin lattices into curved structures $^{88}$. Avinoam and colleagues for instance, demonstrate that membrane area covered by clathrin does not change as curvature progressively increased throughout the lifetime of a CCP; suggesting the invagination of pre-formed flat lattices ${ }^{88}$. In addition, using correlative light and electron microscopy (CLEM), Bucher and colleagues determined that flat lattices generally initiate curvature upon acquiring 
$\sim 70 \%$ of the final clathrin content, further supporting this model ${ }^{86}$. Although no clear consensus has been reached, the contribution of clathrin to membrane bending is widely established as clathrin ablation leads to a loss in coated pits, and concomitant decrease in both TfR and EGFR endocytosis ${ }^{89}$.

In addition to clathrin, actin similarly contributes in generating membrane curvature during formation of $\mathrm{CCVs}^{51}$. Previously discussed, actin polymerization is directed by the Arp2/3 complex, which is activated by WASP family proteins ${ }^{90}$. WASP binds to PIP 2 located around the rim of the endocytic site, thereby facilitating peripheral actin assembly. From the rim, actin polymerizes into thin filaments growing around the budding membrane, towards the bottom edge of the invaginating vesicle ${ }^{51}$. Several proteins anchor actin to the plasma membrane and clathrin, forming a link through which mechanical forces from actin polymerization are transferred for membrane shaping. Epsin and the Huntingtininteracting protein 1-related protein (HIP1R) are known to bind $\mathrm{PIP}_{2}$, clathrin, and actin filaments, effectively tethering actin onto the endocytic machinery ${ }^{51}$. As such, deletion of epsin or HIP1R result in arrested endocytosis coupled with unproductive actin polymerization at endocytic sites in mammalian cells ${ }^{91,92}$. Other proteins such as myosin I help tether actin to the plasma membrane, although its exact role and mechanism remain unclear ${ }^{51}$. Despite some evidence for this model, many mammalian cells undergo $\mathrm{CME}$ in absence of actin polymerization, suggesting actin-independent mechanisms for membrane bending ${ }^{72,78}$.

As previously described, BAR domain proteins are endocytic components which also induce membrane curvature, stabilize pre-existing curvature, and recruit cytoplasmic proteins to curvature sites $^{93}$. Most BAR domains are composed of three elongated amphipathic $\alpha$-helices, which upon dimerization with another BAR protein assumes an arc-like conformation ${ }^{81}$. These arc-shaped domains are naturally adapted to binding membranes with positive curvatures such that of endocytic structures ${ }^{63}$. Upon binding, BAR proteins can bend membranes simply by imposing its shape onto the membrane substrate ${ }^{93}$. In addition, amphipathic segments within the BAR domain act as wedges which inserts into the inner leaflet of the bilayer, causing local membrane buckling and curvature generation ${ }^{94}$. Curvature generated from BAR proteins further recruit additional BAR-domain effectors in a positivefeedback manner, thus, progressively inducing membrane bending ${ }^{94}$. Early module proteins such as epsin and CALM clustered by clathrin polymerization, work synergistically with BAR proteins of which respective ENTH (epsin N-terminal homology) and ANTH (AP180 N-terminal homology) domains also promote curvature by amphipathic helix insertion into lipid membranes ${ }^{51,95}$. 
Variations in arc depth exists amongst BAR domain proteins, where some are shallow (F-BAR; radius of $60-80 \mathrm{~nm}$ ), while others are highly curved (N-BAR; radius of $10 \mathrm{~nm})^{51}$. As such, the recruitment sequence of BAR proteins is hypothesized to depend on their recognition of various curvature states of the endocytic site ${ }^{51}$. F-BAR proteins including $\mathrm{FCHo1/2}$ for example-due to their shallow arc-recognize membranes with less extreme curvatures, and thus, arrive at earlier stages of the endocytic process; exemplified by its role in endocytic initiation ${ }^{76}$. In contrast, the high curvature of N-BAR proteins including amphiphysins, endophilins, and sorting nexin 9 (SNX9) recognize highly curved membranes such as the neck of a late CCP, and therefore, recruited towards the final stages of the vesicle formation ${ }^{51}$. Many BAR proteins contain other functional segments including the SH3 domain ${ }^{69}$. The SH3 domain recognizes proline-rich sequences in endocytic proteins such as the GTPase dynamin, and the phosphoinositide phosphatase synaptojanin, which are recruited by N-BAR proteins to the endocytic site $^{50}$. As such, BAR domain proteins not only generate membrane curvature, but are also critical for the recruitment of endocytic proteins required for pit maturation.

(iv) The scission event marks the maturation and productivity of a CCP, which through a dynamin-based machinery, is released from the plasma membrane into the cytosol ${ }^{51,78}$. As mentioned, dynamin is a mechano-chemical enzyme which oligomerizes and constricts the neck of an endocytic vesicle in a GTP-dependent manner. While three isoforms exist in vertebrates, only Dyn2 is ubiquitously expressed at high levels, with Dyn1 and Dyn3 enriched mostly in neurons ${ }^{78}$. All dynamin isoforms exhibit a high degree of structural similarity and functionality in regulating endocytic scission. Dyn1 and Dyn2 however, have distinct biochemical properties which account for their tissue-specific functions ${ }^{78}$. Dyn1, is rapidly activated by calcium/calcineurin-dependent dephosphorylation (described in section 1.5) and can strongly induce de novo membrane curvature-ideally suited for its role in rapid endocytic recycling following exocytosis in neuronal systems ${ }^{96}$. In contrast, membrane fission catalyzed by Dyn2 requires other curvature generating endocytic component such as N-BAR proteins. The SH3 domain of N-BAR proteins strongly interact with the proline-rich region of Dyn2, resulting in its recruitment to highly curved necks of matured CCPs ${ }^{78}$. Through its interaction with N-BAR proteins, Dyn2 is thus, more efficiently targeted to nascent CCPs than Dyn1 in non-neuronal cells. Recruitment of Dyn2 to the neck of mature CCPs by BAR proteins, is proposed to coordinate the progressive acquisition of bilayer curvature with the recruitment of factors driving constriction, fission, and immediate post-fission processing of endocytic vesicles ${ }^{71}$. 
(v) Upon scission from the plasma membrane, the newly formed intracellular CCV is rapidly uncoated of its clathrin-coat. This uncoating event is marked by disassembly of endocytic protein machineries, and subsequent traffic of the 'naked' vesicle within the endomembrane system ${ }^{51}$. Two mechanisms have been described which act synergistically in coordinating the uncoating process. The first mechanism involves the ATPase heat shock cognate 70 (HSC70) chaperone protein and its cofactor auxilin (or cyclin G-associated kinase (GAK) in non-neuronal tissues) ${ }^{51}$. Upon scission, auxilin is recruited onto CCVs through its tensin and clathrin-binding domain, which respectively interact with dynamin and clathrin ${ }^{97}$. A C-terminal J domain in auxilin recruits an ATP-bound HSC70 (ATP-HSC70), forming the auxilin-HSC70 complex on coated vesicles ${ }^{97}$. Although mechanistically not well-understood, the formation of the auxilin-HSC70 complex induces vesicle uncoating through two proposed models. In the 'steric wedge' model, auxilin-HSC70 is described to act as a wedge, of which direct interaction with clathrin causes molecular crowding, leading to loosening and disassembly of clathrin-clathrin interactions ${ }^{51}$. HSC70 is an ATPase which hydrolyzes ATP to ADP required for clathrin uncoating-albeit through an unresolved mechanism ${ }^{98}$. The second 'wrecking ball' model however, suggests that ATP hydrolysis by HSC70 serves in inducing a conformational shift within its globular structure, resulting in its movement and collision with clathrin-owing to its attachment to clathrin via auxilin-leading to clathrin lattice disassembly ${ }^{51}$. Blocking recruitment of HSC70 via auxilin depletion result in the accumulation of CCVs in the cytosol, further supporting the role of the auxilin-HSC70 complex in the uncoating process ${ }^{97}$.

The second mechanism of endocytic vesicle uncoating involves the conversion of $\mathrm{PIP}_{2}$, into PI4P (phosphatidylinositol-4-phosphate) by the lipid phosphatase synaptojanin (Sjn). Discussed in 1.3.5, PIP 2 is one of, if not the most important phosphoinositide for endocytosis, which binds many endocytic proteins recruited throughout the lifetime of an endocytic vesicle ${ }^{51}$. As previously described, $\mathrm{PIP}_{2}$ binds several pioneer proteins including FCHo1/2, CALM, epsin, eps15, and AP2; components of the actin module such as WASP and HIP1R; and scission proteins including dynamin ${ }^{51}$. Indeed, several studies which deplete $\mathrm{PIP}_{2}$ concomitantly demonstrate impairments in $\mathrm{CME}$, presumably due to the uncoupling of endocytic machineries ${ }^{99,100}$. As such, $\mathrm{PIP}_{2}$ serve as an ideal regulatory hub of which turnover is exploited in the uncoating stages of CME-largely mediated by Sjn activity ${ }^{51}$.

Further described in section 1.3.5.2, mammalian cells express two isoforms of Sjn which are both involved in the regulation of CME. The longer isoform, Sjn1-170 (170kDa), is found throughout the 
formation and maturation of CCPs, owing to a C-terminal NPF (asparagine-proline-phenylalanine) domain which bind both AP2 and clathrin ${ }^{101}$. The exact functional roles of Sjn1-170 in clathrin-coat maturation however, is currently unknown; although it is presumed to regulate dynamic protein recruitment during clathrin-coat assembly via $\mathrm{PIP}_{2}$ turnover ${ }^{51,101}$. In contrast, the $\mathrm{C}$-terminal truncated Sjn1-145 (145kDa) isoform is recruited during the late stages of CCP formation-appearing in a transient burst $^{101}$. In addition to recruiting dynamin, the N-BAR domain protein endophilin also recruits Sjn1-145 via a PRD at its C-terminus ${ }^{51}$. Indeed, endophilin, dynamin, and Sjn-145 accumulate within mature endocytic pits under a similar time course, indicative of coordination between vesicle scission and membrane uncoating processes ${ }^{101}$. It is postulated that Sjn-145 destabilizes coat-membrane interactions which promotes uncoating immediately after fission ${ }^{101}$. Furthermore, PI4P generated from Sjn activity, are converted into $\mathrm{PI}, 4 \mathrm{P}_{2}$ (phosphatidylinositol-3,4-bisphosphate) by PIK3C2A (phosphatidylinositol 4phosphate 3-kinase C2 domain-containing subunit $\alpha$ ) -later converted into PI3P (phosphatidylinositol-3phosphate) by phosphatidylinositol 4-phosphatases, switching the vesicle from having a plasma membrane $\mathrm{PIP}_{2}$ profile, to an endosomal PI3P profile (see section 1.3.5.1) ${ }^{51}$. PI3,4 $\mathrm{P}_{2}$ and $\mathrm{PI} 3 \mathrm{P}$ are recognized by the PTEN domain present in auxilin, which effectively couples Sjn-mediated PIP $_{2}$ turnover with recruitment of the auxilin-HSC70 uncoating machinery ${ }^{51}$. As such, genetic deletion of Sjn in neurons of mice resulted in the accumulation of both $\mathrm{PIP}_{2}$ and coated synaptic vesicles ${ }^{102}$.

These five distinct morphological stages characterize the canonical progression of the $\mathrm{CME}$ pathway. This linear depiction of CME however, is an oversimplification of an otherwise highly complex process, which can be more accurately defined as the simultaneously progressions of the distinct endocytic stages ${ }^{12}$. Cargo recruitment, membrane bending and clathrin-polymerization for instance, often occur in parallel rather than sequentially, mediated by the overlapping activities of functional endocytic modules ${ }^{12}$. Previously mentioned, cargo-specific endocytic proteins may also be involved in regulating $\mathrm{CCP}$ dynamics, further adding to the complexity of CME. For example, CCP size-indicative of the degree of clathrin/AP2 recruitment, and hence stabilization-can be influenced by cargo composition as demonstrated by the formation of larger CCPs, upon recruitment of LDLR and its cognate adaptors Dab2 and $\mathrm{ARH}^{80}$. Similarly, lifetimes of CCPs on the plasma membrane may also be influenced by cargo content, exemplified by the longer lifetimes of CCPs containing GPCR and its respective $\beta$ arrestin adaptor ${ }^{103}$. Longer lifetimes are indicative of 'productive' CCPs, which successfully matures into CCVs and are internalized into the cell; in contrast to 'abortive' CCPs which have shorter lifetimes due to a lack in structure stabilization-resulting in premature disassembly ${ }^{104}$. Lastly, different CME cargoes 
have distinct internalization requirements, which impacts the maturation rate of CCPs within which they reside. EGFR but not TfR clathrin-mediated endocytosis for instance, is regulated by phosphatidic acid, while gene silencing or sequestration of AP2 strongly impairs TfR clathrin-mediated endocytosis but not that of EGFR in some cells ${ }^{74,105,106}$. Thus, despite the conserved endocytic stages underlying CCP formation, distinct cargo-specific regulatory mechanisms may fine-tune CCP dynamics, yielding structurally diverse endocytic assemblies. Indeed, microscopic visualization reveal striking heterogeneity amongst CCP structures which differ in size and lifetime, reflecting in part differences in their cargo protein constituents ${ }^{1}$.

Given their role in influencing CCP dynamics, the recruitment of cargo within clathrin-coated structures remain a crucial event in the stabilization and formation of productive CCPs. Nearly $70 \%$ of nascent CCPs are abortive structures, which quickly disassemble upon formation due to a lack in efficient cargo recruitment and stabilization ${ }^{107}$. Approximately $60-70 \%$ of these short-lived structures termed 'early-abortive' CCPs, rapidly turnover (time constant of $\sim 5 s$ ) due to their complete deficiency in endocytic cargoes and hence, appropriate structural stabilization ${ }^{78}$. The second sub-population of abortive pits termed 'late-abortive' CCPs ( $\sim 15 s)$, recruit cargo but nonetheless fail to mature into productive CCVs, likely due to failure in structural stabilization imposed by defects in other endocytic components $^{78}$. The remaining $30 \%$ of all CCP formations thus, represent productive CCPs (<80s) which are stabilized by efficient recruitment of cargo, adaptors, clathrin, and other endocytic accessory proteins which prevent coat disassembly and abortive turnover ${ }^{6}$. As such, CME has been proposed to be gated by a hypothetical endocytic checkpoint, through which only stable, cargo-containing CCPs proceed into maturation, and subsequent scission and internalization into the cell ${ }^{107}$.

While molecular identities and functions of most endocytic accessory proteins have been widely defined, mechanistic details on their spatiotemporal organization is lacking. As well, how distinct contributions of endocytic proteins are quantitatively graded in vesicle formation is unclear; preventing deterministic modeling of the endocytic process. Nevertheless, core components of the endocytic machinery have been identified, namely clathrin, the tetrameric adaptor protein AP2, and the membrane lipid $\mathrm{PIP}_{2}$, of which depletions exhibit catastrophic effects on the endocytic network ${ }^{12}$. Discussed in the following sections, these three components have disproportionately more interactions than any other proteins in the endocytic pathway, and thus, described as the major 'hubs' of CMEserving as important protein-protein interaction platforms for the endocytic process (Figure 1.4) ${ }^{12}$. 


\subsection{3 - Clathrin is a hallmark hub of CME}

Clathrin is the hallmark protein which coats endocytic vesicles during CME, and is one of its major hubs upon which several endocytic protein interactions converge ${ }^{12}$. Clathrin is a well-conserved protein found within the eukaryotic cytoplasm, characterized by its three-legged appearance referred to as a 'triskelion' ${ }^{85,108}$. The clathrin triskelion is comprised of three $190 \mathrm{kDa}$ clathrin heavy chain (CHC) subunits which radiate from a central vertex in forming most of the clathrin structure (Figure 1.5) ${ }^{85}$. In addition, each $\mathrm{CHC}$ is associated with a $\sim 25 \mathrm{kDa}$ clathrin light chain $(\mathrm{CLC})$ which stably binds the proximal region near the $\mathrm{CHC}$ vertex; which can only be detached from $\mathrm{CHCs}$ in vivo by proteolytic action ${ }^{109}$. This trimeric clathrin assembly of three heavy chains associated to light chains form the basic assembly unit of a clathrin-coat-which interacts with other endocytic components in facilitating $\mathrm{CME}^{85}$. It is worth noting that while best understood to coat endocytic vesicles from the plasma membrane, clathrin also facilitates endocytosis from the TGN (trans-Golgi network) network to the endosomes, of which mechanistic details and function are further described in [108].

\subsubsection{1 - Clathrin structure: CHC and CLC subunits make up the clathrin protein}

Clathrin heavy chain serve as the major architectural backbone accounting for the cage-like properties of clathrin lattice assemblies. In humans, two $\mathrm{CHC}$ isoforms exists named from their encoding chromosomes: $\mathrm{CHC} 17$ and $\mathrm{CHC} 22^{110}$. Both isoforms exhibit identical structural features and are $85 \%$ similar at the amino acid level; differing however, in their tissue distribution and cellular functions ${ }^{85}$. $\mathrm{CHC17}$ is well-characterized and is the major $\mathrm{CHC}$ isoform expressed ubiquitously amongst different tissues. It is involved in many intracellular membrane traffic pathways of which depletion globally impairs $\mathrm{CME}^{111}$. In contrast, $\mathrm{CHC} 22$ isoform is predominantly found in cardiac and skeletal muscles, though their expression levels are eight- to ten-fold lower than $\mathrm{CHC} 17^{109}$. The exact roles of $\mathrm{CHC} 22$ is yet to be defined, however, they have been implicated in intracellular membrane traffic rather than in endocytic processes. In particular, $\mathrm{CHC} 22$ has been described in targeting GLUT4 transporters from the endoplasmic reticulum (ER) to intermediate storage compartments within the Golgi, critical in glucose metabolism ${ }^{85,108,110}$. Thus, CHC17 remains the primary isoform involved in CME (from here on in referred to as $\mathrm{CHC}$, and $\mathrm{CHC17}$ only when necessary to distinguish it from $\mathrm{CHC22}$ ) of which assembly into clathrin lattices is further discussed in later sections.

Each CHC molecule displays a 'kinked' conformation and can be divided into three regions: a proximal region, an intermediate distal region, and a globular $\mathrm{N}$-terminal domain (TD) ${ }^{85}$. Briefly, the 
proximal region is an $\alpha$-solenoid structure composed of 10 helices of 10-12 residues connected by loops-designated as the clathrin heavy chain repeat (CHCR) motif ${ }^{110}$. This motif is responsible for the trimerization of three $\mathrm{CHC}$ subunits, forming the central C-terminal vertex, and serve as the binding site for clathrin light chain association ${ }^{110}$. The intermediate distal region is also comprised of CHCR motifs critical for clathrin-clathrin interactions during coat assembly ${ }^{85}$. Lastly, the globular N-terminal domain known as the 'feet' of the triskelion, act as the main endocytic protein interaction module, connecting clathrin to the endocytic machinery ${ }^{85}$. It has a seven-bladed $\beta$-propeller structure which within a clathrin lattice, projects towards the membrane, away from the clathrin-coat ${ }^{112}$. This conformation allows the terminal domain to interact within various adaptors and endocytic proteins important for cargo recruitment and vesicle stabilization. Lemmon and Traub describes that over 30 endocytic proteins physically bind clathrin through the terminal domain, of which interaction motifs have not all been precisely mapped ${ }^{112}$. Several sites on the clathrin TD however, have been identified, one of which recognizes and binds the 'clathrin-box' motif ( $L \varnothing X \varnothing[D / E]$, where $\varnothing$ is a bulky hydrophobic residue) present in endocytic adaptor proteins including AP2 and $\beta$-arrestins ${ }^{113,114}$. A second interaction site on this domain can also bind the W-box motif (PWXXW), found in N-BAR domain proteins such as SNX9 and amphiphysin ${ }^{112,113}$. Thus, through several interactions with endocytic proteins, clathrin is effectively linked and assembled on the plasma membrane-which in itself lacks membrane binding capabilities ${ }^{85}$.

The clathrin light chain is the secondary component of a clathrin molecule, which functions as a regulatory module that affect trimerization of the clathrin triskelion ${ }^{108}$. Similar to the heavy chains, clathrin light chains exists as two isoforms in humans: clathrin light chain -a and -b (CLCa and $\mathrm{CLCb})^{85}$. Both isoforms are found in most tissue types, though their expression ratios may vary; with CLCa enriched in lymphoid tissue and CLCb dominant in the brain ${ }^{109}$. Structurally, they are more divergent than $\mathrm{CHCs}$ exhibiting $60 \%$ similarity in protein sequence ${ }^{85}$. Despite this divergence, the physiological difference between $\mathrm{CLCa}$ and $\mathrm{CLCb}$ is not well-understood; wherein both isoforms are known to exclusively associate with $\mathrm{CHC} 17$ and not $\mathrm{CHC} 22$-indicative of their roles in endocytic clathrin assemblies $^{110}$.

CLCs can be divided into seven regions: 1) an N-terminal segment; 2) a conserved region between $\mathrm{CLCa}$ and $\mathrm{CLCb}$ with $100 \%$ sequence homology; 3) an Hsc70 binding domain in CLCa; 4) a calcium-binding domain; 5) a CHC binding site; 6) a splice site for neuronal protein inserts; and 7) a calmodulin-binding domain at the $\mathrm{C}$-terminus ${ }^{85}$. $\mathrm{CLC}$ binds $\mathrm{CHC}$ with high affinity mediated by interaction 
between its central $\mathrm{CHC}$ binding site, and the $\mathrm{CHC}$ proximal domain; although some reports suggest that CHCs may not be saturated by CLCs in every tissue ${ }^{85,110}$. Bound CLC is oriented with the $\mathrm{N}$ - and Cterminal portions directed towards the distal and vertex region of $\mathrm{CHC}$, respectively ${ }^{85}$. Although yet to defined, $\mathrm{CLC}$ is proposed to negatively regulate $\mathrm{CHC}$ assembly as demonstrated by the coat assembly of CHC (with bound CLCs) upon addition of calcium (non-physiological levels) in vitro ${ }^{110}$. In contrast, depletion of CLCs by siRNA from mammalian cells does not produce measurable effects in clathrin vesicle phenotype, and internalization of classical CME cargoes including TfR, LDLR, and EGFR ${ }^{108,109}$. CLCs were however, implicated in the uptake of three GPCRs and other large particles including viruses and bacteria-indicating their requirement for cargo-specific internalization ${ }^{109}$.

An interesting role for CLC in CME emerges from its interaction with several endocytic components including HIP1R and HSC70. As previously described, HIP1R binds PIP2, clathrin, and actin filaments, effectively tethering actin onto the endocytic machinery ${ }^{51}$. Recruitment of HIP1R to the plasma membrane is proposed to be mediated by HIP1R binding to the $\mathrm{CHC}$ binding region of $\mathrm{CLC} \mathrm{C}^{109}$. HIP1R proteins are then transferred onto plasma membranes via $\mathrm{PIP}_{2}$ where it coordinates actin assembly around an endocytic pit $^{110}$. Indeed, deletion of CLCs produce clear phenotypes in mammalian cells involving changes in the organization of the actin cytoskeleton ${ }^{110}$. This observation is consistent with CLCs role in facilitating internalization of large particles which require stronger mechanical forces in overcoming plasma membrane tensile strength, provided by actin filament polymerization ${ }^{109}$. Further highlighting its role in negatively regulating clathrin assembly, CLCa contains a unique HSC70 region which binds and stimulates the activity of the auxilin-HSC70 complex ${ }^{85}$. Young and colleagues showed that loss of clathrin light chains reduces the efficiency with which auxilin-HSC7O facilitates clathrin uncoating $^{115}$. The exact role of this HSC70 binding site however, remain unclear, as clathrin-coats free of light chains continue to form and disassemble normally in vitro ${ }^{85}$. Thus, CLCs may have context-specific roles in regulating clathrin assembly or disassembly.

\subsubsection{2 - Clathrin assembles into geometrical lattices in CME}

Clathrin is an intrinsically self-assembling protein of which concentration on the plasma membrane result in the formation of geodesic clathrin cages $^{85}$. Adaptor proteins such as AP2, concentrate clathrin within endocytic sites, promoting clathrin-clathrin interactions which create fivesided pentagonal, and six-sided hexagonal rings ${ }^{51}$. The assembly of hexatonic rings lead to the formation of flat clathrin lattices, while assembly of pentagons form structures with curvature. The assembly of 
either ring is contingent on the number of clathrin molecules involved, and the organization of their interactions $\mathrm{s}^{51,85}$. Briefly, cryo-electron microscopy studies reveal that the proximal and distal domains of a CHC subunit within a triskelion, serve as the primary interaction site for reciprocal domains of another clathrin molecule ${ }^{85}$. The proximal and distal domain is separated by a 'knee' region which bends or 'kinks' the CHC structure ${ }^{110}$. Due to its kinked shape, both domains contribute to the formation of two polygon edges within a ring structure (i.e., pentagonal rings have five edges connected by five vertices; hexagonal rings with six edges connected by six vertices). A polygonal edge is comprised of four intertwining clathrin leg sections from different triskelia: an anti-parallel pair of proximal domains from two triskelia of which vertices lie on opposite ends of the edge; and an anti-parallel pair of distal regions from two triskelia with vertices one edge away ${ }^{85}$. These ring structures serve as the basic building block unit in forming higher-ordered clathrin-coat assemblies.

The number of hexagons and pentagons within a clathrin lattice defines the size and shape of the overall structure ${ }^{72}$. Clathrin cages reconstituted and visualized in vitro are typically $>90 \mathrm{~nm}$ in diameter, of which three types have been defined: a 'mini-coat' containing 28 triskelia arranged in a tetrahedral symmetry; a 'hexagonal barrel' comprised of 36 triskelia with a dihedral symmetry; and the 'soccer ball' structure with 60 triskelia with icosahedral symmetry ${ }^{85,116}$. The mini-coat structure is the smallest achievable clathrin cage that can be produced, containing 12 pentagons and 8 hexagonsalbeit, probably too small to accommodate a membrane vesicle in vivo ${ }^{85}$. The hexagonal barrel is the most common clathrin lattice observed in in vitro assays, containing 12 pentagons and 8 hexagons of which size corresponds to synaptic vesicles ${ }^{51,81}$. Other cell types including fibroblast cells, may prefer larger clathrin cages such as the icosahedral soccer ball structure, containing 12 pentagons and 20 hexagons-proposed to be the structure of many coated vesicles ${ }^{108}$. These prototypical clathrin lattice structures may exist in various sizes within the cell, reflecting the structural diversity of endocytic vesicles containing various cargo types ${ }^{98,116}$.

Despite its intrinsic ability in forming curved geometric structures, the exact mechanisms which govern clathrin-coat assembly remain unclear. Specifically, how clathrin lattices form around endocytic membranes and their contributions to membrane bending remain controversial; where two opposing

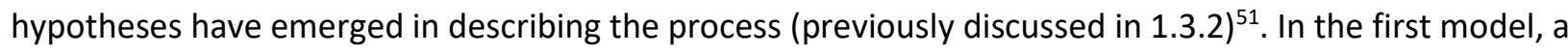
$\mathrm{CCV}$ is proposed to initiate as a flat lattice which progressively gains curvature, forming a CCP ${ }^{86}$. This structural transition involves the conversion of hexagonal rings into pentagonal structures, to 
accommodate the spherical geometry of a budding membrane ${ }^{86}$. Although readily visualized under electron microscopy, this model is challenging from an energetics perspective, due to the costly process of breaking and forming rigid clathrin-clathrin interactions ${ }^{86}$. Nonetheless, several studies including recent work by Bucher and colleagues have provided experimental evidence for this model ${ }^{86,88}$. Using correlative light and electron microscopy, Bucher and colleagues determined that flat clathrin lattices are reorganized into curved structures upon reaching a clathrin density threshold of $\sim 70 \%$ of total clathrin content ${ }^{86}$. The authors describe that clathrin accumulation scaffolds other endocytic components including adaptors and membrane bending proteins, which act against the plasma membrane tension in initiating membrane curvature ${ }^{86}$. Similar studies from Avinoam and colleagues have corroborated this hypothesis, demonstrating the existence of pre-assembled flat lattices which become increasingly curved while maintaining a constant surface area ${ }^{88}$. Thus, coated vesicle budding appears to involve the dynamic bending of pre-assembled clathrin-coats.

In contrast, the second model proposes that clathrin triskelia are dynamically recruited onto endocytic sites - progressively accumulating around invaginating membranes ${ }^{51}$. Several in vitro studies support this hypothesis, demonstrating that clathrin recruitment and assembly does not occur on plasma membranes under high membrane tension ${ }^{51,87}$. Briefly, plasma membrane tension (PMT) is a force maintaining bilayer rigidity, which counteracts inward forces such that generated by endocytosis ${ }^{51}$. Under high tensile conditions, membrane budding is therefore inhibited, which was demonstrated to prevent polymerization of clathrin into geometric structures-indicative of the dynamic recruitment of clathrin onto budding membranes during endocytosis ${ }^{87}$. In addition, using a refined polarized total internal reflection fluorescence microscopy (pol-TIRF) technique, Scott and colleagues recently demonstrated the accumulation of clathrin within progressively invaginating endocytic sites, further validating this model of clathrin assembly ${ }^{117}$. Surprisingly, the authors similarly report the presence of pre-assembled flat lattice structures, which bend into endocytic pits within the same system ${ }^{117}$. Thus, despite the thermodynamically unfavourable nature of the initial model, both models appear to facilitate CME presumably under different physiological contexts ${ }^{51}$. Specifically how cells decide between the two modes however, remain unclear, but believed to be dependent on the careful orchestration of local biochemical, and -physical factors during the process ${ }^{117}$. 


\subsubsection{3 - Clathrin is an essential hub of CME}

Mapping the CME network identifies clathrin as a central hub, due to its several interactions with endocytic accessory proteins and adaptors mediating membrane traffic ${ }^{85}$. Clathrin interaction partners include at least 30 different endocytic proteins, most of which physically bind clathrin via the globular N-terminal domain ${ }^{12}$. Proteins from different endocytic modules are organized by clathrin during distinct stages of vesicle formation; including pioneer proteins such as FCHo1/2, CALM, intersectin, eps15, and AP2; actin and membrane bending components such as HIP1R, amphiphysin, and epsin; and scission and uncoating proteins including Sjn, auxilin and $\mathrm{HSC}^{118,119}$. Due to these key interactions, inhibition or dysregulation of clathrin is often catastrophic to the overall endocytic process $^{89}$. Indeed, disrupting CHC expression in eukaryotic cells (with the exception of yeast) result in decreased growth and proliferation, concomitant with a decrease in CME cargo internalization ${ }^{85}$. In multi-cellular organisms, $\mathrm{CHC}$ knockout is often embryonic lethal owing to impairments in endocytosisattesting to the role of clathrin in propagating $l i f e^{50}$.

Interestingly, despite its diverse interaction profile and robust recruitment during endocytosis, clathrin lacks an intrinsic membrane binding domain, and on its own is unable to bind plasma membranes ${ }^{120}$. Instead, clathrin is recruited to endocytic sites by binding protein adaptors containing clathrin binding motifs. Adaptors such as AP2 effectively binds and links clathrin to the membrane; by simultaneously binding plasma membrane constituents including lipids and cargoes ${ }^{120}$. Discussed in the next section, AP2 therefore, is an essential component in initiating clathrin lattice assembly and function-serving fundamental roles in bridging clathrin with cargoes destined for $\mathrm{CME}^{120}$.

\subsection{4 - Adaptor protein-2 (AP2) complex is a major adaptor hub of CME}

After clathrin, the adaptor protein-2 (AP2) complex is the most abundant protein found exclusively within plasma membrane-derived $\mathrm{CCVs}^{121} . \mathrm{AP} 2$ is a heterotetrameric protein comprised of four subunits forming a large globular core structure, with two appendage domains connected via long flexible linkers ${ }^{79}$. Each AP2 subunit binds various endocytic components including phospholipids and surface cargo-which recruits AP2 to the plasma membrane ${ }^{121}$. In addition, AP2 also binds clathrin, thereby facilitating its recruitment and assembly within plasma membrane endocytic sites. Able to simultaneously bind membranes, cargo molecules and clathrin, the AP2 complex therefore acts a central 
hub in $\mathrm{CME}$, which in addition to its role in crosslinking clathrin to the membrane, also recruits and concentrates cargo within clathrin-coated structures ${ }^{121,122}$.

\subsubsection{1 - Adaptor protein complexes including AP2 regulates membrane traffic}

AP2 belongs to a functionally diverse family of adaptor protein (AP) complexes, of which five types have been identified in mammalian cells; designated adaptor protein-1 through -5 (AP1-5) (Figure 1.6A) ${ }^{81}$. These adaptors have distinct roles in membrane traffic including both endocytic and secretory pathways-recruiting and enriching cargo within budding vesicles ${ }^{83}$. All adaptor complexes are heterotetrameric structures composed of two large subunits (X and $\beta)$, one medium-sized subunit $(\mu)$, and one small-sized subunit $(\sigma)$ arranged in a general $X \beta \mu \sigma$ structure, where ' $X$ ' is a variable subunit ${ }^{120}$. Each subunit, termed 'adaptins', have various isoforms of which combinatorial assembly give rise to the five distinct AP types; AP1-5 respectively consists of one of the unique $\gamma / \alpha / \delta / \varepsilon / \zeta$ large adaptin ( 100 $150 \mathrm{kDa})$, one of the large $\beta 1-5$ adaptin ( $\sim 100 \mathrm{kDa})$, one of the medium-sized $\mu 1-5$ adaptin ( $\sim 50 \mathrm{kDa})$, and one of the small-sized $\sigma 1-5$ adaptin $(\sim 20 \mathrm{kDa})^{83}$. The complex of subunits form a general structure resembling a 'Mickey mouse' head, where the head (or trunk/core) is formed by the N-terminal segments of the large subunits, with the 'ears' (or appendage) composed of their C-terminal domains connected to the head via a flexible neck (or linker/hinge); the medium and small adaptins contribute to the formation of the head ${ }^{113}$. Despite their similarity in structural composition and arrangement, AP complexes are functionally diverse adaptors which are differentially recruited to specific intracellular locales, driving vesicular membrane transport between different intracellular compartments ${ }^{83}$.

AP2 is a well-studied AP type which exclusively localizes to the plasma membrane in facilitating $\mathrm{CME}^{83}$. Further discussed in the next section, AP2 recognizes cytosolic motifs present on a wide range of surface proteins including receptors, nutrient transporters and adhesion molecules, enabling their recruitment and internalization into the cell ${ }^{83}$. Through AP2, clathrin is also recruited to the plasma membrane of which lattice assembly and protein scaffolding remain crucial for initiating endocytosis (see section 1.3.3). In contrast, other AP types such as the AP1 complex primarily localizes within intracellular compartments, including recycling endosomes and the TGN-mediating bi-directional cargo transport between these structures ${ }^{83}$. Similarly, AP3 and AP4 localizes and facilitates vesicular trafficking between the TGN and endosomal membranes. Specifically, AP3 mainly localizes on endosomal membranes, facilitating the delivery of cargo from tubular endosomes to late endosomes; and is involved in the generation of lysosome-related organelles (LRO) ${ }^{83}$. AP4 on the other hand, is 
preferentially localized to the TGN where it mediates cargo transport from the TGN to endosomes. Lastly, the most recently discovered AP5, is found on late endosomes of which exact function however, remains to be uncovered ${ }^{83}$. Most AP complexes are known to recruit protein scaffolds such as Vps41 and SPG11/15, which are involved in facilitating cargo transport by AP3 and AP5, respectively; and clathrin which is used in cargo sorting by AP1, AP2 and AP3 ${ }^{83}$. Due to its preferential localization to the plasma membrane however, the AP2 complex remains the predominant AP involved in CME-and therefore, will be the focus of the following discussions.

\subsubsection{2 - The basic structure of the AP2 complex}

The AP2 complex is the primary AP of CME composed of the large $\alpha$ and $\beta 2$ adaptins, the medium $\mu 2$, and the small $\sigma 2$ adaptin ${ }^{123}$. Previously discussed, the assembly of these subunits into a multimeric complex result in the formation of a 'Mickey mouse' like structure with a brick-like 'head' attached with 'ears' as described by Peden and colleagues ${ }^{124}$. Under electron microscopy, the head referred to as the 'core/trunk' of AP2 ( $200 \mathrm{kDa})$, appears as a highly compact 'rectangular' structure partly derived from the $\mathrm{N}$-terminal domains of the two large $\alpha$ and $\beta 2$ adaptins ${ }^{122,125,126}$. The $\alpha$ and $\beta 2$ adaptins exhibit a similar topology, respectively made up of 29 and $28 \alpha$-helices which are connected in forming a highly curved superhelical-solenoid arrangement ${ }^{125}$. The ear or 'appendage' region of AP2 $(\sim 30 \mathrm{kDa})$ is similarly derived from the same adaptin components albeit from their C-terminal regions, forming the $\alpha$ - and $\beta 2$-ear domains - connected to the core via a flexible unstructured linker ${ }^{122}$. Described later, the core serves as the primary membrane binding module recognizing phospholipids and cargo molecules, with the ear domains imparting functional versatility to AP2 by binding other key protein components involved in $\mathrm{CME}^{12}$.

The $\mu 2$ and $\sigma 2$ subunits are the smaller constituents of the AP2 complex, which contributes to the formation of the AP2 core domain structure ${ }^{125}$. The $\mu 2$ subunit is made up of an N-terminal (N- $\left.\mu 2\right)$ and a C-terminal ( $\mathrm{C}-\mu 2)$ domain held together by a disordered linker. The $\mu 2$ and $\sigma 2$ subunit lay central within the core by binding of $\mathrm{N}-\mu 2$ with the $\beta 2$ adaptin, and $\sigma 2$ to $\alpha$ adaptin-forming the $\mathrm{N}-\mu 2 / \beta 2$ and $\sigma 2 / \alpha$ subunit heterodimer interface ${ }^{125}$. The $\mathrm{N}-\mu 2$ and the $\sigma 2$ subunit are almost identical in structure composed of a five-stranded $\beta$ sheet flanked by $\alpha$ helices, indicative of their similar roles in the structural stabilization of the AP2 core ${ }^{125}$. Furthermore, the $\mathrm{N}-\mu 2 / \beta 2$ and $\sigma 2 / \alpha$ subunit heterodimers are brought together in a multimeric arrangement, forming a shallow central dish wherein the $\mathrm{C}-\mu 2$ domain sits (Figure 1.7) ${ }^{127}$. Discussed in the following section, the $C-\mu 2$ domain is an essential component of AP2 
which binds a key motif present in several CME cargoes, essential for their recruitment and enrichment within $\mathrm{CCPs}^{125}$. This structural arrangement is not limited to AP2 of which multimeric assembly can be adapted for other AP types; albeit not as structurally defined ${ }^{125}$.

\subsubsection{3 - The AP2 complex bind several components of the endocytic machinery}

As a main constituent of a CCV and one of the central hubs of CME, AP2 serves a key role in the spatiotemporal organization of several endocytic components (Figure 1.6B) ${ }^{12}$. Through its several subunits, AP2 is recruited exclusively to the plasma membrane where it recruits motif-containing cargo proteins for incorporation within $\mathrm{CCVs}^{126}$. The $\mathrm{N}$-terminal $\alpha$ adaptin trunk of AP2 is the primary membrane interaction site which binds both $\mathrm{PIP}_{3}$ (phosphatidylinositol 3,4,5- triphosphate) and $\mathrm{PIP}_{2}$; of which binding to the latter is indispensable for $\mathrm{CME}^{125,126}$. Further described in section 1.3.5, $\mathrm{PIP}_{2}$ is an essential component of $\mathrm{CME}$, enriched at the plasma membrane, which serves as a substrate for the localization of AP2 to the cell surface ${ }^{128}$. Through $x$-ray crystallography, it was shown that the PIP 2 binding site exists within the first 80 amino acids of the $\alpha$ adaptin, which contains several basic residues compatible for interaction with the anionic lipid ${ }^{125}$. Specifically, site-directed mutagenesis indicates that lysine residues at position 55, 56 and 57 ( $\alpha$ Lys55, -56, and -57) form a basic triad which serves as the putative $\alpha$ adaptin PIP $_{2}$ binding region ${ }^{129}$. Indeed, AP2 mutants with glutamines in place of the lysine triad are not recruited to plasma membranes, concomitant with impairments in internalization of classical CME cargoes such as $\operatorname{TfR}^{123,129}$. Chang and colleagues also demonstrated that $\alpha$ adaptin alone can recruit onto the plasma membrane through $\mathrm{PIP}_{2}$ interactions, further demonstrating its role in localizing the AP2 complex to the cell surface ${ }^{130}$. Nonetheless, although the $\alpha$ adaptin serve as the strongest determinant for localizing AP2 to the plasma membrane, two additional $\mathrm{PIP}_{2}$ binding sites have been identified present in the $\beta 2$ adaptin and the $C-\mu 2$ domain, both of which are required for efficient progression of $\mathrm{CME}^{79,128}$.

The crystal structure of AP2 produced by Collins and colleagues initially indicated the inability of the $\beta 2$ adaptin trunk in binding phospholipids such as $\mathrm{PIP}_{2}{ }^{125}$. The authors describe that the $\beta 2$ adaptin is unable to bind the plasma membrane due to a missing $\mathrm{N}$-terminal $\alpha$-helix structure containing basic side chains; which is present and used by the $\alpha$ adaptin trunk in binding $\mathrm{PIP}_{2}{ }^{125}$. More recent crystallographic studies on AP2 however, reveal basic lysine residues ( $\beta 2$ Lys5, $-12,-26,-27,-29,-36$ ) from the $N$ terminus of $\beta 2$ adaptin which interacts with $\mathrm{PIP}_{2}$-containing membranes ${ }^{127}$. Similar to the lysine triad of the $\alpha$ adaptin trunk, mutation of these lysine sites to glutamate inhibits AP2 recruitment to the plasma 
membrane, indicative of their role in the cellular localization of $A P 2^{127}$. This finding was further corroborated by Kadlecova and colleagues who demonstrated a decrease in the initiation rate of CCPs in vivo, in cells expressing a $\beta 2$ adaptin mutant deficient in binding $\mathrm{PIP}_{2}$; concomitant with the inhibition of TfR internalization ${ }^{79}$. Thus, despite its controversial ability to bind $\mathrm{PIP}_{2}$, the $\beta 2$ adaptin trunk nonetheless, is an essential component of AP2 required for the proper initiation of CME.

The $C-\mu 2$ domain is the third phospholipid binding component of AP2, which similarly contains basic regions capable of $\mathrm{PIP}_{2}$ binding ${ }^{127}$. This $\mathrm{PIP}_{2}$ binding site however, is not initially accessible by the plasma membrane due to the position of the $\mathrm{C}-\mu 2$ domain within the AP2 core structure ${ }^{79}$. Further discussed in section 1.3.4.4, AP2 exists in a 'locked' cytosolic state which 'tucks' the C- $\mu 2$ domain within the AP2 core, unable to interact with the plasma membrane ${ }^{79}$. The initial binding of AP2 to the plasma membrane via $\alpha$ and $\beta 2$ adaptin 'unlocks' and activates AP2, releasing the $C-\mu 2$ domain from obstruction-leading to its interaction with plasma membrane components ${ }^{127}$. Specifically, 10 lysine residues (lys330, -334, -350, -352, -354, -356, -365, -367, -368, -373) in the C- 22 domain have been identified as the putative $\mathrm{PIP}_{2}$ binding region, of which mutations into glutamate weakens the binding of AP2 to PIP 2 -containing membranes ${ }^{127}$. This decrease in AP2 binding however, was modest and does not decrease the initiation rates of CCPs, in contrast to mutations in the $\alpha$ and $\beta 2$ adaptins ${ }^{79,127}$. Instead, binding of the $\mathrm{C}-\mu 2$ domain to $\mathrm{PIP}_{2}$ appears to be an auxiliary event which functions in the subsequent stabilization and activation of plasma membrane-bound $\mathrm{AP} 2^{127}$. Thus, the secondary lipid binding role of $C-\mu 2$ functions downstream of $\alpha$ - and $\beta 2-P_{1} P_{2}$ interactions, which promotes full activation of AP2 required for later stages in $\mathrm{CME}$; including enhancement of CCP growth and maturation ${ }^{79}$.

In addition to its intrinsic plasma membrane binding capability, AP2 functions as an essential hub in CME due to its role in binding and concentrating transmembrane cargo within clathrin-coated structures $^{12}$. Alluded to in section 1.3.2, AP2 is a crucial cargo-recruiting component which recognizes short linear amino acid sequences found in the cytosolic tails of transmembrane proteins ${ }^{77}$. These residues include the widely used and best-characterized tyrosine-based $(Y X X \emptyset)$ and dileucine-based ([DE]XXXL[LI]) sorting motifs ( $\mathrm{X}$ is any amino acids and $\varnothing$ is a bulky hydrophobic amino acid), respectively recognized by the $C-\mu 2$ domain and the $\sigma 2$ adaptin (although controversial due to reports of a $\beta 2$ adaptin dileucine binding site) ${ }^{79,83,126}$. The tyrosine motif is commonly found in classical CME cargoes including TfR and EGFR; with the latter also containing a dileucine-based sorting signal ${ }^{74,131,132}$. Recognition of these cargo motifs however, occurs only after AP2 interacts with plasma membrane 
$\mathrm{PIP}_{2}{ }^{127}$. As such, depletion of PIP 2 fails to recruit AP2 onto the plasma membrane, despite the presence of endocytic sorting motif-containing transmembrane cargoes ${ }^{79,127}$. This observation indicates that cargo recognition must follow an initial $\mathrm{PIP}_{2}$ binding event-which further described in 1.3.4.4-activates AP2 by inducing a conformational shift in its structure ${ }^{122}$.

Endocytic accessory proteins required for the progression of CME are similarly recruited and concentrated by AP2 within budding $\mathrm{CCVs}^{78}$. The appendage domains of AP2 derived from the Cterminal region of the $\alpha$ adaptin, and to a lesser extent the $\beta 2$ adaptin, serve as the primary interaction site for accessory proteins ${ }^{78}$. Both $\alpha$ - and $\beta 2$-appendages have a bilobal structure which recognizes short linear peptide sequences including the DP[FW], FxDxF and WxxF motifs present in several endocytic components $^{128}$. Endocytic components including CALM, eps15, epsin, amphiphysin, auxilin, synaptojanin and AAK1 ( $\alpha$-adaptin-associated kinase 1 ) contain these motifs which are recognized by the $\alpha$ appendage domain; with some binding overlap on the $\beta 2$-appendage ${ }^{125,133}$. Described in 1.3.1, these endocytic components define distinct stages in $\mathrm{CME}$, and are crucial for the initiation and maturation of $\mathrm{CCPs}^{51}$. Indeed, deletion of the $\alpha$-appendage domain resulted in increased formation of flat clathrin lattices, concomitant with a higher rate of CCP turnover and overall dysregulation of CME dynamics ${ }^{5}$. Interestingly, uptake of Tfn is not significantly impaired by deletion of the $\alpha$-appendage domain, suggestive of compensatory mechanisms which facilitate overall cargo internalization ${ }^{5}$. Nonetheless, depletion of the AP2 complex severely inhibits Tfn internalization, largely due to the ablation of clathrin lattice assembly within endocytic sites on the plasma membrane ${ }^{74}$.

Lastly, AP2 is a crucial component of CME due to its ability in binding and promoting the selfassembly of clathrin on the plasma membrane ${ }^{79}$. The $\beta 2$ adaptin contains the main clathrin binding site located on its 'unstructured' linker region, connecting the $\beta 2$ trunk to its appendage domain ${ }^{122}$. Previously, the role of the $\beta 2$ linker as a potential clathrin binding site had been dismissed due to great divergence in sequence between other $\beta$ adaptin isoforms; since the clathrin binding site was hypothesized to be more conserved ${ }^{134}$. Shih and colleagues however, using derivatives of recombinant AP2 constructs, demonstrated the presence of a highly conserved $\sim 50$ residue region in the middle of the $\beta 2$ linker structure ${ }^{134,135}$. This region was determined as the canonical clathrin box motif (L $\varnothing \times \varnothing[D / E]$, where $\varnothing$ is a bulky hydrophobic residue) which is recognized and bound by the N-terminal region of CHCs (see section 1.3.3.1) ${ }^{135}$. Similar to the $\mathrm{C}-\mu 2$ domain and $\sigma 2$ adaptin, the clathrin binding site on the $\beta 2$ linker however, is tucked within the AP2 core in its closed cytosolic state. The recruitment and 
assembly of clathrin lattices therefore, requires structural reorganization of AP2 into an open conformation, promoted by plasma membrane binding discussed in the following section ${ }^{135}$.

\subsubsection{4 - A large-scale conformational rearrangement in AP2 is required for CME}

X-ray crystal structure analysis reveals at least two conformations adopted by AP2, designated as the inactive 'closed', and active 'open' states (Figure 1.7) ${ }^{127}$. In the cytoplasm, AP2 assumes an inactive autoinhibited arrangement, with its cargo and clathrin binding sites structurally obstructed within the core region ${ }^{79}$. In facilitating CME of surface cargo, AP2 must therefore undergo structural rearrangements to relieve its key interaction sites from steric inhibition ${ }^{125}$. Indeed, several studies including that of Jackson and colleagues, indicate large scale conformational changes in the AP2 structure during its transition from a closed cytosolic, to an open membrane-bound state-of which striking feature includes the release of the $\mathrm{C}-\mu 2$ and $\sigma 2$ cargo binding domains ${ }^{127}$. Similarly, Kelly and colleagues demonstrates the exposure of the $\beta 2$ linker containing the clathrin box motif, upon activation and localization of AP2 on PIP 2 containing plasma membranes ${ }^{135}$. Thus, the functional significance of AP2 on plasma membranes and in CME, is largely imparted by its intrinsic autoinhibitory mechanisms, which is relieved only upon encountering physiologically relevant plasma membranes ${ }^{135}$.

As previously described, the $\alpha$ and $\beta 2$ adaptin serve as the primary contact points between AP2 and the plasma membrane, of which binding to $\mathrm{PIP}_{2}$ is required for the efficient progression of $\mathrm{CME}^{136}$. These AP2 adaptins readily recognize and bind plasma membrane $\mathrm{PIP}_{2}$, forming the initial steps in the allosteric rearrangement and activation of the AP2 structure ${ }^{79}$. Briefly, the inactive state of AP2 is stabilized by portions of the $\beta 2$ trunk, termed the latch, which sterically blocks the $C-\mu 2$ and $\sigma 2$ domains within the AP2 core ${ }^{127}$. Displacement of the $\beta 2$ latch from the AP2 core therefore, is amongst the initial events involved in the 'opening' and activation of AP2, induced by its initial binding to the plasma membrane ${ }^{127}$. Tucked within the AP2 core, rearrangement of the $\beta 2$ latch causes the release or 'flippingout' of the $\mathrm{C}-\mu 2$ domain, thereby exposing its essential tyrosine-motif and $\mathrm{PIP}_{2}$ binding sites to the plasma membrane ${ }^{127}$. The 'unlatched' conformation of the C- $\mu 2$ domain is further stabilized by AAK1, which phosphorylates $\mathrm{C}-\mu 2$ on Thr156 upon recruitment by the AP2 $\alpha$-appendage domain in a clathrindependent manner ${ }^{79}$. The phosphorylation of $\mathrm{C}-\mu 2$ on Thr156 increases the internalization rate of CME cargoes including Tfn-likely due to its role in stabilizing the open conformation of AP2 ${ }^{136}$. Thus, the initial binding of AP2 on plasma membranes induces a conformational shift, which unblocks and 
activates key interaction sites on AP2-required for efficient AP2 stabilization and recruitment of cargo destined for $\mathrm{CME}^{123}$.

The large-scale conformational shift in AP2 upon plasma membrane binding causes the release from autoinhibition of both its cargo binding motifs, which orients collinearly with the $\mathrm{PIP}_{2}$ binding regions-facilitating the simultaneous interaction of $\mathrm{AP} 2$ with $\mathrm{PIP}_{2} /$ cargo-containing plasma membranes ${ }^{127}$. In addition to its functional role in recruiting cargo within CCPs, recognition and binding of AP2 to cargo sorting-motifs imparts a stabilizing effect in the open conformation of the AP2 structure ${ }^{136}$. Specifically, binding of the $C-\mu 2$ domain to the tyrosine-based $Y X X \emptyset$ motif reinforces the unlatched structure of $\mathrm{AP} 2$ required for efficient $\mathrm{CME}^{79}$. Kadlecova and colleagues further demonstrates that deletion of this cargo binding site on C- $\mu 2$ strongly impairs endocytosis of $\mathrm{YXX} \emptyset$ - and dileucinebased cargoes, and as well as other classes of CME cargoes including LDLR ${ }^{79}$. This decrease is endocytic efficiency is likely due to the destabilization of AP2 on the plasma membrane, corroborated by decreased CCP initiation rates and clathrin recruitment in C- $\mu 2$ cargo-defective mutants ${ }^{79}$. In contrast, the dileucine-based [DE]XXXL[LI] binding site of the $\sigma 2$ adaptin appears to play a lesser role in AP2 stabilization, of which deletion does not significantly influence overall cargo endocytosis and CME dynamics $^{79}$. In support of its role in stabilizing AP2, YXX $\varnothing$ motifs are more common in plasma membrane cargoes than $[D E] X X X L[L I]$ motifs, suggestive of the tyrosine-based binding requirement for AP2 activation ${ }^{136}$. Taken together, the AP2 structure is intrinsically built to bind and recognize appropriate plasma membrane structures-containing both $\mathrm{PIP}_{2}$ and cargo components-ensuring fidelity of cargo recruitment within appropriate CME sites.

The activation of AP2 on the plasma membrane imposed by conformational rearrangements, enable the binding and stabilization of clathrin lattice assembly indispensable for $\mathrm{CME}^{79}$. Previously discussed, clathrin binds AP2 via the $\beta 2$ linker, which Kelly and colleagues describes as an event enabled only by the open conformation of $A P 2^{135}$. Similar to the $\mathrm{C}-\mu 2$ domain, the $\beta 2$ linker region is tucked and occluded within the AP2 core in its closed cytosolic state. Kelly and colleagues determined that the stabilization of $A P 2$ via $\mathrm{PIP}_{2}$ and cargo interactions, is an absolute pre-requisite in releasing the $\beta 2$ linker for clathrin recruitment ${ }^{135}$. The mere in vitro immobilization of AP2 on synthetic substrates (devoid of $\mathrm{PIP}_{2}$ and cargo motifs) through GST/His6 tags therefore does not promote clathrin recruitment and assembly, likely due to the absence of structural reorganization of AP2-induced only by $\mathrm{PIP}_{2}$ and cargo binding ${ }^{135}$. As such, reincorporation of $\mathrm{PIP}_{2}$ and cargo motifs within these artificial substrates efficiently 
restored clathrin assembly, demonstrating the necessity for the hierarchical activation of AP2 in facilitating $\mathrm{CME}^{79,135}$. Thus, while essential in recruiting and assembling clathrin lattices, the built-in autoinhibitory mechanisms of AP2 prevent unwanted cytosolic clathrin interactions, which are facilitated only upon interaction with physiological membrane ligands ${ }^{135}$.

In summary, the hierarchical activation of AP2 into an open state is defined by sequential allosterically regulated conformational changes, initially triggered by $\mathrm{PIP}_{2}$ binding of the $\alpha$ and $\beta 2$ adaptins ${ }^{127}$. The subsequent release of the $\mathrm{C}-\mu 2$ domain provides additional $\mathrm{PIP}_{2}$ and cargo binding sites, further stabilizing the open conformation of AP2 ${ }^{127}$. Finally, as AP2 completes its transition from a closed to an open state, clathrin subunits are recruited and assembled on the plasma membrane forming clathrin-coated structures. Through its appendage domains, AP2 recruits endocytic accessory proteins which aid in membrane curvature, cargo stabilization and CCP maturation ${ }^{137}$. Thus, the AP2 complex is an essential hub of $\mathrm{CME}$, which in addition to linking clathrin to the phospholipid membrane, organizes the spatiotemporal recruitment of cargo and other endocytic components indispensable for the efficient initiation and progression of $\mathrm{CME}^{128}$.

\subsection{5 - Phosphatidylinositol-(4,5)-bisphosphate $\left(\mathrm{PIP}_{2}\right)$ is a CME lipid hub} Phosphatidylinositol-(4,5)-bisphosphate $\left(\mathrm{PIP}_{2}\right)$ is a phospholipid species enriched in the inner leaflet of the plasma membrane, which functions as an essential organizing hub indispensable for the process of $\mathrm{CME}^{6}$. The importance of $\mathrm{PIP}_{2}$ in $\mathrm{CME}$ had been thoroughly implied in the previous sections, emphasized as a membrane ligand able to recruit and localize endocytic components to the plasma membrane. Specifically, $\mathrm{PIP}_{2}$ plays a crucial role in CCP nucleation, recruiting pioneer proteins including FCHO1/2, CALM, epsin, eps15, and AP2, which together coordinates the initiation of bona fide CCPs ${ }^{51,138}$. Its role in stabilizing endocytic assemblies is further exemplified by its requisite hydrolysis during the late stages of $\mathrm{CME}$, which results in the disassembly of clathrin components-leading to the uncoating of mature CCVs. Indeed, several studies in mammalian cells report a dramatic loss in CCP initiation and assembly upon depletion of plasma membrane $\mathrm{PIP}_{2}$; likely attributed to the failure in recruitment of CCP initiating factors to the plasma membrane ${ }^{138}$. Thus, $\mathrm{PIP}_{2}$ represents a critical juncture in the regulation of $\mathrm{CME}$, of which synthesis and turnover are implicated in the fine-tuning of the endocytic process ${ }^{6}$. 


\subsubsection{1 - PIP 2 is a phosphoinositide marker of the plasma membrane}

Phosphatidylinositol (PtdIns) is one of the minor phospholipids found concentrated within the cytosolic surface of cell membranes, involved in several important cellular processes. Ptdlns is composed of a glycerol backbone attached to two non-polar fatty acid chains and a D-myo-inositol polar head group ${ }^{139}$. The fatty acid constituents of PtdIns insert into the plasma membrane with the inositol head group protruding into the cytosol, representing $10-20 \%$ of total cellular phospholipids in eukaryotic cells ${ }^{140,141}$. Although yet to be experimentally verified, Ptdlns is thought to be largely made in ER membranes where the inositol head group is attached to the diacylglycerol backbone by a phosphatidylinositol synthase (PIS) enzyme ${ }^{140,142}$. Ptdlns in the ER are then distributed throughout the cell presumably by several phosphatidylinositol transfer proteins (PITP) at membrane contact sites, or possibly through vesicular carriers ${ }^{142,143}$. In general, Ptdlns serve as precursors in generating various phospholipid derivatives including phosphoinositides (PI), of which abundance is about one order of magnitude less than the overall Ptdlns content ${ }^{141}$. Despite comprising only a small fraction of the total cellular phospholipids, PIs are implicated in numerous cellular processes-controlling almost every aspect of cellular life ${ }^{140}$.

Phosphoinositides are the phosphorylated derivatives of Ptdlns generated by various phosphatidylinositol kinases and phosphatases ${ }^{140}$. Phosphorylation of Ptdlns occurs on the hydroxyl residues at the D3, D4 and D5 positions of the inositol head group, of which combinatorial phosphorylation give rise to seven phosphoinositide species (Figure 1.8) ${ }^{144}$. Each PI species has a unique subcellular distribution, enriched within distinct membrane domains which contributes to the 'identity' of different intracellular membrane compartments ${ }^{141,145}$. In addition, each PI species are recognized by subsets of functionally diverse protein effectors mostly via low affinity interactions, thereby compartmentalizing distinct biological activities within different cellular locales ${ }^{146}$. For instance, $\mathrm{PIP}_{2}$ is the primary phosphoinositide enriched within the plasma membrane which recruits protein effectors involved in CME. Previously discussed, the localization of $\mathrm{PIP}_{2}$ targets AP2 and hence, clathrin assembly towards appropriate endocytic sites on the plasma membrane ${ }^{79}$. Other PI species including PI4P and PI3P serve as signature landmarks for distinct membranes including the Golgi complex and endosomal structures, respectively, where they direct organelle-related processes ${ }^{141}$. Nevertheless, minor PI overlap may exist within common membrane domains, serving as coincident localization signals for the specific recruitment of effector proteins (i.e. low amounts of PI4P and PI3P are found in $\mathrm{PIP}_{2}$-enriched plasma membranes) ${ }^{145}$. Due to its specific enrichment in the plasma membrane and essential role in 
$\mathrm{CME}, \mathrm{PIP}_{2}$ will therefore be the focus of the following discussions with the interested reader directed to the following references detailing the biological roles of other PI species $[140,141,145]$.

$\mathrm{PIP}_{2}$ is derived from the phosphorylation of Ptdlns at the D4 and D5 positions of the inositol ring, comprising $\sim 1 \%$ of the total phospholipid content from a typical mammalian plasma membrane ${ }^{146}$. Aside from its role in regulating $\mathrm{CME}, \mathrm{PIP}_{2}$ is an important lipid anchor involved in many other cellular processes. For instance, $\mathrm{PIP}_{2}$ serves as a precursor for the generation of secondary messengers including inositol triphosphate, diacylglycerol, and $\mathrm{PIP}_{3}$ (phosphatidylinositol-3,4,5-triphosphate) of which involvement in cell signaling is well-documented in literature ${ }^{147}$. Further discussed in section 1.4, inositol triphosphate is a by-product of $\mathrm{PIP}_{2}$ hydrolysis which binds calcium receptors on the ER triggering increases in cytosolic calcium levels ${ }^{148}$. Calcium is a ubiquitous signaling molecule controlling numerous processes including cell proliferation, differentiation and survival/death-supporting the pleiotropic roles of $\mathrm{PIP}_{2}$ in regulating basic physiological functions ${ }^{149}$. In addition, $\mathrm{PIP}_{2}$ acts as a tether which attaches the cytoskeleton to the plasma membrane by binding actin-related protein components including WASP and HIP1R, thereby essential in maintaining cell rigidity and motility ${ }^{145}$. Given its diverse physiological roles, $\mathrm{PIP}_{2}$ is therefore a tightly regulated molecule of which critical balance between synthesis and turnover, is essential for maintaining optimal cellular physiology.

\subsubsection{2 - Distinct kinases and phosphatases regulate PIP 2 levels on the plasma membrane}

$\mathrm{PIP}_{2}$ is synthesized from Ptdlns via lipid kinases which catalyzes the sequential phosphorylation of the D4 and D5 positions of the inositol head group ${ }^{139}$. Phosphatidylinositol-4 kinase (PI4K) enzymes for instance, phosphorylates Ptdlns at the D4 inositol ring position creating PI4P, which is subsequently phosphorylated by type I phosphatidylinositol phosphate kinases (PIPKI) at the D5 position forming $\mathrm{PIP}_{2}{ }^{139}$. Alternatively, $\mathrm{PIP}_{2}$ can also be synthesized from an initial phosphorylation of Ptdlns at the D5 position by the type III PIPK (PIPKIII), of which PI5P (phosphatidylinositol-5-phosphate) product is converted into $\mathrm{PIP}_{2}$ by the type II PIPK (PIPKII) via phosphorylation of the D4 inositol ring position ${ }^{139}$. Although unlikely to majorly contribute to the pool of plasma membrane $\mathrm{PIP}_{2}, \mathrm{PIP}_{3}$ (phosphatidylinositol-3,4,5-triphosphate) can likewise be converted to $\mathrm{PIP}_{2}$ by phosphoinositide 3phosphatases which dephosphorylates the D3 phosphate of its inositol ring ${ }^{144}$. Despite the multiple routes in $\mathrm{PIP}_{2}$ production, phosphorylation of PI4P by PIPKI serves as the primary route in generating the majority of plasma membrane $\mathrm{PIP}_{2}$, largely due to the limited amounts of basal PI5P and $\mathrm{PIP}_{3}$ on most mammalian cell membranes ${ }^{141}$. 
PIPKIs are cytosolic proteins found peripherally associated on cell membranes responsible for the phosphorylation of PI4P into PIP $_{2}$; commonly referred to as phosphatidylinositol-4-phosphate-5kinases (PIP5Ks) ${ }^{139}$. PIP5Ks are encoded by three genes generating three mammalian isozymes involved in CME: PIP5K $\alpha,-\beta$ and $-\gamma$. While all isoforms are widely expressed and share a conserved kinase domain, differences in their relative tissue distribution and cellular localization give rise to their distinct roles in cellular processes ${ }^{139}$. PIP5K $\gamma$ for instance, is a neuronal specific isoform serving as the major producer of $\mathrm{PIP}_{2}$ in the brain. Within neurons, $\mathrm{PIP}_{2}$ is an important molecule which couples exo- and endocytic pathways necessary for retrieval of synaptic vesicles during neurotransmission ${ }^{141}$. Outside the brain, PIP5K $\alpha$ and PIP5K $\beta$ serve similar roles in contributing to efficient endocytosis of surface cargoes. PIP5K $\beta$ regulates routine constitutive endocytosis of the TfR by increasing $\mathrm{PIP}_{2}$ concentrations for AP2 binding ${ }^{139}$. PIP5K $\alpha$ on the other hand appears to participate in the stimulated-induced endocytosis of plasma membrane receptors such that of the EGFR ${ }^{143}$. Indeed, mutations in PIP5K $\alpha,-\beta$ and $-\gamma$ causes endocytic deficiencies, of which the latter resulting in embryonic neural defects owing to reductions in $\mathrm{PIP}_{2}$ production ${ }^{139}$. As an essential component of $\mathrm{CME}, \mathrm{PIP}_{2}$ production and hence, spatiotemporal regulation of PIP5Ks become critical in regulating CCP initiation, stabilization and maturation ${ }^{6}$.

Equally important in the regulation of $\mathrm{CME}$ and other cellular processes, the production of $\mathrm{PIP}_{2}$ must be accompanied with its subsequent down-regulation or turnover. While essential in CCP initiation, overexpression of PIP5K and subsequent accumulation of $\mathrm{PIP}_{2}$ affects actin dynamics, shown to decrease CME efficiency as measured by cargo internalization ${ }^{6,143}$. Although playing a minor role, turnover of $\mathrm{PIP}_{2}$ can be achieved by phosphorylation of its D3 position by type I phosphatidylinositol-4,5bisphosphate 3-kinase (PIP3K) producing PIP $_{3}$ essential in growth signaling pathways (see section 1.6.3 $)^{139,143}$. Conversely, $\mathrm{PIP}_{2}$ turnover can occur through dephosphorylation of either the phosphate on the D4 or D5 position creating PI5P and PI4P, respectively. Phosphoinositide 4-phosphatases such as the transmembrane protein TMEM55 mediates the dephosphorylation of $\mathrm{PIP}_{2}$ at the D4 position-of which functional contribution to CME however, remain unclear ${ }^{139}$. In contrast, phosphoinositide 5phosphatases play a predominant role in $\mathrm{PIP}_{2}$ turnover via dephosphorylation of its $\mathrm{D} 5$ phosphate, which was previously described a necessary event in the progression of $\mathrm{CME}^{139}$.

Phosphoinositide 5-phosphatases or Inositol polyphosphate 5-phosphatases (INPP5s), share a conserved inositol 5-phosphatase (5-Ptase) domain critical for the dephosphorylation of the D5 phosphate on $\mathrm{PIP}_{2}{ }^{139}$. Several INPP5s have been implicated in membrane traffic processes including CME 
due to their ability in binding endocytic proteins. These INPP5s include the 1) SH2-domain-containing inositol 5-phosphatase (SHIP2) which bind to the CCP protein component intersectin, the 2) OCRLa phosphatase (Oculocerebrorenal Syndrome of Lowe) which binds to clathrin and AP2, and 3) Sjn which binds to clathrin, AP2 and endophilin ${ }^{6}$. Of particular interest is Sjn which has been demonstrated to localize within CCPs throughout their lifetime, and as previously discussed, an essential CCP uncoating component which directly influences CME dynamics ${ }^{6,139}$.

In mammalian cells, Sjn exists as two isozymes, Sjn1 and Sjn2 encoded by two different genes. Both isozymes share a conserved $\mathrm{N}$-terminal Sac domain and a central 5-Ptase domain, varying only in their C-terminal region ${ }^{139}$. As a result of alternative splicing, each of Sjn1 and Sjn2 further give rise to various isoforms each containing unique C-terminal regions. For Sjn1, the major splice variants are the 145-kDa (Sjn1-145) and the 170-kDa (Sjn1-170) isoforms which both contain a C-terminal proline-rich region $^{139}$. The Sjn1-170 variant however contains an additional 30-kDa asparagine-proline-phenylalanine (NPF) domain responsible for binding specific endocytic proteins such as the eps15, AP2 and clathrin ${ }^{139}$. Sjn2 on the other hand gives rise to three variants (Sjn2-A, -B1, -B2) which structurally differ in their Cterminal regions. All Sjn phosphatases use their central 5-Ptase domains to dephosphorylate $\mathrm{PIP}_{2}$ and other phosphoinositide species at the D5 position of the inositol ring ${ }^{139}$. In addition to being a 5phosphatase, the N-terminal Sac domains of Sjn1 and Sjn2 may also function in removing the D3 and D4 phosphates from phosphoinositides, which is important for the conversion of $\mathrm{PIP}_{2}$ into other PI species during $\mathrm{CME}$ as described in section 1.3.2. In addition, variations in the C-terminal domains of Sjn enzymes allow for their unique localizations and interactions-diversifying their physiological roles within the cell ${ }^{6,139}$.

As key enzymes essential for $\mathrm{PIP}_{2}$ turnover, both Sjn isozymes have been implicated in the regulation of CME. Both Sjn variants localize within clathrin-coated structures, however, Sjn1 appears to be the predominant isoform found in most CCPs, with Sjn2 found only within small subsets ${ }^{6}$. Specifically, the longer Sjn1-170 isoform localizes within CCPs throughout their lifetime, of which exact contribution to $\mathrm{CME}$ however, remain unclear. Nevertheless, Sjn1-170 has been proposed to promote the formation of bona fide cargo-containing CCP structures by destabilizing defective endocytic assemblies ${ }^{6}$. Briefly, defective endocytic assemblies are nascent CCP structures forming solely via adaptor-PIP 2 interactions, which fail to recruit cargo along with appropriate endocytic proteins required for structural stabilization-and as such, susceptible to structural collapse upon $\mathrm{PIP}_{2}$ turnover by Sjn1 ${ }^{6}$. Productive CCP 
structures on the other hand, are stabilized by the efficient recruitment and reciprocal interactions between AP2, cargo, and endocytic accessory proteins, of which maturation into CCVs persists despite local PIP 2 turnover ${ }^{6}$. Discussed in section 1.3.2, $\mathrm{PIP}_{2}$ turnover nevertheless, is a necessary step in the uncoating of mature CCVs, mediated by the Sjn1-145 isoform recruited within CCPs-albeit during the late stages of $\mathrm{CME}^{101}$. The assembly dynamics of CCPs and overall CME is therefore dependent on the spatiotemporal organization of PIPKs and Sjns, which respectively regulates $\mathrm{PIP}_{2}$ synthesis and turnover ${ }^{6}$.

\subsubsection{3 - Phosphoinositide recognition domains recognize plasma membrane $\mathrm{PIP}_{2}$}

$\mathrm{PIP}_{2}$ serves as a plasma membrane anchor for key regulatory proteins required in CCP initiation and maturation, recognized and bound by several phosphoinositide recognition domains. The pleckstrin homology (PH) domain for instance, is a structurally well-characterized module of approximately 120 amino acids which binds several phosphoinositide species (e.g. $\mathrm{PI}, 4 \mathrm{P}_{2}, \mathrm{PIP}_{3}$ ) including $\mathrm{PIP}_{2}{ }^{150}$. Many proteins ( 250 proteins) in the human proteome including endocytic components (e.g. intersectin and dynamin) have been identified containing the $\mathrm{PH}$ domain, structurally characterized by two perpendicular anti-parallel $\beta$ sheets connected by a loop, followed by a C-terminal amphipathic helix ${ }^{150}$. Variations in loop length, surrounding amino acid sequences, and structural folds impart distinct phosphoinositide, and hence, $\mathrm{PIP}_{2}$ binding specificities and affinities to various $\mathrm{PH}$ domain-containing proteins-conferring diverse biological roles ${ }^{150}$. Nonetheless, $\mathrm{PIP}_{2}$ binding by the $\mathrm{PH}$ domain is largely driven by a conserved basic motif $\left[K-X_{n}-(K / R)-X-R\right]$ centered on the connecting loop, in which the basic lysines and arginines serve essential roles in forming hydrogen bonds with the anionic head group ${ }^{151}$. Other PIP 2 binding domains including the ENTH and ANTH modules are also found within endocytic proteins (e.g. epsin and AP180/CALM, respectively), providing additional PIP $_{2}$ recognition sites as a part of a multi-domain interaction via coincidence detection ${ }^{140}$. Previously discussed, characteristic folds and polybasic linear sequences also form binding sites for $\mathrm{PIP}_{2}$ as demonstrated by the AP2 subunits, further attesting to the robust role of $\mathrm{PIP}_{2}$ in directing endocytic assemblies ${ }^{152}$.

\subsubsection{4 - Regulation of CME by control of PIP 2}

As previously described, studies which experimentally involve the direct reduction of available $\mathrm{PIP}_{2}$ on the plasma membrane, prove detrimental to the process of $\mathrm{CME}$, causing major defects in cargo internalization along with dysregulation of clathrin lattice assembly ${ }^{99,153,154}$. Jost and colleagues for instance, reduced available $\mathrm{PIP}_{2}$ by introducing $\mathrm{PH}$ domain-containing probes into cells, which strongly binds and sequesters the anionic lipid ${ }^{153}$. Specifically, the neomycin antibiotic compound or the $\mathrm{PH}$ 
domain segment of phospholipase $C-\delta$ (further discussed in section 1.4) both effectively sequestered $\mathrm{PIP}_{2}$, significantly reducing AP2 binding to $\mathrm{PIP}_{2}$-causing defects in TfR clathrin-mediated endocytosis ${ }^{155}$. Several studies have also induced the acute depletion of plasma membrane $\mathrm{PIP}_{2}$ in cells, using a rapamycin/FRB/FKBP12 system. Briefly, this system involves the expression of the plasma membranetargeted FRB (fragment of mammalian target of rapamycin [mTOR] that binds FKBP12) protein in cells, along with a cytosolic 5-phosphatase fused FKBP12 (FK506 binding protein 12) protein, which heterodimerizes upon addition of the rapamycin compound ${ }^{154}$. The addition of rapamycin cross-links the two proteins thereby bringing the 5-phosphatase module to the plasma membrane, where it is demonstrated to catalyze the massive dephosphorylation of $\mathrm{PIP}_{2}{ }^{99}$. Indeed, depletion of $\mathrm{PIP}_{2}$ using this system was associated with a complete blockade of both Tfn and EGF internalization, corroborated by the dramatic loss of clathrin structures-demonstrating the critical importance of $\mathrm{PIP}_{2}$ in clathrin-coat dynamics and $\mathrm{CME}^{99,154}$.

While these studies involve the artificial sequestration and depletion of $\mathrm{PIP}_{2}$, distinct cellular processes nonetheless exist within cells which physiologically act on $\mathrm{PIP}_{2}$ in similar manners. As mentioned, many other proteins contain $\mathrm{PIP}_{2}$ binding domains, which in theory may compete with endocytic protein binding on the plasma membrane ${ }^{152}$. The MARCKS (myristoylated alanine-rich Ckinase substrate) protein for instance, is a cytosolic protein known to bind acidic membrane lipids such as $\mathrm{PIP}_{2}$, effectively sequestering them from other proteins ${ }^{156}$. MARCKS contains a basic effector domain composed of basic residue clusters, capable of electrostatically interacting with $\mathrm{PIP}_{2}^{157}$. Interestingly, MARCKS and $\mathrm{PIP}_{2}$ are present at similar concentrations in the cell suggesting the potential sequestration of a significant fraction of $\mathrm{PIP}_{2}{ }^{156}$. Whether or not this impacts $\mathrm{CME}$ similar to that exhibited by artificial sequestration however, is not well-understood.

Likewise, PIP 2 depletion is physiologically facilitated mainly by phosphodiesterase proteins including the phospholipase $\mathrm{C}$ enzyme ${ }^{13}$. Phospholipase $\mathrm{C}$ is activated by many signaling pathways, which binds and catalyzes the hydrolysis of $\mathrm{PIP}_{2}$ - regarded as a major pathway in $\mathrm{PIP}_{2}$ turnover ${ }^{13}$. Given the requirement for $\mathrm{PIP}_{2}$ in the assembly of endocytic machineries, the fundamental question arises of how phospholipase $\mathrm{C}$ activation may impact $\mathrm{CME}$ through enzymatic $\mathrm{PIP}_{2}$ depletion. In the following sections, the potential role of phospholipase $\mathrm{C}$ in CME will be discussed by first describing the biology of the enzyme to some detail, followed by a discussion on the potential contribution of its enzymatic action and products in regulating the process. 


\section{4- Phospholipase C (PLC) and its potential role in CME regulation}

Phospholipase C (PLC) is a phosphodiesterase enzyme which selectively catalyzes the hydrolysis of the rare phospholipid $\mathrm{PIP}_{2}{ }^{13}$. In doing so, $\mathrm{PLC}$ may directly regulate $\mathrm{CME}$ due to the requirement for $\mathrm{PIP}_{2}$ in organizing and initiating the process (see section 1.3.5). The potential role of PLC in CME however, is not well-understood and is one of the key questions investigated in this thesis. In addition, the hydrolysis of $\mathrm{PIP}_{2}$ generates secondary molecules that are involved in a variety of cellular functions including cell signaling, membrane traffic, and cytoskeleton regulation ${ }^{158}$. Of interest and further described in later sections, secondary messengers produced by PLC also induces cytosolic calcium increases which may similarly impact $\mathrm{CME}$-indicated by the established role of calcium in augmenting neuronal endocytosis during neurotransmission (further described in section 1.5) ${ }^{96}$. Whether calcium increases generated by PLC is implicated in non-neuronal CME however, is not well-understood and is also a key question investigated in this work. Thus, the role of PLC in CME might be bimodal in nature, with one functional arm acting on the direct depletion of $\mathrm{PIP}_{2}$, with the other producing secondary molecules which may interact and upregulate endocytic components (Figure 1.9A). As such, this section will describe PLC biology in context of their diversity in regulation, activation, and potential role in CME regulation.

Several isotypes of PLC have been identified in mammalian cells which are classified into six subfamilies: PLC $-\beta,-\gamma,-\delta,-\varepsilon,-\zeta$ and $-\eta$ (Figure 1.9B) ${ }^{13}$. While all capable of catalyzing PIP $_{2}$ hydrolysis, each PLC types possesses distinctive domains which impart unique modes of activation and localization mechanisms; and thus, might differentially contribute to CME. Briefly, PLC is activated by various signaling molecules including calcium, heteromeric $\mathrm{G}$ proteins, small $\mathrm{G}$ proteins, and receptor/nonreceptor tyrosine kinases-contingent on their unique regulatory domains ${ }^{158}$. Each isotype is expressed widely throughout the body, except for PLC- $\zeta$ and $-\eta$, respectively found in vertebrate sperm and neuronal cells ${ }^{13}$. Individual isotypes further generate splice variants in forming up to 30 different PLC isoforms, of which 13 have been characterized exhibiting unique tissue and subcellular distributions ${ }^{158}$. Due to their diversity, different PLC isoforms have been implicated in diverse functions including cell growth and migration, wherein PLC- $\gamma$ has been found frequently enriched and mutated in various cancers $^{159}$. Thus, differences between PLC isozyme structures impart differing regulatory and functional roles, which however, may or may not depend on their lipase activity ${ }^{160-162}$. 


\subsection{1 - The conserved core structure of PLCs facilitates PIP 2 hydrolysis}

As a phosphodiesterase enzyme, PLC catalyzes the hydrolysis of $\mathrm{PIP}_{2}$ on the glycerol side of its phosphodiester bond. This reaction generates two secondary messengers: diacylglycerol (DAG) which remain on the plasma membrane, and inositol 1,4,5-triphosphate $\left(\mathrm{IP}_{3}\right)$ which diffuses within the cytosol $^{161}$. Later discussed, these secondary messengers serve functional roles in the propagation of downstream signaling pathways, of which the latter triggers cytosolic calcium increases ${ }^{13}$. All PLC isozymes contain a highly conserved catalytic $X$ and $Y$ TIM barrel domain attached together by a negatively charged flexible linker. The flexible linker is believed to serve as an autoinhibitory region which blocks the $\mathrm{PIP}_{2}$ catalytic $X$ and $Y$ sites $^{13}$. Indeed, deletion of this flexible linker increases PLC activity by configuring them in a constitutively active state ${ }^{163}$. This catalytic region is further flanked by four EF-hand domains on the N-terminal and a single C2 domain on the C-terminal side-both of which bind calcium which contributes to enzyme regulation and activity ${ }^{163,164}$. The $\mathrm{N}$-terminal region contains the PH domain which is present in all but the PLC- $\zeta$ isoform ${ }^{13}$. The PH domain as previously described, recognizes and binds phosphoinositide species including $\mathrm{PIP}_{2}$ to varying degrees, illustrated by the different specificities and affinities of the PH domains of PLCs ${ }^{165}$. The PH domain of PLC- $\delta$ for instance, has emerged as one of, if not the strongest known recognition domains of PIP 2 , targeting PLC- $\delta$ to the

$\mathrm{PIP}_{2}$-enriched plasma membrane ${ }^{150,165}$. Specifically, due to its strong affinity to $\mathrm{PIP}_{2}$, the PLC- $\delta \mathrm{PH}$ domain fused to a green fluorescent protein (GFP), is a widely used probe for the in vivo localization of the PI species $^{152}$. Tuzi and colleagues demonstrates that the PLC- $\delta$ PH domain structure is unique in containing an additional hydrophobic binding module which increases membrane interaction ${ }^{166}$. These unique auxiliary membrane binding mechanisms might also be present in other PLC isotypes exhibiting strong PI sequestration, of which PH domain structures however remain to be elucidated ${ }^{166}$. In contrast, the PLC- $\beta$ $\mathrm{PH}$ domain binds only weakly to $\mathrm{PIP}_{2}$, of which plasma membrane localization is largely facilitated by interaction of its unique domain inserts with other protein components ${ }^{167}$.

\subsection{2 - PLCs are characterized by distinct domain inserts and extensions}

Comprised of a conserved catalytic core, all PLC isotypes share a common trait in $\mathrm{PIP}_{2}$ hydrolysis, albeit serving different roles in diverse cellular processes due to their differential modes of activation and localization. It is worth noting that although similarly conserved amongst PLC isotypes, the noncatalytic PH, C2, and EF-hand domains exhibit functional variability between different PLC types, uniquely regulating PLC activation and function ${ }^{158}$. In addition, PLCs diverge into various isoforms with 
the addition of domain inserts and extensions within their amino acid sequences ${ }^{140}$. Variation in these inserts serve as the basis in classifying PLC isoforms within respective sub-families ${ }^{13}$. Importantly, these additional domains confer unique regulatory modulations in activating and localizing PLCs within distinct sub-cellular compartments, which are discussed next.

(i) The PLC- $\beta$ subtype includes four members encoded by different genes designated as PLC $\beta 1-4$, which are activated by G-protein coupled receptors (GPCRs) ${ }^{140}$. The PLC- $\beta$ family is structurally defined by a unique coiled-coil (CC) domain extension at its $\mathrm{C}$-terminus which play several regulatory roles ${ }^{13}$. For instance, the CC domain is folded in a manner which occludes the PLC- $\beta$ catalytic site, acting as an autoinhibitory module ${ }^{158}$. The CC domain however, also provides a binding site for the $\mathrm{G}_{\mathrm{q}}$ subunit of the $\mathrm{G}_{\mathrm{q}}$-coupled GPCR on plasma membranes. Binding of the $\mathrm{G \alpha}_{\mathrm{q}}$ subunit to the $\mathrm{CC}$ domain induces a conformational rearrangement which relives PLC- $\beta$ of autoinhibition, thereby stabilizing its catalytic function within the vicinity of activated GPCRs ${ }^{13}$. The $\mathrm{CC}$ domain contains polybasic residues which is presumed to further aid in its PLC $\beta$ recruitment to the plasma membrane ${ }^{164}$.Through its PH domain, PLC- $\beta$ binds and similarly activated by proteins including G $\beta$, Rac, and Cdc42; and stabilized on the plasma membrane via PIP 2 and PI3P interactions ${ }^{158,168}$. Like all PLC isotypes, PLC- $\beta$ is stimulated by calcium binding, although its effects on overall activation with respect to other PLC- $\beta$ activators have not been studied in detail ${ }^{13}$. PLC- $\beta$ isoforms are widely expressed in many cell types, with mammalian cells commonly expressing at least two or three isoforms ${ }^{13}$. Differential tissue enrichment between isoforms however exists, with PLC- $\beta 1$ and $-\beta 4$ preferentially in the neural tissue; PLC- $\beta 2$ within hematopoietic tissues; and PLC- $\beta 3$ broadly expressed ${ }^{13,167}$. Indeed, differences in PLC- $\beta$ cellular localization and regulation result in its diverse physiological roles, implicated in cardiovascular function, chemotaxis, and neuronal signaling ${ }^{167}$.

(ii) The PLC- $\gamma$ subtype includes two members encoded by different genes designated as the PLC$\gamma 1$ and $-\gamma 2$ isoforms. PLC- $\gamma 1$ is the predominant isoform widely expressed in non-hematopoietic cells, with PLC- $\gamma 2$ found primarily in immune cells ${ }^{13}$. Both isoforms however, are characterized by a large ( 450) multi-domain insert comprised of two SH2 domains ( $\mathrm{nSH} 2$ and $\mathrm{cSH} 2$ ), one SH3 domain, and a split $\mathrm{PH}(\mathrm{sPH})$ domain of which halves flank the insert at the $\mathrm{N}$ - and $\mathrm{C}$-terminal ends ${ }^{13}$. This multi-domain segment is situated within the linker region between the $X-Y$ catalytic core, which play essential roles in PLC- $\gamma$ regulation and activation by cytosolic/receptor tyrosine kinases ${ }^{13,162}$. The $\mathrm{SH} 2$ domains for instance, serve critical roles in the activation of PLC- $p 1$, wherein $n S H 2$ recognizes and binds specific 
tyrosine-phosphorylated motifs on a variety of activated receptor tyrosine kinases (see section 1.6.3) ${ }^{140}$. Upon binding to receptor tyrosine kinases, PLC- $\gamma 1$ is phosphorylated on several tyrosine (Tyr) residues including: Tyr472, $-771,-775,-783$, and $-1254^{140}$. Specifically, phosphorylation of PLC- -1 on Tyr783 located between the $\mathrm{CSH} 2$ and $\mathrm{SH} 3$ domain, has been demonstrated essential for its activation ${ }^{140}$. Briefly, PLC- $\gamma$ exists in an inactive, autoinhibited state imparted by the $\mathrm{CSH} 2$ domain folding which occludes the catalytic X-Y-site ${ }^{158}$. Phosphorylation of PLC- $\gamma 1$ on Tyr783 induces a conformational shift relieving autoinhibition, largely due to the reorganization and binding of the $\mathrm{cSH} 2$ domain to the phosphorylated site ${ }^{13}$. PLC- $\gamma 2$ is also activated through a similar mechanism via phosphorylation of Tyr759, albeit downstream of cytosolic receptor tyrosine kinases ${ }^{13}$.

Plasma membrane targeting of PLC- $\gamma 1$ is achieved by recruitment to receptor tyrosine kinases present on the surface. However, several domains contribute to its stabilization on plasma membranes including the conserved N-terminal PH domain, which is known to bind both $\mathrm{PIP}_{2}$ and $\mathrm{PIP}_{3}$ present on the surface ${ }^{168}$. Later described, $\mathrm{PIP}_{3}$ is a secondary metabolic product of receptor tyrosine kinase activation, of which concentration on the plasma membrane significantly increases upon stimulation ${ }^{141}$. Studies indicate that relatively high concentrations of $\mathrm{PIP}_{3}$ are required to activate $\mathrm{PLC}-\gamma 1$ in vitro, thereby coupling its activity with receptor tyrosine kinase activation ${ }^{13}$. In addition, the sPH domain within the insert folds into a tertiary structure resembling a canonical PH domain, which was similarly shown to interact with phosphoinositides including $\mathrm{PIP}_{2}{ }^{169}$. Membrane binding of the $\mathrm{SPH}$ domain however, is conflicted by the crystal structure of PLC- $\gamma 1$ which reveals a missing lipid-binding domain in the sPH segment, essential for phosphoinositide binding ${ }^{162}$. Despite its controversial lipid-binding role, the SPH domain nonetheless, has been implicated as a regulatory module which binds small GTPases including Rac1, essential for enzyme activation-however, only in the PLC- $\gamma 2$ isoform ${ }^{13}$. Although unclear, the EF-hand, $\mathrm{C} 2$, and SH3 domains appear to similarly regulate PLC- $\gamma$ activity, with the latter providing a recognition site for proline-rich sequences present in adaptor and scaffolding proteinsfurther stabilizing PLC- $\gamma$ on the plasma membrane ${ }^{13,143,168}$.

Due to its ubiquitous expression in various tissues and predominant activation by receptor tyrosine kinases, PLC- $\gamma 1$ is commonly implicated in several mitogenic processes including cellular growth, differentiation, migration and invasion ${ }^{170}$. Indeed, PLC- $\gamma 1$-deficient mice died prematurely due to defects in vasculogenesis and erythrogenesis ${ }^{158}$. With its proliferative roles, dysregulation of PLC- $\gamma 1$ is often implicated in various cancer types where it is found frequently enriched and mutated ${ }^{159}$. 
Important to this thesis, the specific activation of the PLC- $\gamma 1$ by receptor tyrosine kinases therefore provides a possible route through which mitogenic signals may regulate $\mathrm{CME}$, important for plasma membrane remodeling. The PLC- $\gamma 1$ isoform will therefore be the focus in considering the potential effects of mitogenic signals in CME regulation, later described in section 1.8.

(iii) PLC- $\varepsilon$ exists only as a single isoform in mammalian cells, expressed ubiquitously in various tissues, most abundantly in the heart ${ }^{168}$. It is structurally defined by an $\mathrm{N}$-terminal CDC25-homology domain and two RA domains (RA1 and RA2) at its C-terminus ${ }^{158}$. The RA2 domain has been observed to bind the GTP-bound form of Ras and Rap signaling molecules, which recruit and activate PLC- $\varepsilon$ on the plasma membrane and the perinuclear region, respectively ${ }^{158}$. While the precise activation mechanism induced by RA2 binding remain unclear, it is thought to involve a similar mechanism observed with the CC domain of PLC- $\beta$, of which binding to GPCR subunits relieve autoinhibition ${ }^{13}$. Importantly, the Ras and Rap signaling molecules are involved in several downstream signaling pathways stimulated by GPCR and receptor tyrosine kinase activation ${ }^{13,143}$. As such, PLC- $\varepsilon$ may reasonably be activated by a diverse set of signaling pathways concomitant with activation of other PLC isoforms including PLC- $\beta$ and $-\gamma 1^{13,170}$. Activation of PLC- $\varepsilon$ is further stabilized by its CDC25-homology domain, which acts as a GEF (guanine nucleotide exchange factor) for Ras - thereby forming a positive feedforward loop in attenuating PLC- $\varepsilon$ activity over many tens of minutes ${ }^{13}$. Consistent with its enrichment within the heart, PLC- $\varepsilon$ is involved in the development and function of cardiomyocytes, with PLC- $\varepsilon$-deficient mice demonstrating impairments in cardiac development ${ }^{158}$.

(iv) In contrast to other PLC isotypes, PLC- $\delta$ which includes the $-\delta 1,-\delta 3$ and $-\delta 4$ isoforms, contain only the minimal PLC building blocks: PH, EF, X-Y and C2 domains ${ }^{143}$. Rather than direct regulation by signaling from various signaling receptors, the regulation of PLC- $\delta$ depends solely on its strong interaction with $\mathrm{PIP}_{2}$ and calcium binding ${ }^{140}$. The PLC- $\delta \mathrm{PH}$ domain appears to be the least sterically hindered amongst PLC isotypes, attached to the catalytic core through a flexible, mobile linker ${ }^{164}$. As previously described, its $\mathrm{PH}$ domain also includes an auxiliary hydrophobic module which increases its specificity and affinity for $\mathrm{PIP}_{2}$ on the plasma membrane ${ }^{13,164}$. $\mathrm{PIP}_{2}$-mediated membrane binding however, is not sufficient to activate PLC- $\delta$, which requires calcium binding for maximal activity $^{13}$. Indeed, PLC- $\delta$ binds four calcium ions at its C2 domain, and one at its catalytic site ${ }^{13}$. Given its dependence on calcium, PLC- $\delta$ is extremely sensitive to calcium fluctuations, reported to be activated by calcium concentrations in the $10-100 \mathrm{nM}$ range, which curiously is the basal calcium concentration in 
most cell types; suggesting the potentially constitutive activation state of PLC- $\delta^{171-173}$. Although the least understood PLC isoform, PLC- $\delta$ is a widely expressed protein which has been proposed to act as a signal amplifier for other PLC isoforms ${ }^{174}$. Described in later sections, $\mathrm{PIP}_{2}$ hydrolysis by PLCs induce cytosolic calcium increases, of which concentration fall within the range for PLC- $\delta$ activation ${ }^{13}$. PLC- $\delta$ may therefore, synergistically act with other PLC isoforms in $\mathrm{PIP}_{2}$ hydrolysis and elevating calcium levels involved in downstream signaling ${ }^{158}$.

Similar to PLC- $\delta$, the (v) PLC- $\zeta$ and (vi) PLC- $\eta$ isoforms also exhibit the basic PLC building blocks, of which the former responds only to calcium due to the absence of a PH domain ${ }^{13}$. Interestingly, the $\mathrm{X}-\mathrm{Y}$ autoinhibitory linker in PLC- $\zeta$ is positively charged, believed to be involved in membrane recruitment, which compensates for its lack of a $\mathrm{PH}$ domain ${ }^{13}$. Furthermore, these isoforms exhibit distinct tissue enrichment with PLC- $\zeta$ found mostly in sperm cells, and PLC $-\eta$ mostly in brain tissues ${ }^{158}$. Thus, although able to catalyze $\mathrm{PIP}_{2}$ hydrolysis, modifications present across PLC isotypes allow each to respond to its own spectrum of regulatory inputs; some of which may overlap between other PLCs ${ }^{13}$.

\subsection{3 - PLCs induce cytosolic calcium increases via PIP2 hydrolysis}

PLCs are excellent catalysts which catalyze the rapid turnover of $\mathrm{PIP}_{2}$. A kinetic quantification of its absolute enzyme activity however, proves difficult to measure due to variabilities in PLC isoforms, plasma membrane lipid composition, and assay constraints. Despite this difficulty, several laboratories have estimated specific activities including that from the PLC- $\beta$ isoform, which was determined in the range of $1000-5000$ molecules/sec at $30^{\circ} \mathrm{C}^{13}$. Apart from its efficient turnover of $\mathrm{PIP}_{2}$, a by-product of $\mathrm{PIP}_{2}$ hydrolysis by PLC involves the subsequent production of cytosolic calcium increases ${ }^{13}$. Calcium is an important signaling molecule which exhibits pleiotropic effects in diverse signaling processes, thereby expanding the role of PLCs beyond the plasma membrane ${ }^{172}$.

As a direct result of $\mathrm{PIP}_{2}$ hydrolysis, DAG and $\mathrm{IP}_{3}$ are produced which respectively act as a secondary protein anchor and signaling messenger. $\mathrm{IP}_{3}$ is an inositol ring cleaved from $\mathrm{PIP}_{2}$, with phosphate groups on the D1, D4 and D5 position. $\mathrm{IP}_{3}$ is a diffusible cytosolic molecule which stimulates the release of calcium from the ER by binding and opening $I P_{3}$ receptors $\left(I P_{3} R\right)^{175}$. Upon opening of $I P_{3} R$ calcium gates, calcium efflux from the ER into the cytosol increases cytosolic calcium concentrations from $100 \mathrm{nM}$ to $500-1000 \mathrm{nM}$, which is implicated in numerous cellular processes including cell 
signaling ${ }^{13,172}$. In contrast, the glycerolipid DAG remains membrane-bound where it serves as an anchor for proteins such as protein kinase $C$ (PKC). Briefly, PKC is a protein kinase which sits at the crossroads of many signaling events where it modifies activities of cellular proteins including receptors, enzymes, cytoskeletal proteins and transcription factors ${ }^{176}$. The PKC family is composed of nine isoforms encoded by nine genes, which can be grouped into three subfamilies based on their cofactor dependence: conventional PKCs $(\alpha, \beta$, and $\gamma)$ are activated by DAG and calcium; novel PKCs $(\delta, \varepsilon, \theta$, and $\eta)$ are activated by DAG; and atypical PKs $(\zeta, \mathrm{l})$ which are activated via protein scaffolds ${ }^{177}$. Regulated by DAG and/or calcium, conventional and novel PKCs, are therefore some of the primary downstream effectors of PLC activity. Aside from PKC, several calcium binding proteins (CBP) exists which bind calcium through any of the following modules: EF-hand domain, C2 domain, and intrinsic calcium binding pockets (further described in section 1.5.2) ${ }^{178}$. As a result, IP 3 generated by PLC activity result in both local and global calcium signals that regulate numerous cellular processes ${ }^{179}$.

\subsection{4 - PLCs may regulate CME via direct PIP2 hydrolysis and/or calcium signals} With PLC activity being a major pathway in $\mathrm{PIP}_{2}$ turnover, it is tempting to hypothesize about its role in CME. To date, it is not well-understood precisely how the direct consumption of $\mathrm{PIP}_{2}$ by PLC impacts CME. As major regulatory hub, $\mathrm{PIP}_{2}$ consumption may however, act in an antagonistic manner against CME, preventing AP2 activation and clathrin assembly which requires $\mathrm{PIP}_{2}$ as a membrane anchor $^{78}$. As previously described, several studies have demonstrated that $\mathrm{PIP}_{2}$ sequestration by either neomycin or the PLC- $\delta 1 \mathrm{PH}$ domain reduces clathrin-mediated endocytosis of $\mathrm{TfR}^{155}$. The acute depletion of $\mathrm{PIP}_{2}$ was also achieved using the inducible rapamycin/FRB/FKBP12 system which was similarly reduces TfR internalization concomitant with the loss of global clathrin puncta structures ${ }^{99,154}$. Although suggestive of its inhibitory role in $\mathrm{CME}$, whether these observations recapitulate the role of PLC in clathrin-mediated endocytosis under physiologically relevant stimuli remain unclear.

The extent and context by which PLCs consume $\mathrm{PIP}_{2}$ on the plasma membrane is not well understood and may vary between isotypes, upstream activators and cellular localization ${ }^{13}$. Briefly, the insulin receptor is a plasma membrane receptor tyrosine kinase which bind the extracellular insulin ligand (see section 1.6) ${ }^{180}$. Insulin binding result in the simultaneous initiation of both insulin receptor signaling, and internalization of the insulin-insulin receptor complex through $\mathrm{CME}^{3,181}$. Carvou and colleagues demonstrated that PLC- $\delta$ activation by ATP-coupled GPCRs diminished insulin internalization 
accompanied with increased inositol production as measured by inositol radiolabelling ${ }^{182}$. PLC- $\delta$ activation was without effect on the CME cargo $\beta_{2}$-glycoprotein I $\left(\beta_{2} \mathrm{gpl}\right)$, which the authors presume due to its basolateral distribution compared to the apical localization of both insulin receptor and ATPcoupled GPCRs. Thus, the authors determined that signaling through PLC interferes with CME through localized $\mathrm{PIP}_{2}$ hydrolysis ${ }^{182}$. In contradiction to a role for PLC in consuming $\mathrm{PIP}_{2}$ to ablate CME, Eichhorn and colleagues show that a component of insulin receptor signaling involves the direct activation of the PLC- $\gamma 1$ isoform ${ }^{183}$. The authors determined that inhibition of PLC- $\gamma 1$ by its molecular inhibitor U73122, resulted in the inhibition of insulin receptor signaling and internalization ${ }^{183}$. Insulin receptor activation by insulin was further shown to increase production of secondary PLC by-products including DAG, suggesting the requirement for PLC- $\nu 1$ activity in insulin signaling ${ }^{183}$.

From the previous studies, despite catalyzing similar enzymatic reactions, PLC- $\delta$ and PLC- $\gamma 1$ appear to impact CME in opposing mechanisms. While likely that the direct reduction of $\mathrm{PIP}_{2}$ by $\mathrm{PLC}$ may negatively regulate cargo internalization, its extent and physiological relevance to CME must be carefully considered given the many cellular contexts through which they are activated. In addition, the role of PLC on CME may be derived from its ability in triggering cytosolic calcium increases via secondary messengers, of which effect on endocytosis is well-understood, albeit in neurons ${ }^{96,184-186}$. Cytosolic calcium increases in neurons is well-established to enhance synaptic vesicle CME during neurotransmission ${ }^{187}$. Neuronal homologues of endocytic proteins-including AP2, amphiphysin, dynamin and synaptojanin - are present in neurons and are activated by calcium signals during depolarization events, enhancing $\mathrm{CME}^{187}$. Whether calcium increases derived from PLC activity similarly impacts $\mathrm{CME}$ in non-neuronal systems however, is not well-understood, of which consideration will provide insight in the possible mechanisms through which PLCs may regulate clathrin-mediated endocytosis.

The following section will thus discuss the role of PLC-derived calcium signals in CME with an initial description of basic calcium biology. Calcium binding proteins and their respective calcium binding domains will subsequently be described, followed by a discussion of their role in augmenting neuronal CME. From here, parallels will be drawn in describing the potential role of calcium in non-neuronal CME. By the end of section 1.5, the reader should conceptualize the bimodal role of PLC in the potential regulation of $\mathrm{CME}$ either by i) direct $\mathrm{PIP}_{2}$ depletion, or by ii) triggering cytosolic calcium increases, or both. 


\section{5- Calcium and endocytosis}

Calcium is a ubiquitous molecule commonly regarded as a pre-requisite for optimal skeletal development. While essential in this facet, the role of calcium ions $\left(\mathrm{Ca}^{2+}\right)$ extend far beyond bone construction, impacting nearly every aspect of cellular life ${ }^{188}$. As a small, universal cation, $\mathrm{Ca}^{2+}$ is diffused throughout the body, found in cells and within their extracellular environments. Along with phosphate, $\mathrm{Ca}^{2+}$ is one of the two main ions which dominate and facilitate signal transductions throughout the life of an organism ${ }^{172,188}$. From fertilization to death, a vast range of cellular functions are modified and directly controlled by $\mathrm{Ca}^{2+}$ levels ${ }^{184,188}$. Through alterations of local electrostatic fields and modifications of protein shapes and charges, $\mathrm{Ca}^{2+}$ increases and binding elicits direct effects in cellular responses to stimuli ${ }^{189}$. Largely dispersed throughout the body, cells however must create a gradient where an increase/influx in $\mathrm{Ca}^{2+}$ concentration can be decoded as a signaling event ${ }^{189}$. As such, cells invest a large amount of energy in creating $\mathrm{Ca}^{2+}$ gradients through its chelation, compartmentalization or extrusion from the cell (Figure 1.10) ${ }^{188}$.

\subsection{1 - Calcium gradients are maintained by channels and pumps}

In utilizing $\mathrm{Ca}^{2+}$ as a secondary signaling molecule, cells have developed strategies in maintaining its low cytosolic levels. At rest, cytosolic $\mathrm{Ca}^{2+}$ concentration is $\sim 100 \mathrm{nM}$ which can rise to $500-1000 \mathrm{nM}$ during signaling events ${ }^{172}$. This increase can be derived from extracellular $\mathrm{Ca}^{2+}$ present in the range of 1$2 \mathrm{mM}$, or through intracellular compartments such as the ER with concentrations in the range of 0.1$1 \mathrm{mM}^{172,188,190}$. The outside-to-inside chemical gradient for $\mathrm{Ca}^{2+}$ is estimated at around 15,000-40,000:1, creating one of the largest known physiological gradients in the body ${ }^{188,191,192}$. This gradient is created through coordinated efforts of various plasma membrane proteins which transport $\mathrm{Ca}^{2+}$ out of the cytosol. ATPase pumps for instance actively pump $\mathrm{Ca}^{2+}$ out of the cell into the extracellular space (via plasma membrane $\mathrm{Ca}^{2+}$ ATPases; PMCA pumps), or into the ER (via sarco-endoplasmic reticular $\mathrm{Ca}^{2+}$ ATPases; SERCA pumps) through ATP hydrolysis ${ }^{188}$. As a second mechanism, $\mathrm{Na}^{+} / \mathrm{Ca}^{2+}$ exchangers (NCX) and $\mathrm{Na}^{+} / \mathrm{Ca}^{2+}-\mathrm{K}^{+}$exchangers (NCKX) pump $\mathrm{Ca}^{2+}$ into the extracellular fluid by respectively exchanging three $\mathrm{Na}^{2+}$ for one $\mathrm{Ca}^{2+}$ ion, and four $\mathrm{Na}^{2+}$ for the co-transport of $\mathrm{C} \mathrm{Ca}^{2+}$ and $\mathrm{K} \mathrm{K}^{+}$ion ${ }^{188}$. Despite its high affinity for $\mathrm{Ca}^{2+}$, PMCA operates at a slow rate, pumping out one $\mathrm{Ca}^{2+}$ ion for every ATP hydrolyzed ${ }^{188}$. However, its high affinity allows PMCAs to remove $\mathrm{Ca}^{2+}$ even at low cytosolic concentrations, maintaining low internal levels over longer durations. Exchangers on the other hand have a low affinity for $\mathrm{Ca}^{2+}$ but operate at a rapid rate of $\sim 5,000 \mathrm{Ca}^{2+} / \mathrm{sec}^{193}$. These ion exchangers respond to rapid 
cytosolic $\mathrm{Ca}^{2+}$ influxes, which make rapid adjustments in subsequently reducing intracellular $\mathrm{Ca}^{2+}$. Thus, within the cell, both ion transporters functionally complement each other in regulating intracellular $\mathrm{Ca}^{2+}$ concentrations.

With a large electrochemical gradient, the regulated opening of $\mathrm{Ca}^{2+}$ channels both on the plasma membrane and within intracellular compartments result in the rapid increase in cytosolic $\mathrm{Ca}^{2+}$ concentrations. Voltage-operated $\mathrm{Ca}^{2+}$-selective channels (VOCs) are the fastest route through which extracellular $\mathrm{Ca}^{2+}$ enters the cell ${ }^{172,188}$. They are activated when a rapid change in voltage occurs across the plasma membrane such that in neuronal signaling or muscle contraction. Due to the large gradient, the opening of VOCs allows the entry of $\sim$ a million $\mathrm{Ca}^{2+} / \mathrm{sec}$, across thousands of channels present on the plasma membrane. Thus, VOCs can rapidly change intracellular $\mathrm{Ca}^{2+}$ levels upon activation. In nonexcitable cells, internal $\mathrm{Ca}^{2+}$ stores within the ER can increase cytosolic $\mathrm{Ca}^{2+}$ through various channels, of which $\mathrm{IP}_{3} \mathrm{R}$ and ryanodine receptor (RYR) families are best characterized ${ }^{171,172}$.

$\mathrm{IP}_{3} \mathrm{R}$ and $\mathrm{RYR}$ are $\mathrm{Ca}^{2+}$ channels on the ER which open in response to $\mathrm{Ca}^{2+}$ binding itself, either on their luminal or cytosolic side; in addition to receptor-specific ligands including $I P_{3}$ which induces $I P_{3} R$ opening upon binding ${ }^{179} . \mathrm{Ca}^{2+}$ in the range of $100-300 \mathrm{nM}$ stimulates $\mathrm{IP}_{3} \mathrm{R}$ of which opening increases cytosolic $\mathrm{Ca}^{2+}$ concentration. Above $300 \mathrm{nM}, \mathrm{Ca}^{2+}$ becomes inhibitory giving $\mathrm{IP}_{3} \mathrm{R}$ a 'bell-shaped' $\mathrm{Ca}^{2+}$ dependent relationship ${ }^{172,193}$. Interestingly, $I P_{3} R$ opening is potentiated through binding of its $I_{3}$ ligand. Briefly, $\mathrm{IP}_{3}$ is a secondary by-product of the PLC-mediated $\mathrm{PIP}_{2}$ hydrolysis, which binds to its cognate receptor $I P_{3} R$ on the ER. Upon $\mathrm{IP}_{3}$ binding, $I \mathrm{P}_{3} \mathrm{R}$ becomes more sensitive to the stimulatory effects of $\mathrm{Ca}^{2+}$ and less on its inhibitory role, shifting from a 'bell-shaped' to a sigmoidal $\mathrm{Ca}^{2+}$-dependent relationship ${ }^{172}$. Thus, signaling events which trigger $\mathrm{IP}_{3}$ production - such that derived from PLC activation-result in sustained $\mathrm{IP}_{3} \mathrm{R}$ opening which largely increases cytosolic $\mathrm{Ca}^{2+}$ concentrations. While both channels are co-expressed in numerous cell types, RYRs are predominant in the sarcoplasmic reticulum (SR) of muscle cells where its opening can be stimulated by extracellular $\mathrm{Ca}^{2+}$ influx, due to its mechanical coupling with voltage-gated channels on the $\mathrm{PM}^{188}$. RYRs may also exhibit dependence on $\mathrm{Ca}^{2+}$ similar to that of $\mathrm{IP}_{3} \mathrm{Rs}$, of which opening is stimulated by low $\mathrm{Ca}^{2+}$ concentrations, with higher concentrations playing an inhibitory role ${ }^{188}$. RYRs are named based on its ligand, ryanodine, made by the Ryania speciosa plant known to be poisonous to humans. In normal human physiology, RYRs are potentiated by binding diffusible second messengers such as the nicotinamide adenine dinucleotide (NAD ${ }^{+}$), cyclic ADP-ribose metabolites, and caffeine ${ }^{188,193-195}$. 


\subsection{2 - Calcium signals are decoded by calcium binding proteins (CBPs)}

Upon opening of $\mathrm{Ca}^{2+}$ channels, the subsequent ion increase is translated into cellular responses through various $\mathrm{Ca}^{2+}$-sensitive processes ${ }^{172}$. The role of $\mathrm{Ca}^{2+}$ as a key signaling molecule relies on calcium binding proteins (CBPs) which bind this ion using specific domains ${ }^{178}$. The major domains involved include the EF hand which binds one $\mathrm{Ca}^{2+}$ ion, and the $\mathrm{C} 2$ domain which depending on the $\mathrm{CBP}$ can bind two or three ${ }^{188}$. CBPs bind $\mathrm{Ca}^{2+}$ through these domains in a reversible process, inducing a conformational shift toggling their intracellular activity. By direct binding, $\mathrm{CBPs}$ decode $\mathrm{Ca}^{2+}$ signals through conformational changes which it relays onto its downstream targets ${ }^{193}$. As such, it is important to emphasize that $\mathrm{Ca}^{2+}$ does not participate in catalysis at the active site of enzymes, but rather act as an allosteric regulator in modulating conformational changes in $\mathrm{CBPs}^{189}$.

The EF-hand domain is a helix-loop-helix motif resembling the perpendicular spread of the thumb and the index finger of a human hand ${ }^{178}$. The structure of the EF-hand has become the prototype for a large family of proteins which have been subdivided into 66 subfamilies $^{189}$. Proteins containing this domain include parvalbumin, calpain, myosin, troponin C, calcineurin and calmodulin, which all exhibit varied affinities for $\mathrm{Ca}^{2+}$, participating in diverse signaling processes ${ }^{193}$. Calmodulin (CALcium MODUlated protelN; $\mathrm{CaM}$ ) for instance is a small, ubiquitous adapter protein capable of amplifying the diminutive size of $\mathrm{Ca}^{2+}$ to the scale of proteins ${ }^{188}$. It contains four EF-hand domains and is the largest and undoubtedly the most important $\mathrm{Ca}^{2+}$-sensing module within the cell, having changed only slightly over 1.5 billion years of evolution ${ }^{188}$. It is involved in several processes including muscle contraction, signal transduction, gene transcription, ion channel modulation and metabolism ${ }^{172}$. Upon binding $\mathrm{Ca}^{2+}, \mathrm{CaM}$ undergoes a conformational shift which exposes hydrophobic residues on its surface, which is then in turn recognized by hundreds of proteins ${ }^{188}$. These proteins include kinases such as the myosin light chain kinase involved in muscle contraction, and the brain-abundant calmodulin dependent kinase II (CaMKII) involved in maintaining synaptic function ${ }^{196}$. For example, $\mathrm{Ca}^{2+} / \mathrm{CaM}$ binding relieves the autoinhibition in the CaMKII catalytic domain which activates its kinase activity ${ }^{188}$. $\mathrm{Ca}^{2+} / \mathrm{CaM}$ also activates phosphatases such as calcineurin (CaN) through a similar mechanism ${ }^{197}$. A downstream target of $\mathrm{Ca}^{2+} / \mathrm{CaM}$, CaN is also an EF-containing CBP which in addition to $\mathrm{CaM}$, also requires $\mathrm{Ca}^{2+}$ binding for full activation ${ }^{198}$. This mode of $\mathrm{Ca}^{2+}$ regulation parallels that of the protein kinase $\mathrm{C}$ (PKC) of which full activation relies on PM and $\mathrm{Ca}^{2+}$ binding, albeit through a $\mathrm{C} 2$ domain ${ }^{199}$. 
Other proteins without the EF-hand domain, or the ability to bind EF-hand domain proteins such as $\mathrm{CaM}$, may also be $\mathrm{Ca}^{2+}$-modulated through the $\mathrm{C} 2$ domain. The $\mathrm{C} 2$ domain is a $\sim 120$ amino acid segment which exhibit a common fold of an eight-stranded antiparallel $\beta$-sandwich connected by variable loops ${ }^{188}$. About two to three $\mathrm{Ca}^{2+}$ ions can bind the $\mathrm{C} 2$ domain with a lower affinity than EF-hand proteins, but similarly induce conformational changes in the CBP. To date, the best characterized C2containing proteins are PKC, PLC and synaptotagmin all of which translate $\mathrm{Ca}^{2+}$ signals into direct responses in phosphorylation, phospholipid hydrolysis and membrane fusion respectively ${ }^{193}$. $\mathrm{Ca}^{2+}$ binding to the $\mathrm{C} 2$ domain of PKC mirrors a similar removal of autoinhibition for full activation briefly alluded to above. Other $\mathrm{C} 2$-containing proteins follow a similar activation pattern requiring additional inputs for full activation in a coincidence manner ${ }^{172,193}$. As such, $\mathrm{Ca}^{2+}$ ability in regulating many processes lie in its speed and versatility, predicated on its large gradient and diversity in interacting CBPs.

\subsection{3 - Calcium is required for endocytosis in excitable cell systems}

The opening of $\mathrm{Ca}^{2+}$ channels and its subsequent influx is a fundamental event in cellular processes. Neurons especially, greatly depend on the fidelity of its $\mathrm{Ca}^{2+}$ channels which participate in synaptic transmission. Briefly, as an action potential propagates towards the terminal axon of neurons, VOCs become activated causing an influx of $\mathrm{Ca}^{2+}$ ions within the presynapse. The presynapse contains 100-200 synaptic vesicles (SVs) filled with neurotransmitters (NTs) which undergoes exocytosis in response to $\mathrm{Ca}^{2+}$ influx ${ }^{200}$. In general, SVs are found docked and tethered on the PM through proteinfusion machines such as SNARE complexes and synaptotagmins ${ }^{188}$. Synaptotagmin is a CBP containing an $\mathrm{N}$-terminal transmembrane domain, followed by a variable linker attached to two C2 domains (C2A and $\mathrm{C} 2 \mathrm{~B})$. In addition to $\mathrm{Ca}^{2+}$ binding, the synaptotagmin $\mathrm{C} 2 \mathrm{~A}$ and $\mathrm{C} 2 \mathrm{~B}$ domains contain additional basic residues which respectively bind phosphatidylserine (PS) on the vesicle and $\mathrm{PIP}_{2}$ on the plasma membrane ${ }^{201}$. These interactions allow SVs to dock on the plasma membrane, primed for their subsequent rapid exocytosis. While the exact mechanism remains unclear, $\mathrm{Ca}^{2+}$ binding to synaptotagmin induces a conformational shift which causes the C2B domain to embed into the inner leaflet of the plasma membrane, acting as a wedge which promotes membrane bending ${ }^{201}$. This synaptotagmin-induced membrane bending result in the fusion of SVs with the PM which triggers further exocytic machineries in NT release ${ }^{201}$. 
The exocytic release of SVs in neurons must be coupled to complementary endocytic events in maintaining synaptic alignment and recycling of NT-containing SVs. In fact, that NT release underlie the basic unit of neuronal communication, neurons have developed one of the fastest mechanisms of SV recycling through fast endocytosis ${ }^{200}$. Due to its speed and diversity in signaling, $\mathrm{Ca}^{2+}$ evolved as the ideal candidate in the coupling of exo- and endocytic events in neurons. Briefly, 'dephosphins' are a set of proteins that are phosphorylated in resting nerve terminals. These proteins include AP180, amphiphysin 1/2, epsin, eps15, Dyn1 and synaptojanin, all of which have been directly implicated as essential for endocytosis (see section 1.3.2) ${ }^{96}$. AP180 is a protein isoform of the predominantly non-neuronal CALM protein, required for membrane bending, in addition to amphiphysin, epsin and eps15 which similarly work in generating membrane curvature ${ }^{10,100}$. Upon pit maturation, Dyn1, a well characterized GTPase excises the vesicle from the plasma membrane, while synaptojanin functions in the uncoating of clathrin from CCVs during CME as described in section 1.3.2 $2^{96,101,107}$. In their phosphorylated state, dephosphins remain inactive, unable to stimulate endocytosis. Their activation, as their name would suggest, result from their coordinated dephosphorylation in response to $\mathrm{Ca}^{2+}$ influx $^{96}$. As discussed, nerve stimulation result in $\mathrm{Ca}^{2+}$ entry which activate CBPs including phosphatases such as $\mathrm{CaN}$. $\mathrm{Ca}^{2+}$ binding to the CaN EFhand domain induces its activation and subsequent interaction with dephosphins. CaN dephosphorylates dephosphins, enabling them to partake in initiating endocytosis. With this role, the inhibition of CaN by its known inhibitor Cyclosporin A (CsA), result in the inhibition of synaptic transmission due to failure in vesicle recycling by endocytosis. Thus, within neurons, $\mathrm{Ca}^{2+}$ acts as an important signaling molecule which coordinates coupling between the exocytic release of SVs, and their subsequent recycling through endocytosis ${ }^{201}$.

The role of $\mathrm{Ca}^{2+}$ in coupling exocytic events with compensatory endocytosis is not limited to neurons, where the mechanism has similarly been observed in other excitable cells. Endocrine cells for instance, also participate in vesicle exocytosis, of which excitation result in the massive fusion of secretory vesicles with the plasma membrane within a short period of time ${ }^{202}$. This process similarly involves calcium binding proteins including $\mathrm{CaM}$ and $\mathrm{CaN}$, which converts $\mathrm{Ca}^{2+}$ signals into the exocytic response ${ }^{202}$. As such, endocrine cells demonstrate a similar dependence on $\mathrm{Ca}^{2+}$ in the regulation of endocytosis where it acts as a mechanism for compensatory membrane retrieval ${ }^{202,203}$. In the (neuro)endocrine insulin-secreting INS-1 cells, MacDonald and colleagues demonstrated the ability of $\mathrm{Ca}^{2+}$ in enhancing endocytosis by increasing endocytic vesicle size and fission speed. Furthermore, the 
authors demonstrate that $\mathrm{Ca}^{2+}$ may regulate INS-1 endocytosis through a similar neuronal $\mathrm{CaN}$ dependent pathway, illustrating the conserved role of $\mathrm{Ca}^{2+}$ as an endocytic activator.

\subsection{4 - The potential role of calcium is non-excitable cell systems}

In general, $\mathrm{Ca}^{2+}$ is regarded as a signaling molecule which couples exocytosis of synaptic-like vesicles with rapid compensatory endocytosis ${ }^{203}$. Studies on the role of $\mathrm{Ca}^{2+}$ in endocytosis however, has been done almost exclusively in excitable, neuronal-like cells ${ }^{204}$. Thus, while research on the subject is lacking, it is tempting to hypothesize on the universal role of $\mathrm{Ca}^{2+}$ in mediating endocytosis through comparable mechanisms outside of the neuronal context. The understanding of how $\mathrm{Ca}^{2+}$ influx may regulate constitutive endocytosis in non-neuronal cells for instance, remain unclear but may reflect a similar mechanism to that in neurons. Derivatives of neuronal endocytic proteins exist in non-neuronal cells and have been identified to similarly respond to $\mathrm{Ca}^{2+}$ either through direct binding modules, or interaction with CBPs, providing a platform for $\mathrm{Ca}^{2+}$ in non-neuronal endocytic regulation. Proteins including AP2, intersectin, eps15, amphiphysin, Dyn2 and Sjn1 are just a few endocytic proteins involved in $\mathrm{CME}$, of which functions may involve a $\mathrm{Ca}^{2+}$ regulatory component ${ }^{50,205}$. Thus, cellular processes which acutely elevate $\mathrm{Ca}^{2+}$ concentrations in non-neuronal cells, may play agonistic roles in the regulation of endocytosis.

Alluded to in section 1.4, PLCs are enzymes which generates localized $\mathrm{Ca}^{2+}$ increases within diverse cell types through $\mathrm{IP}_{3}$ production. As such, the activation of PLCs represents an acute process by which localized $\mathrm{Ca}^{2+}$ increases can be generated in non-neuronal cells. The precise role of PLC-mediated $\mathrm{Ca}^{2+}$ increases in endocytosis however, remains to be elucidated and indeed, a question addressed in this work. Based on these discussions, the role of PLCs in regulating CME my therefore be bimodal in nature, facilitated by either or both mechanisms: 1) PIP 2 hydrolysis and 2) cytosolic $\mathrm{Ca}^{2+}$ increases.

Described in section 1.2.1, mitogenic signals induce a diverse set of cellular processes which requires, to some degree, both local and global plasma membrane reorganization events. How mitogenic signals may induce plasma membrane remodeling however, is mechanistically not wellunderstood $^{59-61}$. As previously described, mitogenic signaling by receptor tyrosine kinases are known to recruit and activate PLC- $\gamma 1$, thereby inducing both $\mathrm{PIP}_{2}$ hydrolysis and cytosolic calcium increases ${ }^{206}$. The activation of PLC- $\gamma 1$ by receptor tyrosine kinases may therefore, serve as a mechanistic route by which 
mitogenic signals may regulate $\mathrm{CME}$; influencing plasma membrane remodeling. The precise role of mitogenic-induced PLC- $\gamma 1$ activities in CME regulation however, is not well-understood, serving as one of the major questions investigated in this work.

In addition to PLC- $\gamma 1$ recruitment, activation of receptor tyrosine kinases also induces their own

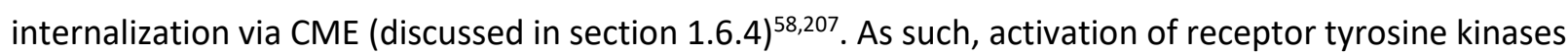
facilitates a temporal overlap between PLC- $\nu 1$ activation and initiation of CME. The regulatory role of PLC- $\gamma 1$ in RTK clathrin-mediated endocytosis however, is not well-defined, and will be the focus of this thesis in understanding how mitogenic signals may regulate overall CME. As an aside, findings from this work will indirectly address the role of acute calcium increases in the regulation of non-neuronal CME.

Receptor tyrosine kinases are robust platforms for a wide array of signaling pathways; one of which involving PLC- $\nu 1$ activation. It is therefore essential to discuss receptor tyrosine kinases in detail, to define the network within which PLC- $\gamma 1$ functions. Doing so will provide indications as to how PLC- $\gamma 1$ may regulate RTK clathrin-mediated endocytosis. The following section will thus, comprehensively describe receptor tyrosine kinases with emphasis on their activation mechanisms, signaling properties, and endocytosis.

\section{6- Receptor tyrosine kinase (RTK)}

Receptor tyrosine kinases (RTK) are the largest identified family of proteins belonging to the superfamily of enzyme-linked receptors which include serine/threonine kinase receptors, guanylyl cyclase receptors, and ligand-gated ion channels ${ }^{208,209}$. Their main function involves the translation of extracellular signals intro intracellular cues through the activation of intracellular signaling messengers ${ }^{210}$. Enzyme-linked receptors are transmembrane proteins found on the plasma membrane characterized by three domains: an extracellular ligand-binding domain, a transmembrane helical domain, and an intracellular domain with intrinsic catalytic activity ${ }^{211}$. RTKs are defined by the same three domain structure and as their name implies, possess an intrinsic tyrosine kinase activity. Its tyrosine kinase is activated upon RTK interaction with extracellular polypeptide signals including growth factors, of which binding elicits a conformational switch leading to tyrosine kinase activation ${ }^{212,213}$. The ligand-induced activation of RTKs lead to the phosphorylation of intracellular RTK segments which 
recruit and activate signaling molecules. This in turn result in the generation of a wide spectrum of signaling cascades which play fundamental roles in cell proliferation, migration, metabolism, differentiation, and survival-and thus, implicated in multiple human pathologies including cancer 208,214 .

\subsection{1 - RTK structure: three prototypic domains}

To date, 58 RTKs have been identified in the human genome which can be divided into 20 subfamilies ${ }^{215}$. Some of the best characterized and prototypic families include EGFR, insulin receptor (IR), platelet-derived growth factor (PDGF) receptor (PDGFR), vascular endothelial growth factor (VEGF) receptor (VEGFR), fibroblast growth factor (FGF) receptor (FGFR), and the neurotrophin receptor (NR) family of which member tropomyosin-related kinase receptor (Trk), binds to the first characterized mitogenic peptide, the nerve growth factor (NGF) (Figure 1.11) ${ }^{211,215}$. All RTKs share the conserved structural arrangement of an extracellular N-terminal ligand-binding domain (ECD), a single hydrophobic $\alpha$-helix transmembrane domain (TMD), and a cytosolic tyrosine kinase domain (TKD) which catalyzes the phosphorylation of tyrosine residues ${ }^{208}$. Beyond these similarities, RTK families diverge in displaying variations within the three main architectural domains, diversifying their modes of regulation, activation and signaling properties.

RTK families for instance, vary in their ECD structures defined by characteristic arrays of structural motifs, capable of binding specific protein-ligands ${ }^{216}$. Structural features such as EGF-like motifs, cysteine-rich motifs, leucine- and glycine-rich repeats, immunoglobulin-like repeats (Ig-like), and fibronectin type III repeats (FNIII) can exist solely or in different combinations on the ECD, conferring unique ligand-binding properties to RTKs. For instance, the EGFR family which consist of four RTKs in humans (EGFR/ErbB1/HER1, ErbB2/HER2/neu, ErbB3/HER3, and ErbB1/HER4) share a common ECD made up of four parts: EGF-like domains I and III, and cysteine-rich domains II and IV ${ }^{217}$. This structure interacts with a family of structurally related ligands which include EGF, transforming growth factor $\alpha$ (TGF- $\alpha$ ), betacellulin (BTC), heparin-binding EGF-like growth factor (HB-EGF), amphiregulin (ARG), epiregulin (EPR), epigen (EGN), and neuregulin (NRG) ${ }^{217}$. Most of these ligands are commonly made as membrane-bound precursor proteins, which are cleaved and released into the extracellular environment via proteases. In general, structurally related ligands may overlap in binding different receptor members, while some remain exclusive to specific receptors such as EGF and NRG, which respectively bind the EGFR and ErbB4 ${ }^{217,218}$. Further described in section 1.6.3.1, ligand binding 
constitutes one of the mechanisms through which RTK signaling may be diversified. Thus, despite sharing a common catalytic tyrosine kinase core, variations in ligand binding and receptor expression allow cells in propagating context-specific RTK signals.

While most conserved amongst RTKs, TKDs similarly exhibit partial variability present either as a full-length or a split tyrosine-kinase, of which the latter is divided into two domains by a 100 amino acid residue insertion termed the kinase insert domain (KID) ${ }^{215}$. While its exact functions remain unclear, KIDs may impose potentially significant regulatory roles as seen with its requirement for VEGFR, PDGFR, and FGFR signaling 215,219,220. In addition, TKDs are also regulated by an intrinsic kinase activation loop (Aloop) built within the domain; with the exception of EGFR (described section 1.6.2.1) ${ }^{11}$. The A-loop can be visualized in an inactive RTK as a hinge blocking the catalytic site of TKDs. Common to most autoinhibitory loops, the A-loop can be modified by phosphorylation resulting in a conformational change which removes its inhibitory role on the TKD ${ }^{221}$. While exact phosphorylation sites may differ between RTK families, A-loops contain three similarly placed tyrosine residues important for activation $^{221}$. Furthermore, TKDs are flanked by additional regulatory segments including the intracellular juxtamembrane (JM) region and the carboxy-terminal tail sequence (carboxy-terminal domain; CD) - both of which differ largely amongst RTK classes ${ }^{219}$.

The JM region separates the transmembrane from the cytosolic domain and is generally not well conserved between RTK families. It is however, highly similar between RTK family members and shown a regulatory target in modulating RTK activity. The JM region of EGFR for instance, is phosphorylated on threonine 654 (Thr654) residue by PKC during 'receptor transmodulation'222,223. Receptor transmodulation is a negative feedback mechanism which modulates the intensity and duration of RTK signaling, as in the case of EGFR following GPCR transactivation ${ }^{219,223}$. Briefly, GPCR agonist binding stimulates the release of ligands including EGF, which bind and activate EGFR on the same cell through an autocrine loop; the transactivation of EGFR is understood to amplify GPCR signaling which includes the Ras/ERK and PI3K/Akt pathways (see section 1.6.3.2) 223. Referred to in section 1.4, GPCRs are known activators of PLC $\beta$ which subsequently activates PKC through $\mathrm{Ca}^{2+}$ influx and DAG production. Santiskulvong and colleagues demonstrated that the GPCR-mediated PKC activation transmodulates and inhibits EGFR signaling by phosphorylating the Thr654 residue on its JM region. In a GPCR context, the EGFR juxtamembrane region serves as a regulatory module in fine-tuning its signaling activity. As well, this major Thr654 phosphorylation site is attributed in the diversion of EGFR trafficking from a 
degradative pathway, to the recycling endosome-which limits the ligand-induced down-regulation of EGFR signaling (see section 1.6.3.4) ${ }^{224}$. In other RTKs, the JM region may fold and act as an autoinhibitory loop blocking the TKD site. Phosphorylation in this segment elicits conformational changes which relieves the catalytic site of autoinhibition, as in the case of the ephrin (Eph) receptor family ${ }^{215,225}$. Thus, the JM region further serves to diversify RTK function by exerting additional regulatory layers to its signaling repertoires.

Lastly, variations between RTK families occur largely within their CDs which remain the most divergent segment of the receptor molecule. CDs serve as the predominant sites of autophosphorylation of which occurrence remain a hallmark in RTK activation ${ }^{11}$. CD phosphorylation typically occurs on tyrosine residues, creating phosphotyrosine (pTyr) epitopes which act as docking sites for SH2 and PTB domain-containing proteins ${ }^{208,226}$. Signaling molecules with intrinsic enzymatic activities for instance, are docked and activated as a result of binding to pTyr residues on specific RTKs. Some of these signaling molecules include the non-receptor Src tyrosine kinases, SHP tyrosine phosphatases, GTPase-activating protein (GAP) for Ras, ubiquitin ligases, and PLC- $\gamma 1$ which may facilitate CME regulation by RTKs investigated in this thesis ${ }^{215}$. Scaffolding proteins are also recruited and subsequently phosphorylated by RTKs including the insulin receptor substrate (IRS) family for IRs, FGF receptor substrate 2- $\alpha$ and $-\beta$ (FRS2- $\alpha,-\beta$ ) for FGF and NGF receptors, and Gab1 or Gab2 for EGFR and several other RTKs, providing additional recognition motifs for downstream effectors ${ }^{215}$. CDs vary between RTK families in terms of size and number of phosphorylation sites, giving receptors specificity in protein recruitment. As such, activated RTKs act as hubs which form stable complexes of signaling proteins involved in the propagation of diverse signaling networks ${ }^{215}$. With their implications to signal transduction, mutations in CDs effect dysregulated RTK signaling as observed with the ligand-independent constitutive activation of EGFR, resulting from a truncated $C^{218,227}$. While poorly characterized, $C D$ s may also act as autoinhibitory segments of which phosphorylation is required for TKD activation ${ }^{215,217,218}$. Thus, despite sharing a similar molecular architecture, RTK families are uniquely regulated by both their upstream ligand binding modules, and as well as their regulatory cytosolic domains-consistent with the key regulatory roles they play in the cell ${ }^{11}$. 


\subsection{2 - RTK activation by ligand-induced dimerization}

In general, RTKs exist on the plasma membrane as monomers in their unbound, inactive state.

Their activation and signal transducing properties rely on their ability to oligomerize into RTK dimers induced by ligand binding. Ligand binding induces a conformational shift, inducing monomers towards self-association with other RTKs through non-covalent dimerization ${ }^{218}$. Dimerization activates RTK activity by the physical juxtaposition of RTK domains including the ECD, TMD, and the TKD along with the $\mathrm{JM}$ and $\mathrm{CD}$ regions ${ }^{215}$. While activation mechanisms may differ between RTK families, it is demonstrated that adjacent TKDs within dimer pairs cross-phosphorylate one another in the process termed 'autophosphorylation'11. With the exception of EGFR, dimerized TKDs of receptor tyrosine kinases generally autophosphorylate adjacent residues including the autoinhibitory A-loop, which initially relieves their catalytic domains from autoinhibition ${ }^{11}$. Previously mentioned, intracellular segments such as the JM region, KID in split-tyrosine kinase RTKs, and CDs may also act as autoinhibitory modules which are similarly phosphorylated in the full activation of tyrosine kinase activity ${ }^{215}$. The ligand-induced dimerization of RTKs is a crucial event in receptor activation which not only serve in releasing the tyrosine kinase from inhibition, but also in the generation of protein docking sites and subsequent self-enhancement of catalytic activity.

\subsubsection{1 - Four mechanisms of RTK dimerization}

Ligand-induced dimerization is a crucial step in RTK signaling which facilitates the strong, noncovalent self-association between two dimerization-competent RTK monomers ${ }^{217}$. While common amongst RTKs, ligand-induced dimerization differs between receptor families-occurring through four possible mechanisms, described below ${ }^{208}$.

(i) In the first mechanism, dimerization occurs due to the dimeric nature of some RTK ligands which simultaneously binds two RTK monomers, bringing them in proximity for autophosphorylation. Through "ligand-mediated" dimerization, receptors do not form direct contacts, but instead are held into a dimeric complex by a bivalent ligand interface ${ }^{11}$. Specifically, the NGF receptor TrkA is the best characterized receptor whose dimerization is found exclusively ligand-mediated. Other RTK families including VEGFR and PDGFR are known receptors of dimeric ligands which however, also require reciprocal interaction between receptors in stimulating receptor activity ${ }^{215}$. 
(ii) The second mechanism of dimerization include both ligand-mediated and receptor-mediated components, of which the latter follows the former event. The VEGFR family for instance, dimerizes by the initial cross-linking of monomers through a dimeric VEGF ligand. In addition, ligand binding induces VEGFR to undergo conformational rearrangements, exposing several domains on the ECD for dimerization. The formation of receptor-receptor interactions in the ECD stabilizes the rigid dimer conformation, enabling the subsequent positioning and activation of intracellular kinase domains ${ }^{228}$. In this model, VEGFR is initially dimerized by ligand binding which initiates the sequential 'zipping' of both the VEGFR membrane proximal ECD domains, and its intracellular kinase. Other related RTKs including the Ephrin (Eph) and PDGF receptor families exhibit similar ECD arrangements, which mediate dimerization through ligand-mediated and receptor-mediated interactions ${ }^{11,228}$.

(iii) The FGFR family of receptors similarly undergo dimerization through interactions mediated by both ligands and receptors. Unique with FGFRs however, is the involvement of an accessory molecule which aids in receptor dimerization. The extracellular cofactor heparin sulfate proteoglycan (HSPG) is a membrane glycoprotein which contacts both the FGF ligand, and the FGFR. The involvement of HSPG within the dimer is thought to aid in both cross-linking FGFR monomers and establishing ligand binding specificities to FGFR family members. As well, HSPG may contribute to the activation of FGFR monomers by shifting them from a 'closed' to a more 'open' conformation. Thus, aside from ligand-receptor and receptor-receptor interactions, accessory molecules such as HSPG may participate in the cross-linking and stabilization of activated RTK dimers as observed with FGFR ${ }^{11}$.

(iv) In contrast to the oligomerizing roles of ligands described in the previous mechanisms, the fourth mechanism of dimerization is mediated entirely by receptor-receptor interactions. In this mechanism, ligands do not physically cross-link receptors but exclusively induces a conformational shift in promoting receptor self-association. Currently, the EGFR family remain the best studied and characterized RTK class, of which dimerization is found to be exclusively mediated by receptor-receptor interactions-and as such, will be the RTK of interest in this thesis, in understanding how PLCs may regulate CME. As one of the first studied RTKs for which the importance of ligand-induced dimerization was established, EGFR were previously thought to dimerize through ligand-mediated cross-linking-and deemed as the 'prototypical' RTK ${ }^{11}$. A series of EGF-bound EGFR crystal structures published in 2002 and 2003 however, provided a thematic shift in the understanding of EGFR dimerization which classified its mechanism as an 'extreme' form-far from being prototypical ${ }^{217}$. These structures show that in contrast 
to other RTKs, activating ligands of EGFR are distantly positioned from the dimer interface and do not engage in ligand-ligand interactions. As such, EGFR ligands do not directly cross-link EGFR monomers, but instead effect receptor dimerization through large-scale conformational shifts within the ECD which influences dimerization of the overall structure ${ }^{217}$.

The EGFR ECD is made up of four domains (DI-IV) wherein the structurally related DI and DIII regions primarily bind activating ligands. The cysteine rich domains II and IV are also structurally related which serve as dimerization modules participating in receptor-receptor interactions. In its inactive, unbound state, EGFR monomers are observed to be in a 'tethered' configuration, where domains II and IV exist in a closed state forming weak electrostatic interactions with one another ${ }^{11}$. Binding of the bivalent EGF ligand on domains I and III pulls domains II and IV apart, exposing the predominant 'dimerization arm' found within the DII region. This dramatic conformational shift creates dimerizationcompetent EGFR monomers which self-associates strongly through the DII dimerization arm, and weakly through the DIV region ${ }^{217}$. Thus, despite their lack of involvement at the dimer interface, EGF ligands drive the receptor-mediated dimerization of EGFR, which subsequently positions their intracellular domains for activation ${ }^{217}$.

Unique amongst RTKs, the activation of EGFR is defined by the allosteric activation of its intracellular TKD, which occurs upon formation of an asymmetric dimer ${ }^{217}$. The asymmetric dimerization of the intracellular domains of EGFR, impart a mode of activation which does not involve the typical phosphorylation of the inhibitory A-loop, common to most RTKs ${ }^{217}$. The TKD of EGFR adopts a bilobed structure forming an $\mathrm{N}$-terminal and a $\mathrm{C}$-terminal lobe, connected by a flexible hinge region which defines an ATP binding site ${ }^{229}$. Upon ligand binding, one EGFR subunit within the dimer assumes an 'activator' role, of which C-terminal TKD lobe interacts with the N-terminal TKD lobe of the adjacent EGFR, termed the 'receiver' kinase. This interaction is thought to induce allosteric changes in the Nterminal lobe of the receiver kinase thus, activating its kinase activity ${ }^{230}$. Although phosphorylation of the A-loop typically required for activation of other RTKs is not involved in this mechanism, the EGFR Aloop, nonetheless, is extruded from the active site due to conformational changes induced by the asymmetric interaction ${ }^{217}$. While sufficient in promoting EGFR activation, this model raises the question as to how the two roles are assigned between the dimeric pair. Previously regarded as a prototypical receptor, EGFR thus, represents the diversity which exists amongst RTKs, of which complete mechanisms of dimerization is yet to be fully uncovered. 


\subsubsection{2 - RTKs may exist on the plasma membrane as pre-formed dimers}

While other modes may exist, these four main modes of RTK activation are generally regarded as the canonical mechanisms of dimerization, of which future studies will identify new variations for many of the 58 human RTKs ${ }^{11}$. It is also worth emphasizing that while dimerization is understood as an event following ligand binding, an increasing number of studies have identified the presence of preformed unbound, inactive RTK dimers ${ }^{215}$. The IR family characterizes this exception which is understood to exist as constitutive dimers in their unbound, inactive configurations; which however, still requires ligand binding for activation 231 .

Interestingly, other receptors are observed to exhibit pre-formed dimers prior to ligand-binding which includes the well characterized EGFR family ${ }^{231,232}$. Seminal work from Yarden and colleagues in 1987, first described the role of ligand binding in the dimerization of RTKs. Specifically, they observed that EGFR quickly assembles into dimers upon addition of the EGF ligand which initially suggested a role for dimerization in activation of RTKs ${ }^{233}$. Recent findings however, have challenged this notion in describing the presence of unbound, inactive EGFR dimers presumed in equilibrium with monomeric structures ${ }^{234}$. Research including that of Moriki and colleagues further describes this equilibrium to favour dimeric structures, illustrating that contrary to previous findings, $>80 \%$ of unbound, inactive EGFR are constitutively dimeric in nature ${ }^{235}$. These findings suggest that EGFR monomers are inherently selfassociating molecules, which do not require the ligand-induced conformational shift within its ECDpreviously described as a requirement for its dimerization ${ }^{217}$. In support of these findings, studies have shown that apart from the ECD, the TM region along with other intracellular domains may promote receptor dimerization in the absence of ligand ${ }^{216,231}$. It remains unclear however, the cellular context by which homotypic interactions between these domains precede ligand binding since their contributions to premature dimerization appear negligible within a full-length EGFR construct ${ }^{216,231,236,237}$. Nonetheless, receptor stoichiometry appears to influence the pre-dimerization of unbound RTKs, wherein elevated expression levels appear to positively correlate with this phenomenon ${ }^{234}$. This is especially true for EGFR which produce unbound dimers at high expression levels, while strictly monomeric under lower expressions ${ }^{236}$. Work from Liu and colleagues however, obscures this finding by demonstrating the presence of pre-formed, unbound EGFR homo- and hetero-dimers at physiological expression levelsfurther supporting this thematic shift in RTK biology ${ }^{238}$. Nonetheless, unbound, inactive EGFR dimers, are likely not the same as their ligand-bound counterpart due to the asymmetric nature of the latter ${ }^{217}$. 
With this disparity, it is also important to consider the plasma membrane identity within which an RTK resides, as receptor assembly and hence dimerization, can be influenced by specific membrane microdomains ${ }^{3}$. EGFR activation and signaling for instance, may be modified depending on the identity of its microdomain which often involve complex lipid-protein interactions ${ }^{3,236}$. Lambert and colleagues for instance found that the ligand-independent dimerization of EGFR can be initiated in cholesterol depleted plasma membranes, suggesting the role of cholesterol and lipid rafts in the spatiotemporal regulation of dimerization amongst $\mathrm{RTKs}^{239}$. Using molecular dynamic simulations, Arkhipov and colleagues further demonstrates the potential requirement for lipid-protein interactions in stabilizing the inactivate state of EGFR ${ }^{237}$. They describe that anionic lipids such as $\mathrm{PIP}_{2}$ may promote and stabilize the homotypic interaction between TM domains in absence of ligands. In addition, they found that the tyrosine kinase domains contain basic patches which are sequestered by anionic lipids on the plasma membrane. Thus, it appears that membrane components including $\mathrm{PIP}_{2}$ may promote the dimerization of unbound RTKs through the TM region, but simultaneously inhibit their tyrosine kinase activity by electrostatic sequestration ${ }^{237}$. This is supported in previous work by Michailidis and colleagues, which describes a reduction in EGFR activation upon ligand binding in plasma membrane domains containing elevated levels of $\mathrm{PIP}_{2}{ }^{240}$. Taken together, the heterogeneic nature of the plasma membrane is likely to impose regulatory features towards RTK activation, which may increase or decrease their propensity for ligand-independent clustering and signaling ${ }^{241}$. More work needs to be done in consolidating these findings, but whether inactive RTKs exist as monomers or dimers, ligand binding remains a requisite event in the stabilization and activation of RTK signaling ${ }^{11}$.

\subsubsection{3 - Heterodimerization as a means of signal diversification and amplification}

While the oligomerization state of unbound RTKs remain controversial, it is generally accepted that a change in dimerization conformation precedes tyrosine kinase activation ${ }^{242}$. Dimerization can take place in one of two ways: between two identical receptors (homodimerization) or between different members of the same receptor family (heterodimerization). Homodimerization however, is commonly regarded as the canonical process through which RTKs dimerize, in part due to the paucity of methods in quantifying heterodimeric formations. Biochemical assays which largely involve the chemical crosslinking and subsequent immunoprecipitation of receptors, have been the primary strategies in studying RTK hetero-interactions ${ }^{216,243}$. While sufficient in identifying heterodimers, these techniques fail to recapitulate physiologically relevant conditions occurring in native PMs; incapable of quantifying rates of 
formation, and classifying heterodimer identities ${ }^{216,243}$. Nonetheless, several RTKs are well-documented in literature to undergo heterodimerization, which include the well-characterized HER2 receptor.

Although mechanistically not well understood, heterodimerization is believed to increase an RTKs ligand-binding repertoire, in addition to the amplification and diversification of its signaling pathways ${ }^{219,243}$. RTK signaling is predicated on tyrosine kinase activities and patterns of autophosphorylation within intracellular domains, which commonly differ between receptors. As such, variations in receptor composition within heterodimeric complexes inevitably generate diverse signaling pathways with varying biological outcomes ${ }^{215}$. Some members of the EGFR family for instance, undergo reciprocal dimerization with one another, forming heterotypic complexes with differing signaling properties $^{244}$. Consistent with its inability to homodimerize under physiological expression, HER2 is the preferred heterotypic partner of all EGFR members, of which activation and signaling occurs potently through heterodimeric complexes ${ }^{217,244}$. In addition, activating ligands had-and has still-not been identified for HER2 after its characterization in 1985, which left researchers uncertain about its role within the cellular signaling network. Interestingly, the chromosomal localization of HER2 was found identical to the neu gene in rats, which during the time had been recognized as an oncogene ${ }^{245}$. It wasn't until two years after its discovery that HER2 was similarly substantiated as an oncogene by Slamon and colleagues, who used antibody probes to measure its expression levels in primary breast tumours ${ }^{245}$. This pivotal work by Slamon was first to correlate HER2 expression with the pathogenesis of human breast cancer, of which receptor amplification was found linked with poor patient survival. Specifically, $30 \%$ of sampled breast tumors had a $~ 2-20$ fold HER2 amplification which corresponded with reduced survival and time to relapse ${ }^{246}$. Since then, HER2 became an established prognostic factor in some forms of human breast cancer, and as well as in other oncogenic manifestations including ovarian carcinomas $^{247}$.

Part of the oncogenicity of HER2 is attributed to its ability in promiscuously heterodimerizing with other EGFR members. While similar to EGFR in overall architectural arrangement, the HER2 crystal structure reveals an extended or 'un-tethered' ECD, of which fixed configuration supersedes the requirement for ligand binding in activation. Specifically, the HER2 ECD exists in an open conformation in where the dimerization arm is constitutively exposed-bypassing its requirement for a ligand-induced conformational shift within the ECD ${ }^{248}$. Garrett and colleagues further describe that the HER2 ectodomain contain a greater distribution of acidic and basic residues compared to EGFR, giving HER2 a 
larger electrostatic potential ${ }^{248}$. This large electrostatic potential is described to prevent HER2 from homotypic dimerization, due to electrostatic repulsions between the dimerization arm and its binding pocket on an adjacent HER2 ${ }^{248}$. Poised for dimerization however, HER2 instead form heterodimers with other ligand-bound EGFR monomers, which subsequently engage in tyrosine kinase autophosphorylation and signaling. Thus, despite the absence of an activating ligand, HER2 receptors can be activated by all other EGFR members, forming heterodimers such as EGFR/HER2 and HER2/ErbB3 - of which the latter is believed the most biologically active and pro-tumorigenic of all ErbB dimer complexes ${ }^{216}$.

Along with HER2, ErbB3 is also unique in requiring ligand-induced heterodimerization for activity. While capable of forming homodimers, ErbB3 lacks critical amino acid residues involved in catalysis and therefore has no-or very low - tyrosine kinase activity ${ }^{215}$. As such, ErbB3 activation rely on heterodimerization with other receptor members including EGFR, HER2 and ErbB4, which provide surrogate tyrosine kinases to compensate for the non-functional catalytic domain of ErbB3 ${ }^{210}$. Within the heterodimer, ErbB3s serve as phosphorylatable scaffolds capable of augmenting protein recruitment-exemplified by its powerful role in amplifying PI3-kinase activity ${ }^{215}$. With this role, ErbB3 is similarly implicated in oncogenic diseases including human breast cancer, of which overexpression is commonly observed together with HER2 ${ }^{244}$. Although mechanistically unclear, other receptors such as VEGFR also produce heterodimeric complexes with differing signaling capabilities, serving similar roles in the diversification and amplification of receptor signaling ${ }^{232}$. Recently, improved methodologies have also identified the intrinsic amino acid residues responsible for RTK heterodimerization, albeit only in FGFR $^{243}$. These improvements involve the powerful method of Forster resonance energy transfer (FRET), coupled to previously established biochemical assays-allowing researchers to quantify rates of formation and as well as in classifying identities of heterodimeric complexes ${ }^{243}$. Thus, although rudimentary in current understanding, future optimizations of research techniques will inevitably uncover the explicit roles of heterotypic interactions in the diversification, amplification, and propagation of RTKs' diverse signaling pathways.

This thesis thematically focuses on the ability of mitogenic signals in regulating CME; and hence influence plasma membrane remodeling. Mitogenic signaling by RTKs is specifically considered due to their ability in binding and activating PLC- $\gamma 1$, of which potential implications on CME was described (see section 1.4 and 1.5). In addition, some RTKs-under various contexts-are internalized via CME upon 
activation, providing an ideal model through which the requirement for PLC- $\gamma 1$ in overall CME may be investigated. The comprehensive discussion on RTK diversity from the previous sections highlights differences in receptor structure and activation mechanisms-contingent on their physiological roles within mammalian systems. Important to this thesis, this diversity presents potentially distinct regulatory mechanisms which not only influences receptor function, but also mechanistic details of internalization via CME. As such, the requirement and role for PLC- $\gamma 1$ in CME of the 20 distinct families of RTKs may vary - of which complete elucidation is both daunting and beyond the scope of this work. The thesis will therefore focus on EGFR which is the best-characterized RTK family, with special focus on EGFR homodimers. Later discussed in section 1.6.4, EGFR (from here used in referring to ErbB1, unless stated otherwise) homodimer internalization largely occurs via $\mathrm{CME}$, of which mechanism is bestdefined amongst RTKs. Focusing on EGFR will thus, enable identification of the potential roles of PLC- $\gamma 1$ in regulating $\mathrm{CME}$, providing clues as to how mitogenic signals may remodel the plasma membrane landscape.

\subsection{3 - RTK signaling}

Previously introduced, the diversity amongst receptor structures enable RTKs in responding to a wide array of extracellular cues-uniquely decoded into intracellular signals directing cell growth, differentiation, and function ${ }^{215}$. While conceptually straightforward, RTK signaling is complex, of which diverse biological processes involve a challenging amount of protein interactions ${ }^{196,249}$. The prototypical signaling network of the well-characterized EGFR family for instance, was determined to involve 122 proteins which form 211 different types of interactions ${ }^{250}$. Quantitative data for the vast majority of these interactions however, is currently lacking which impedes the full deterministic modeling of RTK signaling ${ }^{11}$. Despite this complexity, extensive research on EGFR have provided useful insights in understanding RTK signaling topology. Specifically, biochemistry tools along with computational modeling have allowed systems biology in elucidating a "bow-tie" or "hourglass" network structure in describing RTK signaling ${ }^{249}$. Three sequential signaling layers make up this network model; the first layer consisting of broad input signals from ligands and multiple RTKs; the narrow second layer serving as the 'core processing' stage conserved amongst RTKs; and the broad third layer representing the diverse biological outputs of RTK signaling ${ }^{249}$. Individual layers of the EGFR signaling architecture will be discussed in this section. 
Important to this thesis, the concomitant CME of EGFR induced upon receptor activation is regulated by its own distinct signaling activities; which the role for the PLC- $\gamma 1$ signaling pathway is currently undefined. The EGFR signaling network is highly interconnected, composed of distinct signaling pathways regulated by overlapping regulatory mechanisms. As such, understanding precisely how PLC1 may regulate EGFR clathrin-mediated endocytosis requires discussion of overall EGFR signaling activities. The following section will therefore discuss the basic signaling components of EGFR, in providing insight into the functional role of PLC- $\gamma 1$ within the EGFR network.

\subsubsection{1 - The first layer of RTK signaling: signal input}

The first layer in the RTK bow-tie signaling topology consists of broad signaling input, derived from ligands and receptors which collectively induce RTK activation ${ }^{11}$. Specifically, the molecular diversity amongst ligands and receptors both contribute to distinct phosphorylation and hence, activation patterns of RTKs ${ }^{249}$. The input layer for EGFR activation for instance, primarily involve 11 ligands and four receptor types, of which individual parameters and ligand-receptor derivatives often drive distinct biological outcomes ${ }^{11}$. The polypeptide growth factor ligands which activate EGFR for example, are extracellular signals of which concentrations vary within cellular microenvironments. All cells synthesize one or more growth factors commonly secreted as soluble molecules, or synthesized as transmembrane proteins-released into the extracellular milieu by proteases ${ }^{211}$. Furthermore, growth ligands may be made locally and provided within regional tissues through a group of mechanisms involving paracrine, autocrine, intracrine and juxtacrine interactions ${ }^{196}$. Growth factors commonly operate in a paracrine manner-synthesized in one cell and exerts its effects on a neighbouring celllocally inducing RTK activities on a cell-to-cell basis ${ }^{211}$. Ligands may also function in an endocrine manner, capable of remotely activating distant RTKs by entering the circulatory system ${ }^{196}$. As such, ligand bio-availability fluctuates within a given tissue depending on their localized production, and remote synthesis. With that, it is worth emphasizing that biological outputs in RTK signaling result from the collective summation of multiple tyrosine kinase activities, of which duration and signaling strength are thereby dependent on extracellular ligand concentrations ${ }^{211}$. For example, PDGF stimulation of

PDGFR leads to both cell migration and proliferation-but within single cells are two mutually exclusive processes $^{251}$. Donatis and Cirri further show that under low PDGF concentration, PDGFRs induce migration while higher concentrations result in cell proliferation. Thus, signaling inputs by ligands quantitatively modulate receptor activation, enabling PDGFR - and likely many other RTKs -in inducing variable signaling outputs ${ }^{251}$. 
In addition to their bio-availability within the cellular microenvironment alluded to above, inherent ligand properties similarly contribute to the initiation of divergent signaling pathways. Many studies have indicated ligands to be 'functionally selective' capable of binding the same receptors-yet producing distinct cellular responses ${ }^{252}$. The 11 activating ligands of the EGFR family for instance, have varying receptor affinities and exclusive binding specificities implicated in distinct cellular processes $^{215,253}$. Of prominence, is the group of ligands which include EGF, TGF- $\alpha$ and AR which competitively bind to EGFR ${ }^{254}$. The respective binding of these ligands however, elicit different responses as demonstrated with $A R$ in stimulating greater motility and invasiveness in human mammary epithelial cells in vitro; in contrast to $\mathrm{EGF}^{253}$. As well, in transgenic mouse models, AR induces epidermal hyperplasia, aberrant differentiation, resistance to apoptosis, and increased inflammation of the epidermis-not observed with TGF- $\alpha^{253}$. In context to human diseases, the predominant expression of EGF in human breast cancer is associated with a more favourable prognosis, whereas TGF- $\alpha$ expression correlate to more malignant outcomes ${ }^{253}$. Similarly, non-small-cell lung carcinoma patients experience better prognosis when exhibiting higher EGF serum concentrations, whereas TGF- $\alpha$ and AR expression correlate to more aggressive tumor forms ${ }^{253}$. Thus, these studies suggest distinctly inherent molecular properties which allow ligands in eliciting diverse biological outputs-despite sharing cognate receptors.

The exact mechanism by which ligands may facilitate functional selectivity is not wellunderstood-although mutagenic analysis of receptor tyrosine kinase ECDs indicate the involvement of ligand-induced conformational rearrangements ${ }^{253}$. Ligand binding induces a conformational shift within the ECD of RTKs, resulting in receptor homo- or hetero- dimerization-activating the tyrosine kinase. Activated tyrosine kinases autophosphorylate intracellular tyrosine residues on the carboxy-terminal tail (CD) of adjacent dimeric partners, creating pTyr epitopes. These epitopes serve as specific docking sites for signaling molecules involved in several signaling cascades and thus, central to RTK signaling ${ }^{11}$. Previously mentioned, CDs are the most divergent segments amongst RTKs which contain distinct phosphorylatable tyrosine sites ${ }^{219}$. The EGFR carboxy-terminal tail for example, contains approximately 20 phosphorylatable tyrosine residues of which dynamic phosphorylation mediate the spatiotemporal recruitment of signaling proteins; PDGFR $\beta$ contains 19 known phosphorylatable tyrosine residues, IR with 13, and TrkA with $6^{254-256}$. It is postulated by Wilson and colleagues that the phosphorylation patterns of these tyrosine sites are specified by distinct ligand-receptor interactions. They elaborate that different ligands stabilize subtly distinct ECD conformations-which through the dimeric interface-is transmitted to the intracellular domains, altering details of activation ${ }^{253}$. As such, diverse 
autophosphorylation patterns can hypothetically be produced contingent on the distinct spatial juxtaposition of intracellular domains. In support of this hypothesis, structural comparisons between EGF-EGFR and TGFa-EGFR bound complexes reveal subtle differences between their ECD structureshighlighting the plausibility of a ligand-induced receptor phosphorylation mechanism ${ }^{253}$. Through this mechanism, distinct ligands are presumed to mediate functional selectivity by creating conformationally-distinct dimers with different complements of phosphorylated tyrosine residuesultimately inducing unique sets of signaling pathways with diverse biological outcomes ${ }^{253}$.

Evidence supporting the ligand-induced receptor phosphorylation hypothesis can be derived from the observation that different EGFR ligands stimulate distinct patterns of EGFR tyrosine phosphorylation. Gilmore and colleagues for instance, showed that EGF potently induce phosphorylation of Tyr1045, $-1068,-1148$, and -1173 in three different cell lines, whereas AR does not $^{257}$. This differential phosphorylation pattern corresponds to distinct intracellular activities resulting from the differential recruitment of signaling proteins. For instance, the abundant EGF-dependent phosphorylation of Tyr1045 creates a docking site for the strong recruitment of the E3 ubiquitin ligase, $\mathrm{Cbl}$ (Casitas B-lineage lymphoma; c-Cbl and $\mathrm{Cbl}-\mathrm{b}$ isoforms) ${ }^{254}$. Cbl is responsible for EGFR ubiquitination which promotes its trafficking towards the lysosomes for degradation-thereby attenuating receptor

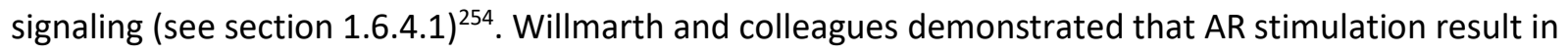
low pTyr1045 which correlate with low EGFR ubiquitination-in contrast to EGF ${ }^{258}$. Consistent with this finding, they find that EGFR remained stable in human mammary epithelial cells overexpressing AR, whereas it was degraded in the presence of EGF-emphasizing the distinct roles of EGF and AR in RTK internalization and signaling ${ }^{258}$. Additionally, EGF and TGF $\alpha$ have similarly been found to induce overlapping cellular processes-however using different protein effectors ${ }^{259}$. Ellis and colleagues adds that EGF and TGFa both induce the equipotent stimulation of migration in fibroblast, however requiring the cell adhesion receptors $\mathrm{CD} 44$, and integrin respectively ${ }^{259}$. Taken together, by inducing distinct patterns of tyrosine phosphorylation through subtle conformational modifications, different ligands can propagate diverse RTK signaling pathways with varying strengths, durations, and overall biological outcomes.

Able to differentially impact EGFR signaling, this thesis will therefore solely focus on the EGF ligand in activating EGFR, due to its role in promoting CME via Cbl binding (further discussed in section 1.6.4.1). As well, EGF results in the phosphorylation of Tyr1173, which is one of the two preferred PLC- $\gamma 1$ 
docking sites; with the other being pTyr992 ${ }^{260}$. The activation of PLC- $\gamma 1$ by EGF stimulation of EGFR is further corroborated by the downstream activation of PKC, which previously discussed, is activated in response to PLC- $\gamma 1$ activity (see section 1.4$)^{261}$.

\subsubsection{2 - The second layer of RTK signaling: core-processing}

Following the first layer, the broad range of signaling input converge into the secondary 'core

processing' stage, where their information is collectively decoded into signaling pathways ${ }^{250}$. Analogous to the center of a bow-tie, the second layer represents the narrow convergence of a wide array of signaling input into small sets of signaling intermediates ${ }^{262}$. Friedlander et al., describes that mammalian signal transduction systems commonly follow this topology, wherein a set of less than 10 intracellular pathways mediate the conversion of information, from hundreds of possible input signals into diverse biological outputs ${ }^{262}$. In context of RTKs, the diverse molecular input from ligands and receptors result in differential receptor activation patterns, which are decoded by the cell into a relatively small number of intracellular signaling cascades ${ }^{11,241}$. As such, only a handful of conserved molecules and interactions mediate the diverse biological outputs generated from all RTKs ${ }^{250}$. It is believed that the evolution of this signaling architecture impart robustness to RTK signaling, which enable core processes to persist despite input perturbations ${ }^{249}$. With that, although individual core processes are well-understood in literature, how diverse input signals are summed into distinct core processes require further work of which complex cross-talk obscures its deterministic understanding; therefore the reader is referred to select publications providing insight on signal transduction modeling [249,250,262,263].

Emphasized in the previous section, information from ligand-binding are translated into receptor docking sites which recruit signaling proteins implicated in diverse RTK signaling pathways. These proteins are the foundation for a handful of canonical core signaling cascades well-defined in literature ${ }^{250}$. While diverse in receptor classes and sub-classes, RTK signaling is collectively limited and such, may be based on the comprehensively studied EGFR family ${ }^{11}$. Briefly, the EGFR signaling pathway is one of the best-investigated signaling systems, of which dysregulation is widely associated with human malignancies; attested by the amount of drugs in clinical use targeting these receptors ${ }^{264}$. At the time of writing, a PubMed search for the term 'EGFR' returned $\sim 50,000$ publications of which vast majority involve uncovering details of receptor activation, expression, and relevance to cancer ${ }^{265}$. Reflecting this importance, its core-processing modules are experimentally and computationally well characterized allowing for its dynamic analysis. Using this multitude of data, Oda and colleagues 
comprehensively compiled a map of the EGFR signaling pathway highlighting key processes involved ${ }^{250}$. It is well understood that other RTK families utilize these canonical pathways with variations in location, intensity and duration ${ }^{220,249}$. Thus, although explicit in describing EGFR signaling, signaling properties of other RTK families may draw parallels from the following discussions; of key protein-protein interactions facilitating intracellular RTK network cascades.

\subsubsection{1 - Activated EGFR recruits a diverse array of signaling proteins}

Activation of EGFR leads to the phosphorylation of its tyrosine residues creating specific docking sites for downstream signaling proteins, containing Src-homology 2 (SH2) and phosphotyrosine-binding (PTB) domains ${ }^{254}$. SH2 and PTB domains recognize pTyr sites and are found in diverse proteins including enzymes of distinct catalytic functions, and adapters with specific interactions ${ }^{226}$. The SH2 and PTB domains however, recognize pTyr sites in an appropriate amino acid context, imparting specificity in binding activated RTKs and other tyrosine phosphorylated effectors ${ }^{226}$. EGFR for instance, bind SH2containing proteins with enzymatic activities such as phosphodiesterase PLC- $\gamma 1$, the non-receptor tyrosine kinase Src, phosphatidylinositol 3-kinase (PI3K), phosphotyrosine phosphatase SHP2, GAP for Ras, and ubiquitin ligase $\mathrm{Cbl}$; and adaptor proteins such as growth receptor-bound protein 2 (Grb2), Grb2-associated binding protein 1 (Gab1), and Src homology domain-containing adaptor protein C $(\mathrm{Shc})^{254}$. Enzymatic effectors directly translate EGFR activation into intracellular responses creating signaling intermediates, while adaptor and scaffold proteins act in recruiting additional signaling proteins to the receptor-of which collective activities stimulate various intracellular signaling pathways ${ }^{266}$. In addition, other structural modules are utilized by these proteins in facilitating distinct biological functions including phospholipid binding domains: PH or FYVE, C1, and C2 which respectively bind $\mathrm{PIP}_{2} / \mathrm{PIP}_{3}, \mathrm{DAG}$, and calcium; and the $\mathrm{SH} 3$ domain recognizing sequences with proline residues important in protein-protein interactions ${ }^{226}$. As such, activated RTKs form unique signaling platforms, of which protein complexes propagate core signaling processes ${ }^{267}$. Later described in section 1.6.4, activated EGFR similarly induces the recruitment of endocytic proteins required for $\mathrm{CME}$, of which binding and assembly may be modulated by several of the mentioned effectors including PLC- $\gamma 1, \mathrm{Cbl}$, and Grb2.

The major EGFR signaling cascades in humans include the (1) MAPK pathway, and the (2) PI3K/Akt pathway; with other key intracellular pathways including the (3) Src, (4) STAT, and the (5) PLCү1 pathway (Figure 1.12) 267,268 . Other RTK families similarly activate these common pathways although 
the location, intensity, and duration of such signaling events exhibit unique patterns contingent on ligand, RTK, and cell-type. In this manner, the signaling system is capable of distinctly controlling cell function despite propagation of conserved core processes 220 .

The following discussions will thus extensively describe key mechanistic events in the propagation of distinct EGFR pathways. Although not explicitly crucial in the investigation of the role of PLC- $\gamma 1$ in EGFR clathrin-mediated endocytosis, discussion of the major EGFR pathways nonetheless, provide contextual insight into potential mechanisms which may interact and influence PLC- $\nu 1$ activities. Later described in section 1.6.4, the role of PLC- $\gamma 1$ in CME may also be indirectly inferred from measurements of EGFR signaling activation (e.g. PI3K/AKT) - thus, warranting comprehensive discussion 4 .

\subsubsection{2 - The MAPK pathway: A high-profile signaling pathway conserved amongst RTKs}

The MAPK pathway is one of the major and best studied RTK signaling pathway involving the mitogen-activated protein kinase (MAPK) which regulate important processes such as cell proliferation, gene expression, differentiation, mitosis, cell survival, and apoptosis ${ }^{269}$. The pathway is triggered by the Grb2 adaptor which may directly bind activated EGFR at pTyr1068 and -1086; or indirectly by binding the Shc adaptor, which binds EGFR at pTyr1148 and -1173 sites $^{267,270}$. Grb2 is an adaptor protein composed mostly of SH2 and $\mathrm{SH} 3$ domains which respectively bind RTKs, and proline-rich sequences of numerous target proteins including the GEF protein, Son of Sevenless (Sos $)^{270}$. In addition to its proline-rich Cterminal region recognized by Grb2, Sos contains an addition $\mathrm{N}$-terminal PH domain capable of binding phosphatidic acid generated upon EGFR activation - further aiding its recruitment to the plasma membrane ${ }^{106,271}$. On the membrane, Sos interacts and activates the monomeric $G$ protein Ras; a small GTPase belonging to the Ras superfamily of proteins ${ }^{271}$. Briefly, Ras is a membrane localized GTPase which cycles between an inactive GDP-bound, and an active GTP-bound state-with similar affinities for GDP and GTP272. The very slow dissociation of GDP however, stabilizes the inactive state of Ras, thus, requiring signal input in provoking an enhanced GDP/GTP exchange ${ }^{272}$. Sos is a known Ras-GEF of which binding causes a conformational shift in Ras-inducing the release of GDP ${ }^{268}$. Due to its high cellular concentration, upon Sos action GTP readily binds Ras, displacing Sos in the formation of an active GTPbound complex. Ras is an essential component of the MAPK pathway, of which aberrant activity has been greatly characterized in malignant transformations ${ }^{271}$. 
The activation of Ras by the Grb2-Sos complex represent a prerequisite step in initiating the MAPK cascade. Activated, Ras-GTP initiates the three-tiered RAF-MEK-ERK cascade which starts with the activation of its major effector-rapidly accelerated fibrosarcoma (Raf) kinase ${ }^{273}$. Raf is a serine/threonine-specific kinase activated by Ras-GTP as a result of an interaction-induced conformational shift ${ }^{274}$. The Raf kinase family (A-, B-, and C-Raf) is defined by a three domain structure: an $\mathrm{N}$-terminal conserved region 1 (CR1), containing the Ras-binding domain (RBD) and the cysteine rich domain (CRD); the conserved region 2 (CR2) rich in serine/threonine sequences; and the C-terminal kinase domain ${ }^{273}$. In its inactive cytosolic state, the Raf CR1 region act as an autoinhibitory segment which folds over the kinase domain inhibiting its catalytic activity. Furthermore, the inactive conformation of Raf is stabilized by binding of 14-3-3 protein on its phosphorylated Ser259 within the CR2 region; a product of negative inhibition by the MAPK pathway ${ }^{273}$. Through yet a poorly understood mechanism, Ras-GTP recruits Raf onto the plasma membrane through the RBD, imposing structural changes relieving its kinase domain of autoinhibition-correlating with Ser259 dephosphorylation and release of 14-3-3 protein ${ }^{273}$. Recent data demonstrate that Raf proteins bound to activated Ras form homo- and hetero-dimers from which various Raf activities originate ${ }^{273}$. Although mechanistically unclear, dimerization is thought to result in full activation of Raf via phosphorylation, enabling the subsequent recruitment and phosphorylation of the MAP/ERK kinase (MEK) which propagates signaling down the three-tiered MAPK module ${ }^{273}$.

The MEK protein represents the second stage of the three-tiered signaling cascade, which remains the only well-established physiological target of Raf ${ }^{275}$. The MEK family is a dual nature serine/threonine/tyrosine kinase consisting of the ubiquitously expressed MEK1 and MEK2 (MEK1/2) proteins, sharing a similar structure: a trifunctional $\mathrm{N}$-terminal sequence containing the ERK binding domain, nuclear export sequence, and an inhibitory segment; a protein kinase domain with the activation segment and a proline-rich region; and a C-terminal domain ${ }^{276}$. Like most kinases, MEK1/2 exists in an autoinhibited conformation in its inactive state, mediated by the $\mathrm{N}$-terminal inhibitory segment. Its activation occurs upon recruitment to Raf proteins which induces a conformational change leading to the phosphorylation of key residues in the activation loop; Ser218/222 and Ser222/226 for MEK-1 and -2, respectively ${ }^{267}$. Catalanotti and colleagues further show that MEK-1 and -2 form a heterodimeric complex upon Raf recruitment, which they found essential for its negative regulation ${ }^{276}$. They highlight that after activation, the heterodimer is attenuated by phosphorylation on the MEK1 Thr292 residue within its proline-rich region by MAPK, stimulating Ser218/222 dephosphorylation; this 
residue is absent in MEK2 ${ }^{276}$. Thus, in MEK1 deficient cells, they find an enhanced MAPK signaling attributed to the loss of negative regulation of MEK and lack in heterodimerization ${ }^{276}$. Activated, MEK1/2 subsequently phosphorylates and activates MAPK which denotes the final stage within the kinase cascade.

MAPK is the final protein within the three-tiered kinase cascade of which activation remain a hallmark in the signaling pathway. MAPK is a serine/threonine kinase currently established as the only physiological substrate of MEK1/2 ${ }^{276}$. MEK1/2 phosphorylates MAPK and as such, denoted as MAPK kinase (MAPKK, MAP2K); in this regard Raf is referred to as MAPK kinase kinase (MAPKKK, MAP3K) ${ }^{255}$. MAPK is also referred to as ERK (extracellular signal-regulated protein kinase) which consists of two ubiquitously expressed proteins ERK-1 and -2 (ERK1/2; p44/p42). Unlike MEK1/2, both ERK isoforms function independently but are often referred to as the single unit-ERK1/2-due to many shared features including mode and degree of activation, substrate specificity, and overall biological output ${ }^{277}$. The structure of ERK-1 and -2 is $84 \%$ identical in sequence made up of an $\mathrm{N}$-terminal domain which bind ATP; a protein kinase domain with an activation segment and catalytic core; and a C-terminal tail important for substrate binding. Upon recruitment to the Ras-Raf-MEK scaffold, ERK1/2 is phosphorylated by MEK1/2 protein on Thr202/185, and Tyr204/187 within their activation segments. Unlike the limited substrates of Raf and MEK, ERK1/2 catalyzes the phosphorylation of more than 175 documented cytoplasmic and nuclear targets including regulatory molecules and transcription factors ${ }^{277}$. With this diverse substrate repertoire, the MAPK pathway robustly regulates key cellular processes in survival and growth of cells-attesting to its role as one of the major RTK core processes.

\subsubsection{3 - The PI3K/Akt pathway: Regulation of metabolism and growth}

The PI3K/Akt pathway is another major signaling cascade conserved amongst RTK signaling networks ${ }^{278}$. This pathway regulates cellular functions involved in maintaining balance between cell survival and apoptosis ${ }^{267}$. Processes in protein synthesis, metabolism, and cell cycle progression are facilitated by this pathway which is initiated by PI3K activation ${ }^{267,279}$. PI3K is a lipid kinase composed of a catalytic subunit (p110) and an adaptor/regulatory subunit (p85) which heterodimerizes in forming the complex ${ }^{279}$. The p85 subunit binds p110 through a p110 binding motif located in between two SH2 domains, which bind activated EGFR ${ }^{254,267}$. The p110 subunit serve as the PI3K catalytic core which phosphorylates the plasma membrane lipid $\mathrm{PIP}_{2}$ at the $\mathrm{D} 3$ inositol position, creating $\mathrm{PIP}_{3}$. Under resting conditions, the p85 subunit inhibits PI3K activity by acting as an autoinhibitory module for $\mathrm{p} 110^{280}$. 
Binding of p85 to activated receptors induces a conformational shift, relieving p110 of autoinhibition, thereby activating PI3K. Furthermore, p85 is a known phosphorylation target of the Src family kinases Lck and $\mathrm{Abl}$ (discussed below)-similarly recruited to activated EGFR ${ }^{280}$. The nature of this phosphorylation in context to PI3K regulation however, remain uncertain, although expression of a constitutively active Src kinase in vitro had been correlated with increased PI3K activity ${ }^{280}$.

The recruitment of PI3K is downstream of most active EGFR dimers ${ }^{244}$. The potency and kinetics of PI3K activation however, may differ between dimeric partners due to the absence of a PI3K binding site in EGFR and HER2 receptors ${ }^{244,268}$. The two SH2 domains of p 85 bind pTyr residues following the specific pYXXM motif ${ }^{281}$. At least six pYXXM motifs have been confirmed in ErbB-3 and -4 which directly bind PI3K, resulting in enhanced PI3K activation-compared to EGFR and HER2 ${ }^{210,282}$. Alternatively, the docking protein Gab1 is recruited by Grb2 adaptors on activated EGFR and HER2 receptors, and contains three canonical pYXXM motifs recognized by PI3K ${ }^{268,281}$. Proline residues on Gab1 interacts with the Grb2 $\mathrm{SH} 3$ domains-becoming rapidly phosphorylated on its tyrosine sites upon receptor recruitment ${ }^{281}$. The pTyr residues generated on Gab1 provides docking sites for multiple $\mathrm{SH} 2$ domain-containing proteins, including $\mathrm{PI}_{3}{ }^{283}$. As such, all activated EGFR result in the production of $\mathrm{PIP}_{3}$ on the plasma membrane through the direct and/or indirect recruitment of $\mathrm{PISK} \mathrm{PIP}_{3}$ serve as a key plasma membrane docking site for two distinct protein-lipid binding domains-the FYVE and PH domains ${ }^{279}$.

Proteins containing PH-domains are critical in mediating PI3K-induced signaling events ${ }^{279}$. Proteins such as Akt (or protein kinase B; Akt/PKB) and its activator 3-Phosphoinositide-dependent kinase 1 (PDK1) for instance, are recruited by $\mathrm{PIP}_{3}$ onto the plasma membrane via PH domains ${ }^{226}$. Akt is a serine/threonine-specific protein kinase which sits at the crossroads of multiple cellular processes. It has three highly homologous members (Akt-1, -2 , and -3) which share a similar structure of an N-terminal segment with a PH-domain, a central kinase core domain with an activation loop, and a C-terminal regulatory domain ${ }^{254,279}$. In its inactive form, the Akt PH domain is inhibitory, assuming a "PH-in" conformation blocking the central kinase core ${ }^{284}$. $\mathrm{PH}$ domain binding to $\mathrm{PIP}_{3}$ result in a "PH-out" conformation which releases the kinase domain, exposing two phosphorylation sites such as Thr308 in the activation loop and Ser473 in the regulatory domain of Akt1; Akt-2 and -3 adopts a similar mechanism exposing Thr309/Ser474 and Thr305/Ser472, respectively ${ }^{279,284}$. Likewise, PDK1 is a serine/threonine-specific kinase of which low basal activity becomes fully activated upon engagement of its $\mathrm{PH}$ domain at the plasma membrane ${ }^{212}$. Plasma membrane-bound, PDK1 phosphorylates Akt1 at 
Thr308, which stabilizes its active conformation and partial activation ${ }^{278,279}$. Full activation of Akt1 requires subsequent phosphorylation at Ser473 residue by the mammalian target of rapamycin (mTOR) complex 2 (mTORC2); of which activation and activity parallels PDK1 ${ }^{284}$. The fully activated Akt is mostly understood to dissociate from $\mathrm{PIP}_{3}$, able to phosphorylate both cytosolic and nuclear targets involved in cell proliferation, and anti-apoptotic processes ${ }^{267,284}$. Related to this thesis, $\mathrm{PIP}_{3}$ also creates a docking site for the N-terminal PH domain of PLC- $\gamma 1$ which stabilizes it on the plasma membrane. Whether or not this has synergistic roles in the potential regulation of PLC- $\gamma 1$ by CME is not well-understood ${ }^{13}$.

A major function of Akt involves the activation of the mammalian target of rapamycin complex 1 (mTORC1). The mTORC1 complex is involved in ribosomal biogenesis, and translation of pro-mitogenic proteins underlying cell growth ${ }^{284}$. It is comprised of three components: the ser/thr kinase mTOR, the scaffolding protein RAPTOR (regulatory-associated protein of mTOR), and the mammalian lethal with Sec13 protein $8(\mathrm{mLST})^{285}$. It is activated by the GTPase Ras homolog enriched in brain (RHEB), of which RHEB-GTP conformation bind and activate the complex ${ }^{286}$. In unstimulated cells, RHEB is kept in an inactive RHEB-GDP state by the tuberous sclerosis complex 2 (TSC2) GAP protein which prevent mTORC1 activation ${ }^{284}$. In response to growth factor stimulation, Akt phosphorylates TSC2, which through a poorly understood mechanism inhibits its GAP activity, thereby promoting GTP loading of RHEB and subsequent activation of $\mathrm{mTORC} 1^{287}$. In parallel to mTORC1 activation, Akt similarly inhibits the proapoptotic transcription factor FOXO through phosphorylation, which sequesters it from the nucleus unable to induce apoptotic responses ${ }^{284}$. As such, $\mathrm{PI3K}$ and $\mathrm{PIP}_{3}$ are considered obligate and rate limiting for proper induction of the Akt pathway, of which dysregulation is commonly implicated in tumorigenesis 278,284 .

\subsubsection{4 - The Src pathway: Synergistic roles in EGFR signaling}

The Src pathway is defined by the activation of the non-receptor protein-tyrosine kinase (nRTK) Src, implicated in mitogenic processes inducing DNA synthesis, cell survival, cytoskeleton rearrangements, cell adhesion and motility ${ }^{255}$. Src is the archetypal member of an 11-gene Src kinase family expressed in vertebrate cells ${ }^{288}$. They can be categorized into three groups (group-I; Src, Fyn, Yes which are expressed ubiquitously, and Fgr; group-II; Blk, Hck, Lck, and Lyn; group-III: Frk, Srm, and Brk) based on structural diversity, albeit sharing a conserved structural arrangement: an N-terminal 14carbon myristoyl group, a 'unique' domain, an SH3 domain, an SH2 domain, an SH2-kinase linker, an SH1 protein-tyrosine kinase domain, and a C-terminal regulatory segment ${ }^{288}$. The myristoyl group facilitates 
localization to plasma membranes where they may be activated and brought in proximity to their catalytic substrates. As its name implies, the unique domain varies between different Src family members, mediating diverse protein-protein interactions involved in both distinct, and overlapping activities $^{289}$. Aside from their respective interactions with proline-rich and pTyr residues, the SH3 and $\mathrm{SH} 2$ domains serve roles in constraining enzyme activity via intramolecular contacts. Briefly, the Src kinase exists in an autoinhibited state in its inactive form, where its $\mathrm{SH} 2$ domain binds intramolecularly to a phosphorylated Tyr530 residue in the C-terminal tail. In this inactive conformation, the SH3 domain similarly interacts with the SH2-kinase linker, stabilizing the structure ${ }^{288}$. Binding of the $\mathrm{Src} \mathrm{SH} 2$ domain to pTyr sites on activated EGFR relieve Src of autoinhibition, subsequently undergoing intermolecular autophosphorylation catalyzed by another proximal Src molecule. Autophosphorylation between Src proteins occur at Tyr419 within the activation loop, which promotes kinase activity ${ }^{288}$.

Despite its involvement in key cellular processes and human diseases, the specific roles and substrate targets of activated Src kinases are still not fully understood. Work from Ferrando and colleagues however, hinted of the potentially wide array of Src target proteins involved in signaling, of which phosphorylation levels increased in response to Src activation ${ }^{290}$. Whether or not these interactions are the direct product of Src phosphorylation nonetheless, remain uncertain. FAK is amongst Src kinases verified effectors of which phosphorylation is involved in cell adhesion and migration ${ }^{254,290}$. Although selective for a pYEEI (where $E$ is an acidic residue, and $I$ is isoleucine) motif, the $\mathrm{Src} \mathrm{SH} 2$ domain can bind a variety of other sequences that do not conform to this optimal pattern ${ }^{288}$. As such, it is recruited by several activated RTKs including EGFR, which interestingly is also one of its verified substrates. In the late 1980s, Src kinases and EGFR were found to behave synergistically in propagating EGF induced mitogenic cell responses. It wasn't until later that specific pTyr sites distinct from EGFR autophosphorylation sites, were attributed to Src kinase phosphorylation activity ${ }^{291}$. Specifically, Tyr845, $-891,-920$, and -1101 were found to be phosphorylated by Src on EGFR residues which were observed to potentiate EGFR signaling. Of interest to this thesis is Tyr845 within the EGFR activation loop, of which phosphorylation by Src was shown to stabilize its active state-corroborated by increased phosphorylation of its downstream effectors including PLC- $\gamma 1$ and Shc ${ }^{292}$. In addition, Tyr920 provide essential docking sites for the activation of STAT transcription factors (discussed below) ${ }^{291,293}$. Thus, although mechanistically unclear, Src kinases function in potentiating EGFR signals through synergistic activation of downstream signaling pathways ${ }^{292}$. 


\subsubsection{5 - The STAT pathway: Direct effector of phenotypic response}

The signal transducers and activators of transcription (STAT) proteins are a family of proteins which facilitate the STAT pathway downstream of EGFR 294 . Activation of STAT effect cellular processes including cell differentiation, proliferation, and invasion, and thus, implicated in oncogenesis and tumor progression ${ }^{294,295}$. The STAT family is comprised of seven members: STAT-1, $-2,-3,-4,-5 a,-5 b$, and -6 which are activated by binding distinct pTyr residues on activated EGFR (STAT-1, -3 , and -5 ), or through the JAK protein during cytokine signaling (STAT-2, -4 , and -6$)^{254,294}$. As such, STAT-1, -3 , and -5 appear to play the most direct roles in tumorigenesis, exhibiting diverse mitogenic functions ${ }^{295}$. STAT-3 and -5 for instance, stimulate cell cycle progression, angiogenesis, and inhibition of apoptosis, with STAT1 initiating cell cycle arrest ${ }^{294}$. All STAT proteins however, share an overall general structure organized into functional modular domains: an N-terminal oligomerization domain facilitating protein-protein interactions, a DNA binding domain involved in nuclear import and gene transcription, a dimerization domain with an SH2 module important for activation, and a C-terminal transactivating region with several binding partners ${ }^{296,297}$. As transcription factors, STATs thus serve as convergence points and terminal effectors of many signal transduction cascades, capable of directly translating upstream signaling events into direct gene responses ${ }^{295}$.

Mechanistically, activation of STAT proteins is relatively simple, wherein phosphorylation of a conserved Tyr residue within the SH2 domain is sufficient in inducing its active conformation. STATs can also be phosphorylated by Src family kinases and/or MAPK which respectively phosphorylates activating Tyr and Ser residues in an EGF-dependent manner; a mechanism commonly exploited in several cancer types ${ }^{296,297}$. With their direct role in gene transcription, STATs are considered oncogenes of which aberrant activity are well-known to contribute to cancer formation and progression-and such, current targets of drugs in clinical use 294,295 .

\subsubsection{6 - The PLC- $\gamma 1$ pathway: induction of pleiotropic calcium signaling}

Lastly, the PLC- $\gamma 1$ pathway is a signaling cascade described in section 1.4, of which hallmark phosphodiesterase PLC- $\nu 1$ enzyme is a known EGFR effector; which is the primary pathway work in this thesis will focus on ${ }^{143,298}$. PLC- $\gamma 1$ was the first protein discovered to be phosphorylated in response to EGF stimulation, providing one of the first clues as to how ligand-induced autophosphorylation propagates RTK signaling ${ }^{266}$. Activation of PLC- $\gamma 1$ augments mitogenic, pro-proliferative effects during RTK stimulation-and as such, implicated in carcinogenesis ${ }^{143,159}$. PLC- $\gamma 1$ contains two SH2 domains- 
$\mathrm{nSH} 2$ and $\mathrm{CSH} 2$ - of which former binds to pTyr992 and pTyr1173 on activated EGFR ${ }^{257,299}$. Upon recruitment, PLC- $p 1$ is typically phosphorylated on several sites including pTyr783 within the catalytic core, necessary for stimulation of its lipase activity ${ }^{13,300}$. Previously discussed, PLC- $\gamma 1$ catalyzes the hydrolysis of $\mathrm{PIP}_{2}$ into secondary messengers, DAG and $\mathrm{IP}_{3}$, which further stimulates the activation of downstream signaling cascades, diversifying the role of PLC- $\gamma 1$ beyond the plasma membrane ${ }^{300}$.

In context to MAPK signaling, DAG may serve as precursor in producing phosphatidic acid enhancing the MAPK pathway by recruiting Sos to the plasma membrane ${ }^{271}$. Described in section 1.4.3, DAG also serve as a plasma membrane docking site for PKC through a C1 domain, of which kinase autoinhibition is relieved by DAG binding ${ }^{177}$. PKC is a family of protein kinases that have been a focus of extensive drug discovery due to their roles in cell proliferation, differentiation, survival, and apoptosis $^{176,301}$. Indicative of this role, PKC proteins upregulate the MAPK pathway by phosphorylating Raf proteins activating MEK1/2-inducing DNA replication, transcription and growth ${ }^{274,302}$. Furthermore, calcium influx generated by the $\mathrm{IP}_{3}$-mediated opening of ER calcium channels, aid in the activation of a

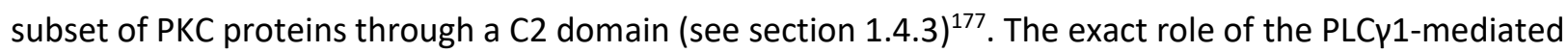
calcium release in distinct signaling processes however, is difficult to clarify due to the ubiquity of calcium and its pleiotropic roles in cell signaling (see section 1.5$)^{188}$. Specifically, its role in EGFR clathrinmediated endocytosis is not well-defined and is a key topic investigated in this thesis.

Furthermore, it is worth noting that PLC- $\gamma 1$ had been observed to play essential roles in cellular proliferation through protein-protein interactions mediated by its $\mathrm{SH} 3$ domain-independent of enzymatic activity ${ }^{303}$. Specifically, the PLC- $\gamma 1$ SH3 domain binds and recruits Sos onto the plasma membrane-enhancing Ras activation and MAPK signaling ${ }^{300}$. This SH3-mediated augmentation of MAPK signaling overlaps with Sos recruitment through DAG; highlighting the role of PLC- $p 1$ as a robust enhancer of the MAPK pathway ${ }^{303}$. The GTPase Dyn1 involved in receptor endocytosis, also binds PLC- $\gamma 1$ serving as a non-conventional GEF in neuronal cells-albeit through an unknown mechanism and physiological context ${ }^{303,304}$. The role of PLC- $\gamma 1$ as a GEF is further highlighted in the brain, where it interacts and promotes GTP loading of the PIKE (PI3Kinase Enhancer) GTPase-regarded as the nuclear counterpart of Ras involved in PI3K signaling ${ }^{305,306}$. Thus, the SH3 domain of PLC- $\gamma 1$ may serve as scaffold for proteins in propagating mitogenic signals independent of lipase activity. The exact mechanisms of these interactions however, remain unclear and require further research in consolidating their contributions to RTK signaling propagated by secondary messengers of PLC- $\nu 1^{303}$. 
EGFR signaling is largely defined by these canonical pathways, of which mechanistic processes are conserved amongst different members of the RTK superfamily ${ }^{11}$. Variations in the spatiotemporal organization of these cascades is contingent on ligand, RTK, and cell-type, result in diverse signaling patterns and biological outcomes ${ }^{220}$. In addition, a dizzying array of regulatory factors including negative/positive feedback loops, determine the duration and strength of respective pathways which impact overall signaling output ${ }^{11}$. Significant cross-talk and redundancy between pathways also occur, of which complex tangled network remain a challenge in the predictive modeling of RTK signaling ${ }^{11}$.

In understanding the role of PLC- $\gamma 1$ in regulating EGFR clathrin-mediated endocytosis, careful consideration of potential pathway-pathway interactions must therefore be made. The PI3K/Akt pathway for instance, rapidly increases $\mathrm{PIP}_{3}$ on the plasma membrane thereby stabilizing PLC- $\gamma 1$ on the surface. In addition, Src kinases stabilizes EGFR activation which leads to increased PLC- $\nu 1$ phosphorylation and presumably, lipase activity. The requirement for these stabilizing factors in PLC- 11 activity however, is not well-understood and to some degree, may impact its potential regulation of EGFR clathrin-mediated endocytosis. Nonetheless, understanding regulatory cross-talk between these specific parameters is beyond the scope of this thesis, and may come secondary upon establishing the primary role of PLC- $\gamma 1$ in EGFR clathrin-mediated endocytosis-addressed in this work.

\subsubsection{3 - The third layer of RTK signaling: signal output}

The third and final layer of the RTK 'bow-tie' network represents the diverse phenotypic output, derived from the summation of RTK-induced core processes ${ }^{249}$. At this stage, terminal effectors of signaling pathways induce gene transcription leading to diverse biological outputs, ranging from cell division and migration, to adhesion, differentiation, and apoptosis ${ }^{244}$. While much progress has been gained in understanding RTK signaling input and core processing layers, knowledge regarding the output layer lags behind due to methodological difficulties ${ }^{249}$. In particular, quantifying individual contributions of signaling pathways to overall phenotypic outcomes, prove difficult due to redundancies in activation of transcriptional processes ${ }^{307}$. Prototypic transcription factors such as c-Fos for instance (discussed below) - while commonly regarded as a MAPK terminal effector-is regulated directly or indirectly by adjacent overlapping pathways including PI3K, STAT, Src, and PLC- $\gamma 1$ cascades (see section 1.6.3.1) ${ }^{277,308}$. Furthermore, positive and negative feedback mechanisms which respectively amplify and attenuate signaling pathways, vary depending on ligand, RTK, and cell-type, which further complicates predictive 
modeling of signal output ${ }^{11,309}$. As such, significant cross-talk occur between RTK core processes, of which individual perturbations likely lead to the concomitant modulation of others ${ }^{307}$.

Currently, an operational understanding of RTK biology is lacking, largely due to difficulties in modeling its complex, multivariate signaling network ${ }^{310}$. Specifically, understanding precisely how cells represent external stimuli into intracellular molecules directing phenotypic responses is not completely understood $^{310}$. How mitogenic cues such as growth factors may regulate CME in remodeling the plasma membrane for instance, is relatively unexplored and is a key question this thesis aims to investigate. The following discussion is therefore limited to description of what is known about the signal output layer, covering topics in: transcription factor activation, regulatory feedback loops, signaling cross-talk, and signal termination.

\subsubsection{1 - RTKs induce activation of mitogenic transcription factors}

Transcription factors (TF) are proteins which recognize and bind specific DNA sequences, in regulating gene transcription ${ }^{311}$. They directly interpret the genome by binding genes at specific sites, inducing the expression or down-regulation of their genetic products ${ }^{311}$. Most TFs recognize small 6-12 bp-long DNA sequences, with a typical human gene $(>20 \mathrm{~kb})$ containing multiple potential binding sites $^{311,312}$. This intrinsically low specificity suggests the involvement of more complex mechanisms, utilized by TFs in DNA regulation - which extends beyond their simple individual affinities for DNA ${ }^{312}$. The same TF can therefore regulate different genes in different cell types, indicative of their dynamic regulatory network ${ }^{311}$. Able to directly interpret the genome, TFs are key regulators of cell behaviour and development of which dysregulation is associated with numerous diseases including cancer; with TFs overrepresented amongst oncogenes ${ }^{313}$. As such, growth factor receptors including EGFR, commonly activate TFs in inducing cellular processes directed by external stimuli.

RTK signaling generally culminate in activation of TFs, which direct cell behaviour in response to input signals. The MAPK pathway for instance, activates several gene regulators including the c-Fos, cJun, and c-Myc TFs ${ }^{244,269}$. Under basal conditions, c-Fos is unstable and rapidly degraded within the cell ${ }^{277}$. Upon growth factor stimulation, activated ERK1/2 translocate into the nucleus where it phosphorylates c-Fos at Ser374 in the C-terminus ${ }^{314}$. The cytoplasmic ribosomal S6 kinase (RSK) effector downstream of ERK1/2 is also activated, which similarly localizes into the nucleus, catalyzing the secondary phosphorylation of c-Fos at Ser362 $2^{314}$. Once phosphorylated, c-Fos is stabilized for a few 
hours acting as a TF for the FOS gene, producing additional c-Fos proteins ${ }^{249}$. If ERK1/2 is still active during this subsequent c-Fos expression, they are similarly phosphorylated and stabilized leading to initiation of phenotypic responses such as cell differentiation ${ }^{314}$. In contrast, if ERK1/2 is activated transiently, cells fail to accumulate stable c-Fos proteins leading to cell proliferations instead of differentiation ${ }^{249}$. While generally unclear, this seminal work by Murphy and colleagues provide a glimpse into how conserved core processes may generate diverse signaling outputs, specifically through temporal control of signaling components ${ }^{314}$.

Other RTK pathways initiated by PI3K, Src, STAT and PLC- $\gamma 1$ also induce activation of TFs including c-Fos, albeit through a less defined mechanism. The TF protein STAT, is directly activated by EGFR resulting in its nuclear localization, where it may form complexes and regulate gene expression with other TFs including c-Fos and c-Jun ${ }^{294}$. PLC- $\gamma 1$ indirectly regulates gene transcription by activating PKC, of which target substrates include the c-Fos ${ }^{315}$. The PI3K pathway also regulates c-Fos in promoting cell survival, in addition to inhibition of apoptosis by inactivating the pro-apoptotic transcription factor FOXO via Akt phosphorylation; which may be synergistically potentiated by the Src pathway ${ }^{280,316}$. Many of the long-term changes in cellular behaviour elicited by growth factors are therefore, largely due to altered programs in gene expression resulting from TF activities, which direct cellular phenotypic changes including plasma membrane remodeling events ${ }^{317}$. A degree of overlap in TF regulation evinced by c-Fos however, is evident amongst core processes and exactly how they distinctly contribute to the overall gene response is not well-understood. Despite this challenge, a general transcriptional program following RTK activation had been described, detailing the canonical expression of primary and secondary response genes governing many of the phenotypic output produced by RTKs ${ }^{317}$.

\subsubsection{2 - Expression of Immediate-early genes (IEG), delayed-early genes (DEG), and secondary response genes (SRG) by RTK signaling}

The transcriptional program induced by RTK activation consists of three stereotypical waves: transcription of immediate-early genes (IEG), delayed-early genes (DEG), and secondary response genes (SRG) responsible for determining cellular outcome ${ }^{318,319}$. IEGs are genes that are rapidly and transiently activated following growth factor stimulation, representing the first transcriptional event preceding phenotypic cellular responses ${ }^{317}$. About 50 IEGs have been identified including FOS, JUN, and MYC, of which transcription are mediated by pre-existing $\mathrm{TFs}^{317}$. The FOS IEG for instance, is transcribed by preexisting c-Fos stabilized by ERK1/2, resulting in further expression and accumulation of the $\mathrm{TF}^{314}$. As well, 
IEG expression do not require de novo protein synthesis evinced by transcription of their mRNA, under the presence of protein synthesis inhibitors ${ }^{318}$. Largely coding for transcriptional activators, IEGs rapidly produce TFs such as c-Fos, c-Jun, c-Myc, and early growth response gene 1, which function in subsequently inducing expression of secondary gene sets ${ }^{269}$.

IEG expression reaches maximal activation within 30-40 minutes following growth factor stimulation-creating transcriptional activators for $\mathrm{SRGs}^{320}$. SRGs are genes of which products determine new cell phenotypes ${ }^{320}$. They encode for proteins including enzymes implicated in metabolism and membrane biogenesis. In contrast to IEGs, SRGs are latently expressed following growth factor stimulation due to their requirement for de novo protein synthesis ${ }^{321}$. Transcription of SRGs occur $\sim 120$ minutes following growth factor stimulation, of which maximal activation is achieved within $\sim 4-5$ hours $^{320}$. As such, RTKs induce the long-range expression of SRGs, of which effects persists well-past initial RTK activation in ultimately determining cellular outcome ${ }^{320}$.

The RTK transcriptional program is comprised of $36 \%$ IEGs, $26 \%$ SRGs, and the remaining $44 \%$ classified as $\mathrm{DEGs}{ }^{317}$. DEGs were once considered a part of the secondary response program due to their latent expression following RTK activation. The transcription of DEGs however-similar to IEGs-do not require de novo protein synthesis, thus, classifying it as an early gene response ${ }^{317}$. DEGs are expressed within 40-240 minutes following growth factor stimulation of which products largely participate in signal attenuation $^{322}$. DEG products commonly repress early wave genes, consisting of phosphatases and transcriptional repressors ${ }^{249,309}$. Transcriptional repressors such as FOSL1 and JUNB for instance, are known DEGs of which latent expression inactivates IEGs upregulated in response to growth factors ${ }^{322}$. Phosphatases including MAPK phosphatases (MKPs) or dual-specificity phosphatases (DUSPs) also comprise the DEG response which attenuates RTK signaling by dephosphorylation of MAPK pathway components ${ }^{322}$. As such, DEG expression is a component of the built-in RTK system control, which include positive and negative feedback mechanisms regulating signaling parameters and overall phenotypic response ${ }^{249}$.

\subsubsection{3 - RTK System controls: Positive feedback mechanisms}

The overall behaviour of RTK signaling is dynamically regulated by system controls fundamentally comprised of positive and negative feedback loops ${ }^{250}$. Positive and negative feedback loops modulate signaling amplitudes, kinetics, and overall output by respectively amplifying and 
attenuating the active state of signaling pathways ${ }^{264}$. The prototypic EGFR for instance, is defined by positive and negative feedback loops which are stimulated concomitant with EGFR activation ${ }^{250}$. EGFR positive circuits include the production of EGFR ligands, which are secreted to re-stimulate and sustain EGFR signaling in an autocrine manner ${ }^{249}$. This mechanism is largely regulated by the integral plasma membrane glycoprotein enzyme ADAM (a disintegrin and metalloprotease), which induces ligand shedding from the plasma membrane through proteolytic catalysis ${ }^{323}$. This mechanism may be activated by EGFR pathway components such as those derived from MAPK signaling, thus, providing positive feedback mechanisms in sustaining the EGFR signaling network ${ }^{11,324}$.

Another positive feedback mechanism induced by EGFR include the production of reactive oxygen species (ROS) derived from activation of the PI3K pathway ${ }^{215}$. ROS are commonly regarded as cytotoxic agents due to the oxidative damage they cause to cellular components. At low concentrations however, ROS may function as secondary messengers capable of augmenting EGFR signaling pathways $^{325}$. Briefly, protein tyrosine phosphatases (PTPs; discussed below) are molecules which antagonize EGFR activity by removing phosphate groups from phosphorylated tyrosine residues of activated receptors ${ }^{11}$. Bae et al., describes that the specific activity of PTPs are 10-1000 fold greater than those of protein tyrosine kinases suggesting that the ligand-mediated activation of a receptor kinase domains may not be sufficient for full EGFR activation ${ }^{325}$. The authors further elaborate that inhibition of PTPs might therefore be necessary for full activation of EGFR which they describe achievable through production of $\mathrm{ROS}^{325}$. Growth factor stimulation upregulates intracellular ROS such as hydrogen peroxide $\left(\mathrm{H}_{2} \mathrm{O}_{2}\right)$, as a consequence of its core signaling processes ${ }^{326}$. The PI3K pathway specifically, is implicated in ROS generation, through several mechanisms highlighted by Silva and colleagues ${ }^{326}$. They describe that $\mathrm{PIP}_{3}$ generated by PI3Ks serve as plasma membrane docking sites for the recruitment of NADPH oxidase, which bind phosphoinositides through a PX domain ${ }^{326,327}$. On the plasma membrane, NADPH oxidase produce $\mathrm{H}_{2} \mathrm{O}_{2}$ which oxidizes a vulnerable cysteine residue within PTP active sites, resulting in their inhibition ${ }^{326}$. PLC- $\gamma 1$ was similarly shown to activate ROS production through the PKCmediated phosphorylation of NADPH oxidase, albeit through a less understood mechanism ${ }^{328}$. Thus, although transient in expression, ROS are potent positive regulators of EGFR signaling, which amplifies downstream pathways by enhancing receptor tyrosine phosphorylation ${ }^{215}$. 


\subsubsection{4 - RTK System controls: Negative feedback mechanisms}

In preventing aberrant activity, negative feedback controls are spatiotemporally integrated within the EGFR signaling network which attenuate signaling components at several levels ${ }^{250}$. Several attenuating processes mediate EGFR signaling which include catalytic inactivation, steric hindrance, and post-translational modification mechanisms ${ }^{264}$. Direct activation of phosphatases for instance, represent an obvious feedback mechanism which directly opposes EGFR signaling through abolishment of binding sites for signaling intermediates and adaptor proteins ${ }^{11,210}$. Several PTPs catalyze the removal of phosphate groups from phosphorylated tyrosine residues on activated receptors ${ }^{320,329}$. The densityenhanced phosphatase-1 (DEP1) for instance, is a transmembrane receptor-like PTP which is proximally activated by EGFR, abrogating phosphorylation of its cytosolic tyrosine residues ${ }^{330,331}$. Further discussed in section 1.6.4, stimulation of EGFR induces their concomitant internalization via endocytosis, creating receptor-containing endosomes. While still incompletely, it is becoming clear that some components of EGFR signaling is preserved within the endosomal system, where activated receptors continue to propagate core signaling processes ${ }^{332}$. PTP1B is an ER resident phosphatase which dephosphorylates EGFR in transit within endosomes-inactivating downstream signaling ${ }^{320}$. These two PTPs represent some of the immediate pre-existing attenuators of EGFR signaling ${ }^{264}$.

Direct protein inhibitors of EGFR have been identified which are upregulated hours following ligand stimulation ${ }^{309}$. The Mitogen-inducible gene 6 (Mig6)/receptor-associated late transducer (RALT) (MIG6/RALT) for instance, is a transcriptionally induced feedback inhibitor of EGFR, which functions directly at the receptor level ${ }^{333}$. MIG6/RALT inhibits signaling by binding EGFR within their kinase domain, thus precluding intracellular dimerization and autophosphorylation ${ }^{334}$. MIG6 further attenuates EGFR signaling by recruiting endocytic adaptors including AP2, which promotes receptor endocytosis

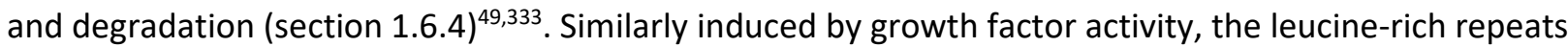
and immunoglobulin-like domains protein 1 (LRIG1) also inhibits EGFR signaling by promoting receptor degradation ${ }^{264}$. LRIG1 is a transmembrane protein which forms a complex with EGFR receptors through ectodomain interactions ${ }^{335}$. The intracellular domain of LRIG1 also provides a docking site for the Cbl, leading to accelerated ubiquitination and degradation of EGFR ${ }^{336}$. Interestingly, LRIG1 reduces EGFR in both basal and ligand-stimulated conditions, which together with other latently expressed inhibitors, provide a long-term mechanism for signaling attenuation ${ }^{335}$. 
In addition to receptor phosphatases and inhibitors which broadly abrogate EGFR activities, pathway-specific negative loops are also induced in response to ligand-stimulation which attenuate EGFR signaling on a local level ${ }^{309}$. The EGFR-mediated MAPK-responses for instance, is regulated by several negative feedback mechanisms given their central role in EGFR signaling ${ }^{11}$. Aside from its activation of transcriptional responses, ERK1/2 also catalyzes the direct phosphorylation of several of its upstream components including Sos. Phosphorylation of Sos impairs its interaction with Grb2 which reduces its recruitment to the plasma membrane-dampening Ras activation ${ }^{11,309}$. ERK1/2 similarly phosphorylates Raf kinase which reduces its kinase activity required for ERK1/2 activation ${ }^{11,273}$. The PI3K pathway as well, is inhibited by ERK1/2 via phosphorylation of Gab1 preventing PI3K recruitment and transactivation of Ras (see section 1.6.3.3.5) ${ }^{273}$. Lastly, ERK1/2 terminates its activation by directly attenuating EGFR activity by phosphorylating Thr669 within the receptors JM region ${ }^{254}$.

Phosphatases and protein inhibitors similarly regulate the activities of several EGFR core processes in attenuating signaling. The PI3K pathway for instance, is primarily inhibited by phosphatase and tensin homologue deleted on chromosome 10 (PTEN) protein at the Akt level ${ }^{279}$. PTEN exists in a closed, autoinhibited state which is relieved by phosphorylation through a mechanism not wellunderstood $^{337}$. EGFR however, had been observed to induce activation of the Rho-associated protein kinase (ROCK), which phosphorylates PTEN ${ }^{337,338}$. In its open conformation, PTEN is recruited onto the plasma membrane via anionic lipid interactions, where it catalyzes the dephosphorylation of $\mathrm{PIP}_{3}$ at the D3 inositol position, producing $\mathrm{PIP}_{2}{ }^{279}$. As previously described, $\mathrm{PIP}_{3}$ is a necessary plasma membrane docking site for the recruitment and subsequent activation of Akt, with its ablation therefore detrimental to PI3K signaling 279 . Indeed, several studies have reported the tumor suppressing properties of PTEN, of which dysregulation is implicated in oncogenic mutations ${ }^{254}$. Other pathways propagated by PLC- $p 1$, Src, and STAT, similarly integrate negative feedback loops involving phosphatases and protein inhibitors, of which mechanisms are reviewed elsewhere $[303,339,340]$.

Lastly, receptor internalization coupled to degradation is considered the most effective, irreversible process which robustly attenuates RTK signaling ${ }^{264}$. It is initiated as a part of the early gene response, which directly attenuates signaling by the endocytic removal of receptors from the plasma membrane, further discussed in section 1.6.4. 


\subsubsection{5 - Extensive crosstalk exists within the RTK signaling network}

Although largely involved in regulating signaling pathways from which their activities are induced, positive and negative feedback mechanisms may overlap in regulating other core processes due to extensive cross-talk between the RTK signaling network ${ }^{250}$. Oda et al., illustrates the complexity of EGFR signaling in their comprehensive map detailing not only linear signaling chains, but also network intersections between different core processes ${ }^{250}$. The MAPK and PI3K pathway provides a clear example of this crosstalk wherein both processes have been observed to reciprocally regulate the other. ERK1/2 for instance, cross-inhibits PI3K by phosphorylating Gab1, which prevents the recruitment and activation of PI3K and its downstream signaling ${ }^{285}$. Reciprocally, Akt phosphorylates Raf kinase upstream of ERK1/2, recognized by the 14-3-3 protein which stabilizes its inactive state-thereby attenuating ERK1/2 activation ${ }^{285}$. In contrast, cross-activation of the PI3K pathway can also be induced by Ras which phosphorylates and activates PI3K recruited on the plasma membrane. The downstream effectors of the PI3K pathway TSC2, is also phosphorylated by ERK1/2, which inhibits its GAP function promoting mTORC1 activity ${ }^{285}$. Both ERK1/2 and RSK are also shown to directly phosphorylate mTORC1 further adding robustness to RTKs signaling activities ${ }^{284}$.

Significant crosstalk also exists between structurally unrelated RTK families - wherein signaling activities from one, regulates the activation of another ${ }^{309}$. The insulin-like growth factor-1 receptor (IGF1R) for instance, activates a cognate Src kinase which was found to induce transactivation of EGFR important for mitogenesis ${ }^{341}$. Crosstalk between EGFR and the receptor for the hepatocyte growth factor, MET, had also been demonstrated in cancer cell lines wherein both receptors were found to positively influence each other; and correspondingly, inhibition of one negatively regulating the other ${ }^{342}$. Lastly, other transmembrane proteins such as GPCRs induce transactivation of EGFR through stimulation of ADAM proteases, promoting ligand shedding and subsequent receptor activation ${ }^{210}$. Furthermore, through PLC $\beta$-mediated calcium influx, GPCRs similarly induce the activation of a cognate Src family kinase, which is shown capable of phosphorylating and activating EGFR 244,250 .

Taken together, distinct RTK signaling pathways exists within a larger, highly interconnected network, of which combinatorial activations and regulations ultimately determine cellular responses ${ }^{210}$. The modulation of common substrates by different pathways highlights this convergence, from which related transcriptional programs are induced in promoting overlapping phenotypic outcomes ${ }^{285}$. System controls which amplify or attenuate distinct signaling cascades, must therefore be considered in context 
of signaling cross-talk, as regulation of one pathway, is likely to result in regulation of others ${ }^{307}$. Due to this high degree of interconnectivity, it is difficult to determine how distinct signaling pathways may contribute towards specific cellular phenotypes, requiring quantitative datasets to better improve deterministic modeling ${ }^{343}$.

In summary, RTK signaling can be modeled with a bow-tie structure comprised of three layers, where a large number of inputs (ligand-, receptor-, cell-type) are converted into a small number of intermediates (conserved core processes), which diverge in generating a large number of outputs (phenotypic outcomes) - contingent on intrinsic molecular rules derived from feedback and crossinteraction mechanisms ${ }^{262}$.

Important to this thesis, mitogenic signaling by RTKs often induce cellular responses requiring phenotypic modifications of the plasma membrane surfaceome ${ }^{59,60}$. Precisely how the distinct RTK signaling pathways are orchestrated in facilitating membrane reorganization however, is not wellunderstood. Thus, although knowledge of RTK biology-in context of their diverse molecular structures, complex modes of regulation, and signaling properties-is extensive, understanding of precise mechanisms and machineries through which RTKs may influence overall cellular morphology is lacking. In this thesis, we attempt to provide a potential pathway through which RTKs may mechanistically influence the surfaceome. Specifically, the PLC- $\nu 1$ pathway may serve as a direct mechanistic link between mitogenic RTK activation, and plasma membrane remodeling via CME regulation. As illustrated by EGFR, other RTK signaling pathways contribute to the regulation of PLC- $\gamma 1$ including Akt (PIP 3 production), MAPK (indirectly via Akt activation), and Src (stabilization of receptor activation). Understanding the precise role of PLC- $\gamma 1$ in regulating EGFR clathrin-mediated endocytosis, will therefore clarify how the distinct RTK pathways - and hence, mitogenic signaling-may modify the plasma membrane in eliciting diverse physiological responses.

Alluded to in the previous sections, activation of RTKs including EGFR, induces their concomitant internalization via CME, and as well as the recruitment and activation of PLC- $\gamma 1$. Initiation of EGFR clathrin-mediated endocytosis is therefore coupled to the activation of PLC- $\gamma 1$ and its lipase activity. The role of PLC- $\gamma 1$ in EGFR clathrin-mediated endocytosis however, is relatively unexplored, of which elucidation may provide insight as to how PLC- $\gamma 1$ may potentially regulate CME on both local and global levels. In the following section, mechanisms of EGFR clathrin-mediated endocytosis will be detailed, in 
identifying potential junctures which may be regulated by PLC- $\gamma 1$ activities. In addition, discussion of the several strategies used by EGFR in enhancing its own internalization, will provide clues whether other potential mechanisms exist-aside from the potential role of PLC- $\gamma 1$-in regulating global plasma membrane remodeling upon mitogenic signaling by RTKs.

\subsection{4 - RTK endocytosis}

Receptor signaling, while essential for cellular function, must be subsequently followed by signal attenuating processes in or order to both prevent aberrant activities, and to allow cells to receive and discriminate between old and new signals ${ }^{344}$. As previously described, activation of RTKs is intimately linked with the simultaneous activation of negative feedback control systems, which involves dephosphorylation and steric inhibition of activated receptors. In addition, activation of RTKs is mechanistically coupled to the recruitment of endocytic proteins, promoting receptor internalizationwhich serve as the major mechanism of RTK signal attenuation ${ }^{345}$. Upon activation, RTKs are internalized within intracellular structures such as early endosomes, of which transit within the endomembrane system result in signal down-regulation ${ }^{11}$. For instance, increasing luminal acidity within endosomal structures may contribute towards RTK inactivation by inducing ligand-receptor dissociation.

Emphasized in section 1.6.3.1, ligand binding induces and maintains the active conformation of RTKs, of which dissociation therefore render receptors inactive ${ }^{345}$. Inactive receptors are unable to support recruitment and scaffolding of signaling proteins which ultimately inhibits RTK signaling. As well, depending on several regulatory parameters, RTKs within endosomes may either be recycled back to the plasma membrane for subsequent stimulation, or delivered within lysosomes for degradation — which ultimately attenuates signaling ${ }^{254}$. While RTKs may undergo constitutive endocytosis in a ligandindependent manner, ligand stimulation nonetheless, rapidly increases the rate of RTK internalization by several folds, ensuring the down-regulation and prevention of aberrant RTK activities ${ }^{345}$.

As the major endocytic mechanism in mammalian cells, CME remains the predominant and bestcharacterized internalization route for the ligand-induced down-regulation of RTKs ${ }^{249}$. While clathrinindependent internalization pathways have been described, their precise mechanisms and roles in RTK signaling are less defined ${ }^{249,345}$. For instance, some studies have demonstrated the internalization of EGFR via clathrin-independent pathways-albeit only when high EGF concentrations are used, and a large amount of ligand-bound receptor complexes are present at the cell surface ${ }^{132}$. In addition, clathrin- 
independent routes may be secondarily preferred by RTKs, upon saturation of the CME pathway during aberrant receptor activation and expression ${ }^{132}$. As such, the contributions of these mechanisms to EGFR endocytosis, in vivo may be minimal ${ }^{132}$. Clathrin-independent internalization pathways of RTKs will therefore not be considered in this section, with the interested reader referred towards excellent reviews detailing their specific processes [53-55].

CME serve as the primary route of RTK internalization, and thus, commonly regarded as the principal pathway involved in attenuating receptor signaling. While largely involved in this facet, an additional role for CME and its clathrin components have been recently proposed. Specifically, work from our laboratory have uncovered a direct role for clathrin and CCPs in regulating and organizing EGFR signaling at the plasma membrane. Activation of the PI3K/Akt signaling pathway for instance, was demonstrated to require clathrin lattice assembly, but not EGFR endocytosis as shown by the respective perturbations of clathrin and dynamin ${ }^{207}$. This finding exhibits the functional role of clathrin assemblies in propagating EGFR signaling at the cell surface independent of receptor internalization. Furthermore, other surface receptors including $\beta 1-A R$ ( $\beta 1$-adrenergic receptor, a GPCR that interacts with hormones and neurotransmitters) have been observed to utilize CCPs in propagating their signaling activities. Eichel and colleagues found that agonist stimulation of $\beta 1-A R$ elicits the enrichment of its downstream $\beta$-arrestin-2 adaptor (component of GPCR-mediated MAPK signaling), but not $\beta 1-A R$, within cell surface clathrin structures, supporting the notion that CCPs function as signaling hubs ${ }^{346}$. Thus, in addition to its role in signal attenuation, clathrin and its clathrin components may also provide a signaling platform on the cell surface, for distinct RTKs which similarly undergo CME upon activation; of which experimental validations however, have yet to be conducted.

Indeed, several other RTKs (IR, IGF1R, MET, and RET) have been described to predominantly undergo ligand-induced $\mathrm{CME}$, however, their specific components and molecular mechanisms remain poorly defined ${ }^{3,249}$. Important to this thesis, most of what is known regarding RTK internalization had been derived from studies of EGFR, serving as the prototypical model for receptor tyrosine kinase $\mathrm{CME}^{249}$. The following section will therefore discuss RTK internalization in context of EGFR, detailing ligand-induced mechanisms which enhances EGFR clathrin-mediated endocytosis, of which details nonetheless may likely be adapted for other RTKs. 


\subsubsection{1 - EGF binding promotes EGFR clathrin-mediated endocytosis through several mechanisms}

EGF binding induces the rapid endocytosis of EGFR of which most obvious function involves signal attenuation ${ }^{254}$. Several lines of evidence demonstrate EGFR internalization to be facilitated predominantly by CME. For instance, studies including those from our laboratory have demonstrated the colocalization and concentration of the EGF:EGFR complex, within clathrin-coated structures upon stimulation with physiologically relevant ligand concentrations ${ }^{4,7}$. Several others have also measured the kinetic rate of EGFR internalization upon EGF stimulation, which was found to be comparable to that of the classical CME cargo, $\mathrm{TfR}^{347}$. Indeed, inhibition studies which mutate key endocytic components including dynamin and clathrin, fully ablate internalization of EGFR-strongly supporting CME as the primary EGFR endocytic route ${ }^{132}$. Although the major steps of EGF:EGFR clathrin-mediated endocytosis are well-established, the precise molecular machinery governing the internalization process remains poorly understood ${ }^{254}$. Nonetheless, several mechanisms have been identified which augments EGFR clathrin-mediated endocytosis, of which details for some are described below (Figure 1.13).

(i) EGF stimulation induces the dimerization and subsequent phosphorylation of tyrosine residues within the intracellular domains of EGFR, enhancing receptor internalization. Emphasized in section 1.6, pTyr residues on EGFR provide docking sites for the recruitment and activation of signaling proteins, some of which include components involved in EGFR clathrin-mediated endocytosis. Later discussed, cytoplasmic substrates including $\mathrm{Grb2}$ and $\mathrm{Cb}$ are amongst the proteins recruited to $\mathrm{pTyr}$ sites on activated EGFR, which have been implicated in receptor internalization ${ }^{132}$. In addition, posttranslational phosphorylation of several tyrosine sites on EGFR cytoplasmic domains, have been implicated in the regulation of EGFR clathrin-mediated endocytosis. Phosphorylation of Ser1046 and 1047 by CaMKII for instance, was demonstrated in vitro to enhance EGFR internalization ${ }^{348}$. The exact mechanisms and implications of CaMKII in EGFR clathrin-mediated endocytosis in vivo however, is not well-understood-but presents an interesting regulatory juncture for the PLC $\gamma 1$-derived calcium signals; relevant to this thesis. In contrast, phosphorylation of the JM region on Thr654 by PKC, transmodulates EGF activity resulting in decreased internalization due to partial kinase inhibition ${ }^{219,223}$. As such, deletion or mutation of the EGFR kinase domain results in the re-routing of EGFR from rapid ligand-induced CME internalization, towards slower clathrin-independent pathways; likely due to failure in proper recruitment and assembly of endocytic proteins ${ }^{132}$. 
(ii) Described in section 1.3, recruitment of cargo within CCPs is primarily mediated by the AP2 complex which recognizes short-linear amino acid motifs within the cytoplasmic tails of transmembrane proteins ${ }^{77}$. EGFR contains a tyrosine-based (YXX $\varnothing$; YRAL) and a dileucine-based (residues Leu1010 and 1011; LL) sorting motif, which are respectively recognized by the $\mu 2$ and $\beta 2$ subunits of AP2 ${ }^{132}$. Although mechanistically unclear, these recognition motifs are believed to be masked in the unbound, inactive EGFR conformation, and are exposed only upon ligand binding and activation of EGFR ${ }^{58}$. EGFR is known to phosphorylate the Tyr6 residue of the $\beta 2$ subunit of AP2, which is predicted to facilitate conformational changes required for its interaction with the EGFR LL motif ${ }^{249}$. Despite the importance of these motifs in AP2 binding, deletion of the primary YRAL sequence on EGFR, or mutations in the $\mu 2$ YRAL recognition domain on AP2, does not interfere with the rate of EGFR clathrin-mediated endocytosis ${ }^{132}$. Similarly, mutations in the LL motif also does not affect EGFR internalizationdemonstrating a mechanistic redundancy in EGFR clathrin-mediated endocytosis ${ }^{132}$. Interestingly, under certain experimental conditions, depletion of AP2 does not affect EGFR clathrin-mediated endocytosis, suggestive of potential alternative EGFR adaptors which may mediate the internalization process ${ }^{74}$. Thus, although important for facilitating AP2 interactions, understanding of the precise roles of the tyrosine and dileucine motifs in EGFR clathrin-mediated endocytosis is incomplete, and remains to be defined $^{249}$.

(iii) Another internalization mechanism induced by EGFR activation involves the recruitment of the E3 ubiquitin ligase, $\mathrm{Cbl}$. Cbl catalyzes the covalent transfer of a ubiquitin molecule to lysine residues on the carboxy-tail of EGFR ${ }^{345}$. This post-translational ubiquitination is implicated in directing EGFR towards the degradative lysosomal route upon internalization ${ }^{220}$. Although known to facilitate protein poly-ubiquitination, $\mathrm{Cbl}$ facilitates the mono-ubiquitination of EGFR which-through a ubiquitin binding domain (UBD) - is recognized by the ESCRT (endosomal sorting complexes required for transport) machinery involved in protein degradation ${ }^{11}$. Other UBD-containing proteins capable of binding the ubiquitin moiety on EGFR includes the endocytic proteins epsin, and eps15. Described in section 1.3, epsin and eps15 belong to the pioneer CME module, required for the initiation of CCP assembly ${ }^{78}$. As such, ubiquitination of EGFR is proposed to induce CME by enhancing recruitment of endocytic machineries. Despite this possible enhancement, the role of EGFR ubiquitination as an internalization signal remains controversial. One study for instance, reports the unperturbed internalization of a ubiquitination-deficient EGFR mutant with full kinase activity ${ }^{349}$. In addition, conflicting reports on the role of epsin and ep15 on CME have emerged; with some studies supporting, while others dismissing 
their absolute requirement for EGFR clathrin-mediated endocytosis ${ }^{132}$. Indeed, evidence supporting the role for ubiquitination in enhancing EGFR clathrin-mediated endocytosis were based on indirect observations of mutations, or down-regulations of components of the ubiquitination system ${ }^{345}$. As such, the precise role of ubiquitin as an internalization signal have not been completely clarified, needing further research.

Despite the controversial role of ubiquitin in receptor internalization, studies which deplete endogenous $\mathrm{Cbl}$ via genetic knockout, siRNA depletion, or overexpression of inactive mutants, collectively demonstrate the inhibition of EGFR clathrin-mediated endocytosis and several other RTKs; implying an alternative role for $\mathrm{Cbl}$ in $\mathrm{CME}$ regulation ${ }^{345}$. $\mathrm{Cbl}$ is a multidomain protein comprised of an $\mathrm{N}$ terminal tyrosine kinase binding domain (TKB; containing the $\mathrm{SH} 2$ domain) important for activation; a RING finger domain mediating its ligase activity; and a divergent C-terminal structure (which includes an $\mathrm{SH} 3$ domain recognition site) facilitating various protein-protein interactions ${ }^{350}$. $\mathrm{Cbl}$ is directly recruited onto activated EGFR via binding of TKB to pTyr1045, or indirectly through Grb2; Cbl is subsequently phosphorylated and activated by EGFR or Src kinases ${ }^{351}$. Direct binding of Cbl to EGFR however, is not as crucial to receptor internalization compared to its interaction with Grb2, as demonstrated by the unperturbed CME of EGFR mutated at the Tyr1045 site ${ }^{349}$. Cellular depletion of Grb2 however, ablated EGFR clathrin-mediated endocytosis, which is rescued by the expression of a chimeric protein comprised of the $\mathrm{Grb} 2 \mathrm{SH} 2$ domain and $\mathrm{Cbl}^{352}$. Indeed, $\mathrm{Grb} 2$ and $\mathrm{Cbl}$ are colocalized and remain bound to activated EGFR within CCPs, indicating their role in the recruitment of EGFR within clathrin structures ${ }^{345}$. Cbl may therefore regulate EGFR clathrin-mediated endocytosis in a ubiquitination-independent manner, of which precise mechanisms however, remain to be elucidated.

(iv) Highlighted by its role in recruiting $\mathrm{Cbl}$, Grb2 is an important component of the EGFR internalization pathway, which enhances receptor CME upon ligand activation. In addition to its interaction with $\mathrm{Cbl}, \mathrm{Grb} 2$ is known to bind several other proteins implicated in CME. The SH3 domain of Grb2 for instance, recognizes and binds the proline-rich region of dynamin, which was previously described an essential component in CCV fission. Indeed, ligand stimulation induced increased Grb2/dynamin association as demonstrated by biochemical assays ${ }^{353}$. In addition, the $\mathrm{SH} 2$ domain of Grb2 was shown to interact with the signaling adaptor protein Tom1L1 (Target of myb1 like 1 membrane trafficking protein), implicated in EGFR clathrin-mediated endocytosis ${ }^{354}$. Briefly, Tom1L1 is a transient interaction partner of EGFR, phosphorylated by Src kinases at Tyr460 upon EGF stimulation. This 
pTyr460 site is subsequently recognized by the Grb2 SH2 domain, which alternatively associates with EGFR via the Shc adapter (see section 1.6.3.2.2); forming the Tom1L1/Grb2/Shc/EGFR complex ${ }^{354}$. Interestingly, the C-terminal tail of Tom1L1 contains a novel clathrin-interacting motif, which interacts with the proximal region of the $\mathrm{CHC}$ subunit ${ }^{354}$. The interaction of Tom1L1 with clathrin was determined required for receptor internalization, as EGFR clathrin-mediated endocytosis in Tom1L1-knockdown cells was rescued by exogenous Tom1L1 ${ }^{354}$. Central to these interactions, Grb2 is therefore a crucial link in receptor internalization which bridges EGFR with several CME-promoting components. How these interactions may mechanistically enhance EGFR clathrin-mediated endocytosis however, is not welldefined and requires further investigation.

While several other possible CME-enhancing processes have been described in $[345,355]$, these aforementioned mechanisms are primarily known to co-ordinate the internalization of EGFR upon EGF stimulation; and likely, CME of other RTKs. It is interesting to ponder however, why several redundant mechanisms have evolved in regulating EGFR clathrin-mediated endocytosis. It can be hypothesized that due to the oncogenic potential of EGFR, and many other RTK members, robust internalization mechanisms become necessary as defects in one pathway may be rescued by the activity of anotherensuring down-regulation and prevention of aberrant mitogenic signaling ${ }^{356}$. Singular perturbations of internalization mechanisms may therefore be insufficient in inhibiting EGFR clathrin-mediated endocytosis, which may explain discrepancies in literature regarding the role of AP2, ubiquitin and its binding adapters (epsin and eps15) in receptor internalization ${ }^{355}$. Indeed, studies have shown that simultaneous mutations in AP2 binding motifs, and multiple ubiquitination and acetylation sites, is necessary to substantially suppress EGFR clathrin-mediated endocytosis ${ }^{345}$. Although the precise contributions of individual internalization mechanisms to overall CME is not well-understood, it is possible that their relative roles may vary depending on cell type and environmental parameters, endowing flexibility in the robust CME of EGFR ${ }^{355}$.

\subsubsection{2 - Mitogenic signaling by RTKS may induce global plasma membrane remodeling via PLC- $\gamma 1$ Mitogenic signaling by RTKs including EGFR, commonly induces both local and global}

reorganization of the plasma membrane required for mitogen-derived cellular processes ${ }^{59-61}$. How EGFR activation may mechanistically induce plasma membrane reorganization however, is not wellunderstood. Able to enhance its own $\mathrm{CME}$, it is tempting to hypothesize whether the same internalization mechanisms used by EGFR may be applied in inducing global reorganization of the 
plasma membrane. Based on the previous discussions however, the internalization strategies induced by EGFR are likely limited within the spatial domains of the activated receptor ${ }^{3}$. Some of the best-studied internalization strategies of EGFR for instance, involve the recruitment of AP2, Grb2, Cbl and TOM1L1 to binding sites on the activated receptor, of which functional roles are transient and terminated upon dissociation from the complex. These CME-enhancing mechanisms thus appear to operate locally in exclusively enhancing EGFR internalization, of which effect on global plasma membrane remodeling may be minimal-raising the question of what other signals generated by EGFR may induce global plasma membrane remodeling.

In contrast to the local CME-enhancing mechanisms induced by EGFR activation, downstream EGFR signaling components may have the potential in influencing global plasma membrane remodeling, of which spatial reach extends beyond the domains of activated receptors. The PLC- $\gamma 1$ pathway for example, is activated downstream of EGFR which presents a direct mechanism in potentially influencing CME on both local and global scales. Previously discussed, the direct recruitment and activation of PLC$\mathrm{Y} 1$ on the plasma membrane may directly impact CME via $\mathrm{PIP}_{2}$ hydrolysis, or indirectly via cytosolic calcium increases. PLCs have been described to catalyze PIP 2 hydrolysis on the plasma membrane via a 'scooting' mechanism, wherein they must slide along the plane of the membrane in order to access another $\mathrm{PIP}_{2}$ substrate-therefore likely to consume $\mathrm{PIP}_{2}$ locally within sites of recruitment ${ }^{357}$. Calcium on the other hand are diffusible signals with pleiotropic cellular roles, of which some may be involved in the global regulation of plasma membrane $\mathrm{CME}$; as demonstrated by the role of calcium in neuronal endocytosis ${ }^{96,102}$. Indeed, the potential role of calcium in augmenting CME in non-neuronal cells (also discussed in section 1.5) can be implied from the regulation of EGFR clathrin-mediated endocytosis by CaMKII and PKC, both of which require calcium for activation as previously described. Nonetheless, the role of PLC-derived calcium increases in regulating CME had not been explicitly studied.

PLC- $\gamma 1$ provides a potential link by which mitogenic signals may regulate both local and global $\mathrm{CME}$, important for plasma membrane remodeling. Knowledge on how PLC- $\gamma 1$ may impact EGFR clathrin-mediated endocytosis is therefore useful in providing insight as to how PLC- $\gamma 1$ may regulate the global plasma membrane. Indeed, this section on RTK endocytosis was prefaced with the goal of identifying potential junctures in EGFR clathrin-mediated endocytosis, which may be regulated by PLC$\gamma 1$ activity. An emerging theme revealed from these discussions however, is the insufficiently researched role of PLC- $\gamma 1$ in mediating receptor internalization. This study will therefore investigate and uncover 
novel roles for PLC- $\gamma 1$ in the regulation of EGFR clathrin-mediated endocytosis, providing further understanding on their potential role as mitogenic effectors of plasma membrane remodeling.

\section{7- Metabolic signals may regulate the plasma membrane via CME} As emphasized in section 1.1, the cell surfaceome collectively describes the proteins and molecules embedded within the plasma membrane, which functions in diverse cellular processes including cell adhesion and migration, nutrient uptake, product release, and intracellular signaling ${ }^{358}$. The surfaceome serve as the primary interface for cell-environment interaction, which senses and responds to physicochemical changes within the extracellular milieu ${ }^{10}$. For instance, under nutrient-rich conditions, the surfaceome exhibits enhanced expression of amino acid and lipid transporters which respectively induces protein and lipid synthesis ${ }^{359}$. Similarly, proliferative conditions stimulate the increased expression of motogenic surface proteins including integrins, which promotes cellular adhesion and migration ${ }^{360}$. In contrast, metabolic stress induced by nutrient scarcity promotes the down-regulation of energy-consuming processes (e.g. removal of $\mathrm{Na}+\mathrm{K}+\mathrm{ATPase}$ pumps from the plasma membrane), along with the concomitant upregulation of glucose uptake (e.g. increased GLUT1 surface expression) ${ }^{361,362}$. As such, the cell surfaceome adequately responds to the dynamic environment, of which modifications are contingent on adaptations necessary for survival.

Highlighted above, fluctuations in nutrient availability represents one of the dynamic parameters defining the cellular environment, which largely regulates the cellular surfaceome. Nutrient availability controls cellular energy levels (e.g. ATP) and overall cellular metabolism, and hence, a major driver of many, if not, all cellular processes. In this regard, reorganization of the surfaceome is tightly coupled to cellular metabolic states, which correspondingly adapts to changing cellular demands imposed by fluctuations in nutrient availability. How perturbations in metabolic states may be interpreted and directly translated into surfaceome remodeling however, is not well-understood, although several studies including work from our laboratory have indicated a role for AMPK (AMPactivated protein kinase) in regulating the process ${ }^{360}$. Briefly, AMPK is a predominant metabolic sensor which is activated upon sensing metabolic stress resulting from reduced energy levels (i.e. decreased cellular ATP). AMPK activation is regarded as a cellular response which stimulates adaptive mechanisms responsible for restoring energy balance and metabolic homeostasis (Figure 1.14). Additionally, previous 
work from our laboratory have shown that AMPK activation induces the global reorganization of the plasma membrane, involving robust reductions in cell surface expression levels of surface proteins; likely as a component of its metabolic repertoire ${ }^{360}$. Nonetheless, how AMPK may directly regulate the plasma membrane has not been explicitly studied, of which elucidation will provide a mechanism as to how metabolic signals may directly regulate the surfaceome.

In this section, I thus aim to describe how AMPK may directly induce remodeling of the plasma membrane, in providing a mechanism through which metabolic signals (e.g. low ATP levels) may directly influence cellular processes. This thematic shift from mitogenic to metabolic signals is largely motivated by previous work from our laboratory demonstrating the AMPK-induced remodeling of the plasma membrane ${ }^{360}$. The precise mechanisms of how AMPK may directly remodel the plasma membrane however, is not well-defined. Given the broad range of cell surface proteins that are controlled by AMPK activation, AMPK might be expected nonetheless to control the plasma membrane via regulation of $\mathrm{CME}$, which previously emphasized, is the major endocytic route in eukaryotic cells. In line with the focus of this thesis, I therefore aim to investigate how AMPK may potentially regulate CME in inducing robust plasma membrane remodeling in mammalian cells. The following section provides an overview of AMPK biology, with emphasis on its regulation and cellular roles, of which understanding will provide insight into its potential role in CME regulation.

\subsection{1 - AMPK is an energy sensor of the cell.}

AMP-activated protein kinase or AMPK is a cellular metabolic sensor which plays a critical role in maintaining energy balance, and homeostasis under conditions of metabolic stress. AMPK is exquisitely sensitive to perturbations in energy states of which kinase activity is directly activated by reduced ATP levels ${ }^{363}$. Activated during metabolic stress, AMPK stimulates stress responses aimed at restoring metabolic homeostasis by inhibiting ATP-consuming anabolic processes, while promoting catabolic processes which generates ATP. Indeed, AMPK has been a major focus in several human pathologies, of which therapeutic activation is a promising target for cancer, type II diabetes, and other illnesses characterized by abnormal utilization of cellular energy ${ }^{359}$. 


\subsubsection{1 - AMPK directly senses cellular energy availability via direct nucleotide binding}

ATP (adenosine triphosphate) is the primary energy currency largely generated from glycolysis and oxidative phosphorylation, which provides the energy required for driving cellular processes in living cells $^{364}$. ATP is structurally comprised of three sequentially connected phosphate molecules $(\alpha, \beta$, and the terminal $\gamma$ phosphate; referred to as triphosphate) covalently attached to an adenine-ribose ring ${ }^{196}$. Two phosphoanhydride bonds connect the triphosphate moiety within which energy derived from ATP is predominantly stored. Specifically, hydrolysis of ATP into ADP (adenosine diphosphate) and an inorganic phosphate-and subsequently into AMP (adenosine monophosphate) and another inorganic phosphate-releases large amounts of energy which is tightly coupled and used as fuel for other cellular processes $^{363}$. Due to its critical role as an energy source, cells therefore sustain high amounts of intracellular ATP relative to ADP, which is maintained at a 10:1 ratio ${ }^{365}$. Nonetheless, perturbations in the ATP:ADP ratio may occur upon cellular exposure to metabolic stress induced by starvation, hypoxia, ischemia, and exercise ${ }^{365-367}$. Under these conditions, ATP consumption exceeds its replenishment thereby reducing its concentration, which concomitantly increases levels of ADP and AMP. As described, cells acutely cope to reduced energy states by activating AMPK which as implied by its name, is activated upon direct binding of AMP or ADP ${ }^{363}$. AMPK is therefore a direct effector of metabolic stress, which restores energy homeostasis upon sensing perturbations in ATP concentrations.

AMPK is a heterotrimeric kinase enzyme comprised of three subunits with differing functions: a catalytic $\alpha$-, scaffolding $\beta$-, and a regulatory $\gamma$-subunit (Figure 1.14A) ${ }^{359}$. In mammals, these subunits are further expressed into different isoforms encoded by multiple genes ( $\alpha 1$ and $\alpha 2 ; \beta 1$ and $\beta 2$; and $\gamma 1-3$ ), of which various combinations give rise to 12 total AMPK complexes ${ }^{366}$. The expression levels and enrichments of AMPK subunit isoforms however, vary amongst tissue types giving rise to tissue-specific AMPK complexes. The $\alpha 2$ and $\beta 2$ subunits for instance, are enriched within skeletal and cardiac muscles serving as the major $\alpha$ and $\beta$ isoforms comprising AMPK within these tissues; whereas they are found at lower levels in other tissue types ${ }^{363}$. Similarly, the $\gamma 2$ and $\gamma 3$ subunits appear to also be restricted in skeletal and cardiac tissues, within which the $\alpha 2 / \beta 2 / \gamma 2$ or $-\gamma 3$ AMPK complexes predominate ${ }^{363}$. In contrast, the $\alpha 1, \beta 1$, and $\gamma 1$ subunits are ubiquitously expressed, forming the prototypical $\alpha 1 / \beta 1 /{ }^{\prime} 1$ complex from which most knowledge regarding AMPK biology is derived ${ }^{359}$. Although the distinct AMPK complexes generally exhibit functional redundancy in sensing and responding to metabolic stress, they may nonetheless, exhibit different biochemical properties of which regulations may differ in a tissuespecific manner; described in [368]. Despite this difference, a general regulatory mechanism has been 
described which details precisely how AMPK complexes may be activated at the onset of metabolic stress.

A reduction in ATP:ADP/AMP ratio largely regulates the activation of AMPK. Specifically, the $\gamma$ subunit serve as the main regulatory module that allows AMPK to sense changes in cellular adenosine nucleotide concentrations. The $\gamma$-subunit is made up of two Bateman domains, each of which contains two cystathionine $\beta$-synthase (CBS) repeats, providing four total sites (sites 1-4) which binds either ATP, $A D P$, or AMP in a competitive manner ${ }^{363}$. Site 2 however, appears to be missing an essential aspartate residue required for nucleotide binding, rendering the site non-functional and commonly devoid of a nucleotide molecule. As well, while able to bind all three adenosine derivatives, site 4 exhibits preferential affinity for AMP which is thought to permanently occupy the nucleotide binding site ${ }^{363}$. In contrast, sites 1 and 3 competitively binds all three nucleotide molecules, therefore serving as the regulatory binding sites capable of monitoring perturbations in nucleotide concentrations. Indeed, AMP, and to a lesser extent ADP, binding to sites 1 and 3 induces a conformational shift promoting AMPK activation, which in contrast, is directly opposed by ATP binding to these regulatory sites ${ }^{363}$.

The $\alpha$-subunit serves as the primary catalytic module of AMPK, of which activation is mediated by AMP binding to the $\gamma$-subunit. The $\alpha$-subunit is comprised of: an $\mathrm{N}$-terminal kinase domain responsible for phosphorylating downstream AMPK targets; an autoinhibitory domain (AID) which regulates AMPK activity; an $\alpha$-linker containing two $\alpha$-RIM (regulatory-subunit-interacting motifs) segments which interacts with the $\gamma$-subunit; and a C-terminal domain that interacts with both the $\gamma$ and $\beta$-subunits, which also contains an ST loop (serine/threonine-rich domain) responsible for finetuning AMPK activity ${ }^{363}$. Crystallographic studies reveal that in its ATP-bound state, AMPK exists in an autoinhibited configuration wherein the kinase domain is occluded by the AID-rendering the enzyme inactive. AMP binding relieves AMPK of autoinhibition by reorganizing the $\alpha$-RIM segments to interact with the empty site 2 and AMP-bound site 3 of the $\psi$-subunit, inducing a large-scale conformational shift displacing the AID from the kinase domain; increasing AMPK activity by $\sim 10-$ fold $^{363}$. In addition, the AMP-mediated conformational shift also promotes the phosphorylation of Thr-172 within the activation loop of the kinase domain, regarded as the hallmark of AMPK activation which enhances AMPK activity in vitro by $\sim 100$-fold ${ }^{363,369}$. Release of autoinhibition and Thr-172 phosphorylation of the $\alpha$-AMPK subunit are thus, essential events for the full activation of AMPK, allosterically mediated by AMP binding. 


\subsubsection{2 - Full activation of AMPK under metabolic stress requires Thr-172 phosphorylation by LKB1}

Several kinases have been identified which phosphorylates AMPK at Thr-172 upon allosteric activation by AMP. LKB1 (liver-kinase-B1) for example, is the major upstream kinase which is believed to exist in a constitutively active state, responsible for phosphorylating AMPK during metabolic stress ${ }^{362,369}$. Interestingly, LKB1 is known to phosphorylate AMPK at Thr-172 even under high cellular ATP levels, which nonetheless, is readily dephosphorylated by phosphatases including protein phosphatase $2 \mathrm{~A}$ and 2C (PP2A and PP2C), and PPM1E ( $\mathrm{Mg}^{2+}-/ \mathrm{Mn}^{2+}$-dependent protein phosphatase $\left.1 \mathrm{E}\right)^{362}$. The conformational shift induced by AMP binding to AMPK however, stabilizes Thr-172 phosphorylation, of which active conformation sterically obstructs phosphatase docking thereby protecting Thr-172 from dephosphorylation ${ }^{363}$. Other AMPK kinases have also been identified including CaMKII (Calmodulin dependent kinase II) and TAK1 (TGFß-activated kinase 1), which similarly phosphorylates Thr-172, albeit under different cellular conditions such that during intracellular calcium increases and mitogenic signaling, respectively ${ }^{362}$. Indeed, LKB1 is the primary kinase which activates AMPK in a nucleotidedependent manner of which deletion in mice significantly reduces survival, likely due to the inhibition of phosphorylation, and hence, activation of AMPK preventing energy maintenance under metabolic stress $^{369,370 .}$

In its AMP-bound, activated state, AMPK phosphorylates several downstream targets which functions in numerous cellular energy-restoring processes (Figure 1.14B). AMPK for instance, limits energy consumption by inhibiting anabolic pathways such as glycogenolysis, lipogenesis, and protein synthesis, via inhibitory phosphorylation of key regulatory proteins including glycogen synthase (GS), acetyl-coA carboxylase (ACC) and mTOR, respectively ${ }^{359,363}$. In parallel, catabolic processes which breakdown macromolecules for ATP regeneration are enhanced by AMPK. Catabolic pathways which breakdown glucose (e.g. glycolysis), fatty acids (e.g. fatty acid oxidation), and proteins (e.g. autophagy) for instance, are upregulated by AMPK phosphorylation of regulatory proteins including PFKFB3 (6phosphofructo-2-kinase/fructose-2,6-bisphosphate 3), ACC, and ULK1 (unc-51-like autophagy-activating kinase 1), respectively ${ }^{359,363}$. Furthermore, AMPK restores energy balance by remodeling the plasma membrane, limiting the expression of ATP-consuming surface proteins such as ion pumps (e.g. $\mathrm{Na}+\mathrm{K}+$ ATPase pumps) and motogenic receptors (e.g. integrin receptor), while increasing surface expression of nutrient transporters such as GLUT-1 and -4, and CD36 (cluster of differentiation 36), respectively increasing glucose and lipid uptake for ATP synthesis ${ }^{362,363}$. While intracellular control of metabolic processes by AMPK is mechanistically well-annotated, understanding of how AMPK may directly 
remodel and regulate the plasma membrane nonetheless, remains poorly understood. Several studies however, suggests the potential involvement of the small GTPase protein, Arf6 (ADP-ribosylation factor 6), which has been demonstrated to promote the assembly of clathrin lattices on the plasma membrane; and of which activation may similarly be regulated by AMPK activity as demonstrated in recent work from our laboratory ${ }^{122,360}$. Arf6 may thus be hypothesized to couple AMPK activation with plasma membrane remodeling, in restoring energy balance during perturbations of cellular metabolic homeostasis-further described in the following section.

\subsection{2 - The Arf family of small GTPases are major regulators of membrane traffic} The ADP-ribosylation factor (Arf) family represents one of the five major sub-families (e.g. Ras, Rho, Ran, Rab and Arf) constituting the Ras superfamily of small GTPases (20-40kDa) ${ }^{371}$. To date, more than 100 small GTPases have collectively been identified in eukaryotes from yeast to humans, with varying regulatory roles in diverse cellular processes ${ }^{371}$. The best-characterized Ras family for instance, is known to regulate gene expression involved in cellular growth, differentiation, and survival (as described in section 1.6.3.2.2); while other families such as Rho and Ran regulates cytoskeletal reorganization and nucleo-cytoplasmic transport during cell division, respectively ${ }^{371,372}$. Rab and Arf proteins on the other hand are major regulators of membrane traffic, with various roles in mediating intracellular vesicle budding and subsequent targeting to subcellular organelles ${ }^{373}$. Importantly, Arf proteins have been largely implicated in the regulation of almost every aspect of membrane traffic in cells, including a role at the plasma membrane in the control of endocytosis ${ }^{372}$. In this section, I will therefore focus on the Arf family and their role in regulating membrane traffic, to provide insight as to how they may mediate plasma membrane remodeling in response to AMPK activation during metabolic stress.

\subsubsection{1 - Arfs proteins regulate distinct membrane traffic processes in a GTP-dependent manner}

The Arf family of small GTPases regulates several aspects of membrane traffic in eukaryotes including vesicle budding, tethering and cytoskeleton organization, important for cell adhesion and migration $^{374,375}$. Six mammalian Arf isoforms (Arf1-6) have been identified which are organized into three classes according to their sequence homology: class I (Arf1-3), class II (Arf4 and 5), and class III (Arf6). All Arf isoforms however, share a similar structural arrangement: an $\mathrm{N}$-terminal amphipathic helix bound to a myristoyl group; and two GTP-binding regions common to small GTPases termed 'switch 1' and 'switch 2 ', which nonetheless, is uniquely separated in Arfs by a mobile 'interswitch' region ${ }^{376}$. Moreover, Arf 
proteins are the founding members of a larger structurally related protein family that also includes Arl (Arf-like), Arp (Arf-related proteins) and Sar (Secretion-associated and Ras-related) proteins, of which role in membrane traffic albeit, remain poorly characterized ${ }^{377}$. In contrast, the six major Arf isoforms have been implicated in regulating distinct intracellular membrane traffic processes which include both secretory, and endocytic pathways. Indeed, single knockout of Arf isoforms in mice such as Arf1 or Arf6, result in embryonic developmental defects likely attributed to dysregulation of membrane traffic ${ }^{376,378 .}$

Arf proteins regulate membrane traffic by directly associating with biological membranes, where they recruit effector molecules necessary for vesicular budding and targeting. Specifically, the myristoylated helix at the $\mathrm{N}$-terminal region of Arfs is shorter compared to other small GTPase membrane-anchoring domains, enabling the close apposition of Arfs and their respective effector molecules to cytosolic membranes ${ }^{376}$. Furthermore, Arfs exhibit various localizations within intracellular membranes allowing for the regulation of distinct membrane traffic pathways. Arf1 for instance, is the best-studied and most abundant Arf isoform predominantly localized to the Golgi complex where it functions in largely regulating the secretory pathway; along with Arf3, Arf4 and Arf5 ${ }^{379,380}$. On the Golgi complex, Arf1 recruits endocytic components including coat (e.g. COPI; coat protein complex I) and adaptor proteins (e.g. AP1) - promoting vesicle budding from the TGN ${ }^{379,381}$. Arf6 on the other hand, is the predominant Arf isoform localized on the plasma membrane due to a larger positive surface charge, where it may regulate CME via several mechanistic routes ${ }^{375}$. Firstly, Arf6 is known to induce production of plasma membrane $\mathrm{PIP}_{2}$ by recruitment and activation of PIP5Ks, thereby enhancing AP2 recruitment and CME (see section 1.3) 379,382. Secondly, Arf6 has also been demonstrated to directly recruit and interact with $\mathrm{AP} 2$, which together with $\mathrm{PIP}_{2}$, coordinates its translocation to the plasma membrane ${ }^{379}$. Thirdly, Arf6 has been demonstrated to directly bind the $\mathrm{CHC}$ terminal domain, strongly indicating its role in CME regulation ${ }^{383}$; although less defined, the Arf6 regulator SMAP (described in the following section) is also known to bind clathrin, further corroborating the role of Arf6 in clathrin recruitment ${ }^{376}$. Indeed, ablation of Arf6 activity inhibits TfR internalization, indicative of its regulatory role in the clathrin-mediated endocytic process ${ }^{379}$. It is important to note however, that the recruitment of effector molecules and thus, regulation of membrane traffic by Arfs, occur in a GTP-dependent mannerpredicated on the intrinsic ability of Arfs in binding GTP ${ }^{376}$. 


\subsubsection{2 - Arf-GEFs and Arf-GAPs regulates Arf nucleotide cycling and control of membrane traffic}

As with other small GTPases, Arf proteins are guanine nucleotide-binding enzymes which cycle between GDP-bound (Arf-GDP) inactive and GTP-bound (Arf-GTP) active states, of which the latter configuration promotes effector molecule recruitment (Figure 1.15) ${ }^{377}$. In their inactive GDP-bound form, Arfs are held in an autoinhibited conformation by their interswitch region, which retracts onto switch 1 and switch 2 preventing GTP binding. In addition, the retracted interswitch segment forms a hydrophobic pocket within which the amphipathic N-terminal helix binds, localizing Arf-GDP in the cytosol unable to interact with biological membranes ${ }^{377}$. GTP binding on the other hand, relieves Arfs from autoinhibition by inducing a large-scale conformational shift resulting in the outward protrusion of the interswitch region, and extrusion of the $\mathrm{N}$-terminal helix for membrane binding; thereby coupling Arf activation with membrane recruitment ${ }^{377}$. While capable of intrinsically binding and hydrolyzing GTP, Arfs nonetheless require the regulatory activities of GEFs which facilitate GDP dissociation promoting GTP binding, and as well as GAPs that stimulate GTP hydrolysis ${ }^{372}$. As such, nucleotide cycling by Arfs is largely mediated by Arf-GEFs and Arf-GAPs, of which spatiotemporal regulations therefore determines the scope by which Arfs may regulate membrane traffic 372,376 .

Several GEFs and GAPs have been identified which regulates the activation states of Arfs in eukaryotes. Specifically, there are six families of Arf-GEFs which share a conserved Sec7 domain responsible for catalyzing Arf-nucleotide exchange, via displacement of bound GDP by a negatively charged 'glutamic finger' motif ${ }^{372}$. Arf-GEF families nevertheless, structurally diverge featuring different domain architectures that enable mediation of diverse cellular activities. The cytohesin/ARNO, EFA6 and IQSEC/BRAG Arf-GEF families for instance, all contain a PH domain which targets their GEF activity towards the $\mathrm{PIP}_{2}$-enriched plasma membrane ${ }^{380}$. Indeed, the PH domain-containing Arf-GEFs have been shown as the predominant Arf6-GEFs, of which concomitant recruitment to the cell surface regulates Arf6-and thus, its regulation of plasma membrane endocytosis ${ }^{371,384}$. Similarly, ten subfamilies of ArfGAPs have been identified in humans, which despite having unrelated structures share a common ArfGAP domain ${ }^{376}$. The Arf-GAP domain contains an arginine-finger motif which inserts into the Arf active site, where it participates in catalyzing GTP hydrolysis ${ }^{376}$. Plasma membrane localized Arf-GAP families including ArfGAP3, ARAP, and SMAP for example, significantly enhances Arf6 GTPase activity, promoting its inactivation and dissociation from the plasma membrane ${ }^{376}$. In this regard, the functional roles of Arf6 in mediating plasma membrane traffic is therefore intimately coupled with the spatiotemporal 
control of its GEFs and GAPs, of which precise mechanistic regulations under various cellular contexts nonetheless, remain poorly annotated.

\subsection{3 - AMPK may induce global plasma membrane remodeling via Arf6}

As previously described, AMPK is the primary energy sensor which upon activation induces global remodeling of the plasma membrane surfaceome. The precise mechanism by which AMPK facilitates cell surface reorganization however, remains poorly understood. Several lines of evidence nevertheless, indicate the potential involvement of Arf6, which described above, may augment CME by facilitating PIP 2 production and AP2 and clathrin recruitment (Figure 1.16) ${ }^{383}$; and perhaps to a lesser extent via SMAP activity ${ }^{379}$. Importantly, we have recently shown that in addition to the down-regulation of ATP-consuming surface proteins, AMPK activation also decreases surface expression of Arf6-GAPs including ArfGAP3 and ARAP, of which the former contains a predicted AMPK phosphorylation sitethereby potentially regulating Arf6 function ${ }^{360}$. In addition, Arf-GEFs including GBF1 and FBXO8 have been demonstrated to be directly phosphorylated by AMPK, which although not specific for Arf6, highlights the regulatory role of AMPK in controlling membrane traffic ${ }^{385,386}$. Lastly, surface expression of the $\beta 1$-integrin receptor was also significantly reduced upon AMPK activation, which further described below, is endocytosed via CME in an Arf6-dependent manner. The precise mechanism by which AMPK may regulate Arf6 in enhancing CME of plasma membrane cargoes including $\beta 1$-integrins however, is poorly understood; of which elucidation will provide a mechanistic link by which AMPK may induce global plasma membrane remodeling under metabolic stress.

\subsubsection{1 - Integrins are cell surface adhesion proteins endocytosed via CME in an Arf6-dependent manner} Integrins are heterodimeric transmembrane surface receptors implicated in functionally diverse processes including cellular growth, differentiation, adhesion, motility and migration ${ }^{387}$. It is comprised of non-covalently associated $\alpha$ and $\beta$ subunits, respectively expressed as 18 and 8 isoforms encoded by different genes in humans; producing 24 different integrin heterodimers ${ }^{388}$. The 24 integrin complexes can be grouped into subfamilies based on ligand-binding and structural properties, which include the predominant $\beta 1$-integrin group (i.e. integrins containing a $\beta 1$-integrin subunit and a variable $\alpha$ subunit $)^{387}$. $\beta 1$-integrins are widely expressed in mammalian tissues, topologically comprised of an exofacial $\mathrm{N}$-terminal domain which binds to the extracellular matrix; a transmembrane domain; and a Cterminal domain which bind components of the cytoskeleton-thereby mechanically anchoring the cell 
to its environment ${ }^{389}$. In addition, $\beta 1$-integrins (and integrins in general) propagate signal transduction from adhesion sites which biochemically provides the cell information on its location, adhesion and local environment state, enabling cells in executing appropriate cellular processes necessary for adaptation and survival ${ }^{390}$. As such, $\beta 1$-integrins are regarded as major drivers of mitogenic and migratory processes, which unsurprisingly, are substantially down-regulated from the cell surface during metabolic stress; likely as an energy conserving mechanism ${ }^{360}$.

While the exact nature of $\beta 1$-integrin endocytosis remains to be fully understood, CME appears to be a major route through which $\beta 1$-integrins are internalized ${ }^{387,391}$. Specifically, $\beta 1$-integrins contain NPXXY/NXXY motifs within the cytoplasmic tail of the $\beta 1$-subunit, which may be recognized by specific clathrin adaptors including AP2, eps8, Numb and Dab2 (see section 1.3.2) ${ }^{392}$. As well, Dyn2 localizes at adhesion sites together with clathrin and integrin, further highlighting CME as a predominant route for $\beta 1$-integrin endocytosis ${ }^{392}$. Interestingly, Arf6 has similarly been implicated in cellular traffic of $\beta 1$ integrins, of which endocytosis becomes significantly impaired upon knockdown of Arf6-GAPs (e.g. ARAP) and Arf6-GEFs (e.g. BRAG2; member of the IQSEC/BRAG GEF family) ${ }^{391,393}$. Nonetheless, precisely how Arf6 may mechanistically regulate $\beta 1$-integrin internalization is not well-understood, although it can be expected to do so via regulation of $\mathrm{PIP}_{2}$ and recruitment of endocytic components as previously described.

In summary, Arf6 may therefore function as a mechanistic link directly coupling AMPK activation, and hence metabolic stress, with the reorganization of the plasma membrane via CME. While knowledge regarding AMPK control of Arf6 is limited, previous studies including our recent findings described above, indicate the potential regulation of Arf6 regulators (i.e. Arf6-GAPs and Arf6-GEFs) by AMPK. These findings are further corroborated by the AMPK-induced down-regulation of $\beta 1$-integrins from the cell surface, which are known to undergo CME in an Arf6-dependent manner. Collectively, these previous studies suggest that it is possible that Arf6 is regulated upon AMPK activation. Thus, AMPK may control Arf6 potentially via nucleotide cycling which can be hypothesized to alter CME dynamics - ultimately providing metabolic signals with a mechanism by which modifications of the plasma membrane may be initiated. 


\section{8- Project goals and rationale}

The plasma membrane is an important organelle which allow cells to sense and interact with the surrounding environment, necessary for adaptation and survival. It is composed of a lipid bilayer embedded with key proteins including carriers and transporters, adhesion molecules, and signaling receptors-collectively termed as the cell surfaceome-which directs fundamental processes in cellular metabolism, migration, growth and proliferation ${ }^{31}$. As an essential environment-interfacing module, the surfaceome constantly undergoes dynamic changes involving constant removal and addition of proteins (e.g. integral and peripheral), in responding and adapting to changes within the extracellular milieu ${ }^{10}$. Endocytosis is the cellular process generally responsible for the reorganization of the surfaceome which directly removes proteins from the plasma membrane. During endocytosis, small regions of the plasma membrane invaginate into pits which pinch off as vesicles into the cytoplasm, capturing extracellular material including specific surface proteins into the cell ${ }^{51}$. Although several forms of endocytosis exist, CME is the predominant and best characterized endocytic route in mammalian cells, largely involved in plasma membrane remodeling, given the ability of CME to selectively concentrate specific receptors and transporters into budding vesicles ${ }^{10,58}$. CME is implicated in many facets of cell physiology and homeostasis, and understanding mechanisms of its regulation is therefore, of great importance ${ }^{57}$.

Diverse cellular cues including mitogenic (e.g. growth factors) and metabolic signals (e.g. cellular energy levels) induce diverse physiological processes in mammalian cells (cellular growth, proliferation, migration and differentiation) requiring some degree of plasma membrane remodeling-and hence, direct regulation of $\mathrm{CME}$. How mitogenic and metabolic signals may regulate $\mathrm{CME}$ however, is not welldefined. In this thesis, I will therefore investigate how i) mitogenic and ii) metabolic signals may regulate $\mathrm{CME}$, providing insight as to how these cellular cues may induce modifications of the plasma membrane.

(i) In addressing the role of mitogenic signals in $\mathrm{CME}$, I will specifically study mitogenic signaling by the RTK family with specific focus on the best-characterized member, EGFR. EGFR is activated upon stimulation by the mitogenic EGF ligand, resulting in the subsequent recruitment and activation of the PLC- $\gamma 1$ enzyme by the activated receptor. The enzymatic activities of PLC- $\gamma 1$ result in both PIP 2 depletion and an increase in cytosolic calcium, both of which functions have not been explicitly studied in context of CME. Several lines of evidence however, imply the potential role of these PLC $\gamma 1$-derived activities in $\mathrm{CME}$ regulation. As well, EGFR stimulation induces their own internalization via $\mathrm{CME}$, thereby coupling PLC- $\gamma 1$ activation with the internalization process. The potential role of PLC- $\gamma 1$ in regulating EGFR 
clathrin-mediated endocytosis however, remains relatively unexplored. Thus, in my thesis, I will investigate the requirement for PLC- $\gamma 1$ in EGFR clathrin-mediated endocytosis, which will provide clues as to how mitogenic signals may regulate $\mathrm{CME}$ in effecting plasma membrane remodeling.

I hypothesize that PLC- $\gamma 1$ is required for the efficient internalization of EGFR via CME, of which enzymatic activities are required in regulating clathrin-coat assemblies. In addressing this hypothesis, the following goals were set:

1) Determine the requirement for PLC- $\gamma 1$ in the clathrin-mediated endocytosis of EGFR.

2) Determine the roles for $P_{P_{2}}$ hydrolysis and calcium signals derived from PLC- $\gamma 1$ activity in regulating the clathrin-mediated endocytosis of EGFR.

3) Determine the requirement for PLC-derived signals in CCP dynamics.

4) Determine a possible mechanism by which PLC-derived calcium signals may impact CCP dynamics; and hence CME.

*This work is published in the peer-reviewed journal Molecular Biology of the Cell in $2017^{1}$, and presented here in Chapters 3 and 4.

(ii) In addressing the role of metabolic signals in CME, I will specifically study AMPK which is considered as the primary energy sensor of the cell. AMPK is activated during metabolic stress induced by a reduction in cellular energy availability. Upon activation, AMPK stimulates global remodeling of the plasma membrane, which restores metabolic homeostasis by limiting energy consuming processes at the cell surface. Precisely how AMPK may mechanistically regulate the plasma membrane however, is not well-understood, although a role for the Arf6 GTPase can be proposed. Arf6 is a small GTPase which has been implicated to enhance CME by stimulating $\mathrm{PIP}_{2}$ production and AP2 and clathrin recruitment on the plasma membrane ${ }^{383}$. Importantly, previous studies including recent findings from our laboratory have demonstrated the potential control of Arf6 by AMPK, via regulation of Arf6-GAPs and Arf6-GEFs. In addition, we have shown that AMPK activation reduces cell-surface levels of $\beta 1$-integrin, which is known to internalize via CME in an Arf6-dependent manner-suggestive of the potential regulation of Arf6, and hence, CME by AMPK. In this thesis, I will thus attempt to determine the role of Arf6 in controlling CME dynamics during AMPK activation, in providing a mechanism by which metabolic signals may regulate the cell surfaceome. 
I hypothesize that AMPK regulates CME dynamics by control of Arf6 nucleotide cycling. Specifically, I hypothesize that AMPK activation toggles Arf6 nucleotide cycling, which impacts CCP dynamics - thereby influencing global CME and plasma membrane remodeling. In addressing this hypothesis, the following goals were set:

1) Determine the role of $A M P K$ in regulating CME dynamics.

2) Determine how AMPK may control Arf6 activity, and its implication in regulation of CME.

3) Determine how AMPK activation might regulate the internalization of CME cargoes; with specific focus on $\beta 1$-integrin.

*This work is currently in preparation and will be submitted to a peer-reviewed journal early 2019, and presented here in Chapter 5. 


\section{9- Figures for Chapter 1}

Signaling receptors

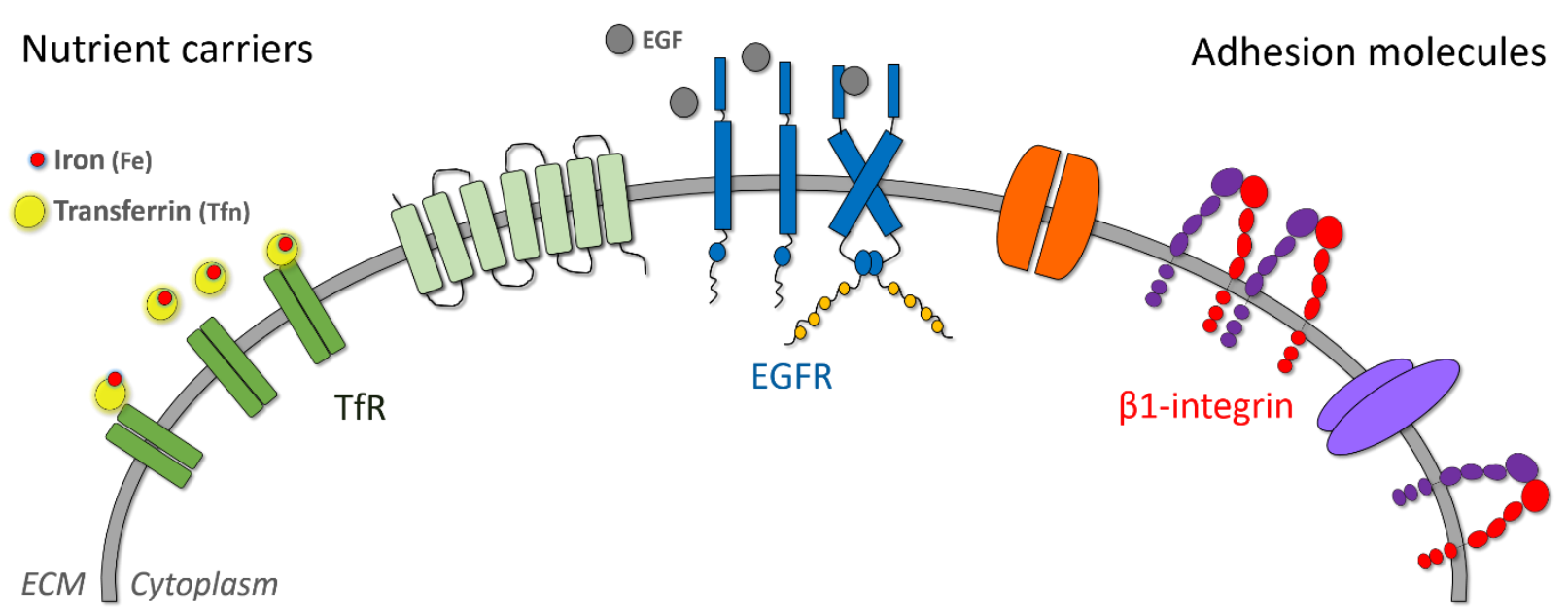

Figure 1.1. The cell surfaceome. The plasma membrane is comprised of a lipid bilayer embedded with surface proteins, collectively termed as the 'cell surfaceome's1. Surface proteins can associate with the plasma membrane either integrally or peripherally (not shown), and include functionally diverse proteins such as nutrient carriers (e.g. Transferrin receptors), signaling receptors (e.g. EGFR), and adhesion molecules (e.g. $\beta 1$-integrin) ${ }^{32}$. As such, the cell surfaceome is involved in a variety of cellular processes including cell-cell and cell-environment communication, adhesion and migration, metabolism, and signal transduction ${ }^{30}$. 


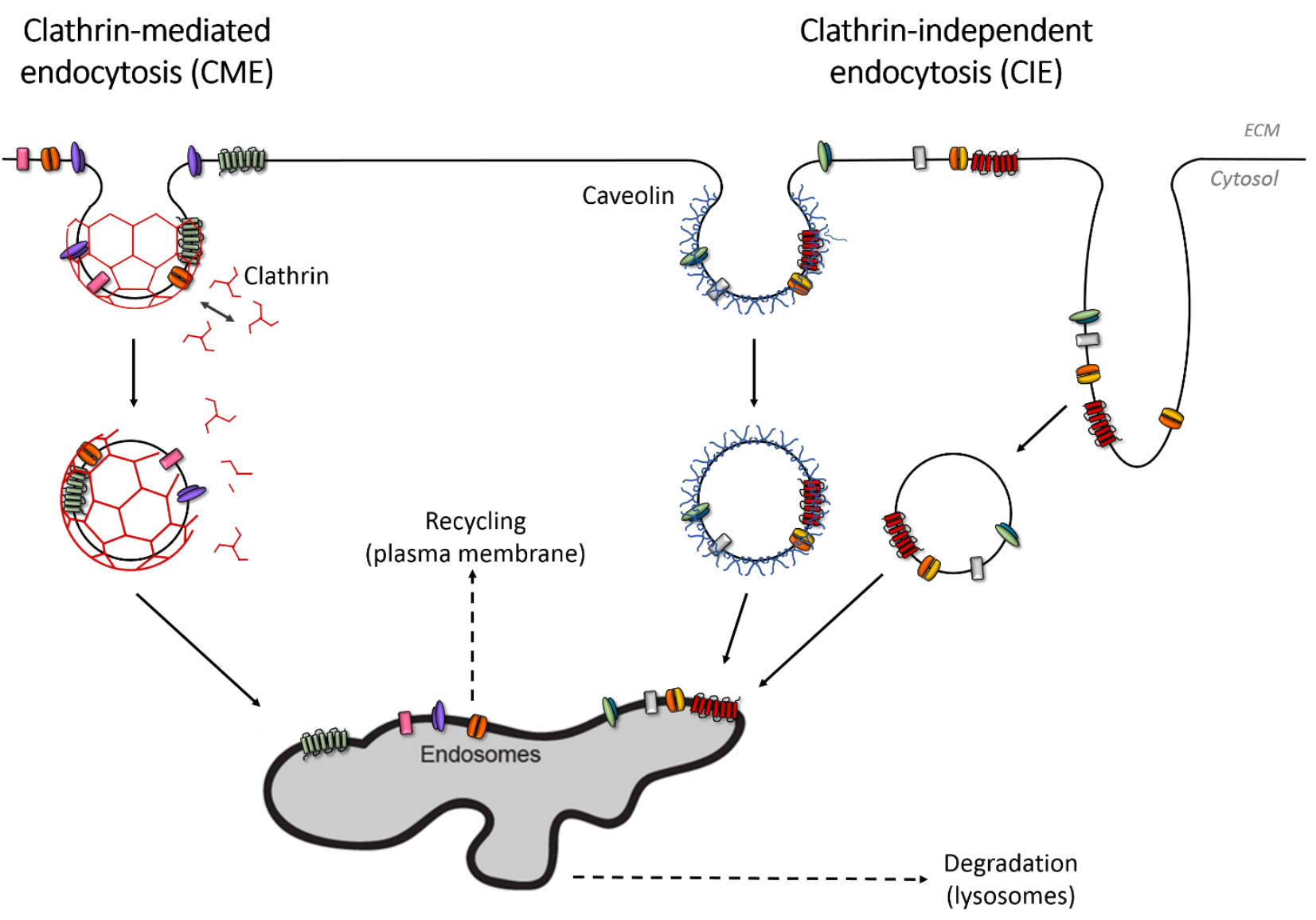

Figure 1.2. Different modes of endocytosis. Endocytosis is the cellular process generally responsible for the reorganization of the surfaceome which directly removes proteins from the plasma membrane. During endocytosis, small regions of the plasma membrane invaginate into pits which pinch off as vesicles into the cytoplasm, capturing extracellular material including specific surface proteins into the cell ${ }^{50}$. Multiple pathways of endocytosis exist in eukaryotic cells including: i) Clathrin-dependent (e.g. clathrin-mediated endocytosis) and ii) Clathrin-independent (e.g. Caveolin-dependent and bulk endocytosis) forms of internalization ${ }^{50}$. All three modes follow a general step-wise sequence starting from plasma membrane invagination which forms into an endocytic vesicle trafficked to endosomes, where their content (termed cargo) are sorted either back to the plasma membrane for recycling or to the lysosomes for degradation. The main differences between these processes lie in the types of cargo they internalize, signal for initiation and as well as the type of endocytic vesicle that is initially formed. Clathrin-mediated endocytosis (CME) is the best-characterized and predominant form of endocytosis in mammals, characterized by the clathrin coat protein which surrounds the budding vesicle; caveolindependent pathway form pits with caveolin while the latter mode is absent of any signature protein ${ }^{50}$. 


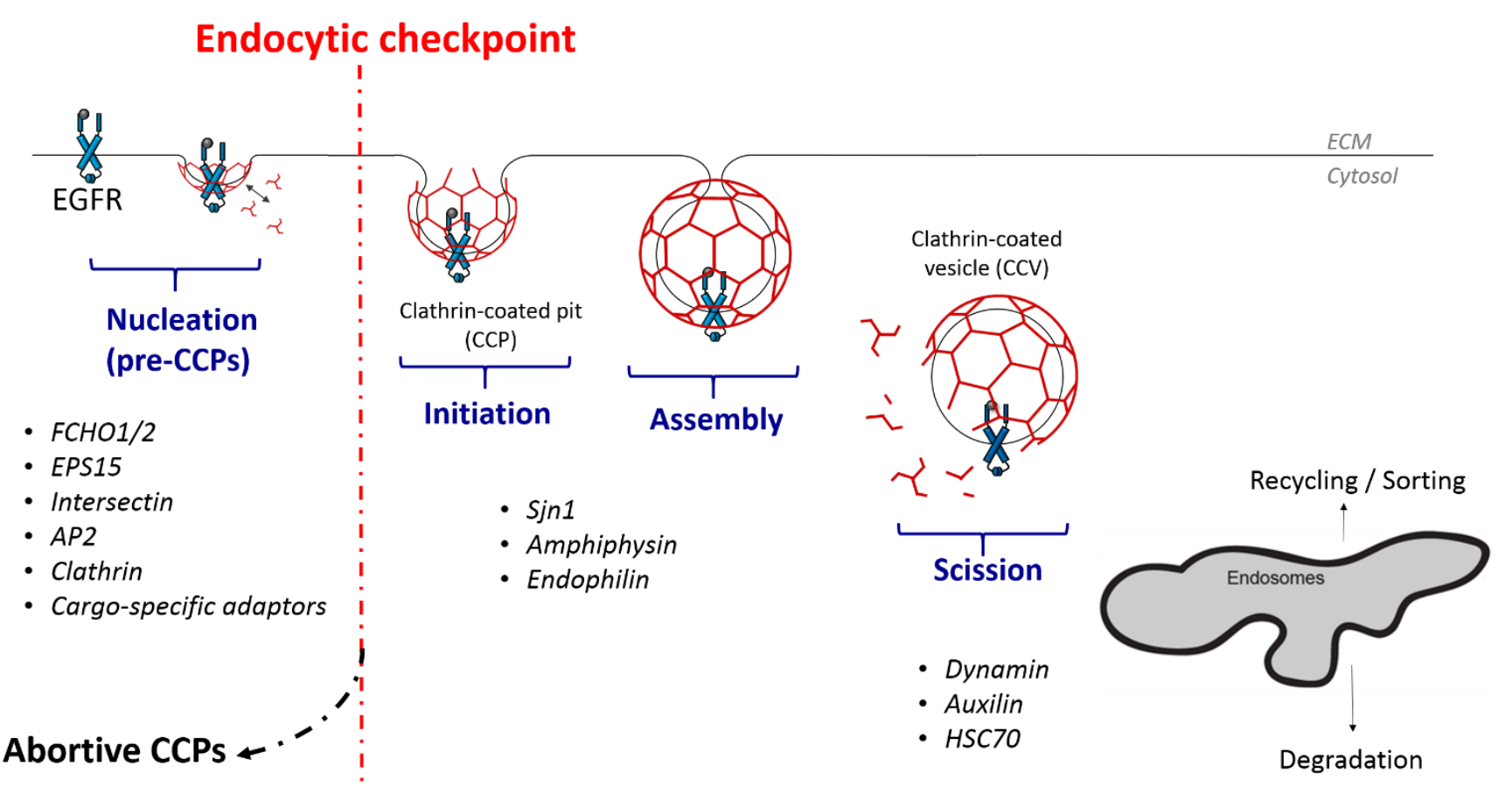

Figure 1.3. Clathrin-mediated endocytosis is defined by morphologically distinct stages. CME proceeds through discrete stages: cargo selection and nucleation, coat initiation and assembly, and scission and uncoating. Each stage is marked by the arrival of functionally distinct protein modules; of which some are listed. Notably, protein modules exhibit overlap and may regulate multiple stages of $\mathrm{CME}$, and as such, are not limited to the stages under which they are listed above. CME is initiated by 'pioneer' module proteins including FCHO1/2, eps15, intersectin, AP2 and clathrin, which define sites on the plasma membrane for CME. Cargo specific adaptors recruit surface proteins including EGFR (shown above) within nascent clathrin structures. Further recruitment and assembly of clathrin, along with other endocytic accessory proteins, promotes the growth of nascent clathrin structures into bona fide cargo-containing clathrin-coated pits (CCPs). CCPs continue to mature and eventually excised from the plasma membrane via dynamin, forming clathrin-coated vesicles (CCVs) within the cytosol. CCVs are then uncoated by Auxilin and $\mathrm{HSC70}$, releasing clathrin and other components of the endocytic machinery for use in succeeding CME events. The progression of CME is hypothesized to be gated by an 'endocytic checkpoint' (red broken line), through which nascent clathrin structures must progress to develop into productive CCPs. Several factors determine progression through this checkpoint including the rate and nature of coat assembly, extent and characteristic of cargo loading, recruitment of appropriate endocytic accessory proteins, and curvature generation-which if are not satisfied, induces the turnover of nascent clathrin structures ${ }^{6}$. 


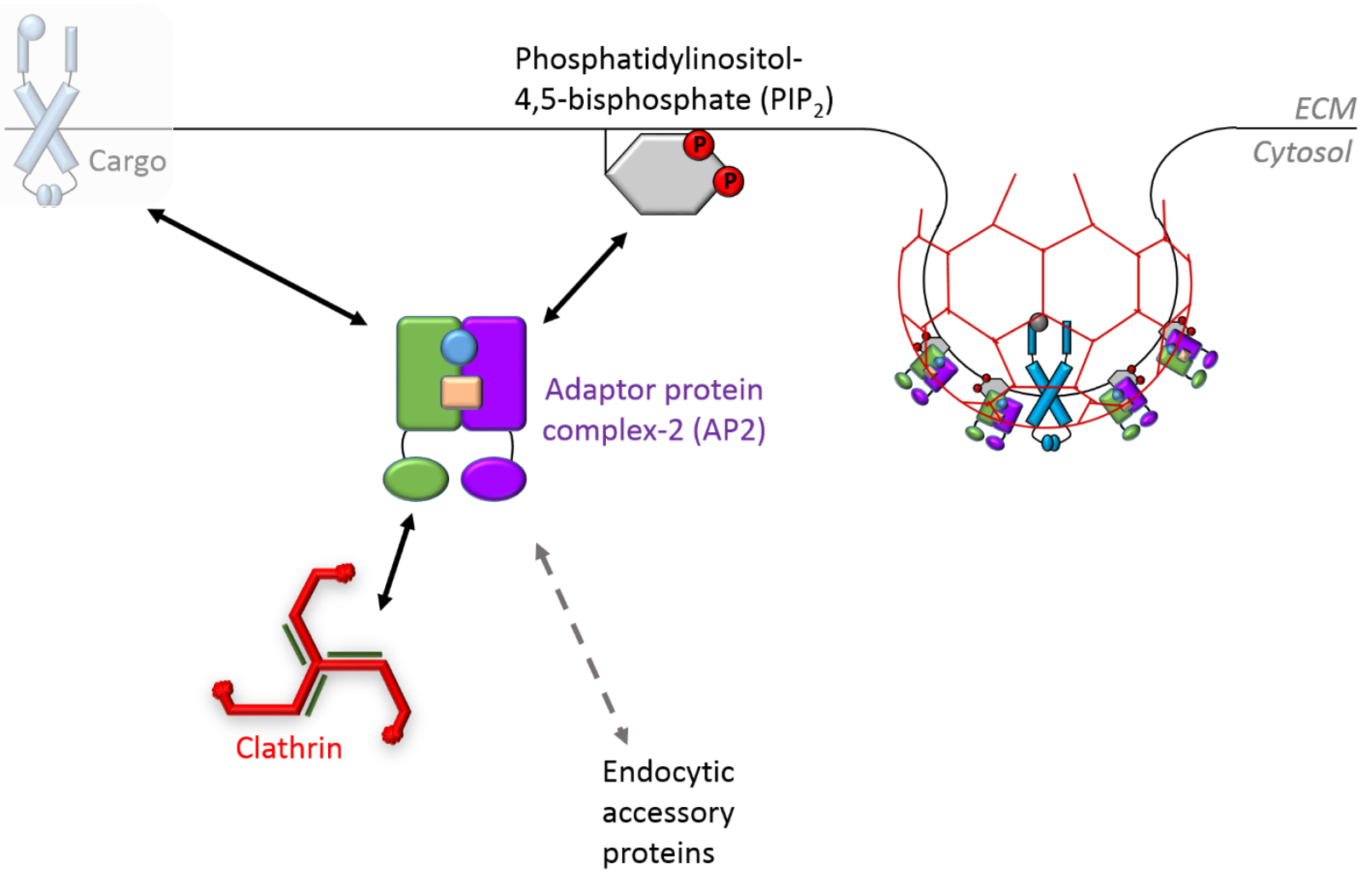

Figure 1.4. Clathrin-mediated endocytosis is regulated by three core hubs. CME is regulated by three core hubs: i) clathrin, ii) adaptor protein-2 complex (AP2), and iii) phosphatidylinositol-4,5-bisphosphate $\left(\mathrm{PIP}_{2}\right)$. Clathrin is the hallmark protein coat which surrounds and provides structural support for the budding endocytic structure. After clathrin, AP2 is the most abundant protein found exclusively within plasma membrane-derived CCVs, which functions in recruiting endocytic accessory proteins and cargo molecules ${ }^{121}$. The membrane lipid $\mathrm{PIP}_{2}$ is an indispensable component of $\mathrm{CME}$, serving as a plasma membrane substrate for several key endocytic components including AP2, FCHO1/2, CALM, epsin, eps15 and dynamin. These core components of CME have disproportionately more interactions than any other proteins in the endocytic pathway, of which depletions exhibit catastrophic effects on the endocytic network ${ }^{12}$. 


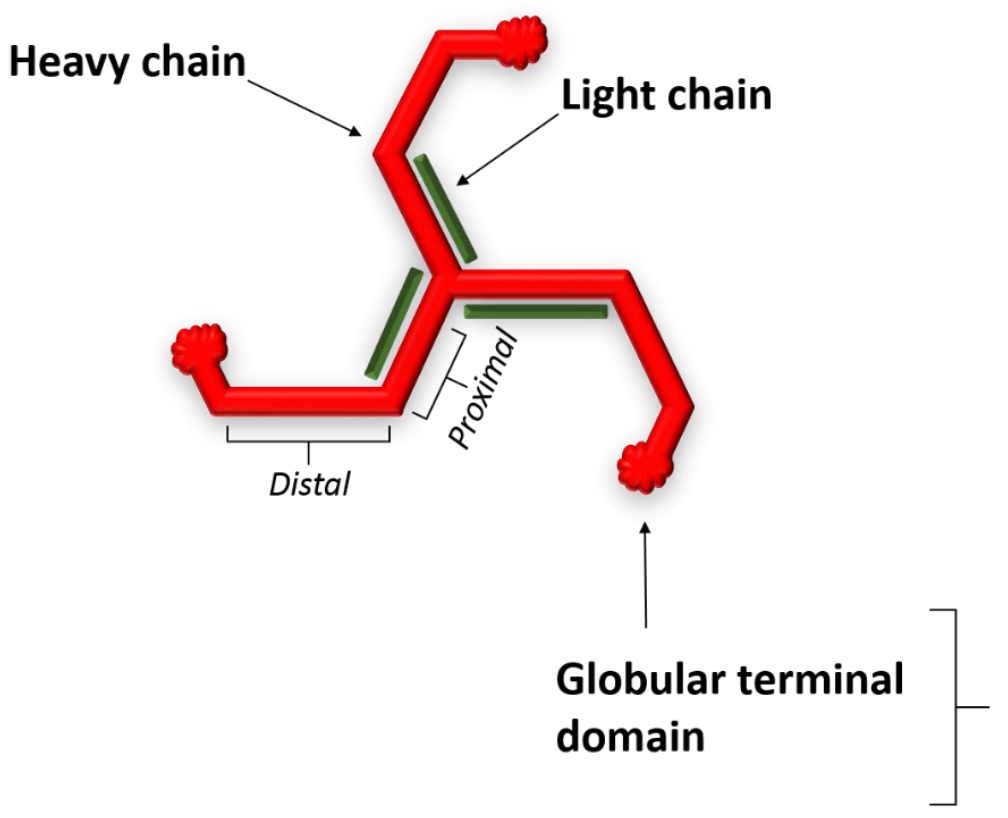

Endocytic accessory proteins:

- AP2

- $\beta$-arrestin

- Epsin

- Amphiphysin

- SNX9

- HIP1R

- Sjn1

- Auxilin

Figure 1.5. The structure of clathrin. Clathrin is the hallmark protein which coats endocytic vesicles during CME, characterized by its three-legged appearance referred to as a 'triskelion'85,108. The clathrin triskelion is comprised of three $\sim 190 \mathrm{kDa}$ clathrin heavy chain (CHC; shown in red), which are each associated with a $25 \mathrm{kDa}$ clathrin light chain (CLC; shown in green). Each CHC molecule can be divided into three regions: a proximal region, an intermediate distal region, and a globular $\mathrm{N}$-terminal domain. The N-terminal domain of clathrin is largely responsible for its interaction with endocytic components including key proteins listed above. 
A

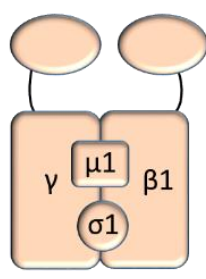

AP1

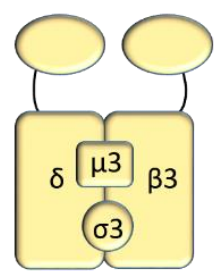

AP3

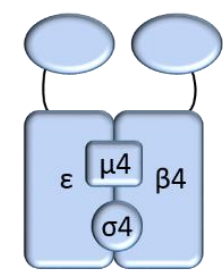

AP4

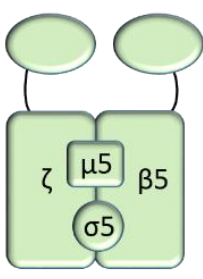

AP5

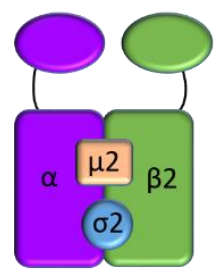

Adaptor protein complex-2 (AP2)

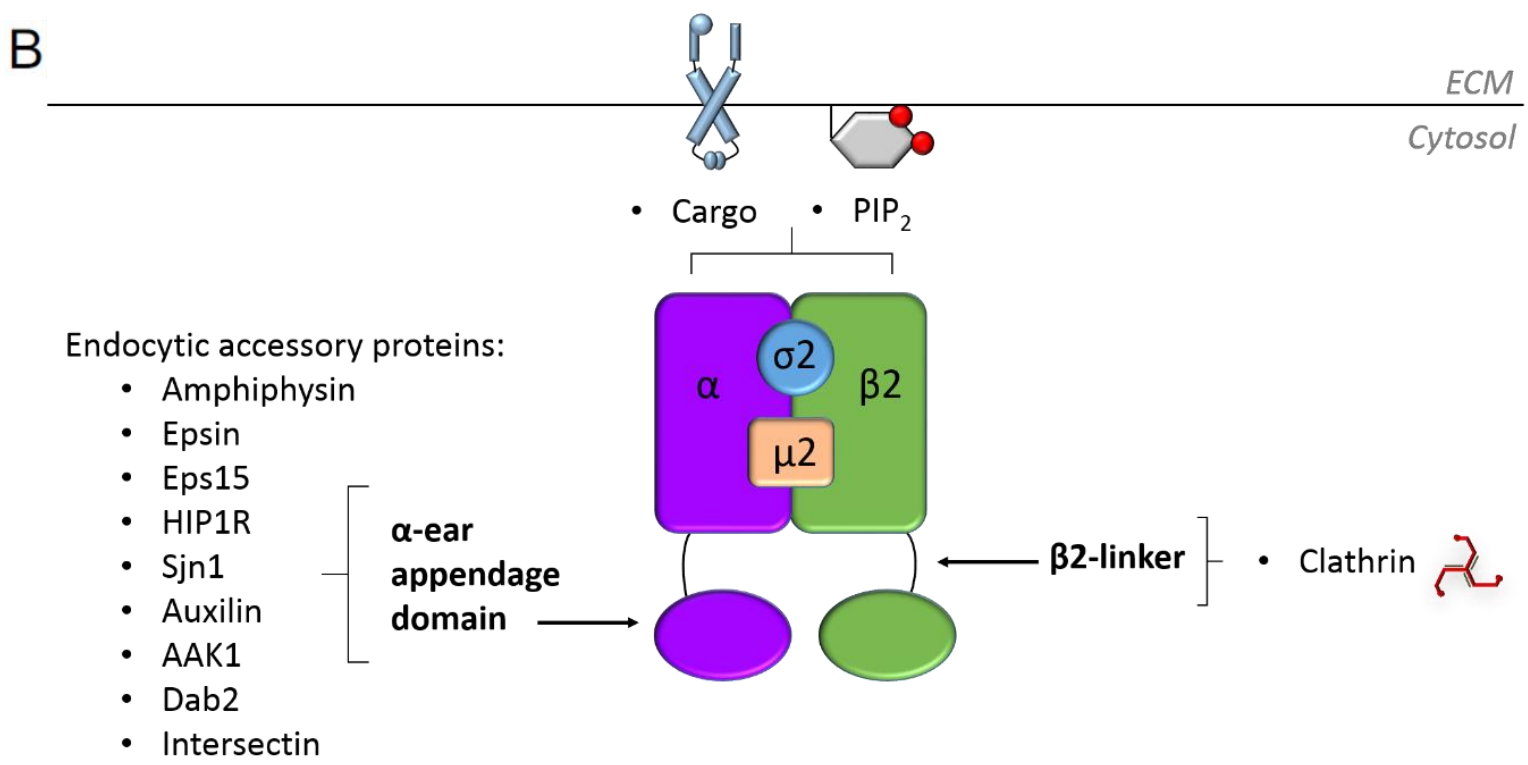

Figure 1.6. The structure of the adaptor protein complex-2. (A) AP2 belongs to a functionally diverse family of adaptor protein (AP) complexes, comprising of five members in mammalian cells; designated adaptor protein-1 through -5 (AP1-5). All APs are heterotetrameric structures composed of isoform specific subunits including two large subunits ( $\mathrm{X}$ and $\beta$; 100kDa each), one medium-sized subunit ( $\mu$; $\sim 50 \mathrm{kDa})$, and one small-sized subunit $(\sigma ; \sim 20 \mathrm{kDa})$ arranged in a general $X \beta \mu \sigma$ structure, where ' $X$ ' is a variable subunit; $\gamma / \alpha / \delta / \varepsilon / \zeta$. AP complexes form a general structure resembling a 'Mickey mouse' head, where the head (or trunk/core) is formed by the N-terminal segments of the large subunits, with the 'ears' (or appendage) composed of their C-terminal domains connected to the head via a flexible neck (or linker/hinge). (B) AP2 is the best studied AP type which exclusively localizes to the plasma membrane in facilitating $\mathrm{CME}$, comprised of the large $\alpha$ and $\beta 2$ adaptins, the medium $\mu 2$, and the small $\sigma 2$ adaptin. AP2 binds a broad repertoire of endocytic components including PIP $_{2}$, cargo, clathrin, and various endocytic accessory proteins via the $\alpha$-ear appendage domain. 


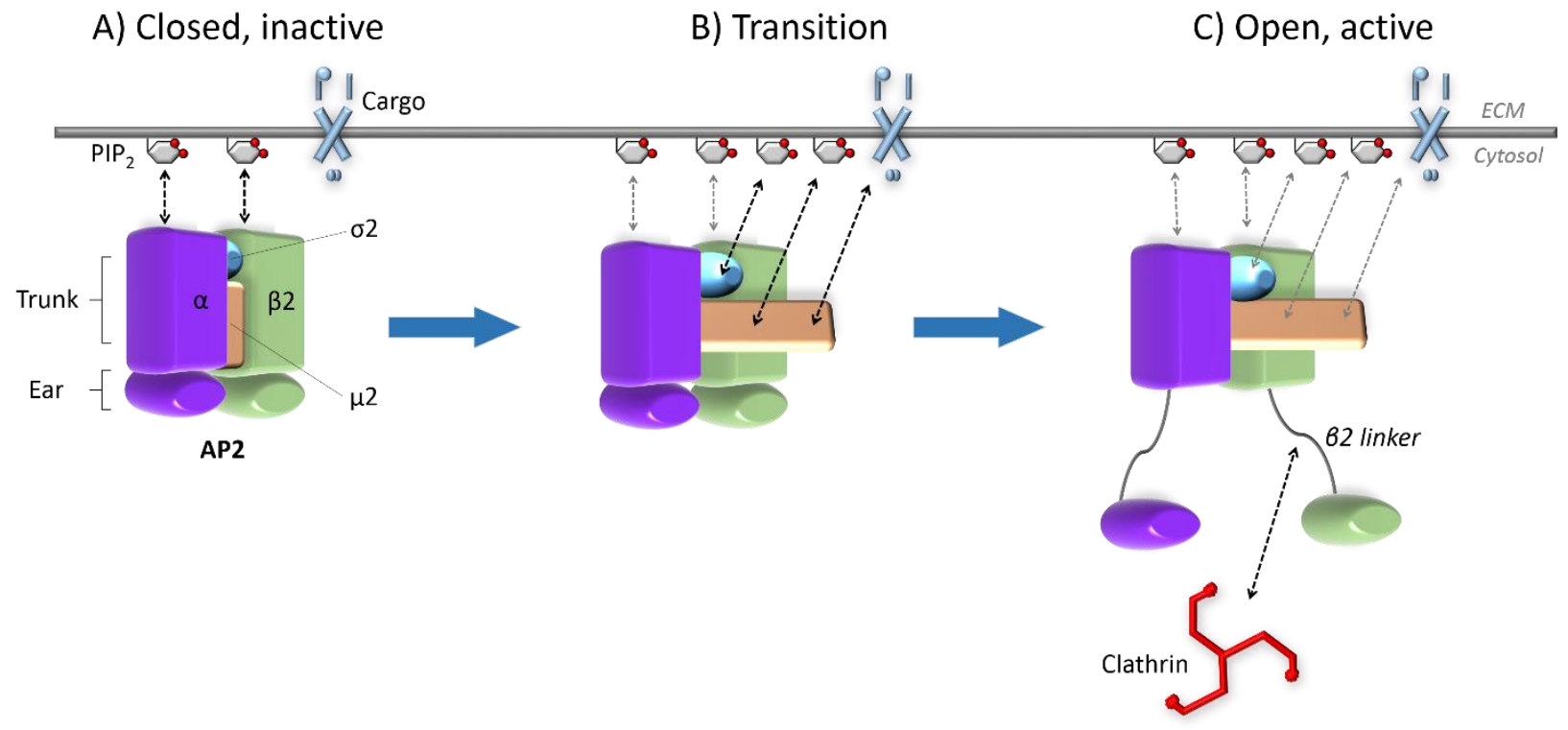

Figure 1.7. A large conformational change is required for the activation and stabilization of AP2. AP2 exists in at least two conformations designated as the inactive 'closed', and active 'open' states. In the cytoplasm, AP2 assumes an inactive autoinhibited arrangement, with its cargo and clathrin binding sites structurally obstructed within the core region. The activation of AP2 requires hierarchical interactions with its ligands, $\mathrm{PIP}_{2}$ and cargo on the plasma membrane. i) The $\alpha$ and $\beta 2$ adaptins of AP2 directly binds $\mathrm{PIP}_{2}$ on the plasma membrane, forming the initial steps in the allosteric rearrangement and activation of the AP2 (A). This in turn causes the ii) release or 'flipping-out' of the C- $\mu 2$ domain from the AP2 core, thereby exposing its essential tyrosine-motif and $\mathrm{PIP}_{2}$ binding sites to the plasma membrane-further stabilizing the active state of AP2 (B). iii) Lastly, the stabilization of AP2 on the plasma membrane via $\mathrm{PIP}_{2}$ and cargo interactions, releases the $\beta 2$ linker for clathrin recruitment and assembly (C). Clathrin assembly promotes the recruitment of AAK1 which further stabilizes the open conformation of AP2 by phosphorylating the $\mathrm{C}-\mu 2$ domain (not shown above). 


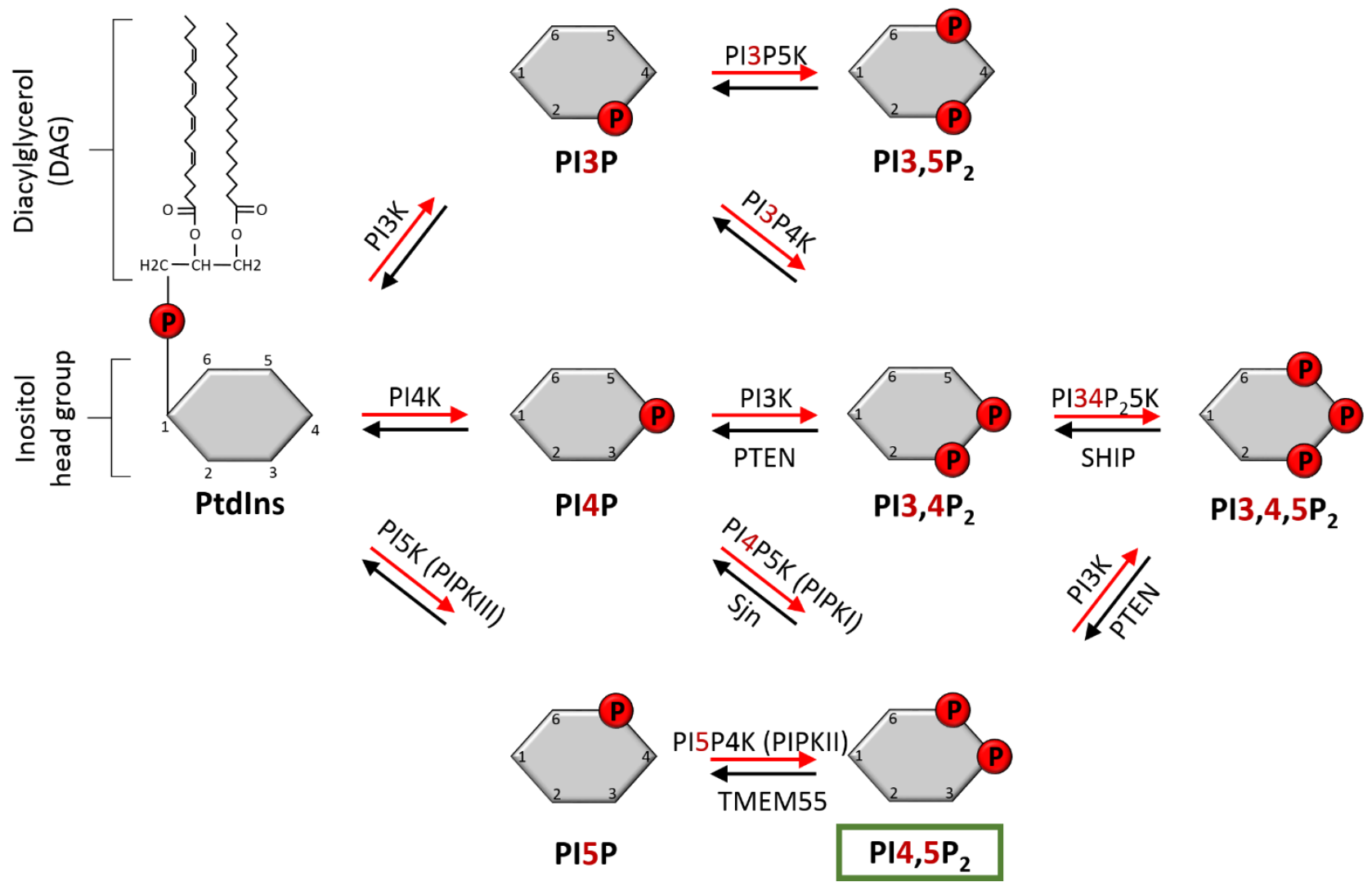

Figure 1.8. The seven derivatives of phosphoinositide. Phosphoinositides (PI) are the phosphorylated derivatives of phosphatidylinositol (Ptdlns) generated by various phosphatidylinositol kinases and phosphatases. The hydroxyl residues at the D3, D4 and D5 positions of the inositol head group can be differentially phosphorylated giving rise to seven PI species. Examples of kinases and phosphatases which respectively catalyze PI phosphorylation and turnover are shown. $\mathrm{PIP}_{2}$ (highlighted in green box) is derived mainly from the phosphorylation of PI4P by PIPKI. 


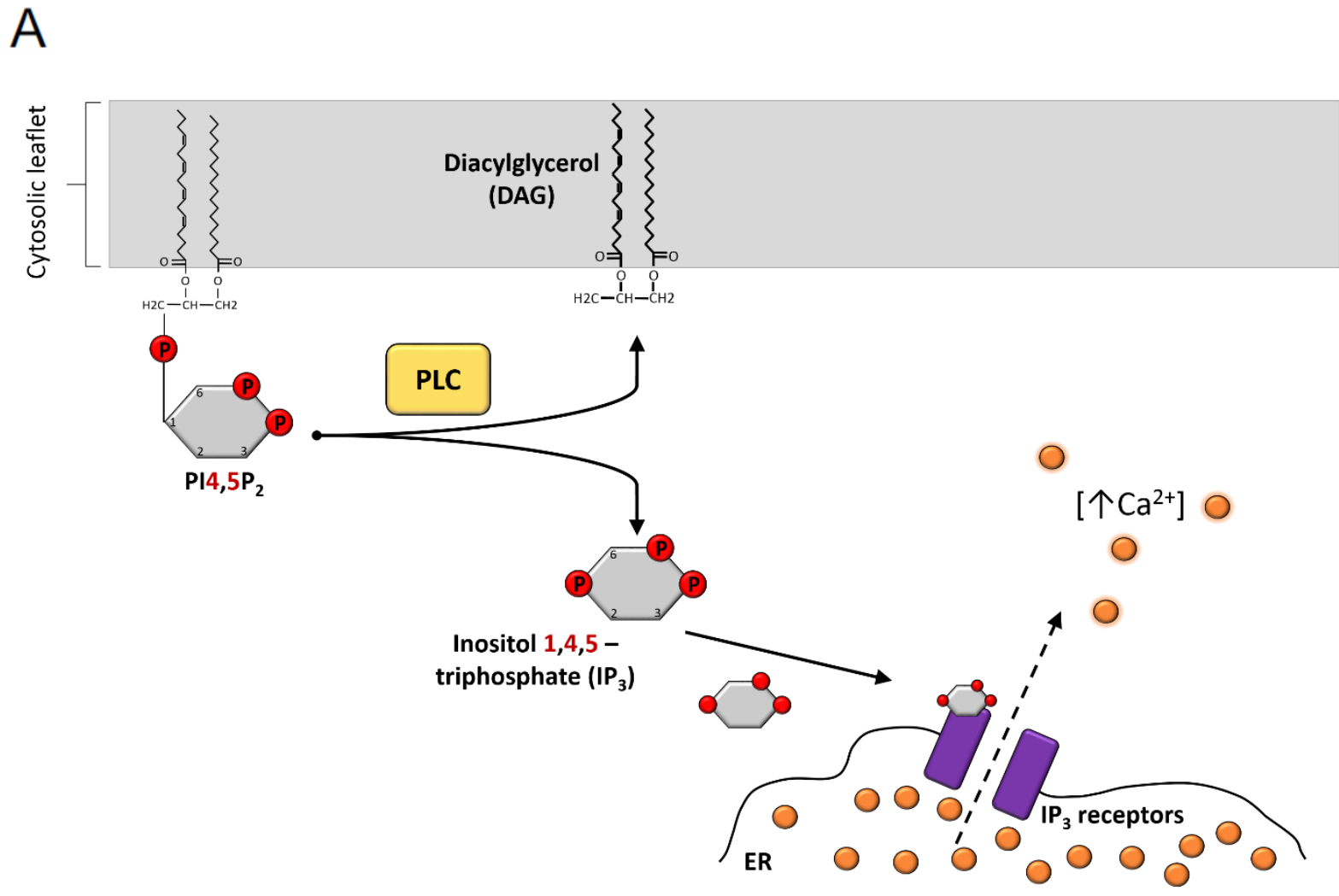

$\mathrm{B}$

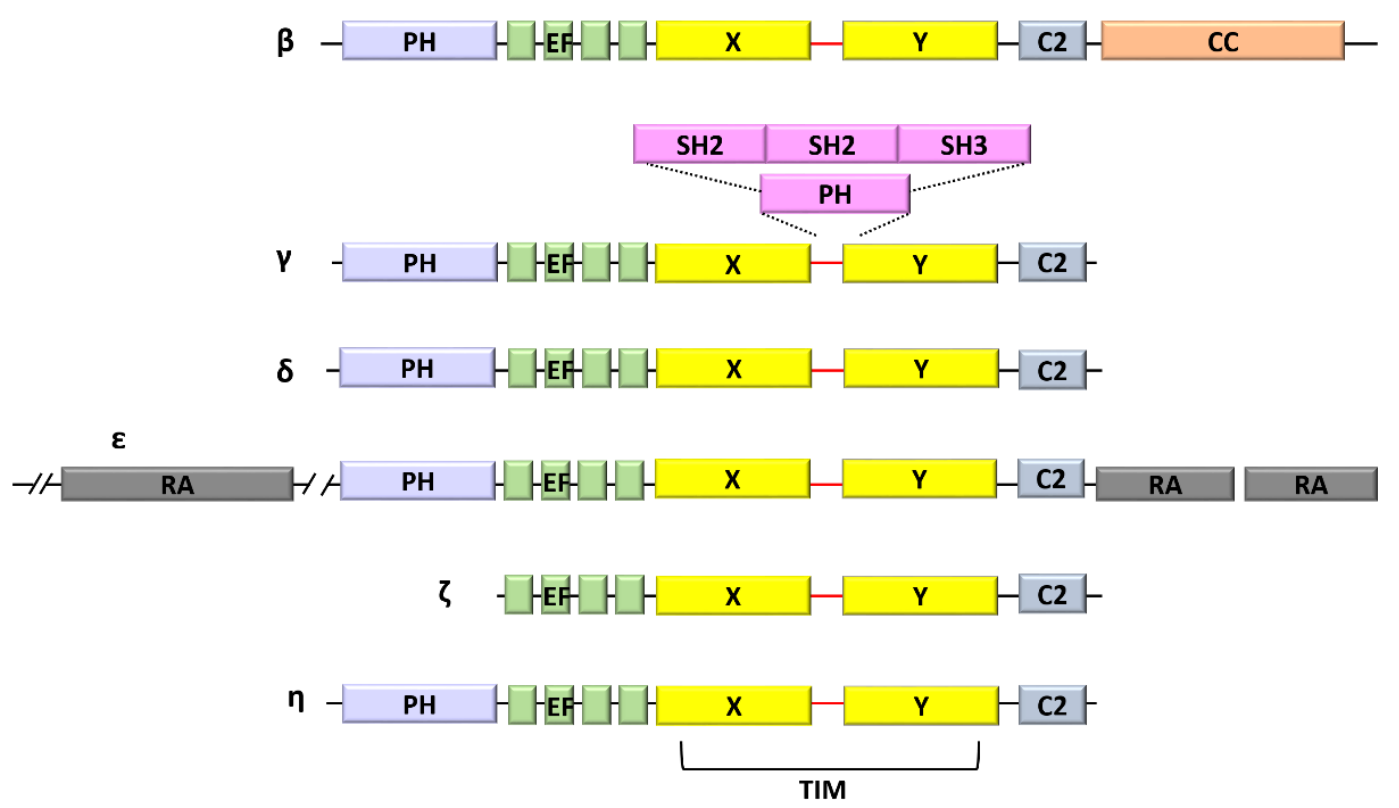

Figure 1.9. Phospholipase $\mathrm{C}$ activity and structure. Phospholipase $\mathrm{C}$ is a phosphodiesterase enzyme which hydrolyzes $\mathrm{PIP}_{2}$ on the plasma membrane. (A) The hydrolysis of $\mathrm{PIP}_{2}$ by PLC generates two 
secondary signaling molecules: i) diacylglycerol (DAG) which remain on the plasma membrane, and iii) inositol 1,4,5-triphosphate $\left(\mathrm{IP}_{3}\right)$ which diffuses within the cytosol. IP 3 is a diffusible cytosolic molecule which stimulates the release of calcium from the ER by binding and opening $I P_{3}$ receptors $\left(I P_{3} R\right)$. Upon opening of $I P_{3} R$, calcium efflux from the $E R$ into the cytosol increases cytosolic calcium concentrationsimplicated in facilitating numerous cellular processes. (B) Several PLC isotypes have been identified in mammalian cells which are classified into six sub-families based on unique domain inserts and extensions: PLC $-\beta,-\gamma,-\delta,-\varepsilon,-\zeta$ and $-\eta$. 


\section{NCKX}

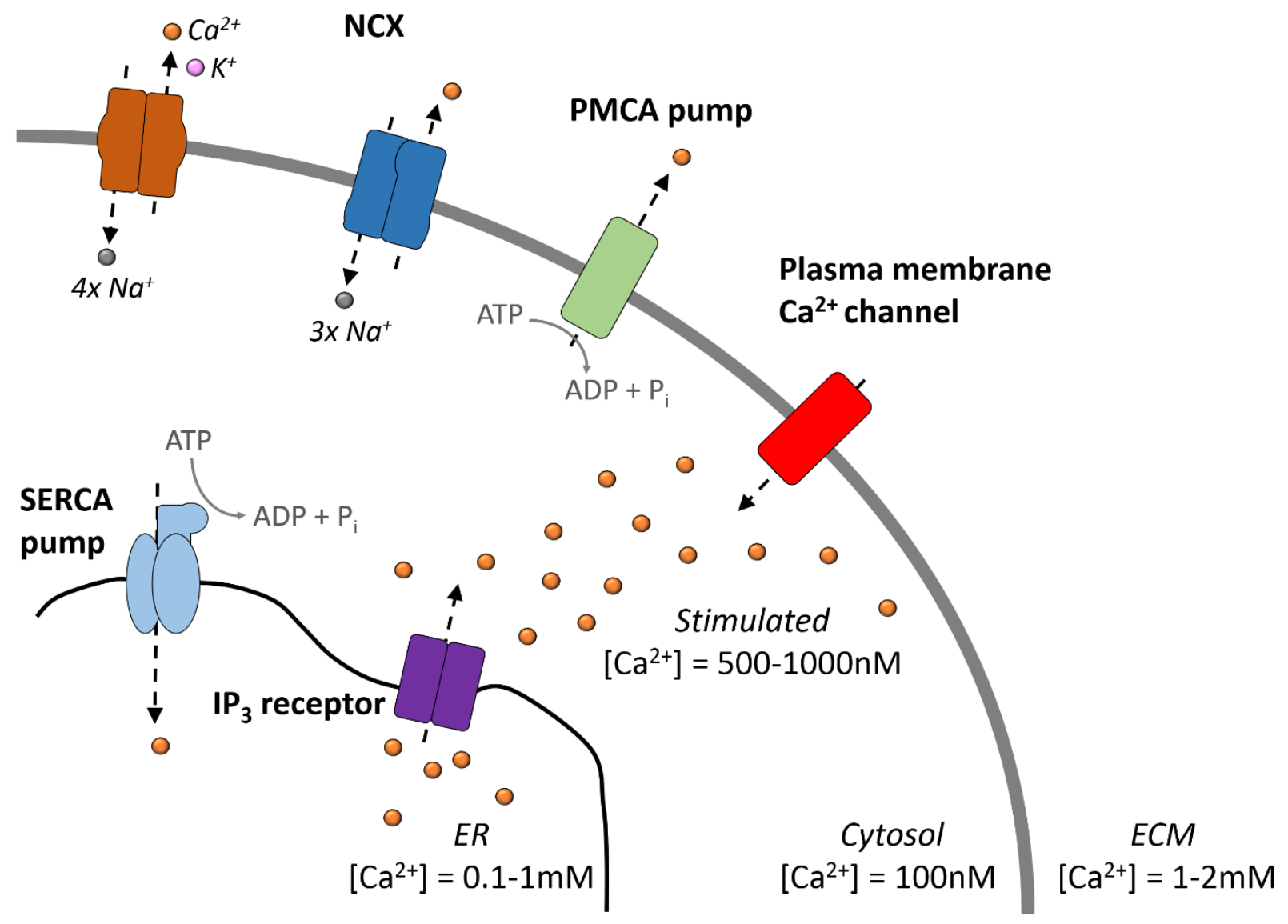

Figure 1.10. Maintenance of the cellular calcium gradient. The outside-to-inside chemical gradient for calcium is estimated at around 15,000-40,000:1, creating one of the largest known physiological gradients in the body. This gradient is created by various plasma membrane proteins which transport $\mathrm{Ca}^{2+}$ out of the cytosol. ATPase pumps actively pump calcium out of the cell into the extracellular space (via PMCA pumps), or into the ER (via SERCA pumps) through ATP hydrolysis. As well, ion exchangers such as NCX and NCKX pump Ca ${ }^{2+}$ into the extracellular fluid by respectively exchanging three $\mathrm{Na}^{2+}$ for one $\mathrm{Ca}^{2+}$ ion, and four $\mathrm{Na}^{2+}$ for the co-transport of a $\mathrm{Ca}^{2+}$ and a $\mathrm{K}^{+}$ion. At rest, cytosolic $\mathrm{Ca}^{2+}$ concentration is $\sim 100 \mathrm{nM}$ which can rise to $500-1000 \mathrm{nM}$ during signaling events. This increase can be derived from extracellular $\mathrm{Ca}^{2+}$ present in the range of $1-2 \mathrm{mM}$ (via plasma membrane calcium channels), or through intracellular compartments such as the ER with concentrations in the range of 0.1-1mM (via $\mathrm{IP}_{3}$ receptor). (PMCA, plasma membrane $\mathrm{Ca}^{2+}$ ATPases; SERCA, sarco-endoplasmic reticular $\mathrm{Ca}^{2+}$ ATPases; $\mathrm{NCX}, \mathrm{Na}^{+} / \mathrm{Ca}^{2+}$ exchangers; $\mathrm{NCKX}, \mathrm{Na}^{+} / \mathrm{Ca}^{2+}-\mathrm{K}^{+}$exchangers). 


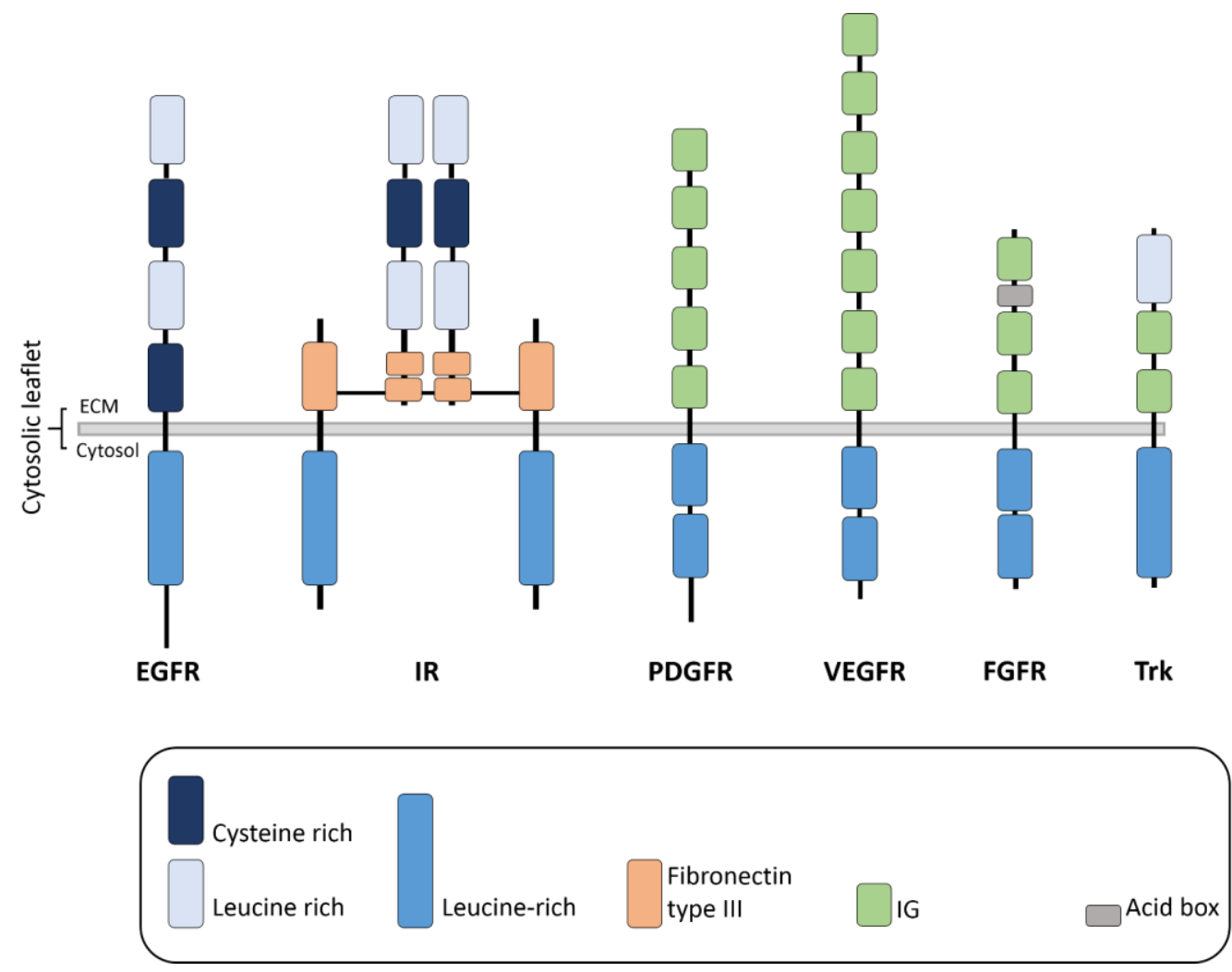

Figure 1.11. The different families of receptor tyrosine kinases (RTK). RTKs are transmembrane proteins found on the plasma membrane characterized by three domains: an extracellular ligandbinding domain, a transmembrane helical domain, and an intracellular domain with intrinsic tyrosine kinase activity. Shown above are the domain architecture of some of the best characterized RTK families including EGFR, IR, VEGFR, PDGFR, FGFR, and the Trk family. Although sharing a conserved structural arrangement, RTKs nonetheless diverge in displaying variations within the three main domainsfeaturing unique combinations of domain inserts and extensions indicated above. (EGFR, epidermal growth factor receptor; IR, insulin receptor; PDGFR, platelet-derived growth factor receptor; VEGFR, vascular endothelial growth factor receptor; FGFR, fibroblast growth factor receptor; Trk, tropomyosinrelated kinase receptor; IG, immunoglobulin-like domain). 


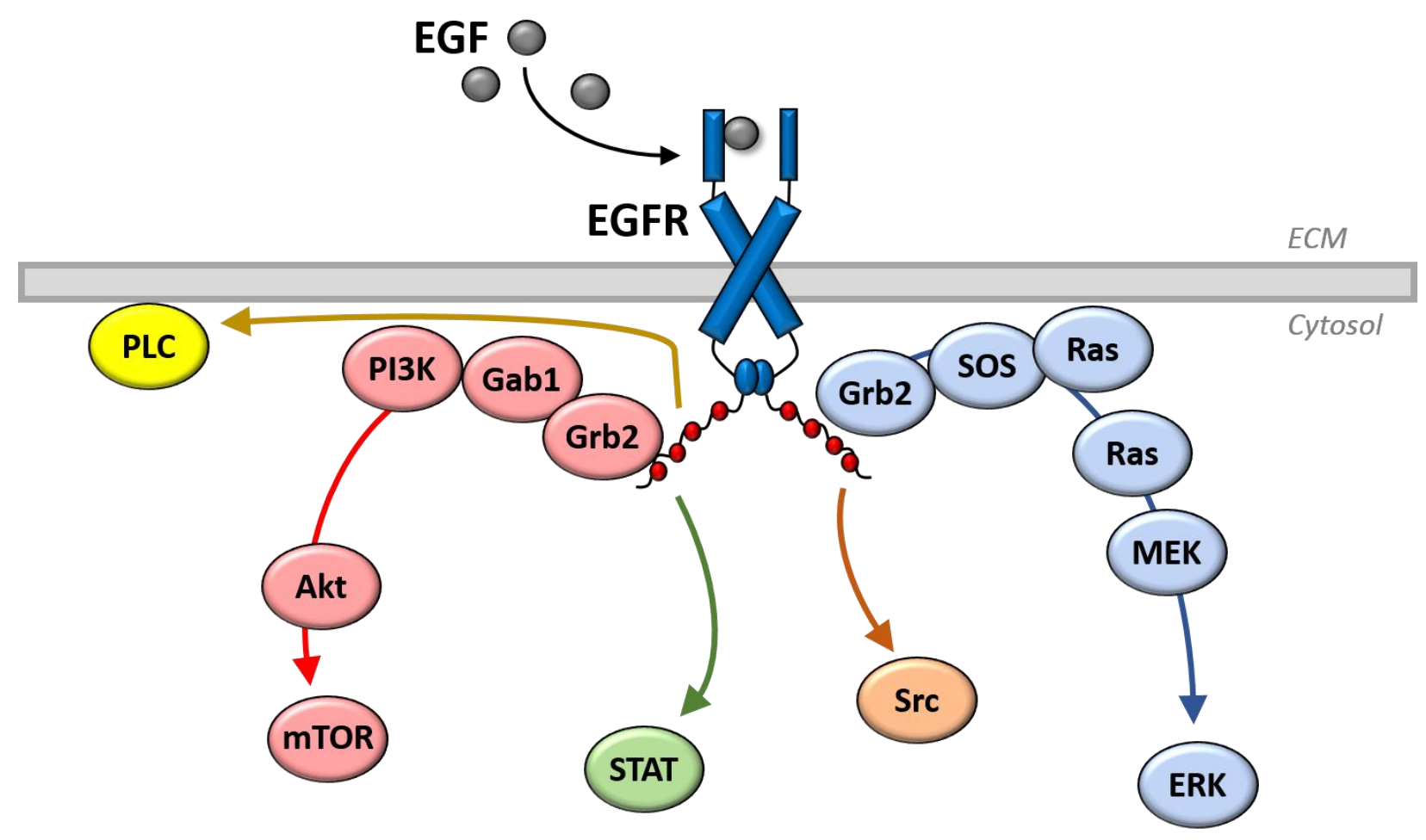

Figure 1.12. The major signaling pathways of EGFR. EGFR is the best characterized family of RTKs of which signaling pathways are well annotated in the literature. Upon EGF ligand binding, EGFR becomes activated and induces autophosphorylation of its intracellular domains, resulting in the generation of a wide spectrum of signaling cascades which play fundamental roles in cell proliferation, migration, metabolism, differentiation, and survival. Shown above are the major EGFR signaling cascades which includes the (1) MAPK (blue arrow) and the (2) PI3K/Akt (red arrow) pathway; with other key intracellular pathways including the (3) Src (orange arrow), (4) STAT (green arrow), and the (5) PLC- $\gamma 1$ (yellow arrow) pathway. 


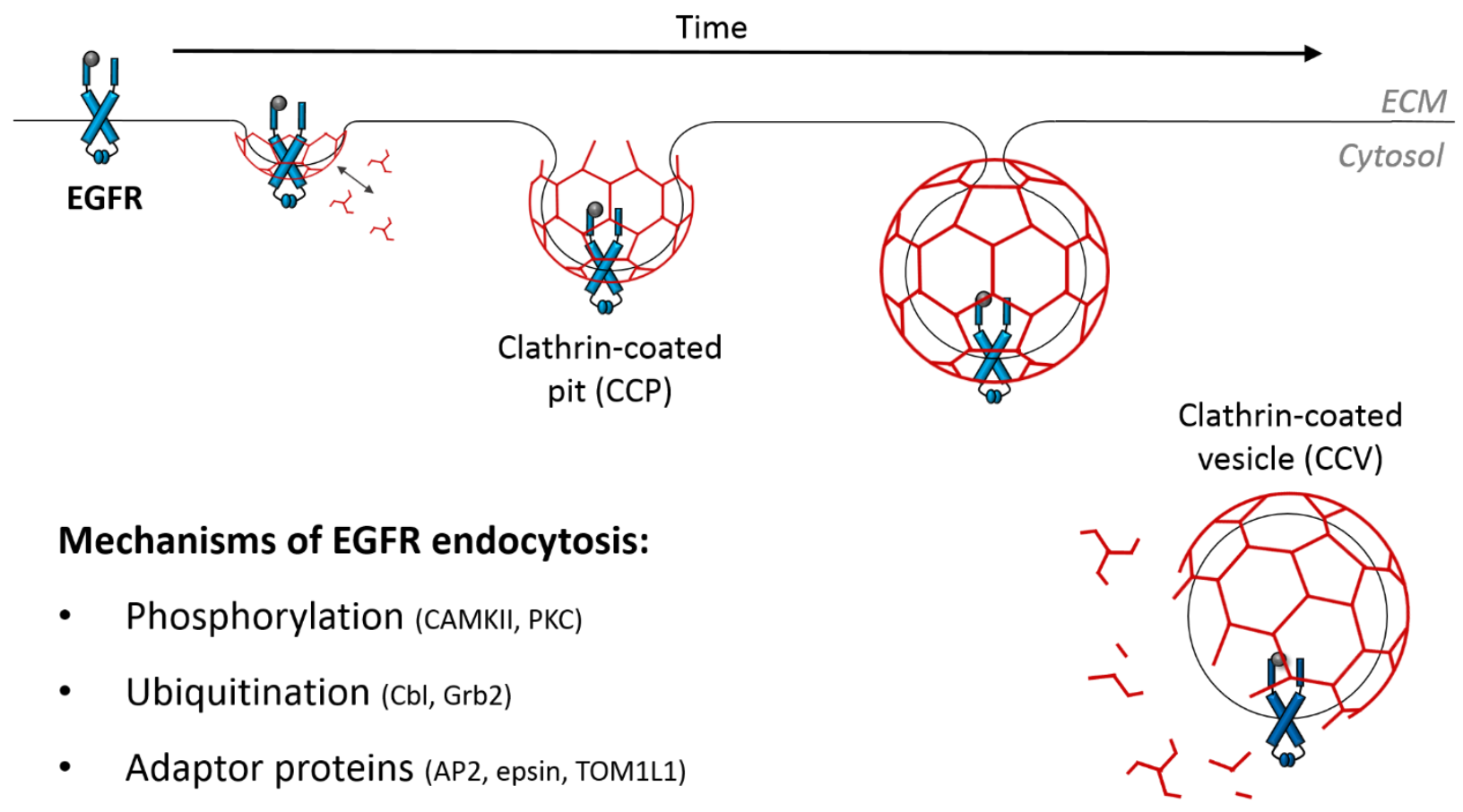

Figure 1.13. EGFR is a cargo of CME. EGF stimulation of EGFR induces its recruitment within clathrin structures, serving as a mechanism for EGFR internalization and signaling attenuation. Listed above are some of the possible mechanisms which may directly augment EGFR clathrin-mediated endocytosis which includes: the i) direct phosphorylation and ubiquitination of EGFR intracellular domains by CAMKII and $\mathrm{Cbl}$, respectively; and the ii) recruitment of endocytic adaptor proteins including AP2, epsin and TOM1L1. 
Autoinhibited, Inactive

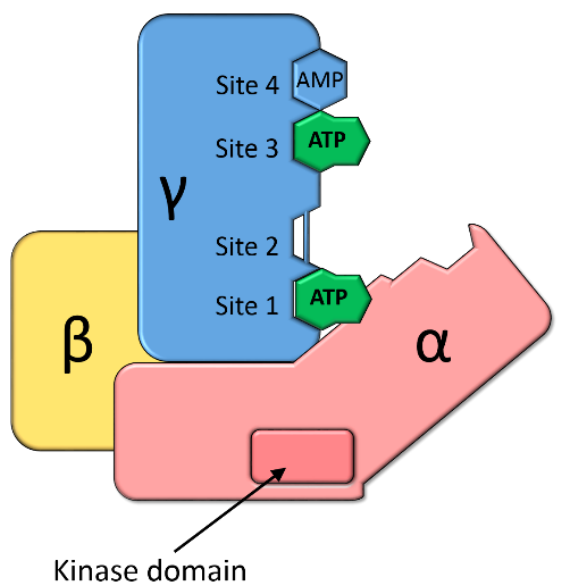

Open, Active

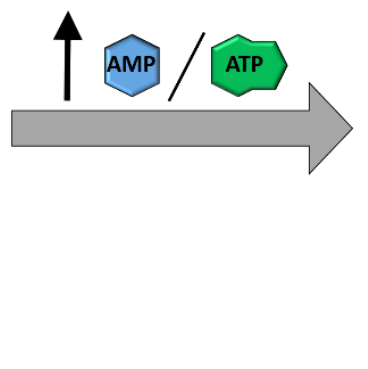

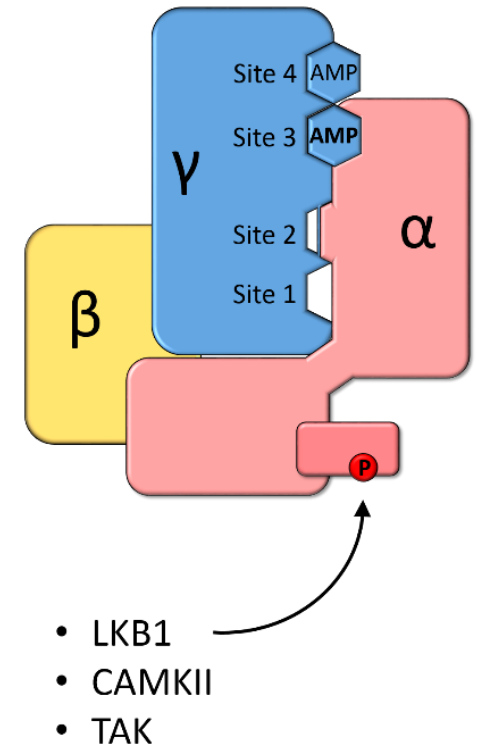

B

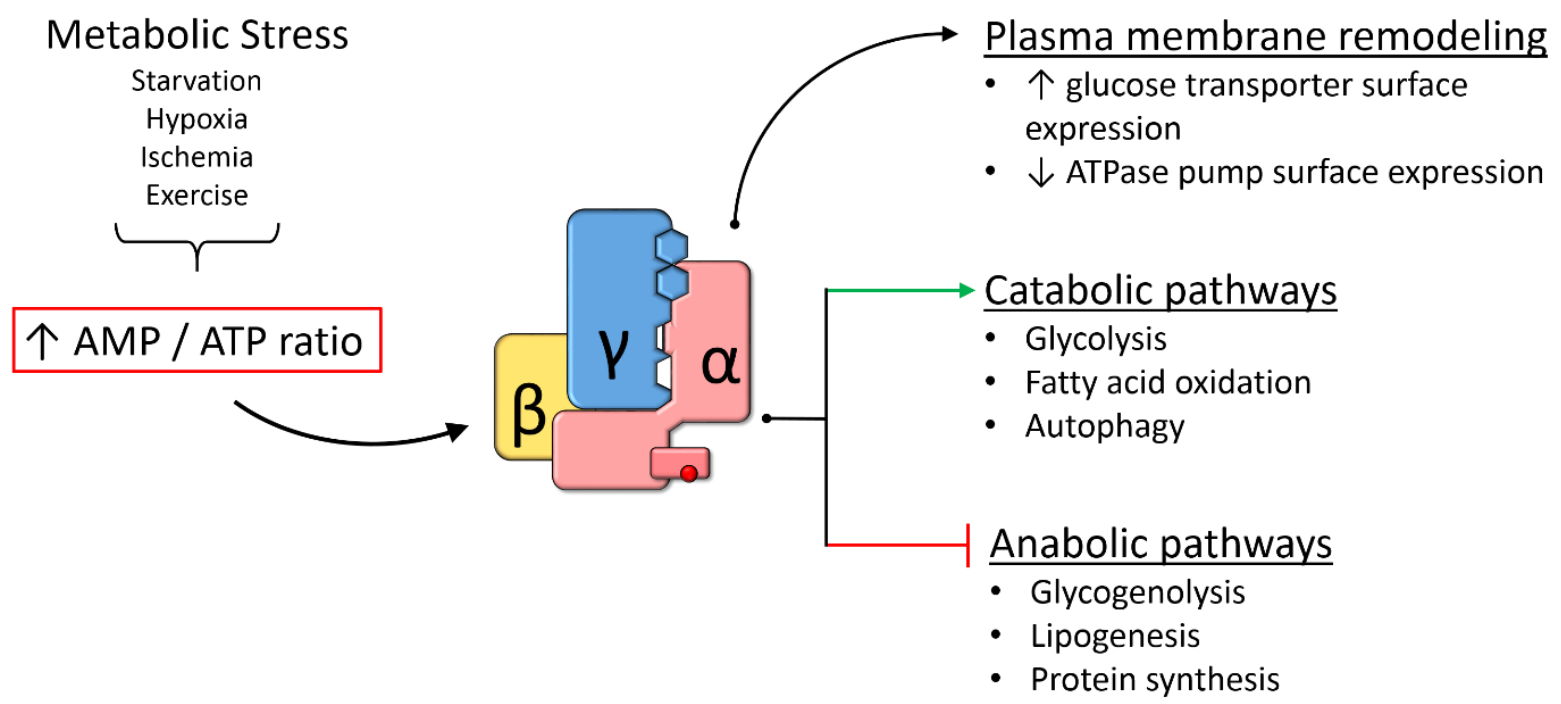

Figure 1.14. AMPK is a major metabolic sensor. (A) AMPK is a heterotrimeric kinase comprised of three subunits: a catalytic $\alpha$-, scaffolding $\beta$-, and a regulatory $\gamma$-subunit. The $\gamma$-subunit contains four total sites (sites 1-4) which binds either ATP, ADP, or AMP in a competitive manner. Site 2 cannot bind nucleotide molecules due to a missing aspartate residue, while site 4 is constitutively bound to an AMP molecule. 
Under high cellular energy states, ATP binds sites 1 and 3, keeping AMPK in an autoinhibited, inactive conformation. In this conformation, the kinase domain of the $\alpha$-subunit is obstructed by an autoinhibitory segment, unable to phosphorylate downstream effectors (left panel). Reduction in cellular energy levels increases the concentration of AMP, outcompeting ATP for $\gamma$-subunit binding. AMP binding to AMPK induces a conformational shift within the $\alpha$-subunit-causing the release of the kinase domain. The kinase domain is subsequently phosphorylated by upstream kinases including LKB1, CAMKII and TAK, which further stabilizes the activated state of AMPK (right panel). (B) Reduction in cellular energy levels can be induced by various metabolic stressors which in turn activates AMPK. Once activated, AMPK triggers several downstream processes which collectively functions in restoring metabolic and cellular homeostasis. These processes involve the respective inhibition and stimulation of anabolic and catabolic pathways, and as well as remodeling of the plasma membrane. 


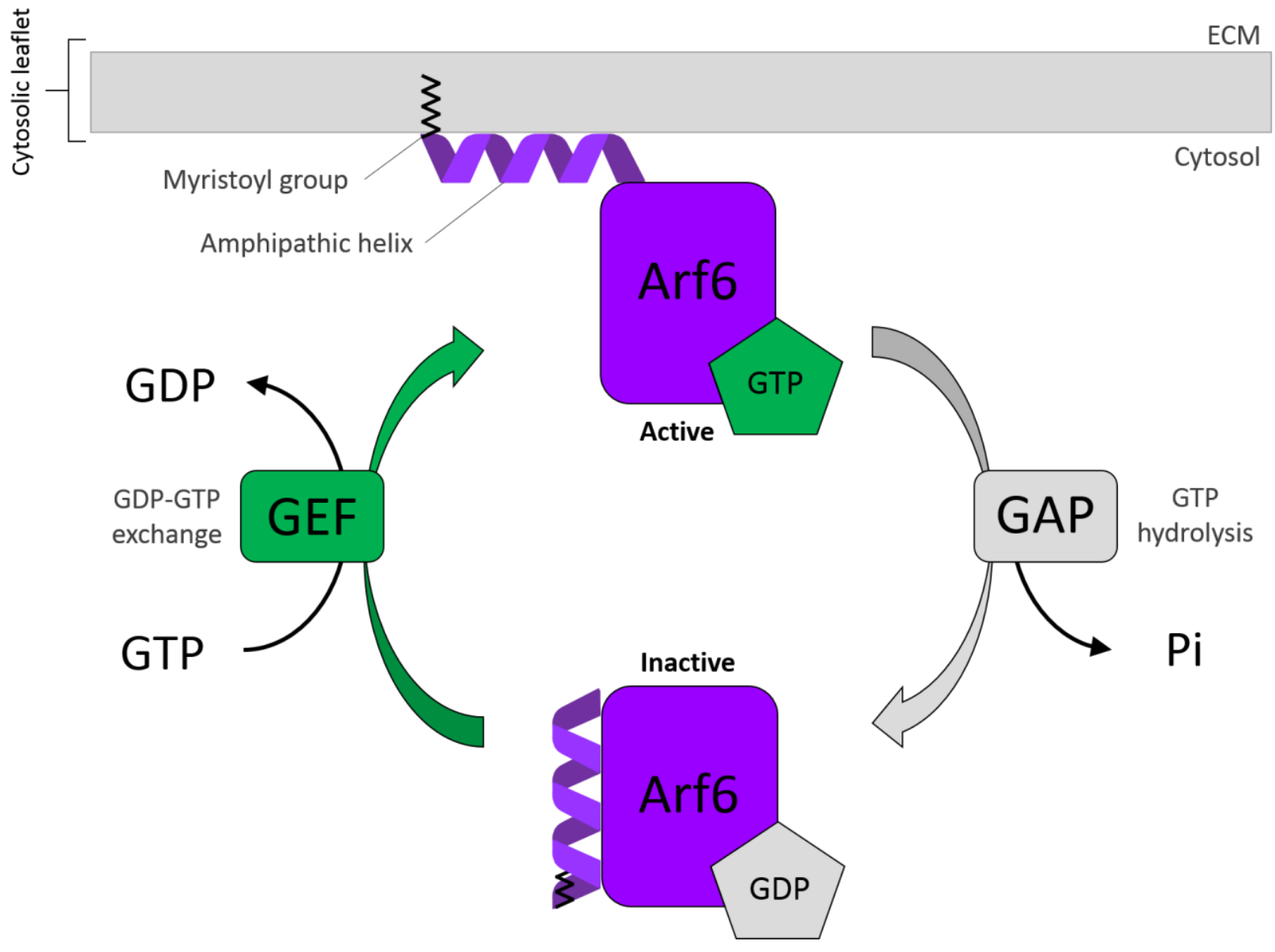

Figure 1.15. The small GTPase Arf6. Arf6 is a small GTPase which cycles between an active GTP-bound, and an inactive GDP-bound state. GTP and GDP binding of Arf6 is regulated by various GAP (e.g. ArfGAP3, ARAP, and SMAP) and GEF (e.g. cytohesin/ARNO, EFA6 and IQSEC/BRAG) interactors. The structure of Arf6 is largely defined by two domain regions: an i) $\mathrm{N}$-terminal amphipathic helix bound to a myristoyl group; and ii) two GTP-binding regions common to small GTPases termed 'switch 1' and 'switch 2', separated in by a mobile 'interswitch' region (not shown). GTP binding induces a conformational shift in Arf6, resulting it its translocation and association with the plasma membrane via the N-terminal region. 


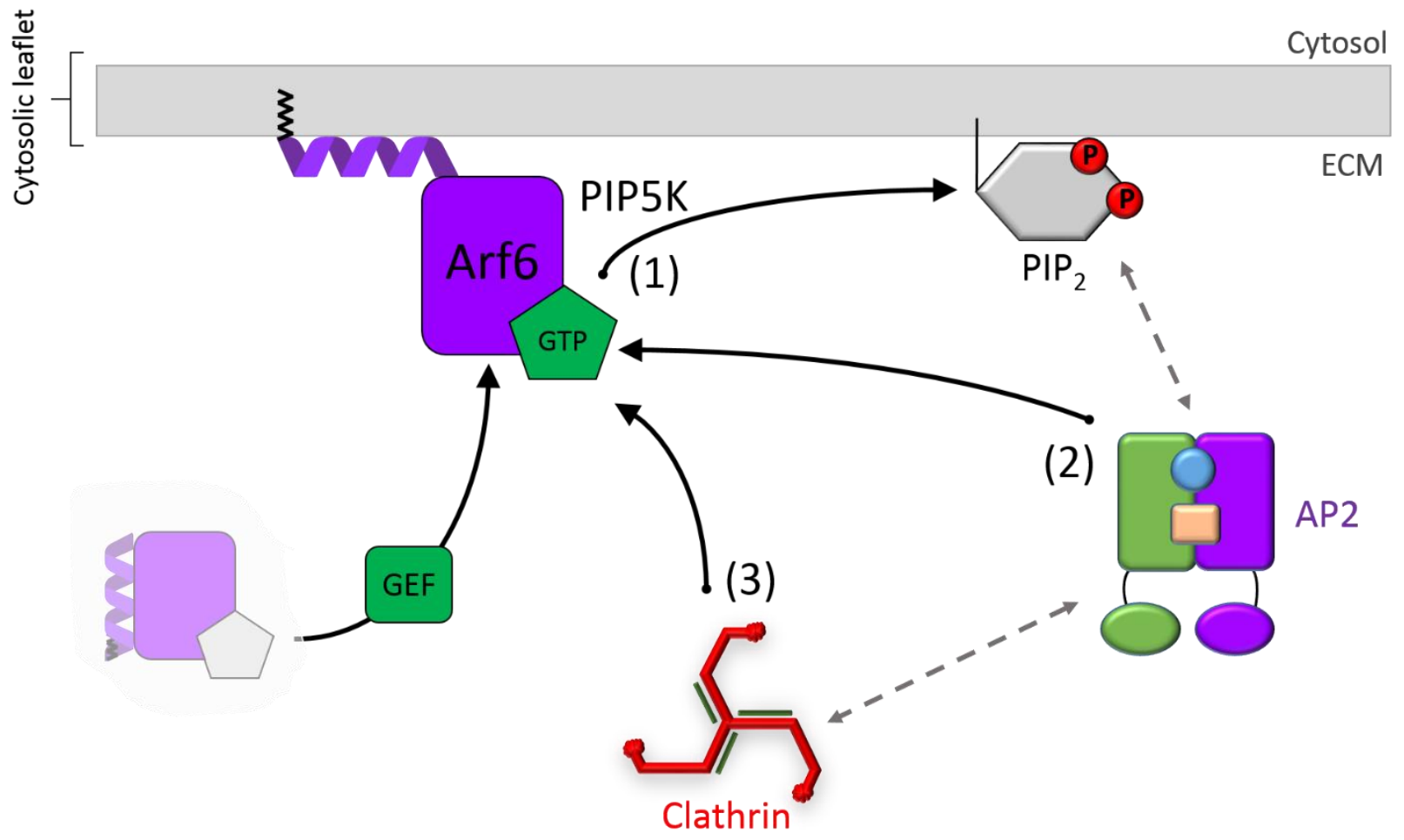

Figure 1.16. Arf6 has been implicated in the regulation of CME. Arf6 is recruited and associates with the plasma membrane via the N-terminal region in a GTP-dependent manner. Arf6 functions in membrane traffic pathways and has been implicated in enhancing CME through mechanisms shown above: 1) Recruitment of PIP5K inducing local PIP 2 production; 2) direct recruitment of AP2; and 3) direct recruitment of clathrin molecules. Broken arrows represent interactions between corresponding molecules. 
Chapter 2. Materials and Methods 


\section{1- Materials}

DMEM/F12, fetal bovine serum (FBS), penicillin/streptomycin solution, insulin-Tfn-seleniumethanolamine solution, and sterile HEPES buffer were obtained from Life Technologies (Carlsbad, CA). IGlutamine was obtained from Sigma-Aldrich (St. Louis, MO). Human EGF (unlabeled), EGF (complexed to Alexa Fluor 555 [A555-EGF] or rhodamine [rhodamine-EGF]), Tfn (complexed to Alexa Fluor 555 [A555Tfn] or Alexa Fluor 647 [A647-Tfn]), biotin-xx-EGF, and biotin-xx-Tfn were obtained from Life Technologies (Carlsbad, CA). Anti-EGF antibodies (used in EGF internalization assay) were from Upstate Biotechnologies (Millipore, Etobicoke, ON, Canada), and Anti-Tfn antibodies (used in Tfn internalization assay) were from Bethyl Laboratories (Montgomery, TX). Avidin and o-phenylenediamine hydrochloride reagent were obtained from Biobasic (Markham, ON, Canada), and biocytin was obtained from Santa Cruz Biotechnology (Dallas, TX). Superblock blocking buffer was obtained from Thermo Fisher (Rockford, IL).

Small molecular inhibitors and activators used were as follows: U73122 (1-(6-((17ß-3methoxyestra-1,3,5(10)-trien-17-yl)amino)hexyl)-1H-pyrrole-2,5-dione) was obtained from Cayman Chemicals (Ann Arbor, MI), BIM (Bisindolylmaleimide I, hydrochloride) and CsA (Cyclosporin A) were obtained from Cell Signaling (Danvers, MA), XeC (Xestospongin C) was obtained from Abcam (Cambridge, MA), BAPTA-AM (1,2-Bis(2-aminophenoxy)ethane- $\mathrm{N}, \mathrm{N}, \mathrm{N}^{\prime}, \mathrm{N}^{\prime}$-tetraacetic acid tetrakis(acetoxymethyl ester)) was obtained from Santa Cruz Biotechnology (Dallas, TX), A7 (A-769662) and SecinH3 was obtained from Abcam (Cambridge, MA), and lonomycin was obtained from SigmaAldrich (St. Louis, MO).

Antibodies used for immunofluorescence (IF) microscopy were as follows: anti-Sjn1 from Synaptic Systems (Goettingen, Germany), and anti- $\beta 1$-integrin from EMD Millipore (Darmstadt, Germany). Antibodies used for Western blotting were obtained as follows: for detection of phospho-Akt (pS473) from Life Technologies (Carlsbad, CA), and for total Akt, phospho-Erk (Thr202/Tyr204), total Erk, phospho-EGFR (Tyr1068), phospho-PLC 1 (Tyr783), total PLC- 1 1, total dynamin 1, and actin from Cell Signaling (Danvers, MA).

cDNA constructs used were as follows: encoding the wild-type pleckstrin homology (PH) domain of PLC- $\delta 1$ fused to mCherry (mCherry-PH WT) or of the PH domain harboring K32A W36A mutations to abolish binding to $\mathrm{PIP}_{2}$ similarly fused to mCherry (mCherry-PH K32A, W36A) were previously described ${ }^{6}$. 
cDNA encoding Sjn1 (170-kDa isoform) fused to mCherry (mCh-Sjn1) was previously described ${ }^{6}$. cDNA constructs encoding recombinant Arf6 proteins were previously described ${ }^{388,394}$. cDNA encoding $\beta 1$ integrin fused to mCherry (mCh- $\beta 1$-integrin) was previously described ${ }^{395}$. Hemagglutinin (HA)-NFAT1(4460)-GFP was a gift from Anjana Rao, Harvard Medical School (Addgene plasmid \#11107) ${ }^{396}$. siRNA sequences used were as follows (sense): Dyn1, 5'-GGCUUACAUGAACACCAACCACGAA ${ }^{397}$; clathrin heavy chain (CHC), GGAAGGAAAUGCAGAAGAAUU ${ }^{207}$; PLC- $\nu 1$, GAGCAGUGCCUUUGAAGAAUU; or non-targeting control siRNA, CGUACUGCUUGCGAUACGGUU; and were obtained from Dharmacon (Lafayette, CO) or Sigma-Aldrich (St. Louis, MO).

\section{2- Cell culture}

Wild-type ARPE-19 (RPE herein) human retinal pigment epithelial cells (RPE-WT) and derivative lines stably expressing clathrin light chain fused to eGFP (RPE eGFP-CLCa) or Tag-RFP-T (RPE RFP-CLCa) were used as previously described ${ }^{5,6}$. RPE cells exhibit a flat morphology with their plasma membranes closely and uniformly apposed to the substratum (e.g. coverslips)-serving as an optimal cell model for studying CME given their largely dispersed clathrin structures which can be individually tracked via software analysis ${ }^{7}$. RPE cells were cultured in DMEM/F12 supplemented with $10 \%$ FBS, $100 \mathrm{U} / \mathrm{ml}$ penicillin, and $100 \mu \mathrm{g} / \mathrm{ml}$ streptomycin at $37^{\circ} \mathrm{C}$ and $5 \% \mathrm{CO}_{2}$. RPE cells engineered to stably express HER2 (RPE-HER2) used for experiments in Figure 4.9C were previously described and cultured similarly as RPEWT cells, with the addition of $1 \mu \mathrm{g} / \mathrm{ml}$ puromycin to the growth medium ${ }^{207}$.

\section{3- CDNA and siRNA transfection}

cDNA transfection (as per Figures 3.6, 3.10, 4.6, 4.7, 5.3 and 5.6) was performed using Lipofectamine 2000 (Life Technologies, Carlsbad, CA), as per the manufacturer's instructions and as previously described ${ }^{142,207}$. Briefly, cells were grown in each well of a six-well plate and allowed to reach $40-60 \%$ confluency. For each well of a six-well plate, $2.5 \mu \mathrm{g}$ of cDNA was pre-complexed with $5 \mu$ l of transfection reagent in Opti-MEM medium (Life Technologies, Carlsbad, CA) at room temperature for 15-20 min. Cells were washed once in 1x phosphate-buffered saline (PBS) and replaced with fresh OPTI- 
MEM medium. The pre-complexed cDNA-reagent is subsequently added dropwise onto each wells and incubated for $4 \mathrm{~h}$, followed by washing and incubation of cells in regular growth medium for 18-24 $\mathrm{h}$ before the start of the experiment.

siRNA gene silencing (as per Figures 3.1, 3.3-3.6, and 4.9) was performed using Lipofectamine RNAiMAX (Life Technologies, Carlsbad, CA), as per the manufacturer's instructions and as previously described ${ }^{207,360}$. Briefly, cells were grown in each well of a 12 -well plate and allowed to reach $40-80 \%$ confluency. For each well of a 12-well plate, each siRNA construct was transfected at $220 \mathrm{pmol} / \mathrm{l}$ precomplexed to the transfection reagent in Opti-MEM medium (Life Technologies, Carlsbad, CA) at room temperature for 15-20 min. Cells were washed once in 1x phosphate-buffered saline (PBS) and replaced with fresh OPTI-MEM medium. The pre-complexed siRNA-reagent is subsequently added dropwise onto each wells and incubated for $4 \mathrm{~h}$, followed by washing and incubation of cells in regular growth medium. siRNA transfections were performed twice ( $72 \mathrm{~h}$ and $48 \mathrm{~h}$ ) before each experiment.

\section{4- Small molecule treatment and EGF stimulation}

For all experiments, cells were subjected to serum starvation for $1 \mathrm{~h}$ before the start of the assay. Following serum starvation, cells were treated with small-molecule pharmacological inhibitors and activators as follows: U73122 (2 $\mu \mathrm{M}$ for $10 \mathrm{~min})^{398}$, BIM $(1 \mu \mathrm{M} \text { for } 30 \mathrm{~min})^{399}$, CSA (10 $\mu \mathrm{M}$ for 30 $\min )^{400}, \mathrm{XeC}(3 \mu \mathrm{M} \text { for } 30 \mathrm{~min})^{401}$, BAPTA-AM (10 $\mu \mathrm{M}$ for $\left.15 \mathrm{~min}\right), \mathrm{A} 7(100 \mu \mathrm{M} \text { for } 15 \mathrm{~min})^{360}$, SecinH3 (20 $\mu \mathrm{M}$ for $10 \mathrm{~min})^{402}$, and lonomycin (10 $\mu \mathrm{M}$ for $20 \mathrm{~min}$ ), or treated in media containing a corresponding volume of vehicle (dimethyl sulfoxide [DMSO], in each case $<0.1 \%$, control condition). All experiments requiring EGF stimulation were performed with an EGF concentration of $5 \mathrm{ng} / \mathrm{ml}$ unless otherwise stated. 


\section{5- TIRF-M measurement of cell surface $\mathrm{PIP}_{2}$ and $\beta 1$-integrin abundance}

RPE cells were seeded onto glass coverslips and transfected with cDNA encoding either the wildtype PH domain of PLC- $\delta 1$ fused to mCherry (mCherry-PH WT), or of the PH domain harboring K32A and W36A mutations to abolish binding to $\mathrm{PIP}_{2}$ similarly fused to mCherry (mCherry-PH K32A, W36A) for experiments in Figure 3.6B as previously described ${ }^{6}$. On the day of the experiment, transfected cells were serum starved for $1 \mathrm{~h}$, then treated (or not; control) with $5 \mathrm{ng} / \mathrm{ml} \mathrm{EGF}$ for $5 \mathrm{~min}$, followed by immediate fixation in $4 \%$ paraformaldehyde (PFA). Cells were then imaged using sequential TIRF-M (Total internal reflection fluorescence-microscopy) and wide-field epifluorescence microscopy using a 150×/1.45 NA objective on an Olympus IX81 instrument equipped with CellTIRF modules (Olympus Canada, Richmond Hill, ON, Canada) using 561-nm (50 mW) laser illumination and an 624/50 emission filter. Images were acquired using a C9100-13 EM-CCD camera (Hamamatsu, Bridgewater, NJ).

Images were analyzed using ImageJ by manually delineating a region of interest corresponding to the cell outline, followed by measurement of the mean pixel intensity within this region for each of the TIRF-M and epifluorescence channels for each cell ${ }^{360,403}$. To obtain the relative cell-surface localization index for the mCherry-PH probes under various conditions, we determined the ratio of TIRF/epifluorescence for each cell. Measurements were subjected to analysis of variance (ANOVA) followed by Tukey's posttest, with a threshold of $p<0.05$ for statistically significant differences between conditions (Figure 3.6B).

For experiments in Figure 5.5A, RPE cells were seeded onto glass coverslips and transfected with cDNA encoding $\mathrm{mCh}$ - $\beta 1$-integrin as previously described ${ }^{395}$. On the day of the experiment, transfected cells were serum starved for $1 \mathrm{~h}$, then treated (or not; control) with A7 (100 $\mu \mathrm{M}$ for $5 \mathrm{~min}$ ). Intact cells were then immuno-labeled with an antibody specific for an exofacial epitope on $\beta 1$-integrin. The cells were subsequently fixed in 4\% PFA and imaged via immunofluorescence microscopy using wide-field epifluorescence as per Figure 3.6B, to acquire single frame images of the cell surface for manual analysis by ImageJ software as per [111]. With ImageJ, cells were manually outlined of which enclosed areas were quantified for mean pixel intensity of fluorescence-providing measurement of $\beta 1$-integrin. The results were subjected to one-way ANOVA followed by Tukey's posttest, with a threshold of $p<0.05$ for statistically significant differences between conditions (Figure 5.5A). 


\section{6- Biochemical measurement of phosphoinositide levels}

RPE cells were incubated for $24 \mathrm{~h}$ in inositol-free DMEM (MP Biomedicals, Santa Ana, CA) with $10 \mathrm{Ci} / \mathrm{ml}$ myo-[2-3 H(N)] inositol (PerkinElmer Life Sciences, Waltham, MA), 10\% FBS, 4 mM I-glutamine, $1 \times$ insulin-Tfn-selenium-ethanolamine $20 \mathrm{mM} \mathrm{HEPES}$, and $1 \times$ penicillin/streptomycin. On the day of the experiment, cells were serum starved for $1 \mathrm{~h}$ and then stimulated with 5 or $20 \mathrm{ng} / \mathrm{ml} \mathrm{EGF}$ for $5 \mathrm{~min}$ or with $10 \mu \mathrm{M}$ ionomycin for $20 \mathrm{~min}$, as indicated. Cells were then treated with $600 \mu \mathrm{l}$ of $4.5 \%$ perchloric acid (vol/vol) on ice for $15 \mathrm{~min}$, scraped, and pelleted at $12,000 \times \mathrm{g}$ for $10 \mathrm{~min}$. Pellets were washed with $1 \mathrm{ml}$ ice-cold 0.1 M EDTA and resuspended in $50 \mu \mathrm{l}$ of water. Phospholipids were deacylated with $500 \mu \mathrm{l}$ of a solution of methanol/40\% methylamine/1-butanol (45.7\% methanol:10.7\% methylamine:11.4\% 1butanol [vol/vol]) for $50 \mathrm{~min}$ at $53^{\circ} \mathrm{C}$. Samples were vacuum-dried and washed twice with water. The dried samples were then resuspended in water, extracted with a solution of 1-butanol/ethyl ether/ethyl formate (20:4:1), vortexed for $5 \mathrm{~min}$, and centrifuged at 12,000 $\times \mathrm{g}$ for $2 \mathrm{~min}$. The bottom aqueous layer was collected and extracted twice more. The aqueous layer was vacuum-dried and resuspended in $50 \mu l$ of water. Equal counts of $3 \mathrm{H}$ were separated by high-performance liquid chromatography (Agilent Technologies, Santa Clara, CA) through an anion exchange $4.6 \times 250 \mathrm{~mm}$ column (Phenomenex, Torrance, $\mathrm{CA}$ ) with a flow rate of $1 \mathrm{ml} / \mathrm{min}$ and subjected to a gradient of water (buffer $\mathrm{A}$ ) and $1 \mathrm{M}$ (NH4)2HPO4, pH 3.8 (buffer B) as follows: 0\% B for $5 \mathrm{~min}, 0-2 \%$ B for $15 \mathrm{~min}, 2 \%$ B for $80 \mathrm{~min}, 2-10 \%$ B for $20 \mathrm{~min}, 10 \% \mathrm{~B}$ for $30 \mathrm{~min}, 10-80 \%$ B for $10 \mathrm{~min}, 80 \%$ B for $5 \mathrm{~min}$, and $80-0 \%$ B for $5 \mathrm{~min}$. The radiolabeled eluate was detected by $\beta$-RAM 4 (LabLogic, Brandon, FL) with a 1:25 ratio of eluate to scintillant (LabLogic) and analyzed using Laura 4 software. Each of the phosphoinositides was normalized against the parent phosphatidylinositol peak. Samples were subjected to ANOVA followed by Tukey's posttest, with a threshold of $p<0.05$ for statistically significant differences between conditions (Figure 3.6A). For further experimental details, the reader is directed to [142].

\section{7- EGF and Tfn ligand internalization assays}

All internalization/uptake assays were performed on RPE-WT cells grown on 12-well plates as previously described ${ }^{207}$. Following siRNA gene silencing (Figures 3.3-3.5) or drug treatments (Figures 3.2, 3.7-3.9, and 5.5), cells were then incubated with either biotin-XX-EGF ( $5 \mathrm{ng} / \mathrm{ml}$ for EGF internalization) or biotin-xx-Tfn ( $1 \mu \mathrm{g} / \mathrm{ml}$ for Tfn internalization) for $0,2,5$, or $10 \mathrm{~min}$ at $37^{\circ} \mathrm{C}$. Following ligand internalization, cells were immediately placed on ice and washed three times in ice-cold PBS2+ 
(phosphate-buffered saline [PBS] supplemented with $1 \mathrm{mM} \mathrm{CaCl} 2$ and $1 \mathrm{mM} \mathrm{MgCl}_{2}$ ) to remove unbound ligand and arrest membrane traffic. Once bound, uninternalized ligands were subsequently quenched by sequential incubation with free avidin for $30 \mathrm{~min}$ on ice $(5 \mu \mathrm{g} / \mathrm{ml}$ avidin for EGF internalization, and 28.6 $\mu \mathrm{g} / \mathrm{ml}$ for Tfn internalization), and biocytin for $15 \mathrm{~min}$ on ice $(7.69 \mu \mathrm{g} / \mathrm{ml}$ biocytin for EGF internalization, and $27.3 \mu \mathrm{g} / \mathrm{ml}$ for Tfn internalization). Cells were subsequently washed repeatedly with ice-cold PBS2+ and solubilized by incubation in a buffer composed of $0.01 \%$ Triton X-100 and $0.01 \%$ SDS in Superblock buffer. Cell lysates were then plated onto enzyme-linked immunosorbent assay (ELISA) plates coated with either anti-EGF or anti-Tfn antibodies, followed by detection of biotin-xx-EGF or biotin-xx-Tfn (free, unbound to avidin) by incubation with streptavidin conjugated to horseradish peroxidase (POD), and detection of bound POD by o-phenylenediamine assay. Absorbance readings corresponding to internalized biotin-xx-EGF or biotin-xx-Tfn readings were then normalized to the total levels of surfaceligand binding measured at $4^{\circ} \mathrm{C}$ and not subjected to avidin-biocytin quenching, performed in parallel for each condition.

To resolve whether any differences in EGF internalization observed using the above assay might be due to changes in EGF:EGFR binding (instead of EGF:EGFR internalization rate), we performed a control experiment to measure cell-associated biotin-xx-EGF (reflecting EGF:EGFR binding) in which the quenching by avidin and biocytin was omitted (Figure 3.8). This assay was performed identically to the above EGF internalization assay, with the exception that, following ligand internalization (at $37^{\circ} \mathrm{C}$ ), placement of cell on ice, and extensive washing in ice-cold PBS2+ to remove unbound ligand, cells were directly solubilized by incubation $0.01 \%$ Triton X-100 and 0.01\% SDS in Superblock buffer. Cell-associated biotin-xx-EGF was detected on EGF-coated ELISA plates, as described above, and normalized to total biotin-xx-EGF bound $\left(\right.$ at $\left.4^{\circ} \mathrm{C}\right)$.

Measurement of biotin-xx-Tfn internalization during EGF stimulation was performed similarly as Tfn internalization described above, but was preceded by the addition of $5 \mathrm{ng} / \mathrm{ml} \mathrm{EGF}$ (unlabeled) $1 \mathrm{~min}$ before addition of Tfn-xx-biotin in each sample. Measurement of Tfn-xx-biotin was performed in the continued presence of unlabeled EGF.

In all EGF or Tfn internalization experiments, the results were subjected to two-way ANOVA followed by Bonferroni's multiple comparison posttest, with a threshold of $p<0.05$ for significant difference between treatment conditions. 


\section{8- Measurement of cytosolic $\left[\mathrm{Ca}^{2+}\right]$ with Fluo4-AM}

RPE-WT cells were seeded onto glass coverslips and then subjected to siRNA silencing as described in section 2.3. At the time of the experiment, cells were loaded with Fluo-4 AM ( $8 \mu \mathrm{M}$ for 15 $\min$ ) at $37^{\circ} \mathrm{C}$ and $5 \% \mathrm{CO}_{2}$; this was followed by washing to remove excess Fluo-4 AM and a further 30 min incubation at $37^{\circ} \mathrm{C}$ and $5 \% \mathrm{CO}_{2}$, during which time some cells were treated with $\mathrm{XeC}$, as indicated. Spinning-disk confocal microscopy of living cells was performed at $37^{\circ} \mathrm{C}$ and $5 \% \mathrm{CO}_{2}$ throughout image acquisition using an Olympus IX81 equipped with a Yokogawa CSU X1 scanhead and a 60x/1.35 NA oil objective using a Hamamatsu C9100-13 EM-CCD camera. Excitation light was provided by 488-nm (100 $\mathrm{mV}$ ) laser illumination, and emitted light was collected following passage through a 525/50 emission filter.

Images of multiple fields of view of unstimulated cells (basal) were obtained, followed by addition of $5 \mathrm{ng} / \mathrm{ml} \mathrm{EGF}$ for $5 \mathrm{~min}$ and acquisition of images of multiple fields of view of the EGFstimulated condition. For testing the maximum intensity of Fluo4-AM fluorescence, subsequent to EGF stimulation, cells were treated with ionomycin $(10 \mu \mathrm{M}$ for $5 \mathrm{~min})$ at $37^{\circ} \mathrm{C}$ in the presence of $5 \% \mathrm{CO}_{2}$, followed by acquisition of images of multiple fields of view of the ionomycin-treated condition. All images were analyzed using ImageJ, measuring the total intensity of Fluo4-AM per cell in each condition (before EGF, after EGF, and after ionomycin). Shown (Figures 3.6, D, F, and G) are the differences in the mean Fluo4-AM fluorescence between the EGF-stimulated and basal conditions and the differences in the mean Fluo4-AM fluorescence between the ionomycin-treated and EGF-stimulated conditions. The results were subjected to one-way ANOVA followed by Tukey's posttest, with a threshold of $p<0.05$ for statistically significant differences between conditions. 


\section{9- TIRF-M and automated software image analysis of CLSs in fixed cells}

\subsection{1 - Fixed sample preparation for CLS detection and analysis by TIRF-M RPE cells stably expressing eGFP-CLCa or RFP-CLCa were seeded onto glass coverslips the day}

before the experiment, and in some cases transfected with cDNA encoding mCh-Sjn1 (Figure 4.6) or recombinant Arf6 proteins (Figure 5.3). Following $1 \mathrm{~h}$ serum starvation and drug treatment as indicated, cells were treated in some cases with appropriate ligands for defined periods of time including rhodamine-EGF $(20 \mathrm{ng} / \mathrm{ml})$ and A647-Tfn $(10 \mu \mathrm{g} / \mathrm{ml})$ alone or in combination (Figure 3.11), either A555EGF $(5 \mathrm{ng} / \mathrm{ml}$ ) or A555-Tfn (1 $\mu \mathrm{g} / \mathrm{ml}$ ) (Figure 4.1), or unlabeled EGF (Figures 4.6 and 4.8 )-followed by immediate fixation in 4\% PFA. For experiments shown in Figure 4.8, endogenous Sjn1 was additionally labeled via immunofluorescence staining following fixation as previously described, using an anti-Sjn1 antibody ${ }^{207}$. For further experimental details, the reader is directed to [7].

\subsection{2 - Image acquisition by TIRF-M}

For experiments shown in Figure 4.1, samples were imaged by TIRF-M using a 150×/1.45 NA objective on an Olympus IX81 instrument equipped with CellTIRF modules (Olympus Canada, Richmond Hill, ON, Canada) using 491- and 561-nm laser illumination and 520/30 and 624/50 emission filters. Images were acquired using a C9100-13 EM-CCD camera (Hamamatsu, Bridgewater, NJ).

For experiments shown in Figures 3.11, 4.6, 4.8, 5.1, and 5.3, samples were imaged using a Quorum (Guelph, ON, Canada) Diskovery TIRF-M, comprising a Leica DMi8 microscope equipped with a 63 $\times / 1.49$ NA TIRF objective with a 1.8x camera relay (total magnification 108x). Imaging was done using 488-, 561-, and 637-nm laser illumination and 527/30, 630/75, and 700/75 emission filters and acquired using a Zyla 4.2Plus sCMOS camera (Hamamatsu). For further experimental details, the reader is directed to [7]. 


\subsection{3 - CLS detection and quantification of protein fluorescence intensity within}

CLSS

For Figures 3.11, 4.1, 4.6, 4.8, 5.1, and 5.3, clathrin-labeled structures or CLSs were detected and quantified using TIRF-M coupled to custom software developed in Matlab (MathWorks, Natick, MA), as previously described ${ }^{5,207}$. CLSs represent all clathrin-containing structures as reported by fluorescent clathrin signals (e.g. RPE cells expressing either eGFP-CLCa or RFP-CLCa) detected by TIRF-M imaging coupled to automated software analysis ${ }^{79}$. Briefly, diffraction-limited CLSs were detected using a Gaussian-based model method to approximate the point-spread function of eGFP-CLCa or RFP-T-CLCa puncta. The amplitude of the Gaussian model of fluorescence intensity of a CLS informs about its size. In some experiments (Figures 3.11, 4.1, 4.6, 4.8, and 5.3), the fluorescence intensity corresponding to ligands (e.g. A555-EGF, rhodamine-EGF, A555-Tfn, or A647-Tfn) or cytosolic proteins (e.g. mCh-Sjn1, endogenous Sjn1, or eGFP-Arf6 recombinant proteins) were also detected and quantified via Gaussian modeling. The localization of the respective ligands and cytosolic proteins within CLSs were then determined based on the amplitude of the Gaussian model for the appropriate fluorescent channel for each CLS. As such, the measurements of fluorescent ligands or proteins within CLSs represent enrichment of the corresponding receptors (EGFR or TfR) or cytosolic proteins (Sjn1 or Arf6) relative to the local background fluorescence in the vicinity of the detected CLS. For further experimental details, the reader is directed to $[5,7]$.

\subsection{4 - Measurement of mean fluorescence of ligand or cytosolic proteins within CLSS}

Measurements of mean rhodamine-EGF and A647-Tfn (Figure 3.11B), A555-EGF (Figure 4.1C), A555-Tfn (Figure 4.1E), mCh-Sjn1 (Figure 4.6B), endogenous Sjn1 (Figure 4.8), or eGFP-Arf6 (Figure 5.3) enrichment within CLSs for each cell were subjected to ANOVA followed by Tukey's posttest, with a threshold of $p<0.05$ for statistically significant differences between conditions.

\subsection{5 - Generation of two-dimensional histogram of EGF and Tfn fluorescence within CLSS}

Measurements of EGF and Tfn fluorescence intensities within CLSs were plotted using a twodimensional histogram, with 50 bins per channel (Figure 3.11B). For normalization of differences in EGF 
and Tfn binding between cells, the fluorescence intensities of EGF or Tfn within CLSs were rescaled by normalization to a value $n=\mu+0.75 \sigma$, where $\mu$ and $\sigma$ are the mean and SD of the EGF or Tfn intensity within CLSs in each image. For further experimental details, the reader is directed to $[5,7]$.

\subsection{6 - Identification of EGF- and Tfn-enriched CLSS}

To measure the properties of CLS subsets enriched in fluorescently conjugated EGF or Tfn (and thus the corresponding receptors, EGFR or TfR, respectively; Figures 3.11C and 4.1, D and F), we established a threshold of the $\sim 85$ th percentile of ligand fluorescence intensity within CLSs in the control (no inhibitor) condition in each experiment ${ }^{207}$. Using this threshold, we defined subsets of CLSs enriched in either fluorescent EGF and/or Tfn for each condition as those with ligand fluorescence intensity above this threshold. This method delineates CLSs as enriched in specific ligands (e.g. EGF+), effectively producing sorting of CLSs into cohorts based on the presence or absence of specific ligands.

For the experiments in Figure 3.11C, within each condition, the CLSs were sorted into cohorts that are either positive for only rhodamine-EGF or A647-Tfn, or for both. For the experiments in Figure 4.1, D and $F$, within each condition, the A555-EGF or A555-Tfn enriched CLS population was used to measure mean eGFP-CLCa fluorescence per CLS in each cell. Measurements (mean eGFP-CLCa within the specified CLS subset for each cell) were subjected to ANOVA followed by Tukey's posttest, with a threshold of $p<0.05$ for statistically significant differences between conditions. For further experimental details, the reader is directed to $[5,7]$.

\subsection{0- TIRF-M and automated image analysis of CCP properties (time- lapse imaging)}

Time-lapse imaging by TIRF-M was performed (Figures 4.3-4.5, 5.1, 5.4, and 5.6) as previously

described ${ }^{5,79,404}$. Briefly, RPE cells stably expressing eGFP-CLCa were seeded onto glass coverslips the day before the experiment, and in some cases transfected with cDNA encoding $\mathrm{mCh}$ - $\beta 1$-integrin (Figure 5.6). On the day of the experiment, the cells are serum starved in DMEM/F12 lacking phenol red (supplemented with 0.1\% FBS for experiments in Figures 5.1, 5.4 and 5.6) for $1 \mathrm{~h}$ followed by 
appropriate drug treatment as indicated. In some cases, the cells were treated with appropriate ligands including rhodamine-EGF $(20 \mathrm{ng} / \mathrm{ml})$ or A647-Tfn (100 ng/ml in the presence of $20 \mathrm{ng} / \mathrm{ml}$ unlabeled EGF) at the time of imaging (Figure 4.3). Towards the end of drug treatment or ligand addition, time lapseimage series were acquired at a frame rate of $1 / \mathrm{s}$ and an exposure time of 80-150 ms using a Quorum Diskovery TIRF-M comprising a Leica DMi8 microscope equipped with a 63×/1.49 NA TIRF objective with a $1.8 \times$ camera relay (total magnification 108x). Imaging was done using 488-, 561-, and/or 637-nm laser illumination and 527/30,630/75, and 700/75 emission filters and acquired using a Zyla 4.2Plus sCMOS camera (Hamamatsu).

Unlike TIRF-M of fixed cell samples described in section 2.9, time-lapse imaging of live cells via TIRF-M coupled to automated software analysis enables the detection of CLS subpopulations-allowing CLSs to be classified as either subthreshold (sCLSs) or bona fide CCP structures ${ }^{79}$. $\mathrm{sCLSs}$ represent shortlived and dim CLSs which fail to grow. In contrast, bona fide CCPs are quantitatively defined by the continuous accumulation of clathrin which grow past a specific fluorescence threshold intensity ${ }^{79}$. The detection, tracking, and analysis of clathrin objects, which allow detection of sCLSs and CCPs, was done as previously described using the cmeAnalysis software package ${ }^{5,79}$. Briefly, diffraction-limited clathrin structures were detected using a Gaussian-based model method to approximate the point-spread function ${ }^{5}$, and trajectories were determined from clathrin-structure detections using u-track software ${ }^{405}$. SCLSs were distinguished from bona fide CCPs as previously described, based on the quantitative and unbiased analysis of clathrin intensity progression in the early stages of structure formation ${ }^{5,79}$. Both SCLSs and CCPs represent nucleation events, but only bona fide CCPs represent structures that undergo stabilization, maturation, and in some cases, scission to produce intracellular vesicles ${ }^{5,79}$. We report the rate of SCLS and CCP formation (Figures 4.3, 5.1, 5.4 and 5.6) and the distribution of CCP lifetimes (Figures 4.4C and 4.5C), and these measurements were subjected to Student's t-test, with a threshold of $p<0.05$ for statistically significant differences between conditions.

Because CCPs are diffraction-limited objects, the amplitude of the Gaussian model of the fluorescence intensity of eGFP-CLCa indicates CCP size (Figures 4.3, 5.1, 5.4 and 5.6). In addition, the fluorescence intensity of receptor ligands (rhodamine-EGF or A647-Tfn) and endogenous receptors (mCh- $\beta 1$-integrin) indicates the recruitment of the corresponding receptor (Figures 4.4, 4.5, and 5.6). Given the heterogeneity of CCP lifetimes, for eGFP-CLCa and ligand/receptor fluorescence intensity 
measurements of CCP size and receptor recruitment, objects were separated into lifetime cohorts. For further experimental details, the reader is directed to $[5,7]$.

\subsection{1- Whole-cell lysates and Western blotting}

Western blotting was performed for experiments in Figures 3.1, 3.5, 4.9, and 4.10 as previously described ${ }^{2,207}$. Following transfection, serum starvation, and/or treatment with inhibitors as indicated, cells were stimulated (Figures 3.1, 4.9, and 4.10) with $5 \mathrm{ng} / \mathrm{ml} \mathrm{EGF}$ for $5 \mathrm{~min}$ (unless otherwise indicated) or left unstimulated (basal). Whole-cell lysates were prepared using Laemmli sample buffer (0.5M Tris, pH 6.8, glycerol, 10\% SDS, 10\% $\beta$-mercaptoethanol, and 5\% bromophenol blue) supplemented with a protease and phosphatase inhibitor cocktail ( $1 \mathrm{mM}$ sodium orthovanadate, $10 \mathrm{nM}$ okadaic acid, and 20nM Protease Inhibitor Cocktail [BioShop, Burlington, ON, Canada]). Lysates were then heated at $65^{\circ} \mathrm{C}$ for $15 \mathrm{~min}$ and passed five times through a 27.5 -gauge syringe. Proteins were resolved by glycine-Tris SDS-PAGE followed by transfer onto polyvinylidene fluoride membranes, which were washed, blocked, and incubated with antibodies as previously described ${ }^{207}$.

The levels of respective phosphorylated protein in each condition were quantified as previously described ${ }^{406}$. This was done by signal integration in an area corresponding to the appropriate lane and band for each condition. The measurement corresponding to the levels of a phosphorylated form of a specific protein was then normalized to the loading control (e.g., actin) signal and subsequently normalized to the measurement of total protein signal, obtained by a similar method either following blot stripping or reblotting. In each experiment, the resulting normalized phospho/total protein (e.g. phospho-Akt/total Akt) signal in each condition is expressed as a fraction of the normalized pAkt/total Akt measurement in the control condition stimulated with EGF for $5 \mathrm{~min}$. Samples were subjected to ANOVA followed by Tukey's posttest, with a threshold of $p<0.05$ for statistically significant differences between conditions (Figures 4.9 and 4.10). 
Chapter 3. Determining the role of PLC- $\gamma 1$ in EGFR endocytosis 
Mitogenic signaling by RTKs, including that by EGFR, results in the initiation of various cellular processes which may induce broad reorganization of the plasma membrane ${ }^{59-61}$. Precisely how EGFR signaling may robustly regulate the cell surface however, remain poorly understood-although it may be expected to do so via control of $\mathrm{CME}$; which serve as the major endocytic route and regulator of the plasma membrane in mammalian cells ${ }^{50,58}$. While knowledge regarding EGFR control of endocytosis is limited, I hypothesize a role of PLC- $\gamma 1$ in coupling EGFR activation with regulation of CME. Upon ligand binding, activated EGFR subsequently recruits and activates PLC- -1 on the plasma membrane. PLC- $\gamma 1$ is a phosphodiesterase enzyme largely responsible for the turnover of $\mathrm{PIP}_{2}{ }^{13}$. Importantly, $\mathrm{PIP}_{2}$ is an essential component of CME of which dysregulation is well-documented in literature to cause endocytic defects $^{138}$. The activation of PLC- $\gamma 1$ by EGFR may therefore function to regulate CME of which context to the overall endocytic process nonetheless, remains to be elucidated. In addition, the hydrolysis and turnover of PIP 2 by PLC- $\gamma 1$ result in the production of secondary by-products such as the plasma membrane-localized DAG and the cytosolic $I \mathrm{P}_{3}$ molecule ${ }^{13}$. IP $\mathrm{P}_{3}$ functions as a ligand which upon binding induces the opening of $I P_{3} R$ on the $E R$, resulting in the release and subsequent increase of calcium within the cytosol ${ }^{13}$. Calcium is a well-known regulator of neuronal endocytosis which enhances CME during synaptic transmission - suggesting a potential role for PLC $\gamma 1$-derived calcium signals in regulating $\mathrm{CME}^{96}$. As such, I hypothesize that by activating PLC- $\gamma 1$, mitogenic signaling by EGFR may regulate CME via PIP 2 hydrolysis and mobilization of calcium, in broadly regulating the plasma membrane.

In this chapter, I elucidate the functional role of PLC- $\gamma 1$ in the potential regulation of CME. In addition to the downstream activation of PLC- $p 1$, activated EGFR also induces its concomitant internalization via CME - thereby providing an ideal model through which the role of PLC- $\gamma 1$ in the endocytic process may be examined. To understand the functional requirement for PLC- $\psi 1$ in CME, I specifically examined the role of PLC 1 1-derived signals in context of EGFR endocytosis. Specifically, I measured the internalization of the EGF ligand (and hence, EGFR endocytosis) using biochemical assays, under control and PLC- $\gamma 1$ perturbed conditions, to determine the functional requirement for PLC- $\gamma 1$ in endocytosis of EGFR. Furthermore, I dissect the functional contributions of $\mathrm{PIP}_{2}$ hydrolysis and increased cytosolic calcium triggered by PLC- $\nu 1$ in EGFR endocytosis, to further define precisely how PLC- $\gamma 1$ may regulate CME. Importantly, the following examinations of the endocytic regulation of EGFR are supplemented by parallel measurements of Tfn internalization (and hence, TfR endocytosis) under identical treatment conditions, to determine whether PLC- $\gamma 1$ may broadly or selectively regulate the endocytic process. I hypothesize that PLC- $\gamma 1$ activation, and both its subsequent hydrolysis of $\mathrm{PIP}_{2}$ and 
mobilization of intracellular calcium signals, regulates endocytosis which may selectively impact CME of specific cargoes including EGFR.

\section{1- PLC- $\gamma 1$ selectively controls clathrin-mediated endocytosis of EGFR EGF stimulation of retinal pigment epithelial (ARPE-19, henceforth RPE) cells elicits} phosphorylation and thus activation of PLC- $\gamma 1$, detectable as early as 1 minute after ligand addition (Figure 3.1A). To initially determine whether PLC- $p 1$ signaling contributes to EGFR endocytosis, I used the known PLC inhibitor compound U73122 in RPE cells stimulated with EGF, and monitored internalization of the ligand at various time points (e.g. 0, 2, 5, and 10 minutes) as per section 2.7. Treatment with U73122 significantly reduced internalization of the EGF ligand at all time points compared to control, which indicates a potential requirement for PLC- $\gamma 1$ in EGFR endocytosis (Figure 3.2). It is important to note however, that U73122 is a pan-PLC inhibitor which broadly inhibits multiple PLC isoforms through a poorly understood mechanism ${ }^{407}$. Thus, the reduction in EGF internalization cannot be directly attributed to the inhibition of PLC- $\gamma 1$ as the non-specific effects of U73122 cannot be readily ruled out. Indeed, several studies have reported the non-specific effects of U73122 which was observed to activate rather than inhibit some human PLCs ${ }^{408}$. Nonetheless, the U73122-induced reduction of EGF internalization preliminarily highlights the potential contribution of PLC- $\gamma 1$ in EGFR clathrin-mediated endocytosis.

In circumventing the difficulty associated with the non-specific effects of U73122, I sought to directly inhibit PLC- $\gamma 1$ expression via small interfering RNA (siRNA)-mediated silencing of PLC- $\nu 1$ in RPE cells, which resulted in an $85.6 \pm 6.4 \%$ reduction $(p<0.05, n=3)$ in PLC- $\gamma 1$ expression (Figure 3.1B). I then monitored the internalization of the EGF ligand which I found significantly impaired in PLC- $\gamma 1$ silenced cells compared to control (non-targeting siRNA), demonstrating a requirement for PLC- $\nu 1$ in EGFR endocytosis (Figure 3.3A). In determining the scope by which PLC- $p 1$ may regulate CME, I also measured the internalization of the classical CME cargo, Tfn, which I found was expectedly unaffected by PLC- $\gamma 1$ silencing under basal, non-EGF stimulated conditions due to the lack of PLC- $\gamma 1$ activation (Figure 3.3B). Surprisingly, the lack of effect of PLC- $p 1$ silencing on Tfn endocytosis was also apparent even in the presence of EGF stimulation (to trigger PLC- $\gamma 1$ activity), ruling out a broad effect of PLC- $\gamma 1$ on CME (Figure 3.3C). Overall, these observations indicate PLC- $\gamma 1$ as a selective regulator of CME, of which 
activities may be required for the efficient internalization of a subset of CME cargoes which includes EGFR, but not TfR.

Described in section 1.6.4, previous studies have indicated the internalization of EGFR via clathrin-independent endocytosis upon stimulation with high concentrations of EGF $(>20 \mathrm{ng} / \mathrm{ml})^{409}$. In assessing the role of PLC- $\gamma 1$ in EGFR endocytosis, it is therefore important to consider the possibility of EGFR internalization via non-clathrin pathways. Indeed, for all experiments, I monitored EGFR internalization under low doses of EGF stimulation $(5 \mathrm{ng} / \mathrm{mL})$ to favour receptor internalization by $\mathrm{CME}^{409}$. In addition, in RPE cells, stimulation with low EGF doses induces EGFR internalization to occur very largely by $\mathrm{CME}^{207}$. Interestingly, I demonstrated that even at saturating levels of EGF stimulation (100 ng/mL EGF), EGFR endocytosis appears to be predominantly clathrin-dependent in RPE cells, as demonstrated by the significant impairment of EGF internalization upon siRNA silencing of clathrin, compared to control (Figure 3.4). These findings indicate that in RPE cells, EGFR may be largely restricted to internalization by CME. I thus conclude that the findings in this section indicate PLC- $\gamma 1$ as a selectively regulator of the clathrin-dependent endocytosis of EGFR.

\section{2- PLCY1-derived intracellular calcium is selectively required for EGFR endocytosis}

Having established the requirement for PLC- $\gamma 1$ in CME of EGFR, I then sought to determine how precisely PLC- $\gamma 1$ may control the endocytic process. Specifically, PLC- $\gamma 1$ may control EGFR endocytosis by consumption of $\mathrm{PIP}_{2}$ or by generation of secondary signaling intermediates such as intracellular calcium. In addition, PLC- $\gamma 1$ has also been proposed to regulate endocytosis by acting as a GEF for Dyn1 in neuronal cells (see section 1.6.3.2.6), which selectively regulates CME of the tumor necrosis factorrelated apoptosis-inducing ligand (TRAIL)-death receptor (DR) ${ }^{304,400,410}$. To determine whether PLC- 11 regulates CME of EGFR by acting as a Dyn1-GEF, I silenced Dyn1 via siRNA and measured EGF internalization. Silencing of Dyn1 however, had no effect on EGF internalization relative to control (Figure 3.5, A and B), indicating that PLC- $\gamma 1$ regulates EGFR endocytosis in a Dyn1-independent manner. Indeed, EGFR internalization is largely mediated by Dyn2 in RPE cells, which currently has not been reported to be directly controlled by PLC- $\gamma 1^{207}$. It can therefore be hypothesized that PLC- $\gamma 1$ may control 
CME of EGFR either through PIP $_{2}$ hydrolysis, or stimulation of cytosolic calcium increases, investigated in this section ${ }^{207}$.

\subsection{1 - PLC- $\gamma 1$ does not broadly regulate plasma membrane $\mathrm{PIP}_{2}$ concentrations}

As an indispensable component of CME, the consumption of $\mathrm{PIP}_{2}$ by PLC- $\gamma 1$ may therefore serve as a potential mechanism by which mitogenic signaling by EGFR may directly regulate endocytosis. With this possibility, we sought to determine the extent by which PLC- $\gamma 1$ may regulate plasma membrane PIP 2 concentrations. Using HPLC (high performance liquid chromatography), we biochemically measured and compared changes in concentrations of PIP 2 under various conditions: basal $(0 \mathrm{ng} / \mathrm{mL})$, low $(5 \mathrm{ng} / \mathrm{mL})$ or high $(20 \mathrm{ng} / \mathrm{mL}$ ) EGF doses. Interestingly, EGF-stimulation at both low and high EGF doses, did not appreciably decrease $\mathrm{PIP}_{2}$ levels compared to the basal condition (Figure 3.6A). In contrast, PIP 2 concentration fell close to undetectable levels upon treatment of cells with ionomycin (calcium ionophore which stimulates calcium influx across the plasma membrane), which is well-known to deplete plasma membrane $\mathrm{PIP}_{2}{ }^{411}$. This finding suggests that $\mathrm{PIP}_{2}$ concentrations are modestly, if at all, regulated by PLC- $\gamma 1$ activity, indicating the minimal contribution of the PLC $\gamma 1$-mediated PIP $_{2}$ hydrolysis in the regulation of endocytosis.

To further corroborate the previous finding, I similarly monitored plasma membrane $\mathrm{PIP}_{2}$ availability by using a genetically-encoded fluorescent $\mathrm{PIP}_{2}$ probe coupled with microscopic imaging. Specifically, the $\mathrm{PIP}_{2}$ probe used is comprised of the PLC- $\delta \mathrm{PH}$ domain fused with a fluorescent mCherry tag (mCh-PH WT), which described in section 1.4.1 is known to strongly bind $\mathrm{PIP}_{2}{ }^{166}$. The mCh-PH WT construct was then transfected into RPE cells and subsequently visualized using total internal reflection fluorescence microscopy (TIRF-M). TIRF-M is a microscopic technique which allows imaging of the immediate cell surface (TIRF field; 200-400nm), and hence, visualization of plasma membrane-bound mCh-PH WT, of which quantification of fluorescence intensities indirectly reports on plasma membrane $\mathrm{PIP}_{2}$ content ${ }^{412,413}$. Consistently, stimulation of $\mathrm{mCh}$-PH WT-expressing RPE cells with low dose EGF, did not appreciably decrease the fluorescence levels of this probe in the TIRF field compared to control, again reflecting modest reductions in plasma membrane PIP 2 mediated by PLC- $\gamma 1$ (Figure 3.6B). In contrast, fluorescence quantification of the mutant $\mathrm{mCH}-\mathrm{PH} \mathrm{K32A/W36A}$ construct (unable to bind $\mathrm{PIP}_{2}$ ) transfected in RPE cells, yielded lower intensity measurements compared to $\mathrm{mCH}-\mathrm{PH}$ WT, indicating that the lack of fluorescence decrease in the EGF-stimulated condition was not due to low sensitivity for 
detection. These experiments thus, indicate that the impact of PLC- $\gamma 1$ on EGFR endocytosis is not due to robust, broad $\mathrm{PIP}_{2}$ depletion.

\subsection{2 - PLC- $\gamma 1$ regulates EGFR endocytosis via stimulation of intracellular calcium}

The findings in the previous sections indicate that PLC- $\gamma 1$ regulates EGFR endocytosis in a manner independent from its potential regulation of dynamin, or control of plasma membrane $\mathrm{PIP}_{2}$ concentrations-suggesting the involvement of an alternate mechanism. Specifically, a mechanism by which PLC- $\gamma 1$ could regulate EGFR endocytosis may be derived from its stimulation of calcium release from the ER. Briefly, hydrolysis of PIP 2 by PLC- $\gamma 1$ results in the production of the secondary IP 3 byproduct, which induces cytosolic calcium increases by binding and opening $I \mathrm{P}_{3} \mathrm{R}$ calcium channels on the $\mathrm{ER}^{13}$. Emphasized in section 1.5.3, calcium is a well-known regulator of neuronal endocytosis which enhances CME during neurotransmission. As such, I then sought to determine whether the PLCY1derived calcium signals may similarly enhance EGFR endocytosis, described in the following.

In elucidating the potential regulation of EGFR endocytosis by PLC $\gamma 1$-derived calcium signals, we used the fluorescent Fluo4-AM calcium probe to initially determine whether PLC- $\gamma 1$ activation does indeed, result in measurable cytosolic calcium increases. Fluo4-AM is a cell-permeable fluorescent molecule which exhibits an increase in fluorescence upon binding calcium, and thus, an ideal reporter of intracellular increases in calcium concentrations ${ }^{414}$. Indeed, EGF stimulation elicits a gain in Fluo4-AM fluorescence intensity as visualized by wide-field epifluorescence microscopy, compared to the basal, unstimulated condition (Figure 3.6C) ${ }^{415,416}$. Expectedly, silencing of PLC- $\gamma 1$ ablated the EGF-induced gain in Fluo4-AM fluorescence, which highlights the requirement for PLC- $\gamma 1$ in stimulating cytosolic calcium increases (Figure 3.6, C and D). To further determine whether the gain in Fluo4-AM fluorescence upon EGF-stimulation was the direct result of PLC $\gamma 1$-mediated calcium release from the ER, we used the $I P_{3} R$ channel inhibitor Xestospongin $\mathrm{C}(\mathrm{XeC})$ and imaged the cells under similar conditions. $\mathrm{XeC}$ is a highly selective, membrane permeable inhibitor of $I P_{3} R$, which although poorly understood, is hypothesized to inhibit IP $R$ by sterically blocking the channel ${ }^{417}$. Interestingly, XeC treatment severely reduced the EGFinduced intracellular calcium increase as reported by measurement of Fluo4-AM intensity, compared to control (Figure 3.6, E and F) ${ }^{418}$. Importantly, in each of these PLC- $\gamma 1$ or IP ${ }_{3} R$ perturbing conditions, subsequent treatment with ionomycin elicited a robust increase in intracellular calcium, indicating that 
these treatments did not alter Fluo4-AM uptake (Figure 3.6G). Collectively, these findings indicate PLC$\gamma 1$ and $I P_{3} R$ as essential requirements for the EGF-stimulated gain in cytosolic calcium.

Having established the role of PLC- $\gamma 1$ in inducing cytosolic calcium increases upon EGF stimulation, I then sought to determine the functional requirement for PLC $\gamma 1$-derived calcium signals in EGFR endocytosis. Specifically, I demonstrated that treatment of RPE cells with XeC significantly abrogated EGF internalization relative to control, measured through identical biochemical assays used previously, highlighting the requirement for PLC 1 1-derived calcium signals in EGFR endocytosis (Figure 3.7A). Furthermore, $\mathrm{XeC}$ treatment did not affect Tfn internalization either in the absence (Figure 3.7B), or presence (Figure 3.7C) of EGF stimulation, demonstrating the selective regulation of EGFR endocytosis by PLC- $\gamma 1$. Interestingly, treatment of RPE cells with the calcium chelator BAPTA-AM (1,2bis(2-aminophenoxy)ethane- $\mathrm{N}, \mathrm{N}, \mathrm{N}^{\prime}, \mathrm{N}^{\prime}$-tetraacetic acid tetrakis(acetoxymethyl ester)), phenocopies the reduction in EGF internalization observed with both PLC- $\gamma 1$ silencing and $I P_{3} R$ inhibition (Figure 3.7D); which again was without effect on Tfn endocytosis in absence (Figure 3.7E) or presence (Figure 3.7F) of EGF stimulation. BAPTA-AM is a cell-permeable compound which binds two calcium ions per molecule, commonly used in chelating intracellular calcium thereby sequestering them from biological processes including endocytosis ${ }^{149,419}$. Importantly, BAPTA-AM treatment did not impact the total amount of cellassociated EGF when the assay was repeated without distinguishing internalized from surfaceassociated EGF (Figure 3.8). This indicates that BAPTA-AM treatment did not impact EGF endocytosis as a result of alteration of EGF:EGFR association, but instead decreased the rate of EGF:EGFR internalization. Collectively, the impairment of EGF internalization by PLC- $\gamma 1$ silencing, IP ${ }_{3} R$ inhibition, and intracellular calcium chelation, suggests the selective requirement for PLC $\gamma 1$-derived calcium signals in the regulation of EGFR endocytosis.

\subsection{3 - PLCY1-calcium control of EGFR endocytosis is mechanistically distinct from that in neuronal systems and may involve PKC. \\ The findings from the previous sections demonstrate that PLC 1 -derived calcium signals are selectively required for the efficient internalization of EGFR. Calcium is a known regulator of endocytosis in neurons, of which influx into the neuronal synapse enhances CME. The mechanism by which calcium augments neuronal endocytosis is well-annotated, involving the activation of $\mathrm{CaN}$, which upon binding calcium subsequently activates components of the clathrin machinery (see section 1.5 .3$)^{400}$. It is}


therefore tempting to hypothesize whether the PLC 1 1-derived calcium signals may function similarly in regulating EGFR endocytosis. To examine whether PLC- $\gamma 1$ control of EGFR internalization recapitulates the control of endocytosis by CaN in neurons, I used the CaN inhibitor cyclosporin A (CsA) and measured the internalization of EGF. CSA is a cyclic, lipid-soluble peptide which forms a molecular complex with cyclophilin, that directly binds and blocks the phosphatase activity of $\mathrm{CaN}^{420}$. Strikingly, treatment of RPE cells with CSA does not appreciably reduce EGF internalization, suggesting that PLC $\gamma 1$-derived calcium increases may regulate EGFR endocytosis through a different mechanism (Figure 3.9A). Importantly, CsA treatment effectively impaired the calcineurin-dependent nuclear translocation of NFAT (nuclear factor of activated T-cells) - detected by transfection and epifluorescence imaging of the NFAT-GFP construct in RPE cells-thereby confirming inhibition of CaN by CsA (Figure 3.10, A and B) ${ }^{420}$. These results thus, indicate that the PLCY1-calcium control of EGFR endocytosis is at least partly distinct from that of enhanced CME elicited by calcium signaling in neurons.

In addition to the activation of calcium sensing proteins such as $\mathrm{CaN}$, calcium signaling is also known to activate several other downstream effectors including the conventional isoforms of PKC (CPKC). Described in section 1.4.3, CPKC proteins are activated by both binding DAG and calcium, which interestingly are both by-products of PLC- $\gamma 1$ activity - and thus, coupled to EGF stimulation and receptor internalization. I therefore sought to determine the potential role of PKCs in the regulation of EGFR endocytosis by PLC 1 -derived calcium, by using the pan-PKC inhibitor BIM (bisindolylmaleimide I). BIM is a cell-permeable compound which broadly inhibits several PKC isoforms including CPKC, by acting as a competitive inhibitor for the ATP binding site thereby preventing full PKC activation ${ }^{421,422}$. Surprisingly, treatment of RPE cells with BIM significantly impaired EGF internalization (Figure 3.9B) comparable to that elicited by PLCY1-silencing, IP ${ }_{3} R$ inhibition, and calcium chelation; which similarly was without effect on Tfn endocytosis measured in the absence (Figure 3.9C) or presence (Figure 3.9D) of EGF stimulation. Although the reduction of EGF internalization cannot be directly attributed to the role of CPKC due to the broad inhibition of other PKC isoforms by BIM, these results nonetheless demonstrate the potential role of CPKC in selectively regulating the clathrin-dependent internalization of EGFR, in a manner similar to PLCY1- and ER-derived cytosolic calcium. 


\section{3- EGFR and TfR localize largely to distinct CLSS}

The findings from the previous sections indicate that EGFR internalization is selectively regulated by PLCY1-derived calcium signals, of which regulation of endocytosis may act via activation of PKC. In addition, these requirements appear to be limited to regulation of EGFR endocytosis, as internalization of other CME cargo such as TfR were largely unaffected. These findings nonetheless, are consistent with the distinct regulatory requirements for CME of EGFR and TfR, described in section 1.3.2 (i.e. EGFR requires epsin, eps15 and phosphatidic acid; while TfR requires AP2 and TTP) $)^{74,105,106,423}$. As such, these observations lead to the hypothesis that EGFR and TfR reside within distinct sub-populations of clathrin structures, each with distinct functional requirements, and hence, regulation by PLC- $\gamma 1^{404,423}$.

To directly and systematically probe whether EGFR and TfR localize to distinct CCP structures, we used RPE cells stably expressing clathrin light chain fused to enhanced green fluorescent protein (eGFP) (eGFP-CLCa), which allows direct visualization of clathrin-containing structures identified by microscopic imaging. We then labeled the cells with the fluorescently conjugated ligands rhodamineEGF and A647-Tfn, which respectively binds to EGFR and TfR; labeled for 5 minutes followed by fixation and subsequent imaging via TIRF-M (Figure 3.11). Using TIRF-M, we observed diffraction-limited clathrin structures on the cell surface as reported by eGFP-CLCa, along with their respective cargo content including EGFR or TfR (reported by rhodamine-EGF and A647-Tfn, respectively; Figure 3.11A). Here I use the term clathrin-labeled structures (CLSs) for clathrin structures observed or detected within single images (frames); it is important to note that identification of bona fide CCPs requires additional analysis of CLS behaviour over time that is possible only when examining live-cell images ${ }^{5,79}$. The set of CLSs includes CCPs and as well as other clathrin structures such as subthreshold CLSs (sCLSs), of which the latter do not give rise to bona fide CCPs representing abortive endocytic structures described in section 1.3. $2^{5,79,404}$. Nevertheless, the images obtained demonstrate that CLSs harboring either EGFR or TfR were more predominant, in contrast to CLSs harboring both receptors which were rarer-suggesting that EGFR and TfR are largely localized within distinct endocytic structures (Figure 3.11A).

To quantitatively analyze the relationship between CLSs containing EGFR (EGF-positive; EGF+) and TfR (Tfn-positive; Tfn+), we subjected the obtained TIRF-M images to automated software detection and analysis described in $[5,6,80,84,106,107,137,207,405]$. Importantly, automated software analysis enabled the systematic, unbiased identification of CLSs, in addition to the quantitative measurement of fluorescent receptors contained therein ${ }^{5,8,207}$. To represent these data, we first plotted the distribution 
of fluorescent EGF and Tfn within all identified CLSs in a two-dimensional histogram (Figure 3.11B). Notably, this approach reveals an apparently mutually exclusive relationship between the content of EGF and Tfn within CLSs. To directly quantify the proportion of CLSs that contain EGFR (EGF+), TfR (Tfn+), or both receptors $(E G F+/ T f n+)$, we sorted the CLSs into cohorts by receptor content, using a systematic cut-off for classification of CLSs as positive for EGF or $\mathrm{Tfn}^{207}$. Importantly, this approach revealed that CLSs exclusively harboring either EGF or Tfn, were approximately three-fold more abundant than CLSs harboring both receptors (Figure 3.11C). In addition, using higher concentrations of rhodamine-EGF (50 $\mathrm{ng} / \mathrm{ml}$ ) increased the percentage of EGF+ CLSs, without altering the percentage of EGF+/Tfn+CLSs, indicating that the distinct localization EGFR within distinct CLSs is not a function of ligand concentration (Figure 3.11C). Collectively, these systematic analyses of the recruitment of EGF and Tfn ligands to CLSs indicates that EGFR and TfR largely reside in distinct endocytic structures, and thus may internalize through distinct CCPs.

\section{4- Summary of Chapter 3}

In this chapter, I demonstrated the role of PLC- $\gamma 1$ as a selective regulator of EGFR endocytosis. Specifically, I found that inhibition of PLC- $\gamma 1$-either broadly by chemical inhibition, or directly by genetic silencing-significantly reduces EGF ligand internalization in RPE cells. Furthermore, EGFR endocytosis occurs largely through CME in these cell lines even upon stimulation with high EGF doses, thereby indicating control of CME by PLC- $\gamma 1$. Importantly, the regulatory role of PLC- $\gamma 1$ on endocytosis appears limited to EGFR, as other CME cargoes including TfR were largely unaffected by PLC- $\gamma 1$ perturbations. As such, I concluded that PLC- $\gamma 1$ is a selective regulator of CME which mediates the internalization of a subset of cargoes including EGFR, but not TfR.

While knowledge regarding PLC- $\gamma 1$ control of endocytosis remain poorly investigated, several studies nonetheless have suggested the role of PLC- $p 1$ as a GEF for Dyn1, thought to promote Dyn1 activation and hence, augmentation of CME in neuronal cells ${ }^{400}$. Nonetheless, the requirement for PLC$\gamma 1$ in EGFR endocytosis was demonstrated to function in a Dyn1-independent manner, due to the unperturbed internalization of EGF under Dyn1-silenced conditions. Indeed, EGFR in RPE cells is primarily endocytosed by CME in a Dyn2-dependent manner, which to date has not been shown to be directly regulated by PLC- $\gamma 1^{207}$. As a phosphodiesterase enzyme, PLC- $\gamma 1$ directly catalyzes the hydrolysis 
of $\mathrm{PIP}_{2}$, which is well-documented in several studies as an indispensable component of $\mathrm{CME}^{6,99,100}$. One possibility of the mechanism by which PLC- $\gamma 1$ may control endocytosis is by directly consuming PIP ${ }_{2}$. However, biochemical measurements of $\mathrm{PIP}_{2}$ concentrations and availability demonstrated a lack of appreciable reduction in $\mathrm{PIP}_{2}$ levels in both basal, and EGF stimulated conditions; highlighting that although a major pathway for PIP 2 turnover, PLC- $\gamma 1$ does not robustly alter $\mathrm{PIP}_{2}$. This indicates that any EGF-stimulated reduction in PIP 2 by PLC- $\gamma 1$ activation may not be sufficient to regulate EGFR endocytosis.

Numerous studies have demonstrated the role of calcium in enhancing CME in neurons during synaptic transmission. Specifically, calcium influx in neuronal synapses greatly enhances CME by promoting the activation of several endocytic components via CaN (see section 1.5.3) ${ }^{96}$. Interestingly, PLC $-\gamma 1$ similarly stimulates intracellular calcium increases via the activation and opening of $I P_{3} R$ calcium channels on the ER, mediated by its enzymatic $\mathrm{IP}_{3}$ by-product-suggesting its potential regulation of EGFR endocytosis via mobilization of calcium signals. Indeed, chelation of calcium or inhibition of $I P_{3} R$ opening impaired EGF internalization to a similar degree elicited by PLC- $\gamma 1$ silencing, indicating the requirement for PLCY1-derived calcium signals in EGFR endocytosis. Furthermore, the mechanistic control of EGFR endocytosis by calcium is partly distinct from that in neurons, independent of CaN activity as demonstrated by the lack of effect of CaN inhibition on EGFR internalization. Instead, calcium may act to regulate EGFR endocytosis by activating PKC, as its chemical inhibition phenocopied the reduction in EGF internalization observed with previous calcium perturbing conditions. Importantly, these treatments were without effect on Tfn internalization, further indicating the selective role of the PLCY1-calcium-PKC signaling axis in the distinct regulation of EGFR endocytosis (Figure 3.12).

The results in this chapter indicate that CME of EGFR and TfR are differentially regulated by PLC$\gamma 1$, consistent with previous studies demonstrating their unique requirements for endocytic regulatory proteins ${ }^{74,105,106,423}$. Together, these findings suggest that EGFR and TfR may be localized within different CCPs which may thus, be differentially regulated in a cargo-dependent manner. Indeed, I have thoroughly demonstrated the distinct localization of EGFR and TfR within distinct clathrin structures, further corroborating the selective role of PLC- $\gamma 1$ in CME regulation. Therefore, I conclude that the internalization of EGFR requires PLCY1-derived calcium signals, which although pleiotropic in nature, may selectively regulate only a subset of CME cargoes. Although PKC was implicated as a potential downstream calcium effector regulating EGFR endocytosis, the precise mechanistic implication of PLC $\gamma 1$ - 
derived calcium on CME dynamics nonetheless, remain to be elucidated, which will be explored in the following chapter. 


\section{5- Figures for Chapter 3}
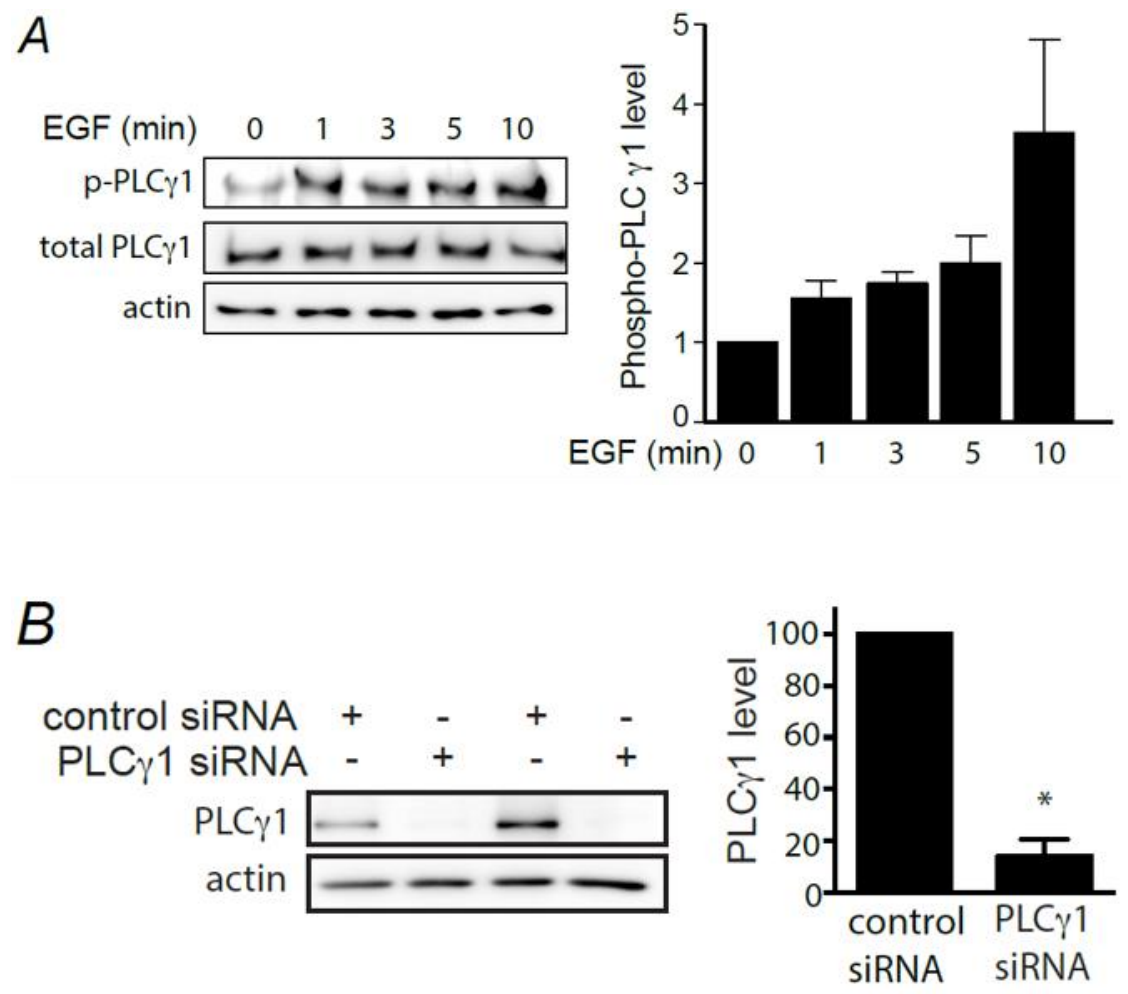

Figure 3.1. EGF-stimulated PLC- $\gamma 1$ activation and PLC- $\gamma 1$ silencing in RPE cells. (A) RPE cells were stimulated with $5 \mathrm{ng} / \mathrm{mL}$ EGF for 0-10 min (as indicated), followed by preparation of whole cell lysates and immunoblotting to detect phospho-PLC $\gamma 1$. Shown are representative immunoblots (left panels), as well as mean \pm SE of the phospho-PLCY1 signal intensity (right panels), $n=3$. (B) RPE cells were transfected with siRNA to silence PLC 1 or non-targeting siRNA (control). Whole cell lysates were resolved by western blotting and probed with anti-PLC 1 specific antibodies or anti-actin antibodies (loading control). Shown are representative immunoblots (left panel), as well as mean \pm SE of the PLC- -1 signal intensity (right panels), $\mathrm{n}=3,{ }^{*}, \mathrm{p}<0.05$. 


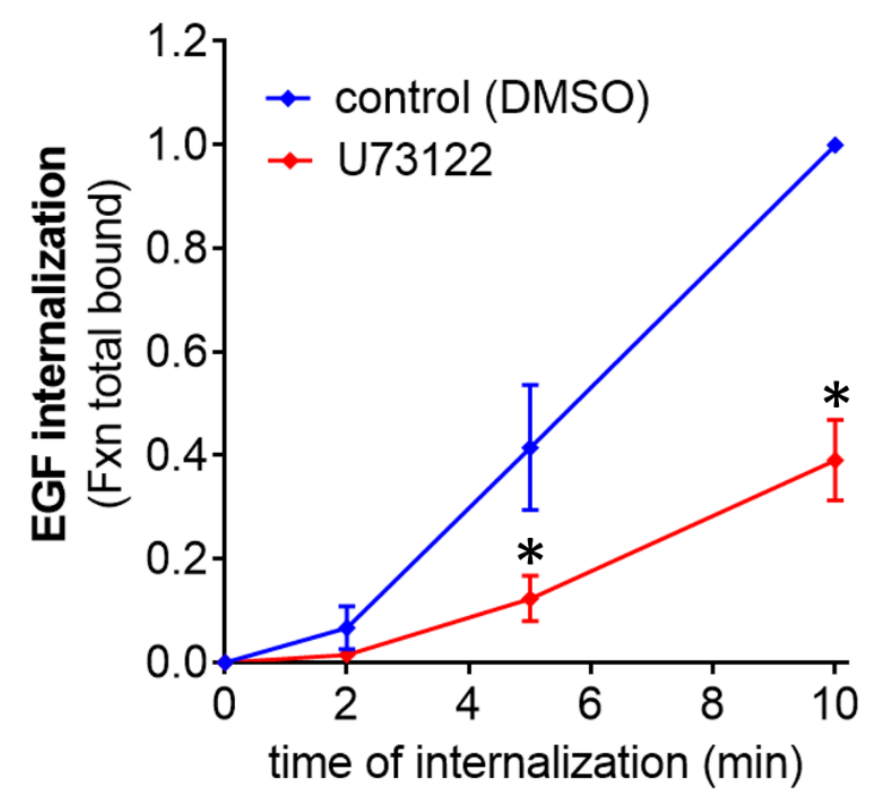

Figure 3.2. The pan inhibition of PLC by U73122 decreases EGF endocytosis. RPE cells were treated with

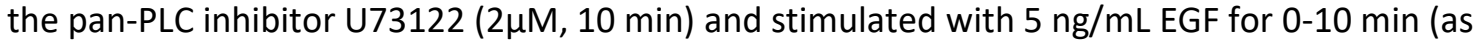
indicated). The amount of EGF internalization was quantified which was significantly reduced in the U73122 treated cells, compared to DMSO (control). Shown are mean \pm SE for $n=3$ independent experiments; ${ }^{*}, \mathrm{p}<0.05$. 

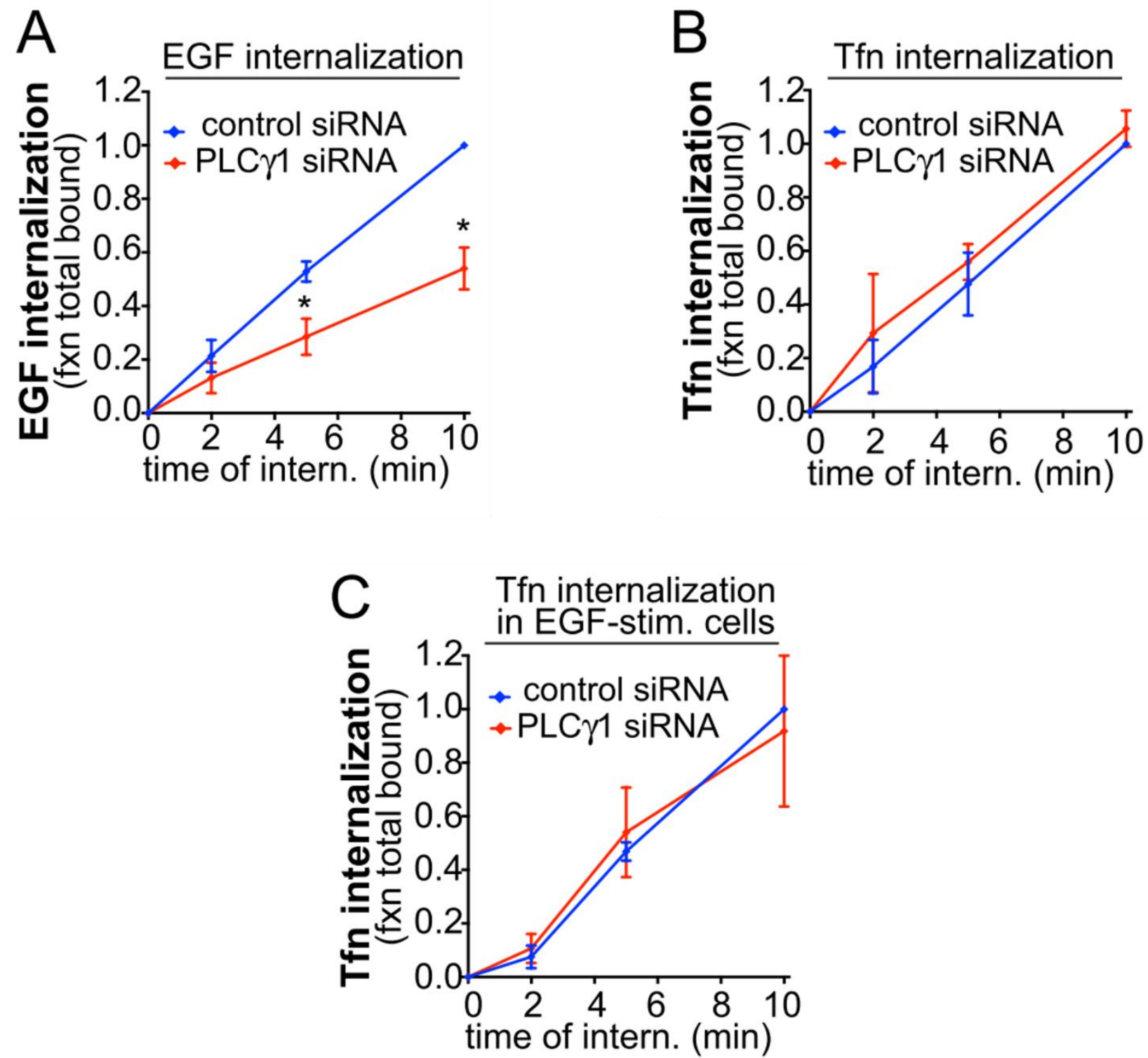

Figure 3.3. PLC- $p 1$ is required for EGF but not Tfn internalization. RPE cells were treated with siRNA targeting PLC- $\gamma 1$ or non-targeting siRNA, followed by measurement of EGF (A), Tfn (B), or Tfn internalization in EGF-stimulated cells (C). RPE cells were stimulated with $5 \mathrm{ng} / \mathrm{mL}$ EGF for 0-10 min (as indicated). Shown are the means \pm SE for $n>3$ independent experiments; ${ }^{*}, p<0.05$. 


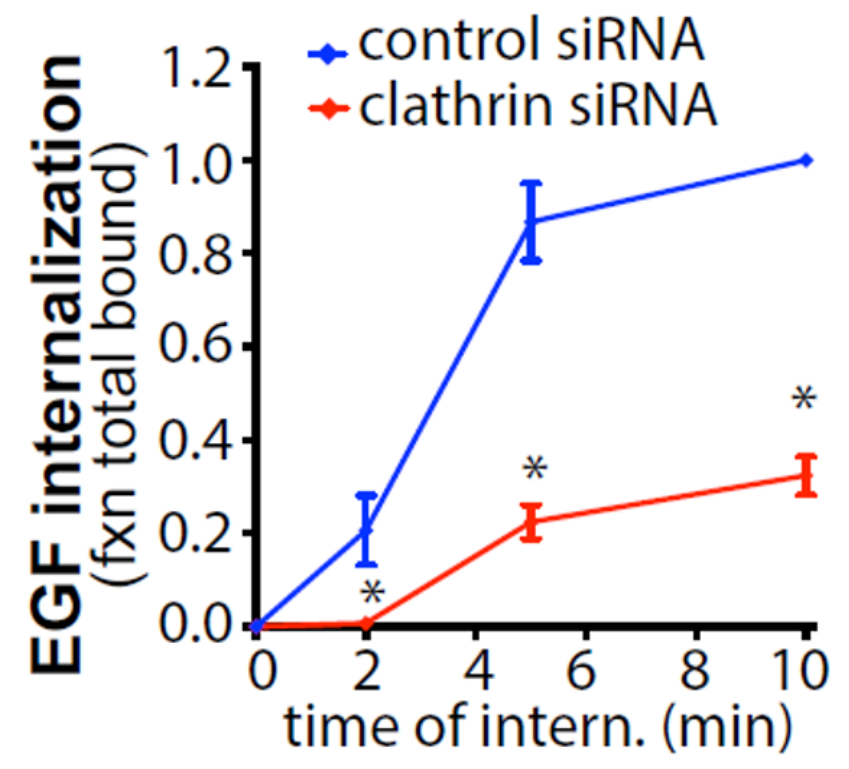

Figure 3.4. Silencing of clathrin broadly reduces EGF internalization. RPE cells were treated with siRNA to silence clathrin heavy chain (clathrin), or non-targeting siRNA (control). EGF internalization was measured at $100 \mathrm{ng} / \mathrm{mL}$ of biotin-Xx-EGF. Shown are the means \pm SE of 4 independent experiments. ${ }^{*}, p$ $<0.05$. While EGFR may internalize by non-clathrin mechanisms under some conditions (i.e. when stimulated with high doses of EGF in some cells ${ }^{409}$ ), these results indicate that EGF internalization is very largely clathrin-dependent in RPE cells, both at low dose of EGF (5 ng/mL, as per [4]) and at high doses $(100 \mathrm{ng} / \mathrm{mL})$ of EGF (this figure). 


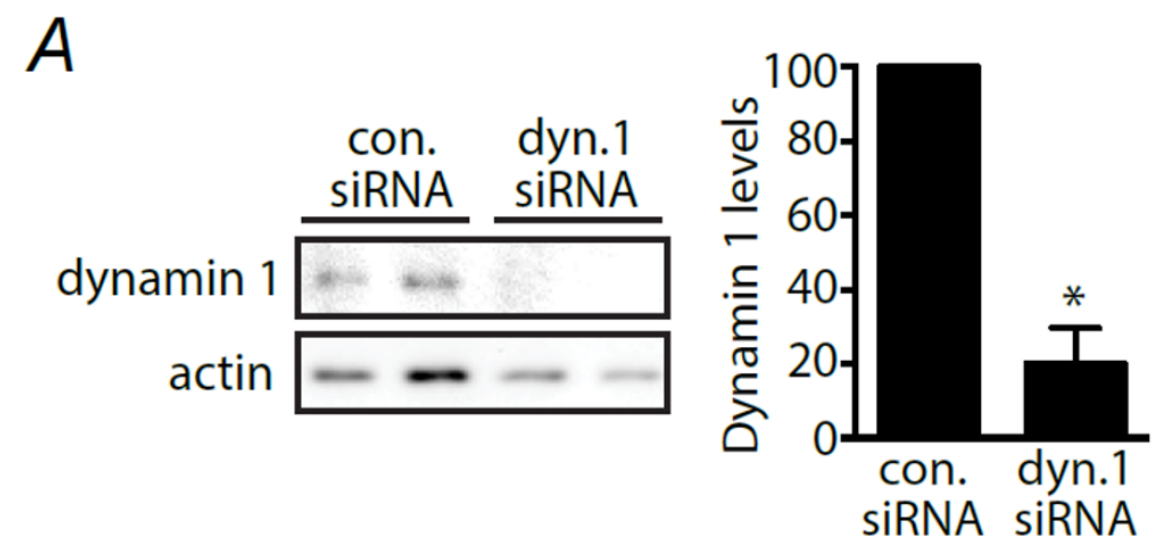

$B$

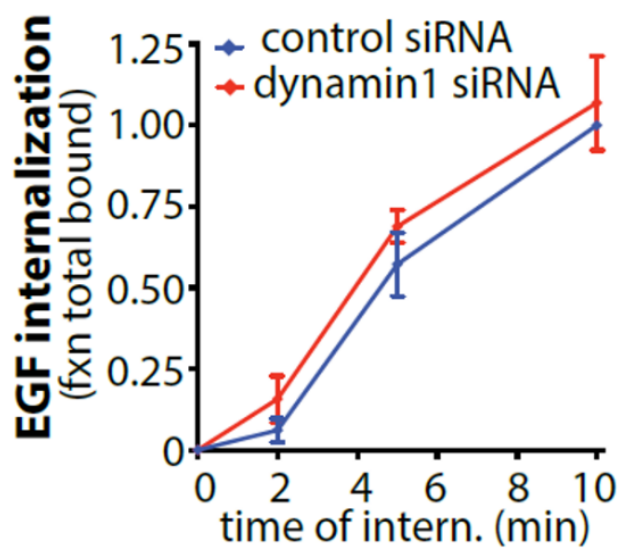

Figure 3.5. Silencing of Dyn1 has no effect on EGF internalization. RPE cells were treated with siRNA to silence Dyn1 (as per[400]), or non-targeting siRNA (control). (A) Whole cell lysates were resolved by western blotting and probed with anti-Dyn1 specific antibodies or anti-actin antibodies (loading control). Shown are representative immunoblots (left panel), as well as mean \pm SE of the Dyn1 signal intensity (right panels), $n=3, *, p<0.05$. (B) EGF internalization was measured (at $5 \mathrm{ng} / \mathrm{mL}$ EGF). Shown are the means \pm SE of 4 independent experiments in each condition. 

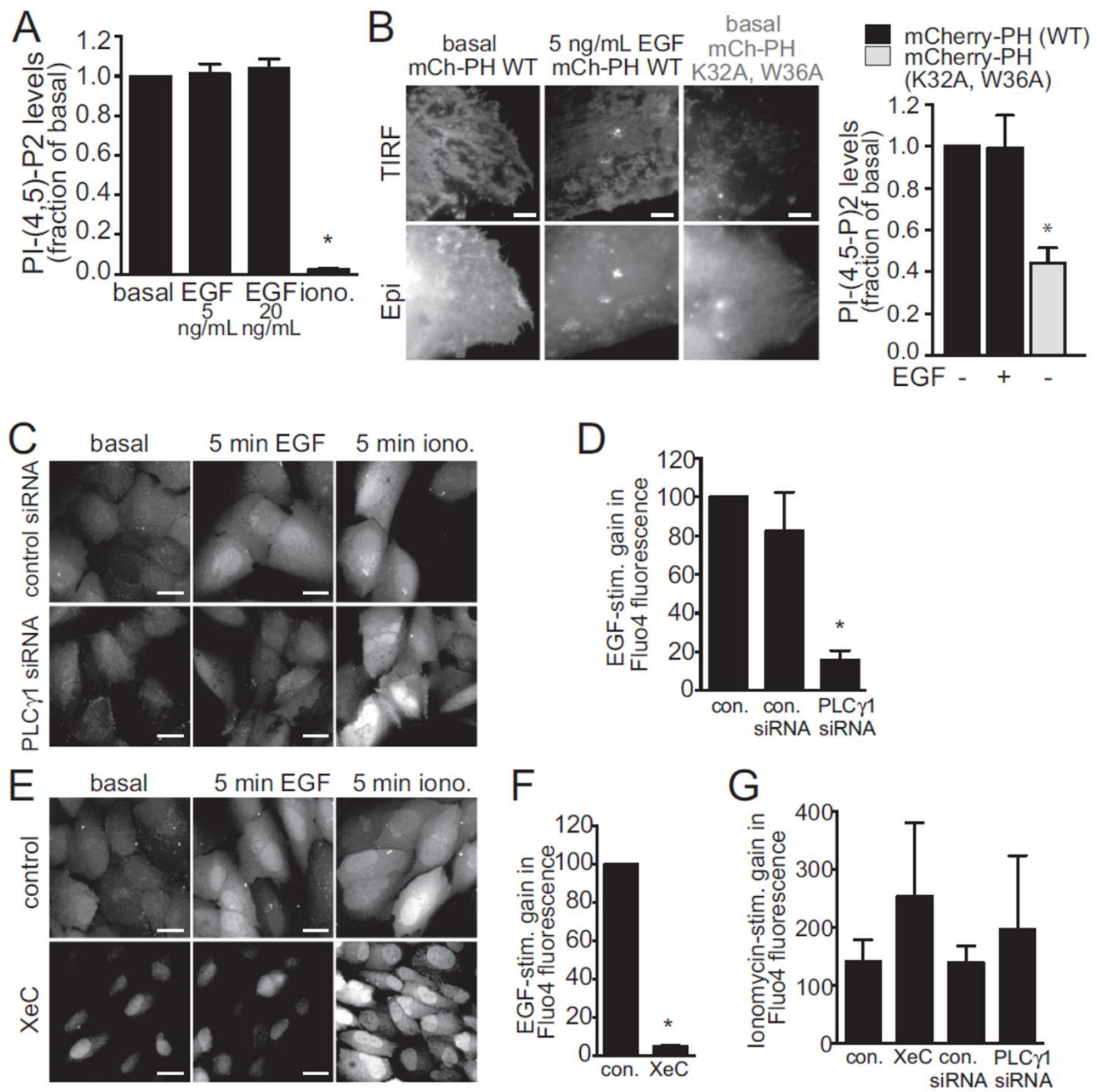

Figure 3.6. EGF stimulation elicits a PLC 1 1-dependent increase in cytosolic $\mathrm{Ca}^{2+}$. (A) Biochemical PIP measurement (means $\pm \mathrm{SE}$ ) in cells treated with $\mathrm{EGF}(5$ or $20 \mathrm{ng} / \mathrm{ml}, 5 \mathrm{~min}$ ) or ionomycin $(10 \mu \mathrm{M}, 20$ $\min$ ) as indicated. $\mathrm{n}=3 ;{ }^{*}, \mathrm{p}<0.05$ (Biochemical measurements performed by Dr. Leslie Bone; Ryerson University, Toronto, ON, Canada). (B) RPE cells transfected with cDNA encoding the wild-type PH domain of PLC- $\delta 1$ fused to mCherry (mCh-PH WT) or a similar fusion with a K32A/W36A mutant PH domain (unable to bind $\left.\mathrm{PIP}_{2}\right)^{6}$. Shown are representative micrographs obtained by wide-field epifluorescence or TIRF microscopy, as indicated and the means \pm SE of the TIRF/epifluorescence ratio of the mCherry signal, indicative of membrane binding by the PH probe. (C-G) RPE cells were treated with siRNA 
targeting PLC- $\gamma 1$ or non-targeting siRNA, or treated with $3 \mu \mathrm{M} \mathrm{XeC}$ for 30 min or left untreated (control). RPE cells were then treated with Fluo4-AM for $30 \mathrm{~min}$. Shown in C and E are representative images obtained by wide-field epifluorescence of Fluo4-AM obtained before further treatments (basal), after stimulation with $5 \mathrm{ng} / \mathrm{ml} \mathrm{EGF}$ for $5 \mathrm{~min}$, and then again after subsequent treatment with $10 \mu \mathrm{M}$ ionomycin for $5 \mathrm{~min}$, as indicated. Scale bars: $20 \mu \mathrm{m}$. Also shown are the EGF stimulated (D, F) or ionomycin triggered $(\mathrm{G})$ gains (means $\pm \mathrm{SE}$ ) in Fluo4-AM fluorescence within cells in the various conditions examined. $\mathrm{n}>3, *, \mathrm{p}<0.05$ (Fluo4-AM imaging performed by Dr. Monica Dayam; Ryerson University, Toronto, ON, Canada). 

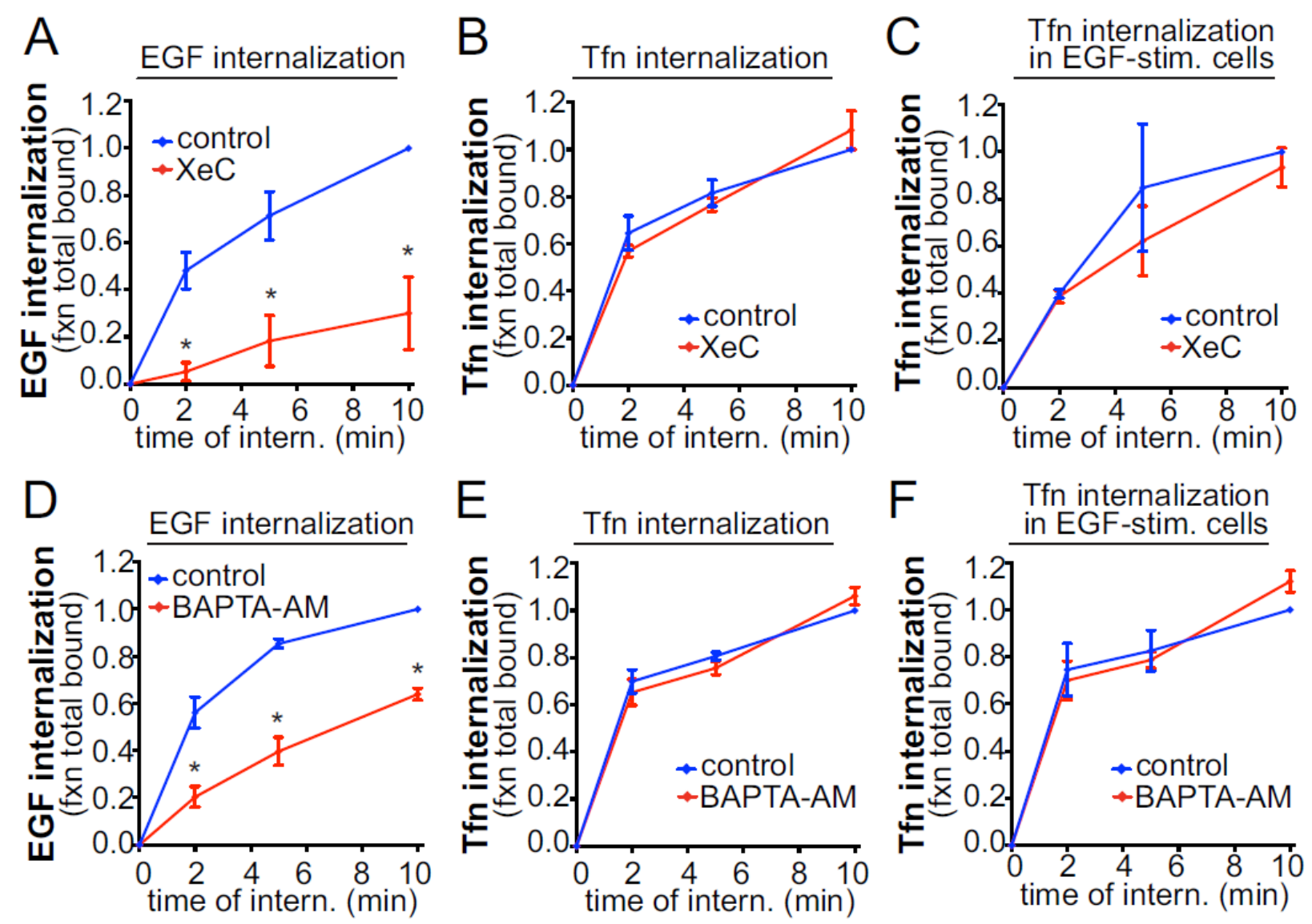

Figure 3.7. IP3 receptor and cytosolic Ca2+ are required for EGF but not Tfn internalization. RPE cells were treated with $3 \mu \mathrm{M} \mathrm{XeC}$ for $30 \mathrm{~min}, 10 \mu \mathrm{M}$ BAPTA-AM for $15 \mathrm{~min}$, or left untreated (control) as indicated, followed by measurement of EGF (A, D), Tfn (B, E) or Tfn internalization in EGF stimulated cells (C, F). RPE cells were stimulated with $5 \mathrm{ng} / \mathrm{mL}$ EGF for 0-10 min (as indicated). Shown are the means $\pm \mathrm{SE}$ for $\mathrm{n}>3$ independent experiments; ${ }^{*}, \mathrm{p}<0.05$. 


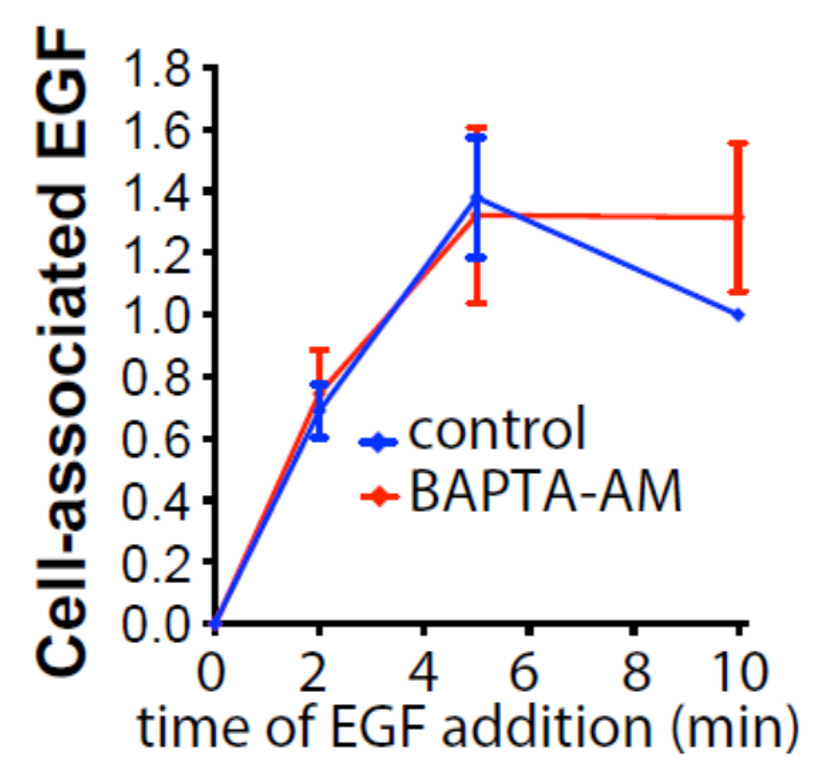

Figure 3.8. BAPTA-AM treatment does not affect EGF ligand binding to EGFR. RPE cells were treated with $10 \mu \mathrm{M}$ BAPTA-AM for $15 \mathrm{~min}$, then subjected to the measurement of cell-associated EGF (using EGF internalization assay modification that omits quenching of non-internalized, surface bound biotin-xxEGF, see Materials and Methods). RPE cells were stimulated with $5 \mathrm{ng} / \mathrm{mL}$ EGF for 0-10 min (as indicated). Perturbation of $\mathrm{Ca}^{2+}$ signaling does not impact the association of EGF ligand with EGFR at $37^{\circ} \mathrm{C}$. Shown are the means \pm SE for $n>3$ independent experiments. 

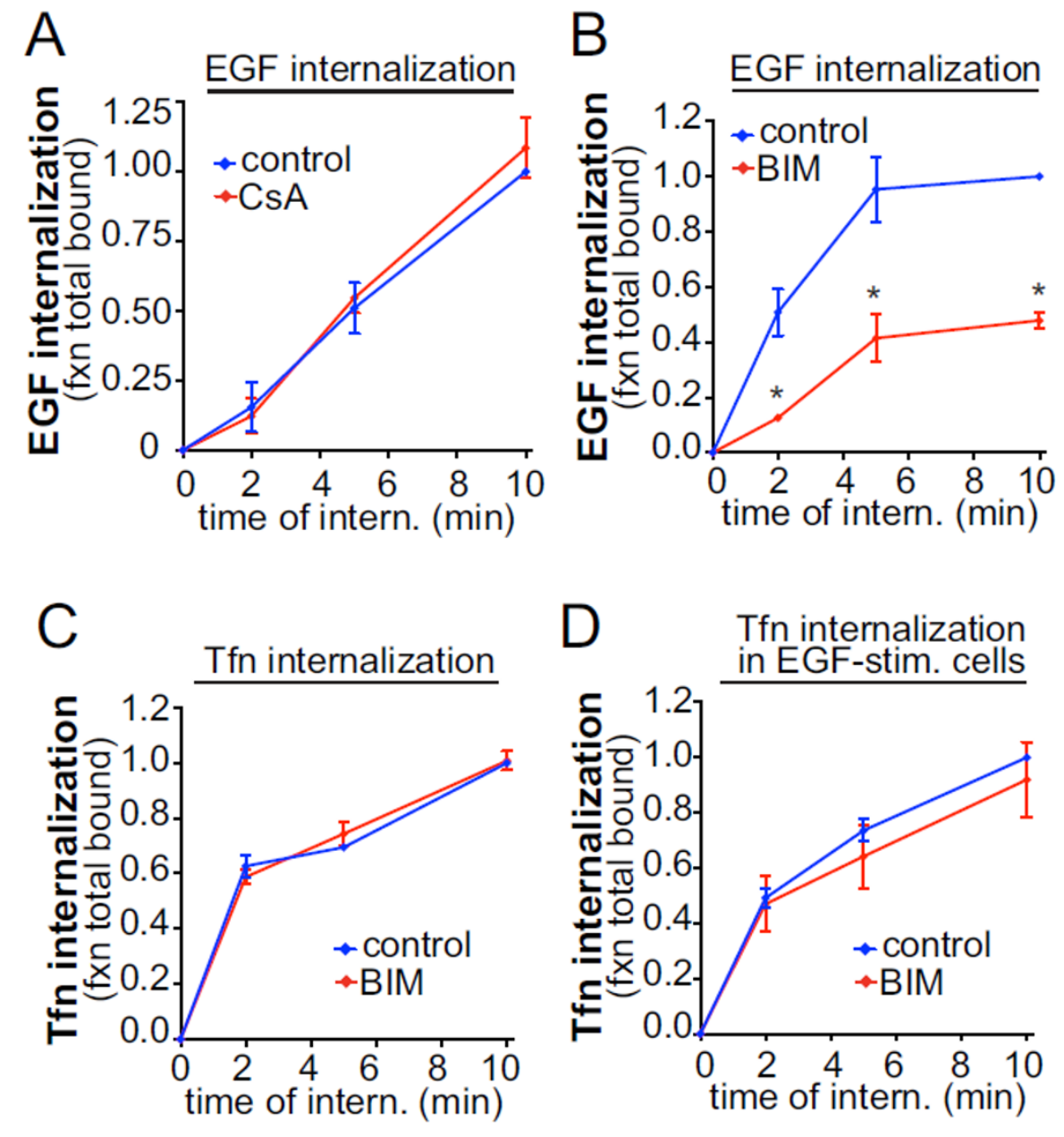

Figure 3.9. PKC, but not calcineurin, is selectively required for EGF internalization. RPE cells were treated with $10 \mu \mathrm{M}$ CsA for 30 min or $1 \mu \mathrm{M}$ BIM for 30 min, or left untreated (control), followed by measurement of EGF (A, B), Tfn (C), or Tfn internalization in EGF-stimulated cells (D). RPE cells were stimulated with $5 \mathrm{ng} / \mathrm{mL}$ EGF for 0-10 min (as indicated). Shown are the means $\pm \mathrm{SE}$ for $\mathrm{n}>3$ independent experiments; ${ }^{*}, \mathrm{p}<0.05$. 


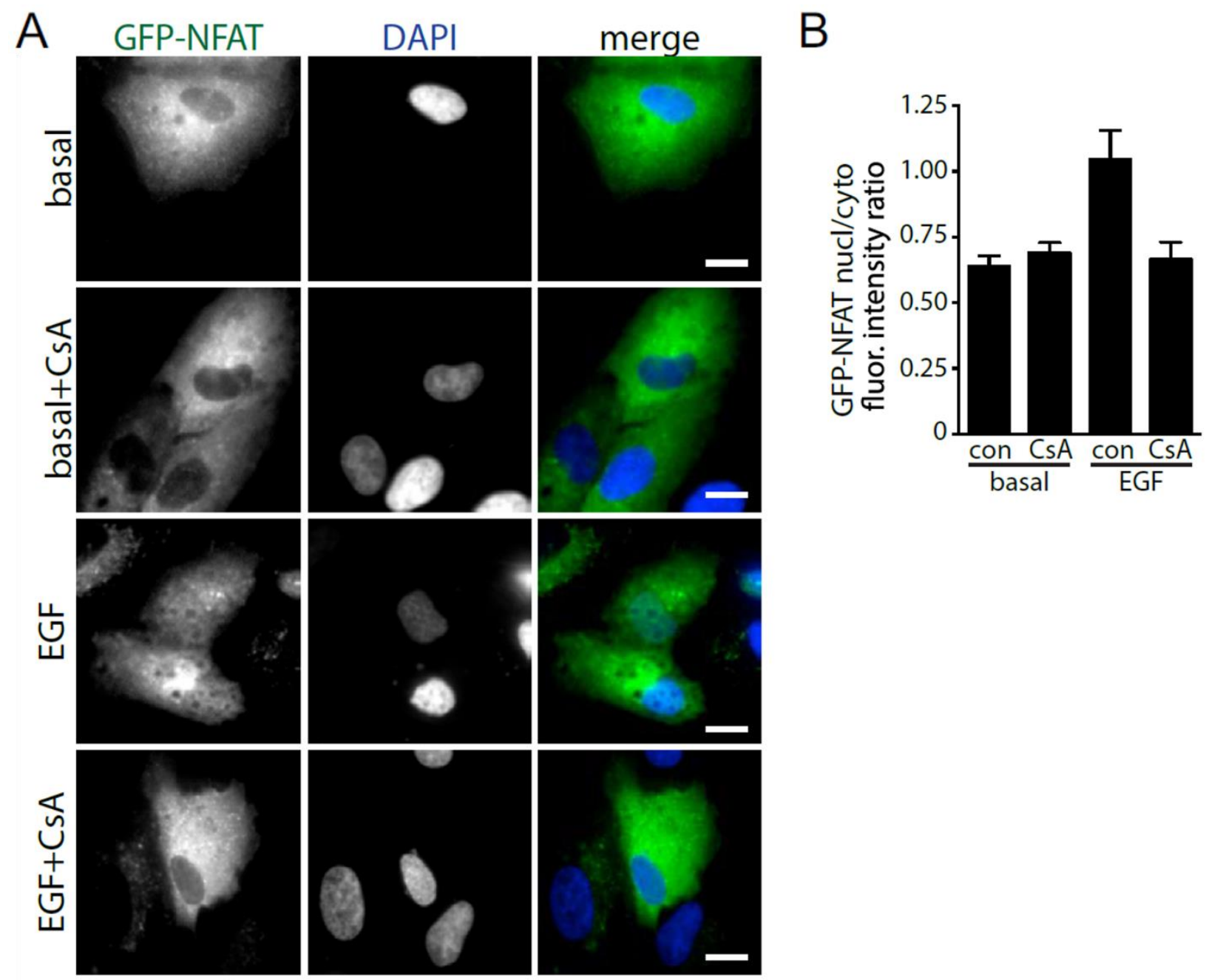

Figure 3.10. Cyclosporin A effectively perturbs calcineurin in RPE cells. NFAT undergoes calcineurindependent nuclear translocation in response to a gain in cytosolic calcium ${ }^{396}$. RPE cells were transfected with cDNA encoding GFP-NFAT, treated with $10 \mu \mathrm{M}$ cyclosporin A (CsA) for $30 \mathrm{~min}$, then treated with 5 $\mathrm{ng} / \mathrm{mL}$ EGF for $5 \mathrm{~min}$, as indicated. Shown in (A) are representative GFP-NFAT and DAPI epifluorescence images obtained using a Leica DM5000 B epifluorescence microscope using a DFC350FX camera (Leica Microsystems, Wetzlar, Germany), Scale bars: $20 \mu \mathrm{m}$, and in (B) the mean \pm SE of cellular nuclear/cytoplasmic GFP-NFAT fluorescence ratio. 

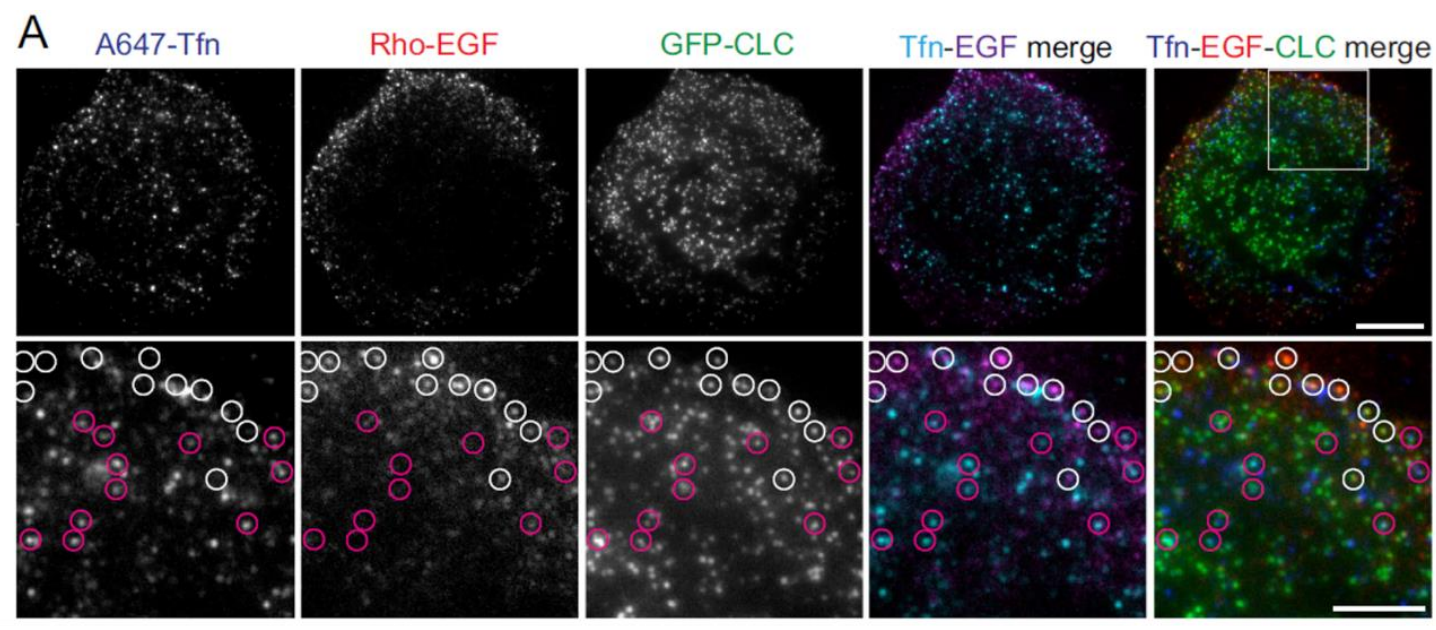

B

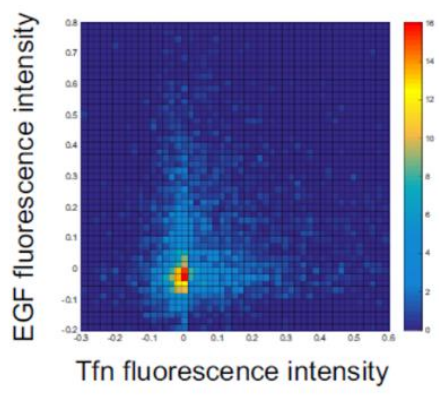

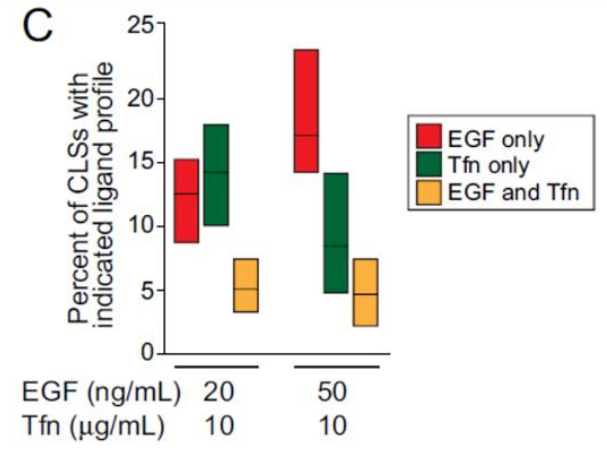

Figure 3.11. EGF and Tfn are recruited to largely distinct CLSs. RPE cells stably expressing clathrin light chain fused to eGFP (eGFP-CLCa) were treated with $20 \mathrm{ng} / \mathrm{ml}$ rhodamine-EGF (Rho-EGF) and $10 \mu \mathrm{g} / \mathrm{ml}$ A647-Tfn for $5 \mathrm{~min}$ or the indicated time, followed by immediate fixation. (A) Shown are representative micrographs obtained by TIRF-M. Scale bars: $10 \mu \mathrm{m}$ (top row); $5 \mu \mathrm{m}$ (bottom row, corresponding to enlarged images of the region shown in the merged image of the top row). White circles depict CLSs that are positive for EGF but devoid of Tfn, and purple circles depict CLSs that are positive for Tfn but not EGF. (B, C) CLSs were subjected to automated detection and analysis as described in Materials and Methods. Shown in (B) is a two-dimensional histogram of the normalized EGF and Tfn fluorescence intensities in each CLS cohort. Shown in (C) are median (bar) and 25th/75th percentiles (boxes) of the proportions of CLSs that are positive for EGF (but not Tfn), Tfn (but not EGF), or both EGF and Tfn. The number of CLSs and cells analyzed, respectively, for each condition are as follows: $20 \mathrm{ng} / \mathrm{ml}$ rhodamineEGF and $10 \mu \mathrm{g} / \mathrm{ml} \mathrm{A647-Tfn:} \mathrm{70,124} \mathrm{and} \mathrm{57;} \mathrm{and} 50 \mathrm{ng} / \mathrm{ml}$ rhodamine-EGF and $10 \mu \mathrm{g} / \mathrm{ml} \mathrm{A647-Tfn:} \mathrm{46,}$ 240 and 37; from a minimum of three independent experiments in each condition. These experiments were performed by Stefanie Lucarelli (PhD. Candidate); Ryerson University, Toronto, ON, Canada. 


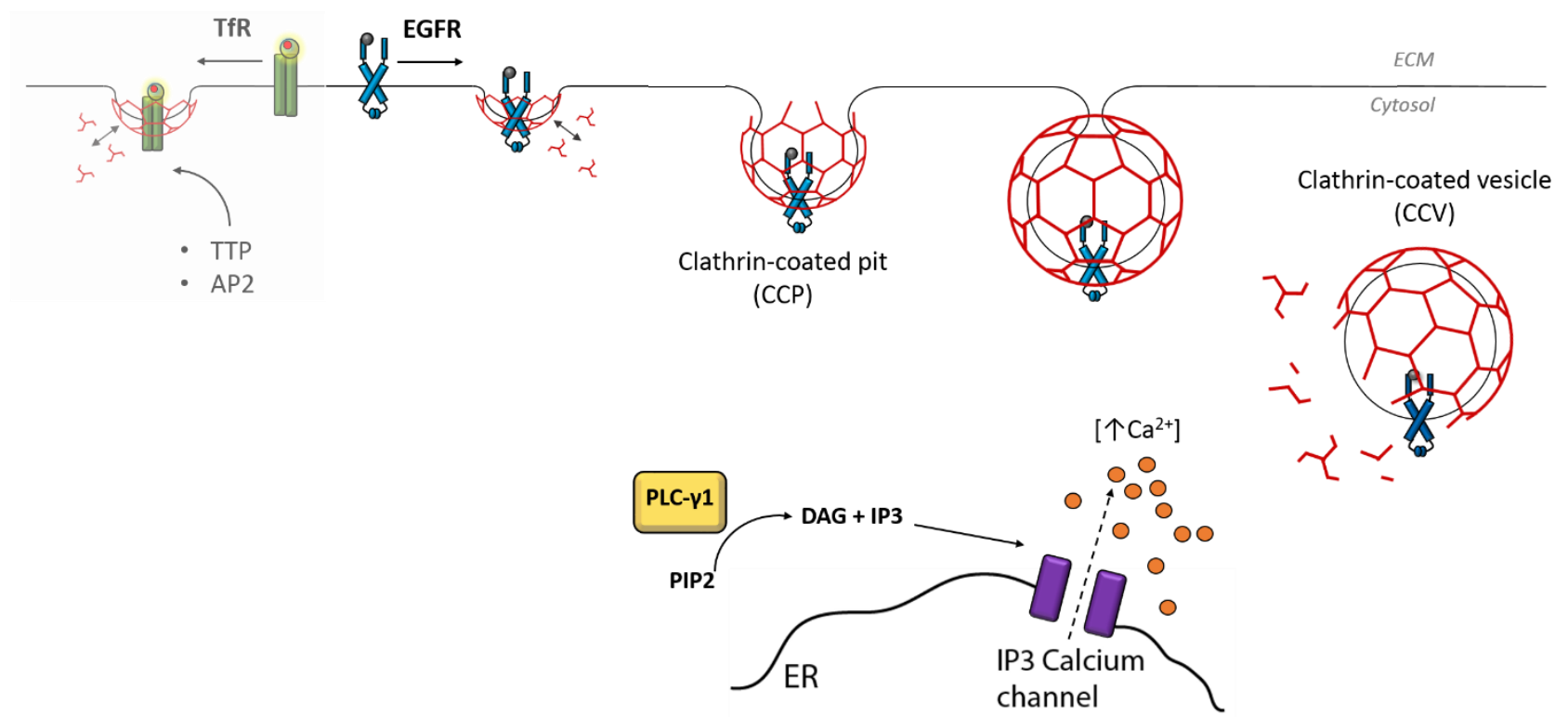

Figure 3.12. PLCY1-derived calcium signaling is selectively required for the internalization of EGFR but not TfR. Shown is the schematic diagram summarizing the findings described in chapter 3. EGF stimulation induces the activation of PLC- $\gamma 1$ which in turn, enzymatically cleaves $\mathrm{PIP}_{2}$ producing the secondary by-products $D A G$ and $I_{3}$. IP $P_{3}$ is a cytosolic secondary messenger which binds its cognate $I_{3}$ receptor on the endoplasmic reticulum. Binding of $\mathrm{IP}_{3}$ subsequently triggers the opening of the $I \mathrm{P}_{3}$ receptor causing the release of the endoplasmic reticulum calcium stores, thereby increasing cytosolic calcium concentrations. Our findings show that PLC 1-derived calcium signals are selectively required for the clathrin-mediated endocytosis of EGFR. In contrast, the clathrin-mediated endocytosis of TfRcontaining clathrin structures is not regulated by PLC 1 1-derived calcium signals, but instead requires other endocytic accessory proteins including $\mathrm{TTP}^{423}$. 
Chapter 4. Understanding how calcium signals impact CME dynamics 
$\mathrm{CME}$ is the major endocytic route in mammalian cells which regulates the plasma membrane by internalization of surface cargo via clathrin-containing structures. Emphasized in section 1.3.2, CME is defined by five distinct stages of which progressions are mediated by functionally diverse endocytic components ${ }^{50}$. The initial i) nucleation and initiation stage for instance, is responsible for spatially defining areas of the plasma membrane to be endocytosed, mediated by the pioneer proteins including $\mathrm{FCHo} 1 / 2$, intersectin, epsin and $\mathrm{AP2} 2^{72,76}$. Cargo molecules are then recruited to endocytic sites by regulatory adaptors which in addition to AP2, may include distinct accessory proteins based on cargospecific requirements for endocytosis (e.g. epsin, Dab2 and ARH, $\beta$-arrestin, and TTP; for EGFR, LDLR, GPCR, and TfR, respectively) $)^{74,105,106,423}$. The ii) recruitment of cargo represents an endocytic checkpoint of which accumulation above a specific concentration threshold, is essential for the stabilization and assembly of bona fide $\mathrm{CCPs}^{80}$. The subsequent iii) assembly and maturation of CCPs is further subjected to regulation of which progression depend on the appropriate recruitment of clathrin, endocytic accessory proteins, and specific regulation of $\mathrm{PIP}_{2}$ by $\operatorname{Sjn} 1^{6,139}$. Described in section 1.3.5.2, Sjn1 is an enzyme that converts $\mathrm{PIP}_{2}$ into PI4P which may be found present within CCPs throughout their lifetime. As such, Sjn1 may be hypothesized as a negative regulator of CCP assembly, of which turnover of PIP 2 may destabilize endocytic structures (i.e. cargo-deficient CCPs) ${ }^{6,139}$. Upon maturation, CCPs are then iv) subjected to scission from the plasma membrane forming intracellular CCVs via a dynamin-dependent mechanism, involving various membrane bending/deforming components (e.g. BAR domain proteins)which are similarly subjected to further regulation (i.e. by $\mathrm{PIP}_{2}$ and calcium) ${ }^{71}$. Lastly, CCVs are v) uncoated of clathrin by an auxilin- and Hsc70-based mechanism, followed by membrane traffic and delivery of the naked vesicle to target organelles ${ }^{50}$. Thus, despite its conceptually simple depiction, CME is a highly complex process of which progression can be defined by the dynamic, multi-layer regulation of its morphologically distinct endocytic stages.

In the previous chapter, I uncover the novel role of PLC- $\gamma 1$ in regulating CME of EGFR. Specifically, I showed that internalization of EGFR requires PLC 1 1-derived calcium signals, of which perturbations significantly impairs EGF ligand endocytosis. Calcium activates various downstream protein effectors including PKC, which I similarly demonstrate to be a potential component of the requirement for PLC- $\gamma 1$ in EGFR internalization. Moreover, I found that the PLC 1 1-calcium-PKC signaling axis is selectively required for the internalization of EGFR, but not other endocytic cargoes including TfR, which I found localized within spatially distinct clathrin-containing structures. The selective control of EGFR endocytosis thus, imply the distinct regulation of EGFR-containing CCPs by PLC- $\gamma 1$-raising the 
question of whether and how PLCY1-derived calcium signals may mechanistically regulate CME dynamics in facilitating the specific internalization of EGFR.

As described above, CME proceeds through distinct morphological stages of which respective regulations may thus, be susceptible to calcium control. Calcium has been implicated in the direct regulation of clathrin, endocytic adaptors (e.g. AP2 and Dab2) and other accessory proteins (e.g. Sjn1, intersection, eps15, amphiphysin, Dyn2), indicating the potential control of CME dynamics by PLCY1derived calcium signals ${ }^{50,110,205}$. In this chapter, I determine whether PLC $\gamma 1$-derived calcium signals regulate EGFR endocytosis by regulation of the distinct stages governing its CME. To assess the functional contribution of PLCY1-derived calcium in CME of EGFR, I employ live-cell imaging techniques coupled to software tracking and analysis of clathrin structures. Using this strategy, I detected bona-fide EGFR-containing CCPs and monitored their endocytic progression under various calcium signaling perturbation conditions. Importantly, this method will allow delineation of the stage(s) of CME regulated by calcium (i.e. cargo recruitment, clathrin assembly and/or maturation), and hence, identify how PLCY1-derived calcium signals may precisely regulate EGFR endocytosis. I hypothesize that PLCY1derived calcium signals regulate the efficient assembly and maturation of EGFR-containing CCPs, through control of resident endocytic proteins sensitive to calcium signals.

\section{1- EGFR-derived calcium signals regulate CCP initiation and assembly} Having demonstrated the localization of EGFR and TfR within spatially distinct CLSs, I next examined how CLSs harboring EGFR or TfR may be distinctly regulated by PLC 1 1-derived calcium signals. The selective regulation of EGF+ CCPs may reflect in part the distinct regulation by calcium of EGFR recruitment to CLSs, or calcium control of subsequent endocytic steps such as nucleation, initiation, assembly, maturation or scission subsequent to receptor recruitment therein ${ }^{5,6,79,106,207,404}$.

\subsection{1 - PLC- $\gamma 1$ does not regulate the recruitment of EGFR within CLSS} In understanding the precise role of calcium signals in regulation of EGFR endocytosis, I first sought to determine its functional role in control of receptor recruitment within CLSs. To do so, I treated 
RPE cells expressing eGFP-CLCa with fluorescent EGF (A555-EGF; Figure 4.1, A, C, and D, and 4.2) and Tfn (A555-Tfn, Figure 4.1, B, E, and F, and 4.2), followed by fixation and imaging via TIRF-M; as performed in section 3.3. Single frame images were obtained and subjected to automated software detection and analysis of CLSs ${ }^{5,7,207}$. Using this strategy, EGF+ and Tfn+ CLSs (i.e. colocalization of fluorescent ligands with eGFP-CLCa) were identified and systematically quantified under PLC- $\gamma 1$ perturbing conditions (e.g. XeC, BAPTA-AM and BIM). Strikingly, treatment of cells with XeC, BAPTA-AM and BIM, each resulted in an increase in EGF+ CLSs relative to control, indicating that PLC 1 1-derived calcium signals is not required for the efficient recruitment of EGFR within endocytic structures (Figure 4.1C). Instead, PLC- $\gamma 1$ may regulate EGFR endocytosis by control of the subsequent endocytic stages following receptor recruitment, of which impairment under the PLC 1 1-perturbed conditions can be implied from the resulting accumulation of detected EGF+ CLSs on the cell surface. Importantly and consistent with ligand-uptake experiments (Figures 3.7 and 3.9), XeC, BAPTA-AM, and BIM treatments did not impact Tfn recruitment to CLSs (Figure 4.1E), showing that the effect of PLC 1 1-calcium-PKC signaling is selective for EGF+ CLSs. As such, regulation of EGFR endocytosis by PLCY1-derived calcium may involve direct control of EGFR clathrin-mediated endocytosis dynamics, independent and subsequent to recruitment of receptors within endocytic sites.

To broadly understand whether PLC- $\gamma 1$ may directly control CME dynamics in regulation of EGFR endocytosis, I subjected the obtained TIRF-M single frame images to systematic analysis of detected EGF+ CLSs. Specifically, the fluorescence intensities of eGFP-CLCa were quantified which provides measurement of clathrin recruitment and assembly, and hence, overall endocytic productivity of detected CLSs. Notably, fluorescence intensity of clathrin in EGF+ CLSs were reduced by XeC, BAPTA-AM, and BIM treatments relative to control, suggesting the requirement for PLC $\gamma 1$-derived calcium signals in promoting clathrin assembly and maturation of CLSs harboring EGFR (Figure 4.1D). Consistently, these treatments were without effect on Tfn+CLSs, which produced comparable eGFP-CLCa fluorescence intensities relative to control (Figure 4.1F). Thus, the PLCY1-calcium-PKC signaling axis may selectively regulate EGFR endocytosis by controlling the process of CCP formation or assembly, independent of EGFR recruitment.

\subsection{2 - PLC- 1 1 regulates the assembly and maturation of EGFR-containing CCPs} The findings from the previous sections demonstrate a role for PLC- $p 1$ in controlling the endocytic dynamics of CLSs containing EGFR, indicating the requirement for PLC $\gamma 1$-derived calcium 
signals in regulation of the distinct endocytic stages. To resolve how PLC- $\gamma 1$ may regulate the nucleation, initiation, assembly, maturation, or scission of CCPs harboring EGFR, I similarly used the previous TIRF-M imaging techniques coupled to automated software detection of CLSs. Importantly, assessment of the distinct endocytic stages requires temporal analysis of CLSs, of which discrete endocytic progression and dynamics may be monitored only by live-cell imaging. Thus, in contrast to previous acquisitions of single frame images of fixed cells, the TIRF-M imaging technique was fitted to capture time-lapse images of live cells, to unbiasedly detect, track and analyze the endocytic dynamics and progression of CLSs ${ }^{5,79,404}$.

Using this approach, I examined RPE cells expressing eGFP-CLCa treated (or not) with BAPTA$A M$, and imaged under the presence of either fluorescent EGF (rhodamine-EGF) or Tfn (A647-Tfn) ligand; the latter in the presence of unlabeled EGF to trigger calcium signaling (Figure 4.3, A and B). Notably, our computational tools allow the detection of clathrin structures harboring specific receptors in twocolor time lapse-image series, based on the presence of a secondary signal within these structures (i.e. presence of secondary ligand fluorescence within detected eGFP-CLCa structures) $)^{5}$. As such, this method allows the selective analysis of EGF+ or Tfn+CLSs in these time-lapse image series. Moreover, this strategy allows further resolution of each CLS as either a sCLS or a bona fide CCP ${ }^{5,79,404}$. Briefly, sCLSs are quantitatively and systematically distinguished from CCPs by the fact that sCLSs fail to meet a minimum clathrin-intensity threshold (reported by eGFP-CLCa) within the early stages of formation, and likely represent either stochastic assembly of clathrin at the plasma membrane or early nucleation events that fail to proceed to formation of bona fide $\mathrm{CCPs}^{5,79,404}$. In contrast, bona fide CCPs represent CLSs that stabilize, assemble, mature, and in some cases, undergo scission from the plasma membrane to produce intracellular CCVs; representing productive endocytic structures described in section 1.3.2.

With this analysis strategy, I found that inhibition of PLC 1 1-derived calcium signals by BAPTA-AM treatment substantially and significantly increased the number of EGF+ sCLSs (which are dim and shortlived), indicating impairments in clathrin assembly and maturation of EGFR-containing clathrin structures (Figure 4.3C) ${ }^{5,79,404}$. In addition, the increase in EGF+ sCLS density is complemented by a reduction in bona fide EGF+ CCPs (longer-lived clathrin structures which grow past an eGFP-CLCa threshold intensity), further corroborating the requirement for PLCY1-derived calcium signals in the efficient formation of EGFR-containing CCPs (Figure 4.3D) ${ }^{79}$. These results therefore suggest that calcium signals control the transition of CLSs from an early nucleation stage, to initiation and subsequent assembly into bona fide CCPs. 
That EGFR endocytosis is maintained to some extent despite perturbations of PLC 1 1-derived calcium signals (Figures 3.7 and 3.9) implies the persistence and formation of bona fide EGFR-containing CCPs capable of internalization, which although limited, were indeed detected as shown by findings in the previous sections (Figure 4.3D). Following their detection, I then sought to determine whether the persisting EGF+ CCPs are to any degree, regulated by PLC 1 1-derived calcium signals. To do so, I employed time-lapse tracking of CLSs which enable detection of bona fide CCPs; representing CLSs that recruit clathrin above a certain threshold and exhibit longer lifetimes on the cell surface relative to $\mathrm{sCLSs}^{79}$. Expectedly, detection and tracking of bona fide CCPs in RPE cells reveal heterogeneity amongst CCP lifetimes, attesting to the heterogeneic nature of CCP formation described in section 1.3.2. Lifetimes of detected CCPs for instance, ranged from 10 to 100 seconds, which were respectively grouped into lifetime cohorts to enable unbiased analysis and comparison of CCPs. The mean fluorescence of clathrin (eGFP-CLCa) within CCPs of respective lifetime cohorts were then quantified in both control and BAPTAAM treated cells, and compared to determine the impact of calcium perturbation on the assembly dynamics of bona fide CCPs. Interestingly, BAPTA-AM treatment decreased the size of bona fide EGFRcontaining CCPs consistently within each lifetime cohort, as indicated by the reduction in mean fluorescence of eGFP-CLCa within EGF+ CCPs, relative to control (Figure 4.3E). Thus, these results indicate that although bona fide EGF+ CCPs form under calcium perturbed conditions, these structures nonetheless exhibit deficiencies in clathrin recruitment and assembly, further highlighting the requirement for PLCY1-derived calcium signals in the efficient CME of EGFR.

In line with the selective regulatory role of PLC- $\gamma 1$ on endocytosis, perturbation of calcium by BAPTA-AM treatment did not affect the initiation rate and density of $\mathrm{Tfn}+\mathrm{sCLSs}$ or bona fide Tfn+CCPs, in contrast to the effects on EGF+ CLSs (Figure 4.3, F and G). Furthermore, BAPTA-AM treatment also did not appreciably alter the size of bona fide TfR-containing CCPs across various lifetime cohorts (Figure 4.3H), consistent with the lack of regulation of TfR endocytosis by PLC 1 1-derived calcium signals (Figures 3.7 and 3.9). As such, PLCY1-derived calcium signals selectively regulate CME of EGFR, but not TfR, via control of CCP initiation and assembly.

Our strategy of live-cell detection and tracking of CLSs enabled identification of bona fide EGF+ and Tfn+ CCPs, which exhibit characteristic gains in eGFP-CLCa fluorescence during early stages of pit formation, and subsequent fluorescence decays during vesicle scission and internalization away from the TIRF field. Importantly, to determine whether detected CCPs were indeed endocytic structures, I 
similarly tracked the fluorescence profile of ligands (rhodamine-EGF or A647-Tfn) contained therein. As expected, EGF or Tfn detected within CCPs similarly demonstrate a characteristic gain and decay in fluorescence, which are largely concomitant with eGFP-CLCa indicating that detected CCPs indeed, give rise to productive endocytic vesicles (Figure 4.4, A and B; 4.5, A and B). As well, the concomitant gain and decay of clathrin and ligand fluorescence, is largely observed particularly for CCPs having lifetimes $<80$ s, which represents the majority of detected structures - further attesting to the endocytic nature of detected CCPs (Figure 4.4C; 4.5C).

Collectively, these results indicate that PLCY1-derived calcium signals are selectively required for the efficient CME of EGFR. In particular, I showed that calcium regulates the initiation and assembly of bona fide EGFR-containing CCPs following clathrin structure nucleation, of which perturbation by BAPTA-AM significantly enhances the frequency of EGF+ SCLSs; while decreasing the density of EGF+ CCPs. In addition, BAPTA-AM treatment reduces the size of bona fide EGF+ CCPs relative to control, indicating the role of calcium in facilitating the efficient assembly of EGFR-containing clathrin structures. Importantly, calcium signals were shown dispensable for the initiation and assembly of CCPs harboring TfR, consistent with the selective regulatory role of PLC- $\nu 1$ on EGFR clathrin-mediated endocytosis. Taken together with the observation that perturbation of calcium signals enhances the abundance of EGFR within all CLSs (Figure 4.3C), these results indicate that calcium signals do not impact recruitment of EGFR to clathrin structures, but are required for initiation and assembly of bona fide CCPs containing EGFR from nascent clathrin structures.

\section{2- PLC- $y 1$ regulates EGFR-containing CCPs by removal of Sjn1}

Consistently emphasized throughout this thesis is the complex nature of CME, of which distinct morphological stages are dynamically regulated by functionally diverse endocytic components. The initiation, assembly, maturation, and scission stages of CCPs for instance, are facilitated and regulated by specific protein modules described in section 1.3.1 (i.e. pioneer, assembly and scission modules) ${ }^{50}$. Importantly, most, if not all endocytic components including endocytic accessory proteins, are organized around the three major CME hubs (clathrin, AP2 and $\mathrm{PIP}_{2}$ ), of which perturbations prove catastrophic to the overall endocytic process ${ }^{12,50,137}$. Highlighted in section 1.3.5.4, manipulations that impact $\mathrm{PIP}_{2}$ synthesis and turnover largely influence CCP dynamics, and hence CME, due to its role as a plasma 
membrane substrate for endocytic proteins. For instance, PIP5K is the major lipid kinase responsible for $\mathrm{PIP}_{2}$ synthesis of which overexpression increases the rate of CCP initiation and size relative to control ${ }^{6}$. Consistently, knockdown of PIP5K result in opposing effects, resulting in reduced initiation of CCPs which are smaller in size-likely due to reduced $\mathrm{PIP}_{2}$ synthesis ${ }^{6}$. Interestingly, the reduction in density and size of bona fide CCPs in PIP5K knockdown cells, mimic the findings from the previous sections of reduced EGF+ CCP initiation and size under calcium perturbed conditions (i.e. BAPTA-AM treatment) (Figure 4.3, D and E). The phenotypic similarities in CCP dynamics imposed by both PIP5K knockdown and calcium inhibition, thus, suggests the potential role of PLC 1 1-derived calcium signals in regulating $\mathrm{PIP}_{2}$ levels-in selectively controlling EGFR endocytosis.

Understanding precisely how PLC 1 1-derived calcium signals may control plasma membrane $\mathrm{PIP}_{2}$ levels in regulation of EGFR clathrin-mediated endocytosis, requires systematic profiling of lipid regulators, of which elucidation of distinct responses to calcium signals however, is beyond the scope of this work. Nonetheless, our findings in the previous chapter demonstrate that PLC- $\gamma 1$ activation-and hence, generation of calcium signals-do not appreciably modify global plasma membrane $\mathrm{PIP}_{2}$ concentrations (see section 3.2.1), indicating that the potential control of $\mathrm{PIP}_{2}$ by PLC $\gamma 1$-derived calcium signals may occur in a localized manner, such that within CCPs. In addition, that perturbation of calcium signals elicits impairments in CCP dynamics comparable to that resulting from reduction in $\mathrm{PIP}_{2}$ levels (i.e. PIP5K knockdown), suggests the potential role of PLCY1-derived calcium in sustaining appropriate $\mathrm{PIP}_{2}$ levels required for the efficient assembly and maturation of CCPs. Largely effecting localized PIP 2 turnover within CCPs, the lipid phosphatase Sjn1 therefore emerges as a potential downstream calcium effector, of which possible regulation by PLC $\gamma 1$-derived calcium signals may thus, directly control plasma membrane $\mathrm{PIP}_{2}$ - and hence, $\mathrm{CME}$.

In contrast to PIP5K, Sjn1 is a CCP-localized lipid phosphatase which negatively regulates CCP formation by conversion of $\mathrm{PIP}_{2}$ into $\mathrm{PI}_{4} \mathrm{P}^{6}$. Catalyzing $\mathrm{PIP}_{2}$ turnover, overexpression of Sjn1 significantly reduces the lifetime of abortive CCP structures, which described in section 1.3.5.2, may largely be due to its destabilizing role in CCP assembly ${ }^{6}$. Conversely, knockdown of Sjn1 expectedly increases the proportion of productive CCPs relative to abortive endocytic structures, likely due to the release of negative regulation imposed by $\operatorname{Sjn} 1$ on $\mathrm{CCPs}^{6}$. Although knowledge regarding $\operatorname{Sjn} 1$ control by calcium is lacking, it is tempting to hypothesize on the role of PLC 1 1-derived calcium signals in promoting EGFR clathrin-mediated endocytosis by either inhibition, or removal of Sjn1 from EGFR-containing CCPs; which 
in turn, relieves these structures of negative regulation thereby promoting their efficient assembly and maturation. In the following, I thus uncover whether PLC 1 1-derived calcium signals may directly regulate Sjn1 activity.

In uncovering whether PLCY1-derived calcium signals may directly regulate Sjn1, I transfected a genetically-encoded fluorescent Sjn1 construct (mCherry-tagged Sjn1; mCh-Sjn1) into eGFP-CLCa expressing RPE cells, which were subsequently imaged by TIRF-M (Figure 4.6A and 4.7) followed by systematic image analysis as performed in the previous sections. With this strategy, the abundance of mCh-Sjn1 within CLSs were quantified under various treatment conditions, stimulated or not (basal) with EGF: control (no treatment), BAPTA-AM and BIM (Figure 4.6B). Strikingly, in the no treatment condition, EGF stimulation resulted in the significant reduction of $\mathrm{mCh}-\mathrm{Sjn} 1$ abundance within CLSs, relative to basal conditions (Figure 4.6A and quantification in Figure 4.6B). Importantly, the respective perturbations of calcium and PKC, by BAPTA-AM and BIM, impaired the reduction of $\mathrm{mCh}$-Sjn1 within CLSs elicited by EGF stimulation. Thus, these results suggest the role of PLC 1 1-derived calcium signals, in regulating the dissociation of Sjn1 from endocytic structures - thereby relieving CCPs of negative regulation required for efficient CME (Figure 4.6B).

To further corroborate these findings, I similarly examined the behaviour of endogenous Sjn1 with anti-Sjn1 antibodies, under identical treatment conditions. Expectedly, I obtained similar results indicating that the observed localization and reduction of Sjn1 within CLSs, were indeed due to the activation of EGF signals (i.e. PLCY1-derived calcium signals) and not due to a transfection-based artefact

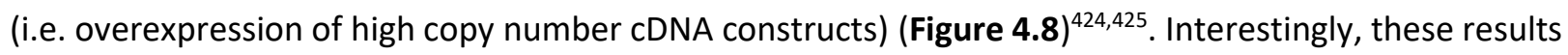
also revealed that BAPTA-AM and BIM treatments further increased Sjn1 labeling in CLSs beyond that in control, unstimulated cells in some conditions (Figure 4.8), suggesting that the recruitment of endogenous Sjn1 may be further modulated by calcium signals. Collectively, these results indicate the role of PLCY1-derived calcium signals in regulating the dissociation of Sjn1 from CCPs, thereby sustaining appropriate $\mathrm{PIP}_{2}$ levels required for the efficient assembly of endocytic structures. Although yet to be demonstrated, the potential localization of Sjn1 within EGFR-containing CCPs (but not TfR), thus provide a mechanism by which PLC 1 1-derived calcium signals may selectively regulate EGFR endocytosis; and potentially other Sjn1-containing endocytic structures. 


\section{3- PLC- $\psi 1$ is required for clathrin-dependent EGFR signaling}

In addition to their predominant role in endocytosis, clathrin structures also function as signaling platforms, which regulates distinct signaling pathways on the cell surface ${ }^{207}$. Described in section 1.6.4, PI3K/Akt signaling by EGFR is one such pathway particularly dependent on the fidelity of clathrin assemblies, as indicated by the perturbation of EGF-stimulated Gab1 and Akt phosphorylation at the plasma membrane, in clathrin-silenced RPE cells ${ }^{207}$. Furthermore, the role for clathrin structures in regulating EGFR signaling is selective, as silencing of clathrin does not impair the phosphorylation of EGFR or ERK1/2207. That PLC 1 1-derived calcium signals regulate the assembly and formation of EGFRcontaining CCPs raises the question of whether PLC- $\gamma 1$ may thus, also control PI3K/Akt signaling by EGFR. In this section, I will examine the requirement for calcium signals in EGFR signaling, of which understanding will further provide insight on the role of PLC- $\gamma 1$ in the regulation of CCP dynamics, and hence, overall CME.

In determining the requirement for PLC- $\nu 1$ in the propagation of EGFR signaling, I employed siRNA-mediated silencing of PLC- $\gamma 1$ in RPE cells, and measured the EGF-stimulated phosphorylation of Gab1 and Akt by immunoblot analysis. Expectedly, PLC- $\gamma 1$ silencing inhibited PI3K/Akt signaling as reported by the impairment of EGF-stimulated Gab1 and Akt phosphorylation (Figure 4.9A), but not that of EGFR and Erk (Figure 4.9B). These results are comparable to the effects of clathrin silencing on $\mathrm{PI3K} /$ Akt signaling, attesting to the role of PLC- $\gamma 1$ signals in regulating the assembly of clathrin structures $^{207}$. Moreover, the selective perturbation of EGF-stimulated Gab1 and Akt phosphorylation was similarly observed upon inhibition of cytosolic calcium by BAPTA-AM (Figure 4.10), indicating that calcium signals generated by PLC- $\gamma 1$ indeed control the assembly of clathrin required for PI3K/Akt signaling by EGFR. Thus, the requirement for PLC- $\gamma 1$ calcium signals in PI3K/Akt signaling is consistent with findings from the previous sections demonstrating its role in regulating the assembly of EGFRcontaining CCPs (Figure 4.3, D and E), of which efficient formation is both required for EGFR endocytosis and signaling.

Discussed in section 1.6.2, EGFR is a member of the ErbB (HER) family of receptor tyrosine kinases which is known to heterodimerize with other members of this family when co-expressed within the same cell-in particular, with HER2 (see section 1.6.2.3). Importantly, EGF stimulation of EGFR/HER2 heterodimers induces the propagation of PI3K/Akt signaling that is independent of clathrin, as demonstrated by the lack of effect of clathrin silencing on the phosphorylation of Gab1 and Akt in EGFR 
and HER2 expressing cells ${ }^{207}$. While RPE cells used in our experiments do not detectably express HER2 levels (thus, largely inducing clathrin-dependent PI3K/Akt signaling from EGFR homodimers), it is nevertheless, important to validate whether control of PI3K/Akt signaling by PLC- $\gamma 1$ is indeed due to its regulation of clathrin assembly, and not due to regulation of clathrin-independent signaling components ${ }^{207}$.

To determine whether PLC- $\gamma 1$ controls PI3K/Akt signaling by regulation of clathrin-independent components, RPE cells engineered to stably express HER2 (RPE-HER2) were used, which had been reported and confirmed to signal largely from EGFR/HER2 heterodimers ${ }^{207}$. RPE-HER2 cells were then subjected to PLC- $\gamma 1$ silencing by siRNA, followed by measurement of EGF-stimulated Akt phosphorylation via similar immunoblot assays used previously. As expected, siRNA-silencing of PLC- $\gamma 1$ did not impact the EGF-stimulated phosphorylation of Akt, likely due to the non-requirement for clathrin and regulation of its assemblies by PLC- $\gamma 1$, in PI3K/Akt signaling by RPE-HER2 cells (Figure 4.9C) ${ }^{207}$. As such, these results collectively suggests that PLC- $\gamma 1$ does not directly regulate components of the $\mathrm{PI3K} /$ Akt signaling pathway at the plasma membrane, but rather modulate the assembly of clathrin structures necessary for PI3K/Akt signaling in HER2-deficient RPE cells.

\section{4- Summary of Chapter 4}

In this chapter I determined how PLC- $\gamma 1$ may precisely control CME of EGFR in promoting its internalization. CME is a complex process which is defined by distinct endocytic stages, each dynamically regulated by a diverse array of endocytic proteins. Prior to this work, it was conceivable that PLC- $\gamma 1$ could regulate EGFR endocytosis by control of one of the morphologically distinct stages: nucleation, cargo recruitment, initiation, assembly, and scission. Examination of eGFP-CLCa expressing RPE cells labeled with fluorescent ligands by TIRF-M imaging revealed that PLC $\gamma 1$-derived calcium signals are not required, and thus, do not regulate receptor recruitment within CLSs, as demonstrated by the increase in EGF+ CLSs on the cell surface upon perturbation of the PLCY1-calcium-PKC signaling axis. The increase in cell surface density of EGF+ CLSs nevertheless, indicate impairments in the efficient internalization of EGFR (subsequent to EGFR recruitment to clathrin structures), suggesting perturbations in subsequent endocytic steps following receptor recruitment, imposed by PLCY1-derived calcium inhibition. Consistent with this, using live-cell imaging coupled to automated detection and tracking of CLSs, I determined a 
robust increase in the frequency of initiation of EGF+ sCLSs, represented by dim, short-lived CLSs, that are likely abortive and fail to internalize into the cell. This finding is corroborated by a concomitant decrease in the rate of formation of bona fide EGF+ CCPs that represent productive clathrin structures, which are brighter and longer-lived relative to SCLSs. In addition, the bona fide EGF+ CCPs that form under calcium perturbed conditions were significantly reduced in size relative to control (untreated condition). Importantly, calcium perturbation was without effect on the dynamics of Tfn clathrinmediated endocytosis, indicating the selective requirement for PLC $\gamma 1$-derived calcium signals in regulating efficient clathrin assembly, formation and subsequent internalization of EGF+ CCPs (Figure 4.11).

The effects of calcium perturbation on CCP dynamics mimic the phenotypic effects on CCPS observed in PIP5K silenced cells-suggesting a role for PLC 1-derived calcium signals in the localized regulation of enzymes within CCPs that regulate $\mathrm{PIP}_{2}{ }^{6,79}$. That activation of PLC- $\gamma 1$ does not elicit broad changes in plasma membrane $\mathrm{PIP}_{2}$ concentrations, indicate the potential regulation of $\mathrm{PIP}_{2}$ by PLC- $\gamma 1$ to occur in a localized manner, such that within CCPs. Sjn1 is a phosphatase localized within CCPs which catalyzes $\mathrm{PIP}_{2}$ turnover, and as such known to negatively regulate clathrin assembly and endocytosis. Removal of Sjn1 from CCPs thus, prevent the dephosphorylation of $\mathrm{PIP}_{2}$ thereby maintaining appropriate $\mathrm{PIP}_{2}$ levels necessary for efficient CCP assemblies. Indeed, I found that PLC 1 1-derived calcium signals promote the broad dissociation of Sjn1 from CLSs, and that this EGF-stimulated loss of Sjn1 from CCPs is abrogated under calcium perturbed conditions.

Lastly, I further corroborated the role of PLC 1 1-derived calcium signals in direct control of clathrin assembly, by examining its role in the clathrin-dependent PI3K/Akt signaling in RPE cells. $\mathrm{PI3K} /$ Akt signaling by EGFR requires the efficient formation of clathrin structures of which perturbation via clathrin silencing, impairs phosphorylation and activation of its signaling components (e.g. Gab1 and Akt ${ }^{207}$. Importantly, I demonstrated that the effects of clathrin silencing on EGFR signaling is recapitulated by silencing of PLC- $\gamma 1$, likely due to impairments in clathrin assemblies resulting from PLC1 knockdown. In addition, knockdown of PLC- $\gamma 1$ was without effect in signaling of RPE-HER2 cells, which is known to propagate $\mathrm{PI3K} / \mathrm{Akt}$ signaling in a clathrin-independent manner. These results indicate that PLC 1 1-derived calcium signals thus, promote PI3K/Akt signaling in HER2-deficient cells (RPE cells) not by direct control of signaling components, but rather by regulation of clathrin assembly consistent with its role in enhancing the assembly and formation of bona fide EGF+ CCPs. 
In summary, PLC- $\gamma 1$ therefore provides a direct mechanism through which mitogenic signaling by RTKs may effect global plasma membrane remodeling. In chapter 3, I demonstrated the requirement for PLC- $\gamma 1$ signals in the regulation of EGFR clathrin-mediated endocytosis. Specifically, this requirement largely involves PLC 1 1-derived calcium signals which selectively regulates EGFR clathrin-mediated endocytosis, but not other endocytic cargoes including TfR; likely due to their localization within distinct CCPs. In chapter 4, I uncovered precisely how calcium signals may distinctly regulate EGFR endocytosis, predominantly by regulating the assembly and formation of EGF+ CCPs (but not Tfn+ CCPs), at a stage subsequent to EGFR recruitment to nucleated clathrin structures. Furthermore, I found that Sjn1 might serve as the primary downstream calcium effector, such that Sjn1 dissociation from CCPs by PLC- $y 1$ calcium signals directly influences local PIP 2 levels promoting the assembly of EGFR-containing CCPs. Importantly, despite the selective regulation of EGFR clathrin-mediated endocytosis by PLC- $\gamma 1$, that Sjn1 broadly localizes to CCPs indicate the broad regulation of CME of cargoes contained therein, which may include other cargo classes apart from EGFR. Thus, through activation of PLC- $\gamma 1$, mitogenic signaling by RTKs may promote clathrin-mediated endocytosis and hence, global remodeling of the plasma membrane via regulation of Sjn1 by PLC 1 1-derived calcium signals.

Having determined how mitogenic cues may regulate the plasma membrane via $\mathrm{CME}$, I will then shift my focus into understanding how metabolic cues such as cellular energy levels may similarly impact the endocytic process. Conceptually the opposite of mitogenic signaling from a cell physiological perspective, metabolic depression in energy levels similarly induces remodeling of the plasma membrane as shown from previous studies in our laboratory ${ }^{360}$. In addition, remodeling of the plasma membrane under metabolic stress is known to be largely regulated by AMPK activation (see section 1.7). However, precisely how AMPK may control the plasma membrane, and whether this may occur through regulation of $\mathrm{CME}$, is not well-understood, serving as the major questions addressed in the following section. 


\section{5- Figures for Chapter 4}
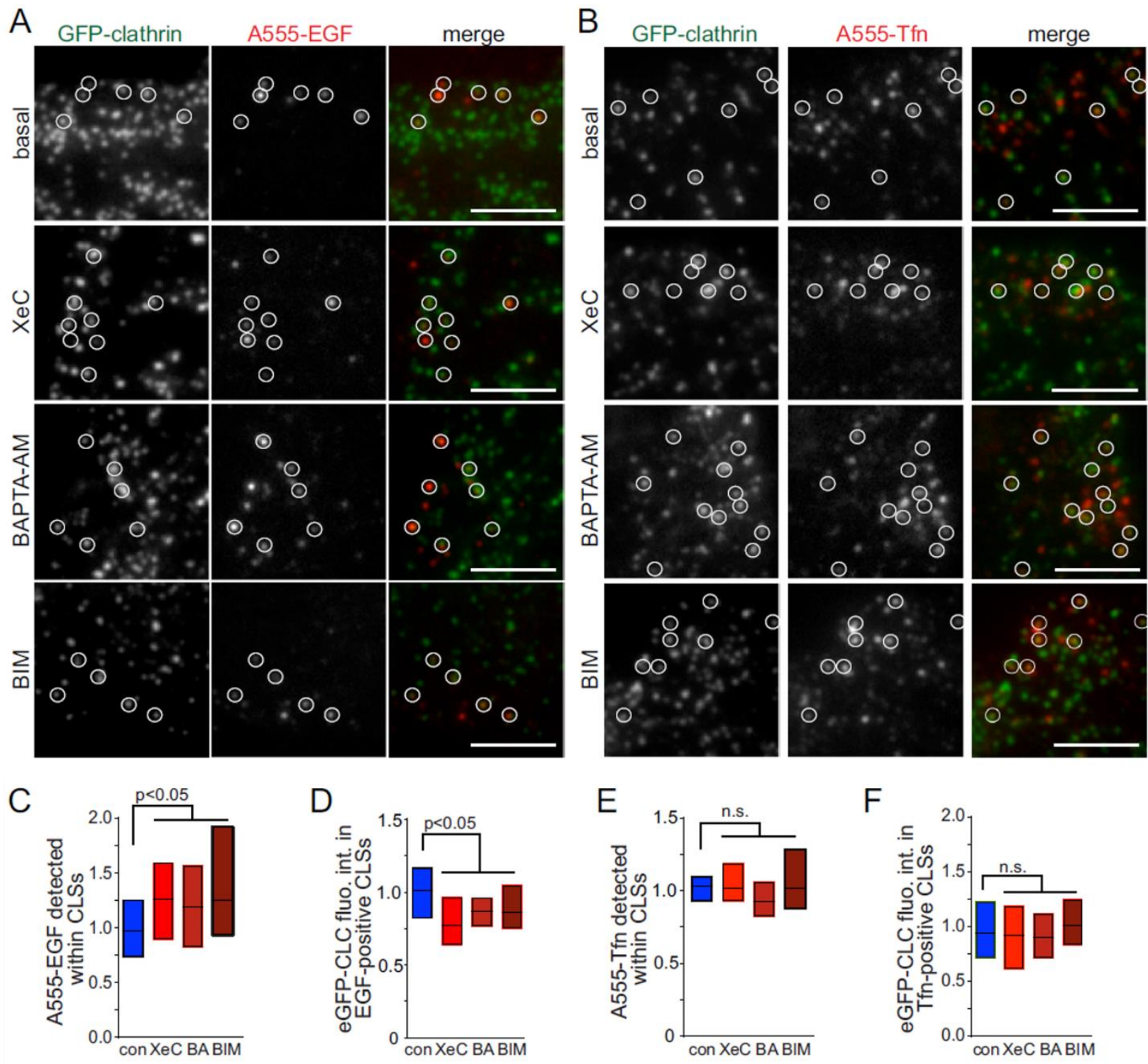

Figure 4.1. Cytosolic Ca2+ and PKC regulate CLSs containing EGFR but not those harboring TfR. RPE cells stably expressing clathrin light chain fused to eGFP (eGFP-CLCa), treated with various inhibitors: 3 $\mu \mathrm{M}$ XeC for 30 min, $10 \mu \mathrm{M}$ BAPTA-AM (BA) for 15 min, $1 \mu \mathrm{M}$ BIM for 30 min, or left untreated (control), and then treated with (A) A555-EGF (5ng/mL for $5 \mathrm{~min}$ ) or (B) A555-Tfn (10 $\mu \mathrm{g} / \mathrm{ml}$ for $3 \mathrm{~min})$. Shown are representative micrographs obtained by TIRF-M. Scale bar: $5 \mu \mathrm{m}$. Full image panels are shown in Figure 4.2. (C-F) TIRF-M images were subjected to automated detection of CLSs, followed by quantification of 
mean A555-conjugated ligand fluorescence intensity therein (C, E). CLSs were sorted into A555-EGFenriched or A555-Tfn-enriched cohorts, followed by quantification of the mean eGFP-CLCa within each CLS cohort (D, F). For C-F, the overall median of the cellular means (bar) and 25th/75th percentiles (boxes) are shown. The number of CLSs and cells analyzed, respectively, for each condition are as follows. EGF control: 14,114 and 114; EGF XeC: 11,322 and 98; EGF BAPTA-AM: 15,786 and 104; EGF BIM: 3860 and 46, Tfn control: 16,983 and 117; Tfn XeC: 10,838 and 98; Tfn BAPTA-AM: 8868 and 64; Tfn BIM: 3718 and 44; from a minimum of three independent experiments in each condition. 

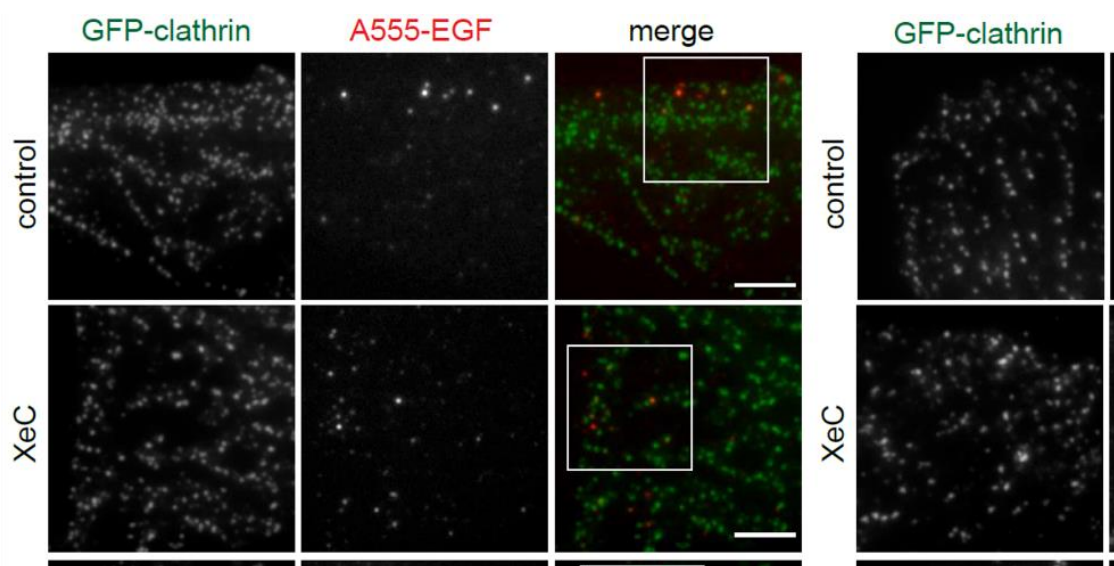

merge
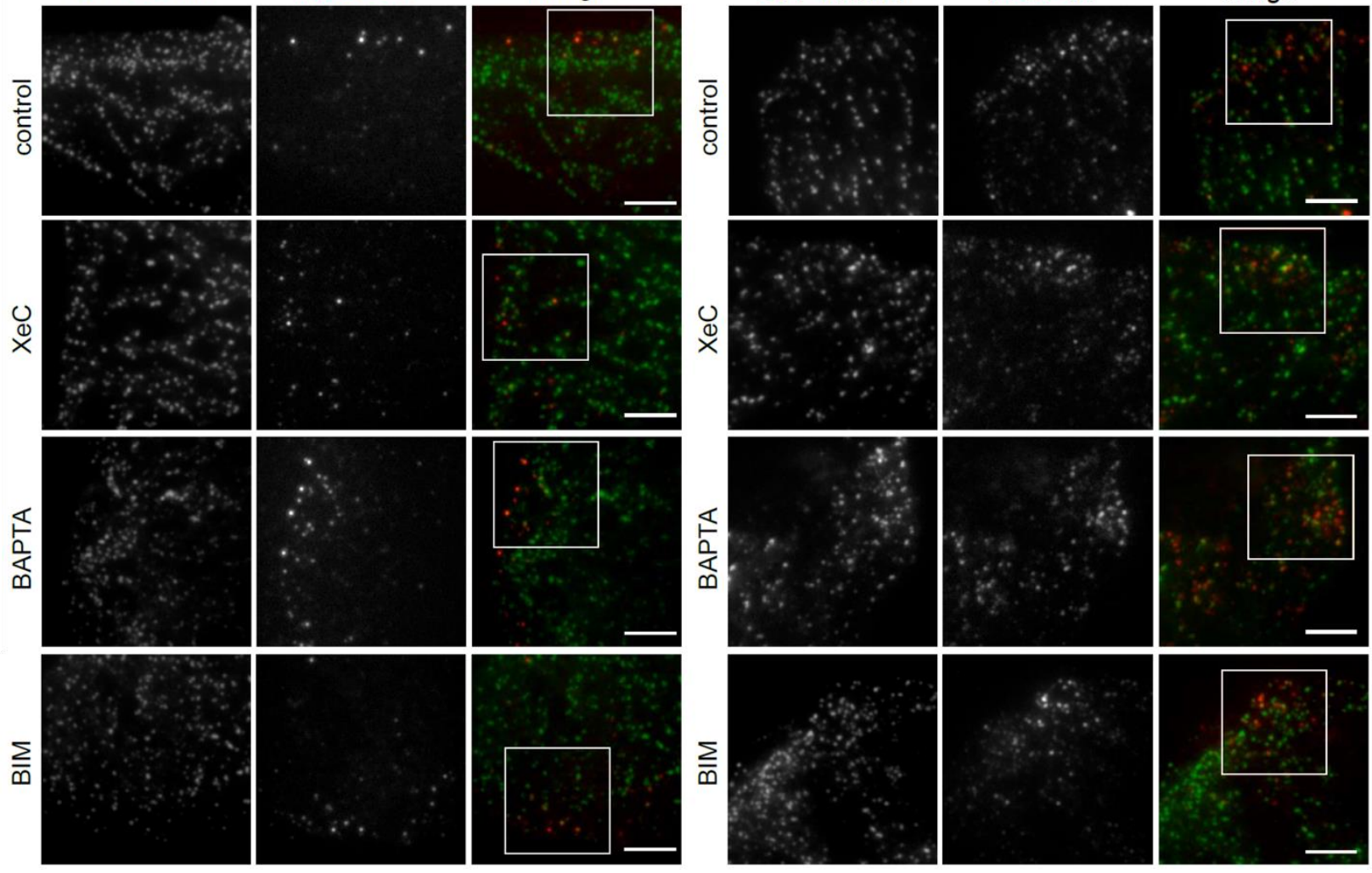

Figure 4.2. Full-size representative TIRF-M images of RPE GFP-CLC cells treated with A555-EGF or

A555-Tfn. Shown in Figure 4.1A-B are images of RPE cells stably expressing eGFP-CLCa and treated with A555-EGF or A555-Tfn for 5 min, followed by fixation and imaging with TIRF microscopy. Shown here are the full-sized images of the representative images shown in Figure 4.1A-B, with a white box showing the area enlarged in Figure 4.1A-B, Scale bar: $20 \mu \mathrm{m}$. 

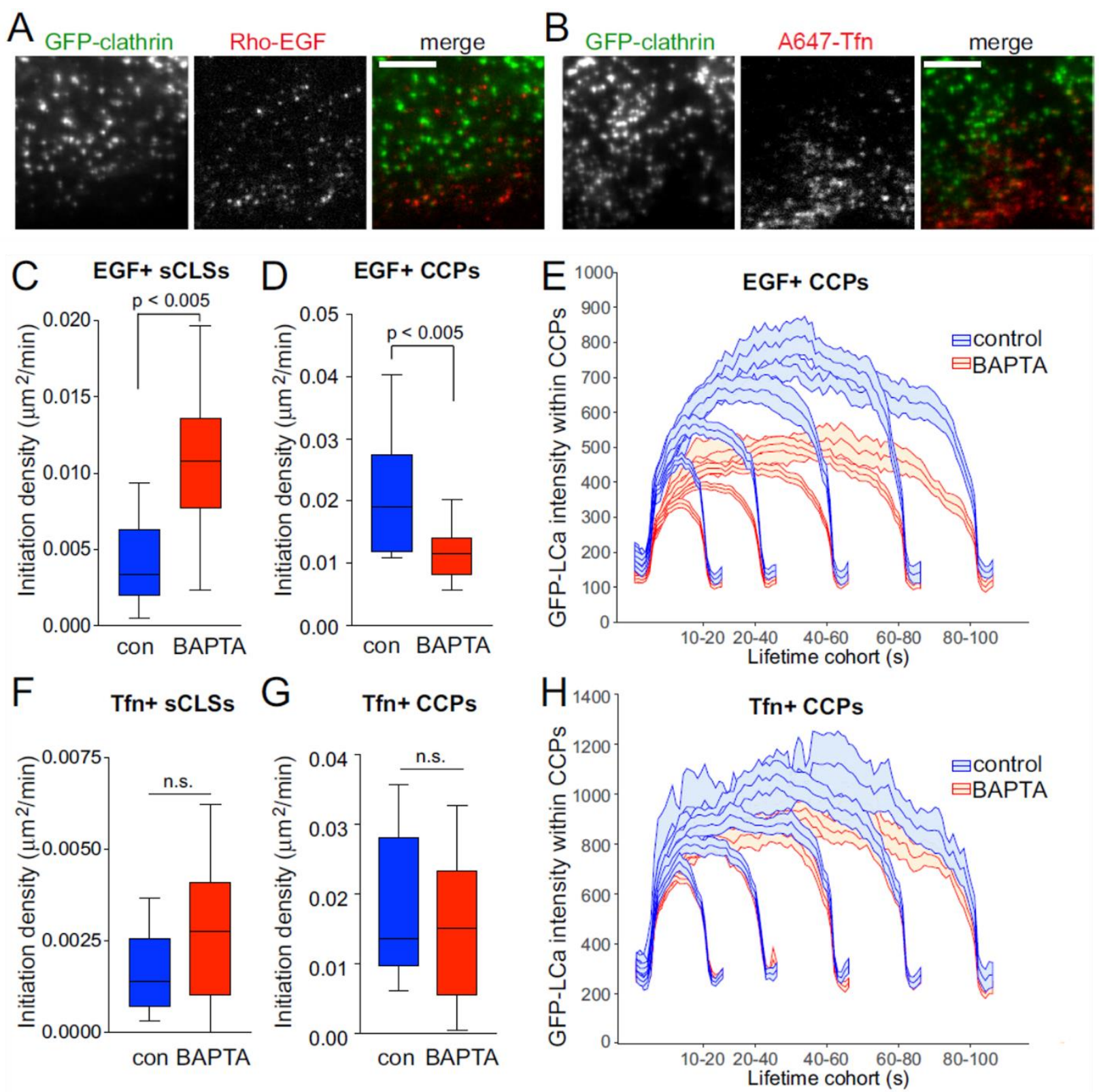

Figure 4.3. Cytosolic Ca2+ selectively controls initiation and assembly of CCPs harboring EGFR. RPE cells stably expressing clathrin light chain fused to eGFP (eGFP-CLCa) were pretreated with $10 \mu \mathrm{M}$ BAPTA-AM for $15 \mathrm{~min}$, and then treated with either $20 \mathrm{ng} / \mathrm{ml}$ rhodamine-EGF (Rho-EGF) or treated with A647-Tfn during time-lapse imaging by TIRF-M. (A, B) Single-frame representative fluorescence micrographs. Scale bar: $5 \mu \mathrm{m}$. Time-lapse TIRF-M image series of cells treated with Rho-EGF (C-E) or A647-Tfn (F-H) were subjected to automated detection, tracking, and analysis of CLSs as described in Materials and Methods, allowing identification of sCLSs and bona fide CCPs as EGF+ or Tfn+, as appropriate. (C-D, F-G) Median, 25th/75th percentiles (boxes) and Tukey range (whiskers) for the 
initiation rate of ligand-positive SCLSs (C, F) or ligand-positive CCPs (D, G) are shown. (E, H) Mean eGFPCLCa fluorescence intensity (reported as arbitrary units [A.U.]) grouped into CCP lifetime cohorts; error bars reflect cell-to-cell variation. The number of total CLS trajectories, CCP trajectories, and cells for each condition are (respectively): Rho-EGF treated (control, DMSO): 24,737, 16,621, and 18; Rho-EGF treated (BAPTA-AM treated): 33,604, 14,731, and 24; A647-Tfn (control, DMSO) 32,693, 22,008, and 24; A647Tfn (BAPTA-AM) 35,287, 20,862, and 25. The breakdown of ligand-positive sCLSs and CCPs is as follows: $5.0 \pm 0.7 \%$ (control) and $8.5 \pm 0.5 \%$ (BAPTA-treated) of sCLSs are EGF+; $13.2 \pm 0.9 \%$ (control) and $10.9 \pm$ $0.9 \%$ (BAPTA-treated) of CCPs are EGF+; $3.1 \pm 0.4 \%$ (control) and $4.0 \pm 0.7 \%$ (BAPTA-treated) of sCLSs are Tfn+; $13.6 \pm 1.9 \%$ (control) and $13.2 \pm 1.9 \%$ (BAPTA-AM) of CCPs are Tfn+. 
$A$
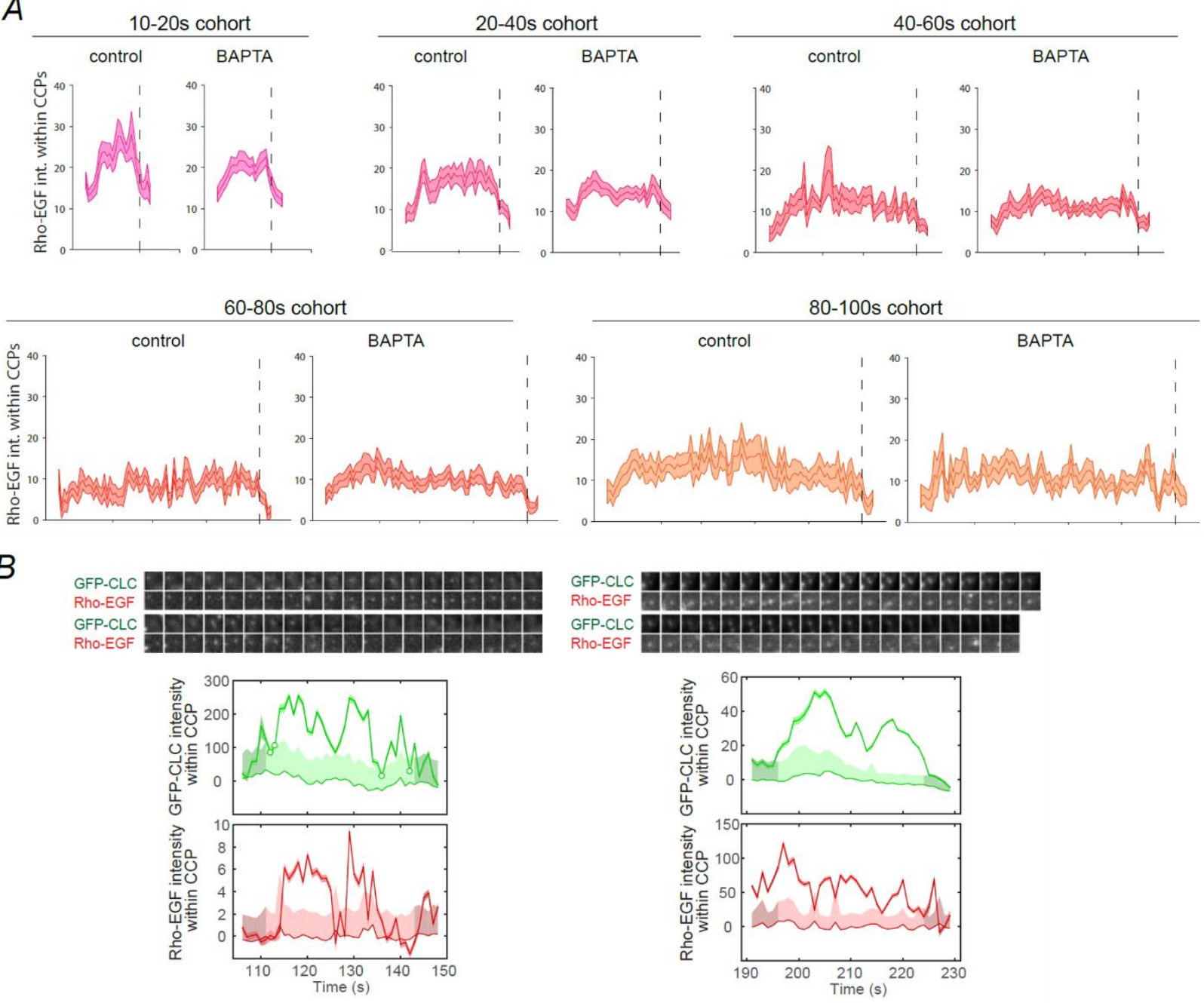

C

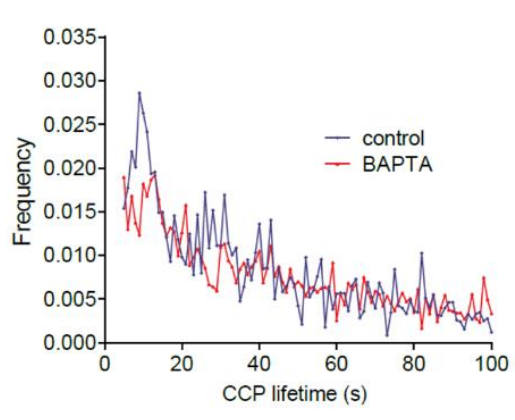

Figure 4.4. Dynamics of EGF+ sCLSs and CCPs. RPE cells stably expressing clathrin light chain fused to eGFP (eGFP-CLCa) were pre-treated with $10 \mu$ M BAPTA-AM for 15 min, and then treated with $20 \mathrm{ng} / \mathrm{mL}$ rhodamine-EGF (Rho-EGF) during time-lapse imaging by TIRF-M. Time-lapse image series were subjected to automated detection, tracking, and analysis of CCPs as described in Materials and Methods, allowing identification of EGF+ sCLSs and CCPs. Shown are (A) the mean Rho-EGF fluorescence intensities within 
CCPs grouped into CCP lifetime cohorts; error bars reflect cell-to-cell variation and dotted lines indicated the disappearance of clathrin from the TIRF field and (B) representative CCP trajectories depicting micrographs obtained by TIRF-M centered on the detected object and the quantification of the fluorescence intensity of eGFP-CLCa and Rho-EGF in these structures. (C) Shown is a histogram depicting the frequency of EGF+ CCPs of indicated lifetimes. 
$A$
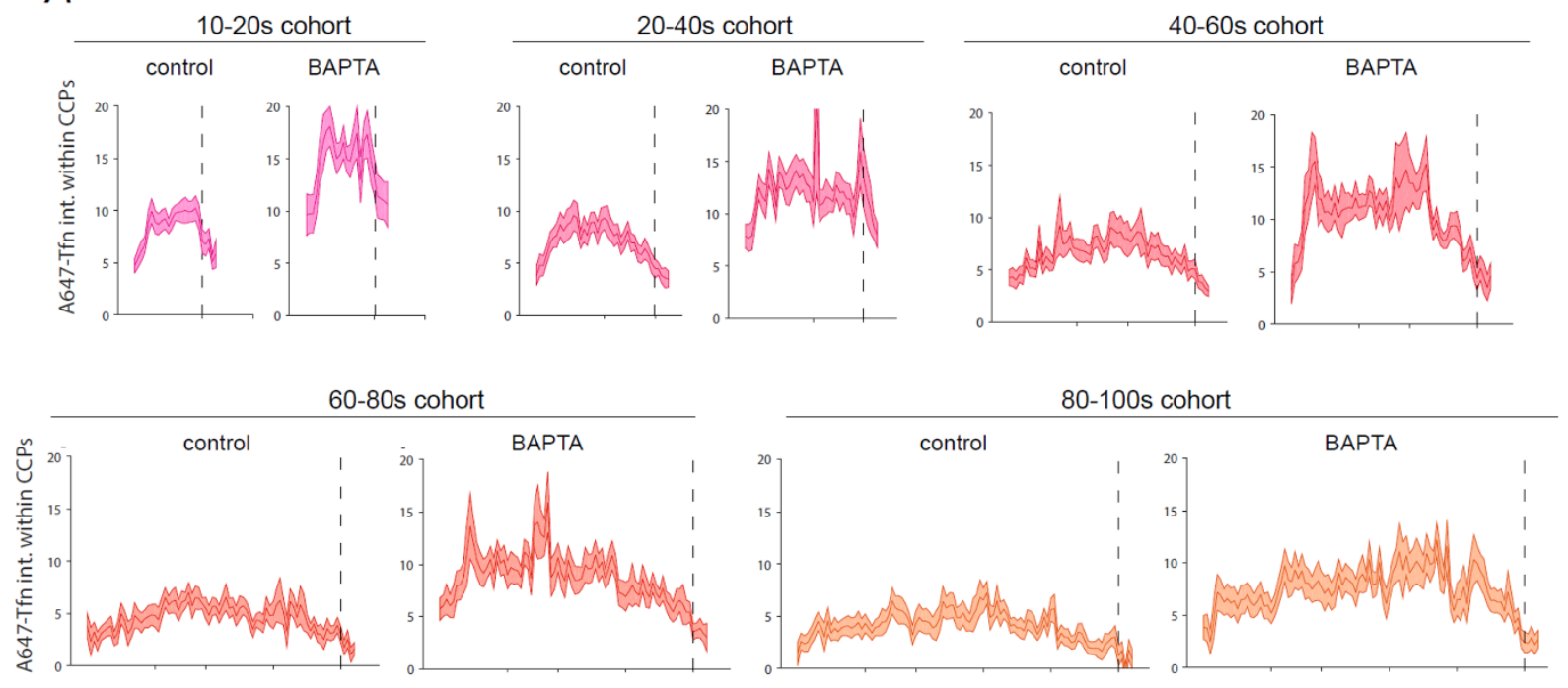

\section{$B$}

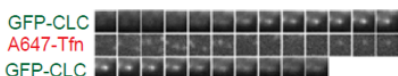

GFP-CLC $=-\infty-\infty-2-2$
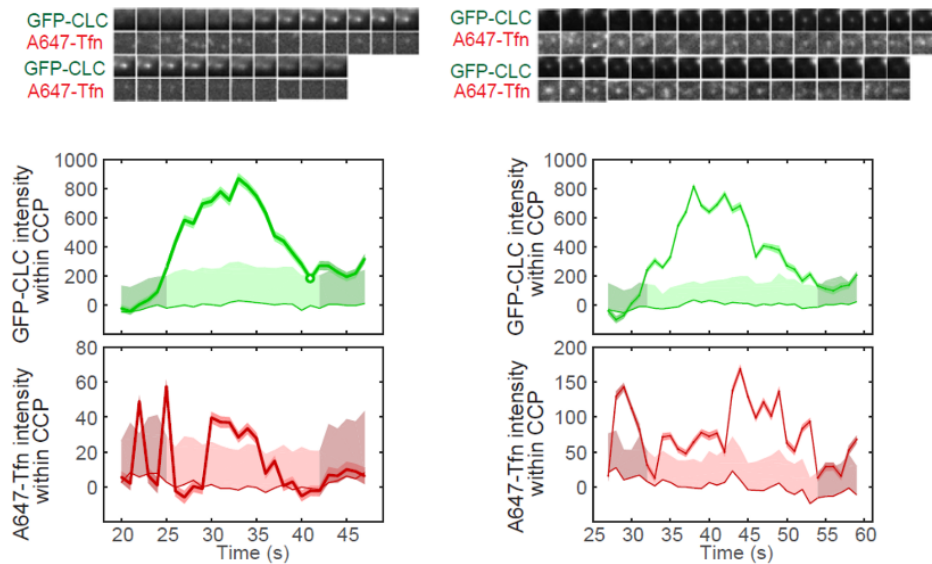

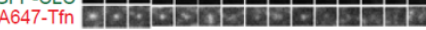

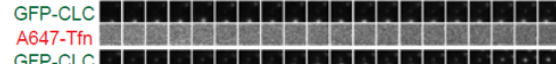

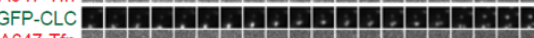
A647-Tfn _ $\quad$ _ GFP-CLC :F: : : : : : :

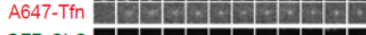

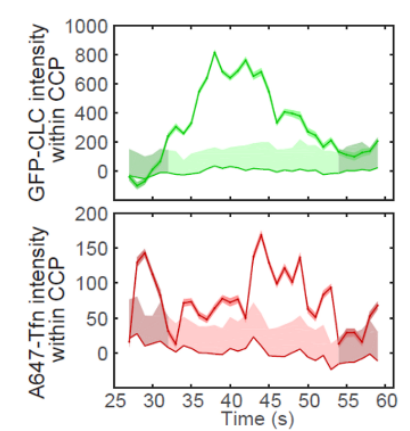
GFP-CLC D

C

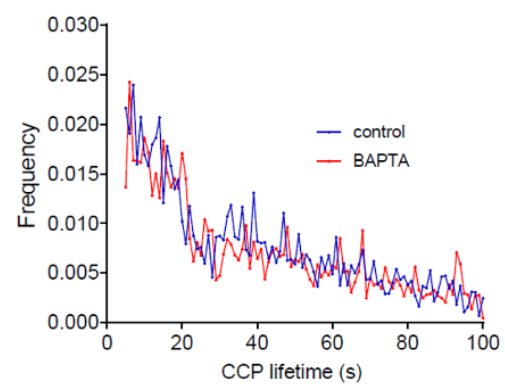

Figure 4.5. Dynamics of Tfn+sCLSs and CCPs. RPE cells stably expressing clathrin light chain fused to eGFP (eGFP-CLCa) were pre-treated with $10 \mu \mathrm{M}$ BAPTA-AM for $15 \mathrm{~min}$, and then treated with $100 \mathrm{ng} / \mathrm{mL}$ A647-Tfn during time-lapse imaging by TIRF-M. Time-lapse image series were subjected to automated detection, tracking, and analysis of CCPs as described in Materials and Methods, allowing identification 
of Tfn+ sCLSs and CCPs. Shown are (A) the mean A647-Tfn fluorescence intensity grouped into CCP lifetime cohorts; error bars reflect cell-to-cell variation and dotted lines indicated the disappearance of clathrin from the TIRF field and (B) representative CCP trajectories depicting micrographs obtained by TIRF-M centered on the detected object and the quantification of the fluorescence intensity of eGFPCLCa and A647-Tfn. (C) Shown is a histogram depicting the frequency of Tfn+ CCPs of indicated lifetimes. 


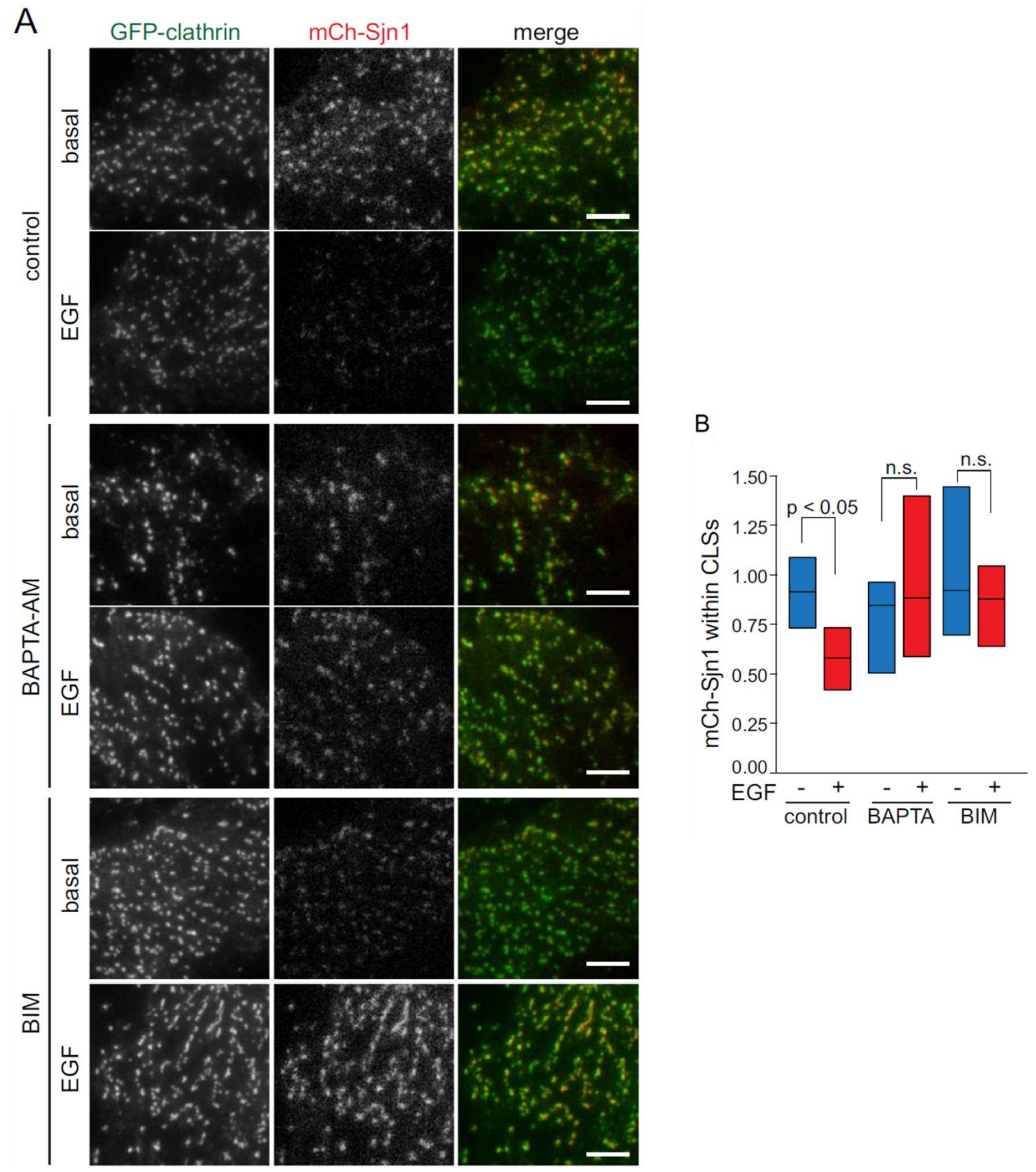

Figure 4.6. mCherry-Sjn1 is depleted from CLSs upon EGF stimulation in a $\mathrm{Ca}^{2+}$ - and PKC-dependent manner. RPE cells stably expressing GFP-CLCa were transfected with mCherry-tagged Sjn1 (mCh-Sjn1, 170-kDa isoform), and then treated with $10 \mu \mathrm{M}$ BAPTA-AM for $15 \mathrm{~min}, 1 \mu \mathrm{M}$ BIM for $30 \mathrm{~min}$, or left untreated (control), followed in some samples by stimulation with $5 \mathrm{ng} / \mathrm{ml} \mathrm{EGF}$, as indicated, then by 
immediate fixation. (A) Shown are representative micrographs obtained by TIRF-M. Scale bars: $10 \mu \mathrm{m}$. Full-image panels, including corresponding epifluorescence images, are shown in Figure 4.7. (B) TIRF-M images were subjected to automated detection of CLSs, followed by quantification of the mean mChSjn1 fluorescence intensity therein (normalized to total mCh-Sjn1 expression determined from epifluorescence images). Shown in B are median (bar) and 25th/75th percentiles (boxes) of mCh-Sjn1 fluorescence intensity within CLSs in each condition. The number of CLSs and cells analyzed, respectively, for each condition are as follows: basal, control (no drug): 43,047 and 50; basal, BAPTAAM: 38,919 and 44; basal, BIM: 35,556 and 46; EGF, control (no drug): 55,482 and 55; EGF, BAPTA-AM: 28,644 and 43; EGF, BIM: 29,637 and 38; from three independent experiments. 


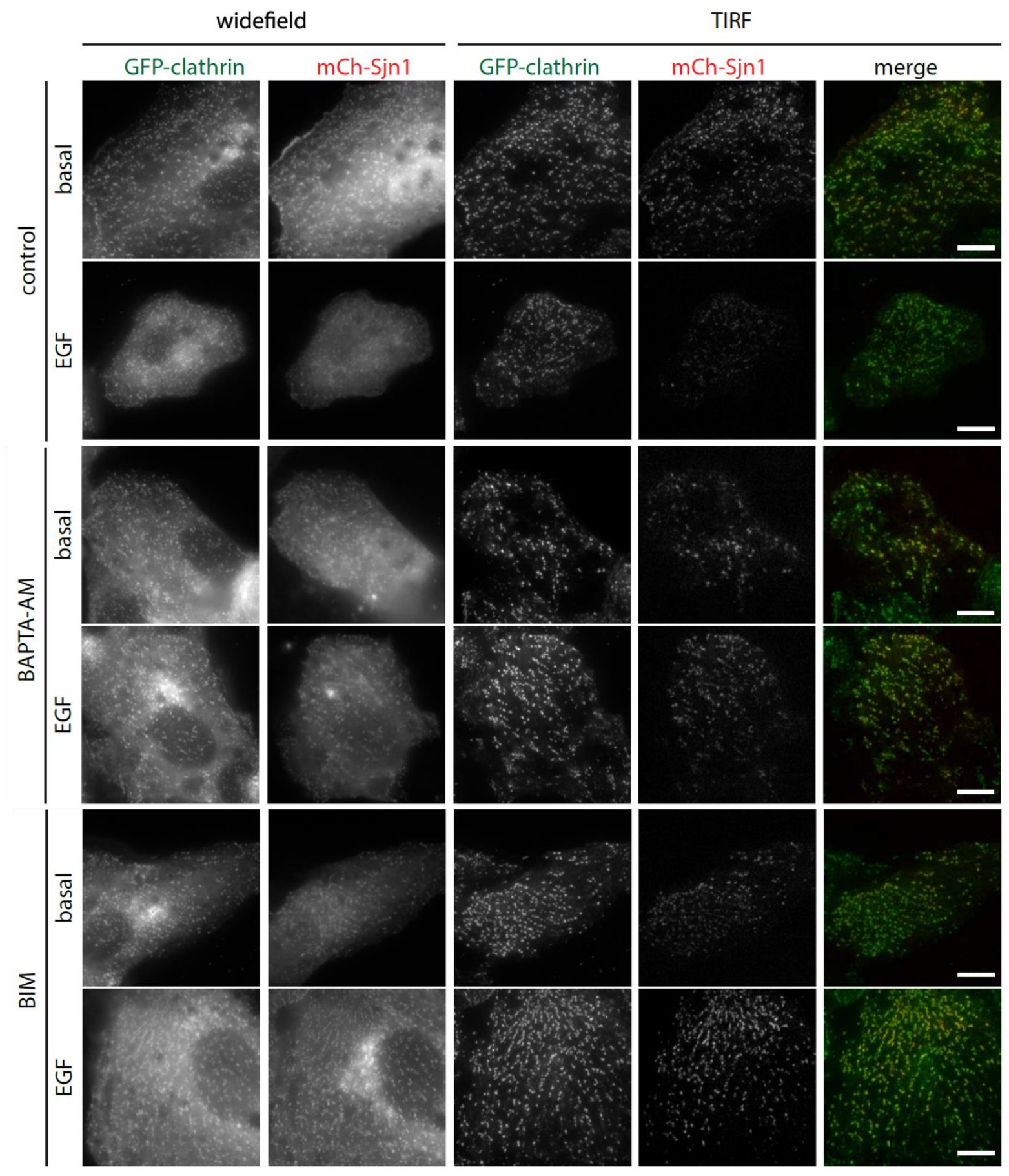

Figure 4.7. Full-size representative TIRF images of RPE eGFP-CLCa cells transfected with mCherry-

Synaptojanin 1. Shown in Figure 4.6A are TIRF-M images of RPE cells stably expressing eGFP-CLCa and transfected with mCherry-Synaptojanin 1 (mCh-Sjn1, $170 \mathrm{kDa}$ isoform). Shown here are the full-sized images of the representative TIRF-M images shown in Figure 4.6A, as well as the corresponding image frames obtained by widefield epifluorescence microscopy, scale, $20 \mu \mathrm{m}$. 
$A$
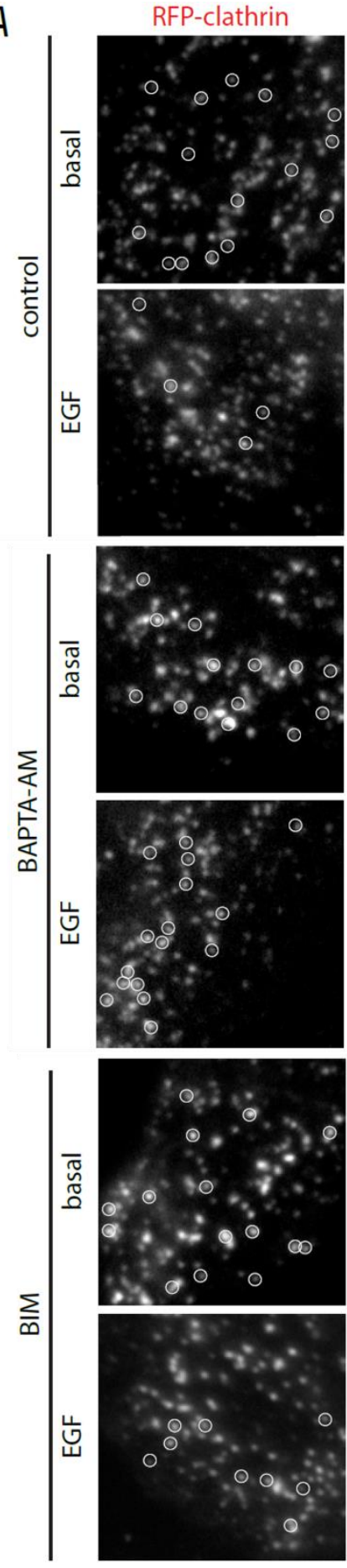

$\operatorname{Sin} 1$
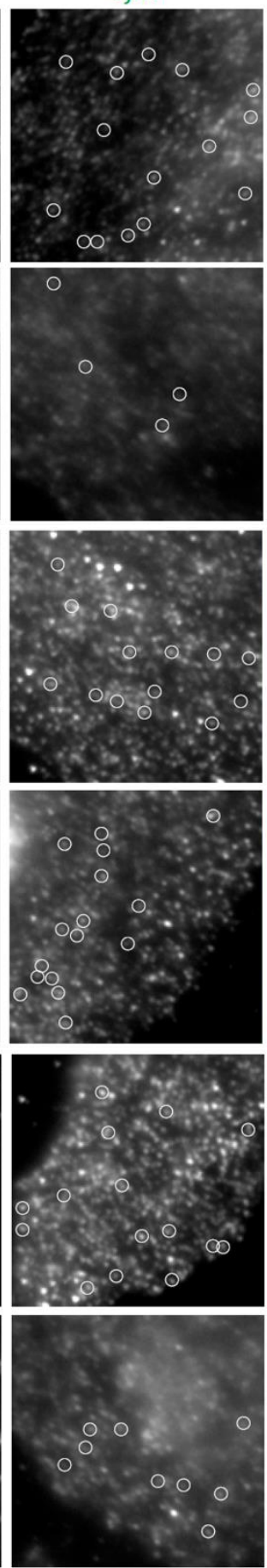

merge
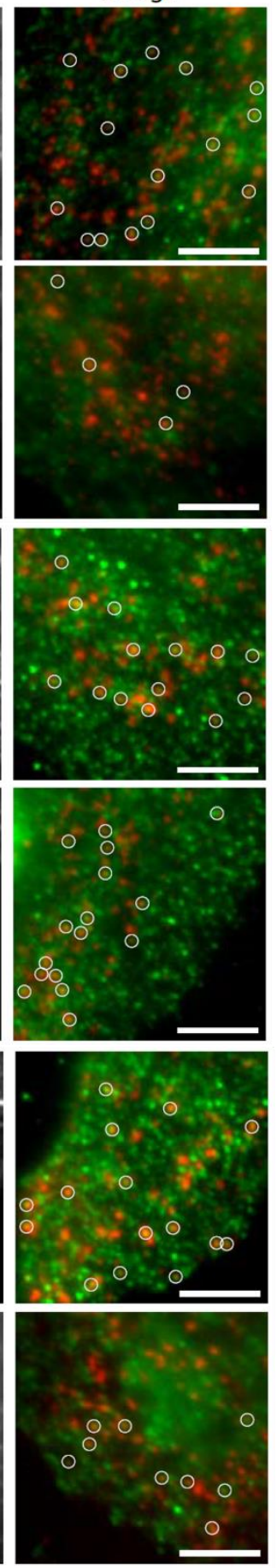

$B$

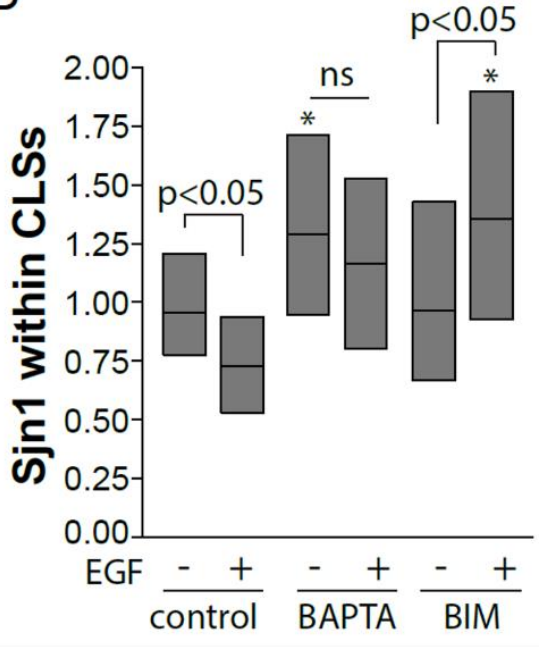

Figure 4.8. Endogenous synaptojanin 1 is depleted from CCPs upon EGF stimulation in a cytosolic $\mathrm{Ca}^{2+}$ dependent manner. RPE cells stably expressing clathrin light chain fused to Tag-RFP-T (RFP-clathrin) were treated with $10 \mu \mathrm{M}$ BAPTA-AM for $15 \mathrm{~min}, 1 \mu \mathrm{M}$ BIM for 30 min or left untreated (control), followed in some samples by stimulation with $5 \mathrm{ng} / \mathrm{mL} E G F$, as indicated, then by immediate fixation and 
staining with anti-synaptojanin 1 (Sjn1) antibodies. (A) Shown are representative fluorescence micrographs obtained by TIRF-M, scale $10 \mu \mathrm{m}$. Circles indicate CCPs positive for endogenous Sjn1, determined manually. (B) TIRF-M images were subjected to automated detection of CCPs, followed by quantification of mean Sjn1 fluorescence intensity therein. Shown in are median (bar) and 25th/75th percentiles (boxes) of Sjn1 fluorescence intensity within CCPs in each condition. The number of CCPs analyzed and cells, respectively, for each condition are as follows: basal, control (no drug): 20547, 68; basal, BAPTA-AM: 9839, 31; basal, BIM: 8843, 31; EGF, control (no drug): 19812, 65, EGF, BAPTA-AM: 7009, 31; EGF, BIM: 7817, 30; from 3 independent experiments. *, $p<0.05$, relative to the control, basal (non-EGF-stimulated) condition. 

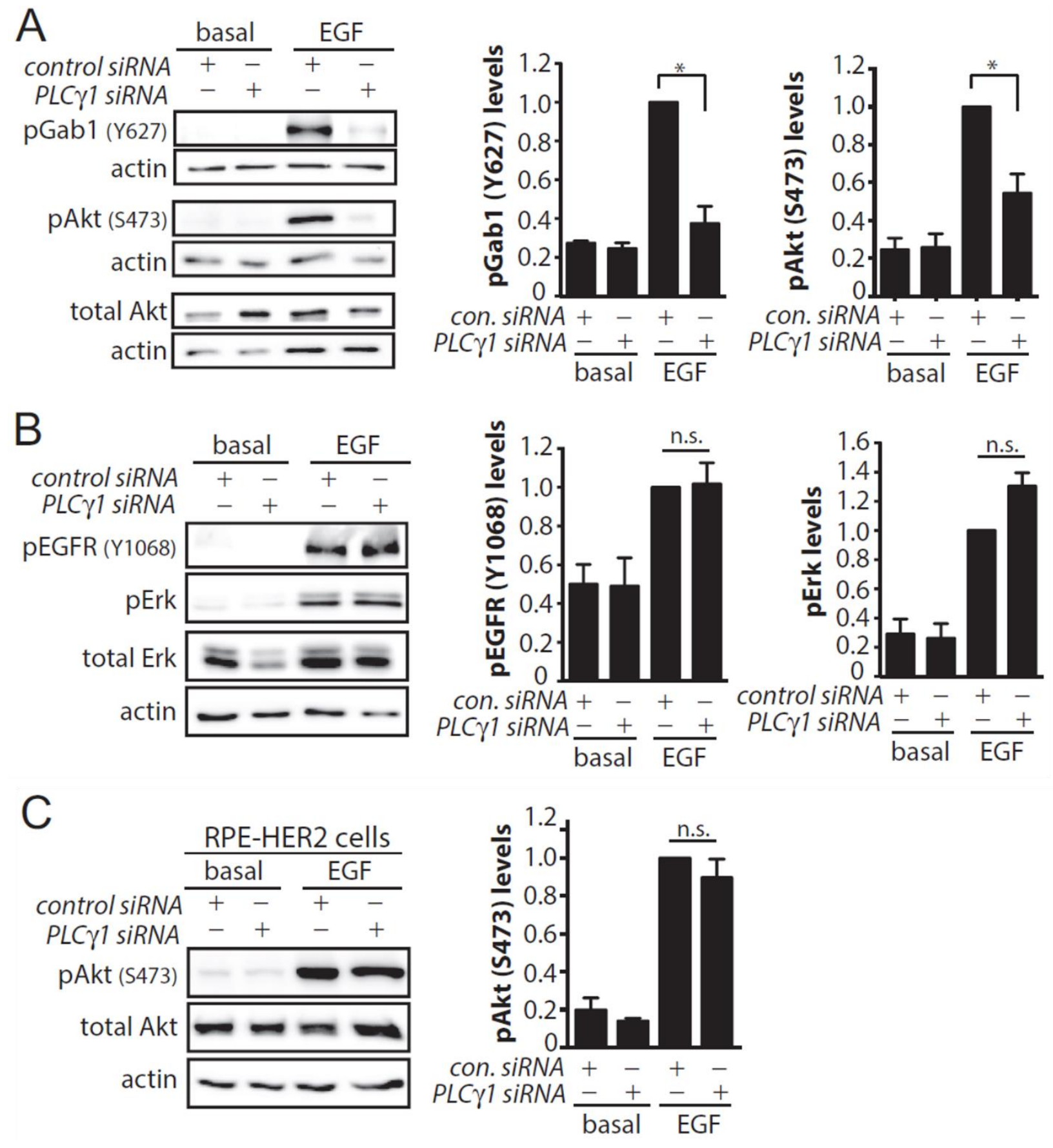

Figure 4.9. PLC- $ү 1$ is required for clathrin-dependent EGFR signaling. RPE cells or RPE cells stably expressing HER2 (RPE-HER2 cells) were subjected to siRNA silencing of PLC- $\gamma 1$ and treated with $5 \mathrm{ng} / \mathrm{ml}$ EGF for $5 \mathrm{~min}$ as indicated, followed by Western blotting of whole-cell lysates with antibodies to detect phosphorylated proteins. Shown are representative immunoblots and the mean \pm SE signal intensity (from $\mathrm{n}>3$ independent experiments; ${ }^{*}, \mathrm{p}<0.05$ ) for (A) pAkt and pGab1 in RPE cells (not expressing HER2), (B) pEGFR and pErk in RPE cells (not expressing HER2) (These experiments on RPE cells were 
performed by Stephen Bautista (PhD. Candidate); Ryerson University, Toronto, ON, Canada), and (C) pAkt in RPE-HER2 cells (These experiments on HER2 cells were performed by John Abousawan (PhD. Candidate); Ryerson University, Toronto, ON, Canada). 


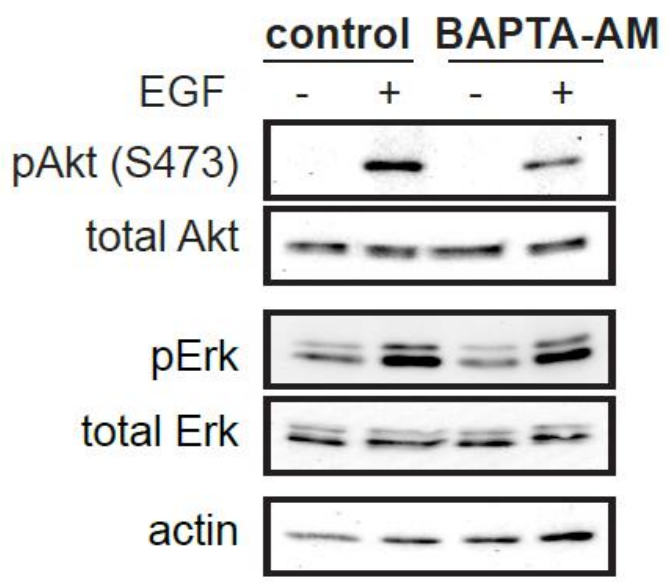

Figure 4.10. Perturbation of intracellular Ca2+ impairs EGF-stimulated Akt phosphorylation. RPE cells were treated with the cytosolic $\mathrm{Ca}^{2+}$ chelator BAPTA-AM (10 $\mu \mathrm{M}$ BAPTA-AM for $15 \mathrm{~min}$ ) and then stimulated with EGF ( $5 \mathrm{ng} / \mathrm{mL}$ for $5 \mathrm{~min}$ ), followed by Western blotting of whole-cell lysates with antibodies to detect phosphorylated proteins. Shown are representative immunoblots for the indicated antibodies. BAPTA-AM treatment impairs EGF-stimulated Akt phosphorylation, but not that of Erk. 


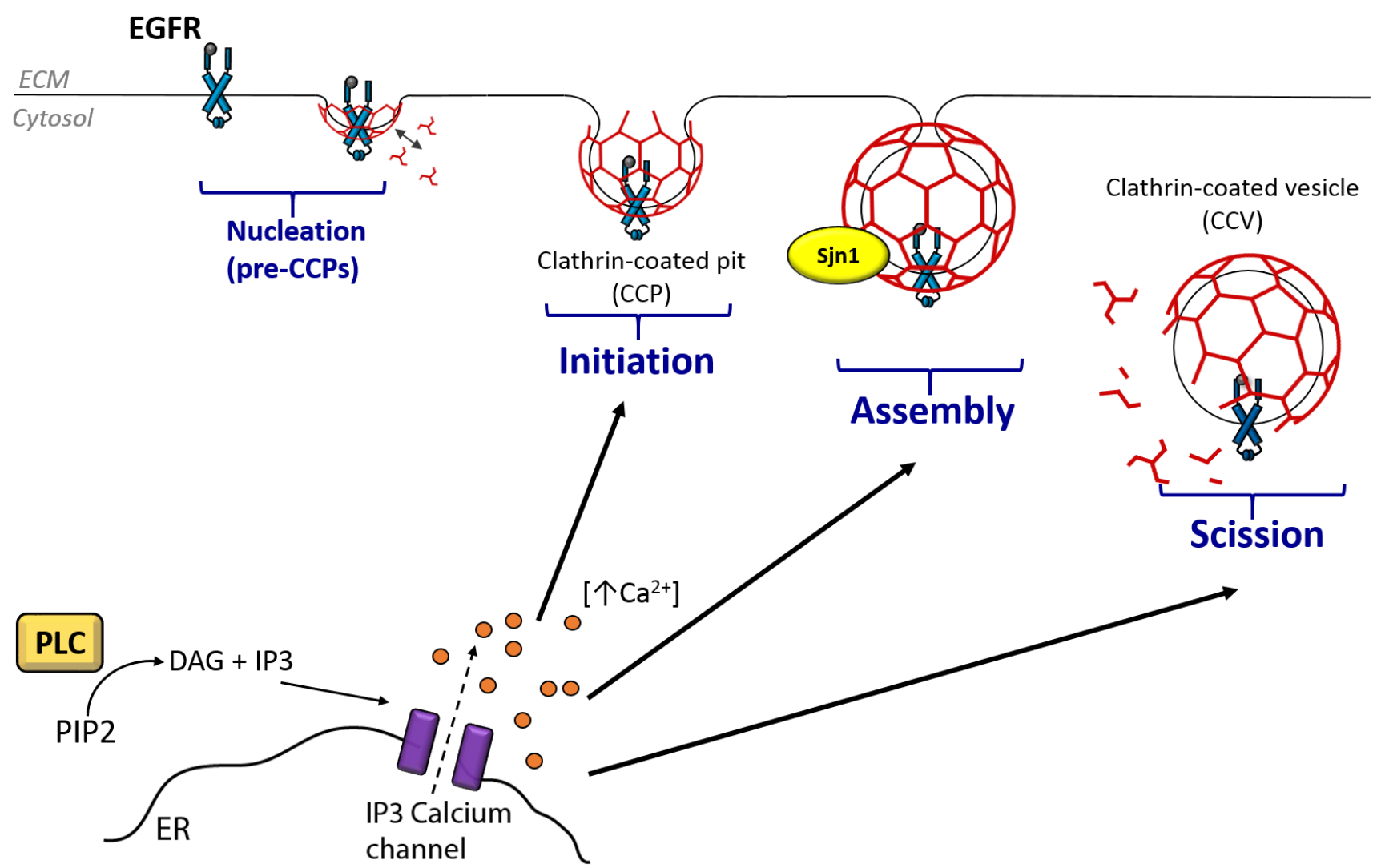

Figure 4.11. PLCץ1-derived calcium signals selectively regulate the efficient formation, assembly and subsequent internalization of EGFR-containing CCPs. Shown is the schematic diagram summarizing the findings described in chapter 4. PLCY1-derived calcium signals were found to control the assembly and internalization of EGFR-containing clathrin structures, but not EGFR recruitment therein. The requirement for calcium signals in CME is selective for EGFR as the formation and assembly of TfRcontaining clathrin structures were largely unaffected (not shown) by perturbation of calcium signals. PLC 1 1-derived calcium signals may control the dynamics of EGFR clathrin-mediated endocytosis by regulating endocytic proteins including Sjn1, which becomes dissociated from clathrin structures upon mobilization of calcium signals; induced by EGF stimulation. 
Chapter 5. Understanding the role of metabolic signals in control of CME 
Nutrient availability represents one of the dynamic environmental parameters which largely determines cellular phenotypes and behaviours via control of cellular metabolism and other cell functions $\mathrm{s}^{358}$. Nutrients such as glucose, proteins and lipids for instance, are taken up and converted by metabolic cellular processes into energy rich ATP molecules-functioning as the primary energy currency of the cell. As the main source of cellular fuel, ATP is responsible for driving fundamental energy-demanding processes (via energy released from ATP hydrolysis) including several pathways occurring at the plasma membrane ${ }^{363}$. The $\mathrm{Na}+/ \mathrm{K}+$ ATPase ion exchange pumps for example, are plasma membrane proteins - which by hydrolysis of ATP - maintains a physiological ion gradient and membrane potential difference across the plasma membrane necessary for cell survival and proliferation ${ }^{361,362}$. Other examples include RTKs and integrins, which upon activation on the plasma membrane similarly promotes ATP hydrolysis by inducing many ATP-consuming processes that contribute to cell growth, proliferation, differentiation, motility and migration ${ }^{59-61}$. As well, proteins involved in mediating energy uptake are similarly found on the plasma membrane including transporters for glucose, lipids, and amino acids ${ }^{42,43}$. As a predominant site for both energy consumption and uptake, the regulation of the plasma membrane is therefore intimately coupled to fluctuations in intracellular ATP concentrations (and hence, nutrient availability), of which perturbations have been demonstrated to specifically effect changes in the surfaceome profile ${ }^{360}$.

Perturbations in intracellular ATP concentrations can result from metabolic stress induced by nutrient starvation, hypoxia, ischemia, and exercise ${ }^{365-367}$. Specifically, these physiological stimuli largely result in the depletion of cellular ATP, thereby resulting in depressed energy states. In coping with metabolic stress, cells often employ a series of adaptive response strategies which functions in restoring cellular energy balance and homeostasis ${ }^{359,363}$. These strategies include the i) stimulation of catabolic processes (e.g. glycolysis) promoting ATP regeneration, and as well as the ii) inhibition of anabolic processes (e.g. glycogenolysis) in reducing ATP consumption. Likewise, reductions in cellular energy concomitantly induces iii) global remodeling of the plasma membrane largely involving the downregulation, and hence, inactivation of ATP-consuming surface proteins and processes; of which some were mentioned previously ${ }^{359,363}$. Importantly, these adaptive responses are the direct effects of AMPK activation, which serves as the primary cellular metabolic sensor activated by AMP bindingreflecting reductions in intracellular ATP levels ${ }^{359,363}$. Indeed, AMPK regulates metabolic pathways (catabolic and anabolic) by direct phosphorylation of several regulatory components discussed in more detail in section 1.7.1. However, the precise mechanism by which AMPK may directly regulate and elicit 
global remodeling of the plasma membrane remain poorly understood, which nevertheless, serve as the major question to be investigated in this chapter.

Previous results from our laboratory indicated the global remodeling of the plasma membrane upon activation of AMPK by its pharmacological agonist A-769662 (here abbreviated as A7) ${ }^{360}$. Through semi-quantitative mass spectrometry analysis, AMPK activation broadly reduced cell surface levels of many proteins including $\mathrm{Na}+/ \mathrm{K}+$ pumps and motogenic proteins such as $\beta 1$-integrins ${ }^{360}$. Precisely how AMPK induces surface protein downregulation, and hence, plasma membrane remodeling however, is not well-understood-although the involvement and mechanistic control of CME may be hypothesized. In addition, AMPK activation reduced surface levels of Arf6-GAPs (i.e. ArfGAP3 and ARAP) known to regulate the activation of the small GTPase, Arf6, on the plasma membrane ${ }^{360}$. Importantly, Arf6 was proposed to enhance CME via stimulation of local PIP 2 production and direct recruitment of AP2, indicating the potential regulation of CME by AMPK, through control of Arf6 GTP-binding ${ }^{379}$. In addition, $\beta 1$-integrins are CME cargoes known to internalize in an Arf6-dependent manner, of which AMPKmediated surface depletion further implies regulation of CME by AMPK, via control of Arf6 GTPbinding ${ }^{391,393}$. Through control of Arf6 GTP-binding, AMPK may thus regulate CME dynamics, thereby serving as a potential mechanism by which metabolic signals may directly effect changes in the cell surfaceome.

In this chapter, I elucidate the biological mechanism by which AMPK may induce global remodeling of the plasma membrane. I hypothesize that by control of CME, AMPK may broadly effect plasma membrane reorganization, which I investigated via TIRF-M imaging coupled to systematic analysis of CLSs used in the previous chapters ${ }^{5,8,207}$. In addition, I determined the requirement for Arf6 in the regulation of CME by AMPK, by probing the contributions of different Arf6 nucleotide states (e.g. Arf6-GTP or Arf6-GDP) on the endocytic process. I hypothesize that AMPK regulates the nucleotide state of Arf6, which in turn results in regulation of CME. Lastly, I determined the implications of AMPK activation on the internalization of CME cargoes, specifically of $\beta 1$-integrin. In doing so, I uncovered the extent by which AMPK may induce broad regulation of the plasma membrane through Arf6-dependent control of CME. Overall, I hypothesize that AMPK robustly remodels the plasma membrane by control of CME via Arf6 regulation-resulting in the downregulation of surface proteins via CME. 


\section{1- Activation of AMPK elicits global reduction in CCP size}

Previous findings from our laboratory indicate the robust reorganization of the plasma membrane protein content (cell surfaceome) upon pharmacological activation of AMPK by A7 in RPE cells $^{360}$. A7 is a chemical compound demonstrated to activate AMPK by direct binding, inducing conformational changes necessary for promoting Thr-172 phosphorylation; and hence, AMPK activation $^{426,427}$. Although the exact binding site of A7 on AMPK has not been elucidated, several studies have shown that treatment of cells with $A 7$ induces the specific activation of AMPK, as commonly reported by the biochemical measurement of phosphorylation of its known downstream effector, ACCof which increase by A7 can be detected as early as 10 minutes in RPE cells ${ }^{360,426,427}$. In the following studies, activation of AMPK, and hence, onset of metabolic stress, will therefore be recapitulated via A7induced activation of AMPK in RPE cells.

That AMPK activation induces broad regulation of the plasma membrane in RPE cells suggests its potential control of $\mathrm{CME}$-regarded as the predominant regulator of the mammalian plasma membrane complement of membrane proteins. To determine whether AMPK regulates the plasma membrane via control of CME, I first sought to determine the broad effects of AMPK activation on CME structure and dynamics. To do so, I used RPE cells expressing eGFP-CLCa treated with A7 for various times (e.g. 0, 5, $10,15,30$, and 60 minutes). The cells were then fixed and imaged via TIRF-M coupled to systematic detection and analysis of CLSs. Interestingly, acquisition and analysis of single frame images reveal a robust and significant reduction in eGFP-CLCa fluorescence intensity within detected CLSs (>10,000 CLSs per conditions) in almost all A7 treated conditions, detectable as early as 5 minutes following treatment; relative to control (0 minute) (Figure 5.1, A and B). The AMPK-induced reduction in CCP size was maintained for up to 30 minutes, upon which eGFP-CLCa fluorescence intensity began to slightly recover to what is seen in basal cells (not treated with A7). This finding therefore provides a preliminary indication of the role of AMPK in inducing broad plasma membrane remodeling by influencing the dynamics of clathrin structure assembly-and hence, overall CME.

While useful in initially determining the effects of AMPK on CME, single frame image detection and analysis nonetheless, remain limited to the broad identification of CLSs, which may or may not represent bona fide endocytic CCP structures ${ }^{1}$. Thus, to better understand the role of AMPK in regulation of CME dynamics, I employed live-cell TIRF-M imaging of eGFP-CLCa expressing RPE cells treated (or not; control) with A7 (10 minutes), to acquire time-lapse images for systematic detection, 
tracking, and analysis of bona fide CCP structures; as implemented in chapters 3 and 4. Interestingly, although A7 did not modify the total number of detected CLSs, A7 nevertheless significantly reduced the initiation density of bona fide CCPs (Figure 5.1C) - suggesting a reduction in efficiency of CCP assembly and formation imposed by A7 treatment. In addition, CCPs detected in A7 conditions exhibited significant reductions in eGFP-CLCa fluorescence intensities across various lifetime cohorts relative to control (Figure 5.1D), consistent with the A7-induced reductions in CLS size previously observed (Figure 5.1B). Importantly, while A7 appears to impair CCP assembly and formation, A7 treatment surprisingly increased CCP lifetimes which were longer-lived relative to control, suggesting increased efficiency of formation of longer-lived productive CCP once these structures initiate (Figure 5.1E). Taken together, A7 treatment-and hence AMPK activation-impairs the earliest stages of clathrin assembly and formation of bona fide CCPs. Those CCPs that do form under A7-treated conditions, are smaller in size, but nonetheless exhibit longer lifetimes suggestive of an enhancement in formation of productive CCPs. This is compatible with the role of AMPK in inducing global remodeling of the plasma membrane proteome via control of $\mathrm{CME}^{360}$.

\section{2- AMPK may regulate CME via control of Arf6 activity}

That A7 treatment effects regulation of CCP size and lifetime indicates the potential role of AMPK in the direct control of CCP dynamics and overall CME. Precisely how AMPK my directly regulate CCP assembly and formation however, is not well-understood. I hypothesize a role for Arf6 in AMPKdependent control of CME. As described in section 1.7.2, Arf6 is a small GTPase protein which upon translocation and activation on the plasma membrane, may enhance CME via stimulation of $\mathrm{PIP}_{2}$ production and recruitment of AP and clathrin to the cell surface ${ }^{379,383}$. Although the direct control of Arf6 by AMPK has not been documented in literature, it has been shown that AMPK activation directly causes the depletion of Arf6 regulators (e.g. ArfGAP3 and ARAP) from the plasma membrane, thereby influencing Arf6 nucleotide cycling and activity ${ }^{360}$. Thus, to determine whether Arf6 activation may serve as the mechanism by which AMPK may regulate $\mathrm{CME}$, in this section I will investigate the potential control of Arf6 by AMPK, and as well as the requirement for Arf6 in the regulation of CCP dynamics. 


\subsection{1 - AMPK may regulate Arf6 by promoting GTP hydrolysis}

Arf6 is a small GTPase which cycles between an inactive Arf6-GDP to an active Arf6-GTP conformation, of which the latter recruits various effector proteins described in section 1.7.2. As such, to first determine the potential regulation of Arf6 by AMPK, we assessed the effects of AMPK activation on the nucleotide state of Arf6 via FRET-FLIM microscopy. FRET-FLIM (Förster resonance energy transfer by fluorescence lifetime imaging) is a microscopic technique which allows identification of molecular interactions and protein binding in vivo, based on a fluorescent donor-acceptor system ${ }^{428,429}$. Specifically, to assess the role of AMPK in regulating Arf6 activation, we transfected RPE cells with a genetically-encoded fluorescent Arf6 construct (CyPET-tagged Arf6; Arf6-CyPET), along with a truncated construct of the downstream Arf6 effector, GGA3 (Golgi-localized gamma-ear containing Arf-binding protein 3), conjugated to fluorescent YPET (YPET-GGA3). Importantly, GGA3 is an established binding partner of Arf6, which is recruited and bound only by the active GTP-loaded Arf6 configuration ${ }^{388}$. The nucleotide state and hence, activation of Arf6, may thus be implicated from measurement of interaction between Arf6-CyPET and YPET-GGA3, detected and quantified via FRET-FLIM microscopy described in the following section (Figure 5.2A).

Fluorophores are characterized by distinct 'fluorescence lifetimes' that describes the duration between photon excitation and subsequent fluorescence emission-which depends on the distinct molecular compositions and dynamic nano-environments of fluorophores ${ }^{428,429}$. For example, the excitation (414nm) and fluorescence emission (475nm) of unbound Arf6-CyPET exhibits a $2 \mathrm{~ms}$ fluorescence lifetime, as measured by FRET-FLIM in RPE cells (Figure 5.2B). Expectedly, co-expression of YPET-GGA3 within the same cells reduced the fluorescence lifetime of Arf6-CyPET to $1.8 \mathrm{~ms}$, likely due to basal interactions between Arf6 and GGA3 (Figure 5.2B). Specifically, Arf6-CyPET (donor) binding to YPET-GGA3 (acceptor) juxtaposes the corresponding fluorophores, resulting in fluorescent quenching of CYPET - thereby reducing its fluorescent lifetime; further elaborated in [429-431]. Interestingly, A7 treatment rescues the reduction in Arf6-CyPET fluorescent lifetime to $1.9 \mathrm{~ms}$ (Figure 5.2B), suggesting the role of A7 in reducing Arf6 interaction with GGA3; and hence, potential enhancement of Arf6 GTP hydrolysis upon A7 treatment. Based on these findings, activation of AMPK may thus result in regulation of Arf6, specifically by enhancing GTP hydrolysis-rendering Arf6 inactive, unable to recruit its various downstream effectors including GGA3. 
The A7-induced increase in Arf6-CyPET fluorescent lifetime is indicative of reduced binding between Arf6 and GGA3, potentially resulting from an increase in Arf6 GTP hydrolysis. To further resolve whether the increase in Arf6-CyPET fluorescent lifetime was indeed, due to the reduction in Arf6-GTP, I used the SecinH3 Arf-GEF inhibitor and similarly measured interaction between Arf6-CYPET and YPETGGA3 via FRET-FLIM. SecinH3 is a well-established chemical inhibitor of Arf-GEFs that binds to the conserved Sec7 domain-impairing GEF activity ${ }^{432,433}$. Arf6-GEFs such as cytohesin/ARNO are amongst the inhibitory targets of SecinH3, which prevents GTP loading thereby increasing GDP-bound Arf6 ${ }^{432}$. Interestingly, SecinH3 treatment of RPE cells co-expressing Arf6-CYPET and YPET-GGA3, increases the fluorescent lifetime of Arf6-CyPET to a degree similar to that elicited by A7, indicating the effects of SecinH3 in reducing Arf6 GTP loading (Figure 5.2B). These results thus indicate that $A 7$, and hence AMPK, reduces Arf6 interaction with GGA3 likely by causing Arf6 inactivation via enhanced GAP activity and/or reduced GEF activity. This in turn suggests that AMPK may control CME as a result of reduced levels of GTP-bound Arf6, consistent with reduced efficiency in CCP dynamics imposed by A7 treatment previously demonstrated (Figure 5.1).

\subsection{2 - Arf6 interacts and localizes within CCPs in a GTP-dependent manner} Arf6 is a known regulator of CME, of which regulation by AMPK, may therefore serve as the

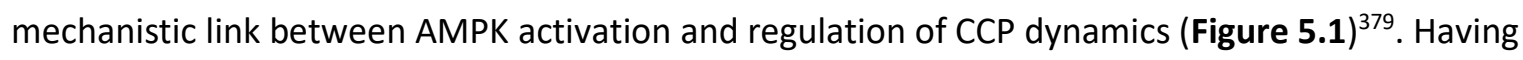
determined that AMPK activation may regulate Arf6 activity, I then sought to determine the role of Arf6 in regulating CME dynamics. Described in section 1.7.2, the activation of Arf6 occurs concomitant to its translocation to the plasma membrane, where it functions in promoting both local production of $\mathrm{PIP}_{2}$ and direct recruitment of AP2 and clathrin-suggesting its localization within CCPs surface ${ }^{379,383}$. Although poorly understood, the potential regulation of CME dynamics by Arf6 may therefore involve its direct interactions and control of various endocytic components required for CCP assembly and formation-which may be hypothesized due to its localization within endocytic structures. Thus, to test this hypothesis, I investigated whether Arf6 does indeed, localize within clathrin structures, and determined the impact of differential nucleotide states (i.e. GTP/GDP binding) on its spatial organization.

To determine the localization of Arf6 within clathrin structures and how GTP/GDP binding may impact its spatial distribution, I employed TIRF-M imaging of fixed RPE cells stably expressing CLCa fused 
to the fluorescent Tag-RFP-T (RFP-CLCa), transfected with various genetically-encoded recombinant Arf6 proteins. Specifically, the Arf6 constructs used were fused to fluorescent eGFP, and exhibited various degrees of GTPase activity, listed in the following: i) Wild-type Arf6 (eGFP-Arf6 ${ }^{\text {WT}}$ ); ii) GTPase-deficient Arf6 mutant that is GTP-locked and constitutively active (eGFP-Arf6 ${ }^{\mathrm{Q} 67 \mathrm{~L}}$ ); and a iii) Fast-cycling Arf6 mutant that is GDP-locked and constitutively inactive (eGFP-Arf6 $\left.{ }^{\mathrm{T} 44 \mathrm{~N}}\right)^{394}$. Single frame images of these cells were acquired and subsequently subjected to automated detection and analysis of CLSs, in addition to quantitative measurement of fluorescent eGFP-Arf6 contained therein-representing Arf6 recruitment ${ }^{5,8,207}$. Strikingly, this strategy reveals the significant enrichment of GTP-locked Arf6 (eGFPArf6 $^{\mathrm{Q} 67 \mathrm{~L}}$ ) within CLSs relative to control (eGFP-Arf6 ${ }^{\mathrm{WT}}$ ) (Figure 5.3), consistent with the enhanced GTPdependent interaction between Arf6 and its downstream effectors including components of the endocytic machinery; as described in section 1.7.2. In contrast, GDP-locked Arf6 (eGFP-Arf6 ${ }^{\text {T44N }}$ ) exhibited reduced CLS localization compared to control, further corroborating the requirement for GTP loading in translocation of Arf6 within clathrin structures (Figure 5.3). As such, these results indicate that Arf6 does indeed localize within clathrin structures on the plasma membrane in a GTP-dependent manner, promoting its interaction with various endocytic components-therefore potentially regulating CME.

That A7 treatment enhances the proportion of the GDP-bound state of Arf6 (Figure 5.2) indicates the potential role of AMPK in inhibiting Arf6 translocation within clathrin structures. Thought to enhance $\mathrm{CME}$, dissociation of Arf6 from clathrin structures by AMPK activation, may therefore be hypothesized to reduce efficiency of CCP assembly and formation; serving as a mechanism by which AMPK may regulate CME dynamics, as previously observed (Figure 5.1). Thus, to determine the role of AMPK in regulating the spatial localization of Arf6, I implemented the identical TIRF-M imaging strategy used above (Figure 5.3), using RFP-CLCa expressing RPE cells, transfected with either of the recombinant eGFP-tagged Arf6 proteins. In addition, the RPE cells were treated (or not; control) with A7 and subsequently fixed, imaged and analyzed as previously performed-to determine the effects of AMPK activation on Arf6 localization. Strikingly, treatment with A7 induces a significant reduction in recruitment of wild-type Arf6 (eGFP-Arf6 ${ }^{\mathrm{WT}}$ ) within CLSs relative to the non-treated, control condition (Figure 5.3), corroborating the reduction in GTP loading of Arf6, imposed by AMPK activation (Figure 5.2B). Correspondingly, A7 was without effect on the localization of both GDP- and GTP-locked Arf6 mutants within CLSs, strongly suggesting the regulation of Arf6 by AMPK predominantly via control of its 
nucleotide state (Figure 5.3). Thus, these findings indicate the role of AMPK in inhibiting Arf6 localization within clathrin structures, via regulated control of Arf6 GTP/GDP binding.

\subsection{3 - Regulation of Arf6 controls clathrin assembly and function}

I showed that the activation of AMPK reduces the formation of bona fide CCPs, in addition to the inactivation and dissociation of Arf6 from clathrin structures. As described, Arf6-GTP directly localizes within CLSs where it may enhance CME by inducing local $\mathrm{PIP}_{2}$ production and recruitment of endocytic proteins (e.g. AP2, clathrin, FCHO, CALM and actin related components) ${ }^{379}$. Thus, the AMPKinduced inactivation and inhibition of Arf6 translocation within CCPs, may serve as a mechanism by which AMPK may robustly regulate CME. To test this hypothesis, I sought to directly determine the effect of Arf6 inactivation on CCP dynamics in eGFP-CLCa expressing RPE cells. Specifically, enhanced GDP binding, and hence, inactivation of Arf6 in RPE cells, was induced via chemical inhibition of Arf6GEFs by SecinH3. Using the similar live-cell TIRF-M imaging strategy implemented in section 5.2.1, timelapse images of cells were acquired and subsequently subjected to automated detection, tracking and analysis of bona fide CCPs. Strikingly, SecinH3 treatment phenocopies the effects of A7-induced activation of AMPK on the dynamics of CCP assembly and formation (Figure 5.4). In particular, SecinH3 treatment did not modify the total number of detected CLSs, but significantly reduced the initiation density of bona fide CCPs to a similar degree elicited by A7 (Figure 5.4B). As with A7, SecinH3 treatment also reduced the size of bona fide CCPs across various lifetime cohorts as indicated by the reduction in eGFP-CLCa fluorescence intensity of detected CCPs (Figure 5.4C). Lastly, SecinH3 also increased the lifetimes of bona fide CCPs, which although smaller in size, nonetheless exhibited a higher mean lifetime relative to the non-treated, control condition (Figure 5.4D). Taken together, the similar phenotypic changes in CCP dynamics elicited by both SecinH3 and A7 treatment, is consistent with the hypothesis that AMPK may indeed act through control of Arf6 to regulate CME.

The regulation of CCP dynamics upon activation of AMPK by A7 results in reduced CCP density and size, while increasing CCP lifetimes. Although control of CME by AMPK is not well-understood, our findings indicate that AMPK may act through Arf6 to elicit broad regulation of CCPs. As a molecular switch, Arf6 toggles between an active GTP- and an inactive GDP-bound state, of which the former was demonstrated to localize within clathrin structures where it may interact with endocytic components ${ }^{379}$. The localization of Arf6-GTP within clathrin structures likely enhances the assembly and formation of 
CCPs as previously discussed, of which inactivation and dissociation may therefore impair the efficient formation of bona fide CCPs. AMPK was demonstrated to reduce Arf6-GTP by enhancing the levels of Arf6 bound to GDP, which concomitantly reduces Arf6 localization within CCPs. That AMPK activation elicits inactivation and depletion of Arf6 from CCPs, in parallel with reductions in CCP density and size, indicates that control of CME by Arf6 may serve as the mechanism through which AMPK may broadly regulate $\mathrm{CME}$, and hence induce broad regulation of the plasma membrane.

\section{3- AMPK selectively regulates CME of surface cargoes}

Activation of AMPK by A7 was originally shown from our previous studies to induce global remodeling of the plasma membrane, causing the depletion of several proteins from the cell surface ${ }^{360}$. Importantly, that A7 treatment was also demonstrated to effect regulation of CME dynamics, imply the potential role of AMPK in enhancing plasma membrane endocytosis in promoting downregulation of surface proteins. However, our current findings suggest otherwise as AMPK activation was demonstrated to reduce CCP initiation and assembly-indicating the potential role of AMPK in impairing and limiting the endocytic process. Although reducing the initiation density and size of bona fide CCPs, AMPK nevertheless effects longer CCP lifetimes, which on the contrary, suggests the role of AMPK in potentially enhancing endocytic productivity and internalization of bona fide CCPs. Thus, to understand and resolve how AMPK control of CME might regulate cargo endocytosis-and hence, broad reorganization of the plasma membrane-in this section, I will determine the effects of AMPK activation on the internalization of various CME cargoes including TfR and EGFR, with specific focus on $\beta 1$-integrin.

\subsection{1 - AMPK selectively enhances the endocytosis of $\beta 1$-integrin containing CCPS} Amongst the surveyed proteins on the plasma membrane, $\beta 1$-integrin was one identified by mass spectrometry analysis to be largely depleted from the cell surface upon A7-induced activation of $\mathrm{AMPK}^{360}$. The depletion of $\beta 1$-integrin from the plasma membrane suggests the potential enhancement of its endocytosis, which described in section 1.7.3.1, may predominantly occur through an Arf6dependent CME pathway. Thus, the previously demonstrated control of Arf6 by AMPK may be hypothesized to enhance $\mathrm{CME}$, leading to the increased endocytosis and depletion of $\beta 1$-integrin from 
the cell surface. To precisely determine the role of AMPK activation on $\beta 1$-integrin CME, we first sought to validate the previous mass spectrometry findings via microscopic visualization and quantification of the AMPK-induced reduction of surface $\beta 1$-integrin. To do so, I labeled cell surface $\beta 1$-integrin of intact RPE cells with fluorescent anti- $\beta 1$-integrin antibodies, which were treated (or not; control) with A7 for a short timescale (e.g. 5 minutes; to eliminate the potential contributions of recycling pathways) ${ }^{434}$. The cells were subsequently fixed and imaged via immunofluorescence microscopy, to acquire single frame images of the cell surface for manual analysis by ImageJ software as per [111]. With ImageJ, cells were manually outlined of which enclosed areas were quantified for mean pixel intensity of fluorescenceproviding measurement of $\beta 1$-integrin labelling and hence, surface expression levels. Indeed, A7 treatment significantly reduced surface $\beta 1$-integrin, as reported by the reduction in mean fluorescence intensity of $\beta 1$-integrin labelling, relative to control (Figure 5.5A). These findings are consistent with the previous mass spectrometry analysis, indicating that the A7-induced depletion of $\beta 1$-integrin, may indeed be due to its enhanced endocytosis elicited by AMPK control of CME.

That AMPK may directly enhance $\beta 1$-integrin endocytosis via control of $\mathrm{CME}$, raises the question of whether AMPK may similarly regulate internalization of other CME cargoes - in eliciting global remodeling of the plasma membrane. Thus, to understand the scope of CME regulation by AMPK, we quantitatively measured the internalization of the established CME cargoes, TfR and EGFR, at various time points (e.g. 0, 2, 5, and 10 minutes) in RPE cells treated (or not, control) with A7-via biochemical ligand internalization assay used in section 3.1. Interestingly, this strategy reveals the lack of effect of A7 treatment on the internalization of both TfR and EGFR relative to control, indicating the selective role of AMPK in enhancing the endocytosis of a subset of CME cargoes such as $\beta 1$-integrin (Figure 5.5B). Collectively, these results nonetheless indicate that although robustly reducing CCP initiation and CCP size, AMPK may selectively maintain (e.g. TfR and EGFR) or enhance (e.g. $\beta 1$-integrin) CME of plasma membrane proteins, of which precise mechanisms nevertheless, remain to be elucidated.

\subsection{2 - AMPK enhances $\beta 1$-integrin recruitment to CCPs and may selectively regulate integrin-containing CCPS}

Treatment of RPE cells with A7 was shown to globally reduce CCP initiation and CCP size, which indicates a role for AMPK in broadly regulating CME. The respective maintenance and enhancement of 
endocytosis of TfR and EGFR, and $\beta 1$-integrin upon A7 treatment however, may suggest the selective role of AMPK in differentially regulating the assembly dynamics of distinct cargo-containing CCPs. Specifically, that surface $\beta 1$-integrin was robustly depleted by $A 7$ treatment, implies the possible lack of effect of AMPK in regulating $\beta 1$-integrin containing CCPs. Thus, to determine whether AMPK may distinctly regulate $\beta 1$-integrin containing CCPs, I transfected a genetically-encoded recombinant $\beta 1$ integrin protein fused with a fluorescent mCherry tag (mCh- $\beta 1$-integrin), into eGFP-CLCa expressing RPE cells. The cells were treated (or not; control) with A7 for 15 minutes and imaged via live-cell TIRF-M, to subsequently obtain time-lapse images for automated software detection, tracking and analysis of bona fide CCPs. With this strategy, CCPs with (integrin+ CCPs) or without (integrin- CCPs) $\beta 1$-integrin were also identified based on signal detection of $\mathrm{mCh}-\beta 1$-integrin fluorescence contained therein ${ }^{207}$. Expectedly, A7 treatment was shown to reduce the overall initiation density of bona fide CCP structures, demonstrated in section 5.1 (Figure 5.1). Strikingly, although robustly reducing the number of bona fide CCPs, A7 treatment was without effect on the initiation density of integrin+ CCPs, of which levels were comparable to the control condition (Figure 5.6A). Consistently, A7 treatment significantly reduced the density of integrin- CCPs; comparable to the broad reduction in CCP initiation (Figure 5.6A). Thus, that A7 treatment reduces overall CCP initiation, but not the formation of $\beta 1$-integrin containing CCPsindicates the selective role of AMPK in regulation of distinct cargo-containing CCPS.

The sustained rate of formation of integrin + CCPs under A7 treatment, may imply their unimpaired assembly dynamics, in contrast to the perturbations in CCP assembly broadly imposed by AMPK on CCPs (Figure 5.1 and 5.6A). Thus, to determine the effects of AMPK activation on the assembly and formation of $\beta 1$-integrin containing CCPs, I subjected the previously acquired time-lapse images to systematic quantification of eGFP-CLCa fluorescence intensity of bona fide CCPs; implemented in section 4.1.2. As expected, integrin+ and integrin- CCPs did not appreciably differ in size (and hence, in assembly dynamics) in the non-treated, control condition, as indicated by their comparable mean eGFP-CLCa fluorescence across various CCP lifetime cohorts (Figure 5.6B). In contrast, A7 treatment significantly reduced the mean fluorescence intensity of eGFP-CLCa within integrin- CCPs, relative to integrin+ CCPs across various lifetime cohorts, indicating the impairment of CCP assembly and size by AMPK, of integrin deficient clathrin structures (Figure 5.6B). Importantly, the mean eGFP-CLCa fluorescence intensity of integrin+ CCPs were largely unaffected by A7 treatment, which were therefore, larger in size relative to integrin- CCPs (Figure 5.6B). Taken together, these results indicate that the assembly and formation of CCPs containing $\beta 1$-integrin, are more resistant to negative regulation of CCP assembly by AMPK than 
$\beta 1$-integrin deficient CCPs - consistent with the lack of effect of A7 treatment on the initiation density of integrin+ CCPs.

Collectively, our results demonstrate the role of AMPK in negatively regulating CCPs by broadly reducing initiation rates, and impairing efficient clathrin assembly. Nonetheless, AMPK does not appear to affect the assembly dynamics of $\beta 1$-integrin containing CCPs, therefore sustaining $\beta 1$-integrin internalization despite broad modifications in CME by AMPK activation. That AMPK does not elicit changes in the rate of formation and assembly of integrin+ CCPs however, raises the question of how A7 treatment may enhance $\beta 1$-integrin endocytosis (and hence surface depletion) to an extent-greater than that observed in non-treated, control conditions (Figure 5.5A). Previously described, the internalization rate of cargoes may be enhanced by increasing cargo recruitment within endocytic structures (see section 4.1), which may provide a mechanism by which AMPK may upregulate $\beta 1$ integrin endocytosis and surface depletion, without affecting the initiation rate and density of bona fide integrin+ CCPs. Thus, to determine whether AMPK enhances the recruitment of $\beta 1$-integrin within CCPs, I similarly subjected the previously obtained time-lapse images of eGFP-CLCa expressing RPE cells transfected with mCh- $\beta 1$-integrin, to automated software detection and analysis of integrin+ CCPs. Specifically, I measured the fluorescence intensity of $\mathrm{mCh}-\beta 1$-integrin within bona fide integrin+ CCPs in both $A 7$ and control treatment, to quantify the amount of $\beta 1$-integrin recruitment within endocytic structures. Strikingly, A7 treatment significantly enhances the mean mCh- $\beta 1$-integrin fluorescence intensity of integrin + CCPs, relative to control across various lifetime cohorts (Figure 5.6C). This finding therefore indicates that although unable to enhance the rate of initiation and assembly of integrin+ CCPs, AMPK nevertheless may enhance $\beta 1$-integrin endocytosis by increasing the rate of receptor recruitment within endocytic structures-of which precise mechanism however, remains to be elucidated.

\section{4- Summary of Chapter 5}

Previous findings from our laboratory showed the robust depletion of specific surface proteins from the plasma membrane upon pharmacological activation of AMPK by A7; as measured by mass spectrometry $^{360}$. AMPK is a phylogenetically conserved metabolic sensor, activated at the onset of metabolic stress. Metabolic stress induced by various physiological stimuli including hypoxia, ischemia, 
nutrient starvation and exercise, reduces cellular energy resulting in the depletion of intracellular ATP ${ }^{365-367}$. Specifically, the reduction in ATP and concomitant increase in AMP levels, directly activates AMPK which restores metabolic homeostasis and optimal cellular energy states-through activation of various adaptive response pathways ${ }^{359,363}$. Adaptive response pathways induced by AMPK largely facilitates the downregulation of energy consuming anabolic processes, and the upregulation of energy producing catabolic pathways which synergistically functions in increasing cellular energy levels ${ }^{359,363}$. Importantly, the robust reorganization of the plasma membrane upon AMPK activation, may similarly be regarded as a component of its energy-conserving repertoire, which inhibits several ATP-consuming proteins and pathways at the cell surface. The biological mechanism by which AMPK may elicit plasma membrane remodeling however, is not well studied-which nevertheless-was found in this chapter to likely involve the AMPK-mediated control of CME.

As a major regulator of the plasma membrane, in this chapter I investigated how AMPK activation by $\mathrm{A} 7$ may regulate the process of $\mathrm{CME}$, in inducing global remodeling of the cell surfaceome. Specifically, I demonstrated that activation of AMPK robustly impairs the initiation rates of CCPs and their corresponding assembly and size, as detected and quantified by TIRF-M imaging coupled to automated software analysis. While these perturbations in CCP dynamics may indicate the role of AMPK in negatively regulating $\mathrm{CME}, \mathrm{CCPs}$ which form under $\mathrm{A} 7$ treated conditions nevertheless, exhibited longer lifetimes relative to control-suggesting the potential enhancement of the efficiency of CCPs that do form to yield productive vesicles upon AMPK activation, despite a reduction in the overall rate of CCP initiation. As such, the regulation of CME appears to be intimately coupled to the activation of AMPK, thus serving as a potential mechanism by which AMPK may induce global reorganization of the plasma membrane.

How AMPK activation may mechanistically regulate CME dynamics is not well-understood, although several lines of evidence including that from our laboratory, suggests the potential involvement of Arf6. Arf6 is a small GTPase which is activated upon translocation to the plasma membrane, known to enhance CME via local PIP 2 production and AP2 and clathrin recruitment ${ }^{379,383}$. Our laboratory has recently shown that regulators of Arf6 are depleted from the plasma membrane upon A7 treatment, suggesting the potential control of Arf6 by AMPK activity. Indeed, FRET-FLIM imaging revealed a reduction in GTP-bound Arf6, and hence, inactivation of Arf6 upon A7 treatment, indicating the negative regulation of Arf6 by AMPK. As a small GTPase, the biological function of Arf6 is contingent 
on its ability to cycle between GDP- and GTP-bound states, of which the latter was hypothesized to interact/associate with components of the endocytic machinery-likely to a greater extent relative to its inactive GDP form. Consistent with this hypothesis, Arf6-GTP was shown to largely localize within clathrin structures wherein it may enhance CCP assembly, in contrast to Arf6-GDP of which enrichment within clathrin structures was not appreciably detected. The pharmacological inhibition of Arf6 activation by SecinH3 was also demonstrated to impair the rates of CCP initiation and assembly, indicating the requirement for Arf6-GTP in the efficient progression of CME. That SecinH3 phenocopies the effects of A7 on CCP dynamics, thus indicate Arf6 inactivation as a likely mechanism by which AMPK may regulate CME. Specifically, by inactivating Arf6, AMPK may inhibit Arf6 CCP localization thereby reducing the rate of initiation and assembly of bona fide CCPs-ultimately resulting in broad regulation of the plasma membrane.

As demonstrated, A7 treatment significantly impairs the rate of initiation and assembly of bona fide CCPs, which nevertheless exhibit longer lifetimes relative to control-suggesting the potential role of AMPK in either inhibiting or enhancing CME; or both. Thus, to resolve how regulation of CME by AMPK may affect the endocytic process, we measured the internalization of established CME cargoes under A7 treatment, including TfR, EGFR and $\beta 1$-integrin. Interestingly, the internalization rates of TfR and EGFR were unaffected by A7 treatment, suggesting that AMPK may maintain CME despite robustly impairing CCP dynamics; of which mechanism, albeit remains to be elucidated. In contrast, AMPK activation significantly enhanced $\beta 1$-integrin endocytosis, consistent with the robust surface depletion of $\beta 1$-integrin by $A 7$ treatment, previously demonstrated via mass spectrometry ${ }^{360}$. Furthermore, the initiation density and size of $\beta 1$-integrin containing CCPs, were largely unaffected by AMPK activation, which however increased the recruitment and concentration of $\beta 1$-integrin contained therein-thereby enhancing $\beta 1$-integrin CME regardless of broad perturbations in CCP dynamics. Taken together, although AMPK robustly impairs CCP dynamics, AMPK may nonetheless differentially regulate distinct cargo-containing CCPs, of which overall regulatory output may result in enhanced cargo endocytosis and downregulation from the cell surface.

In summary, the activation of AMPK and its subsequent control of CME thus, provide a mechanism through which metabolic signals may induce global reorganization of the plasma membrane (Figure 5.7). It has been demonstrated that the pharmacological activation of AMPK by A7 causes the depletion of surface proteins, which in this chapter was demonstrated may be due to the AMPK- 
mediated regulation of CME. It was shown that by inactivating Arf6, AMPK robustly impairs the rate of initiation and assembly of bona fide CCPs, which nevertheless may be limited to certain CCP subsets as $\beta 1$-integrin containing CCPs were largely unaffected. Furthermore, despite the resulting impairments in CCP dynamics, endocytosis of CME cargoes was largely unaffected (e.g. TfR and EGFR) by AMPK activation, and in certain cases was enhanced as exemplified by the increased endocytosis and recruitment of $\beta 1$-integrin within CCPs. While the precise mechanisms by which AMPK may differentially regulate (e.g. maintain, enhance or diminish) CME of distinct cargoes remain to be elucidated, our findings nevertheless demonstrate the role of AMPK in broadly regulating plasma membrane endocytosis-consistent with previous findings on its role in inducing global remodeling of the cell surfaceome. 


\section{5- Figures for Chapter 5}

A
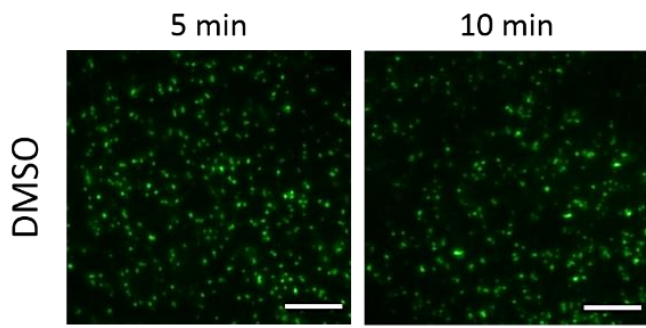

这
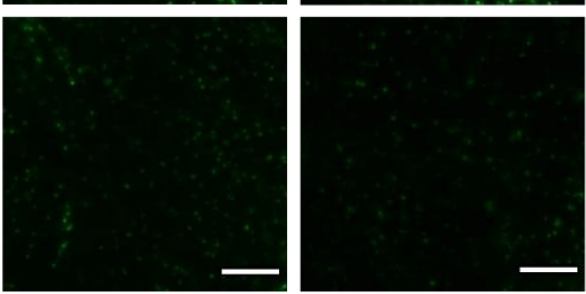

C

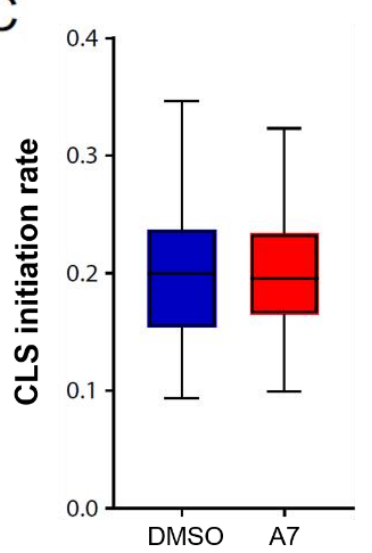

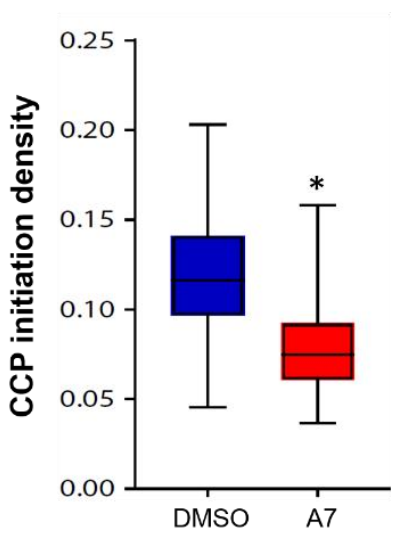

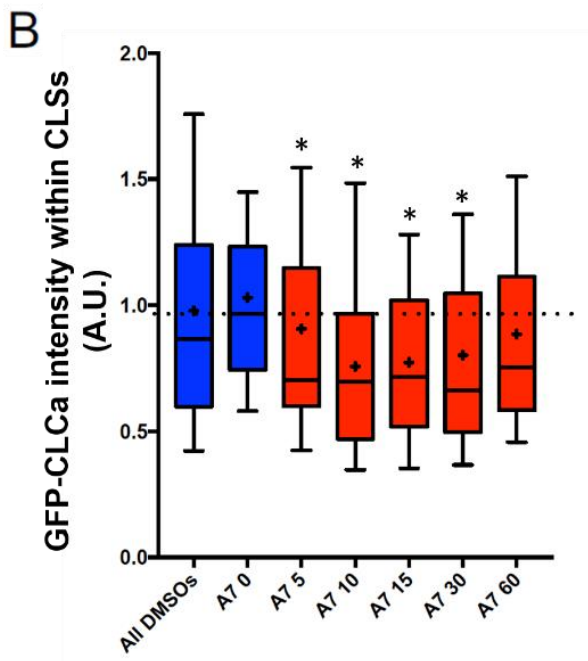

E

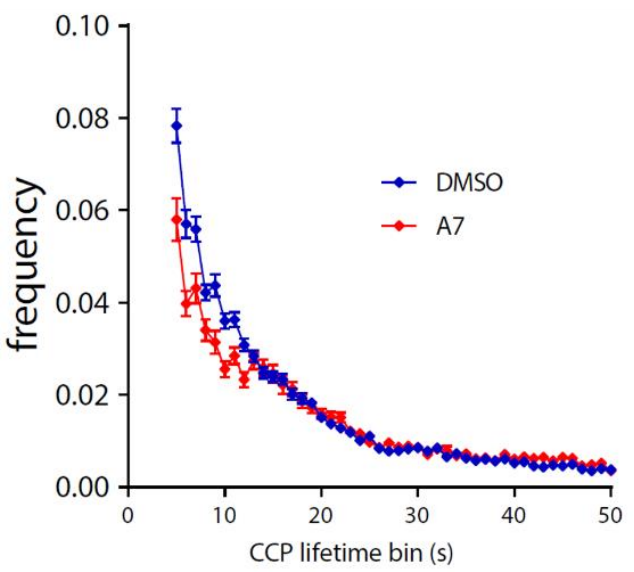

D
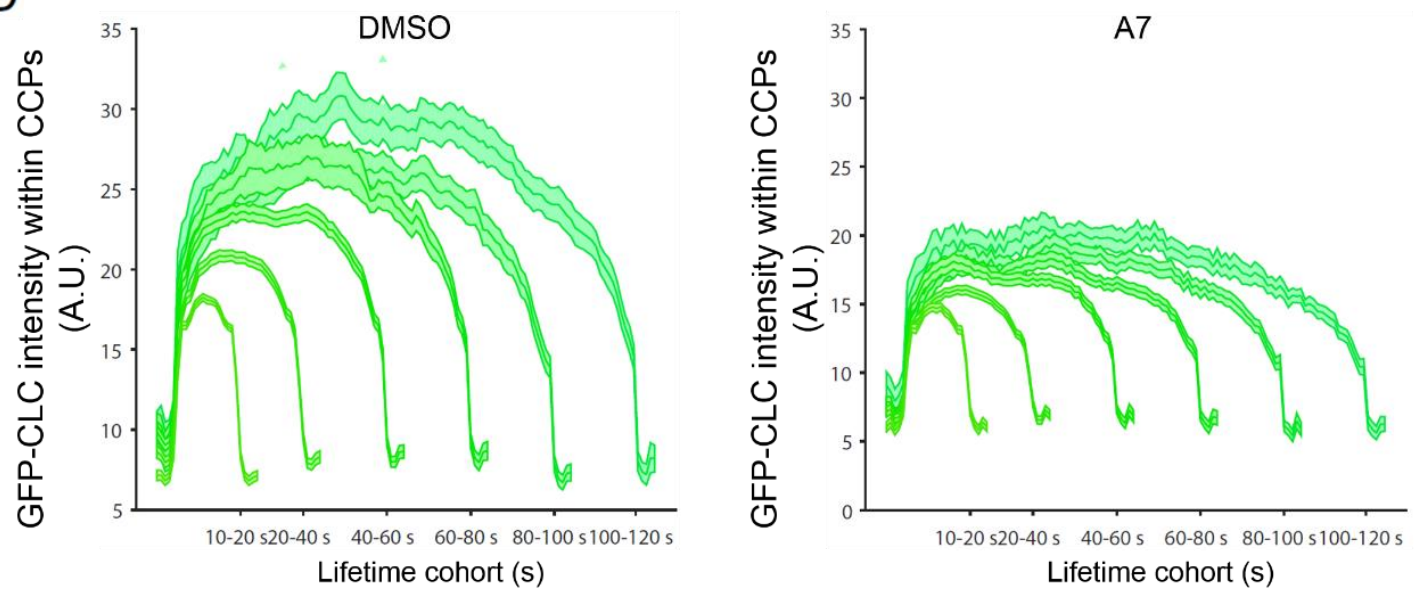
Figure 5.1. A7 treatment regulates the rate of initiation and assembly of CCPs. (A-B) RPE cells stably expressing clathrin light chain fused to eGFP (eGFP-CLCa) were pretreated with $100 \mu \mathrm{M}$ A7 for various time points (e.g. 0, 5, 10, 15, 30 and $60 \mathrm{~min}$ ), and then subjected to fixation and imaging by TIRF-M. (A) Shown are representative single frame fluorescence micrographs. Scale bar: $5 \mu \mathrm{m}$. (B) The fluorescence intensity of eGFP-CLCa within detected clathrin structures were computationally quantified (reported as arbitrary units [A.U.]) as described in Materials and Methods; from a minimum of three independent experiments in each condition. ${ }^{*}, \mathrm{p}<0.05$ vs DMSO control, $\mathrm{k}$ (cells) > 30, n (CLSs) > 10,000 per condition. (C-E) RPE cells stably expressing clathrin light chain fused to eGFP (eGFP-CLCa) were pretreated with $100 \mu \mathrm{M} \mathrm{A7}$ for 15 min and simultaneously subjected to time-lapse imaging via TIRF-M. (C) Time-lapse TIRF-M image series of cells were subjected to automated detection, tracking, and analysis of CLSs as described in Materials and Methods, allowing identification of sCLSs and bona fide CCPs. Median, 25th/75th percentiles (boxes) and Tukey range (whiskers) for the initiation rate of CLSs (left panel) and initiation density of CCPs (right panel) are shown. (D) Mean eGFP-CLCa fluorescence intensity traces (reported as arbitrary units [A.U.]) in lifetime cohorts of CCPs detected in (C), from RPE cells treated (right panel) or not (left panel) with A7. (E) Lifetime distributions of all detected bona fide CCPs in (C), from RPE cells treated (red line) or not (blue line) with A7. (C-E) From a minimum of three independent experiments in each condition. ${ }^{*}, \mathrm{p}<0.05$ vs DMSO control, $\mathrm{k}$ (cells) > 30, $\mathrm{n}$ (CLSs) > 10,000 per condition. 


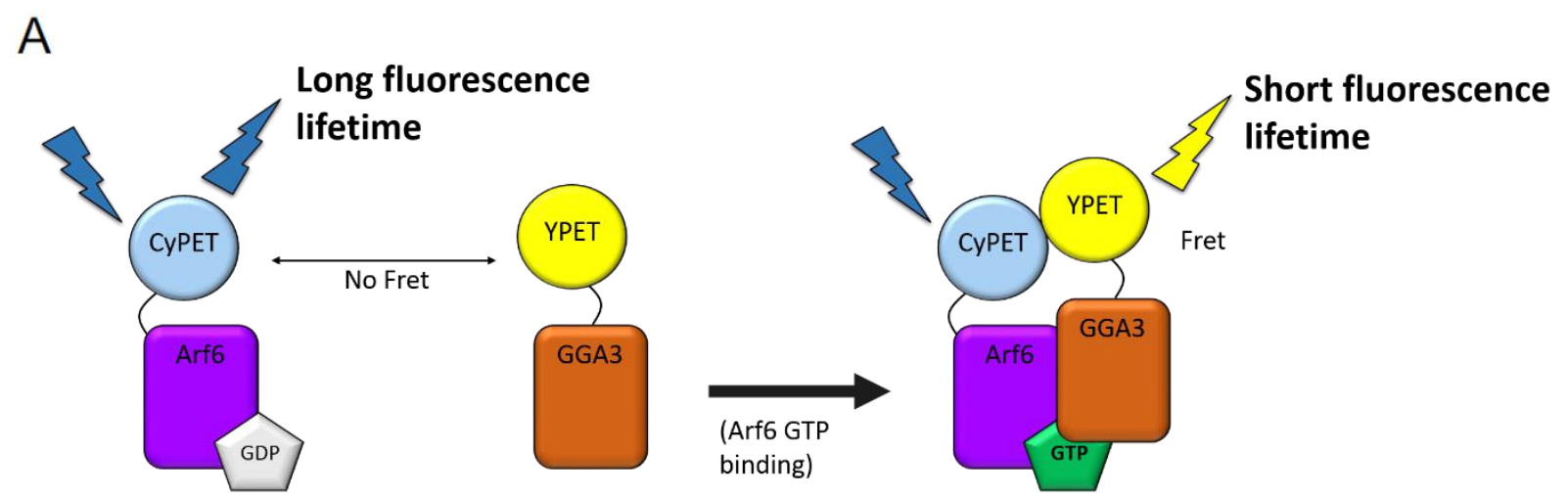

B

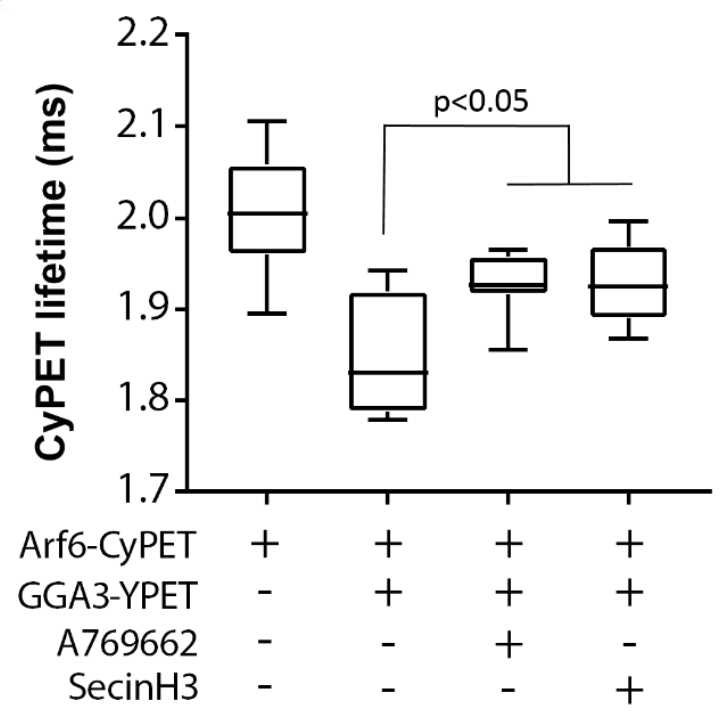

Figure 5.2. AMPK activation regulates Arf6 GTP/GDP binding. Wild-type RPE cells were transfected with a genetically-encoded fluorescent Arf6 construct (CyPET-tagged Arf6; Arf6-CyPET), along with a truncated construct of the downstream Arf6 effector, GGA3 (Golgi-localized gamma-ear containing Arfbinding protein 3), conjugated to fluorescent YPET (YPET-GGA3). Interaction between CyPET-Arf6 (donor) and YPET-GGA3 (acceptor) was measured via FRET-FLIM microscopy as per methods described in [429-431]. (A) Shows a schematic diagram of the FRET-FLIM experimental design, for assay of Arf6 interaction with GGA3. (B) Shown are the fluorescence lifetimes (ms) of CYPET under various conditions, representing the degree of Arf6 and GGA3 interaction as measured via FRET-FLIM. Derived from one experiment in each condition. $\mathrm{p}<0.05$ vs DMSO control, $\mathrm{k}$ (cells) $>30$. These experiments were performed by Farnaz Fekri (PhD. Candidate); Ryerson University, Toronto, ON, Canada. 


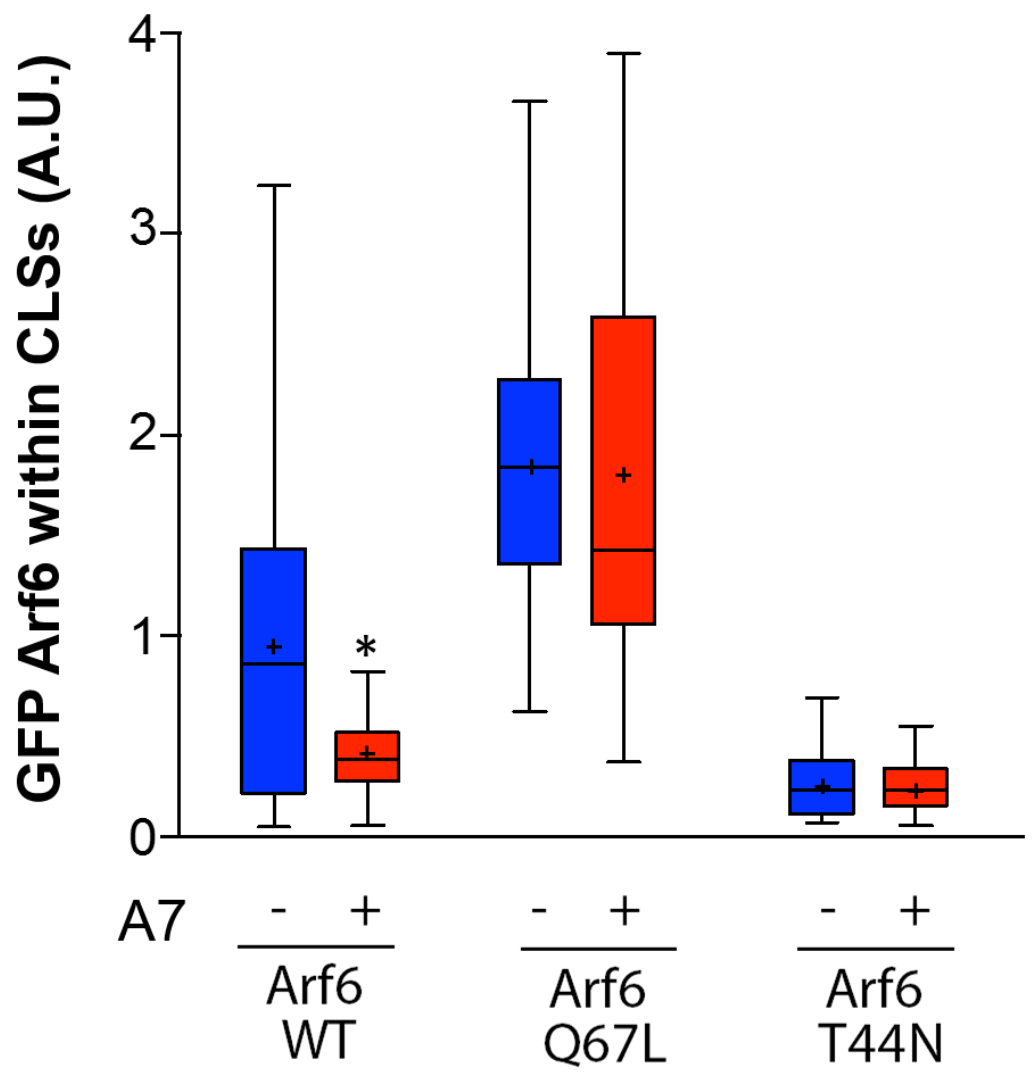

Figure 5.3. Arf6 associates with clathrin-coated pits in a GTP-dependent manner. RPE cells stably expressing CLCa fused to the fluorescent Tag-RFP-T (RFP-CLCa) were transfected with geneticallyencoded recombinant Arf6 proteins fused to fluorescent eGFP: i) Wild-type Arf6 (eGFP-Arf6'WT); ii) GTPase-deficient Arf6 mutant that is GTP-locked and constitutively active (eGFP-Arf6 ${ }^{\text {Q67L }}$ ); and a iii) Fast-

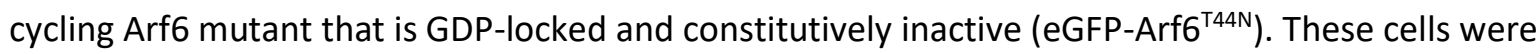
pretreated with $100 \mu \mathrm{M} \mathrm{A7}$ for $15 \mathrm{~min}$, and then subjected to fixation and imaging by TIRF-M. The fluorescence intensity of eGFP-Arf6 (reported as arbitrary units [A.U.]) within detected clathrin structures were computationally quantified as described in Materials and Methods; from a minimum of three independent experiments in each condition. ${ }^{*}, \mathrm{p}<0.05$ vs DMSO control, $\mathrm{k}$ (cells) $>30, \mathrm{n}$ (CLSs) $>$ 10,000 per condition. 
A

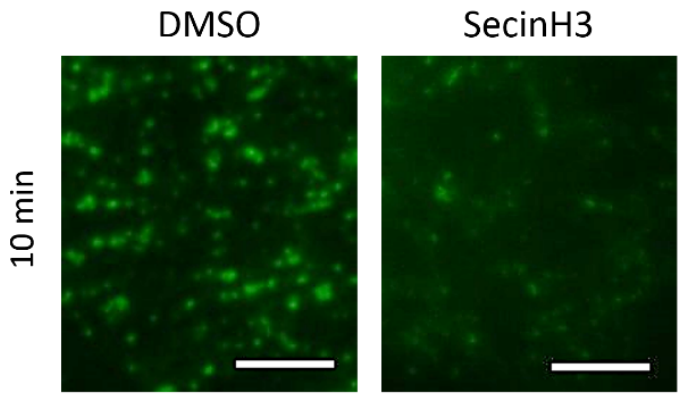

C

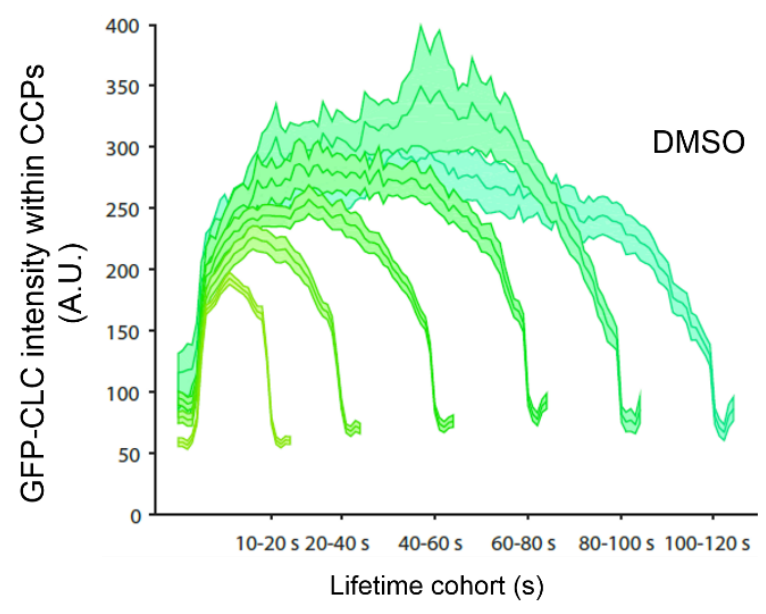

D

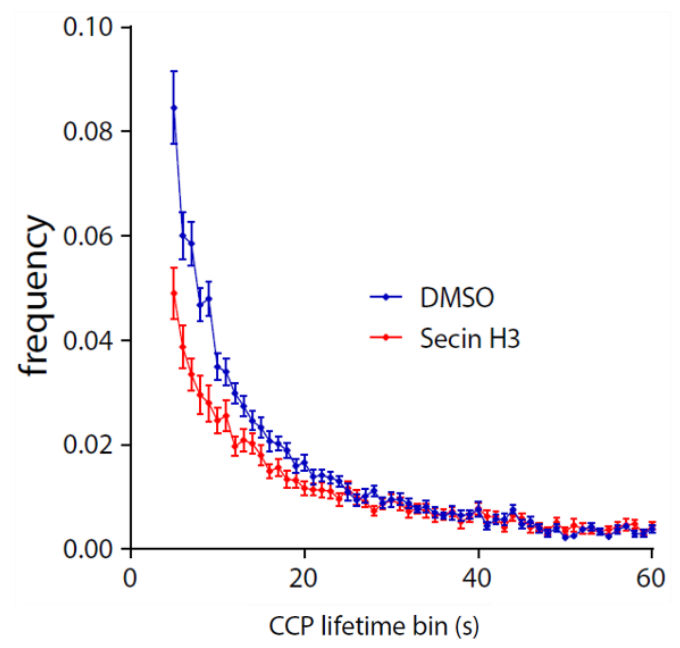

C
B

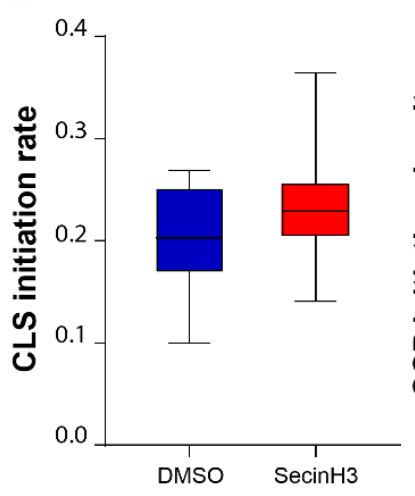

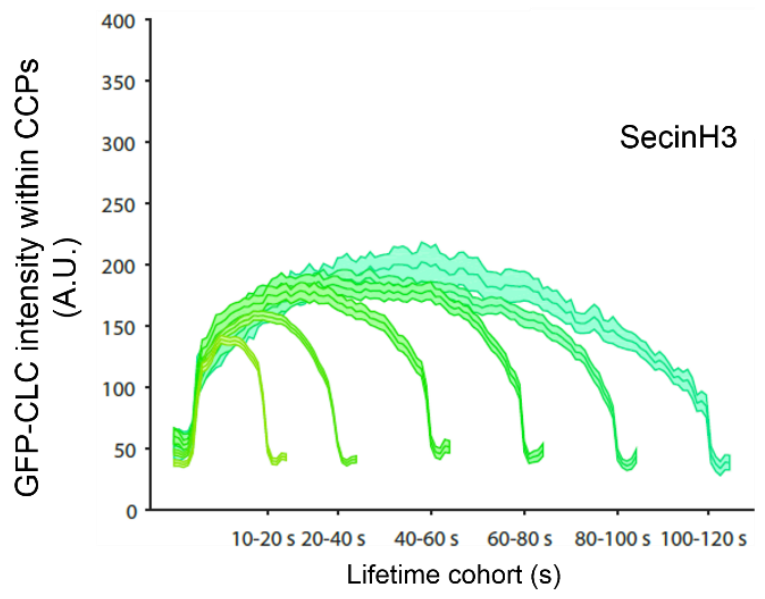

Figure 5.4. Arf6 regulates CME dynamics in a GTP-dependent manner. RPE cells stably expressing clathrin light chain fused to eGFP (eGFP-CLCa) were pretreated with $20 \mu \mathrm{M}$ SecinH3 for $10 \mathrm{~min}$ and simultaneously subjected to time-lapse imaging via TIRF-M. (A) Shown are single-frame representative 
fluorescence micrographs at the 10 minute timepoint. Scale bar: $5 \mu \mathrm{m}$. (B) Time-lapse TIRF-M image series of cells were subjected to automated detection, tracking, and analysis of CLSs as described in Materials and Methods, allowing identification of sCLSs and bona fide CCPs. Median, 25th/75th percentiles (boxes) and Tukey range (whiskers) for the initiation rate of CLSs (left panel) and initiation density of CCPs (right panel) are shown. (C) Mean eGFP-CLCa fluorescence intensity (reported as arbitrary units [A.U.]) traces in lifetime cohorts of CCPs detected in (B), from RPE cells treated (right panel) or not (left panel) with SecinH3. (D) Lifetime distributions of all detected bona fide CCPs in (B), from RPE cells treated (red line) or not (blue line) with A7. From a minimum of three independent experiments in each condition. ${ }^{*}, \mathrm{p}<0.05$ vs DMSO control, $\mathrm{k}$ (cells) $>30, \mathrm{n}$ (CLSs) $>10,000$ per condition. 

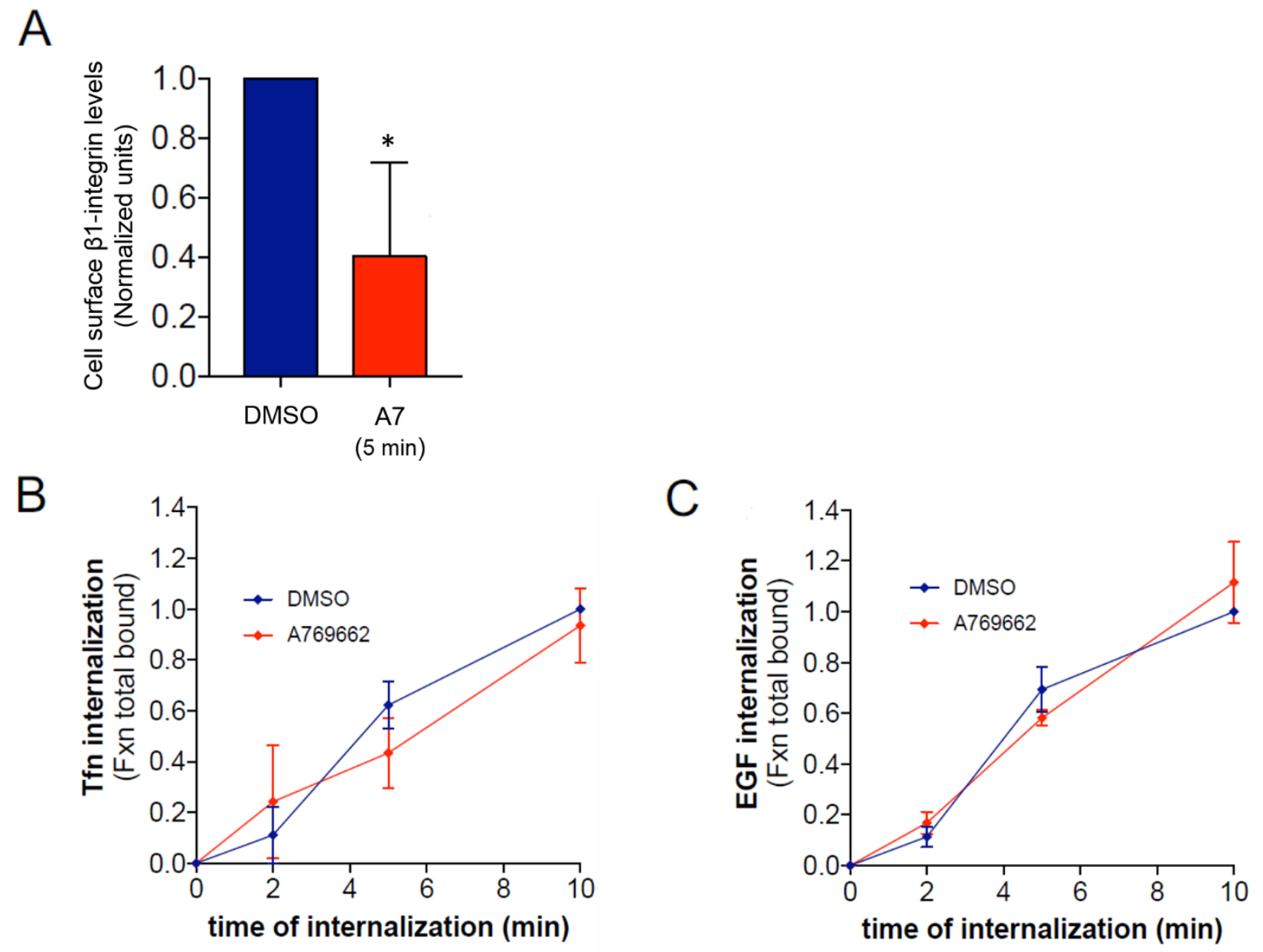

Figure 5.5. AMPK activation selectively regulates the endocytosis of CME cargoes. (A) Wild-type RPE cells were treated (or not; DMSO control) with $100 \mu \mathrm{M}$ A7 for 5 min. Intact cells were then immunolabeled with an antibody specific for an exofacial epitope on $\beta 1$-integrin. The cells were subsequently fixed and imaged via immunofluorescence microscopy, to acquire single frame images of the cell surface for manual analysis by ImageJ software as per [111]. With ImageJ, cells were manually outlined of which enclosed areas were quantified for mean pixel intensity of fluorescence-providing measurement of $\beta 1$ integrin; shown are the means $\pm S E$ of cell surface $\beta 1$-integrin fluorescence intensity in each condition, for $n>3$ independent experiments, each experiment $>20$ cells per condition; ${ }^{*}, p<0.05$. (B-C) Wild-type RPE cells were treated (or not; DMSO control) with $100 \mu \mathrm{M}$ A7 for various time points (e.g. 0, 2, 5, and $10 \mathrm{~min}$ ), followed by measurement of Tfn (B) or EGF (C) internalization as described in Materials and Methods. Shown are the means \pm SE for $n>3$ independent experiments. These experiments were performed by Sadia Rahmani (MSc. Candidate); Ryerson University, Toronto, ON, Canada. 

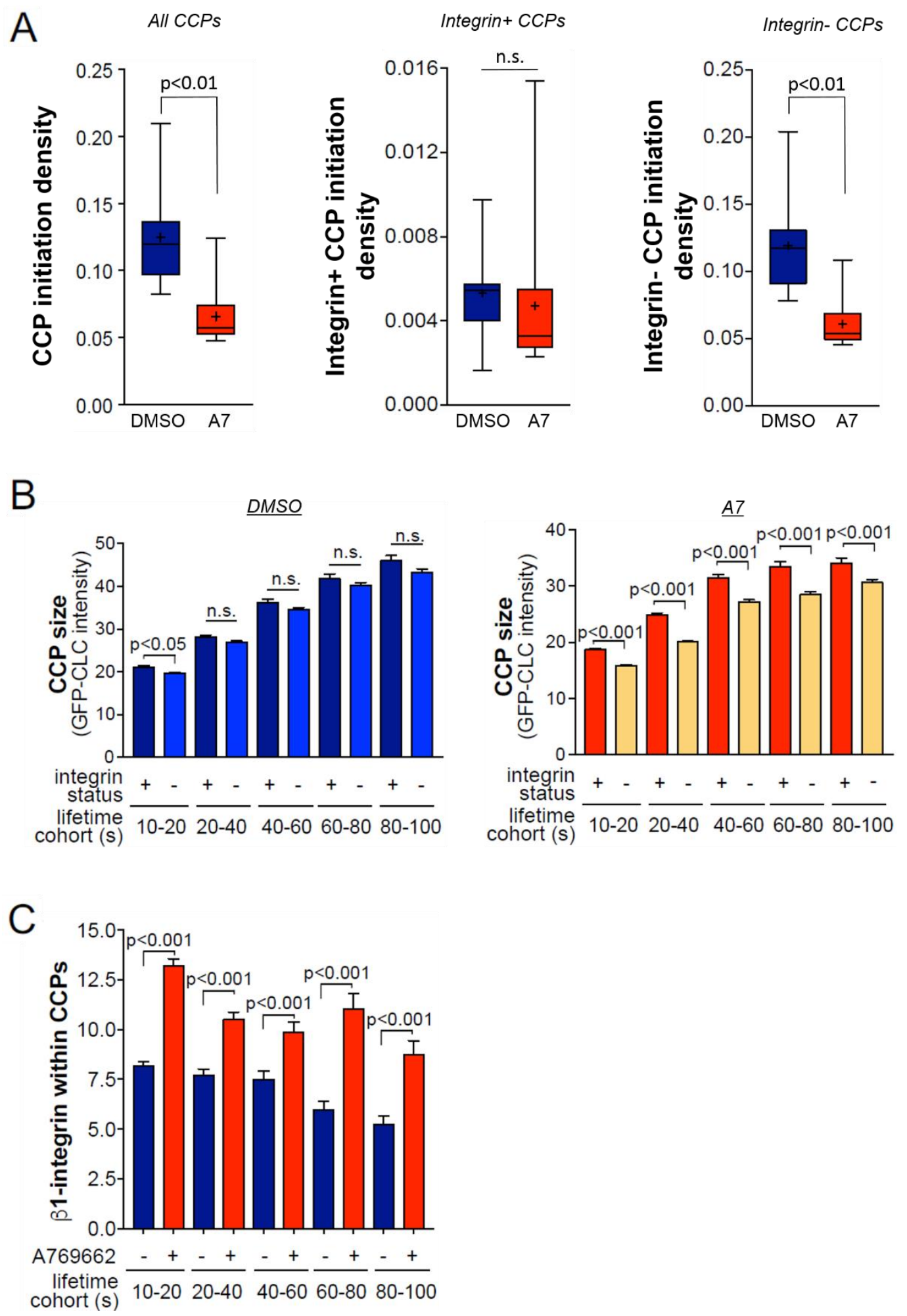

Figure 5.6. AMPK activation differentially regulates integrin+ and integrin- clathrin structures. (A-C) RPE cells stably expressing clathrin light chain fused to eGFP (eGFP-CLCa) were transfected with a 
genetically-encoded recombinant $\beta 1$-integrin protein fused with a fluorescent mCherry tag (mCh- $\beta 1$ integrin). The cells were treated (or not; DMSO control) with $100 \mu \mathrm{M}$ A7 for 15 minutes and subjected to time-lapse imaging via TIRF-M. Time-lapse TIRF-M image series of cells were subjected to automated detection, tracking, and analysis of CLSs as described in Materials and Methods, allowing identification of bona fide CCPs as integrin+ or integrin-, as appropriate. (A) Median, 25th/75th percentiles (boxes) and Tukey range (whiskers) for the initiation density of all CCPs (left panel), integrin+ CCPs (middle panel), and integrin- CCPs (right panel) are shown. (B) Shown are the mean eGFP-CLCa fluorescence intensity in lifetime cohorts of integrin+ and integrin- CCPs detected in (A), from RPE cells treated (right panel) or not (left panel) with A7. Shown in (C) are the mean $\mathrm{mCh}$ - $\beta 1$-integrin fluorescence intensity in lifetime cohorts within detected integrin+ CCPs, from RPE cells treated (right panel) or not (left panel) with A7. (A-C) From a minimum of three independent experiments in each condition, $k$ (cells) $>30, n$ (CLSs) $>10,000$ per condition. 

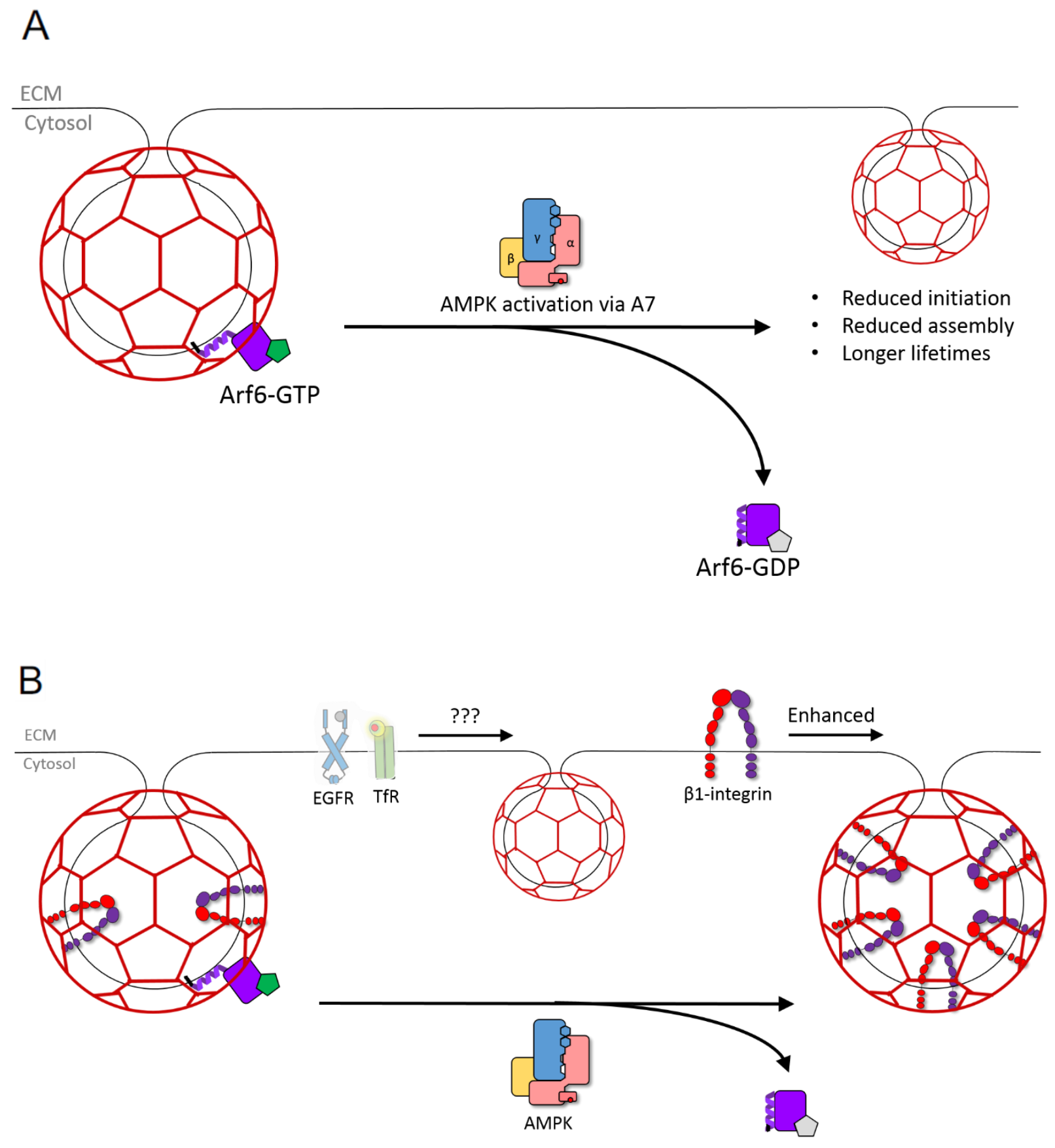

Figure 5.7. AMPK activation induces the modulation of CME dynamics. Shown is the schematic diagram summarizing the findings described in chapter 5. (A) Pharmacological activation of AMPK by A7 induces the broad regulation of CME via control of the small GTPase Arf6. AMPK was shown to inhibit GTP binding of Arf6 resulting in its dissociation from clathrin structures. The dissociation of Arf6 occurs concomitant to modulation of CME by AMPK, resulting in reductions in the rate of initiation and assembly of CCPs, which demonstrated longer lifetimes on the plasma membrane. (B) The control of 
CME dynamics by AMPK did not impact the internalization rates of surface receptors including TfR and EGFR, but enhanced $\beta 1$-integrin endocytosis. Consistently, AMPK activation did not modulate the formation and assembly of $\beta 1$-integrin containing CCPs (shown above) which exhibited enhanced $\beta 1$ integrin recruitment. Precisely how TfR and EGFR recruitment and subsequent CME dynamics are regulated by AMPK remains poorly understood, representing an important area for future research. 
Chapter 6. General discussion 
The plasma membrane is a major environment-interfacing organelle through which cells sense, respond and adapt to their dynamic local surroundings. The plasma membrane is host to functionally diverse protein components (e.g. intrinsically and peripherally associated proteins) collectively termed as the cell surfaceome, which functions in directing fundamental processes in cellular metabolism, migration, growth and proliferation ${ }^{31}$. Importantly, the cell surfaceome continually undergoes remodeling via protein addition and removal, in response to changing environmental parameters; facilitating the necessary cellular adaptations and behaviours important for cell survival and whole body homeostasis ${ }^{10}$. Endocytosis is the cellular process serving as the major mechanism through which cells acutely remodels the surfaceome, by invaginating and internalizing regions of the plasma membrane into intracellular vesicles-effectively and selectively capturing and removing proteins from the cell surface $^{51}$. While several modes of endocytosis exist, CME is the predominant and best characterized endocytic route in mammalian cells, largely involved in plasma remodeling which selectively concentrates surface proteins into budding clathrin-containing vesicles ${ }^{10,58}$. As a major regulator of the mammalian plasma membrane, CME functions as a versatile cellular tool involved in diverse biological processes mediating cellular physiology and homeostasis - of which fundamental and regulatory understanding is therefore of great importance ${ }^{50,358}$.

Diverse cellular cues including mitogenic (e.g. growth factors) and metabolic (e.g. cellular energy levels) signals induce an array of functionally diverse cellular processes (e.g. cellular growth, proliferation, migration and differentiation), which often require some extent of plasma membrane remodeling ${ }^{59-61}$. As the major regulator of the mammalian plasma membrane, CME therefore serves a crucial role in facilitating mitogenic- and metabolic-induced cellular processes. Precisely how CME may be directly regulated by mitogenic and metabolic signals in effecting global reorganization of the plasma membrane however, had remained relatively unexplored which thus, served as the major question I investigated in this work. Specifically, I found that i) mitogenic signaling by RTKs directly regulates CME via stimulation of PLCY1-derived calcium signals, which effects regulation of calcium sensitive endocytic components such as Sjn1. As well, I found that by regulation of the small GTPase Arf6, ii) metabolic signaling by AMPK directly regulates CME, in a manner consistent with previous findings demonstrating the AMPK-induced remodeling of the plasma membrane ${ }^{360}$. Collectively, these findings therefore provide direct mechanisms by which mitogenic and metabolic signals may exert control over $\mathrm{CME}$, in eliciting global reorganization of the plasma membrane-further discussed in the following sections. 


\section{1- Mitogenic signals directly regulate CME}

In chapters 3 and 4 , I uncovered how mitogenic signaling may directly regulate CME by investigating the role of PLCY1-derived calcium signals-activated by EGFR -in regulating the endocytic process. That clathrin-mediated endocytosis of EGFR occurs concomitant with PLC- $\gamma 1$ activation, I specifically studied the requirement for PLCY1-derived calcium signals in EGFR internalization. Using multiple independent approaches including genetic silencing of PLC- $\gamma 1$ (via siRNA), pharmacological antagonism of IP ${ }_{3} R$ (via XeC) and chelation of cytosolic calcium (via BAPTA-AM), I identified that PLCY1derived calcium signaling is required for the selective clathrin-mediated endocytosis of EGFR but not of other receptors such as TfR. While it is possible that each of these approaches has off-target effects, the strikingly similar phenotype of each perturbation on the selective impairment of EGFR clathrin-mediated endocytosis strongly indicates that the effect of each is specific to perturbation of PLC $\gamma 1$-derived calcium signaling. Consistent with the distinct control of the clathrin-mediated endocytosis of EGFR (but not that of TfR) by these calcium signals, we found that EGFR and TfR reside largely in distinct clathrin structures at the cell surface-highlighting the selective role of calcium signals in regulating distinct subsets of cargo-containing CCPs. Indeed, perturbation of intracellular calcium selectively impaired the earliest stages of initiation and assembly of CCPs harboring EGFR, without affecting receptor recruitment therein. Notably, calcium signaling controlled the recruitment of certain endocytic proteins including Sjn1 and clathrin to CCPs. Lastly, the role of PLC- $\gamma 1$ in regulating EGFR clathrin-mediated endocytosis, was further corroborated by the impairment in clathrin-dependent PI3K/Akt signaling of EGFR upon perturbation of PLC- $\gamma 1$ (via siRNA) and calcium (via BAPTA-AM), demonstrating that PLC $\gamma 1$-derived calcium signals do indeed regulate the assembly of CCPs containing EGFR-essential for both EGFR endocytosis and signaling.

\subsection{1 - Regulation of CCPs and clathrin-mediated endocytosis by intracellular calcium}

Described in section 1.5, the role of calcium in regulating CME has been appreciated for some time in pre-synaptic neurons and other excitable cell systems ${ }^{202}$. Specifically, membrane depolarization in neurons induces the opening of calcium channels on the plasma membrane triggering calcium influx, which significantly increases cytosolic calcium concentrations. This increase in intracellular calcium during depolarization stimulates robust calcium-dependent exocytosis of synaptic vesicles, which is coupled to enhanced, compensatory $\mathrm{CME}^{202,435}$. The enhancement of $\mathrm{CME}$ by calcium signals in neurons 
is thought to occur, in part, via the CaN-dependent dephosphorylation of Sjn1, Dyn1, amphiphysin, Eps15 and endophilin-activating these endocytic components to initiate $\mathrm{CME}^{96,204}$.

Notably, neurons express unique isoforms of endocytic proteins such as Dyn1 and AP180, making it unclear if calcium signals could also regulate CME in non-neuronal systems ${ }^{96,204}$. Here we reveal that calcium signals also control $\mathrm{CME}$ in non-neuronal cell lines, and that these signals are selectively required for the endocytosis of EGFR. Moreover, CaN and Dyn1 were dispensable for the internalization of EGFR (Figures 3.5 and 3.9A), suggesting that calcium-dependent control of clathrinmediated endocytosis in RPE cells does not rely on acute dephosphorylation of endocytic proteins such as Dyn1 by CaN; a phenomenon that regulates the clathrin-mediated endocytosis of TRAIL/death receptor ${ }^{304,400,410}$. Indeed, recent work by Reis and colleagues corroborate our findings which similarly observed the lack of effect of the siRNA-mediated knockdown of Dyn1 on CCP dynamics and TfR endocytosis in RPE cells ${ }^{397}$. Nonetheless, they demonstrate Dyn1 as a regulator of the rapid, compensatory CME in RPE cells ${ }^{397}$ - consistent with the role of Dyn1 in enhancing CME in neuronswhich albeit does not appear involved in the enhancement of EGFR endocytosis elicited by PLC 1 derived calcium signals. Given that the control of endocytosis by calcium in RPE cells is at least partly distinct from that thought to occur in neurons independent of $\mathrm{CaN}$ and/or Dyn1, how may calcium signals control clathrin-mediated endocytosis of EGFR in non-neuronal cells?

The perturbation of PLCY1-derived calcium signals does not prevent the nucleation of EGFRcontaining clathrin structures, as observed by the increase in EGF+ sCLSs upon intracellular calcium inhibition (Figure 4.3C), which nevertheless impacts the subsequent initiation and assembly of bona fide CCPs harboring EGFR (Figure 4.3, D and E). Both SCLSs and CCPs represent nucleation of clathrin structures ${ }^{5,79,404}$. Interestingly, mutation of the cargo-binding motifs of AP2 reduces the initiation rate of both sCLSs and bona fide CCPs, measured by similar methods as per [79], indicating that cargo recruitment to clathrin structures contributes to clathrin-structure nucleation, which precedes the requirement for calcium signals ${ }^{78,107}$. Indeed, perturbation of calcium signals does not impair the total recruitment of EGFR to CLSs (Figure 4.1C), of which a large fraction are sCLSs that do not give rise to bona fide CCPs ${ }^{5}$. This suggests that calcium signals do not control EGFR activation or recruitment to nascent clathrin nucleations. Instead, calcium signals control the subsequent initiation of bona fide CCPs harboring EGFR from nascent nucleations of clathrin structures (Figure 4.3D). Calcium signals may thus broadly control the assembly or function of many proteins within CCPs, or more selectively control a 
small number of endocytic proteins. While broad examination of all endocytic accessory proteins recruited to CCPs is beyond the scope of this study, I provide evidence that calcium and PKC signals control the assembly of at least one such endocytic accessory protein, Sjn1.

I demonstrated that Sjn1 recruitment to CCPs is regulated by calcium signals and PKC (Figures 4.6, 4.7 and 4.8). Sjn1 has two isoforms, a neuronal-enriched $145 \mathrm{kDa}$ isoform and a ubiquitous $170 \mathrm{kDa}$ isoform ${ }^{139}$. Both Sjn1-145 and -170 have NPF and PRD domains that interact with Eps15 and endophilin, while Sjn1-170 also interacts with AP2 and clathrin ${ }^{101}$. While both isoforms are recruited to CCPs, Sjn1145 is largely recruited near the end of the lifetime of a CCP, while Sjn1-170 is recruited along with clathrin and AP2 and is present throughout the lifetime of CCPs ${ }^{6,101}$. Sjn1 has 5-phosphatase and Sac1 domains important for the conversion of $\mathrm{PIP}_{2}$ to $\mathrm{PI} 4 \mathrm{P}$ and $\mathrm{PI}$, a process critical in uncoating of clathrincoated vesicles once internalized ${ }^{6,102,139}$. Furthermore, due to recruitment at early stages of clathrin structure formation, Sjn1 also negatively regulates the stabilization of nascent clathrin structure in a manner that requires the 5-phosphatase domain ${ }^{6}$. Hence, the turnover of $\mathrm{PIP}_{2}$ by Sjn1 within nascent CCPs impairs CCP formation. As such, the EGF-stimulated reduction in Sjn1 recruitment to CCPs may increase CCP initiation and efficiency of CCP internalization, thus promoting EGFR endocytosis. Notably, it appears that EGF stimulation elicits a broad depletion of Sjn1 from the majority of visible CCPs

(Figures 4.6A and 4.8), suggesting that depletion of $\operatorname{Sjn} 1$ is not restricted to EGF+ clathrin structures. As Sjn1 silencing impacted CCP dynamics but not TfR internalization as demonstrated in [6], we conclude that clathrin structures harboring EGFR are more sensitive to the regulation of Sjn1 recruitment than those harboring TfR.

While elucidation of the precise mechanism by which calcium signals may control Sjn1 lie beyond the scope of this study, it may nevertheless be hypothesized to occur via direct regulation of Sjn1 or by broader re-programming of CCP assembly. Consistent with the former, previous studies have shown that the receptor tyrosine kinase EphB, upon ligand activation, induces the phosphorylation of Sjn1 at a tyrosine site within its NPF domain ${ }^{436}$. As previously described, the NPF domain of Sjn1 binds to several endocytic components including eps15, endophilin, AP2 and clathrin, of which interactions with Sjn1 were inhibited upon phosphorylation of the NPF domain-which in turn, increased endocytosis in neurons ${ }^{139,436}$. Although yet to be tested, PLC $\gamma 1$-derived calcium signals may therefore cause dissociation of Sjn1 from CCPs in non-neuronal cells, by inducing phosphorylation of Sjn1, impairing its interactions with endocytic proteins. Interestingly, the phosphorylation and inactivation of Sjn1 can be induced by 
the cyclin dependent kinase $5(\mathrm{CDK} 5)^{437}$, which although mostly enriched in neurons, is nonetheless expressed in RPE cells and other non-neuronal cells ${ }^{438,439}$. Importantly, activation of CDK5 was demonstrated to require both calcium and PKC signals in neuronal cells, thus indicating the potential role of CDK5 activation as a mechanism by which the PLC 1 1-calcium-PKC signaling axis may induce depletion of Sjn1 from CCPs, in non-neuronal systems ${ }^{440}$. Notably, PKC may also directly phosphorylate and inactivate Sjn1 in RPE cells, as demonstrated to occur in nerve terminals ${ }^{96}$.

In addition to the role of calcium in selectively regulating CME via Sjn1, calcium and PKC may also more broadly control CCP assembly and clathrin-mediated endocytosis by control of other endocytic target molecules. Specifically, calcium may control endocytosis by direct binding to clathrin light chain which enhances clathrin assembly in vitro ${ }^{110,441,442}$, or by regulation of annexin 2 and 6 , which control AP2 recruitment to the cell surface ${ }^{443,444}$. The control of endocytosis by calcium may also occur in yeast, as the N-BAR domain protein Rvs167 is regulated by calmodulin, impacting release of vesicles from the plasma membrane ${ }^{445}$; this mechanism may have parallels in mammalian cells. Furthermore, our findings that calcium signals control the assembly and size of certain CCPs is consistent with the glucose-induced enhancement of endocytosis in INS-1 cells ${ }^{203}$. While clathrin assembly or clathrinmediated endocytosis was not directly examined, the glucose-induced calcium signals in INS-1 cells was shown to enhance endocytosis and control the size of endocytic vesicles. In addition, although we show here that PKC is required for regulation of clathrin-mediated endocytosis of EGFR, a previous study concluded that PKC regulated EGFR recycling but not internalization ${ }^{224}$. This study examined the effect of treatment of cells with phorbol 12-myristate 13-acetate (PMA; which results in broad activation of PKC), which did not enhance nor impair EGFR internalization ${ }^{224}$. Our observations indicate however, that EGFinduced calcium signals are sufficient to activate specific isoforms of PKC, and that these PKC isoforms are required for EGFR internalization even in the absence of PMA treatment.

Given the complexity and heterogeneity of CCPs, including their lifetime and composition, dissecting the mechanism by which calcium and PKC signals control CCP assembly, maturation and scission from the cell surface is beyond the scope of this study, and represents an important area of investigation for future research. Nevertheless, I establish that PLC 1 1-derived calcium and PKC signals control the clathrin-mediated endocytosis of EGFR, as a result of regulation of the initial endocytic stages in initiation and assembly of bona fide EGFR+ CCPs. 
Interestingly, Sigismund and colleagues demonstrated that PLC $\gamma 1$-derived calcium signals control non-clathrin endocytosis of EGFR ${ }^{446}$, which occurs in some cells upon stimulation with high doses of EGF (>20-50 ng/mL) ${ }^{409}$. Nonetheless, our work establishes that PLC- $\gamma 1$ and calcium, controls clathrinmediated endocytosis of EGFR as i) EGF internalization is very largely clathrin-dependent in RPE cells ${ }^{4}$, even at high EGF doses (100ng/mL) (Figure 3.4); ii) direct observation of clathrin structures and CCP dynamics resolves the question of whether calcium signals control CCP initiation and assembly (Figures 4.1 and 4.3); and iii) calcium signals are shown to control the recruitment of specific proteins (e.g. Sjn1) into CCPs (Figures 4.6, 4.7 and 4.8). Taken together, these studies suggest that calcium signals may represent a broad, common regulator of EGFR internalization through multiple endocytic pathways.

\subsection{2 - Distinct regulation of clathrin-mediated endocytosis of EGFR versus TfR} Several lines of evidence support the internalization of EGFR and TfR through largely distinct CCPs. Silencing of AP2 subunit components ${ }^{74}$ or sequestration of AP2 by overexpression of AAK1 ${ }^{75}$ results in near-complete impairment of TfR endocytosis, with minimal effect on clathrin-mediated endocytosis of EGFR. However, others have reported complete inhibition of both TfR and EGFR endocytosis upon AP2 silencing ${ }^{447,448}$, consistent with the fact that AP2 is found in virtually all CCPs ${ }^{449}$. Taken together, these studies suggest that AP2 may be required for internalization of both TfR and EGFR, but that the endocytosis of each receptor may use AP2 in a functionally distinct manner under some contexts. Furthermore, phosphatidic acid controls CCP dynamics but impacts only the clathrinmediated endocytosis of EGFR, and not that of $\mathrm{TfR}^{106}$.

Here, we employed labeling of TfR and EGFR using fluorescently conjugated ligands, coupled to automated and unbiased image analysis to resolve that these two receptors are localized largely within distinct CCPs (Figure 3.11). We find that CCPs harboring either significant levels of EGFR or TfR (but not both) are detected approximately three times as frequently as CCPs that harbour both receptors. Importantly, this relationship was not altered by increasing the levels of fluorescently labeled EGF used for stimulation in this experiment (Figure 3.11C). Thus, incomplete labeling of TfR or EGFR by labeling with their respective fluorescent ligands does not readily explain the observed enrichment of EGFR and TfR into largely distinct CCPs. Instead, these observations suggest that EGFR and TfR, and not just their ligands, are recruited to largely separate sets of CCPs. 
Most CCPs that are positive for TfR contain multiple copies of this receptor, including when labeled using an exofacial synthetic ligand that is comparable in size to fluorescent $\mathrm{Tfn}$ (and thus also much larger than fluorescent EGF ${ }^{84,423}$. Thus, it is very unlikely that our observation of fluorescent EGF and Tfn enrichment within distinct CCPs is due to steric constraints limiting the inclusion of multiple ligands into the same clathrin structure. Indeed, examination of the EGF and Tfn intensity distribution in CCPs (Figure 3.11B) reveals that a range of ligand fluorescence intensities within CCPs exists for each receptor ligand, indicating that many CCPs have more than one of their respective ligands (EGF or Tfn).

The recruitment of EGFR and TfR to largely distinct CCPs is consistent with some previous studies. These two ligands were observed in largely distinct CCPs with methods similar to those we employed here relying on fluorescently conjugated Tfn and EGF coupled to TIRF-M ${ }^{423}$. While CCPs harboring both EGFR and TfR can indeed be detected by electron microscopy ${ }^{450}$, labeling of EGFR and TfR using antibodies specific for each receptor and immunoelectron microscopy revealed that these receptors were far more frequently detected within distinct CCPs than in the same $\mathrm{CCPs}^{423}$. And consistently, while CCPs were not being examined directly, fluorescent EGF and Tfn were shown to exhibit reduced overlap in the initial stages of internalization ${ }^{451}$. These studies support our conclusion, based on systematic and unbiased analysis of CCPs, that EGFR and TfR are largely found in distinct CCPs.

The recruitment of EGFR and TfR to largely distinct CCPs may occur as a result of stochastic recruitment of receptors to preformed early clathrin structures, that are intrinsically capable of interaction with and subsequent internalization of diverse cargo receptors. Alternatively, the selective incorporation of EGFR into CCPs that have unique properties compared with CCPs harboring TfR suggests that a deterministic cargo-selection mechanism may gate the early stages of clathrin-structure nucleation, initiation, and assembly. Consistent with this interpretation, EGF stimulation of AP2 silenced cells resulted in the formation of CCPs that contained EGF, EGFR, and clathrin but not $\mathrm{TfR}^{452}$. If the stochastic segregation of receptors into largely distinct CCPs predominates, then this phenomenon may be subject to cell context-specific parameters, such as receptor expression levels.

Regardless of which aforementioned cargo-recruiting mechanisms predominate, EGFR and TfR reside largely within distinct clathrin structures in RPE cells. This cargo segregation is likely not restricted to RPE cells, as similar findings were observed in HeLa cells ${ }^{423}$. Notably, the incorporation of cargo within specific CCPs alters the properties of those CCPs, as shown for LDLR ${ }^{453}$ and certain GPCRs ${ }^{103}$. These 
studies suggest that the recruitment of each receptor to particular clathrin structures may be coupled to unique engagement of endocytic accessory proteins that impact CCP formation, assembly, maturation and scission. As such, CCPs harboring EGFR are dependent on contributions from PLC 1 -derived calcium signals for CCP initiation and assembly, and eventual productive formation of intracellular vesicles. In contrast, CCPs harboring TfR are not regulated by PLC 1 1-derived calcium signals.

\subsection{3 - Regulation of EGF-stimulated Akt phosphorylation by PLCY1-derived signals}

We recently uncovered that clathrin structures, but not receptor endocytosis, are required for EGFR signaling leading to Gab1 and Akt phosphorylation ${ }^{3,4,8}$. Perturbations of clathrin selectively impaired EGF-stimulated phosphorylation of Gab1 and Akt, but were without effect on phosphorylation of EGFR or of other signaling pathways such as MAPK ${ }^{4}$. Importantly, perturbations of Dyn2, which allowed formation of CCPs and recruitment of EGFR therein, but not receptor internalization into vesicles, did not impair EGF-stimulated Akt phosphorylation ${ }^{4}$. We proposed that in addition to forming endocytic portals, a subset of clathrin structures also function as signaling microdomains, required for the enrichment of specific receptor signals. Consistent with this, we observed the enrichment of phosphorylated Gab1 within CCPs upon stimulation with ligands of EGFR or Met ${ }^{4,8}$.

Here we find that PLC- $\gamma 1$ and intracellular calcium are required for EGF-stimulated activation of clathrin-dependent signals (phosphorylation of Gab1 and Akt) but not clathrin-independent signals (phosphorylation of EGFR and MAPK). Given that PLC- $\gamma 1$ and intracellular calcium control the assembly of CCPs harboring EGFR, we propose that these signals are also required to prime clathrin structures to function as signaling microdomains required for Akt activation.

The requirement for PLC- $\gamma 1$ for EGF-stimulated Akt phosphorylation may reflect the requirement for PLC- $\gamma 1$ and calcium in the initiation and assembly of EGFR+ clathrin structures, which are required for signaling leading to Akt activation, or other mechanism(s). We observed that EGFstimulated Akt phosphorylation in RPE cells engineered to stably express HER2 (which signal largely from EGFR/HER2 heterodimers) does not require PLC- $\gamma 1$ for EGF-stimulated Akt phosphorylation (Figure 4.9C). Because clathrin is dispensable for EGF-stimulated Akt phosphorylation in RPE-HER2 cells ${ }^{4}$, the requirement for PLC- $\gamma 1$ for activation of Akt by EGFR may be limited to cellular contexts in which EGFR signaling is clathrin-dependent. This result strengthens our conclusion that PLC- $\nu 1$ and calcium- 
dependent signals regulate clathrin-mediated endocytosis of EGFR, as well as clathrin-dependent EGFR signaling. The largely unaltered EGF-stimulated Akt phosphorylation observed in PLC $11-/-$ MEF cell lines $^{454}$ may thus reflect a different cellular context (e.g. HER2 co-expression) in which EGF-stimulated Akt phosphorylation occurs in a clathrin-independent manner, or another long-term adaptation to PLCү1 genetic knockout.

We thus conclude that the regulation of signaling and endocytosis of EGFR is reciprocal, as the regulation of CCP initiation and assembly by EGF-stimulated PLC 1 1-derived calcium signals in turn control EGF-stimulated Akt signaling. Elucidating the mechanisms by which clathrin structures may integrate a number of intracellular cues, which in turn regulate signaling by EGFR and other cell surface receptors, should be the subject of future research in the function and regulation of CCPs.

In summary, we reveal an unprecedented dimension by which functionally distinct subsets of CCPs can be differentially regulated by diffusible signals (e.g. calcium) (Figure 4.11). The specific and distinct regulation of subsets of CCPs by cellular signals provides the basis for how the versatile assembly of CCPs can be modulated, to accomplish distinct regulation of the diverse proteins of the cell surfaceome. As such, this study uncovers the cellular mechanism by which mitogenic signaling may elicit modifications of the plasma membrane-required for facilitating functionally diverse cellular processes $^{59-61}$. By triggering PLC 1 1-derived calcium signals, mitogenic signals directly regulate CME via control of Sjn1-of which broad, yet selective distribution amongst clathrin structures impart calcium signals the ability in eliciting broad regulation of $\mathrm{CME}$, and hence, remodeling of the surfaceome landscape.

\section{2- Metabolic signals directly regulate CME}

In chapter 5, I investigated how activation of AMPK may induce direct regulation of CME in effecting global remodeling of the cell surface. Previous findings from our laboratory have demonstrated the depletion of specific surface proteins from the plasma membrane upon activation of AMPK ${ }^{360}$, which I proposed may be due to the AMPK-mediated enhancement of CME. By pharmacological activation of AMPK by A7, I observed the direct regulation of CME via TIRF-M coupled to automated software detection and analysis of clathrin structures. Specifically, with this strategy I observed the AMPK- 
induced reduction in the initiation density of bona fide CCPs, which was without effect on the total number of clathrin structures (which includes both SCLCs and CCPs) ${ }^{5,79}$. In addition, CCPs which form under A7 treated conditions exhibited reduced clathrin recruitment and assembly which nonetheless, had higher mean lifetimes. Using multiple independent approaches including FRET-FLIM microscopy, transient transfection strategies, and pharmacological antagonism by SecinH3, I identified Arf6 as a protein regulated by AMPK - through which AMPK may elicit control of CME. Interestingly, the direct regulation of CME upon AMPK activation did not impact the rate of internalization of CME cargoes including TfR and EGFR. However, $\beta 1$-integrin internalization was enhanced by A7 treatment which upon closer examination may likely be due to the enhanced recruitment of $\beta 1$-integrin within CCPs. Importantly, AMPK activation was without effect on $\beta 1$-integrin containing CCPs which did not exhibit deficiencies in initiation rates and assembly, highlighting the selective role of AMPK in regulating CME of surface proteins-and hence, reorganization of the plasma membrane.

\subsection{1 - AMPK activation directly regulates CME}

The thematic focus on AMPK as a metabolic signal which may directly regulate CME, was inspired by previous findings from our laboratory demonstrating the robust reorganization of the plasma membrane upon AMPK activation. Specifically, acute pharmacological activation of AMPK by A7 (for 90 minutes) resulted in the acute depletion of $\sim 600 \mathrm{GO}$ (Gene Ontology) annotated proteins from the cell surface, of which $\sim 80$ were transmembrane proteins ${ }^{360}$. The study however, did not delineate the cellular mechanism by which AMPK activation may induce plasma membrane remodeling. This work was guided by the hypothesis that the altered surfaceome was due in part to the regulation of CME by AMPK. While knowledge regarding AMPK control of CME is still lacking, previous studies have shown that the activation of AMPK by the onset of metabolic stress (e.g. reduced cellular ATP levels) enhances the permanence of glucose transporters (e.g. GLUT1) ${ }^{361}$, and as well as the clearance of $\mathrm{Na}+\mathrm{K}+\mathrm{ATPase}$ pumps from the plasma membrane ${ }^{455}$ - of which mechanisms to some extent likely involves regulation of endocytosis ${ }^{456,457}$. In addition, recent work by Rangaraju and colleagues highlighted CME as one of the largest consumers of ATP within presynaptic neurons, of which regulation by AMPK during metabolic stress may thus be expected in restoring cellular homeostasis ${ }^{458}$.

Here, we demonstrate that the activation of AMPK does indeed effect regulation of CME evinced by broad modifications in CCP dynamics upon A7 treatment (Figure 5.1). Specifically, activation 
of AMPK by $A 7$ induces a substantial reduction in clathrin recruitment and assembly-and hence sizeof clathrin structures (Figure 5.1A), of which density on the plasma membrane nonetheless remained relatively unimpaired (Figure 5.1C). Concomitant to the resulting impairments in assembly of clathrin structures, AMPK activation reduced the initiation density (and size) of bona fide CCPs (Figure 5.1, C and D), which taken together, may imply AMPK as a negative regulator of CME. This preliminary interpretation however, is incompatible with our previous findings demonstrating the acute and robust depletion of surface proteins from the plasma membrane upon A7-induced activation of AMPK ${ }^{360}$. Indeed, live-cell TIRF-M imaging and automated analysis of clathrin structures reveal CCPs that form under A7 treatment, to exhibit longer mean lifetimes on the plasma membrane relative to control (nontreated condition) (Figure 5.1E), which highlighted in section 1.3.2-is indicative of enhanced endocytic productivity ${ }^{78}$. Thus, despite globally altering CCP assembly dynamics, AMPK activation nevertheless enhances the mean lifetime and hence, productivity of CCPs-indicating the role of AMPK in augmenting certain aspects of CME.

These findings bring into focus the question of why AMPK might induce alteration of CCP assembly, while simultaneously enhancing CCP productivity. As the master sensor and regulator of cellular metabolism, the biological programs induced by AMPK can be hypothesized to function in collectively restoring metabolic homeostasis ${ }^{363}$ - which this work revealed, also includes the direct regulation of CME. Recent findings by Rangaraju and colleagues demonstrates CME as one of the largest consumers of cellular ATP in presynaptic neurons, which readily depletes ATP if not simultaneously replenished (via compensatory glycolytic pathways) ${ }^{458}$. While yet to be investigated, the efficient progression of CME in non-neuronal systems (including in RPE cells) may thus represent a large energy burden, of which energy requirements likely cannot be sustained by cells under metabolic stress ${ }^{458}$. Described in section 1.3.2, the efficient formation and internalization of CCPs require regulatory input from several ATP-consuming processes such as the dynamic assembly/disassembly of the clathrin coat (via auxilin-HSC70 complex), and the dynamin-mediated fission of CCVs (via hydrolysis of GTP derived from ATP); amongst several others ${ }^{51}$. As such, CME presents an excellent regulatory juncture of which alteration by AMPK (i.e. reducing clathrin recruitment and CCP size) during reduced energy states, may reflect a mechanism by which cellular ATP levels may be conserved ${ }^{50,58}$.

CME is a process essential for life of which complete ablation is often embryonic lethal in multicellular organisms such as Drosophila melanogaster, Caenorhabditis elegans, and Mus musculus ${ }^{50}$. Thus, 
cells must actively prioritize CME progression despite physiological perturbations; such that imposed by metabolic stress. The observed reduction in assembly and size of CCPs, which nevertheless exhibit enhanced endocytic productivity (i.e. longer lifetimes), may therefore reflect an adaptive mechanism by which cells may maintain CME despite reductions in cellular energy-facilitated by AMPK. Firstly, by limiting clathrin structure assembly and size, and forming fewer CCPs, energy consumed by CME may effectively be reduced in preserving intracellular ATP levels during metabolic stress. Secondly, consistent with the essential requirement for $\mathrm{CME}$, the CCPs which form - although smaller and limited-exhibit longer mean lifetimes on the plasma membrane which may enhance (or reflect enhancement of) cargo recruitment and internalization, consequently sustaining the capacity and progression of $\mathrm{CME}^{84,103,104,459}$. While precise mechanisms by which AMPK may effect smaller CCPs with longer lifetimes remain to be elucidated (this may involve Arf6 as described in the following section), previous studies may indicate AMPK to function as a regulatory break which may inhibit distinct regulatory mechanisms in CCP assembly. Kadlecova and colleagues for instance, similarly demonstrated the reduction in size of productive CCPs, in RPE cells expressing recombinant AP2 with impaired $\mathrm{PIP}_{2}$ binding ${ }^{79}$ - highlighting the potential role of AMPK in eliciting modulation of clathrin structure assembly by removal of endocytic regulatory mechanisms. In addition, the enhancement of CCP lifetimes has been demonstrated in epithelial BSC1 cells depleted of endocytic components including Hip1R, Eps15 or intersectin, which further indicates the role of AMPK in reducing the regulation of CCP assembly ${ }^{137}$. Taken together, AMPK activation directly regulates $\mathrm{CME}$ by reducing the density and size of bona fide CCPs, yet the CCPs that do form nonetheless exhibit longer lifetimes and may be more productive-that collectively, may reflect an adaptive response mechanism through which cells may sustain CME despite perturbations in cellular energy states.

\subsection{2 - AMPK may regulate CME via control of Arf6 activity}

Described in section 1.7.1.2, AMPK is a master kinase which modulates several downstream effectors in inducing processes regulating cellular energy homeostasis ${ }^{359,363}$. As such, the regulation of CME by AMPK observed in this study, may potentially be derived from control of many such proteins by AMPK. While broad examination and systematic profiling of individual AMPK targets is beyond the scope of this study, we provide evidence that AMPK directly controls at least one such downstream effector through which AMPK may regulate CME-the small GTPase Arf6. Previous findings from our laboratory demonstrated the depletion of several surface proteins from the plasma membrane upon activation of 
$\mathrm{AMPK}^{360}$. Amongst the downregulated proteins include regulators of Arf6 including ArfGAP3 and ARAP, of which the former also contains a predicted AMPK phosphorylation site-thereby suggesting the potential regulation of Arf6 by $\mathrm{AMPK}^{360}$. Importantly, previous studies have described the role of Arf6 in augmenting $\mathrm{CME}$ by facilitating $\mathrm{PIP}_{2}$ production, and $\mathrm{AP} 2$ and clathrin recruitment-indicating Arf6 as a potential AMPK target which may effect regulation of $\mathrm{CME}^{379,383}$.

Here we show that Arf6 is indeed regulated by AMPK activation (Figure 5.2). Using FRET-FLIM microscopy, we determined that AMPK induces the inactivation of Arf6 by promoting its GDP-bound state. Described in section 1.7.2.1, Arf6 is a molecular switch of which interactions with downstream effectors - and hence role in augmenting $\mathrm{CME}$-is predicated on its ability to bind GTP, inducing activation ${ }^{376}$. Although preliminary $(n=1)$, our FRET-FLIM results are therefore consistent with the observed reduction in CCP density and assembly elicited by AMPK activation (Figure 5.1), which may be hypothesized to result from the inactivation and loss of endocytic enhancements by Arf6. Through transient transfection of fluorescent Arf6 recombinant proteins, I also observed the physiological localization of Arf6 within clathrin structures-corroborating the role of Arf6-GTP in enhancing CME (Figure 5.3) 379,383 . Consistently, AMPK activation by A7 treatment induces the dissociation of Arf6 from clathrin structures, which together with the FRET-FLIM results, may be presumed likely due to its inactivation by AMPK (Figures 5.2 and 5.3). Collectively, these findings indicate that AMPK activation directly regulates Arf6 by enhancing the fraction of Arf6 in its GDP-bound state, preventing its localization and enhancement of clathrin structure assembly-therefore serving as a potential mechanism by which AMPK may effect regulation of CME as described in section 6.2.1.

The exact mechanism by which AMPK may directly influence Arf6 nucleotide cycling and inactivation however, was not investigated in this study, which albeit may be hypothesized to occur through control of ArfGAP3. Specifically, our previous findings demonstrate AMPK activation to reduce plasma membrane expression of ArfGAP3, of which primary function involves inactivation of Arf6 by promoting GTP hydrolysis ${ }^{360,372,460}$. Described in section 1.7.2.2, ArfGAP3 inactivates Arf6 via direct binding, which may thus imply the transit and colocalization of ArfGAP3 within Arf6-containing clathrin structures ${ }^{376}$. While yet to be tested, the AMPK induced surface depletion of ArfGAP3 and concomitant inactivation of Arf6, may thus suggest the potential translocation of ArfGAP3 within clathrin structureswithin which it i) may encounter and inactivate Arf6, and similarly ii) be internalized and removed from the plasma membrane (consistent with the AMPK-induced surface depletion of ArfGAP3). How exactly 
AMPK might induce translocation of ArfGAP3 within Arf6-containing clathrin structures remains to be elucidated, which nonetheless may be hypothesized to occur via direct phosphorylation of a predicted AMPK phosphorylation site on ArfGAP3 ${ }^{360}$. This proposed mechanistic control of ArfGAP3 by AMPK may therefore result in Arf6 inactivation, and hence, direct regulation of CME previously observed (Figure 5.1). Indeed, the hypothesized role of ArfGAP3 in regulating CME via Arf6 inactivation is supported from findings by Dembla and colleagues, who demonstrated the significant inhibition of endocytosis in neuronal synapses overexpressing ArfGAP3 ${ }^{461}$. Taken together, these findings highlight ArfGAP3 as a potential target through which AMPK may elicit inactivation of Arf6, and alteration of CME dynamics.

In contrast to ArfGAP3, the activity of Arf6 may similarly be regulated by the action of Arf6-GEFs including the cytohesin/ARNO, EFA6 and IQSEC/BRAG Arf-GEF families, which promote GTP loading and activation of $\mathrm{Arf6}^{380}$. The inactivation of Arf6 elicited by AMPK may therefore be similarly derived from the potential AMPK-mediated control and inhibition of Arf6-GEFs. While control of Arf6-GEFs by AMPK has yet to be demonstrated, previous studies have shown the direct phosphorylation of GBF1 and FBXO8 by AMPK, which although functioning as GEFs for Arf isoforms other than Arf6-nevertheless indicates the potential regulation of Arf6-GEFs by AMPK ${ }^{385,386}$. Indeed, I demonstrated that the paninhibition of Arf6-GEFs by SecinH3 phenocopies (Figure 5.4) the effects elicited by A7 treatment on CME dynamics (Figure 5.1). The inhibition of Arf6-GEFs by AMPK may thus, similarly provide a mechanism by which AMPK may induce inactivation of Arf6 in regulating CME, which albeit, remains to be experimentally demonstrated. Collectively, these results nonetheless demonstrate that AMPK activation regulates Arf6 by promoting its GDP-bound state, which may occur via i) activation of Arf6-GAPs and/or ii) inactivation of Arf6-GEFs.

The inactivation (via enhancement of Arf6-GDP) and dissociation of Arf6 from CCPs may reflect, in part, the mechanism by which AMPK may induce regulation of CME-reducing the number and size of bona fide CCPs (Figure 5.1). This mechanism however, does not readily explain the enhancement of lifetimes exhibited by CCPs which do form under A7 treatment. Work by Paleotti and colleagues revealed that Arf6-GTP directly binds and strongly promotes the recruitment of AP2 to the plasma membrane in vivo; even in the absence of phosphoinositides in vitro ${ }^{379}$. Described in section 1.3.4, AP2 is a central hub of CME which binds and recruits endocytic accessory proteins including Hip1R, Eps15 and intersectin-amongst several others ${ }^{12}$. Importantly, the depletion of Hip1R, Eps15 or intersectin in BSC1 cells, was demonstrated by Mettlen and colleagues to effect longer lifetimes of productive CCPs ${ }^{137}$. As 
such, the stabilization of Arf6-GDP by AMPK may be hypothesized to not only induce dissociation of Arf6 from clathrin structures, but to also indirectly prevent recruitment of AP2 and its downstream endocytic effectors-thus, potentially increasing the lifetimes of productive $\mathrm{CCPs}^{137}$. In addition, Poupart and colleagues also demonstrated the direct binding of clathrin with Arf6-GTP, of which inactivation into Arf6-GDP may therefore slow the maturation rate of CCPs, hence, increasing CCP lifetimes ${ }^{379,383}$. Nevertheless, while we demonstrate Arf6 as a direct effector of AMPK capable of modulating CME, given the broad array of proteins phosphorylated by AMPK, other mechanisms may likely be involved of which contribution to $\mathrm{CME}$ regulation, albeit remains to be elucidated.

\subsection{3 - AMPK activation may differentially regulate CME of surface cargoes}

Described in section 1.3.2, the internalization of CME cargoes may be regulated by unique sets of proteins, which distinctly impacts the formation and assembly of CCPs within which they reside. Indeed, microscopic visualization of CCP structures reveal structurally diverse endocytic assemblies which morphologically differ in size and lifetimes, reflecting in part differences in their cargo protein constituents ${ }^{1}$. The internalization of CME cargoes including EGFR, TfR and LDLR for example, are functionally regulated by phosphatidic acid ${ }^{106}$ and calcium signals (see chapter 6), AP2 and TTP423,447,448, and Dab2 and $A R H^{80}$, respectively. That AMPK activation globally regulates $C C P$ assembly dynamics (Figure 5.1) therefore raises the question of whether AMPK might distinctly control (i.e. broadly or selectively, or both) the internalization of CME cargoes.

Using biochemical ligand internalization assays, we reveal that AMPK activation does not impact the clathrin-mediated endocytosis of EGFR and TfR, of which internalization rates were unchanged by $A 7$ treatment in RPE cells (Figure 5.5, B and C). In contrast, the significant depletion of $\beta 1$-integrin from the plasma membrane was observed via immunofluorescence microscopy upon acute ( 5 minutes) activation of AMPK by $A 7$ (Figure 5.5A)-indicating the AMPK induced enhancement of $\beta 1$-integrin CME; consistent with the A7-induced downregulation of cell surface $\beta 1$-integrin described in [360]. These findings thus, indicate that despite broadly controlling CCP assembly dynamics, AMPK may nonetheless selectively regulate the internalization of distinct CME cargoes.

The enhancement of $\beta 1$-integrin CME, but not that of EGFR or TfR, indicates the distinct regulation of $\beta 1$-integrin containing CCPs by AMPK activation. Previously demonstrated, AMPK 
activation reduces the number and size of bona fide CCPs (Figure 5.1), which interestingly was not observed for integrin+ CCPs of which initiation density and corresponding size were largely unimpaired, relative to integrin- CCPs (Figure 5.6A). These findings suggest that despite broadly regulating CME, AMPK may nonetheless - through poorly understood mechanisms-selectively control the assembly dynamics of distinct cargo-containing CCPs. Consistently, AMPK had been demonstrated to distinctly regulate CME of several surface proteins including the GLUT4 glucose transporter and the $\mathrm{Na}+\mathrm{K}+$ ATPase pump. Specifically, AMPK activation was found to inhibit clathrin-mediated endocytosis of GLUT1 by triggering the degradation (via phosphorylation) of its endocytic regulator TXNIP (Thioredoxininteracting protein)-which consequently enhances GLUT1 expression on the plasma membrane ${ }^{462}$. In

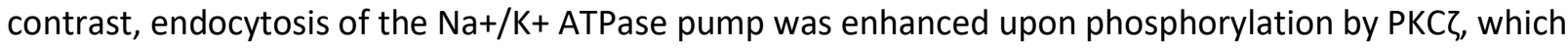
was shown directly phosphorylated and activated by AMPK ${ }^{455,463}$. Taken together, AMPK may therefore broadly and selectively regulate CME through phosphorylation and regulation of functionally diverse endocytic effectors, which may distinctly control the internalization properties of CME cargoes; including $\beta 1$-integrin.

The effect of A7 treatment on the initiation density and assembly dynamics of integrin+ CCPs, raises the question of how $\beta 1$-integrin endocytosis may similarly be enhanced under these conditions (Figure 5.6, $A$ and B). We observed that $A 7$ treatment significantly increases the $\beta 1$-integrin content of integrin+ CCPs relative to control (Figure 5.6C) - suggesting that AMPK activation may increase $\beta 1$ integrin endocytosis by enhancing its recruitment within clathrin structures. Given the complexity and heterogeneity of mechanisms governing distinct cargo $\mathrm{CME}$, dissecting precisely how AMPK activation may enhance $\beta 1$-integrin recruitment within clathrin structures is beyond the scope of this study, and represents an important area of investigation for future research. Nevertheless, it has been demonstrated that siRNA-mediated knockdown of Arf6 significantly reduces surface integrin levels in HeLa cells, suggesting the possible control of $\beta 1$-integrin recruitment and endocytosis, by the AMPKmediated inactivation of Arf6 (Figure 5.2) ${ }^{391}$. Furthermore, the enhanced recruitment of $\beta 1$-integrin to CCPs upon AMPK activation may be hypothesized to involve, to some extent, control of the endocytic adaptor Dab2 which recruits and localizes with $\beta 1$-integrin - within clathrin structures ${ }^{387,434,464}$. Interestingly, Dab2 is known to enhance the size of clathrin structures ${ }^{453}$, of which inclusion within integrin + CCPs may be proposed to negate the modulation of CCP assembly broadly imposed by AMPK (Figure 5.1); consistent with the unablated assembly and size of integrin+ CCPs (Figure 5.6, A and B). Yet to be experimentally validated, the sustained endocytosis of EGFR and TfR (Figure 5.5, B and C) may 
thus similarly reflect, in part, an enhancement in receptor recruitment within clathrin structures, of which net output may effect in unperturbed rates of internalization.

From a physiological context, the robust downregulation of $\beta 1$-integrin upon AMPK activation represents a mechanism by which AMPK may maintain and restore cellular energy levels. $\beta 1$-integrin is a transmembrane protein that mechanistically anchors the cellular cytoskeleton with the extracellular matrix, which promotes energy-demanding processes including cell adhesion, motility, migration, growth and proliferation ${ }^{387}$. The robust downregulation of $\beta 1$-integrin from the cell surface, thus enable cells in reducing energy expenditure during metabolic stress, emphasized in $[360,465]$. The selective endocytic regulation of surface proteins (e.g. maintain, enhance or diminish)-together with the broad modulation of $\mathrm{CME}$ - therefore comprises some of the cellular programs elicited by AMPK, which collectively, functions in maintaining and restoring metabolic homeostasis under conditions of metabolic insufficiency.

In summary, we reveal the direct regulation of CME by AMPK - thereby providing a biological mechanism by which AMPK activation may induce broad regulation of the plasma membrane, as previously reported (Figure 5.7) ${ }^{360}$. The control of CME by AMPK provides a mechanistic basis for how metabolic signals including those derived from metabolic stress, may induce remodeling of the surfaceome in mediating functionally diverse processes necessary for cellular adaptation and survival ${ }^{59-}$ ${ }^{61}$. Through control of Arf6-and likely several other downstream effectors-AMPK may induce both broad and selective regulation of CME dynamics, in distinctly controlling the internalization and expression of surface proteins, and hence, remodeling of the surfaceome landscape.

\section{3- Conclusion}

This thesis investigated the distinct mechanisms by which diverse cellular cues including mitogenic and metabolic signals may regulate CME. This focus is largely motivated by the diverse physiological responses mediated by mitogenic (e.g. growth factors) and metabolic signals (e.g. cellular energy levels) including cellular growth, proliferation, migration and differentiation. These cellular processes, to some extent, involve both local and global cell surfaceome remodeling events ${ }^{59-61}$-raising the question of whether and how mitogenic and metabolic signals may directly regulate the plasma 
membrane. CME is a cellular tool which regulates surface proteins, serving as the predominant regulator of the plasma membrane in mammalian cells; and hence, a likely mechanism through which mitogenic and metabolic signals may induce plasma membrane remodeling. However, the mechanisms by which mitogenic and metabolic signals may directly regulate CME remain relatively unexplored; serving as the major topic investigated in my research.

The work presented in this thesis describes the mechanisms by which mitogenic and metabolic signals may regulate CME. Mitogenic signaling by EGFR triggers the mobilization of PLC 1 1-derived calcium signals, which I demonstrated to directly control CME dynamics. Specifically, I found that PLC 1 1derived calcium signals are required for EGFR clathrin-mediated endocytosis-likely via calcium control of PKC and Sjn1. Consistently, I demonstrated that inhibition of the PLC 1 1-calcium-PKC signaling axis impairs the rate of formation and assembly of clathrin structures containing EGFR, supporting the direct requirement for calcium signals in EGFR clathrin-mediated endocytosis. Importantly, although explicit in examining EGFR clathrin-mediated endocytosis, I hypothesize that the internalization of other surface proteins may similarly be regulated by PLC 1 1-derived calcium signals, due to its broad control of Sjn1which was demonstrated present within other subsets of clathrin structures not harboring EGFR. Thus, this work provides i) a novel mechanism by which EGFR signaling-and hence, mitogenic signals - can directly regulate CME dynamics, in eliciting broad remodeling of the plasma membrane. To my knowledge, my work also ii) provides the first description of how intracellular calcium increases may regulate $\mathrm{CME}$ outside of a neuronal context, as calcium signals was demonstrated to directly control the dynamics of formation and assembly of clathrin structures in non-neuronal, RPE cells.

Previous findings from our laboratory demonstrated the robust remodeling of cell surface as measured via mass spectrometry analysis, upon activation of the predominant metabolic sensor, $\mathrm{AMPK}^{360}$. My work expounds on these findings by elucidating the biological mechanism by which AMPK, and hence metabolic signals, may remodel the plasma membrane. The work presented in this thesis demonstrates the direct regulation of CME upon AMPK activation. I show that AMPK regulates the small GTPase protein, Arf6, which importantly, has been hypothesized to enhance CME by facilitating local $\mathrm{PIP}_{2}$ production and recruitment of endocytic components (e.g. AP2 and clathrin) $379,382,383$. Specifically, I demonstrate that AMPK causes the inactivation of Arf6, resulting in its dissociation from clathrin structures. Consistently, AMPK activation concomitantly reduces the rate of initiation density and assembly of CCPs-which I hypothesize due to the AMPK-induced inactivation of Arf6. Nonetheless, 
CCPs which form under AMPK activated conditions demonstrate enhanced productivities evinced by both longer CCP lifetimes, and the maintenance (e.g. TfR and EGFR) and in some cases, enhancement (e.g. $\beta 1$-integrin) of cargo CME. As such, I hypothesize that although reducing the number and size of CCPs, AMPK nonetheless causes the formation of more efficient clathrin structures - which from a metabolic perspective-sustains CME with lower energy input. Thus, my work i) provides the first description of the direct regulation of CME by AMPK, and ii) adds a component to the metabolic repertoire by which AMPK maintains metabolic and cellular homeostasis.

My work elucidated the distinct mechanisms by which mitogenic and metabolic signals may directly regulate $\mathrm{CME}$-in inducing control of the cell surface. Inevitably, several biological and mechanistic details remain to be investigated due to limitations in our study, which are highlighted and described in the following section (Section 6.4-Future directions). Nevertheless, this study presents important implications for research in human health and disease. Disease states including diabetes, cancer and neurological disorders have been demonstrated to exhibit modulation of CME to some extent $^{10,50,342}$. The pathogenesis of Alzheimer's disease for instance, is marked by endocytic mutations resulting in surface accumulation and hallmark deposition of the $\beta$-amyloid protein ${ }^{10}$. Similarly, several cancer types have been found to exhibit reduced expression of endocytic proteins including PLC- $\gamma 1$, Dab2, Numb, and HIP1R-likely impairing EGFR endocytosis, supporting oncogenic transformation ${ }^{50,159,466}$. Thus, the novel mechanistic regulations of CME elucidated in my work, contributes to advancing our understanding of $\mathrm{CME}$, presenting novel therapeutic targets in treatment of human diseases.

\section{4- Future directions}

The findings presented above reveal novel mechanisms through which cellular cues including mitogenic and metabolic signals may directly regulate $\mathrm{CME}$-in eliciting global regulation of the plasma membrane. Nevertheless, limitations in our studies leave several open questions which discussed in the following sections, represent important areas for future research. 


\subsection{1 - Mitogenic signals}

In chapters 3 and 4, I demonstrated that mitogenic signaling by EGFR directly controls CME via PLC 1 1-derived calcium signals, which I found selectively required for internalization of EGFR but not TfR (Figure 3.6). Specifically, I demonstrated that calcium signals may enhance EGFR endocytosis by regulating endocytic resident proteins including Sjn1. Sjn1 is a negative regulator of clathrin structure assembly, which our work, and previous studies have shown to localize within CCPs (Figure 4.6) throughout distinct endocytic stages ${ }^{5}$. Importantly, I demonstrated that PLC 1 1-derived calcium signals induce the dissociation of Sjn1 from clathrin structures, which I hypothesize to enhance the assembly, growth, and hence, internalization of CCPs (including those containing EGFR) due to the removal of negative regulation imposed by Sjn1 (Figures 4.6 and 4.8). Sjn1 was also found localized within the majority of clathrin structures, which imparts to calcium, and hence mitogenic signaling, the ability to broadly control CME. Nevertheless, the requirement for PLC 1 1-derived calcium signals in CME is selective, as inhibition of calcium was without effect on the formation and internalization of TfRcontaining clathrin structures; which may be hypothesized due to minimal recruitment of Sjn1 within TfR-containing CCPs. While our findings provide a mechanistic basis for how mitogenic signals may regulate $\mathrm{CME}$, several questions remain regarding i) precisely how calcium signals my directly control Sjn1, and whether the ii) enhancement in EGFR clathrin-mediated endocytosis is indeed the result of Sjn1 dissociation from EGFR-containing CCPs; which together with iii) understanding of whether mitogenic signaling by other RTKs induces similar regulation of CME, represents important areas for further research discussed in the following sections.

\subsubsection{1 - Calcium signals may directly control Sjn1 via activation of CDK5}

While the precise mechanism by which PLC 1 1-derived calcium signals may cause dissociation of Sjn1 from CCPs was not in investigated in this work, the involvement of CDK5 may nonetheless be hypothesized as described in section 6.1.1. CDK5 is a protein kinase which although mostly enriched in neurons, is also expressed in other cell types including RPE cells ${ }^{438,439}$. The function of CDK5 has been extensively studied in neuronal systems which includes regulation of the cytoskeleton, axon guidance, membrane transport and synaptic function ${ }^{467}$. In addition, CDK5 has been shown by Lee and colleagues to directly phosphorylate Sjn1, causing its inactivation and inhibition of interaction with endocytic components including endophilin-which in synaptosomes was demonstrated to antagonize endocytosis ${ }^{437}$. Importantly, several studies have documented the regulation of CDK5 by PKC including work by Zhen and colleagues, which demonstrated the requirement for PLC- $\beta$ generated calcium signals 
and PKC, in the activation of CDK5 in PC-12 cells $s^{440}$. PLC 1 1-derived calcium signals activate PKC in RPE cells, which I hypothesize to induce activation of CDK5-proposed to directly phosphorylate and induce dissociation of Sjn1 from clathrin structures.

To test the requirement for CDK5 in the EGF-stimulated dissociation of Sjn1 from CCPs, the potent CDK5 inhibitor Roscovitine may be used, which directly blocks the ATP binding site of CDK5 preventing its phosphorylation and activation ${ }^{468}$. Using the same biochemical ligand internalization assays described in section 3.1, one can measure the internalization rates of EGFR in Roscovitine treated (and non-treated) cells, to initially determine the role of CDK5 in EGFR clathrin-mediated endocytosis. Similarly, siRNA-mediated knockdown of CDK5 may be implemented to resolve the effects of Roscovitine on EGFR internalization. Importantly, these assays should be supplemented with measurement of TfR internalization under similar treatments, to determine Roscovitine specificity in uncovering whether CDK5 activation may indeed, serve as a mechanism through which PLCY1-derived calcium signals may regulate Sjn1-in selectively controlling the assembly dynamics of CCP subsets (including those containing EGFR). Overall, I hypothesize that inhibition CDK5 will selectively impair EGFR clathrin-mediated endocytosis (but not TfR) due to the control of Sjn1 by CDK5 ${ }^{437}$. Furthermore, if CDK5 is indeed downstream of the PLCY1-calcium-PKC signaling axis, inhibition of CDK5 can therefore be hypothesized to phenocopy the effects of XeC, BAPTA-AM and BIM treatment on EGFR internalization.

Though yet to be clarified, several mechanisms have been described by which CDK5 may become activated. Reviewed in [467], phosphorylation of CDK5 on Tyr15 was shown to stimulate and increase CDK5 kinase activity-which is further augmented upon phosphorylation of its Ser159 site. The activation of CDK5 under EGF stimulation, can therefore be measured via immunoblot analysis, using commercially available anti-phospho-Tyr15 and anti-phospho-Thr160 antibodies, as per $[469,470]$. In addition, some studies have also measured the phosphorylation of downstream CDK5 targets including DARPP-32 (also known as PPP1R1B; Protein phosphatase 1 regulatory subunit 1B), to indirectly measure CDK5 activation ${ }^{440}$; and as such may similarly be implemented. I hypothesize that EGF stimulation will increase the phosphorylation, and hence activation of CDK5, to be reported by an increase in pTyr15 and/or pSer159 signals detected by immunoblotting. Furthermore, I hypothesize that the EGF-induced activation of CDK5 requires PLCY1-derived calcium signals and PKC as per [440], of which individual pharmacological inhibitions (i.e. by XeC, BAPTA-AM and BIM treatment) are expected to prevent CDK5 phosphorylation by EGF stimulation. Overall, I hypothesize that through the PLC 1 1-calcium-PKC signaling 
axis, EGF stimulation may directly induce activation of CDK5, which in turn is proposed to phosphorylate Sjn1.

The impact of the PLCY1-calcium-PKC signaling axis on the phosphorylation, and hence, regulation of Sjn1 was not investigated in the results described in chapters 3 and 4 . The observed dissociation of Sjn1 from clathrin structures may nonetheless, be proposed as a result of its direct phosphorylation by $\mathrm{CDK}^{437}$. Specifically, Lee and colleagues identified Ser1144 as a potential CDK5 phosphorylation site present within the NPF domain of Sjn1, which albeit, was discovered via in vitro autoradiography assays ${ }^{437}$. To interrogate the potential phosphorylation of Sjn1 by CDK5 in RPE cells, FRET-FLIM analysis of CyPET/YPET-hybrid fusion proteins (i.e. Sjn1-CyPET and YPET-CDK5) may be implemented as described in section 5.2.1. With this strategy, the interaction between CDK5 and Sjn1 may be measured which is hypothesized to increase upon EGF stimulation, reflecting the direct interaction and likely phosphorylation of Sjn1 by CDK5; which should conversely be inhibited by Roscovitine treatment. Similarly, to determine whether the interaction and phosphorylation of Sjn1 by CDK5 indeed requires the upstream PLCY1-calcium-PKC signaling axis, pharmacological inhibitors of the pathway (e.g. XeC, BAPTA-AM and BIM) should similarly be used, which I propose will inhibit the interaction between CDK5 and Sjn1, to be reported by FRET-FLIM analysis. Overall, I hypothesize that the PLCY1-calcium-PKC signaling axis will induce phosphorylation and activation of CDK5, which in turn is proposed to phosphorylate Sjn1 via direct interaction-resulting in Sjn1 inactivation and hence, dissociation from clathrin structures.

Lastly, to directly determine whether CDK5 activation serves as the mechanism through which PLCY1-calcium-PKC signaling may induce depletion of Sjn1 from clathrin structures, TIRF-M imaging coupled to automated software analysis may be implemented as described in section 4.2. This strategy will allow quantification of Sjn1 colocalization within clathrin structures, which our present work (Figure 4.6) and previous studies have demonstrated ${ }^{5}$. Furthermore, our current findings indicate that EGFstimulation induces the robust depletion of Sjn1 from clathrin structures which requires PLC 1 1-calciumPKC signaling. If CDK5 is indeed the downstream effector through which the PLC 1 1-calcium-PKC signaling axis may regulate $\operatorname{Sjn} 1$, inhibition of CDK5 with Roscovitine can therefore be hypothesized to prevent the EGF-stimulated depletion of Sjn1 from clathrin structures-to a similar extent observed under XeC, BAPTA-AM and BIM treatments. 
The proposed experiments described above will determine: i) the requirement for CDK5 in EGFR (and TfR) clathrin-mediated endocytosis, ii) the activation of CDK5 by EGF stimulation, iii) the direct interaction and potential phosphorylation of Sjn1 by CDK5 in RPE cells, and iv) the functional role of CDK5 activation in the EGF-induced depletion of Sjn1 from clathrin structures. Although previous studies support the potential role of CDK5 in regulating Sjn1 in RPE cells, it is possible for other mechanisms to be involved (e.g. CaMKII) ${ }^{440}$ given the diversity of downstream calcium and PKC targets, of which potential regulatory roles in CME represents an important area for future research.

\subsubsection{2 - Sjn1 may distinctly localize within EGFR-containing CCPs but not TfR}

Our current findings demonstrate the selective requirement for PLC $\gamma 1$-derived calcium signals in the internalization of EGFR (Figure 3.1). Consistently, I found that the PLC 1 1-calcium-PKC signaling axis, is required for the efficient initiation and assembly of EGFR+ CCPs, which become impaired upon pharmacological inhibition by XeC, BAPTA-AM and BIM (Figures 4.2 and 4.3). Sjn1 is a resident protein and a negative regulator of clathrin assemblies, which through PLC 1-derived calcium signals is broadly depleted from clathrin structures upon EGF stimulation (Figures 4.6 and 4.8). As a negative regulator of clathrin assembly, the depletion of Sjn1 from clathrin structures therefore likely enhances the assembly (and hence, internalization) of CCPs, of which some may include subsets harboring EGFR. The enhancement of EGFR clathrin-mediated endocytosis elicited by PLC 1-derived calcium signals, may thus, in part, reflect the dissociation of Sjn1 from EGFR+ clathrin structures, which nevertheless was not investigated in our current work-representing an area for further investigation.

To determine the effect of the EGF-induced depletion of Sjn1 on the distinct formation and internalization of EGFR+ clathrin structures, similar TIRF-M imaging and analysis strategies described in section 4.2 may be employed. Specifically, the localization of Sjn1 within EGFR+ clathrin structures may be determined via triple color TIRF-M imaging of fixed RPE cells with fluorescent clathrin (e.g. eGFP-CLCa expressing RPE cells) ${ }^{1}$, Sjn1 (e.g. mCherry-tagged Sjn1 transfection) ${ }^{1}$ and EGFR (e.g. immuno-labeling of EGFR ectodomain by far-red Alexa Fluor 647$)^{7}$, of which single frame images may be subsequently subjected to automated software analysis, to detect and quantify Sjn1 content of EGFR+ clathrin structures. I hypothesize that with this strategy, Sjn1 will be preferentially enriched within EGFR+ clathrin structures, consistent with the broad localization of Sjn1 described in our current study (Figure 4.8). Furthermore, I hypothesize that EGF stimulation will reduce the Sjn1 content of EGFR+ clathrin structures relative to basal, non-stimulated conditions. Our current work indicates the requirement for 
PLCY1-derived calcium signals in the EGF-induced dissociation of Sjn1 from clathrin structures (Figure 4.8). Thus, I further hypothesize that the depletion of Sjn1 from EGFR+ clathrin structures require the PLCY1-calcium-PKC signaling axis; investigated via pharmacological inhibition (i.e. by XeC, BAPTA-AM and BIM treatment).

Using the above strategy, the assembly and size of EGFR+ clathrin structures may similarly be quantified, and correlated to Sjn1 content detected therein - to demonstrate the functional role of Sjn1 on the formation of EGFR+ clathrin structures. I hypothesize that the detection of Sjn1 negatively correlates with the size of EGFR+ clathrin structures, due to the antagonistic role of Sjn1 on clathrin assembly ${ }^{101}$. In addition, it is important to perform parallel experiments investigating Sjn1 localization within TfR-containing clathrin structures under similar treatment conditions-which I hypothesize to exhibit reduced Sjn1 content relative to subsets containing EGFR - to resolve whether Sjn1 dissociation, indeed forms the mechanistic basis by which PLCY1-derived calcium signals may selectively regulate EGFR clathrin-mediated endocytosis. Overall, the experiments proposed in this section will establish the functional role of Sjn1, in the selective enhancement of assembly and internalization of EGFR+ clathrin structures elicited by EGF stimulation.

\subsubsection{3 - Mitogenic signaling by other RTKs may elicit similar regulation of CME}

The mitogenic control of the plasma membrane via CME has been one of the key themes investigated in this thesis. Work presented in chapters 3 and 4 focused on mitogenic signaling by EGFR, which to date is the best characterized member of the RTK family ${ }^{11}$. We discovered that through activation of PLC- $\gamma 1$, EGFR triggers intracellular calcium increases which we demonstrated to directly control its internalization via clathrin-mediated endocytosis-and likely other subsets of clathrin structures-via Sjn1. Our findings provide a novel mechanism by which mitogenic signals may control $\mathrm{CME}$, and hence, induce remodeling of the cell surfaceome. Importantly, this discovery raises the question of whether CME may also be regulated by other RTK members (e.g. VEGFR, PDGFR and FGFR) given that most have been documented to similarly activate PLC $-\gamma 1^{11,13,266}$. Thus, although the present study was limited to examination of EGFR, the regulation of CME by other RTKs may similarly be hypothesized, of which collective roles in regulating the plasma membrane, represents an important area for future research. 
While beyond the scope of this study, the regulation of CME by other RTK members may nevertheless be investigated using similar experimental strategies implemented in chapters 3 and 4 . Biochemical internalization assays used in chapter 3 for instance, may be performed with other commercially available RTK ligands, to uncover the functional requirement for PLC 1 1-derived calcium signals in the internalization of RTKs other than EGFR in RPE cells. In addition, the spatial organization of other RTK members may similarly be examined via TIRF-M imaging of fluorescently-conjugated cognate ligands as per chapter 4, which may be hypothesized to localize within distinct clathrin structuresreflecting in part, differences in the internalization requirements of distinct cargo receptors ${ }^{74,105,106,423}$. With this strategy, the formation and assemblies of specific RTK-harboring clathrin structures may be investigated under identical conditions presented in chapter 4 , to determine whether RTK members other than EGFR may regulate CME via PLCY1-derived calcium signals. Lastly, the involvement of Sjn1 may also be probed using the strategies highlighted in section 4.2, in resolving whether other RTKs may broadly regulate CME similar to that elicited by EGFR. Overall, I hypothesize that the control of CME via PLC $\gamma 1$-derived calcium signals may not be exclusive to EGFR signaling, as PLC- $\gamma 1$ is a common downstream target of several other RTKS - which in turn, may be hypothesized to effect regulation of $\mathrm{CME}$ and hence, induce remodeling of the plasma membrane.

\subsection{2 - Metabolic signals}

In chapter 5, we demonstrated that metabolic signaling by AMPK directly controls CME, of which pharmacological activation by A7 effects broad reductions in the rate of assembly and density of CCPs; which nevertheless exhibited enhanced productivities (i.e. longer CCP lifetimes) (Figure 5.1). Specifically, we found that AMPK may elicit direct regulation of CME through control of the small GTPase Arf6, which localizes to the plasma membrane where it is known to induce local $\mathrm{PIP}_{2}$ production and promote recruitment of endocytic components including AP2 and clathrin ${ }^{379,382,383}$. Indeed, I found that Arf6 localizes within clathrin structures specifically in its active, GTP-bound form, which strikingly, was inhibited by AMPK evinced by the broad depletion of Arf6 from clathrin structures following A7 treatment (Figure 5.3). Consistently, we demonstrated that AMPK inactivates Arf6 by promoting its GDP-bound form, which may provide a mechanistic basis for its A7-induced dissociation from clathrin structures (Figure 5.2). The dissociation of Arf6 from clathrin structures may be hypothesized to downregulate clathrin assembly, providing a mechanism by which AMPK may elicit modulation of CME dynamics observed in this study (Figure 5.1). Interestingly, the alteration of CME by AMPK was without 
effect on the internalization rates of CME cargoes such as TfR and EGFR; which in contrast, increased $\beta 1$ integrin internalization (Figure 5.5). The enhanced internalization of $\beta 1$-integrin may be explained by the lack of effect of $A 7$ treatment on the density and size of integrin + CCPs, which nonetheless exhibited enhanced $\beta 1$-integrin content (Figure 5.6) - potentially enhancing $\beta 1$-integrin clathrin-mediated endocytosis.

While our findings indicate Arf6 as the potential intermediate through which AMPK may directly regulate $\mathrm{CME}$, several open questions remain to be addressed to precisely uncover how control of Arf6 may effect regulation of CME dynamics; and how AMPK activation may induce broad regulation of the plasma membrane through this mechanism. Further discussed in the following sections, these research questions will address: i) the functional requirement for Arf6 in the assembly dynamics of CCPs; ii) the interaction of Arf6 with endocytic components and the effects of GTP/GDP binding on this process; iii) the specific control of ArfGAP3 by AMPK and its role in the AMPK-induced inactivation of Arf6; and lastly, iv) the implication of CME control by AMPK on the formation and assembly dynamics of other CCP subsets containing distinct cargoes-including TfR and EGFR.

\subsubsection{1 - Understanding the functional role of Arf6 in the AMPK-induced regulation of CME}

Our findings demonstrate that pharmacological activation of AMPK by A7 significantly decreases the formation and assembly of CCPs (Figure 5.1). Several mechanisms described in previous studies indicate the potential role of Arf6 in enhancing $\mathrm{CME}^{379,382,383}$ - which together with the surface depletion of its direct regulator ArfGAP3 elicited by A7 treatment ${ }^{360}$ - provide preliminary evidence suggesting Arf6 as a likely intermediate through which AMPK may directly regulate CME. Consistently, inhibition of Arf6 by inhibition of Arf6-GEFs via SecinH3 treatment phenocopies the alteration of CME dynamics observed under A7 treatment (Figure 5.4)-indicating that AMPK may indeed act through Arf6 in regulating CME. Furthermore, we demonstrate that AMPK activation induces the inactivation of Arf6 (Figure 5.2), preventing its localization within clathrin structures (Figure 5.3). Nonetheless, the exact functional requirement for Arf6 on CCP formation and assembly was not directly investigated in this work, of which elucidation will further establish Arf6 as the direct AMPK effector regulating CME.

To elucidate the functional requirement of Arf6 on CME dynamics, several approaches which involve direct perturbation of Arf6 may be implemented. For instance, the recently identified pharmacological inhibitor of Arf6, NAV-2729, may be used which prevents activation of Arf6 by binding 
and competing for the Arf6-GEF binding site ${ }^{471}$. The use of NAV-2729 was demonstrated to inhibit downstream activation of Arf6 targets as described in [471]. As well, direct inhibition of Arf6 may also be performed in a separate experiment via siRNA-mediated knockdown. Using these Arf6 perturbation strategies, together with live-cell TIRF-M imaging and automated software analysis of eGFP-CLCa expressing RPE cells (as per section 5.1), the role of Arf6 in the formation and assembly of CCPs may be demonstrated. I hypothesize that the inhibition of Arf6 (via NAV-2729 or siRNA) will phenocopy the effects of $\mathrm{A} 7$ and SecinH3 treatment on $\mathrm{CME}$, causing broad reductions in the rate of assembly and density of CCPs - of which elucidation will further our understanding of the role of Arf6 as an intermediate through which AMPK may induce regulation of CME dynamics.

\subsubsection{2 - Arf6 may enhance CME via interaction with various components of the endocytic machinery} Our findings indicate that Arf6 localizes within clathrin structures predominantly in its active, GTP-bound form (Figure 5.3). Although poorly understood, the potential regulation of CME by Arf6 may therefore involve its direct interaction and control of various endocytic components required for CCP assembly and formation. Consistently, Poupart and colleagues demonstrated the direct interaction of Arf6 with AP2 and clathrin, indicating its direct engagement of the clathrin machinery which likely includes other endocytic components ${ }^{383}$. Thus, to further define the functional role of Arf6 in regulation of $\mathrm{CME}$, it is important to investigate its potential interactions with endocytic components. Specifically, the use of the well-established BiolD (Proximity-dependent biotinylation) screening strategy may be recommended, to investigate the proximal interactions between Arf6 and components of CME.

BiolD is a unique method used for screening protein-protein interactions, which is not limited to detection of direct protein binding, but may also include identification of proximal protein associations in living cells ${ }^{472,473}$. Specifically, the protein of interest (bait) is fused to a prokaryotic biotin ligase (BirA*) which upon expression in cells, promiscuously biotinylates the amine groups of neighbouring proteins (candidates) within a 10nm spatial radius upon addition of excess biotin-including directly bound and proximally associated proteins ${ }^{472,473}$. The candidates may then be purified using a biotin-streptavidin system, and subsequently analyzed and characterized by mass spectrometry cells ${ }^{472,473}$. In uncovering the interactions of Arf6 with endocytic components, a genetically-encoded wild-type Arf6 fused to BirA* (Arf6 ${ }^{\mathrm{WT}}$-BirA*) may be used and transfected into RPE cells, which may subsequently be processed for screening biotinylated candidates as per [474]. With this strategy, the interaction partners of Arf6 may be revealed which I hypothesize to include several components of the endocytic machinery including 
AP2 and clathrin, as previously demonstrated ${ }^{383}$. As well, the identification of biotinylated CCP-localized endocytic proteins will thus, indicate that Arf6 does indeed localize with CCPs where it may associate and regulate components of the endocytic machinery-thereby regulating CME.

As a molecular switch, it is also important to investigate how nucleotide binding states (GTP/GDP) might regulate the interaction of Arf6 with endocytic components. To do so, the previous BiolD strategy may be implemented using various genetically-encoded recombinant Arf6 proteins; described in section 5.2.2. Specifically, a GTPase-deficient Arf6 mutant that is GTP-locked and constitutively active ( Arf6 $^{\mathrm{Q} 67 \mathrm{~L}}$ ) may be used, in addition to a fast-cycling Arf6 mutant that is GDP-locked and constitutively inactive (Arf6 $\left.{ }^{T 44 N}\right)^{394}$. Both recombinant Arf6 proteins can similarly be fused to BirA* (e.g. Arf6 ${ }^{\mathrm{Q67L}}-\mathrm{BirA}^{*}$ and Arf6 ${ }^{\mathrm{T44N}}$-BirA*) and transfected into RPE cells, and subsequently screened for candidate biotinylation as per [474]. With this strategy, I hypothesize that Arf6 ${ }^{\mathrm{Q} 67 \mathrm{~L}}$ will robustly increase the biotinylation of AP2 and clathrin in addition to other endocytic components, due to the localization of Arf6-GTP within clathrin structures. Furthermore, I hypothesize that Arf6 ${ }^{\mathrm{T} 44 \mathrm{~N}}$ will not appreciably increase the biotinylation of endocytic components, consistent with its observed depletion from clathrin structures demonstrated in this study (Figure 5.3). Overall, I hypothesize that Arf6 interacts with endocytic components and localizes within clathrin structures predominantly in its active, Arf6-GTP form. As such, the AMPK-induced dissociation of Arf6 from clathrin structures may be hypothesized due to Arf6 inactivation and subsequent disengagement from components of the endocytic machinery.

The experiments proposed in this section - together with the findings to be generated from section 6.4.2.1-will further establish the role of Arf6 as a direct regulator of CME. Collectively, these results will indicate whether Arf6, and its depletion from clathrin structures, indeed form the mechanistic basis by which AMPK may induce broad regulation of CME.

\subsubsection{3 - AMPK may act through ArfGAP3 in inducing Arf6 inactivation}

Previous studies from our laboratory indicated the robust depletion of surface proteins upon activation of AMPK by A7, which included the specific Arf6 regulator ArfGAP3 ${ }^{360}$. ArfGAP3 directly inhibits Arf6 activity by enhancing GTP hydrolysis, thereby promoting its GDP-bound configuration. While the precise mechanism by which AMPK may induce direct regulation of Arf6 was not explored in this work, the A7-induced depletion of ArfGAP3 from the plasma membrane ${ }^{360}$, may be hypothesized to reflect the consequential regulation of Arf6 induced by AMPK. The direct regulation of ArfGAP3 by 
AMPK, and its specific role in the AMPK-induced inactivation of Arf6 however, was similarly not investigated in our current study, representing an important area for future research-of which elucidation will further characterize how AMPK may broadly regulate CME via Arf6.

To initially understand the functional requirement for ArfGAP3 in the AMPK-induced regulation of CME (via Arf6), the direct inhibition of ArfGAP3 via siRNA-mediated knockdown may be implemented. If ArfGAP3 is indeed the intermediate through which AMPK acts in inactivating Arf6 to regulate CME, knockdown of ArfGAP3 is therefore expected to prevent the alteration of CME elicited by AMPK activation. To test this possibility, siRNA-mediated knockdown of ArfGAP3 may be performed in RPE cells expressing eGFP-CLCa. These cells may then be subjected to live-cell TIRF-M imaging coupled to automated software detection and analysis of bona fide CCPs, in both A7 treated and non-treated (control) conditions. I hypothesize that in ArfGAP3 knockdown cells, the density and assembly rate of CCPs will not be affected by A7 treatment, due to the inability of AMPK to induce inactivation of Arf6 as previously observed (Figure 5.2). Using this strategy, the role of ArfGAP3 as a potential regulator of Arf6 during AMPK activation may therefore be resolved-providing a mechanism by which AMPK may induce inactivation of Arf6 in regulating CME.

The direct control of ArfGAP3 by AMPK was not investigated in our current work and similarly has not been demonstrated in the literature; of which elucidation is imperative in establishing the role of ArfGAP3 in regulating Arf6 during AMPK activation. Nevertheless, a consensus AMPK phosphorylation site can be identified in ArfGAP3 via online sequence analysis tools ${ }^{386,475}$, suggesting its direct control by AMPK. Since phospho-specific ArfGAP3 antibodies have yet to be developed, to determine whether ArfGAP3 is directly phosphorylated by AMPK, the use of Phos-Tag ${ }^{\mathrm{TM}}$ western blotting can be recommended. Briefly, Phos-Tag ${ }^{\mathrm{TM}}$ is a compound containing two metallic ions (i.e. Manganese or Zinc) which may be complexed to polyacrylamide on SDS-PAGE gels ${ }^{476,477}$. Phos-Tag ${ }^{\mathrm{TM}}$ directly binds phosphate groups on proteins independent of amino acid sequence ${ }^{476}$, therefore reducing the mobility of phosphoproteins during SDS/PAGE relative to the corresponding dephosphoprotein ${ }^{478}$. As such, Phos$\mathrm{Tag}^{\mathrm{TM}}$ western blotting may separate proteins based on their phosphorylation status, of which implementation may identify the phosphorylation of ArfGAP3 upon AMPK activation by A7 treatment. Using this strategy, I hypothesize that A7 treatment will increase the amount of phospho-ArfGAP3 relative to control (non-treated), due to the direct phosphorylation of ArfGAP3 by AMPK. 
While useful in elucidating the phosphorylation state and hence, regulation of ArfGAP3 by AMPK activation, Phos-Tag ${ }^{\mathrm{TM}}$ analysis does not resolve whether ArfGAP3 may directly be phosphorylated by AMPK. As such, mutagenesis of ArfGAP3 on the predicted AMPK phosphorylation site may be implemented, of which phosphorylation status under A7 treatment may similarly be determined via Phos-Tag ${ }^{\mathrm{TM}}$ analysis. Using this strategy, I predict that mutation of the predicted AMPK phosphorylation site on ArfGAP3 will prevent the phosphorylation of ArfGAP3 by A7 treatment. To further corroborate the proposed role of AMPK in directly phosphorylating ArfGAP3, FRET-FLIM microscopic analysis may similarly be used as per section 5.2.1. Using CYPET/YPET-hybrid fusion proteins (i.e. AMPK-CYPET and YPET-ArfGAP3), the interaction between AMPK and ArfGAP3 may be measured which can be hypothesized to increase upon A7 treatment, reflecting the interaction and phosphorylation of ArfGAP3 by AMPK. Overall, I hypothesize that AMPK interacts and phosphorylates ArfGAP3, resulting in its activation and inhibition of Arf6-of which elucidation is discussed in the following.

The activation of ArfGAP3 by AMPK may represent the mechanism by which AMPK may induce inactivation of Arf6, observed in our current study (Figure 5.2). Described in section 6.2.2, the depletion of ArfGAP3 from the plasma membrane upon A7 treatment ${ }^{360}$ may reflect its direct regulation by AMPK, causing its translocation within Arf6-containing clathrin structures-wherein it i) may bind and inactivate Arf6, and similarly ii) be internalized and removed from the plasma membrane. It is therefore important to investigate how AMPK may regulate the spatial organization of ArfGAP3, to resolve its role in regulating Arf6. To do so, similar TIRF-M imaging and analysis strategies described in section 4.2 may be employed. Specifically, the localization of ArfGAP3 within Arf6-containing clathrin structures may be determined via triple color TIRF-M imaging of fixed RPE cells with fluorescent clathrin (e.g. RFP-CLCa expressing RPE cells) ${ }^{1}$, Arf6 (e.g. eGFP-tagged Arf6 ${ }^{\mathrm{WT}}$ transfection) ${ }^{394}$ and ArfGAP3 (e.g. immuno-labeling of ArfGAP3 by far-red Alexa Fluor 647), of which single frame images may be subsequently subjected to automated software analysis, to detect and quantify ArfGAP3 colocalization within Arf6-containing clathrin structures. I hypothesize that with this strategy, AMPK activation upon A7 treatment will induce the subsequent activation and localization of ArfGAP3 within Arf6-containing structures, mediated by the direct binding of activated ArfGAP3 with Arf6.

Overall, I hypothesize that AMPK directly phosphorylates and regulates ArfGAP3, causing its activation and translocation within Arf6-containing clathrin structures. The translocation of ArfGAP3 within these clathrin subsets may therefore be proposed to result in direct Arf6 inactivation and 
dissociation from clathrin structures. The dissociation of Arf6 from clathrin structures by ArfGAP3, may thus, form the mechanistic basis through which AMPK may induce direct regulation of CME.

\subsubsection{4 - AMPK may regulate the formation and assembly of other cargo-containing CCP subsets}

$\mathrm{CME}$ is the predominant regulator of the plasma membrane in mammalian cells which governs the expression levels of surface proteins ${ }^{49}$. Our current work demonstrates that through Arf6, AMPK may directly control CME dynamics, therefore suggesting its concomitant role in regulating surface protein endocytosis. Indeed, I demonstrated that AMPK activation directly influences the endocytosis of CME cargoes including TfR and EGFR, and $\beta 1$-integrin, of which internalization rates were respectively maintained and enhanced upon acute A7 treatment (Figure 5.5). Our studies indicate that the enhancement in $\beta 1$-integrin internalization may be attributed to i) the lack of effect of AMPK activation on the rate of formation and assembly of integrin+ CCPs (Figure 5.6, A and B), and the ii) enhancement of $\beta 1$-integrin recruitment within integrin+ CCPs (Figure 5.6C). Thus, although broadly inducing alteration of CME, AMPK may enhance $\beta 1$-integrin endocytosis through these mechanistic strategies, of which precise molecular details nevertheless, remain to be characterized. Importantly, although not investigated in our study, the maintenance of TfR and EGFR endocytosis under A7 treatment may similarly reflect, in part, the lack of effect of AMPK activation on TfR+ and EGFR+ CCPs, and the respective enhancements in TfR and EGFR recruitment therein. As such, investigation of CCP subsets harboring TfR and EGFR, and their corresponding regulation by AMPK activation is recommended, in order to further define the effects of CME control by AMPK - on the broad regulation and remodeling of the plasma membrane.

To understand how AMPK activation may distinctly regulate the CME dynamics of TfR and EGFR, similar imaging strategies used to investigate $\beta 1$-integrin CME may be implemented; as per section 5.3. Specifically, eGFP-CLCa expressing RPE cells labeled with fluorescent TfR (A647-Tfn) or EGFR

(rhodamine-EGF) ligands may be visualized by live-cell TIRF-M imaging, to acquire time-lapse images for automated software detection and analysis of clathrin structures ${ }^{5,207}$. Using this strategy, CCPs with TfR (TfR+ CCPS) or EGFR (EGFR+ CCPs) may be identified based on signal detection of the respective fluorescent ligands contained therein ${ }^{207}$. Importantly, to determine the effects of AMPK activation on TfR and EGFR CME dynamics, detected TfR+ and EGFR+ CCPs may be subsequently subjected to systematic quantification of eGFP-CLCa fluorescence intensity following A7 treatment; as per section 5.3.1. As well, the same strategy may be applied to measure the effects of AMPK activation on the 
respective recruitment of TfR and EGFR within TfR+ and EGFR+ CCPs, via systematic quantification of ligand fluorescence intensities contained therein; as per section 5.3.2. Due to the lack of effect of AMPK activation on the internalization rates of TfR and EGFR (Figure 5.5, B and C), the following possibilities can be hypothesized: i) AMPK activation will not affect the assembly and receptor recruitment of TfR+ and EGFR+ CCPs (maintaining basal rates of receptor endocytosis); ii) AMPK activation will reduce the density and size of TfR+ and EGFR+ CCPs, while enhancing receptor recruitment therein (maintaining basal rates of receptor endocytosis). Overall, these results will provide indication as to how AMPK may broadly, yet selectively regulate cargo endocytosis, in effecting global remodeling of the plasma membrane as reported in [360].

\section{5- Additional contributions}

During the course of my graduate studies, I focused on elucidating the mechanisms by which mitogenic and metabolic signals may directly regulate CME. Specifically, I investigated how metabolic signaling by EGFR may directly impact the clathrin-mediated endocytic process, as presented in chapters 3 and 4 of this thesis. This work resulted in a first-author publication in the peer-reviewed journal, Molecular Biology of the Cell in 2017:

Delos Santos RC, Bautista S, Lucarelli S, et al. Selective regulation of clathrin-mediated epidermal growth factor receptor signaling and endocytosis by phospholipase $\mathrm{C}$ and calcium. Mol Biol Cell. 2017;28(21):2802-2818. doi:10.1091/mbc.E16-12-0871.

In addition, I investigated how metabolic signaling by AMPK may similarly regulate CME, of which findings are presented in chapter 5 of this thesis. While extensive work has been done in addressing how AMPK may induce CME regulation, several experiments still require completion which include some of the recommendations presented in section 6.4.2. As such, this work is currently in preparation of which completion and submission is projected for April 2019.

I have also engaged in a several collaborative projects throughout my graduate studies, of which some have been published in peer-reviewed journals. These publications are described in the following, with the extent of my contribution highlighted. 
i) Fekri F, Delos Santos RC, Karshafian R, Antonescu CN. Ultrasound microbubble treatment enhances clathrin-mediated endocytosis and fluid-phase uptake through distinct mechanisms. PLoS One. 2016;11(6):1-22. doi:10.1371/journal.pone.0156754.

This project investigated the effects of USMB treatment (Ultrasound in combination with microbubbles) on the clathrin-mediated endocytosis of RPE cells. Briefly, microbubbles are gas bubbles that are less than $5 \mu \mathrm{m}$ in diameter, consisting of a gas core encapsulated by a lipid layer. Due to their small size, microbubbles may be integrated within the extracellular environment where they may come in close proximity to the cell surface. Importantly, ultra-sonic stimulation of microbubbles via highenergy ultrasound pulses induces microbubble bursting (USMB treatment), which in turn induces sheer stress on the plasma membrane of nearby cells. Sheer stress induced by USMB leads to transient pore formation or disruption of the plasma membrane, which was demonstrated to enhance material uptake. In addition, USMB treatment was hypothesized to enhance endocytosis via CME, which indeed was demonstrated in this work, evinced by the USMB-induced enhancement in TfR internalization. To supplement this finding, I contributed my TIRF-M imaging expertise in elucidating the functional regulation of CME upon USMB treatment. Specifically, I investigated how USMB treatment may affect the initiation density and assembly of clathrin structures. To do so, I used TIRF-M imaging coupled to automated software analysis to visualize and analyze clathrin structures in fixed eGFP-CLCa expressing RPE cells labeled with A555-Tfn (fluorescent TfR ligand). Consistently, I found a significant enhancement in the assembly of clathrin structures following USMB treatment, as reported by an increase in mean intensity of eGFP-CLCa fluorescence detected therein. In addition, I found that USMB did not increase the recruitment of TfR within clathrin structures suggesting that the observed USMB-induced enhancement of TfR CME, may be due to the role of USMB in broadly reprogramming CME dynamics. This finding contributed a figure to the published work, as shown in the following: 


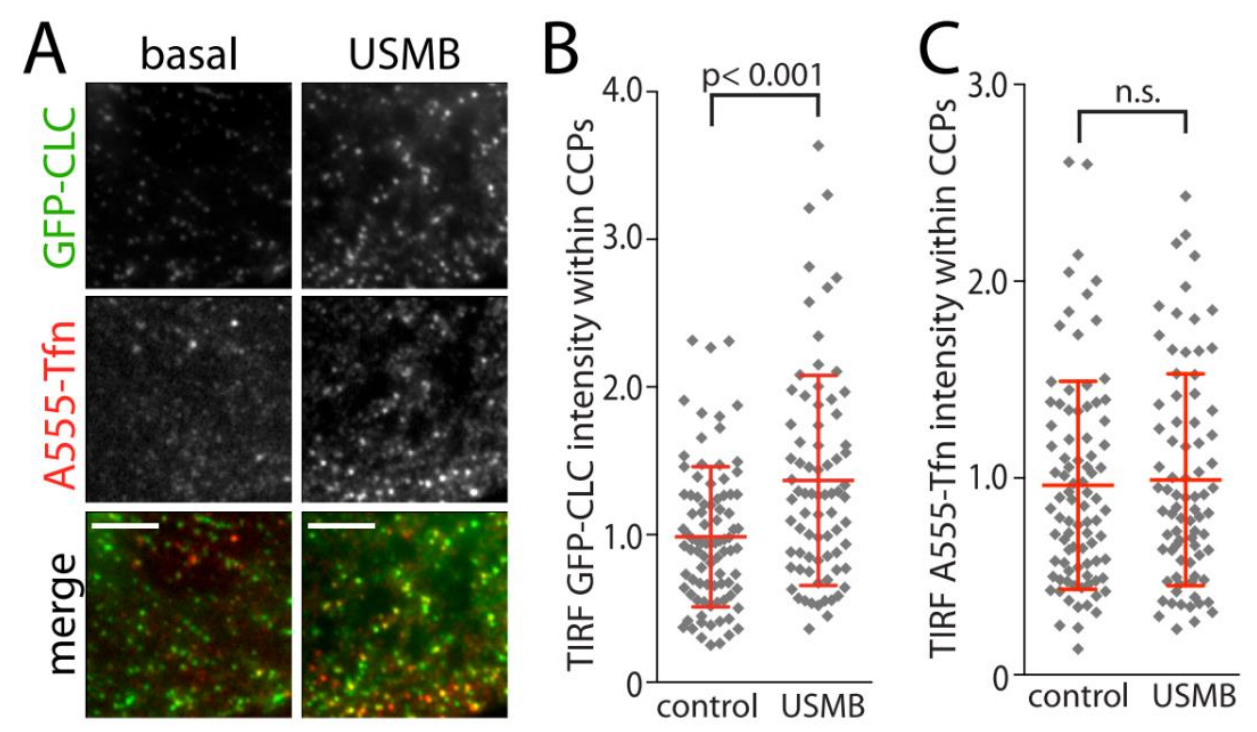

Figure 3. USMB treatment alters the properties of clathrin-coated pits.

Reproduced with permission from [9].

ii) Delos Santos RC, Garay C, Antonescu CN. Charming neighborhoods on the cell surface: Plasma membrane microdomains regulate receptor tyrosine kinase signaling. Cell Signal. 2015;27(10):1963-1976. doi:10.1016/j.cellsig.2015.07.004.

This work is a review article detailing unique plasma membrane microdomains (PM $\mu \mathrm{D})$ on the cell surface, with specific focus on how each may modulate the signaling properties of RTKs contained therein. Emphasized in this work, $\mathrm{PM} \mu \mathrm{Ds}$ are discrete areas on the plasma membrane defined by unique lipid/protein profiles which include lipid rafts, clathrin-coated structures, tetraspanin-enriched and actin-dependent microdomains. This review elaborated on how the localization of RTKs within distinct PM $\mu D$ s may modulate their signaling output-attributed to the enrichment of unique resident proteins contained therein, which may distinctly influence RTK signaling. In this work, I was mainly responsible for discussions regarding lipid-ordered microdomains and lipid rafts. As well, I discussed how RTK signaling may be distinctly modulated by these microdomains; in addition to providing insight for discussions found in other sections. 
iii) Lucarelli S*, Delos Santos RC*, Antonescu CN. Measurement of Epidermal Growth Factor ReceptorDerived Signals Within Plasma Membrane Clathrin Structures. Methods Mol Biol. 2017;1652:191-225. doi:10.1007/978-1-4939-7219-7_15. *Equal contribution to this work

This publication is a methods chapter in the book titled, "ErbB Receptor Signaling" by Wang and colleagues, which reviewed the current methods and protocols for studying EGFR. Specifically, our written chapter provided experimental strategies to microscopically visualize and systematically analyze EGFR on the plasma membrane. My main contribution to this work was in describing the fluorescent labeling, microscopic visualization, and analysis of EGFR and its subsequent recruitment to clathrin structures on the plasma membrane (shown below). In addition, I contributed to the development of a figure (shown below) and provided discussion (i.e. EGFR biology and plasma membrane microdomains) and content for other sections throughout this work. The figures generated from my contributions are shown in the following:

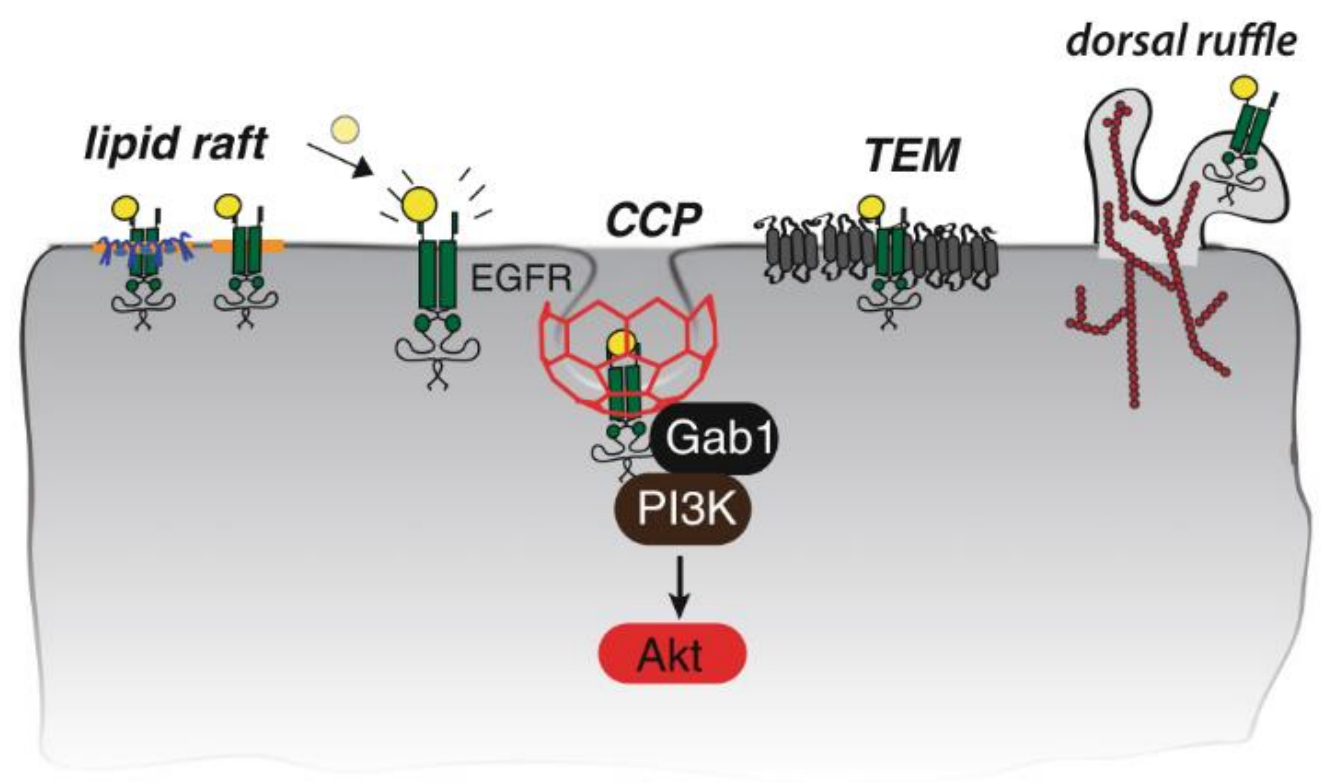

Figure 2. EGFR signaling within plasma membrane microdomains.

Reproduced with permission from [7]. 


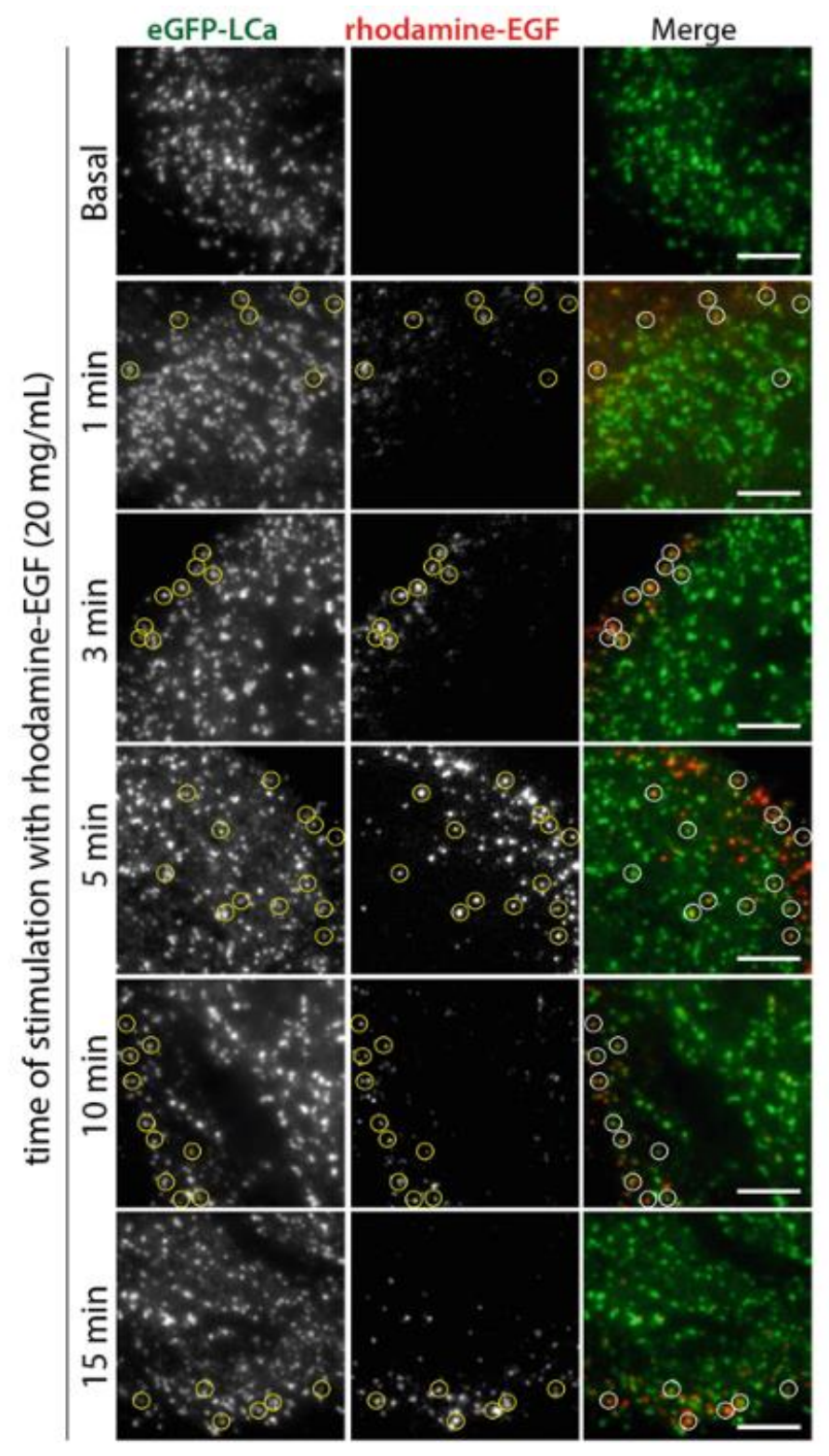

Figure 3. Rhodamine-EGF is rapidly recruited to clathrin-coated pits (CCPs). Reproduced with permission from [7]. 


\section{References}

1. Delos Santos RC, Bautista S, Lucarelli S, et al. Selective regulation of clathrin-mediated epidermal growth factor receptor signaling and endocytosis by phospholipase $\mathrm{C}$ and calcium. Mol Biol Cell. 2017;28(21):2802-2818. doi:10.1091/mbc.E16-12-0871.

2. Bautista SJ, Boras I, Vissa A, et al. mTOR complex 1 controls the nuclear localization and function of glycogen synthase kinase 3ß. J Biol Chem. 2018;293(38):14723-14739. doi:10.1074/jbc.RA118.002800.

3. Delos Santos RC, Garay C, Antonescu CN. Charming neighborhoods on the cell surface: Plasma membrane microdomains regulate receptor tyrosine kinase signaling. Cell Signal. 2015;27(10):1963-1976. doi:10.1016/j.cellsig.2015.07.004.

4. Garay C, Judge G, Lucarelli S, et al. Epidermal growth factor-stimulated Akt phosphorylation requires clathrin or ErbB2 but not receptor endocytosis. Mol Biol Cell. 2015;26(19):3504-3519. doi:10.1091/mbc.E14-09-1412.

5. Aguet F, Antonescu C, Mettlen M, Schmid S, Danuser G. Advances in analysis of low signal-tonoise images link dynamin and AP2 to the functions of an endocytic checkpoint. Dev Cell. 2013;26(3):279-291. doi:10.1016/j.devcel.2013.06.019.

6. Antonescu CN, Aguet F, Danuser G, Schmid SL. Phosphatidylinositol-(4,5)-bisphosphate regulates clathrin-coated pit initiation, stabilization, and size. Mol Biol Cell. 2011;22(14):2588-2600. doi:10.1091/mbc.E11-04-0362.

7. Lucarelli S, Delos Santos RC, Antonescu CN. Measurement of Epidermal Growth Factor ReceptorDerived Signals Within Plasma Membrane Clathrin Structures. Methods Mol Biol. 2017;1652:191225. doi:10.1007/978-1-4939-7219-7_15.

8. Lucarelli S, Pandey R, Judge G, Antonescu CN. Similar requirement for clathrin in EGF- and HGFstimulated Akt phosphorylation. Commun Integr Biol. 2016;9(3):e1175696. doi:10.1080/19420889.2016.1175696.

9. Fekri F, Delos Santos RC, Karshafian R, Antonescu CN. Ultrasound microbubble treatment enhances clathrin-mediated endocytosis and fluid-phase uptake through distinct mechanisms. PLoS One. 2016;11(6):1-22. doi:10.1371/journal.pone.0156754.

10. Doherty GJ, McMahon HT. Mechanisms of Endocytosis. Annu Rev Biochem. 2009;78(1):857-902. doi:10.1146/annurev.biochem.78.081307.110540.

11. Lemmon MA, Schlessinger J. Cell signaling by receptor tyrosine kinases. Cell. 2010;141(7):11171134. doi:10.1016/j.cell.2010.06.011.

12. Schmid EM, McMahon HT. Integrating molecular and network biology to decode endocytosis. Nature. 2007;448(7156):883-888. doi:10.1038/nature06031.

13. Kadamur G, Ross EM. Mammalian phospholipase C. Annu Rev Physiol. 2013;75:127-154. doi:10.1146/annurev-physiol-030212-183750.

14. Brown P. Loneliness at the bench. Is the PhD experience as emotionally taxing as it is mentally 
challenging? EMBO Rep. 2013;14(5):405-409. doi:10.1038/embor.2013.35.

15. CDS. Meme especially made for Costin. I'm sorry, I couldn't help it. Thank you for the adventure!. 1(1):1. https://photos.app.goo.gl/pF7fSgq9BR8Snrgy9.

16. CDS. Thank you Rob! Thank you being brutally honest with everything. 2019;1(1):1. https://photos.app.goo.gl/tS4znqXAcSdvmPKK7.

17. CDS. Camilo Garay, Christopher Choy, Michael Winkler, Krishna Chintaluri, GM Islam, Alejandro Saettone, Kamlesh Mistry, Shane Seepersad, Alex Somerville, Amir Tehrani, Marwan Althagafi, and Philip Abela. Life in 319. We really turned up. Thank you for the memories. To Nurs. 2019;319(23):7. https://photos.app.goo.gl/VZM6nV3ntM912VkL7.

18. CDS. But first, we have to do cuba doe pt.2 and have lots of tasties! To Cuba crew. 2019;500(25):16. https://photos.app.goo.gl/VoNboRRRX3itfcdG6.

19. CDS. Nah, Not really. \#Brolove and for honour. To \#Brolove crew. 2019;23(7):16. https://photos.app.goo.gl/8Ra8or3VFwUPvR7R6.

20. CDS. Mr. Clouthier would be proud of us Stephen Seepersad and Jeffrey Polouse. To my Wildboyz. 2019;2009(16):30. https://photos.app.goo.gl/x6qQEdR8EDztRJkr7.

21. CDS. I cannot wait to return the favour in AYCE sushi vouchers and surprise vacations! To Denise. 2019;1(16):29. https://photos.app.goo.gl/Eucg3Jx11X6cj77t5.

22. CDS. Thank you to Andrew, Julie, Carter, Chris, Dexter, Bryan and Sylvia. The Colegates. 2019;1(16):30. https://photos.app.goo.gl/SDTrb1uy5hMQvuDt8.

23. DelosSantos. One day, I hope you charge it on the Titanium World Elite. Exaggerating. 2019;1(1):1. https://photos.app.goo.gl/2YCBpKDEw5FiJgwo9.

24. DelosSantos. Whatsdat? Thank you for letting me stay at your condo and making me drive you so early in the morning :). Whatsdat. 2019;1(1):1. https://photos.app.goo.gl/b6KMoXED8e5UgPtc9.

25. DelosSantos. Bartholomew and Baltimow forever. Im just wonderii. 2019;1(1):1. https://photos.app.goo.gl/syLonWrwCEUEgEa59.

26. DelosSantos. Poncho is my bun of nine years. Poncho. 2019;1(1):1. https://photos.app.goo.gl/2ftgjAMJZ6U1CEqG9.

27. CDS. Thank you to Joey Basilo, Jason Basilio, all my titos and titas, my cousins, Lola Caring, Lolo Fermin, Lola Cora, and Lolo King-king. To my Fam. 2019;1(1):1. https://photos.app.goo.gl/H6att4wJ1JdmEZwj7.

28. CDS. For embodying genuine brotherhood, thank you Christopher Jovellanos, Mark Guina, Kelvin Vicente, Jamie Gonzaga, Ryan Mendoza, Paul Corteza, Njelle Roxas. Adoboys for life. To Adoboys crew. 2013;1(369):10. https://photos.app.goo.gl/UJU2stB6WQPAU6r76.

29. CDS. Thank you to Leslie Bone, Stefanie Lucarelli, Stephen Bautista, Farnaz Fekri, Nora Dannah, Golam Saffi, Victoria Hipolito, Monica Dayam, Yasmin Awadeh, Sadia Rahmani, Ivan Boras, Biprajit Sanyal, and Elliot Somerville. To my Colleagues. 2019;1(1):1. https://photos.app.goo.gl/pLhhqXiDzWWN3ji57.

30. Engel A, Gaub HE. Structure and Mechanics of Membrane Proteins. Annu Rev Biochem. 
2008;77(1):127-148. doi:10.1146/annurev.biochem.77.062706.154450.

31. Bausch-Fluck D, Hofmann A, Bock T, et al. A mass spectrometric-derived cell surface protein atlas. PLoS One. 2015;10(4):1-22. doi:10.1371/journal.pone.0121314.

32. Reithmeier RAF. Chapter 16 Assembly of Proteins into Membranes. Vol 31. Elsevier Masson SAS; 1996. doi:10.1016/S0167-7306(08)60523-2.

33. Cho W, Stahelin R V. Membrane-Protein Interactions in Cell Signaling and Membrane Trafficking. Annu Rev Biophys Biomol Struct. 2005;34(1):119-151.

doi:10.1146/annurev.biophys.33.110502.133337.

34. Whited AM, Johs $A$. The interactions of peripheral membrane proteins with biological membranes. Chem Phys Lipids. 2015;192:51-59. doi:10.1016/j.chemphyslip.2015.07.015.

35. Marcu LG, Harriss-Phillips WM. In silico modelling of treatment-induced tumour cell kill: Developments and advances. Comput Math Methods Med. 2012;2012(i). doi:10.1155/2012/960256.

36. Buzi G, Lander AD, Khammash M. Cell lineage branching as a strategy for proliferative control. BMC Biol. 2015;13(1):1-15. doi:10.1186/s12915-015-0122-8.

37. Zhang Y, Song J, Zhang X, Xiao Y. Ligand-Receptor Interactions and Drug Design. Biochem insights. 2015;8(Suppl 1):21-23. doi:10.4137/BCl.S37978.

38. Hilger D, Masureel M, Kobilka BK. Structure and dynamics of GPCR signaling complexes. Nat Struct Mol Biol. 2018;25(1):4-12. doi:10.1038/s41594-017-0011-7.

39. Zinser ER, Lindell D, Johnson ZI, et al. Choreography of the transcriptome, photophysiology, and cell cycle of a minimal photoautotroph, Prochlorococcus. PLoS One. 2009;4(4). doi:10.1371/journal.pone.0005135.

40. McCracken AN, Edinger AL. Nutrient transporters: The Achilles' heel of anabolism. Trends Endocrinol Metab. 2013;24(4):200-208. doi:10.1016/j.tem.2013.01.002.

41. Chen C, Qiao R, Wei R, et al. A comprehensive survey of copy number variation in 18 diverse pig populations and identification of candidate copy number variable genes associated with complex traits. BMC Genomics. 2012;13(1):1. doi:10.1186/1471-2164-13-733.

42. Sahoo S, Aurich MK, Jonsson JJ, Thiele I. Membrane transporters in a human genome-scale metabolic knowledgebase and their implications for disease. Front Physiol. 2014;5 MAR(March):1-24. doi:10.3389/fphys.2014.00091.

43. Lin L, Yee SW, Kim RB, Giacomini KM. SLC transporters as therapeutic targets: Emerging opportunities. Nat Rev Drug Discov. 2015;14(8):543-560. doi:10.1038/nrd4626.

44. Gkouvatsos K, Papanikolaou G, Pantopoulos K. Regulation of iron transport and the role of transferrin. Biochim Biophys Acta - Gen Subj. 2012;1820(3):188-202. doi:10.1016/j.bbagen.2011.10.013.

45. Cavallaro U, Dejana E. Adhesion molecule signalling: Not always a sticky business. Nat Rev Mol Cell Biol. 2011;12(3):189-197. doi:10.1038/nrm3068.

46. Wojciechowski M, Krawczyk T, Śmigielski J, Malinowski A. CD44 expression in curettage and 
postoperative specimens of endometrial cancer. Arch Gynecol Obstet. 2015;291(2):383-390. doi:10.1007/s00404-014-3407-1.

47. Bjorge JD, Kudlow JE. Epidermal growth factor receptor synthesis is stimulated by phorbol ester and epidermal growth factor. Evidence for a common mechanism. J Biol Chem.

1987;262(14):6615-6622. http://www.ncbi.nlm.nih.gov/pubmed/3494731.

48. Haeusler RA, McGraw TE, Accili D. Biochemical and cellular properties of insulin receptor signalling. Nat Rev Mol Cell Biol. 2017. doi:10.1038/nrm.2017.89.

49. Mellman I, Yarden Y. Endocytosis and Cancer. 2013.

50. McMahon HT, Boucrot E. Molecular mechanism and physiological functions of clathrin-mediated endocytosis. Nat Rev Mol Cell Biol. 2011;12(8):517-533. doi:10.1038/nrm3151.

51. Kaksonen M, Roux A. Mechanisms of clathrin-mediated endocytosis. Nat Rev Mol Cell Biol. 2018. doi:10.1038/nrm.2017.132.

52. Ferreira APA, Boucrot E. Mechanisms of Carrier Formation during Clathrin-Independent Endocytosis. Trends Cell Biol. 2017:1-13. doi:10.1016/j.tcb.2017.11.004.

53. Mayor S, Pagano RE. Pathways of clathrin-independent endocytosis. Nat Rev Mol Cell Biol. 2007;8(8):603-612. doi:10.1038/nrm2216.

54. Mayor S, Parton RG, Donaldson JG. Clathrin-independent pathways of endocytosis. Cold Spring Harb Perspect Biol. 2014;6(6):1-20. doi:10.1101/cshperspect.a016758.

55. Sandvig K, Pust S, Skotland T, van Deurs B. Clathrin-independent endocytosis: Mechanisms and function. Curr Opin Cell Biol. 2011;23(4):413-420. doi:10.1016/j.ceb.2011.03.007.

56. Buss F, Kendrick-Jones J. How are the cellular functions of myosin VI regulated within the cell? Biochem Biophys Res Commun. 2008;369(1):165-175. doi:10.1016/j.bbrc.2007.11.150.

57. Aguilar RC, Wendland B. Endocytosis of membrane receptors: two pathways are better than one. Proc Natl Acad Sci U S A. 2005;102(8):2679-2680. doi:10.1073/pnas.0500213102.

58. Bergeron JJM, Di Guglielmo GM, Dahan S, Dominguez M, Posner BI. Spatial and Temporal Regulation of Receptor Tyrosine Kinase Activation and Intracellular Signal Transduction. Annu Rev Biochem. 2016;85(1):573-597. doi:10.1146/annurev-biochem-060815-014659.

59. Vega-Cabrera LA, Pardo-López L. Membrane remodeling and organization: Elements common to prokaryotes and eukaryotes. IUBMB Life. 2017;69(2):55-62. doi:10.1002/iub.1604.

60. Paolo P, Fiore D, Zastrow M Von. Endocytosis, Signaling, and Beyond. 2014.

61. Kosmalska AJ, Casares L, Elosegui-Artola A, et al. Physical principles of membrane remodelling during cell mechanoadaptation. Nat Commun. 2015;6. doi:10.1038/ncomms8292.

62. Godlee C, Kaksonen M. From uncertain beginnings: Initiation mechanisms of clathrin-mediated endocytosis. J Cell Biol. 2013;203(5):717-725. doi:10.1083/jcb.201307100.

63. Itoh T, Erdmann KS, Roux A, Habermann B, Werner H, De Camilli P. Dynamin and the actin cytoskeleton cooperatively regulate plasma membrane invagination by BAR and F-BAR proteins. Dev Cell. 2005;9(6):791-804. doi:10.1016/j.devcel.2005.11.005. 
64. Smythe E, Ayscough KR. Actin regulation in endocytosis. J Cell Sci. 2006;119(22):4589-4598. doi:10.1242/jcs.03247.

65. Joseph JM, Fey P, Ramalingam N, et al. The actinome of Dictyostelium discoideum in comparison to actins and actin-related proteins from other organisms. PLoS One. 2008;3(7). doi:10.1371/journal.pone.0002654.

66. Lappalainen P. Actin-binding proteins: the long road to understanding the dynamic landscape of cellular actin networks. Mol Biol Cell. 2016;27(16):2519-2522. doi:10.1091/mbc.E15-10-0728.

67. Suetsugu S, Takenawa T. Regulation of cortical actin networks in cell migration. Int Rev Cytol. 2003;229:245-286.

http://www.ncbi.nlm.nih.gov/entrez/query.fcgi?cmd=Retrieve\&db=PubMed\&dopt=Citation\&list _uids=14669958.

68. Köster D V., Mayor S. Cortical actin and the plasma membrane: Inextricably intertwined. Curr Opin Cell Biol. 2016;38:81-89. doi:10.1016/j.ceb.2016.02.021.

69. Stanishneva-Konovalova TB, Derkacheva NI, Polevova S V., Sokolova OS. The Role of BAR Domain Proteins in the Regulation of Membrane Dynamics. Acta Naturae. 2016;8(4):60-69.

70. Antonny B, Burd C, De Camilli P, et al. Membrane fission by dynamin: what we know and what we need to know. EMBO J. 2016;35(21):2270-2284. doi:10.15252/embj.201694613.

71. Ferguson SM, De Camilli P. Dynamin, a membrane-remodelling GTPase. Nat Rev Mol Cell Biol. 2012;13(2):75-88. doi:10.1038/nrm3266.

72. Kirchhausen T, Owen D, Harrison SC. Molecular structure, function, and dynamics of clathrinmediated membrane traffic. Cold Spring Harb Perspect Biol. 2014;6(5):a016725-a016725. doi:10.1101/cshperspect.a016725.

73. Valus Y, Wigge P, Marks B, Evans PR, McMahon HT. Importance of the pleckstrin homology domain of dynamin in clathrin-mediated endocytosis. Curr Biol. 1999;9(5):257-260. doi:10.1016/S0960-9822(99)80114-6.

74. Motley A, Bright N a, Seaman MNJ, Robinson MS. Clathrin-mediated endocytosis in AP-2depleted cells. J Cell Biol. 2003;162(5):909-918. doi:10.1083/jcb.200305145.

75. Conner SD, Schröter T, Schmid SL. AAK1-mediated $\mu 2$ phosphorylation is stimulated by assembled clathrin. Traffic. 2003;4(12):885-890. doi:10.1046/j.1398-9219.2003.0142.x.

76. Henne WM, Boucrot E, Meinecke M, et al. FCHo proteins are nucleators of Clathrin-Mediated endocytosis. Science (80- ). 2010;328(5983):1281-1284. doi:10.1126/science.1188462.

77. Traub LM, Bonifacino JS. Cargo recognition in clathrin-mediated endocytosis. Cold Spring Harb Perspect Biol. 2013;5(11):1-23. doi:10.1101/cshperspect.a016790.

78. Mettlen M, Chen P-H, Srinivasan S, Danuser G, Schmid SL. Regulation of Clathrin-Mediated Endocytosis. Annu Rev Biochem. 2018;87(1):871-896. doi:10.1146/annurev-biochem-062917012644.

79. Kadlecova Z, Spielman SJ, Loerke D, Mohanakrishnan A, Reed DK, Schmid SL. Regulation of clathrin-mediated endocytosis by hierarchical allosteric activation of AP2. J Cell Biol.

2017;216(1):167-179. doi:10.1083/jcb.201608071. 
80. Mettlen M, Loerke D, Yarar D, Danuser G, Schmid SL. Cargo- and adaptor-specific mechanisms regulate clathrin-mediated endocytosis. J Cell Biol. 2010;188(6):919-933.

doi:10.1083/jcb.200908078.

81. Kirchhausen T, Owen D, Harrison SC. Molecular Structure, Function, and Dynamics of ClathrinMediated Membrane Traffic. Cold Spring Harb Perspect Biol. 2014;6(5):a016725-a016725. doi:10.1101/cshperspect.a016725.

82. Ehrlich M, Boll W, Van Oijen A, et al. Endocytosis by random initiation and stabilization of clathrin-coated pits. Cell. 2004;118(5):591-605. doi:10.1016/j.cell.2004.08.017.

83. Park SY, Guo X. Adaptor protein complexes and intracellular transport. Biosci Rep. 2014;34(4). doi:10.1042/BSR20140069.

84. Liu AP, Aguet F, Danuser G, Schmid SL. Local clustering of transferrin receptors promotes clathrincoated pit initiation. J Cell Biol. 2010;191(7):1381-1393. doi:10.1083/jcb.201008117.

85. Royle SJ. The cellular functions of clathrin. Cell Mol Life Sci. 2006;63(16):1823-1832. doi:10.1007/s00018-005-5587-0.

86. Bucher D, Frey F, Sochacki KA, et al. Clathrin-Adaptor ratio and membrane tension regulate the flat-To-curved transition of the clathrin coat during endocytosis. Nat Commun. 2018;9(1):1109. doi:10.1038/s41467-018-03533-0.

87. Saleem M, Morlot S, Hohendahl A, Manzi J, Lenz M, Roux A. A balance between membrane elasticity and polymerization energy sets the shape of spherical clathrin coats. Nat Commun. 2015;6. doi:10.1038/ncomms7249.

88. Avinoam O, Schorb M, Beese CJ, Briggs JAG, Kaksonen M. Endocytic sites mature by continuous bending and remodeling of the clathrin coat. Science (80- ). 2015;348(6241):1369-1372. doi:10.1126/science.aaa9555.

89. Merrifield CJ, Kaksonen M. Endocytic Accessory Factors and Regulation of Clathrin-Mediated Endocytosis. Cold Spring Harb Perspect Biol. 2014;6(11):1-16. doi:10.1101/cshperspect.a016733.

90. Higgs HN, Pollard TD. Regulation of Actin Filament Network Formation Through ARP2/3 Complex: Activation by a Diverse Array of Proteins. Annu Rev Biochem. 2001;70(1):649-676. doi:10.1146/annurev.biochem.70.1.649.

91. Messa M, Fernández-Busnadiego R, Sun EW, et al. Epsin deficiency impairs endocytosis by stalling the actin-dependent invagination of endocytic clathrin-coated pits. Elife. 2014;3(August2014):125. doi:10.7554/eLife.03311.

92. Engqvist-Goldstein AEY. RNAi-mediated Hip1R Silencing Results in Stable Association between the Endocytic Machinery and the Actin Assembly Machinery. Mol Biol Cell. 2004;15(4):16661679. doi:10.1091/mbc.E03-09-0639.

93. Pollard TD, Earnshaw WC, Lippincott-Schwartz J, Johnson GT. Secretory Membrane System and Golgi Apparatus. In: Pollard TD, Earnshaw WC, Lippincott-Schwartz J, Johnson GT, eds. Cell Biology. 3rd ed. Elsevier; 2017:351-376. doi:https://doi.org/10.1016/B978-0-323-34126-4.000219.

94. Mim C, Unger VM. Membrane curvature and its generation by BAR proteins. Trends Biochem Sci. 
2012;37(12):526-533. doi:10.1016/j.tibs.2012.09.001.

95. Wood LA, Royle SJ. Zero Tolerance: Amphipathic Helices in Endocytosis. Dev Cell. 2015;33(2):119120. doi:10.1016/j.devcel.2015.04.007.

96. Cousin M a., Tan TC, Robinson PJ. Protein phosphorylation is required for endocytosis in nerve terminals: Potential role for the dephosphins dynamin I and synaptojanin, but not AP180 or amphiphysin. J Neurochem. 2001;76:105-116. doi:10.1046/j.1471-4159.2001.00049.x.

97. Lemmon SK. Clathrin uncoating: Auxilin comes to life. Curr Biol. 2001;11(2):49-52. doi:10.1016/S0960-9822(01)00010-0.

98. Heymann JB, Iwasaki K, Yim YI, et al. Visualization of the binding of Hsc70 ATPase to clathrin baskets: Implications for an uncoating mechanism. J Biol Chem. 2005;280(8):7156-7161. doi:10.1074/jbc.M411712200.

99. Zoncu R, Perera RM, Sebastian R, et al. Loss of endocytic clathrin-coated pits upon acute depletion of phosphatidylinositol 4,5-bisphosphate. Proc Natl Acad Sci U S A. 2007;104(10):37933798. doi:10.1073/pnas.0611733104.

100. Posor Y, Eichhorn-Grünig M, Haucke V. Phosphoinositides in endocytosis. Biochim Biophys Acta Mol Cell Biol Lipids. 2015;1851(6):794-804. doi:10.1016/j.bbalip.2014.09.014.

101. Perera RM, Zoncu R, Lucast L, De Camilli P, Toomre D. Two synaptojanin 1 isoforms are recruited to clathrin-coated pits at different stages. Proc Natl Acad Sci U S A. 2006;103(51):19332-19337. doi:10.1073/pnas.0609795104.

102. Cremona O, Di Paolo G, Wenk MR, et al. Essential Role of Phosphoinositide Metabolism in Synaptic Vesicle Recycling. Cell. 1999;99(2):179-188. doi:10.1016/S0092-8674(00)81649-9.

103. Puthenveedu MA, von Zastrow M. Cargo Regulates Clathrin-Coated Pit Dynamics. Cell. 2006;127:113-124. doi:10.1016/j.cell.2006.08.035.

104. Banerjee A, Berezhkovskii A, Nossal R. Stochastic model of clathrin-coated pit assembly. Biophys J. 2012;102(12):2725-2730. doi:10.1016/j.bpj.2012.05.010.

105. Conner SD, Schmid SL. Identification of an adaptor-associated kinase, AAK1, as a regulator of clathrin-mediated endocytosis. J Cell Biol. 2002;156(5):921-929. doi:10.1083/jcb.200108123.

106. Antonescu CN, Danuser G, Schmid SL. Phosphatidic acid plays a regulatory role in clathrinmediated endocytosis. Mol Biol Cell. 2010;21(16):2944-2952. doi:10.1091/mbc.E10-05-0421.

107. Loerke D, Mettlen M, Yarar D, et al. Cargo and dynamin regulate clathrin-coated pit maturation. PLoS Biol. 2009;7(3):e57. doi:10.1371/journal.pbio.1000057.

108. Kirchhausen T. Clathrin. Annu Rev Biochem. 2000;69(1):699-727. doi:10.1146/annurev.biochem.69.1.699.

109. Wu S, Majeed SR, Evans TM, et al. Clathrin light chains' role in selective endocytosis influences antibody isotype switching. Proc Natl Acad Sci. 2016;113(35):9816-9821. doi:10.1073/pnas.1611189113.

110. Brodsky FM. Diversity of Clathrin Function: New Tricks for an Old Protein. Annu Rev Cell Dev Biol. 2012;28(1):309-336. doi:10.1146/annurev-cellbio-101011-155716. 
111. Majeed SR, Vasudevan L, Chen CY, et al. Clathrin light chains are required for the gyratingclathrin recycling pathway and thereby promote cell migration. Nat Commun. 2014;5:3891. doi:10.1038/ncomms4891.

112. Lemmon SK, Traub LM. Getting in touch with the clathrin terminal domain. Traffic. 2012;13(4):511-519. doi:10.1111/j.1600-0854.2011.01321.x.

113. Popova N V., Deyev IE, Petrenko AG. Clathrin-mediated endocytosis and adaptor proteins. Acta Naturae. 2013;5(18):62-73.

114. ter Haar E, Harrison SC, Kirchhausen T. Peptide-in-groove interactions link target proteins to the beta-propeller of clathrin. Proc Natl Acad Sci U S A. 2000;97(3):1096-1100. doi:10.1073/pnas.97.3.1096.

115. Young A, Stoilova-McPhie S, Rothnie A, et al. Hsc70-induced changes in clathrin-auxilin cage structure suggest a role for clathrin light chains in cage disassembly. Traffic. 2013;14(9):987-996. doi:10.1111/tra.12085.

116. Fotin A, Kirchhausen T, Grigorieff N, Harrison SC, Walz T, Cheng Y. Structure determination of clathrin coats to subnanometer resolution by single particle cryo-electron microscopy. J Struct Biol. 2006;156(3):453-460. doi:10.1016/j.jsb.2006.07.001.

117. Scott BL, Sochacki KA, Low-Nam ST, et al. Membrane bending occurs at all stages of clathrin-coat assembly and defines endocytic dynamics. Nat Commun. 2018;9(1):419. doi:10.1038/s41467018-02818-8.

118. Lafer EM. Clathrin-protein interactions. Traffic. 2002;3(8):513-520. http://www.ncbi.nlm.nih.gov/pubmed/12121414.

119. Dell'Angelica EC. Clathrin-binding proteins: Got a motif? Join the network! Trends Cell Biol. 2001;11(8):315-318. doi:10.1016/S0962-8924(01)02043-8.

120. Twyman RM. Clathrin and Clathrin-Adaptors. Encycl Neurosci. 2010:1013-1017. doi:10.1016/B978-008045046-9.01364-4.

121. Boucrot E, Saffarian S, Zhang R, Kirchhausen T. Roles of AP-2 in clathrin-mediated endocytosis. PLoS One. 2010;5(5):e10597. doi:10.1371/journal.pone.0010597.

122. Jackson LP, Kelly BT, McCoy AJ, et al. A large-scale conformational change couples membrane recruitment to cargo binding in the AP2 clathrin adaptor complex. Cell. 2010;141(7):1220-1229. doi:10.1016/j.cell.2010.05.006.

123. Motley AM, Berg N, Taylor MJ, et al. Functional analysis of AP-2 alpha and mu2 subunits. Mol Biol Cell. 2006;17(12):5298-5308. doi:10.1091/mbc.E06-05-0452.

124. Peden AA, Rudge RE, Lui WWY, Robinson MS. Assembly and function of AP-3 complexes in cells expressing mutant subunits. J Cell Biol. 2002;156(2):327-336. doi:10.1083/jcb.200107140.

125. Collins BM, McCoy AJ, Kent HM, Evans PR, Owen DJ. Molecular Architecture and Functional Model of the Endocytic AP2 Complex. Cell. 2002;109(4):523-535. doi:10.1016/S00928674(02)00735-3.

126. Höning S, Ricotta D, Krauss M, et al. Phosphatidylinositol-(4,5)-Bisphosphate Regulates Sorting Signal Recognition by the Clathrin-Associated Adaptor Complex AP2 tional change favored by 
[?2Thr156 phosphorylation. The binding of AP2 to acidic-dileucine motifs occurs. Mol Cell. 2005;18:519-531. doi:10.1016/j.molcel.2005.04.019.

127. Jackson LP, Kelly BT, McCoy AJ, et al. A large-scale conformational change couples membrane recruitment to cargo binding in the AP2 clathrin adaptor complex. Cell. 2010;141(7):1220-1229. doi:10.1016/j.cell.2010.05.006.

128. Owen DJ, Collins BM, Evans PR. ADAPTORS FOR CLATHRIN COATS: Structure and Function. Annu Rev Cell Dev Biol. 2004;20(1):153-191. doi:10.1146/annurev.cellbio.20.010403.104543.

129. Gaidarov I, Keen JH. Phosphoinositide-AP-2 interactions required for targeting to plasma membrane clathrin-coated pits. J Cell Biol. 1999;146(4):755-764. doi:10.1083/jcb.146.4.755.

130. Chang MP, Mallet WG, Mostov KE, Brodsky FM. Adaptor self-aggregation, adaptor-receptor recognition and binding of alpha-adaptin subunits to the plasma membrane contribute to recruitment of adaptor (AP2) components of clathrin-coated pits. EMBO J. 1993;12(5):2169-2180.

131. Kurten RC. Sorting motifs in receptor trafficking. Adv Drug Deliv Rev. 2003;55(11):1405-1419. doi:10.1016/j.addr.2003.07.003.

132. Sorkin A, Goh LK. Endocytosis and intracellular trafficking of ErbBs. Exp Cell Res. 2009;315(4):683696. doi:10.1016/j.yexcr.2008.07.029.

133. Ohno H. Clathrin-associated adaptor protein complexes. J Cell Sci. 2006;119(18):3719-3721. doi:10.1242/jcs.03085.

134. Shih W, Gallusser A, Kirchhausen T. A clathrin-binding site in the hinge of the $\beta 2$ chain of mammalian AP-2 complexes. J Biol Chem. 1995;270(52):31083-31090. doi:10.1074/jbc.270.52.31083.

135. Kelly BT, Graham SC, Liska N, et al. AP2 controls clathrin polymerization with a membraneactivated switch. Science (80- ). 2014;345(6195):459-463. doi:10.1126/science.1254836.

136. Frazier MN, Jackson LP. Watching real-time endocytosis in living cells. J Cell Biol. 2017;216(1):911. doi:10.1083/jcb.201611115.

137. Mettlen M, Stoeber M, Loerke D, Antonescu CN, Danuser G, Schmid SL. Endocytic Accessory Proteins Are Functionally Distinguished by Their Differential Effects on the Maturation of Clathrin-coated Pits. Lemmon S, ed. Mol Biol Cell. 2009;20(14):3251-3260. doi:10.1091/mbc.e0903-0256.

138. Merrifield CJ, Kaksonen M. Endocytic accessory factors and regulation of clathrin-mediated endocytosis. Cold Spring Harb Perspect Biol. 2014;6(11):a016733. doi:10.1101/cshperspect.a016733.

139. Sasaki T, Takasuga S, Sasaki J, et al. Mammalian phosphoinositide kinases and phosphatases. Prog Lipid Res. 2009;48(6):307-343. doi:10.1016/j.plipres.2009.06.001.

140. Balla T. Phosphoinositides: tiny lipids with giant impact on cell regulation. Physiol Rev. 2013;93(3):1019-1137. doi:10.1152/physrev.00028.2012.

141. Di Paolo G, De Camilli P. Phosphoinositides in cell regulation and membrane dynamics. Nature. 2006;443(7112):651-657. doi:10.1038/nature05185. 
142. Bone LN, Dayam RM, Lee M, et al. The acyltransferase LYCAT controls specific phosphoinositides and related membrane traffic. Mol Biol Cell. 2017;28(1):161-172. doi:10.1091/mbc.E16-09-0668.

143. Balla T. Phosphoinositides: Tiny Lipids With Giant Impact on Cell Regulation. Physiol Rev. 2013;93(3):1019-1137. doi:10.1152/physrev.00028.2012.

144. Takasuga S, Sasaki T. Phosphatidylinositol-3,5-bisphosphate: metabolism and physiological functions. J Biochem. 2013;154(3):211-218. doi:10.1093/jb/mvt064.

145. Schink KO, Tan K-W, Stenmark H. Phosphoinositides in Control of Membrane Dynamics. Annu Rev Cell Dev Biol. 2016;32(1):143-171. doi:10.1146/annurev-cellbio-111315-125349.

146. Falkenburger BH, Jensen JB, Dickson EJ, Suh BC, Hille B. Phosphoinositides: Lipid regulators of membrane proteins. J Physiol. 2010;588(17):3179-3185. doi:10.1113/jphysiol.2010.192153.

147. Johnson CM, Chichili GR, Rodgers W. Compartmentalization of phosphatidylinositol 4,5bisphosphate signaling evidenced using targeted phosphatases. J Biol Chem. 2008;283(44):29920-29928. doi:10.1074/jbc.M805921200.

148. Loew LM. Where does all the PIP ${ }_{2}$ come from? J Physiol. 2007;582(3):945-951. doi:10.1113/jphysiol.2007.132860.

149. $\mathrm{Xu} \mathrm{B}, \mathrm{Chen} \mathrm{S}$, Luo $\mathrm{Y}$, et al. Calcium signaling is involved in cadmium-induced neuronal apoptosis via induction of reactive oxygen species and activation of MAPK/mTOR network. PLoS One. 2011;6(4). doi:10.1371/journal.pone.0019052.

150. Lemmon MA. Pleckstrin homology (PH) domains. In: Handbook of Cell Signaling, 2/E. Vol 2. ; 2010:1093-1101. doi:10.1016/B978-0-12-374145-5.00136-4.

151. Stahelin R V. Lipid binding domains: more than simple lipid effectors. J Lipid Res. 2009;50(Supplement):S299-S304. doi:10.1194/jlr.R800078-JLR200.

152. Lemmon MA. Phosphoinositide recognition domains. Traffic. 2003;4(4):201-213. https://onlinelibrary.wiley.com/doi/pdf/10.1034/j.1600-0854.2004.00071.x. Accessed April 25, 2018.

153. Jost M, Simpson F, Kavran JM, Lemmon MA, Schmid SL. Phosphatidylinositol-4,5-bisphosphate is required for endocytic coated vesicle formation. Curr Biol. 1998;8(25):1399-1404. doi:10.1016/S0960-9822(98)00022-0.

154. Varnai P, Thyagarajan B, Rohacs T, Balla T. Rapidly inducible changes in phosphatidylinositol 4,5bisphosphate levels influence multiple regulatory functions of the lipid in intact living cells. J Cell Biol. 2006;175(3):377-382. doi:/10.1083/jcb.200607116.

155. Jost M, Simpson F, Kavran JM, Lemmon MA, Schmid SL. Phosphatidylinositol-4,5-bisphosphate is required for endocytic coated vesicle formation. Curr Biol. 1998;8(25):1399-1404. doi:10.1016/S0960-9822(98)00022-0.

156. McLaughlin S, Wang J, Gambhir A, Murray D. PIP(2) and proteins: interactions, organization, and information flow. Annu Rev Biophys Biomol Struct. 2002;31:151-175. doi:10.1146/annurev.biophys.31.082901.134259.

157. Sundaram M, Cook HW, Byers DM. The MARCKS family of phospholipid binding proteins : regulation of phospholipase D and other cellular components 1 , 2. 2004;200:191-200. 
doi:10.1139/003-087.

158. Nakamura Y, Fukami K. Regulation and physiological functions of mammalian phospholipase C. J Biochem. 2017;161(4):315-321. doi:10.1093/jb/mvw094.

159. Jang HJ, Suh PG, Lee YJ, Shin KJ, Cocco L, Chae YC. PLCY1: Potential arbitrator of cancer progression. Adv Biol Regul. 2018;67:179-189. doi:10.1016/j.jbior.2017.11.003.

160. Yin HL, Janmey PA. Phosphoinositide Regulation of the Actin Cytoskeleton. Annu Rev Physiol. 2003;65(1):761-789. doi:10.1146/annurev.physiol.65.092101.142517.

161. Suh PG, Park JI, Manzoli L, et al. Multiple roles of phosphoinositide-specific phospholipase C isozymes. BMB Rep. 2008;41:415-434.

162. Wen W, Yan J, Zhang M. Structural characterization of the split pleckstrin homology domain in phospholipase C- -1 and its interaction with TRPC3. J Biol Chem. 2006;281(17):12060-12068. doi:10.1074/jbc.M600336200.

163. Fukami K, Inanobe S, Kanemaru K, Nakamura Y. Phospholipase $\mathrm{C}$ is a key enzyme regulating intracellular calcium and modulating the phosphoinositide balance. Prog Lipid Res. 2010;49(4):429-437. doi:10.1016/j.plipres.2010.06.001.

164. Harden TK, Waldo GL, Hicks SN, Sondek J. Mechanism of activation and inactivation of Gq/phospholipase C-B signaling nodes. Chem Rev. 2011;111(10):6120-6129. doi:10.1021/cr200209p.

165. Lemmon MA, Fergusonti KM, O 'brient R, Sigler PB, Schlessinger J. Specific and high-affinity binding of inositol phosphates to an isolated pleckstrin homology domain (phospholipase C81/titration calorimetry/phospholipids). Biochemistry. 1995;92:10472-10476. http://www.pnas.org/content/pnas/92/23/10472.full.pdf. Accessed May 1, 2018.

166. Tuzi S, Uekama N, Okada M, Yamaguchi S, Saitô H, Yagisawa H. Structure and dynamics of the phospholipase C- $\delta 1$ pleckstrin homology domain located at the lipid bilayer surface. J Biol Chem. 2003;278(30):28019-28025. doi:10.1074/jbc.M300101200.

167. Lyon AM, Tesmer JJG. Structural Insights into Phospholipase C- Function. Mol Pharmacol. 2013;84(4):488-500. doi:10.1124/mol.113.087403.

168. Rhee SG. Regulation of phosphoinositide-specific phospholipase C. Annu Rev Biochem. 2001;70(1):281-312. doi:10.1146/annurev.biochem.70.1.281.

169. Kim S-K, Wee S-M, Chang J-S, et al. Point mutations in the split PLC-gamma1 PH domain modulate phosphoinositide binding. J Biochem Mol Biol. 2004;37(6):720-725. http://www.ncbi.nlm.nih.gov/pubmed/15607032.

170. Cocco L, Follo M, Manzoli L, Suh P-G. Phosphoinositide-specifi c phospholipase C in health and disease. J Lipid Res. 2015;56. doi:10.1194/jlr.R057984.

171. Pinto MCX, Kihara AH, Goulart VAM, et al. Calcium signaling and cell proliferation. Cell Signal. 2015;27(11):2139-2149. doi:10.1016/j.cellsig.2015.08.006.

172. Berridge MJ, Lipp P, Bootman MD. The versatility and universality of calcium signalling. Nat Rev Mol Cell Biol. 2000;1(1):11-21. doi:10.1038/35036035. 
173. Ellis MV, James SR, Perisic O, Downes CP, Williams RL, Katan M. Catalytic domain of phosphoinositide-specific phospholipase C (PLC). J Biol Chem. 1998;273:11650.

doi:10.1074/jbc.273.19.11650.

174. Yang YR, Follo MY, Cocco L, Suh P-G. The physiological roles of primary phospholipase C. Adv Biol Regul. 2013;53(3):232-241. doi:10.1016/j.jbior.2013.08.003.

175. Putney JW, Tomita T. Phospholipase C signaling and calcium influx. Adv Biol Regul. 2012;52(1):152-164. doi:10.1016/j.advenzreg.2011.09.005.

176. Breitkreutz D, Braiman-Wiksman L, Daum N, Denning MF, Tennenbaum T. Protein kinase C family: On the crossroads of cell signaling in skin and tumor epithelium. J Cancer Res Clin Oncol. 2007;133(11):793-808. doi:10.1007/s00432-007-0280-3.

177. Callender JA, Newton AC. Conventional protein kinase $\mathrm{C}$ in the brain: 40 years later. Neuronal Signal. 2017;1(2):NS20160005. doi:10.1042/NS20160005.

178. Yáñez M, Gil-longo J, Campos-toimil M. Calcium Signaling. 2012;740:461-482. doi:10.1007/97894-007-2888-2.

179. Foskett JK, White C, Cheung K, Mak DD. Inositol Triphosphate Receptor Ca2+ Release Channels. Physiol Rev. 2007;87(2):593-658. doi:10.1152/physrev.00035.2006.Inositol.

180. Di Guglielmo GM, Drake PG, Baass PC, et al. Insulin receptor internalization and signalling. Mol Cell Biochem. 1998;182(1-2):59-63. doi:10.1023/A:1006883311233.

181. Brännmark C, Palmér R, Glad ST, Cedersund G, Strålfors P. Mass and information feedbacks through receptor endocytosis govern insulin signaling as revealed using a parameter-free modeling framework. J Biol Chem. 2010;285(26):20171-20179. doi:10.1074/jbc.M110.106849.

182. Carvou N, Norden AGW, Unwin RJ, Cockcroft S. Signalling through phospholipase C interferes with clathrin-mediated endocytosis. Cell Signal. 2007;19(1):42-51.

doi:10.1016/j.cellsig.2006.05.023.

183. Eichhorn J, Kayali AG, Austin DA, Webster NJG. Insulin activates phospholipase C- -1 via a PI-3 kinase dependent mechanism in 3T3-L1 adipocytes. Biochem Biophys Res Commun. 2001;282(2):615-620. doi:10.1006/bbrc.2001.4616.

184. Simons TJB. Calcium and neuronal function. Neurosurg Rev. 1988;11(2):119-129. doi:10.1007/BF01794675.

185. Burgoyne RD, Haynes LP. Understanding the physiological roles of the neuronal calcium sensor proteins. Mol Brain. 2012;5(1). doi:10.1186/1756-6606-5-2.

186. Neher E, Sakaba T. Multiple Roles of Calcium Ions in the Regulation of Neurotransmitter Release. Neuron. 2008;59(6):861-872. doi:10.1016/j.neuron.2008.08.019.

187. Cousin MA, Robinson PJ. Ca(2+) influx inhibits dynamin and arrests synaptic vesicle endocytosis at the active zone. $J$ Neurosci. 2000;20(3):949-957. http://www.ncbi.nlm.nih.gov/pubmed/10648699.

188. Clapham DE. Calcium Signaling. Cell. 2007;131(6):1047-1058. doi:10.1016/j.cell.2007.11.028.

189. Brini M, Calì T, Ottolini D, Carafoli E. Neuronal calcium signaling: Function and dysfunction. Cell 
Mol Life Sci. 2014;71(15):2787-2814. doi:10.1007/s00018-013-1550-7.

190. Putney JW, Tomita T. Phospholipase C Signaling and Calcium Influx. Adv Biol Regul. 2011;52(1):152-164. doi:10.1016/j.advenzreg.2011.09.005.Phospholipase.

191. Egelman DM, Read Montague P, Montague PR. Calcium dynamics in the extracellular space of mammalian neural tissue. Biophys J. 1999;76(April 1998):1856-1867. doi:10.1016/S00063495(99)77345-5.

192. Farber JL. The Role of Calcium Ions in Toxic Cell Injury. Environ Health Perspect. 1990;84:107-111. https://ehp.niehs.nih.gov/wp-content/uploads/84/ehp.9084107.pdf. Accessed May 5, 2018.

193. Carafoli E, Santella L, Branca D, Brini M. Generation, control, and processing of cellular calcium signals. Crit Rev Biochem Mol Biol. 2001;36(2):107-260. doi:10.1080/20014091074183.

194. Rogers EF, Koniuszy FR, Shavel J, Folkers K. Plant Insecticides. I. Ryanodine, A New Alkaloid from Ryania Speciosa Vahl. J Am Chem Soc. 1948;70(9):3086-3088. doi:10.1021/ja01189a074.

195. Kong H, Jones PP, Koop A, Zhang L, Duff HJ, Chen SRW. Caffeine induces Ca2+ release by reducing the threshold for luminal Ca2+ activation of the ryanodine receptor. Biochem J. 2008;414(3):441452. doi:10.1042/BJ20080489.

196. Dennis EA. Handbook of Cell Signaling. Vol 1-3.; 2003. doi:10.1016/B978-0-12-124546-7.X5358-3.

197. Rumi-Masante J, Rusinga FI, Lester TE, et al. Structural basis for activation of calcineurin by calmodulin. doi:10.1016/j.jmb.2011.11.008.

198. Steiner H, Tseng KY. Handbook of Basal Ganglia Structure and Function: A Decade of Progress. Handb Basal Ganglia Struct Funct. 2010;20:700. doi:10.1016/B978-0-12-374767-9.00042-1.

199. Steinberg SF. Structural Basis of Protein Kinase C Isoform Function. 2008:1341-1378. doi:10.1152/physrev.00034.2007.

200. Royle SJ, Lagnado L. Clathrin-mediated endocytosis at the synaptic terminal: bridging the gap between physiology and molecules. doi:10.1111/j.1600-0854.2010.01104.x.

201. Koch M, Holt M. Coupling exo- and endocytosis: An essential role for PIP2 at the synapse. Biochim Biophys Acta - Mol Cell Biol Lipids. 2012;1821(8):1114-1132. doi:10.1016/j.bbalip.2012.02.008.

202. Ma C, Zhou Q, Chen L, Liang K, Wei L. Exocytosis, Endocytosis, and Their Coupling in Excitable Cells. Front Mol Neurosci Artic Front Mol Neurosci. 2017;10(10). doi:10.3389/fnmol.2017.00109.

203. MacDonald PE. Calcium increases endocytotic vesicle size and accelerates membrane fission in insulin-secreting INS-1 cells. J Cell Sci. 2005. doi:10.1242/jcs.02685.

204. Leitz J, Kavalali ET. Ca ${ }^{2+}$ Dependence of Synaptic Vesicle Endocytosis. Neurosci. 2016;22(5):464476. doi:10.1177/1073858415588265.

205. Hinshaw JE. DYNAMIN AND ITS ROLE IN MEMBRANE FISSION. Annu Rev Cell Dev Biol. 2000;16:483-519. https://www.ncbi.nlm.nih.gov/books/NBK2233/pdf/Bookshelf_NBK2233.pdf. Accessed May 11, 2018.

206. Wilde JI, Watson SP. Regulation of phospholipase C gamma isoforms in haematopoietic cells: why one, not the other? Cell Signal. 2001;13(10):691-701. 
http://www.ncbi.nlm.nih.gov/pubmed/11602179.

207. Garay C, Judge G, Lucarelli S, et al. Epidermal growth factor-stimulated Akt phosphorylation requires clathrin or ErbB2 but not receptor endocytosis. Mol Biol Cell. 2015;26(19):3504-3519. doi:10.1091/mbc.E14-09-1412.

208. Shih TL, Khakoo AY. Receptor Tyrosine Kinases in Cardiac Muscle. Vol 1. First Edit. Elsevier Inc.; 2012. doi:10.1016/B978-0-12-381510-1.00009-0.

209. Alberts B, Johnson A, Lewis J et al. Signaling through Enzyme-Linked Cell-Surface Receptors. In: Molecular Biology of the Cell. 4th ed. New York: Garland Science; 2002.

https://www.ncbi.nlm.nih.gov/books/NBK26822/.

210. Wieduwilt MJ, Moasser MM. The epidermal growth factor receptor family: Biology driving targeted therapeutics. Cell Mol Life Sci. 2008;65(10):1566-1584. doi:10.1007/s00018-008-7440-8.

211. Zimmer DB. Intercellular Signaling. Med Cell Biol Third Ed. 2007:227-248. doi:10.1016/B978-0-12370458-0.50012-8.

212. Klingmüller U, Schilling M, Depner S, D’Alessandro LA. Biological Foundations of Signal Transduction, Systems Biology and Aberrations in Disease. Second Edi. Elsevier Inc.; 2013. doi:10.1016/B978-0-12-405926-9.00004-6.

213. Lodish H, Berk A, Zipursky SL, Matsudaira P, Baltimore D, Darnell J. Receptor Tyrosine Kinases and Ras. In: Molecular Cell Biology. 4th Edition. 4th editio. New York: W. H. Freeman; 2000. https://www.ncbi.nlm.nih.gov/books/NBK21720/.

214. Cui JJ, McTigue M, Kania R, Edwards M. Case History. Xalkori (Crizotinib), a Potent and Selective Dual Inhibitor of Mesenchymal Epithelial Transition (MET) and Anaplastic Lymphoma Kinase (ALK) for Cancer Treatment. Vol 48. 1st ed. Copyright (C) 2013 Elsevier Inc. All rights reserved.; 2013. doi:10.1016/B978-0-12-417150-3.00025-9.

215. Heldin CH, Lennartsson J. Receptor Tyrosine Kinases and Their Ligands. Vol 3. Elsevier Ltd.; 2015. doi:10.1016/B978-0-12-394447-4.30001-3.

216. He L, Hristova K. Physical-chemical principles underlying RTK activation, and their implications for human disease. Biochim Biophys Acta - Biomembr. 2012;1818(4):995-1005. doi:10.1016/j.bbamem.2011.07.044.

217. Lemmon MA, Schlessinger J, Ferguson KM. The EGFR family: Not so prototypical receptor tyrosine kinases. Cold Spring Harb Perspect Biol. 2014;6(4). doi:10.1101/cshperspect.a020768.

218. Maruyama I. Mechanisms of Activation of Receptor Tyrosine Kinases: Monomers or Dimers. Cells. 2014;3(2):304-330. doi:10.3390/cells3020304.

219. Bafico A, Aaronson SA. Growth Factor Receptors with Tyrosine Kinase Activity. In: Holland-Frei Cancer Medicine. 6th Edition. Hamilton (ON): BC Decker; 2003.

http://www.ncbi.nlm.nih.gov/books/NBK13088/.

220. Critchley W, Pellet-Many C, Ringham-Terry B, Harrison M, Zachary I, Ponnambalam S. Receptor Tyrosine Kinase Ubiquitination and De-Ubiquitination in Signal Transduction and Receptor Trafficking. Cells. 2018;7(3):22. doi:10.3390/cells7030022.

221. Cunningham ME, Stephens RM, Kaplan DR, Greene LA. Autophosphorylation of activation loop 
tyrosines regulates signaling by the TRK nerve growth factor receptor. J Biol Chem. 1997;272(16):10957-10967. doi:10.1074/jbc.272.16.10957.

222. Lund KA, Lazar CS, Chen WS, et al. Phosphorylation of the epidermal growth factor receptor at threonine 654 inhibits ligand-induced internalization and down-regulation. J Biol Chem. 1990;265(33):20517-20523.

223. Santiskulvong C, Rozengurt E. Protein kinase C $\alpha$ mediates feedback inhibition of EGF receptor transactivation induced by Gq-coupled receptor agonists. Cell Signal. 2007;19(6):1348-1357. doi:10.1016/j.cellsig.2007.01.006.

224. Bao J, Alroy I, Waterman H, et al. Threonine phosphorylation diverts internalized epidermal growth factor receptors from a degradative pathway to the recycling endosome. J Biol Chem. 2000;275(34):26178-26186. doi:10.1074/jbc.M002367200.

225. Hubbard SR. Juxtamembrane autoinhibition in receptor tyrosine kinases. Nat Rev Mol Cell Biol. 2004;5(6):464-470. doi:10.1038/nrm1399.

226. Wagner MJ, Stacey MM, Liu BA, Pawson T. Molecular mechanisms of SH2- and PTB-Domaincontaining proteins in receptor tyrosine kinase signaling. Cold Spring Harb Perspect Biol. 2013;5(12). doi:10.1101/cshperspect.a008987.

227. Manni S, Kisko K, Schleier T, Missimer J, Ballmer-Hofer K. Functional and structural characterization of the kinase insert and the carboxy terminal domain in VEGF receptor 2 activation. FASEB J. 2014;28(11):4914-4923. doi:10.1096/fj.14-256206.

228. Stuttfeld E, Ballmer-Hofer K. Structure and function of VEGF receptors. IUBMB Life. 2009;61(9):915-922. doi:10.1002/iub.234.

229. Ogiso H, Ishitani R, Nureki O, et al. Crystal structure of the complex of human epidermal growth factor and receptor extracellular domains. Cell. 2002;110(6):775-787. doi:10.1016/S00928674(02)00963-7.

230. Burgess AW, Cho HS, Eigenbrot $C$, et al. An open-and-shut case? Recent insights into the activation of EGF/ErbB receptors. Mol Cell. 2003;12(3):541-552. doi:10.1016/S10972765(03)00350-2.

231. Endres NF, Barros T, Cantor AJ, Kuriyan J. Emerging concepts in the regulation of the EGF receptor and other receptor tyrosine kinases. Trends Biochem Sci. 2014;39(10):437-446. doi:10.1016/j.tibs.2014.08.001.

232. Mac Gabhann F, Popel AS. Dimerization of VEGF receptors and implications for signal transduction: A computational study. Biophys Chem. 2007;128(2-3):125-139. doi:10.1016/j.bpc.2007.03.010.

233. Yarden Y, Schlessinger J. Epidermal growth factor induces rapid, reversible aggregation of the purified epidermal growth factor receptor. Biochemistry. 1987;26(5):1443-1451. doi:10.1021/bi00379a035.

234. Nagy P, Claus J, Jovin TM, Arndt-Jovin DJ. Distribution of resting and ligand-bound ErbB1 and ErbB2 receptor tyrosine kinases in living cells using number and brightness analysis. Proc Natl Acad Sci. 2010;107(38):16524-16529. doi:10.1073/pnas.1002642107. 
235. Moriki T, Maruyama H, Maruyama IN. Activation of preformed EGF receptor dimers by ligandinduced rotation of the transmembrane domain. J Mol Biol. 2001;311(5):1011-1026.

doi:10.1006/jmbi.2001.4923.

236. Endres NF, Das R, Smith AW, et al. Conformational Coupling Across the Plasma Membrane in Activation of the EGF receptor. January. 2013;31(1523):543-556.

https://www.ncbi.nlm.nih.gov/pmc/articles/PMC3718647/pdf/nihms-459034.pdf. Accessed June $5,2018$.

237. Arkhipov A, Shan $\mathrm{Y}$, Das R, et al. Architecture and membrane interactions of the EGF receptor. Cell. 2013;152(3):557-569. doi:10.1016/j.cell.2012.12.030.

238. Liu P, Sudhaharan T, Koh RML, et al. Investigation of the dimerization of proteins from the epidermal growth factor receptor family by single wavelength fluorescence cross-correlation spectroscopy. Biophys J. 2007;93(2):684-698. doi:10.1529/biophysj.106.102087.

239. Lambert S, Ameels H, Gniadecki R, Hérin M, Poumay Y. Internalization of EGF receptor following lipid rafts disruption in keratinocytes is delayed and dependent on p38 MAPK activation. $J$ Cell Physiol. 2008;217(3):834-845. doi:10.1002/jcp.21563.

240. Michailidis IE, Rusinova R, Georgakopoulos A, et al. Phosphatidylinositol-4,5-bisphosphate regulates epidermal growth factor receptor activation. Pflugers Arch Eur J Physiol. 2011;461(3):387-397. doi:10.1007/s00424-010-0904-3.

241. Casaletto JB, McClatchey Al. Spatial regulation of receptor tyrosine kinases in development and cancer. Nat Rev Cancer. 2012;12(6):387-400. doi:10.1038/nrc3277.

242. Ferguson KM. Extracellular domains drive homo- but not hetero-dimerization of erbB receptors. EMBO J. 2000;19(17):4632-4643. doi:10.1093/emboj/19.17.4632.

243. Del Piccolo N, Sarabipour S, Hristova K. A new method to study heterodimerization of membrane proteins and its application to fibroblast growth factor receptors. J Biol Chem. 2017;292(4):11881301. doi:10.1074/jbc.M116.755777.

244. Yarden Y, Sliwkowski MX. Untangling the ErbB signalling network. Nat Rev Mol Cell Biol. 2001;2(2):127-137. doi:10.1038/35052073.

245. Gschwind A, Fischer OM, Ullrich A. Timeline: The discovery of receptor tyrosine kinases: targets for cancer therapy. Nat Rev Cancer. 2004;4(5):361-370. doi:10.1038/nrc1360.

246. Slamon DJ, Clark GM, Wong SG, Levin WJ, Ullrich A, Mcguire WL. Human Breast Cancer: Correlation of Relapse and Survival with Amplification of the HER-2Ineu Oncogene. Science (80- ). 1987;235(4785):177-182. doi:10.1126/science.3798106.

247. Tuefferd M, Couturier J, Penault-Llorca F, et al. HER2 status in ovarian carcinomas: A multicenter GINECO study of 320 patients. PLoS One. 2007;2(11). doi:10.1371/journal.pone.0001138.

248. Garrett TPJ, McKern NM, Lou M, et al. The crystal structure of a truncated ErbB2 ectodomain reveals an active conformation, poised to interact with other ErbB receptors. Mol Cell. 2003;11(2):495-505. doi:10.1016/S1097-2765(03)00048-0.

249. Wheeler DL, Yarden Y. Receptor Tyrosine Kinases: Structure, Functions and Role in Human Disease.; 2015. doi:10.1007/978-1-4939-2053-2. 
250. Oda K, Matsuoka Y, Funahashi A, Kitano H. A comprehensive pathway map of epidermal growth factor receptor signaling. Mol Syst Biol. 2005;1:2005.0010. doi:10.1038/msb4100014.

251. De Donatis A, Cirri P. Understanding the specificity of receptor tyrosine kinases signaling. Commun Integr Biol. 2008;1(2):156-157. doi:10.4161/cib.1.2.7098.

252. Singh B, Carpenter G, Coffey RJ. EGF receptor ligands: recent advances. F1000Research. 2016;5(0):2270. doi:10.12688/f1000research.9025.1.

253. Wilson KJ, Gilmore JL, Foley J, Lemmon MA, Riese DJ. Functional selectivity of EGF family peptide growth factors: Implications for cancer. Pharmacol Ther. 2009;122(1):1-8. doi:10.1016/j.pharmthera.2008.11.008.

254. Huang Y, Chang Y. Epidermal Growth Factor Receptor (EGFR) Phosphorylation, Signaling and Trafficking in Prostate Cancer. Prostate Cancer - From Bench to Bedside. 2011;4(Figure 1). doi:10.5772/27021.

255. Ségaliny Al, Tellez-Gabriel M, Heymann MF, Heymann D. Receptor tyrosine kinases: Characterisation, mechanism of action and therapeutic interests for bone cancers. $J$ Bone Oncol. 2015;4(1):1-12. doi:10.1016/j.jbo.2015.01.001.

256. Hanke S, Mann M. The Phosphotyrosine Interactome of the Insulin Receptor Family and Its Substrates IRS-1 and IRS-2. Mol Cell Proteomics. 2009;8(3):519-534. doi:10.1074/mcp.M800407MCP200.

257. Gilmore JL, Scott JA, Bouizar Z, et al. Amphiregulin-EGFR signaling regulates PTHrP gene expression in breast cancer cells. Breast Cancer Res Treat. 2008;110(3):493-505. doi:10.1007/s10549-007-9748-8.

258. Willmarth NE, Baillo A, Dziubinski ML, Wilson K, Riese DJ, Ethier SP. Altered EGFR localization and degradation in human breast cancer cells with an amphiregulin/EGFR autocrine loop. Cell Signal. 2009;21(2):212-219. doi:10.1016/j.cellsig.2008.10.003.

259. Ellis IR, Schor AM, Schor SL. EGF AND TGF- $\alpha$ motogenic activities are mediated by the EGF receptor via distinct matrix-dependent mechanisms. Exp Cell Res. 2007;313(4):732-741. doi:10.1016/j.yexcr.2006.11.016.

260. Jorissen RN, Walker F, Pouliot N, Garrett TPJ, Ward CW, Burgess AW. Epidermal growth factor receptor: Mechanisms of activation and signalling. Exp Cell Res. 2003;284(1):31-53. doi:10.1016/S0014-4827(02)00098-8.

261. Sweeney C, Fambrough D, Huard C, et al. Growth Factor-specific Signaling Pathway Stimulation and Gene Expression Mediated by ErbB Receptors. J Biol Chem. 2001;276(25):22685-22698. doi:10.1074/jbc.M100602200.

262. Friedlander T, Mayo AE, Tlusty T, Alon U. Evolution of Bow-Tie Architectures in Biology. PLoS Comput Biol. 2015;11(3):1-19. doi:10.1371/journal.pcbi.1004055.

263. Adrain C, Freeman M, Hunter T, et al. Regulation of Receptor Tyrosine Kinase Ligand Processing. 2014. doi:10.1101/cshperspect.a008995.

264. Citri A, Yarden Y. EGF-ERBB signalling: Towards the systems level. Nat Rev Mol Cell Biol. 2006;7(7):505-516. doi:10.1038/nrm1962. 
265. Bakker J, Spits M, Neefjes J, Berlin I. The EGFR odyssey - from activation to destruction in space and time. J Cell Sci. 2017;130(24):4087-4096. doi:10.1242/jcs.209197.

266. Schlessinger J. Receptor tyrosine kinases: Legacy of the first two decades. Cold Spring Harb Perspect Biol. 2014;6(3):1-13. doi:10.1101/cshperspect.a008912.

267. Ségaliny Al, Tellez-Gabriel M, Heymann MF, Heymann D. Receptor tyrosine kinases: Characterisation, mechanism of action and therapeutic interests for bone cancers. J Bone Oncol. 2015;4(1):1-12. doi:10.1016/j.jbo.2015.01.001.

268. Scaltriti M, Baselga J. The Epidermal Growth Factor Receptor Pathway : A Model for Targeted Therapy The Epidermal Growth Factor Receptor Pathway : A Model for T argeted Therapy. Clin Cancer Res. 2006;12(18):5268-5272. doi:10.1158/1078-0432.CCR-06-1554.

269. Shaul YD, Seger R. The MEK/ERK cascade: From signaling specificity to diverse functions. Biochim Biophys Acta - Mol Cell Res. 2007;1773(8):1213-1226. doi:10.1016/j.bbamcr.2006.10.005.

270. Lewitzky M, Kardinal C, Gehring NH, et al. The C-terminal SH3 domain of the adapter protein Grb2 binds with high affinity to sequences in Gab1 and SLP-76 which lack the SH3-typical P-x-x-P core motif. Oncogene. 2001;20(9):1052-1062. doi:10.1038/sj.onc.1204202.

271. Pierre S, Bats AS, Coumoul X. Understanding SOS (Son of Sevenless). Biochem Pharmacol. 2011;82(9):1049-1056. doi:10.1016/j.bcp.2011.07.072.

272. Simanshu DK, Nissley D V., McCormick F. RAS Proteins and Their Regulators in Human Disease. Cell. 2017;170(1):17-33. doi:10.1016/j.cell.2017.06.009.

273. Lavoie H, Therrien M. Regulation of RAF protein kinases in ERK signalling. Nat Rev Mol Cell Biol. 2015;16(5):281-298. doi:10.1038/nrm3979.

274. Pearson G, Robinson F, Beers Gibson T, et al. Mitogen-Activated Protein (MAP) Kinase Pathways: Regulation and Physiological Functions ${ }^{1}$. Endocr Rev. 2001;22(2):153-183. doi:10.1210/edrv.22.2.0428.

275. Leicht DT, Balan V, Kaplun A, et al. Raf kinases: Function, regulation and role in human cancer. Biochim Biophys Acta - Mol Cell Res. 2007;1773(8):1196-1212. doi:10.1016/j.bbamcr.2007.05.001.

276. Roskoski R. MEK1/2 dual-specificity protein kinases: Structure and regulation. Biochem Biophys Res Commun. 2012;417(1):5-10. doi:10.1016/j.bbrc.2011.11.145.

277. Roskoski R. ERK1/2 MAP kinases: Structure, function, and regulation. Pharmacol Res. 2012;66(2):105-143. doi:10.1016/j.phrs.2012.04.005.

278. Hemmings BA, Restuccia DF. PI3K-PKB/Akt pathway. Cold Spring Harb Perspect Biol. 2012;4(9):a011189. doi:10.1101/cshperspect.a011189.

279. Osaki M, Oshimura M, Ito H. PI3K-Akt pathway: its functions and alterations in human cancer. Apoptosis. 2004;9(6):667-676. doi:10.1023/B:APPT.0000045801.15585.dd.

280. Cuevas BD, Lu Y, Mao M, et al. Tyrosine Phosphorylation of p85 Relieves Its Inhibitory Activity on Phosphatidylinositol 3-Kinase. J Biol Chem. 2001;276(29):27455-27461. doi:10.1074/jbc.M100556200. 
281. Mattoon DR, Lamothe B, Lax I, Schlessinger J. The docking protein Gab1 is the primary mediator of EGF-stimulated activation of the PI-3K/Akt cell survival pathway. BMC Biol. 2004;2:1-12. doi:10.1186/1741-7007-2-24.

282. Mason A, Kinugasa T. East Asian Economic Development: Two Demographic Dividend. J Asian Econ. 2009;19(5-6):389-399. doi:10.1016/j.asieco.2008.09.006.EAST.

283. Wang W, Xu S, Yin M, Jin ZG. Essential roles of Gab1 tyrosine phosphorylation in growth factormediated signaling and angiogenesis. Int J Cardiol. 2015;181(4):180-184. doi:10.1016/j.ijcard.2014.10.148.

284. Manning BD, Toker A. AKT/PKB Signaling: Navigating the Network. Cell. 2017;169(3):381-405. doi:10.1016/j.cell.2017.04.001.

285. Mendoza MC, Er EE, Blenis J. The Ras-ERK and PI3K-mTOR pathways: Cross-talk and compensation. Trends Biochem Sci. 2011;36(6):320-328. doi:10.1016/j.tibs.2011.03.006.

286. Avruch J, Long X, Lin Y, et al. Activation of mTORC1 in two steps: Rheb-GTP activation of catalytic function and increased binding of substrates to raptor ${ }^{1}$. Biochem Soc Trans. 2009;37(1):223-226. doi:10.1042/BST0370223.

287. Ma L, Chen Z, Erdjument-Bromage H, Tempst P, Pandolfi PP. Phosphorylation and functional inactivation of TSC2 by Erk: Implications for tuberous sclerosis and cancer pathogenesis. Cell. 2005;121(2):179-193. doi:10.1016/j.cell.2005.02.031.

288. Roskoski R. Src protein-tyrosine kinase structure, mechanism, and small molecule inhibitors This paper is dedicated to the memory of Prof. Donald F. Steiner (1930-2014) - Advisor, mentor, and discoverer of proinsulin. Pharmacol Res. 2015;94:9-25. doi:10.1016/j.phrs.2015.01.003.

289. Lau L, Huganir R. Protein Tyrosine Kinases. In: Siegel G, Agranoff B, Albers R, et al., eds. Basic Neurochemistry: Molecular, Cellular and Medical Aspects. 6th ed. Philadelphia: Lippincott-Raven; 1999. https://www.ncbi.nlm.nih.gov/books/NBK27987/.

290. Ferrando IM, Chaerkady R, Zhong J, et al. Identification of Targets of c-Src Tyrosine Kinase by Chemical Complementation and Phosphoproteomics. Mol Cell Proteomics. 2012;11(8):355-369. doi:10.1074/mcp.M111.015750.

291. Chen Z, Oh D, Dubey AK, et al. EGFR family and Src family kinase interactions: mechanics matters? Curr Opin Cell Biol. 2018;51:97-102. doi:10.1016/j.ceb.2017.12.003.

292. Biscardi JS, Maa M-C, Tice D a, Cox ME, Leu T-H, Parsons SJ. C-Src-mediated Phosphorylation of the Epidermal Growth Factor Receptor on Tyr 845 and Tyr 1101 Is Associated with Modulation of Receptor Function. J Biol Chem. 1999;274(12):8335-8343. doi:10.1074/jbc.274.12.8335.

293. Stover DR, Becker M, Liebetanz J, Lydon NB. Src phosphorylation of the epidermal growth factor receptor at novel sites mediates receptor interaction with Src and P85a. J Biol Chem. 1995;270(26):15591-15597. doi:10.1074/jbc.270.26.15591.

294. Quesnelle KM, Boehm AL, Grandis JR. STAT-mediated EGFR signaling in cancer. J Cell Biochem. 2007;102(2):311-319. doi:10.1002/jcb.21475.

295. Heppler LN, Frank DA. Targeting Oncogenic Transcription Factors : Therapeutic Implications of Endogenous STAT Inhibitors. TRENDS in CANCER. 2017; xx:1-12. 
doi:10.1016/j.trecan.2017.10.004.

296. Rawlings JS. The JAK/STAT signaling pathway. J Cell Sci. 2004;117(8):1281-1283. doi:10.1242/jcs.00963.

297. Lim CP, Cao X. Structure, function, and regulation of STAT proteins. Mol Biosyst. 2006;2(11):536550. doi:10.1039/b606246f.

298. Haugh JM, Schooler K, Wells A, Wiley HS, Lauffenburger DA. Effect of epidermal growth factor receptor internalization on regulation of the phospholipase C-gamma1 signaling pathway. J Biol Chem. 1999;274(13):8958-8965.

http://www.ncbi.nlm.nih.gov/entrez/query.fcgi?cmd=Retrieve\&db=PubMed\&dopt=Citation\&list _uids=10085141.

299. Nogami M, Yamazaki M, Watanabe H, et al. Requirement of autophosphorylated tyrosine 992 of EGF receptor and its docking protein phospholipase $C \gamma 1$ for membrane ruffle formation. FEBS Lett. 2003;536(1-3):71-76. doi:10.1016/S0014-5793(03)00013-9.

300. Suh P-G, Park J-I, Manzoli L, et al. Multiple roles of phosphoinositide-specific phospholipase C isozymes. BMB Rep. 2008;41(6):415-434. doi:10.5483/BMBRep.2008.41.6.415.

301. Mochly-Rosen D, Das K, Grimes KK V. Protein kinase C, an elusive therapeutic target? Nat Rev Drug Discov. 2012;11(12):937-957. doi:10.1038/nrd3871.Protein.

302. Mackay HJ, Twelves CJ. Targeting the protein kinase $\mathrm{C}$ family: are we there yet? Nat Rev Cancer. 2007;7(7):554-562. doi:10.1038/nrc2168.

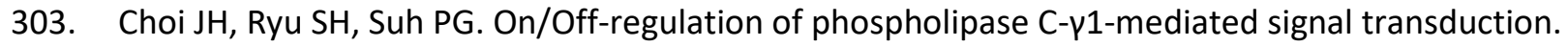
Adv Enzyme Regul. 2007;47:104-116. doi:10.1016/j.advenzreg.2006.12.010.

304. Choi JH. Phospholipase C- 1 is a guanine nucleotide exchange factor for dynamin-1 and enhances dynamin-1-dependent epidermal growth factor receptor endocytosis. J Cell Sci.

2004;117(17):3785-3795. doi:10.1242/jcs.01220.

305. Ahn JY, Ye K. PIKE GTPase signaling and function. Int J Biol Sci. 2005;1(2):44-50. doi:10.7150/ijbs.1.44.

306. Ye K, Hurt KJ, Wu FY, et al. Pike. A nuclear gtpase that enhances PI3kinase activity and is regulated by protein 4.1N. Cell. 2000;103(6):919-930. doi:10.1016/S0092-8674(00)00195-1.

307. Lazzara MJ, Lauffenburger DA. Quantitative modeling perspectives on the erbb system of cell regulatory processes. Exp Cell Res. 2009;315(4):717-725. doi:10.1016/j.yexcr.2008.10.033.

308. Wang Y, Falasca M, Schlessinger J, et al. Activation of the c-fos serum response element by phosphatidyl inositol 3-kinase and rho pathways in HeLa cells. Cell Growth Differ. 1998;9(7):513522. http://www.ncbi.nlm.nih.gov/pubmed/9690619.

309. Volinsky N, Kholodenko BN. Complexity of receptor tyrosine kinase signal processing. Cold Spring Harb Perspect Biol. 2013;5(8):a009043-a009043. doi:10.1101/cshperspect.a009043.

310. Antebi YE, Nandagopal N, Elowitz MB. An operational view of intercellular signaling pathways. Curr Opin Syst Biol. 2017;1:16-24. doi:10.1016/j.coisb.2016.12.003.

311. Lambert SA, Jolma A, Campitelli LF, et al. The Human Transcription Factors. Cell. 2018;172(4):650- 
665. doi:10.1016/j.cell.2018.01.029.

312. Spitz F, Furlong EEM. Transcription factors: From enhancer binding to developmental control. Nat Rev Genet. 2012;13(9):613-626. doi:10.1038/nrg3207.

313. Vaquerizas JM, Kummerfeld SK, Teichmann SA, Luscombe NM. A census of human transcription factors: Function, expression and evolution. Nat Rev Genet. 2009;10(4):252-263. doi:10.1038/nrg2538.

314. Murphy LO, MacKeigan JP, Blenis J. A network of immediate early gene products propagates subtle differences in mitogen-activated protein kinase signal amplitude and duration. $\mathrm{Mol} \mathrm{Cell}$ Biol. 2004;24(1):144-153. doi:10.1128/MCB.24.1.144.

315. Carpenter G, Ji QS. Phospholipase C-gamma as a signal-transducing element. Exp Cell Res. 1999;253(1):15-24. doi:10.1006/excr.1999.4671.

316. Zhang X, Tang N, Hadden TJ, Rishi AK. Akt, FoxO and regulation of apoptosis. Biochim Biophys Acta - Mol Cell Res. 2011;1813(11):1978-1986. doi:10.1016/j.bbamcr.2011.03.010.

317. Tullai JW, Schaffer ME, Mullenbrock S, Sholder G, Kasif S, Cooper GM. Immediate-Early and Delayed Primary Response Genes Are Distinct in Function and Genomic Architecture. J Biol Chem. 2007;282(33):23981-23995. doi:10.1074/jbc.M702044200.

318. Bahrami S, Drabløs F. Gene regulation in the immediate-early response process. Adv Biol Regul. 2016;62(7491):37-49. doi:10.1016/j.jbior.2016.05.001.

319. Vasudevan HN, Mazot P, He F, Soriano P. Receptor tyrosine kinases modulate distinct transcriptional programs by differential usage of intracellular pathways. Elife. 2015;4(MAY):1-22. doi:10.7554/eLife.07186.

320. Avraham R, Yarden Y. Feedback regulation of EGFR signalling: Decision making by early and delayed loops. Nat Rev Mol Cell Biol. 2011;12(2):104-117. doi:10.1038/nrm3048.

321. Trent Fowler, Ranjan Sen and ALR. Regulation of Primary Response Genes. 2012;44(3):617-636. doi:10.1016/j.molcel.2011.09.014.

322. Amit I, Citri A, Shay T, et al. A module of negative feedback regulators defines growth factor signaling. Nat Genet. 2007;39(4):503-512. doi:10.1038/ng1987.

323. Blobel CP. ADAMs: Key components in egfr signalling and development. Nat Rev Mol Cell Biol. 2005;6(1):32-43. doi:10.1038/nrm1548.

324. Schulze A, Lehmann K, Jefferies HBJ, McMahon M, Downward J. Analysis of the transcriptional program induced by Raf in epithelial cells. Genes Dev. 2001;15(8):981-994.

doi:10.1101/gad.191101.

325. Bae YS, Kang SW, Seo MS, et al. Epidermal growth factor (EGF)-induced generation of hydrogen peroxide. J Biol Chem. 1997;272(1):217-221. doi:10.1074/jbc.272.1.217.

326. Silva A, Gírio A, Cebola I, Santos Cl, Antunes F, Barata JT. Intracellular reactive oxygen species are essential for PI3K/Akt/mTOR-dependent IL-7-mediated viability of T-cell acute lymphoblastic leukemia cells. Leukemia. 2011;25(6):960-967. doi:10.1038/leu.2011.56.

327. Zhan Y, Virbasius J V., Song X, Pomerleau DP, Wayne Zhou G. The p40phox and p47phox PX 
domains of NADPH oxidase target cell membranes via direct and indirect recruitment by phosphoinositides. J Biol Chem. 2002;277(6):4512-4518. doi:10.1074/jbc.M109520200.

328. Rossi M a, Di Mauro C, Dianzani MU. Action of lipid peroxidation products on phosphoinositide specific phospholipase C. Mol Aspects Med. 1993;14(3):273-279.

http://www.ncbi.nlm.nih.gov/pubmed/8264343.

329. Tautz L, Critton DA, Grotegut S. Phosphatase Modulators. Vol 1053.; 2013. doi:10.1007/978-162703-562-0.

330. Xu Y, Tan L-J, Grachtchouk V, Voorhees JJ, Fisher GJ. Receptor-type Protein-tyrosine PhosphataseK Regulates Epidermal Growth Factor Receptor Function. J Biol Chem. 2005;280(52):4269442700. doi:10.1074/jbc.M507722200.

331. Spring K, Chabot C, Langlois S, et al. Tyrosine phosphorylation of DEP-1 / CD148 as a mechanism controlling Src kinase activation, endothelial cell permeability, invasion and capillary formation. Blood. 2012;120(13):2745-2756. doi:10.1182/blood-2011-12-398040.

332. Murphy JE, Padilla BE, Hasdemir B, Cottrell GS, Bunnett NW. Endosomes: A legitimate platform for the signaling train. Proc Natl Acad Sci. 2009;106(42):17615-17622. doi:10.1073/pnas.0906541106.

333. Frosi Y, Anastasi S, Ballarò C, et al. A two-tiered mechanism of EGFR inhibition by RALT/MIG6 via kinase suppression and receptor degradation. J Cell Biol. 2010;189(3):557-571. doi:10.1083/jcb.201002032.

334. Kovacs E, Zorn JA, Huang Y, Barros T, Kuriyan J. A Structural Perspective on the Regulation of the Epidermal Growth Factor Receptor. Annu Rev Biochem. 2015;84(1):739-764. doi:10.1146/annurev-biochem-060614-034402.

335. Laederich MB, Funes-Duran M, Yen L, et al. The leucine-rich repeat protein LRIG1 is a negative regulator of ErbB family receptor tyrosine kinases. J Biol Chem. 2004;279(45):47050-47056. doi:10.1074/jbc.M409703200.

336. Ledda F, Paratcha G. Negative Regulation of Receptor Tyrosine Kinase (RTK) Signaling: A Developing Field. Biomark Insights. 2007;2:45-58. http://www.ncbi.nlm.nih.gov/pubmed/19662191\%5Cnhttp://www.pubmedcentral.nih.gov/articl erender.fcgi?artid=PMC2717834.

337. Song MS, Salmena L, Pandolfi PP. The functions and regulation of the PTEN tumour suppressor. Nat Rev Mol Cell Biol. 2012;13(5):283-296. doi:10.1038/nrm3330.

338. Nakashima M, Adachi S, Yasuda I, et al. Inhibition of Rho-associated coiled-coil containing protein kinase enhances the activation of epidermal growth factor receptor in pancreatic cancer cells. Mol Cancer. 2011;10(1):79. doi:10.1186/1476-4598-10-79.

339. Schultheiss KP, Suga H, Ruiz-trillo II, Miller WT. Lack of Csk-mediated negative regulation in a unicellular Src kinase Lack of Csk-mediated negative regulation in a unicellular Src kinase. Biochemistry. 2012;51(41):8267-8277. doi:10.1021/bi300965h.

340. Murphy JM, Tannahill GM, Hilton DJ, Greenhalgh CJ. The Negative Regulation of Jak/Stat Signaling. Vol 2. Second Edi. Elsevier Inc.; 2010. doi:10.1016/B978-0-12-374145-5.00064-4. 
341. Jones HE, Gee JMW, Hutcheson IR, Knowlden JM, Barrow D, Nicholson RI. Growth factor receptor interplay and resistance in cancer. Endocr Relat Cancer. 2006;13(Supplement 1):S45-S51. doi:10.1677/erc.1.01275.

342. Guo A, Villen J, Kornhauser J, et al. Signaling networks assembled by oncogenic EGFR and c-Met. Proc Natl Acad Sci. 2008;105(2):692-697. doi:10.1073/pnas.0707270105.

343. Xu AM, Huang PH. RECEPTOR TYROSINE KINASE COACTIVATION NETWORKS IN CANCER. Cancer. 2010;70(10):3857-3860. doi:10.1158/0008-5472.CAN-10-0163.RECEPTOR.

344. Wiley HS, Burke PM. Regulation of receptor tyrosine kinase signaling by endocytic trafficking. Traffic. 2001;2(1):12-18. doi:10.1034/j.1600-0854.2001.020103.x.

345. Goh LK, Sorkin A. Endocytosis of receptor tyrosine kinases. Cold Spring Harb Perspect Biol. 2013;5(5):a017459. doi:10.1101/cshperspect.a017459.

346. Eichel $K$, Jullié $D$, von Zastrow M. $\beta$-Arrestin drives MAP kinase signalling from clathrin-coated structures after GPCR dissociation. Nat Cell Biol. 2016;18(3):303-310. doi:10.1038/ncb3307.

347. Resat H, Ewald JA, Dixon DA, Wiley HS. An integrated model of epidermal growth factor receptor trafficking and signal transduction. Biophys J. 2003;85(2):730-743. doi:10.1016/S00063495(03)74516-0.

348. Countaway JL, Nairn a C, Davis RJ. Mechanism of desensitization of the epidermal growth factor receptor protein-tyrosine kinase. J Biol Chem. 1992;267(2):1129-1140.

349. Huang F, Goh LK, Sorkin A. EGF receptor ubiquitination is not necessary for its internalization. Proc Natl Acad Sci U S A. 2007;104(43):16904-16909. doi:10.1073/pnas.0707416104.

350. Swaminathan G, Tsygankov AY. The Cbl family proteins: Ring leaders in regulation of cell signaling. J Cell Physiol. 2006;209(1):21-43. doi:10.1002/jcp.20694.

351. Volinsky N, Kholodenko BN. Complexity of Receptor Tyrosine Kinase Signal Processing. Cold Spring Harb Perspect Biol. 2013;5(8):a009043-a009043. doi:10.1101/cshperspect.a009043.

352. Huang F, Sorkin A. Growth factor receptor binding protein 2-mediated recruitment of the RING domain of $\mathrm{Cbl}$ to the epidermal growth factor receptor is essential and sufficient to support receptor endocytosis. Mol Biol Cell. 2005;16(3):1268-1281. doi:10.1091/mbc.e04-09-0832.

353. Wang Z, Moran MF. Requirement for the adapter protein GRB2 in EGF receptor endocytosis. Science. 1996;272(5270):1935-1939. http://www.ncbi.nlm.nih.gov/pubmed/8658166.

354. Liu NS, Loo LS, Loh E, Seet LF, Hong W. Participation of Tom1L1 in EGF-stimulated endocytosis of EGF receptor. EMBO J. 2009;28(22):3485-3499. doi:10.1038/emboj.2009.282.

355. Goh LK, Huang F, Kim W, Gygi S, Sorkin A. Multiple mechanisms collectively regulate clathrinmediated endocytosis of the epidermal growth factor receptor. J Cell Biol. 2010;189(5):871-883. doi:10.1083/jcb.201001008.

356. Gur G, Zwang Y, Yarden Y. Endocytosis of Receptor Tyrosine Kinases: Implications for Signal Transduction by Growth Factors. In: Madame Curie Bioscience Database. Austin (TX): Landes Bioscience. https://www.ncbi.nlm.nih.gov/books/NBK6589/.

357. Gresset A, Sondek J, Harden TK. The phospholipase C isozymes and their regulation. Subcell 
Biochem. 2012;58:61-94. doi:10.1007/978-94-007-3012-0_3.

358. Antonescu CN, McGraw TE, Klip A. Reciprocal regulation of endocytosis and metabolism. Cold Spring Harb Perspect Biol. 2014;6(7). doi:10.1101/cshperspect.a016964.

359. Kim J, Yang G, Kim Y, Kim J, Ha J. AMPK activators: Mechanisms of action and physiological activities. Exp Mol Med. 2016;48(4):e224-12. doi:10.1038/emm.2016.16.

360. Ross $E, A$ ta $R$, Thavarajah $T$, et al. AMP-activated protein kinase regulates the cell surface proteome and integrin membrane traffic. PLoS One. 2015;10(5):1-29.

doi:10.1371/journal.pone.0128013.

361. Antonescu CN, McGraw TE, Klip A. Reciprocal regulation of endocytosis and metabolism. Cold Spring Harb Perspect Biol. 2014;6(7):a016964. doi:10.1101/cshperspect.a016964.

362. Jeon SM. Regulation and function of AMPK in physiology and diseases. Exp Mol Med. 2016;48(7):e245. doi:10.1038/emm.2016.81.

363. Garcia D, Shaw RJ. AMPK: Mechanisms of Cellular Energy Sensing and Restoration of Metabolic Balance. Mol Cell. 2017;66(6):789-800. doi:10.1016/j.molcel.2017.05.032.

364. Vazquez A, Oltvai ZN. Molecular crowding defines a common origin for the warburg effect in proliferating cells and the lactate threshold in muscle physiology. PLoS One. 2011;6(4):1-9. doi:10.1371/journal.pone.0019538.

365. Hardie DG, Scott JW, Pan DA, Hudson ER. Management of cellular energy by the AMP-activated protein kinase system. FEBS Lett. 2003;546(1):113-120. doi:10.1016/S0014-5793(03)00560-X.

366. Herzig S, Shaw RJ. AMPK: Guardian of metabolism and mitochondrial homeostasis. Nat Rev Mol Cell Biol. 2018;19(2):121-135. doi:10.1038/nrm.2017.95.

367. Ke R, Xu Q, Li C, Luo L, Huang D. Mechanisms of AMPK in the maintenance of ATP balance during energy metabolism. Cell Biol Int. 2018;42(4):384-392. doi:10.1002/cbin.10915.

368. Ross FA, Jensen TE, Hardie DG. Differential regulation by AMP and ADP of AMPK complexes containing different subunit isoforms. Biochem J. 2016;473(2):189-199.

doi:10.1042/BJ20150910.

369. Richter EA, Ruderman NB. AMPK and the biochemistry of exercise: implications for human health and disease. Biochem J. 2009;418(2):261-275. doi:10.1042/BJ20082055.

370. Shan T, Xiong Y, Kuang S. Deletion of Lkb1 in adult mice results in body weight reduction and lethality. Sci Rep. 2016;6:1-9. doi:10.1038/srep36561.

371. Takai Y, Sasaki T, Matozaki T. Small GTP-binding proteins. Physiol Rev. 2001;81(1):153-208.

372. Cherfils J, Zeghouf M. Regulation of Small GTPases by GEFs, GAPs, and GDIs. Physiol Rev. 2013;93(1):269-309. doi:10.1152/physrev.00003.2012.

373. Donaldson JG, Johnson DL, Dutta D. Rab and Arf G proteins in endosomal trafficking and cell surface homeostasis. Small GTPases. 2016;7(4):247-251. doi:10.1080/21541248.2016.1212687.

374. Casalou C, Faustino A, Barral DC. Arf proteins in cancer cell migration. Small GTPases. 2016;7(4):270-282. doi:10.1080/21541248.2016.1228792. 
375. Donaldson JG. Multiple Roles for Arf6: Sorting, Structuring, and Signaling at the Plasma Membrane. J Biol Chem. 2003;278(43):41573-41576. doi:10.1074/jbc.R300026200.

376. Gillingham AK, Munro S. The Small G Proteins of the Arf Family and Their Regulators. Annu Rev Cell Dev Biol. 2007;23(1):579-611. doi:10.1146/annurev.cellbio.23.090506.123209.

377. Pasqualato S, Renault L, Cherfils J. Arf, Arl, Arp and Sar proteins: A family of GTP-binding proteins with a structural device for "front-back" communication. EMBO Rep. 2002;3(11):1035-1041. doi:10.1093/embo-reports/kvf221.

378. Hayakawa N, Ogoh $\mathrm{H}$, Sumiyoshi $\mathrm{M}$, et al. The ADP-ribosylation factor 1 gene is indispensable for mouse embryonic development after implantation. Biochem Biophys Res Commun. 2014;453(4):748-753. doi:10.1016/j.bbrc.2014.10.014.

379. Paleotti O, Macia E, Luton F, et al. The small G-protein Arf6GTP recruits the AP-2 adaptor complex to membranes. J Biol Chem. 2005;280(22):21661-21666. doi:10.1074/jbc.M503099200.

380. Jackson CL, Bouvet S. Arfs at a Glance. J Cell Sci. 2014;127(19):4103-4109. doi:10.1242/jcs.144899.

381. Sauvageau E, McCormick PJ, Lefrancois S. In vivo monitoring of the recruitment and activation of AP-1 by Arf1. Sci Rep. 2017;7(1):1-14. doi:10.1038/s41598-017-07493-1.

382. Schweitzer JK, Sedgwick AE, D'Souza-Schorey C. ARF6-mediated endocytic recycling impacts cell movement, cell division and lipid homeostasis. Semin Cell Dev Biol. 2011;22(1):39-47. doi:10.1016/j.semcdb.2010.09.002.

383. Poupart M, Fessart D, Cotton M, Laporte SA, Claing A. ARF6 regulates angiotensin II type 1 receptor endocytosis by controlling the recruitment of AP-2 and clathrin. Cell Signal. 2007;19(11):2370-2378. doi:10.1016/j.cellsig.2007.07.015.

384. D'Souza-Schorey C, Chavrier P. ARF proteins: Roles in membrane traffic and beyond. Nat Rev Mol Cell Biol. 2006;7(5):347-358. doi:10.1038/nrm1910.

385. Miyamoto T, Oshiro N, Yoshino K, et al. AMP-activated Protein Kinase Phosphorylates Golgispecific Brefeldin A Resistance Factor 1 at Thr ${ }^{1337}$ to Induce Disassembly of Golgi Apparatus. J Biol Chem. 2008;283(7):4430-4438. doi:10.1074/jbc.M708296200.

386. Marin TL, Gongol B, Martin M, et al. Identification of AMP-activated protein kinase targets by a consensus sequence search of the proteome. BMC Syst Biol. 2015;9(1):1-12. doi:10.1186/s12918015-0156-0.

387. Bridgewater RE, Norman JC, Caswell PT. Integrin trafficking at a glance. J Cell Sci. 2012;125(16):3695-3701. doi:10.1242/jcs.095810.

388. Ratcliffe CDH, Sahgal P, Parachoniak CA, Ivaska J, Park M. Regulation of Cell Migration and $\beta 1$ Integrin Trafficking by the Endosomal Adaptor GGA3. Traffic. 2016;17(6):670-688.

doi:10.1111/tra.12390.

389. Barczyk M, Carracedo S, Gullberg D. Integrins. Cell Tissue Res. 2010;339(1):269-280. doi:10.1007/s00441-009-0834-6.

390. Harburger DS, Calderwood DA. Integrin signalling at a glance. J Cell Sci. 2009;122(9):1472-1472. doi:10.1242/jcs.052910. 
391. Dunphy JL, Moravec R, Ly K, Lasell TK, Melancon P, Casanova JE. The Arf6 GEF GEP100/BRAG2 regulates cell adhesion by controlling endocytosis of $\beta 1$ integrins. Curr Biol. 2006;16(3):315-320. doi:10.1016/j.cub.2005.12.032.

392. Paul NR, Jacquemet G, Caswell PT. Endocytic Trafficking of Integrins in Cell Migration. Curr Biol. 2015;25(22):R1092-R1105. doi:10.1016/j.cub.2015.09.049.

393. Chen PW, Luo R, Jian X, Randazzo PA. The Arf6 GTPase-activating proteins ARAP2 and ACAP1 define distinct endosomal compartments that regulate integrin $\alpha 5 \beta S 1$ traffic. J Biol Chem. 2014;289(44):30237-30248. doi:10.1074/jbc.M114.596155.

394. Klein S, Franco M, Chardin P, Luton F. Role of the Arf6 GDP/GTP cycle and Arf6 GTPase-activating proteins in actin remodeling and intracellular transport. J Biol Chem. 2006;281(18):12352-12361. doi:10.1074/jbc.M601021200.

395. Son S, Moroney GJ, Butler PJ. $\beta 1$-Integrin-Mediated Adhesion Is Lipid-Bilayer Dependent. Biophys J. 2017;113(5):1080-1092. doi:10.1016/j.bpj.2017.07.010.

396. Aramburu J, Yaffe MB, López-Rodríguez C, Cantley LC, Hogan PG, Rao A. Affinity-driven peptide selection of an NFAT inhibitor more selective than cyclosporin A. Science (80- ). 1999. doi:10.1126/science.285.5436.2129.

397. Reis CR, Chen P, Srinivasan S, Aguet F, Mettlen M, Schmid SL. Crosstalk between Akt / GSK 3 b signaling and dynamin- 1 regulates clathrin-mediated endocytosis. EMBO J. 2015. doi:10.15252/embj.

398. Hardwick JC. Ionic mechanisms of histamine-induced responses in guinea pig intracardiac neurons. AJP Regul Integr Comp Physiol. 2006;290(1):R241-R250. doi:10.1152/ajpregu.00498.2005.

399. Wijesekara N, Tung A, Thong F, Klip A. Muscle cell depolarization induces a gain in surface GLUT4 via reduced endocytosis independently of AMPK. Am J Physiol Endocrinol Metab. 2006. doi:10.1152/ajpendo.00573.2005.

400. Reis CR, Chen P-H, Bendris N, Schmid SL. TRAIL-death receptor endocytosis and apoptosis are selectively regulated by dynamin-1 activation. Proc Natl Acad Sci. 2017. doi:10.1073/pnas.1615072114.

401. Miyamoto S, Izumi M, Hori M, Kobayashi M, Ozaki H, Karaki H. Xestospongin C, a selective and membrane-permeable inhibitor of IP3receptor, attenuates the positive inotropic effect of $\alpha$ adrenergic stimulation in guinea-pig papillary muscle. Br J Pharmacol. 2000. doi:10.1038/sj.bjp.0703358.

402. Ikenouchi J, Umeda M. FRMD4A regulates epithelial polarity by connecting Arf6 activation with the PAR complex. Proc Natl Acad Sci. 2010;107(2):748-753. doi:10.1073/pnas.0908423107.

403. Schneider CA, Rasband WS, Eliceiri KW. NIH Image to ImageJ: 25 years of image analysis. Nat Methods. 2012;9(7):671-675. doi:10.1038/nmeth.2089.

404. Mettlen M, Danuser G. Imaging and modeling the dynamics of clathrin-mediated endocytosis. Cold Spring Harb Perspect Biol. 2014;6(12):a017038. doi:10.1101/cshperspect.a017038.

405. Jaqaman $\mathrm{K}$, Loerke $\mathrm{D}$, Mettlen $\mathrm{M}$, et al. Robust single particle tracking in live cell time-lapse 
sequences. Nat Methods. 2008;5(8):695-702. doi:10.1038/nmeth.1237.Robust.

406. Antonescu CR, Besmer P, Guo T, et al. Acquired Resistance to Imatinib in Gastrointestinal Stromal Tumor Occurs Through Secondary Gene Mutation Cancer Therapy : Preclinical Acquired Resistance to Imatinib in Gastrointestinal Stromal Tumor Occurs Through Secondary Gene Mutation. 2005;11(11):4182-4190. doi:10.1158/1078-0432.CCR-04-2245.

407. Wong R, Fabian L, Forer A, Brill JA. Phospholipase $C$ and myosin light chain kinase inhibition define a common step in actin regulation during cytokinesis. BMC Cell Biol. 2007;8:1-10. doi:10.1186/1471-2121-8-15.

408. Klein RR, Bourdon DM, Costales CL, et al. Direct activation of human phospholipase $\mathrm{C}$ by its well known inhibitor u73122. J Biol Chem. 2011;286(14):12407-12416. doi:10.1074/jbc.M110.191783.

409. Sigismund S, Argenzio E, Tosoni D, Cavallaro E, Polo S, Di Fiore PP. Clathrin-Mediated Internalization Is Essential for Sustained EGFR Signaling but Dispensable for Degradation. Dev Cell. 2008;15(2):209-219. doi:10.1016/j.devcel.2008.06.012.

410. Choi JH, Park JB, Bae SS, et al. Phospholipase C-gamma1 is a guanine nucleotide exchange factor for dynamin-1 and enhances dynamin-1-dependent epidermal growth factor receptor endocytosis. J Cell Sci. 2004. doi:10.1242/jcs.01220.

411. Wong R, Hadjiyanni I, Wei HC, et al. PIP2 hydrolysis and calcium release are required for cytokinesis in Drosophila spermatocytes. Curr Biol. 2005;15(15):1401-1406. doi:10.1016/j.cub.2005.06.060.

412. Mattheyses AL, Simon SM, Rappoport JZ. Imaging with total internal reflection fluorescence microscopy for the cell biologist. J Cell Sci. 2010;123(Pt 21):3621-3628. doi:10.1242/jcs.056218.

413. Axelrod D. Total internal reflection fluorescence microscopy in cell biology. Methods Enzymol. 2003;361(2):1-33. http://www.ncbi.nlm.nih.gov/pubmed/12624904.

414. Schneidereit D, Vass H, Reischl B, Allen RJ, Friedrich O. Calcium sensitive fluorescent dyes fluo-4 and fura red under pressure: Behaviour of fluorescence and buffer properties under hydrostatic pressures up to 200 MPa. PLoS One. 2016;11(10):1-18. doi:10.1371/journal.pone.0164509.

415. Fu T, Xu Y, Jiang $W$, Zhang $H$, Zhu P, Wu J. EGF receptor-mediated intracellular calcium increase in human hepatoma BEL-7404 cells*. Cell Res. 1994. doi:10.1038/cr.1994.15.

416. Bryant JA, Finn RS, Slamon DJ, Cloughesy TF, Charles AC. EGF activates intracellular and intercellular calcium signaling by distinct pathways in tumor cells. Cancer Biol Ther. 2004;3(12):1243-1249. doi:10.4161/cbt.3.12.1233.

417. Schaloske R, Schlatterer C, Malchow D, Neuron IN. A Xestospongin C-sensitive Ca 2 \%. Store Is Required for CAMP- induced Ca $2 \%$ Influx and cAMP Oscillations in Dictyostelium *. 2000;275(12):8404-8408. doi:10.1074/JBC.275.12.8404.

418. Oka T, Sato K, Hori M, Ozaki H, Karaki H. Xestospongin C, a novel blocker of IP3receptor, attenuates the increase in cytosolic calcium level and degranulation that is induced by antigen in RBL-2H3 mast cells. Br J Pharmacol. 2002. doi:10.1038/sj.bjp.0704662.

419. Jameson D. Ion probes. In: Introduction to Fluorescence. 1st ed. Boca Raton, FL: Taylor \& Francis; 2014:233-234. https://books.google.ca/books?id=SI- 
IAgAAQBAJ\&pg=PA233\&lpg=PA233\&dq=BAPTA+binds+two+calcium+ions\&source=bl\&ots=bzYnE ODb9j\&sig=aA-2WcWQBQTASz_bpbAsekEQHpU\&hl=en\&sa=X\&ved=2ahUKEwjGxu-

XwbbdAhUF3IMKHfA_BagQ6AEwBXoECAMQAQ\#v=onepage\&q=BAPTA binds two calcium io.

420. Matsuda S, Koyasu S. Mechanisms of action of cyclosporine. Immunopharmacology. 2000;47(23):119-125. doi:10.1016/S0162-3109(00)00192-2.

421. Parmentier JH, Ahmed A, Ruan Y, Gandhi GK, Saeed AE, Malik KU. Calcium and protein kinase $C(P K C)$-related kinase mediate $1 \mathrm{~A}$ - adrenergic receptor-stimulated activation of phospholipase D in rat-1 cells, independent of PKC. J Pharmacol Exp Ther. 2002;303(3):1206-1215. doi:10.1124/jpet.102.041384.been.

422. Gould CM, Antal CE, Reyes G, et al. Active site inhibitors protect protein kinase $C$ from dephosphorylation and stabilize its mature form. J Biol Chem. 2011;286(33):28922-28930. doi:10.1074/jbc.M111.272526.

423. Tosoni D, Puri C, Confalonieri S, et al. TTP specifically regulates the internalization of the transferrin receptor. Cell. 2005;123(5):875-888. doi:10.1016/j.cell.2005.10.021.

424. Rigó G, Papdi C, Szabados L. Transformation using controlled cDNA overexpression system. Methods Mol Biol. 2012;913(1):277-290. doi:10.1007/978-1-61779-986-0_19.

425. Prelich G. Gene overexpression: Uses, mechanisms, and interpretation. Genetics. 2012;190(3):841-854. doi:10.1534/genetics.111.136911.

426. Göransson O, McBride A, Hawley SA, et al. Mechanism of action of A-769662, a valuable tool for activation of AMP-activated protein kinase. J Biol Chem. 2007;282(45):32549-32560. doi:10.1074/jbc.M706536200.

427. Sanders MJ, Ali ZS, Hegarty BD, Heath R, Snowden MA, Carling D. Defining the mechanism of activation of AMP-activated protein kinase by the small molecule A-769662, a member of the thienopyridone family. J Biol Chem. 2007;282(45):32539-32548. doi:10.1074/jbc.M706543200.

428. Becker W. Fluorescence lifetime imaging - techniques and applications. J Microsc. 2012;247(2):119-136. doi:10.1111/j.1365-2818.2012.03618.x.

429. Day RN, Davidson MW. Fluorescent proteins for FRET microscopy: monitoring protein interactions in living cells. Bioessays. 2013;34(5):341-350. doi:10.1002/bies.201100098. Fluorescent.

430. Hall B, McLean MA, Davis K, Casanova JE, Sligar SG, Schwartz MA. A fluorescence resonance energy transfer activation sensor for Arf6. Anal Biochem. 2008;374(2):243-249. doi:10.1016/j.ab.2007.11.032.

431. Bajar BT, Wang ES, Zhang S, Lin MZ, Chu J. A guide to fluorescent protein FRET pairs. Sensors (Switzerland). 2016;16(9):1-24. doi:10.3390/s16091488.

432. Benabdi S, Peurois F, Nawrotek A, et al. Family-wide Analysis of the Inhibition of Arf Guanine Nucleotide Exchange Factors with Small Molecules: Evidence of Unique Inhibitory Profiles. Biochemistry. 2017;56(38):5125-5133. doi:10.1021/acs.biochem.7b00706.

433. Bi X, Schmitz A, Hayallah AM, Song JN, Famulok M. Affinity-based labeling of cytohesins with a bifunctional SecinH3 photoaffinity probe. Angew Chemie - Int Ed. 2008;47(49):9565-9568. 
doi:10.1002/anie.200803962.

434. Teckchandani A, Toida N, Goodchild J, et al. Quantitative proteomics identifies a Dab2/integrin module regulating cell migration. J Cell Biol. 2009;186(1):99-111. doi:10.1083/jcb.200812160.

435. Wu L-G, Hamid E, Shin W, Chiang H-C. Exocytosis and Endocytosis: Modes, Functions, and Coupling Mechanisms. Annu Rev Physiol. 2014;76(1):301-331. doi:10.1146/annurev-physiol021113-170305.

436. Irie $\mathrm{F}$, Okuno $\mathrm{M}$, Pasquale EB, Yamaguchi Y. EphrinB-EphB signalling regulates clathrin-mediated endocytosis through tyrosine phosphorylation of synaptojanin 1. Nat Cell Biol. 2005;7(5):501509. doi:10.1038/ncb1252.

437. Lee SY, Wenk MR, Kim Y, Nairn AC, De Camilli P. Regulation of synaptojanin 1 by cyclindependent kinase 5 at synapses. Proc Natl Acad Sci. 2004;101(2):546-551. doi:10.1073/pnas.0307813100.

438. Wu P-C, Tai M-H, Hu D-N, et al. Cyclin-dependent kinase inhibitor roscovitine induces cell cycle arrest and apoptosis in rabbit retinal pigment epithelial cells. J Ocul Pharmacol Ther. 2008;24(1). doi:10.1089/jop.2007.0044.

439. Tang D, Yeung J, Lee KY, et al. An isoform of the neuronal cyclin-dependent kinase 5 (Cdk5) activator. J Biol Chem. 1995;270(45):26897-26903. doi:10.1074/jbc.270.45.26897.

440. Zhen X, Goswami S, Abdali SA, Gil M, Bakshi K, Friedman E. Regulation of Cyclin-Dependent Kinase 5 and Calcium / Calmodulin-Dependent Protein Kinase II by Phosphatidylinositol-Linked Dopamine Receptor in Rat Brain. Mol Pharmacol. 2004;66(6):1500-1507. doi:10.1124/mol.104.002279.receptors.

441. Mooibroek MJ, Michiel DF, Wang JH. Clathrin light chains are calcium-binding proteins. J Biol Chem. 1987;262(1):25-28.

442. Nathke I, Hill BL, Parham P, Brodsky FM. The calcium-binding site of clathrin light chains. J Biol Chem. 1990;265(30):18621-18627.

443. Creutz CE, Snyder SL. Interactions of annexins with the mu subunits of the clathrin assembly proteins. Biochemistry. 2005;44(42):13795-13806. doi:10.1021/bi051160w.

444. Cornely R, Rentero C, Enrich C, Grewal T, Gaus K. Annexin A6 is an organizer of membrane microdomains to regulate receptor localization and signalling. IUBMB Life. 2011;63(11):10091017. doi:10.1002/iub.540.

445. Myers MD, Ryazantsev S, Hicke L, Payne GS. Calmodulin Promotes N-BAR Domain-Mediated Membrane Constriction and Endocytosis. Dev Cell. 2016;37(2):162-173. doi:10.1016/j.devcel.2016.03.012.

446. Caldieri G, Barbieri E, Nappo G, et al. Reticulon 3-dependent ER-PM contact sites control EGFR nonclathrin endocytosis. Science. 2017;356(6338):617-624. doi:10.1126/science.aah6152.

447. Huang F, Khvorova A, Marshall W, Sorkin A. Analysis of Clathrin-mediated Endocytosis of Epidermal Growth Factor Receptor by RNA Interference. J Biol Chem. 2004;279(16):16657-16661. doi:10.1074/jbc.C400046200.

448. Rappoport JZ, Simon SM. Endocytic trafficking of activated EGFR is AP-2 dependent and occurs 
through preformed clathrin spots. J Cell Sci. 2009;122(Pt 9):1301-1305. doi:10.1242/jcs.040030.

449. Keyel PA, Mishra SK, Roth R, Heuser JE, Watkins SC, Traub LM. A single common portal for clathrin-mediated endocytosis of distinct cargo governed by cargo-selective adaptors. Mol Biol Cell. 2006;17(10):4300-4317. doi:10.1091/mbc.e06-05-0421.

450. Lamaze C, Baba T, Redelmeier TE, Schmid SL. Recruitment of epidermal growth factor and transferrin receptors into coated pits in vitro: differing biochemical requirements. Mol Biol Cell. 1993;4(7):715-727. doi:10.1091/mbc.4.7.715.

451. Leonard D, Hayakawa A, Lawe D, et al. Sorting of EGF and transferrin at the plasma membrane and by cargo-specific signaling to EEA1-enriched endosomes. J Cell Sci. 2008. doi:10.1242/jcs.031484.

452. Johannessen LE, Pedersen NM, Winther K, Madshus IH, Stang E, Pedersen KW. Activation of the epidermal growth factor ( EGF) receptor induces formation of EGF receptor- and Grb2-containing clathrin-coated pits. Mol Cell Biol. 2006;26(2):389-401. doi:10.1128/MCB.26.2.389.

453. Mettlen M, Loerke D, Yarar D, Danuser G, Schmid SL. Cargo- and adaptor-specific mechanisms regulate clathrin-mediated endocytosis. J Cell Biol. 2010;188(6):919-933. doi:10.1083/jcb.200908078.

454. Ji QS, Ermini S, Baulida J, Sun FL, Carpenter G. Epidermal growth factor signaling and mitogenesis in Plcg1 null mouse embryonic fibroblasts. Mol Biol Cell. 1998;9(4):749-757.

455. Gusarova GA, Trejo HE, Dada LA, et al. Hypoxia Leads to Na,K-ATPase Downregulation via Ca2+ Release-Activated Ca2+ Channels and AMPK Activation. Mol Cell Biol. 2011;31(17):3546-3556. doi:10.1128/MCB.05114-11.

456. Waldhart AN, Dykstra H, Peck AS, et al. Phosphorylation of TXNIP by AKT Mediates Acute Influx of Glucose in Response to Insulin. Cell Rep. 2017;19(10):2005-2013. doi:10.1016/j.celrep.2017.05.041.

457. Khundmiri SJ, Bertorello AM, Delamere NA, Lederer ED. Clathrin-mediated Endocytosis of $\mathrm{Na}+, \mathrm{K}+-\mathrm{ATP} a \mathrm{se}$ in Response to Parathyroid Hormone Requires ERK-dependent Phosphorylation of Ser-11 within the a1-Subunit. J Biol Chem. 2004;279(17):17418-17427. doi:10.1074/jbc.M311715200.

458. Rangaraju V, Calloway N, Ryan TA. Activity-driven local ATP synthesis is required for synaptic function. Cell. 2014;156(4):825-835. doi:10.1016/j.cell.2013.12.042.

459. Weigel A V., Tamkun MM, Krapf D. Quantifying the dynamic interactions between a clathrincoated pit and cargo molecules. Proc Natl Acad Sci. 2013;110(48):E4591-E4600. doi:10.1073/pnas.1315202110.

460. Mishra AK, Lambright DG. Invited review: Small GTPases and their GAPs. Biopolymers. 2016;105(8):431-448. doi:10.1002/bip.22833.

461. Dembla M, Wahl S, Katiyar R, Schmitz F. ArfGAP3 Is a Component of the Photoreceptor Synaptic Ribbon Complex and Forms an NAD(H)-Regulated, Redox-Sensitive Complex with RIBEYE That Is Important for Endocytosis. J Neurosci. 2014;34(15):5245-5260. doi:10.1523/JNEUROSCI.383713.2014. 
462. Wu N, Zheng B, Shaywitz A, et al. AMPK-Dependent Degradation of TXNIP upon Energy Stress Leads to Enhanced Glucose Uptake via GLUT1. Mol Cell. 2013;49(6):1167-1175. doi:10.1016/j.molcel.2013.01.035.

463. Gusarova GA, Dada LA, Kelly AM, et al. 1-AMP-Activated Protein Kinase Regulates HypoxiaInduced Na,K-ATPase Endocytosis via Direct Phosphorylation of Protein Kinase C. Mol Cell Biol. 2009;29(13):3455-3464. doi:10.1128/MCB.00054-09.

464. Teckchandani A, Mulkearns EE, Randolph TW, Toida N, Cooper JA. The clathrin adaptor Dab2 recruits $\mathrm{EH}$ domain scaffold proteins to regulate integrin 1 endocytosis. Mol Biol Cell. 2012;23(15):2905-2916. doi:10.1091/mbc.E11-12-1007.

465. Ata R, Antonescu CN. Integrins and cell metabolism: An intimate relationship impacting cancer. Int J Mol Sci. 2017;18(1). doi:10.3390/ijms18010189.

466. Leung E, Hung JM, Barker D, Reynisson J. The effect of a thieno[2,3-b]pyridine PLC- $p$ inhibitor on the proliferation, morphology, migration and cell cycle of breast cancer cells. Med Chem Commun. 2014;5(1):99-106. doi:10.1039/C3MD00290J.

467. Dhavan R, Tsai LH. A decade of CDK5. Nat Rev Mol Cell Biol. 2001;2(10):749-759. doi:10.1038/35096019.

468. Cicenas J, Kalyan K, Valius M. Roscovitine in cancer and other diseases Cellular effects and preclinical tests. 2015;9(19):1-12. doi:10.2210/pdb2a4l/pdb.

469. Odemuyiwa SO, Ilarraza R, Davoine F, et al. Cyclin-dependent kinase 5 regulates degranulation in human eosinophils. Immunology. 2015;144(4):641-648. doi:10.1111/imm.12416.

470. Kobayashi H, Saito T, Sato K, et al. Phosphorylation of Cyclin-dependent kinase 5 (Cdk5) at Tyr-15 is inhibited by $\mathrm{Cdk} 5$ activators and does not contribute to the activation of Cdk5. J Biol Chem. 2014;289(28):19627-19636. doi:10.1074/jbc.M113.501148.

471. Yoo JH, Shi DS, Grossmann AH, et al. ARF6 Is an Actionable Node that Orchestrates Oncogenic GNAQ Signaling in Uveal Melanoma. Cancer Cell. 2016;29(6):889-904. doi:10.1016/j.ccell.2016.04.015.

472. Roux KJ, Kim DI, Burke B. BiolD: A Screen for Protein-Protein Interactions. In: Current Protocols in Protein Science. Vol 74. Hoboken, NJ, USA: John Wiley \& Sons, Inc.; 2013:19.23.1-19.23.14. doi:10.1002/0471140864.ps1923s74.

473. Liu X, Salokas K, Tamene F, et al. An AP-MS- and BiolD-compatible MAC-tag enables comprehensive mapping of protein interactions and subcellular localizations. Nat Commun. 2018;9(1). doi:10.1038/s41467-018-03523-2.

474. Hesketh GG, Youn J-Y, Samavarchi-Tehrani P, Raught B, Gingras A-C. Parallel Exploration of Interaction Space by BiolD and Affinity Purification Coupled to Mass Spectrometry. Methods Mol Biol. 2017;1550:115-136. doi:10.1007/978-1-4939-6747-6_10.

475. Ata R. Regulation of $\beta 1$ integrin traffic and Arf6 GTPase by AMP-activated protein kinase. 2016:114.

476. Kinoshita E, Kinoshita-Kikuta E, Koike T. Separation and detection of large phosphoproteins using phos-tag sds-page. Nat Protoc. 2009;4(10):1513-1521. doi:10.1038/nprot.2009.154. 
477. Horinouchi T, Terada K, Higashi T, Miwa S. Using Phos-Tag in Western Blotting Analysis to Evaluate Protein Phosphorylation. Methods Mol Biol. 2016;1397:267-277. doi:10.1007/978-14939-3353-2_18.

478. O'Leary B, Rao SK, Plaxton WC. Phosphorylation of bacterial-type phosphoenolpyruvate carboxylase at Ser 425 provides a further tier of enzyme control in developing castor oil seeds. Biochem J. 2011;433(1):65-74. doi:10.1042/BJ20101361. 